\title{
Final Program \\ The International Neuropsychological Society, Federation of Spanish Societies of Neuropsychology, Spanish Neuropsychological Society, Spanish Psychiatry Society Joint Mid-Year Meeting \\ July 4-7, 2007 \\ Bilbao, Spain
}

WEDNESDAY, JULY 4, 2007

10:00 AM-1:00 PM $\begin{aligned} & \text { Morning Continuing Education Workshops } \\ & \text { Refer to CE Program for room assignments }\end{aligned}$

1:00-3:00 PM Lunch Break

on your own

3:00-6:00 PM Afternoon Continuing Education Workshops

Refer to CE Program for room assignments

6:00-6:30 PM Coffee Break

Hall Exposiciones

6:30-8:00 PM

Symposium 1

Obsessive-Compulsive Disorder : From Neurobiology to Neurocognition.

Chair: Jose M Menchon

Sala A4

1. MENCHON MAGRIÑA, J

2. SORIANO-MAS, C

3. DEL PINO ALONSO, M

4. MATAIX-COLS, D

5. SEGALÀS, C

6:30-8:00 PM

1. FLETCHER, JM

2. PAPANICOLAOU, AC

3. CASTILLO, EM

4. JURANEK, JJ

5. FLETCHER, JM

6:30-8:00 PM
Obsessive-Compulsive Disorder: From Neurobiology to Neurocognition.

Neuroanatomical Models of Obsessive-Compulsive Disorder.

Genetic Vulnerability and Neurocognition in Obsessive-Compulsive Disorder.

Symptom Dimensions in Obsessive-Compulsive Disorder and Neuropsychological Performance.

Verbal and Non-Verbal Memory Processing in Obsessive-Compulsive Disorder.

Symposium 2

Neuroimaging Studies of Neural Plasticity: New Modalities and Methods in Focal,

Generalized, and Developmental Brain Disorders

Chair: Jack Fletcher

Sala 43

Neuroimaging studies of neural plasticity: New modalities and methods in focal, generalized, and developmental brain disorders.

Representation of Receptive Language Functions: Relations with Age, Gender, Task, and Mode of Stimulus Presentation.

Aberrant Cortical Functionality of Sensorimotor and Linguistic Networks in Stroke.

Anatomical and Functional Differences in Children with Spina Bifida: MRI and MEG Studies.

Neural Reorganization in Spina Bifida: Evidence from Diffusion Tensor Imaging.

Symposium 3

Literacy and Metalinguistic Awareness in Different Languages

Chair: Esmeralda Matute

Sala $A 2$

Literacy and Metalinguistic Awareness in Different Languages.

Phonological and Orthographic Word Processing in Children with Hyperlexia.

Phonological Awareness and Reading Skills in Spanish-Speaking Children.

Playa, blaya, mlaya. The Influence of Item Type in the Spanish Dyslexics' Reading.

Morphological Awareness and Writing: A Comparison of Spanish- and French-Speaking Children. 


\title{
THURSDAY, JULY 5, 2007
}

\author{
9:00-9:45 AM \\ Invited Plenary: Aging and Neurocognitive Plasticity \\ Speaker: Art Kramer \\ Sala A1
}

9:00-11:00 AM

1. APPELS, B

2. BARROSO. J

3. BERARDI, A

4. BRUNA, O

5. GIANNAKOU, M

6. HIRD, KM

7. LEATHEM, J

8. LeSueur, L

9. LLANERO, M

10. LLEBARIA, G

11. MACEDO, EC

12. MALLOY-DINIZ, LF

13. MALLOY-DINIZ, LF

\section{Poster Session 1 \\ Assessment, Cross Cultural Issues, Forensics, Emotion \\ Hall Exposiciones}

\section{Assessment/Psychometrics}

The Diagnostic Accuracy of Dementia Screening Instruments with an Administration Time of Ten to Thirty Minutes: a Systematic Review.

Verbal fluency: practice effects and use of alternate forms in serial neuropsychological assessment.

Virtual Reality-related Sickness Symptoms in Neurological Patients.

Early detection of cognitive impairment. Adaptation of the Alzheimer's Quick Test to the Spanish population.

Developing a Visual Test Examining Mentalizing Abilities for Psychiatric and Neurological Patients.

Should Aphasia Diagnosis be Shaped for Evidence Based Practise?

How many know the date and why: A preliminary evaluation.

Confusion in learning dysfunction classification: Toward a tighter theory.

Preliminary data to standardize an easy-administration Go-NoGo Task in normal aging, mild cognitive impairment, and Alzheimer's disease.

Utility of the Mattis Dementia Rating Scale for screening dementia in Parkinson's disease. Validation of the instrument in a Spanish population.

Neuropsychological assessment of developmental dyslexia: Psychometric tests and eye movements.

Brazilian Adaptation of Iowa Gambling Task.

Performance of Brazilian Children on the Tower of London Test: comparison by age, gender and scholar attendance.

14. MARTIN ARAGONESES, M A Cognitive Approach to the Assessment of Sentence Comprehension: the ECCO Test.

15. RUIZ, J Discriminant analysis on screening dementia tests in the Spanish: usefulness to early diagnose mild cognitive impairment and Alzheimer's disease.

16. PINO, O

Is the SCIP-S an adequate tool for

17. PINO, O

Is the SCIP-S a reliable tool for a neuropsychological brief assessment?

18. RUIZ, J

19. SANCHEZ-CUBILLO, I

Early Alzheimer's disease diagnose and mild cognitive impairment: How useful is the Trail Making Test in the Spanish?

The validity of Trail Making Test in assessing executive functions: contributions of cognitive and perceptual factors. Multipart models produce visual segmentations and verbal confabulations.

"Five Digit Test": A digital version of the Stroop test appropriate for multicultural use.

"Kiddy Words": Five little steps on the lexical road.

The Factor Structure of the Coughlan Adult Memory \& Information Processing Battery (AMIPB).

The Neuropsychological profile of Diffuse Traumatic Axonal Injury using an Automated Test Battery: Insights from the Brain Resource International Database.

Effects of Stimulus Repetition and Recall Order on Spatial Span Performance.

\section{Cross-Cultural Issues}

Writing Acquisition in a Bilingual Setting: Mutual Interferences of Portuguese and German.

Self-awareness and cultural influence in a normal sample.

Analysis the use or presence of self regulation when otomi children carried out a reading comprehension activity with expositive texts.

7. RAMÍREZ, M

28. SALVADOR, J

29. SAN MIGUEL-MONTES, L Verbal Memory and Executive Functions of Puerto Ricans with Parkinson's, Traumatic Brain Injury, Epilepsy, Mild Cognitive Impairment, Dementia and Cerebrovascular disease.

\section{Cross-Cultural Test Development}

30. GORISSEN, M

31. JOHNSON, LG

32. MIRANDA, MC

The Use of Effort Tests in non native speakers: Risk for False Positives with the French WMT?

CNS Vital Signs (CNSVS): A Valid and Reliable Computerized Neuropsychological Battery for the Evaluation of Mild Cognitive Impairment.

Are the Computerized and Manual Versions of the Wisconsin Card Sorting Test Equivalent? A Comparative Preliminary Study on Samples of North American and Brazilian children.

\section{Emotion}

33. BALCONI, M

34. BOZIKAS, VP

35. EVANS, JJ

36. LEE, GP

37. NAHUM, L

38. PASSAGLIO, KT
Cross-modal (Voice and Face) Perception of Emotion. ERPs and Behavioral Analyses.

Emotion Perception in Obsessive-Compulsive Disorder.

The Frequency and Nature of Deficits in Facial Emotion Perception After Brain Injury.

Emotional Neural Networks as Revealed by fMRI and Electrical Stimulation of Implanted Electrodes in the Human Brain.

Temporally Dissociated Cortical Processing of Inherent Emotional Salience and Behavioural Relevance of Visual Stimuli.

An Empiric-theoretician Assay about the Possible Psychological and Physiological Benefits of the Laugh on the School Environment. 


\author{
39. ARIAS, N \\ 40. BILBAO, AB \\ 41. OSMON, DC \\ 42. VILAR-LÓPEZ, R \\ 43. FONSECA, LE \\ 44. RIVLIN, E
}

10:00-10:45 AM

10:45-11:15 AM

11:15 AM-12:15 PM

1. LUNDERVOLD, AJ

2. UZZELL, BP

3. SCHNIDER, A

4. SOLESIO, E

\section{1:15 AM-12:15 PM}

1. WESTRUPP, EM

2. RIS, M

3. RAZ, S

4. BENJAMIN, C

11:15 AM-12:15 PM

1. ARZOLA, GM

2. MILDERS, M

3. MARRENECA, S

4. GRAY, J

11:30 AM-1:30 PM

1. MARTINEZ, S

2. GARCIA-SANCHEZ, C

3. HIRVIKOSKI, T

4. WILLIAMS, H

5. BLOOMER, RH

6. MACHADO, M

7. SARA, PM
Forensic Neuropsychology

Clinical, neuropsychological, and psychophysiological profiles in impulsive and premeditated Mexican inmates. Factors influencing the simulation of memory deficits.

Attention and Symptom Validity Testing: Surprising Results in a Simulation Study.

Detection of Malingering in a Spanish Traumatic Brain Injury Population using the Rey Complex Figure Test.

Language: Other (e.g., Naming, Fluency, Reading)

LEE Test de Lectura y Escritura en Español.

Other

Multi-cultural medico-legal issues in the neuropsychological assessment of children/young adults in India and the UK.

Invited Plenary: Global Gene Expression Profiling in Neurocognitive Disorders Speaker: Ian Everall

Sala A1

\section{Coffee Break}

Paper Session 1

Memory: Imaging \& Electrophysiology

Sala $A \dot{3}$

Hippocampal Volume and Delayed Recall Performance in Older Adults.

Memory Loss in Young Adulthood: Too Young for DAT.

Cortical Signature of Amnesia: a High-Resolution Event-Related Potential Study.

Retroactive interference modulates brain magnetic activity in normal aging: a magnetoencephalography approach.

Paper Session 2

Childhood Acquired Disorders

Sala $A 4$

Adult Psychiatric Outcomes of Very Low Birth Weight Survivors.

Neurobehavioral Outcome in Extracerebellar Low Grade Astrocytomas in Children.

Extreme Prematurity: Do Two Additional Weeks of Gestation Matter at the Edge of Viability?

Pre- and Post-Encephalitic Neuropsychological Profile of a 7 Year-old Girl.

Paper Session 3

Neuropsychology of Depression

Sala A2

Effects of personality on executive functioning in refractory depression.

Emotional Expression Recognition in Affective Disorder: A Longitudinal Study.

Emotional Processing in Major Depression.

Mood Congruent Biases in the Perception of Facial Affect in Individuals with Bipolar Disorder while Depressed.

Poster Session 2

Medical Disorders, Psychopathology, Psychopharmacology

Hall Exposiciones

Autoimmune Disorders (e.g., CFS, Lupus, fibromyalgia)

Is there progressive cognitive dysfunction in Sjögren syndrome?

Behavioral Neurology

Usefulness of the Frontal Systems Behavior Scales in the assessment of decline behavioral disorders associated with Parkinson's Disease.

\section{Endocrine Disorders/Hormones}

Prenatal Dexamethasone Treatment of Children at Risk for Congenital Adrenal Hyperplasia Affects Cognitive Functions.

\section{Epidemiology}

A 6 month prospective study of immediate and general risk factors for presenting to an Emergency Department with head versus orthopaedic injury.

\section{Genetic disorders}

Hyperekplexia, Genetic abnormal glycene channels: A Review and Case Study.

Neuropsychological, linguistic (and genetic) profile of a family with severe language impairment, transmitted as an autosomic-dominant trait.

Neuropsychological evaluation in the Apert syndrome. A case report. 


$\begin{array}{ll}\text { 8. } & \text { BARAK, O } \\ \text { 9. } & \text { CYSIQUE, LA }\end{array}$

10. LOJEK. E

11. GELB, S

12. HOPKINS, RO

13. HUIDOBRO, A

14. SÁNCHEZ, S

15. BEKKEN, K

16. CASAS. L

17. POUTIAINEN, E

18. SAAVEDRA, G

19. SEGURA. B

20. VIRUDHAGIRINATHAN, K

21. WANG, M

22. BOZIKAS, VP

23. BUTTERFIELD, LC

24. CABALLERO, P

25. GRAY, J

26. HAALAND, V

27. HALL, NM

28. LAWRENCE, AJ

29. MALLOY-DINIZ, LF

30. RAMOS. I

31. SANTACANA, M

32. ANDRES, S

33. CAPOVILLA, AC

34. CROWE, SF

35. JURADO, R

36. GALLAGHER, P

37. BOUDREAU, VG

39. DUARTE ARMOLEA, A Neuropsychological effects of cannabis abuse in first episode psychosis patients.

40. GONZALEZ-BLANCH, C Lack of association between clinical and cognitive change in first-episode psychosis: The first six weeks of treatment.

41. KIM, M

42. OJEDA, N

43. OJEDA, N

44. PEÑA. J

45. PUIG, O

46. SANZ, J

47. VARGAS, ML

48. VAZ, L

49. ZEIFERT. PD

\section{Infectious disease: HIV/AIDS}

Depression and Coping in Persons Infected with HIV.

Predictors of neuropsychological improvement in $\mathrm{HIV}+$ individuals initiating antiretroviral therapy.

Infectious disease: Other (e.g., hepatitis C, West Nile virus)

Neuropsychological Functioning in HIV/HCV Co-infected Subjects Undergoing the Interferon and Ribavirin Therapy.

Medical Disorders

Kidney Transplantation: Neuropsychological Abilities as a Mediator of the Relationship Between Health Status and Everyday Problem Solving.

Predictors of Depression and Anxiety in Acute Respiratory Distress Syndrome Survivors.

Is there a place for Neuropsychology in the Emergency Room? Gershwin revisited.

Cognitive Function In Mexican End Stage Renal Disease Population.

Other

The Role of Memantine (Namenda) in the Treatment of Mental Dysfunction in Patients with Fibromyalgia (FM). Cognitive function in patients with severe carotid stenosis: Evaluation of cognitive mesures before treatment of revascularation.

Does Intensive Reduction in Blood Glucose Levels at the Acute State Improve Cognitive Outcome in Patients Resuscitated from Cardiac Arrest?

One Case of Heatstroke and Neuropsychological Sequelae.

Metabolic Syndrome and Psychomotor Speed Deficit.

India.

Neuropsychological Aspects of General Anaesthesia: A Western Perspective.

\section{Psychopathology/Neuropsychiatry/Other}

Humor Appreciation of Captionless Cartoons in Obsessive-Compulsive Disorder.

The Influence of Apathy and Depression on Cognitive Functioning in Parkinson's Disease.

Visual Memory Deficits In The Early Phases Of Bipolar Disorder.

Subjective organization and impaired verbal learning learning in bipolar disorder while euthymic.

Executive Functions in Patients with Borderline Personality Disorder.

Neuropsychological Markers of Multisymptomatic Functional Disorder.

Neuropsychological Functioning and Varieties of Impulsivity in Problem Gamblers and Alcoholics.

Neuropsychological aspects of bipolar disorder: analyses according history of suicide attempt and category of suicide attempt (violent $\mathrm{X}$ non-violent).

Neuropsychological subtypes in schizophrenia

Is it possible to identify a Kraepelian subtype of schizophrenia in the early stages of the illness?

\section{Psychopathology: Anxiety/Stress}

Response Inhibition in adolescents with Obsessive-Compulsive Disorder and Anorexia Nervosa.

Attencional Deficits in Panic Disorder: Brazilian Version of Computerized Emotional Stroop Test.

An Examination of the Relationship betweeen State Worry. Trait Anxiety and Working Memory Performance in a Non-clinical Sample.

Attencional Deficits in Posttraumatic Stress Disorder and Panic Disorder.

\section{Psychopathology: Depression}

Spatial Memory Impairments in Depressed Patients with Bipolar Affective Disorder.

\section{Psychopathology: Schizophrenia}

The effect of organizational strategy on visual memory in schizophrneic patients.

Processing Speed Mediates The Relationship Between Verbal Fluency And Functional Disability In Schizophrenia. Reasoning Is Related To Verbal Fluency And Processing Speed In Chronic Patients With Schizophrenia. Significant Differences between First Episode Psychosis and Chronic Patients With Schizophrenia in Semantic Fluency, Verbal Memory and Processing Speed.

Memory Dysfunction In Adolescents With Schizophrenia Spectrum Disorders.

Dysexecutive Syndrome and Illness Severity in Patients with Schizophrenia.

Cognitive Adverse Effects of Mild Extrapyramidal Symptoms in Schizophrenia.

Psychopharmacology

Cortisol-Induced Impairments of Working Memory Planning Task in Healthy Young Men.

\section{Stroke}

The Effect of Moyamoya Disease on Neuropsychological Functioning. 


\section{2:30-1:30 PM Paper Session 4 Language \& Aphasia Sala $A 4$}

1. ANSALDO, AI

2. HIRD, KM

3. LAGANARO, M

4. CANCELLIERE, A
Adapted Brain Plasticity in Chronic Aphasia: The Neural Correlates of Efficient Naming.

Anomia in Primary Progressive Aphasia: Specific or General Deficit?

Electrophysiological Correlates of Recovery from Anomia in Aphasic Patients with Semantic or Phonological Impairment.

The Definition Naming Test (DNT): A Written-Verbal Alternative to the Boston Naming Test (BNT).

\section{2:30-1:30 PM}

1. KAUFMANN, L

2. SÁNCHEZ-CARRIÓN ABASCAL, R

3. IBARRETXE, $\mathrm{N}$

4. REMINGER, SL
Paper Session 5 Structural \& Functional Neuroimaging Sala $A 3$

Is There A Functional Overlap in Numerical and Spatial Processing? A Developmental fMRI Study.

Working memory activation following severe diffuse traumatic brain injury: A functional MRI study.

Memory Impairment and Gray Matter Loss in the Hippocampus of Patients with Parkinson's Disease and Visual Hallucinations.

Hippocampal Volume and Memory Function in Breast Cancer Survivors.

12:30-1:30 PM

Paper Session 6

Parkinson's Disease

Sala $A 2$

1. SANCHEZ-CASTAÑEDA, C Differences Between Neuropsychological Profile and Gray Matter Atrophy in Individuals with Dementia with Lewy Bodies and Parkinson Disease with Dementia.

2. MATTHEWS, C

3. OGDEN, JA

4. TORRALVA, T

Cognitive Flexibility in Patients with Parkinson's Disease: The Role of the Basal Ganglia.

Does Bradyphrenia Exist in Parkinson's Disease OFF or ON L-dopa?

Detection of Executive Deficits in Patients with Early Parkinson Disease.

1:30-3:30 PM

\section{3:30-5:00 PM}

1. BOLLA, KI

2. BOLLA, KI

3. DOM, G

4. QUEDNOW, B

5. VERDEJO-GARCIA, A

6. CLARK, L

\section{3:30-5:00 PM}

1. BOBES GARCÍA, J

2. GARCÍA-PORTILLA, M

3. CRESPO-FACORRO, B

4. SEGARRA, R

5. BOMBÍN, I

\section{3:30-5:00 PM}

1. PAPANICOLAOU, A

2. CASTILLO, E

3. LEÓN-CARRIÓN, J

4. NORTHOFF, G
Lunch Break on your own

\section{Symposium 4 \\ Impulsivity and Decision-Making in Substance Abusers Chair: Karen Bolla Sala $A 4$}

Impulsivity and Decision-Making in Substance Abusers.

Decision-Making in Chronic, Heavy Marijuana Users.

Decision-making deficits in alcohol-dependent patients with and without comorbid personality disorder. Impulse control and decision-making cognition in Ecstasy users.

Emotion processing and decision-making in cocaine and heroin abusers.

Neuropsychological mechanisms of impulsivity in chronic amphetamine users.

Symposium 5

Neuropsychological Dysfunction in Early Phases of Psychosis

Discussant: Julio Bobes; Discussant: Miguel Gutiérrez

Sala $A 2$

Neuropsychological Dysfunction in Early Phases of Psychosis.

Cognitive Dysfunction in Schizophrenic Outpatients under Antipsychotic Treatment.

Neuropsychology and Neuroimaging Correlations in First Episode Psychosis.

Course and Outcome in First Schizophrenic Episode. The Role of Insight and Therapeutic Compliance.

Cognitive Profile, Neurological Signs and its Evolution in First-Episode Psychotic Patients: A Two-Year Follow-up.

Invited Symposium

Studies on Cognition and Emotion Using Neuroimaging

Chair: Jose Leon-Carrion

Sala $A 3$

Magnetoencephalography as a Functional Neuroimaging method.

Exploring the language comprehension mechanisms in the Normal and Lesioned Brain.

Does Dorsolateral Prefrontal Cortex (DLPFC) Activation Return to Baseline when Sexual Stimuli Cease? The Role of DLPFC in Visual Sexual Stimulation.

Altered Prefrontal Cortical Function during Perception and Judgment of Emotions in Depression. 


\section{Poster Session 3 \\ Memory, Attention, Visuospatial Functioning Hall Exposiciones}

\section{Attention}

The Melbourne Effect: Differences In Sustained Attention In Urban And Regional Australian Children. Improving Cognitive-Motor Dual Tasking After Brain Injury.

Characteristics of adults with ADHD on the Attention Network Test.

How Does Attention Develop? A study of 6 to 12 Year Old Children From Four Different Cultural Backgrounds. Auditory Sustained Attention Development.

Funciones ejecutivas en niños con TDAH predominantemente inatentos.

Attentional Control and Speed of Information Processing: Dissociations in Four Different Samples.

Impulsivity Effect in Attentional Function on Morbid Obese Patients with Binge Eating Disorder.

\section{Cognitive Neuroscience}

Case Report: Attentional Deficits in a Pacients with Left Temporo-Frontal Lobe Damage.

\section{Memory}

A Case of Developmental Amnesia: Dissociation between Impaired Episodic and Unimpaired Semantic Memory. Imagery Encoding Reduces False Recognition: An Event-Related Brain Potentials (ERPs) Study.

Memory Span for Tones, Words and Nonwords in Amateurs Singers.

Memory Assessment in a Virtual Reality Environment in Patients with Stroke and Multiple Sclerosis.

Semantic Memory Organization: Differential Weighting of Perceptual and Functional Features.

Free Recall and Aging: Bridging Short-Term and Long-Term Memory.

Rapid Forgetting and 'Rapid Remembering' in a Heterogenous Adult Clinical Sample.

Use of Orthographic Coding in Verbal Short-term Memory : Evidence from a Single Case Study.

A New Virtual Task to Evaluate Human Place Learning.

Retrieval Mechanisms for Autobiographical Memories after Severe Traumatic Brain Injury.

Relation of Working Memory Capacity and Free Recall of Semantically Related Words in Alzheimer's Disease.

Re-acquisition of person knowledge in semantic memory disorders.

An investigation of a non-selective imagery impairment on strategic and autobiographical memory processes following posterior frontal lobe craniotomy.

The Rivermead Behavioural Memory Test: an ecological assessment of memory in mild cognitive impairment and Alzheimer's disease.

23. ESPINOSA, A

24. ESTEVEZ-GONZALEZ, A Relation between semantic memory and achievement outcomes in a sample of adolescents.

25. FIGUEIREDO, $\mathrm{P}$

26. GARCIA ORZA, J

27. GARCIA ORZA, J

28. GOMEZ-PEREZ, E

29. KANG, Y

30. KIKUCHI, H

31. LUCCHELLI, F

32. MUÑOZ MARRÓN, E

33. O'CONNOR, MG

34. PINHO, S

35. QUIROZ, YT

36. RASPALL, T

37. REIS, AI

38. SIMÕES, MR

39. STIVANIN, L

40. SUZUKI, M

41. TRAMONI, E

42. VAN ASSELEN, M

43. ZANINOTTO, AL

44. BERARDI, A

45. DE HAAN, EH

46. MONTORO, P

47. O'REILLY, M

48. NYS, GM

50. ABE, $\mathrm{N}$

49. STAMENOVA, V
Dissociable Memory Impairments after Selective Bilateral Fornix Damage.

Disproportionate Retrograde Amnesia in a Traumatic Brain Injury Patient.

Severe Anterograde Amnesia after Damage to Hypothalamus.

Development of Factors Contributing to Age Effects in Memory Tasks.

A Comparison of the Prospective Memory among College Students, Old Adults, and Parkinson's Disease Patients. Brain Mechanisms Underlying Dissociative Retrograde Amnesia: an fMRI Study.

Processes Underlying the Temporal Gradient in Mild Cognitive Impairment and Alzheimer's Disease: A LatentVariable Stochastic Analysis.

Visual versus verbal memory deficit in spina bifida and hydrocephalus.

ECT and Memory Consolidation: A Dissociation between Anterograde and Retrograde Memory.

Verbal Memory Performance of Children with Learning Disabilities on the Coimbra Neuropsychological Assessment Battery.

Semantic Context Interference During a Working Memory Task.

Reliable Mesial Temporal Lobe Activation Using a Classical Neuropsychological Memory Paradigm. A fMRI Study. Implicit learning of structured auditory sequences: An advantage for verbal stimulus.

Assessment of Non-Verbal Memory in Children with Learning Disabilities on the Coimbra Neuropsychological Assessment Battery.

Comparison of ADHD and Typical Children in Implicit Memory.

The neural network for temporal order memory retrieval: evidence from lesion and PET studies.

Can Psychogenic Amnesia Occur without Concomitant Brain Damage?

Implicit context learning in patients with Parkinson's disease.

Articulatory suppression effect on working memory throught free recall test.

\section{Visuospatial Abilities}

Assessment of Spatial and Motor Abilities in Patients with Stroke and Multiple Sclerosis using a Virtual Reality Environment.

Brightness agnosia: a disorder of light- and darkness recognition.

Effect of density and connectedness of local elements on the perception of hierarchical patterns: evidence from a simultagnosic patient.

Infant's Use of Facial Motion to Discriminate Gender.

\section{Neglect}

Mechanisms of Perseverative Behaviour Towards Ipsilesional Locations in Patients with Hemi-neglect.

\section{Other}

A peculiar form of delusional misidentification: a neuropsychological case study providing a window on person identification.

Hemisphere effects in gesture production in patients with Corticobasal Syndrome. 


$\begin{array}{ll}\text { 5:15-6:15 PM } & \text { Paper Session } 7 \\ & \text { Cross-cultural Issues } \\ \text { Sala A2 }\end{array}$

1. CONSTANTINIDOU, F

Age and Language effects on Word Fluency tasks: A cross-cultural comparison between Greek-Cypriot and American-English adults.

2. LOZANO, A

Years of Education vs Quality of Education in a Spanish Speaking Sample.

3. PADILLA-VELEZ, MM Neuropsychological Tests Predict IADL Ability Differently Among Non-Demented Spanish And English Speakers In A Community Setting.

4. BILBAO, AB The Spanish Hopkins Verbal Learning Test: Development, validation and standardization in Spanish population.

\section{5:15-6:15 PM Paper Session 8 \\ Cognitive Rehabilitation \\ Sala $A 3$}

1. PAVAN KUMAR, V Remediation of Dysgraphia in Two Patients with Primary Progressive Aphasia (PPA).

2. FERNÁNDEZ GUINEA, S Benefits of a visualization strategy training in elderly people with memory complains.

3. VINK, M Changes in Self-image, Coping and Social Participation after Holistic Neuropsychological Rehabilitation.

4. MATEER, $\mathrm{C} \quad$ It's just one brain: Integrating cognitive, metacognitive and affective interventions.

\section{5:15-6:15 PM}

1. SULLIVAN, BK

2. HARRISON, AG

3. OSMON, D

6:15-6:45 PM

$6: 45-7: 45$ PM

8:30 PM-11:30 PM
Paper Session 9

Symptom Validity in Dyslexia \& ADHD Sala A4

Symptom Exaggeration by College Adults in ADHD and Learning Disorders Assessments.

Identifying Students Faking either Dyslexia or ADHD: Preliminary Findings and Strategies for Detection. The many faces of effort: Specific symptom validity, attention and anosadiaphoria.

\section{Coffee Break}

Birch Lecture: Working Memory and the Perception-Action Cycle Speaker: Joaquin Fuster Sala A1

Dinner at Sidreria Ibarra Sagardotegi (reservations required) Bus leaves Euskalduna Convention Center 


\section{FRIDAY, JULY 6, 2007}

\author{
9:15-10:15 AM \\ LOPEZ-GARCIA, P \\ 2. ROBLES ARANDA, O \\ 3. BOMBIN, I \\ 4. KRAKOWSKI, MI
}

\section{9:15-10:15 АМ}

1. PEIXOTO, B

2. BRANDS, I

3. VAN DEN BERG, E

4. BECKER, JT

\section{9:15-10:15 AM}

1. BATE, $\mathrm{S}$

2. SEPULCRE, J

3. MCFARLAND, K

4. JOANETTE, Y

\section{9:15-11:15 АM}

1. BARROSO, J

2. GIOGKARAKI, E

3. MACNIVEN, J

4. STERN, S

5. VOLKERT, J

6. AGUILAR MEJIA, OM

7. ALVAREZ-CARRILES, JC

8. KLAAS, PA

9. LOPEZ-ROLON, A

10. LUNA RODRIGUEZ, A

11. RICHARDSON, K

12. WRIGHT, I

13. WRIGHT, I

14. DE NÓBREGA, E

15. KAZANDJIAN, S

16. NARBERHAUS, A

17. NISHIMURA, $R$

18. GOMEZ-RUIZ, MI

19. GÓMEZ, JD

20. JUÁREZ, J

21. RODRÍGUEZ SALGADO, D

22. SABUCEDO, $\mathrm{M}$
Paper Session 10

Schizophrenia

Sala $A 4$

Impact of COMT Genotype on Cognition in Schizophrenia Spectrum Disorders.

Lack Of Cognitive Performance Specificity Among Subdiagnoses Of Early-Onset First Episode Psychosis.

Relationship between Cognition, Soft Neurological Signs, and Symptoms with Insight into Schizophrenia.

Atypical Antipsychotic Agents, Neuropsychological Deficits and Violence in Schizophrenic Patients.

\section{Paper Session 11}

Neuropsychology of Medical Disorders

\section{Sala $A 2$}

Executive Functions in the Spectre of Hepatic Dysfunction.

Type 1 and Type 2 Diabetes: Differential Effects on the Brain?

Cognitive Dysfunction in Type 2 Diabetes Mellitus: Onset in Pre-Diabetic Stages?

Brain Functional Alterations in HIV/AIDS Analysed by the Method of Partial Least Squares.

\section{Paper Session 12 \\ Cognitive Neuroscience \\ Sala A3}

Evidence of an Eye Movement-Based Memory Effect in Congenital Prosopagnosia.

Mechanism of lexical access strategies: evidence from lexical networks in neurological diseases.

The Many Faces of Prosopagnosia.

The Functional Reorganization of the Brain Underlying Cognitive Successful Aging for Language Is Multi-dimensional and Task Dependent.

\section{Poster Session 4 \\ Traumatic Brain Injury, Epilepsy, CNS Abnormalities Hall Exposiciones}

\section{Demyelinating Disease/Multiple Sclerosis/ALS}

Neuropsychological assessment and regional MRI lesion volume in multiple sclerosis patients treated with IFN beta. A four factor model for executive function in MS.

Stroop Performance in Multiple Sclerosis: Information Processing, Selective Attention or Executive Functioning? Cognitive Deficits in Multiple Sclerosis Patients Correlated with Diffusion Tensor MRI Derived Measurements of the Corpus Callosum.

Fatigue in Multiple Sclerosis: Objective Assessment of a Subjective Symptom.

\section{Epilepsy}

Protocol of Neuropsychological follow up in childrens during the epilepsy surgery process.

C Dual-task Performance in Frontal and Temporal Lobe Epilepsy.

Predictors of Post-Surgical Behavioral Outcome in Children with Intractable Epilepsy.

Numerical Cognition and Temporal Lobe Epilepsy: Dot Size Congruency in Large Numerosity Discrimination.

Changes in Neuropsychological Scores after Anterior Temporal Lobectomy plus Amygdalohippocampectomy.

Memory Training for Epilepsy Patients: A Preliminary Investigation.

Sleep Problems In Childhood Epilepsy.

\section{Genetic disorders}

Cognitive Variability in Children with Epilepsy.

\section{Hemispheric Asymmetry/Laterality/Callosal Studies}

Verb Generation in Patients with Hereditary Cerebellar Ataxia.

Hemispheric Specialization in Aesthetic Preference in Homonymous Hemianopia.

Verbal Fluency Impairment in Very Preterm Subjects Correlates with the Corpus Callosum Genu.

\section{Lobar Degeneration}

Selective Attention is Modulated by Independent Resource of Each Hemisphere.

\section{Other}

Primary Progressive Aphasia in a Bilingual Patient: A Case Report.

\section{Sex Differences/Sex Hormones}

Language monitoring during surgery in brain eloquent areas.

Sex Differences in the Effects of Opioid Antagonist Treatment in Alcoholic Patients.

Sex Differences in Frontal Measures. 


23. BEKKEN, K
24. BOLOGNANI, S
25. DE GUISE, E
26. DIAZ, M
27. DIKMEN, S
28. DOUGLAS, JM
29. FAIRJONES, SE
30. FERNÁNDEZ-ESPEJ
31. GARCIA-MOLINA,
32. GARZÓN, B
33. HUNT, C
34. KNIGHT, R
35. KWAPIL, KJ
36. MCLINDEN, A
37. MEACHEN, SJ
38. MEACHEN, SJ
39. MILLER, H
40. NEWSOME, MR
41. NOGUERA, P
42. PLOWRIGHT, B
43. PONSFORD, JL
44. SKILBECK, C
45. SKILBECK, C
46. TATE, RL
47. WATERLOO, K
48. WHITEFIELD, VJ

\section{0:30-11:30 AM}

1. KAUFMANN, L

2. SINZ, H

3. BAFFALUY, MG

4. WOOD, G

5. KUTRZEBA, C

\section{0:30-11:30 AM}

1. EGELAND, J

2. BUCHHOLZ, J

3. HEBER, IA

4. PERAKAKIS, $\mathrm{P}$

\section{0:30-11:30 AM}

1. KORKMAN, M

2. HUIJBREGTS, S

3. CHEUNG, M

4. TOLEDO-PIZA, $\mathrm{C}$

\section{Traumatic Brain Injury}

Development of Fibromyalgia (FM) Following Mild Traumatic Brain Injury (TBI).

The Dysexecutive Syndrome Questionnaire as a Measure of Self Awareness and Rehabilitation Outcome.

Should TBI Patients with Premorbid Psychosocial Issues be Excluded from Neuropsychological Neurotrauma Studies?

Demographic Characteristics and Neurocognitive Performance among Puerto Rican Traumatic Brain Injury Patients from Car-Related Accidents.

Retrospectively Assessed Post-Traumatic Amnesia: Is it of any Value?

'I don't like being alone...': Quality of life and Social Support after Severe Traumatic Brain Injury (TBI).

Predicting Cognitive Outcome Following Mild Traumatic Brain Injury: Applying Reserve Theory.

Working Memory correlates with White Matter decrease in diffuse Traumatic Brain Injury.

Community integration as an outcome of traumatic brain injured rehabilitation.

Voxel based diffusion tensor analysis shows white matter alterations in severe traumatic brain injury patients without neuroradiological lesions.

An Experimental Examination Of Disorders Of Control And Drive Following Traumatic Brain Injury.

Recall of Autobiographically Significant Episodes Involving Famous Names by Persons with Traumatic Brain Injury.

Neuropsychological Outcome following Mild Traumatic Brain Injury: Clinical Significance and Reliable Change Indices.

Using the London Handicap Scale to Measure Quality of Life Following TBI.

Internal Consistency and Test-Retest Reliability of the Brief Symptom Index 18 (BSI-18) in a Sample of Traumatic Brain Injury Survivors.

Rasch Analysis of the BSI-18 in Traumatic Brain Injury.

Duration of Post-Traumatic Amnesia (PTA) is a Better Predictor of Cognitive Outcome After Paediatric Traumatic Brain Injury (TBI) than Admission Glasgow Coma Score (GCS).

Working Memory Brain Activation following Severe Traumatic Brain Injury.

Recovering Verbal Memory after Post-Traumatic Amnesia: a 6-Month Prospective Study.

Early Cognitive Outcome from Trauma in Older Adults.

Psychiatric Disorders Following Traumatic Brain Injury.

The Factor Structure of the Rivermead Post-Concussion Symptom Questionnaire (RPCSQ) and Relationships with Demographic and Clinical Variables.

The Factor Structure of the Hospital Anxiety \& Depression Scale (HADS) in TBI, and Relationships with Demographic and Clinical Variables.

Patterns of Recovery of Memory Function in the Post-acute Stages after Traumatic Brain Injury.

Five-year outcome after mild traumatic brain injury: a prospective controlled study.

Neuropsychological Profile of Contact Versus Noncontact Sports Players at Grade 12 Level.

\section{Paper Session 13 \\ Numerical Representations \\ Sala $A 2$}

Developmental trajectories of magnitude and interference control: an fMRI study.

Decision Making and Processing of Probability in Mild DAT.

Consequences of playing a musical instrument in finger gnosis and arithmetic in children.

Effects of problem size and carry-over on fMRI signal in an addition task.

Exercise training improves executive functions of patients with heart failure.

\section{Paper Session 14 \\ Attention \\ Sala A4}

Differentiating attention deficit in adult ADHD and schizophrenia.

An Attentional Orienting Deficit in Adults with Dyslexia.

Pseudoneglect in virtual space.

Distinguishing Visual Search and Working Memory via Autonomic and Cortical Responses.

\section{Paper Session 15}

Childhood Developmental Disorders

Sala $A 3$

Social Perception in Children with Asperger Syndrome.

Maternal Prenatal Smoking, Inhibitory Control, and Externalizing Behavior in 7- to 9-year-old Offspring.

External organization reduces hyper intrahemispheric theta coherence in children with Autistic Spectrum Disor$\operatorname{der}(\mathrm{ASD})$.

Performance of Dyslexic Children in Pseudoword Repetition.

11:30 AM-12:00 PM Coffee Break 


\section{1:30 AM-1:30 PM Poster Session 5 \\ Imaging, Electrophysiology, Cognitive Neuroscience, Executive Functioning Hall Exposiciones}

Cognitive Neuroscience

2. ATKINSON, TM

A Study about the Efficiency of Alerting, Orienting and Executive Control Functions in a Nine-Year-Old Boy with Landau-Kleffner Syndrome.

Gender and Sport Differences in High School Athletes in Phonemic Switching on the Written COWA-FAS. An ERP Analysis of Iconic Language and Iconic Thinking. The Case of Metaphor.

Neuropsychological functioning in stabilized patients after a first psychotic episode: Bipolar versus Schizophrenia. Attentional Networks Functioning in School-Age Children with ADHD: Differences between ADHD Subtypes.

4. CASADO, M

5. DAZA, M

6. GONZÁLEZ GARRIDO, AA Early ERP Components Index Emotional Contexts Preceding A Detectable Change In Auditory Stimulus Stream.

7. KING, BA

8. MAMELI, F

9. MEMBRIVES, S

10. NAVARRETE, G Semantic Priming in Deep Dyslexia: Investigating the Integrity of the Semantic System.

Prefrontal Cortex Stimulation Interferes with Lying: Evidence for Multiple Neural Mechanisms of Deception. Relationship between anterior cingulate gray matter density and arithmetic performance: a VBM study.

Use of Neural Networks and Feature Selection in Neuropsychological Research: Identifying Relevant Variables on MCI.

11. PERRY, $W$

12. VAN GALEN, M

13. VAN VUGT, PE

The relationship between neuropsychological and psychophysiological measures in the assessment of inhibition among people with Bipolar Disorder.

Developing Automatic Access to Number Magnitude Information.

Tactile Agnosia without Disturbed Tactile Imagery, a Case Study.

14. BALCONI, M

15. BRITO, D

16. CIVAI, C

17. LITTLE, JS

18. VELEZ, A

\section{Electrophysiology/EEG/ERP}

Neuropsychological Processes in Verbal Irony Comprehension: an Event-related Potentials (ERPs) Investigation. Psychobiology of Art: An Exploratory Study with Event Related Potentials.

General and Specific Knowledge in the Brain.

Investigations of Memory Impairment Following Mild Head Injury Using Event Related Potentials.

Electrophysiological study of moral emotions.

\section{Executive Abilities/Frontal System}

19. BESNARD, J

20. CADAVID, $\mathbf{N}$

A study of utilisation behaviour in patients with frontal lobe lesions.

Assessing Executive Functioning in Colombian Children.

21. CAPOVILLA, AG

Executive Functions and Attention Deficit Hyperactivity Disorder in children.

Training of Task Switching Ability in Young and Older Adults.

22. COSTARDI, D

The Effects of Long-Term Cannabis Use on Executive Functioning in Treatment-Seeking Adults.

23. CREAN, RD

24. FERREIRA, D

Qualitative Analysis of Phonemic and Semantic Fluency Performance in MCI Patients.

25. GOMEZ BELDARRAIN, M Sleep Improves Sequential Motor Learning and Performance in Patients with Frontal Lobe Lesions. A pilot study.

26. HOEROLD, D

Self-Awareness,Sustained Attention and Error Processing in Focal Lesion Patients: Neuropsychological and Electrophysiological Correlates.

27. LUETHI, M Selective Planning Impairment After Theta Burst rTMS Over the Right Dorsolateral Prefrontal Cortex: An Eye Movement Study.

28. MALLOY-DINIZ, LF

29. MARCHADOUR, D

30. MARTIN, JE Aplicability of five digits and oral trail tests on the assessment of executive functions of adults with ADHD.

A study of spontaneous and reactive flexibility in patients with frontal lobe lesions and Parkinson's disease.

31. MARTIN ARAGON Card Sorting Test Performance in a Closed Head-Injured Population: The Role of Visual Working Memory.

32. MCLACHLAN, J

33. ORSATI, F

34. ORTIZ-GIL, J

35. PLUCK, G

36. ROJAS, $\mathrm{S}$

37. TORRALVA, T

38. VERDEJO-GARCÍA, AJ

39. BARTRÉS-FAZ, D

Working Memory Span: A Comparison between Measures.

Normative Five Digit Test Data on Adult Clinical Examinees.

Executive Deficits in Pervasive Developmental Disorders.

The Memory and Dysexecutive Deficits in Schizophrenia Are Neuropsychologically Specific.

Frontal Systems Behavior and Psychiatric Symptoms among Homeless Adults.

Neuropsychological Post Surgical Rehabilitation in a Patient with a Cerebral Frontal Lobe Tumour.

Buenos Aires Waiters: Strategies to enhance memory capacity.

Emotional feedback modulates decision-making performance in the Iowa gambling task.

\section{Hydrocephalus}

Voxel-based Morphometry Analysis of CSF before and after Shunting in Normal Pressure Hydrocephalus. Relation with Clinical and Cognitive Measures.

\section{Imaging: Functional}

40. GARCÍA-BLAZQUEZ, M Structural and Functional Neuroimaging Correlates of Clinical Findings in Four Cases of Infrequent Encephalopathies.

41. HALEY, AP

Family History of Hypertension is Associated with Reduced fMRI Response to a Visuospatial Working Memory Task in Healthy Young Adults.

Top-down Processing in Face Perception Detected by Seeing-as-face Task in fMRI Study.

42. HAYASHI, M

43. TOMIOKA, H

Driving evaluation for older adult drives using NIRS and driving simulator.

44. ZABALA, A

Cognitive Correlates of N-Acetyl-Aspartate Alterations in Dorsolateral Prefrontal Cortex in Adolescents with First-Episode Schizophrenia.

\section{Imaging: Structural}

45. RAMETTI, G

46. REIS, AI

Correlations between Verbal Memory and Hippocampal Anisotropy in Schizophrenic Patients.

47. SORIA-PASTOR, S

The impact of reading and writing skills on brain morphometry: A voxel-based morphometry study.

Neuroanatomical Basis of Performance IQ Deficits in Adolescents with History of Prematurity. 
48. BOGGIO, PS

49. SALGUERO, M

12:00-1:30 PM

1. DIAMOND, A

2. RAZ, A

3. DIAMOND, A

4. HOGAN, A

5. RUEDA, C

12:00-1:30 PM

1. OJEDA DEL POZO, N

2. BRANDT, J

3. BECKER, J

4. BERTHIER, M

12:00-1:30 PM

1. OSTROSKY, $\mathrm{F}$

2. WILSON, B

3. CLARE, L

4. FÉRNANDEZ GUINEA, S

5. RAMÍREZ, M

1:30-3:30 PM

3:30-4:15 PM

3:30-5:30 PM

1. BERNABEU, J

2. GROGAN, M

3. VELLA, K

4. EMOND, A

5. NAIR, RD

6. SIMÕES, MR
Other

Effects of transcranial direct current stimulation on working memory.

Neuroanatomic and functional differences between numerical reasoning and calculation.

Invited Symposium

Developmental Cognitive Neuroscience of the Executive Functions Dependent on the Frontal Lobe: Challenging Long-held Beliefs

Chair: Adele Diamond

Sala $A 3$

Developmental Cognitive Neuroscience of the Executive Functions dependent on the Frontal Lobe: Challenging Long-held Beliefs.

The Developmental Cognitive Neuroscience of Suggestion and its Relation to Improving Attention and Self-Regulation. Novel Methods for Improving and Assessing Executive Functions in Young Children.

Impact of Frontal Lobe Disconnection on Performance-Monitoring in Adolescents and Young Adults.

Development of Attentional Aspects of Executive Functions during Childhood.

Invited Symposium

Early Signs in the Identification, Diagnosis and Intervention in Dementia Discussant: Natalia Ojeda Del Pozo

Sala $A 2$

Early Signs in the Identification, Diagnosis and Intervention in Dementia.

Dementia of Huntington's Disease, and Subtle Cognitive Impairment in People at Risk.

Earliest Manifestations of Dementia in a Community Cohort.

Language Dissolution in Alzheimer's Disease and Primary Progressive Aphasia.

Symposium 6

Neurobiology Of The Self: How The Brain Creates A "Self", And What Happens

When Things Go Wrong

Chair: Feggy Ostrosky

Sala A4

Neurobiology Of The Self: How The Brain Creates A "Self", And What Happens When Things Go Wrong.

The Man Who Believes He Has Been Unconscious Or Dead For Twenty Years.

Awareness in people with moderate to severe dementia living in residential care.

Self-Awareness in Brain Injury: Decision-Making and Activities of Daily Living.

Assessment of awareness: A Cross-Cultural Study.

Lunch Break

on your own

Invited Plenary: Brain Imaging and Neurobehavioral Recovery from Traumatic

Brain Injury

Speaker: Harvey Levin

Sala A1

Poster Session 6

Childhood \& Developmental Disorders, Substance Exposure

Hall Exposiciones

Child - Acquired Disorder: other

Neurocognitive sequelae in pediatric oncology: neuropsychological profiles and rehabilitation.

Long-term Neuropsychological Effects of Low-grade Brain Tumours in Children: A Specific Focus on Attention, Executive Functioning and Quality of Life.

Child Brain Injury Research Programme: Preliminary data on predictive validity of the Health Utilities Index (HUI) in children with acquired brain injury.

Child - Acquired Disorder: TBI

Performance of Primary School Children on Measures of Post-Traumatic Amnesia (PTA).

Sexuality in Adolescents following Traumatic Brain Injury: A Systematic Review of Literature and Research Proposal. Attention and Executive Outcomes after Traumatic Brain Injury in Childhood: A Prospective Study with Coimbra Neuropsychological Assessment Battery. 
8. GALTIER. I

9. JANSEN, $\mathrm{P}$

10. MELLO, CB

11. MIRANDA. MC

12. MUSZKAT, M

Performance in Attention and Memory Neuropsychological Tests and its Relation with Academic Achievement in Children.

Developmental Aspect of Verbal Fluency: an Analysis of Clustering and Switching of School-Aged Children. Cognitive Processing Patterns and Reading Outcomes in a Sample of South African Children.

Gender and sociocultural differences in categorization and memory performance: A study in brazilian children.

A Comparative Study of Performance in ihe Conner's Continuous Performance Test (CPT-II) between Brazilian and North American Children.

Clinical and neuropsychological findings in children with low academic achievement: Preliminary results from a Brazilian study.

13. SHERESHEVSKY, G

Examination of the Internal Structure of the DSS-ROCF Organization Scoring System in a Clinical Sample.

\section{Child - Developmental Disorders}

14. ALVAREZ-CARRILES, JC Long-term Verbal and Non-verbal Functioning in Landau-Kleffner Syndrome: A Case Report.

15. ANTEQUERA TORRES, M Dementia in a patient with diagnosis of a generalized disorder of the development.

16. BOTTING, N

17. KISELEV, S

18. LOPEZ-SALA, A

19. LOPEZ-SALA, A

20. ROLDÁN-TAPIA, MD

21. VAN HOOREN. S

22. VAN ITERSON, L

23. ZOTTER. S

24. CABEZUDO, M

25. CROWE, SF

26. NILSEN, HK

27. OLIVEIRA, CC

28. PARADA, M

29. PARADA, M

30. REMY, S

31. RODRÍGUEZ-Á

32. WILKINS, JN

33. INOZEMTSEVA. O

34. BOIX LLUCH, C

35. CAPOVILLA, AG

36. COLOME, $\mathrm{R}$

37. HUTCHINSON, AD

38. RODRIGUES, CC

39. STIVANIN, L

40. TAPIA YEO, ME

41. TOLEDO PIZA, C

42. VIRUDHAGIRINA

43. WOLFSON, K

44. BARROS NETO, R

45. VALDEZ, $\mathrm{P}$

46. LECKY, $\mathrm{F}$

47. TÁRNOKY, CA

48. VIRUDHAGIRINATHAN, B Neuropsychological assessment and management of learning disability in India

49. OLIVEIRA, L pairment (SLI).

Neuropsychological profile of 35 children with Asperger's Syndrome.

Neuropsychological profile of dyslexia: a sample of 150 Spanish children. Epilepsy.

Drug/Toxin Exposure (incl. alcoholism)

The Long Term Neurocognitive Consequences of Benzodiazepine Use.

\section{Genetic disorders}

Learning Disabilities Spectrum in Congenital Adrenal Hyperplasia (CAH) girls.

\section{Learning Disabilities/ADHD}

Review of neuropsychologycal profile in a sample of 125 ADHD children Barcelona. Spain. Calculation Learning Disabilities.

Verbal and Visual-Spatial Working Memory Abilities in Dyslexic Children.

\section{Other}

Developmental Changes in Performance of a Stroop Task.

A service for children recovering from ABI.

Psychopharmacology

Effects of Current and Past Crack Cocaine Use on Neurocognitive Functioning.
The relationship between language and affective disorder in individuals with and without specific language im-

Investigation of Visuospatial Abilities in SLI Children with Poor Grammar Understanding.

Study of the visuoperceptive, executive and motor functions of a sample of children born with great prematurity. Inhibition, attention and simple reaction time of children with attention deficit hyperactivity disorder.

Kaufman Factors in three groups of Special school children: Learning Disabilities, Childhood Psychiatry, and

Frontoexecutive Functions in Five Adolescentes with Asperger Syndrome: Preliminary Findings.

Multidisciplinary treatment of Wernicke-Korsakoff encephalopathy: a case study.

Effects of Chronic non-malignant Pain and of Long-term Opioid Therapy on Neuropsychological Functioning.

Autobiographical and semantic memory of drug users and non users adolescents: Gender comparison.

Effects of Alcohol Binge Drinking on College Students' Memory: Preliminary Results.

Performance of Alcoholic Patients on Behavioral Assessment of the Dysexecutive Syndrome (BADS).

Relations Between Sensation Seeking, Cannabis and Tobacco Use in Young Adults.

Neuropsychological Findings and Work Function in Alcohol Dependent Subjects.

Nature of Reading Difficulties in Brazilian Children with Developmental Dyslexia.

Non Verbal Learning Disorder: Neuropsychological Assessment of 40 Children. R Colome MSC, A Sans MD, A Lopez-Sala MSC, C Boix MSC, R Gassio M Alquezar MSC Department of Neurology Sant Joan de Déu Hospital.

Corpus Callosum Morphology in Children and Adolescents with Attention Deficit Hyperactivity Disorder.

The influence of verbal and performance abilities in IQ measures in dyslexic children.

Processing time and characteristics of picture naming from Brazilian children with reading disorders.

Effects of Phonological Awareness and Visuospatial Distribution Training in a Child with Reading, Writing and

.

Advances in distinguishing clinical profiles using two scoring systems for the Rey-Osterrieth complex figure.

Theorical and Practical Inquiry of Motor Development in 2 to 11 Years Old Children.

The incidence of school instability in 13-22 year olds in full time education following traumatic brain injury. 
4:30-5:15 PM

5:15-5:45 PM

5:45-6:45 PM

7:00-8:00 PM
Invited Plenary: Cognition in Multiple Sclerosis: The Present and The Future Speaker: Maria Ron Sala A1

\section{Coffee Break}

Presidential Address: Alcohol and the Brain: The Good, the Bad, and the Ugly INS President: Igor Grant

Sala $A 1$

INS Awards and Business Meeting

Sala $A 1$ 


\section{SATURDAY, JULY 7, 2007}

\section{9:15-10:15 АM \\ 1. BAHAR-FUCHS, A \\ 2. BARBEAU, EJ \\ 3. MOORTHY, T \\ 4. BAYNES, K}

\section{9:15-10:15 AM}

1. Sistiaga, A

2. FLORES LAZARO, JC

3. GIOVAGNOLI, AR

4. BOMBIN, I

\section{9:15-10:15 AM}

1. LAH. SS

2. WALL, SE

3. MILLER. H

4. NEWSOME, MR

\section{9:15-11:15 AM}

1. NIJBOER. T

2. VAN ZANDVOORT. M

3. BULTITUDE, JH

4. ENCINAS, SM

5. ESPEL-TRIAS, G

6. GARCIA-MOLINA, A

7. HARDY. R

8. HODDER, KI

9. MACKAY, A

10. MORERA, A

11. OTERO, A

12. TRINIDAD, L

13. VINK, M

\section{Paper Session 16 \\ Alzheimer's Disease Sala $A 4$}

Decline of Olfactory Identification in MCI and AD: Evidence for the Breakdown of Olfactory "Semantics".

Early Diagnosis of Alzheimer's Disease Using a Visual Recognition Memory Task.

Predicting Alzheimer Disease in English- and French- Speaking Canadians.

Idea Density as a Measure of Communicative Skill in Alzheimer's Disease.

\section{Paper Session 17}

Frontal Systems \& Executive Functioning Sala $A 2$

Neuropsychological Profile in Myotonic Dystrophy (DM1).

Development of Neuropsychologycal Functions related to the Anterior Prefrontal Cortex.

The Characteristics of Theory of Mind in Patients with Frontal Variant Frontotemporal Dementia.

Dysexecutive versus Frontal Syndromes: Clinical Implications and Outcome Relevancy.

\section{Paper Session 18}

\section{Pediatric Traumatic Brain Injury}

Sala 43

Implicit memory in children who have sustained severe traumatic brain injury: Preliminary findings.

The Assessment of Empathy and Theory of Mind Following Childhood Brain Injury and the Implications for Neurorehabilitation.

Relationship Between Linguistic and Cognitive Functioning in Children Who Have Sustained Traumatic Brain Injury (TBI) Compared With that Seen in Non-injured Controls.

Brain Activation during Working Memory after Traumatic Brain Injury in Children.

\section{Poster Session 7 \\ Stroke, Cognitive Rehabilitation, Language \\ Hall Exposiciones}

\section{Agnosia/Disordered Representations}

Brightness Agnosia: a Disorder of Bright- and Darkness Recognition.

\section{Apraxia/Motor Sequencing}

Aphemia after a Right Hemisphere Stroke Due to Generalised Serial Order Impairment.

\section{Cognitive Intervention/Rehabilitation}

Can Adaptation to Vertically Shifting Prisms Ameliorate Gait Disturbance? A Preliminary Investigation using Healthy Participants.

Formative Groups: a formative and therapeutic resource for caregivers of mild dementia patients.

Application of a Neuropsychological Rehabilitation Program of the Executive Function in Patients with Schizophrenia.

Therapeutic effectiveness of a Computerized Cognitive Rehabilitation Program in neurorehabilitation of traumatic brain injury.

An Innovative Approach to the Cognitive Rehabilitation of Traumatic Brain Injury in Previously High Functioning Individuals.

The Method of Choice in Memory Rehabilitation: Contributions of Errorless Learning, Vanishing Cues and Spaced Retrieval.

Dual-Task Training in Older Adults.

Traumatic Brain injury by precipitation. Benefits of a neuropsychological rehabilitation associated to a program of social integration.

Development of Cognitive Abilities: An Educational Experience.

Neuroconstructivistic Intervention for Attention Deficit Hyperactive Disorder.

Changes in Executive functions after Holistic Neuropsychological Rehabilitation. 


\section{Language: Aphasia}

14. BALASUBRAMANIAN, V

15. CASTRO, SF

16. EL KADIB. J

17. LAUTERBACH. M

18. ANDRESEN, E

19. CANCELLIERE, A

20. FAÍSCA, L

21. GÓMEZ VELÁZQUEZ, F

22. MONTIEL, T

23. OTERO, A

24. STIVANIN, L

25. ZARABOZO, D

26. ÁLVAREZ, D

27. DIJKERMAN, C

28. LOETSCHER, T

29. ТНІММ, М

30. FEDOROV, A

31. SOBANSKA, M

32. VUKOVIC, M

33. ARRABAL, C

34. CHE DIN, N

35. DESMARAIS, G

36. DUMBRAVA, A

37. DUMBRAVA, A

38. GUTIÉRREZ, L

39. JIMÉNEZ I

40. JIMÉNEZ, I

41. LINARES, GO

42. MARTOS, P

43. PASCUAL, M

10:30-11:30 AM

1. BLANCO-CAMPAL, A

2. KESSELS, RP

3. MAITZ, M

4. PERDICES, M

10:30-11:30 AM

1. CONSTANTINIDOU, F

2. BESSELL, AL

3. DEAN, TA

4. CICERONE, KD
Auditory Comprehension of Lexical versus Deep Structure Ambiguities in Three Groups of Brain Damaged Adults. Portuguese Version of Psycholinguistic Assessments of Language Processing in Aphasia, PALPA-P. Cognitive Linguistic Assessment for Arabic-Speaking Patients in Morocco: Presentation of a Battery. Intensive vs Conventional speech therapy in aphasia due to ischaemic stroke: a randomized controlled trail.

\section{Language: Other (e.g., Naming, Fluency, Reading)}

"Semantic" Priming: A Meta-Analysis.

The Effects of Aging and Education upon Word Finding Ability: Data from a New Test.

The role of working memory in the interpretation of predicative metaphors.

Longitudinal Study Demonstrates that Naming Speed is the Best Early Predictor of Reading Abilities in Spanishspeaking Children.

Metacognition, Reading ability and Schooling.

Lectura Inteligente: An educational software that favors speed, understanding and efficient reading in Mexican secondary school students.

Correlation between Latency Time and Reading Efficiency of 2nd, 3rd and 4th Grades Children.

Evaluating Pseudo-Homophones With Event Related Brain Potentials In Spanish-speaking Children.

\section{Neglect}

A Study about Diagnosis and Evolution in a Patient with Balint's Syndrome by Encephalitis.

The effects of prism adaptation on the landmark task and the mental number line in healthy subjects.

Stars in the Sky: Assessing Inattention and Hyperattention in Spatial Neglect.

Effects of a New Computerised Exploration Training on Neural Recovery from Spatial Neglect.

\section{Other}

Low vision patients rehabilitation. Neurophysiological basis of the recovery from optic nerve lesion.

The Preserved Multiplication Fact Retrieval in an Alexic Patient: A Case Report.

Types and evolution of aphasia following closed head injury.

\section{Stroke}

Patients' Clinical Evolution in the Stroke Unit.

Factors Influencing Depression among Stroke Patients.

Using Structure to Improve Memory for Visual Material: Linking Encoding Approaches to Memory Deficits for Visual Information in a Patient with a Focal Lesion to the Right Frontal Lobe.

Apathy More Than Depression Seems to Interfere with Early Motor Recovery After Stroke.

Motor Symptoms of Post-Stroke Depression.

Subcortical ischaemic vascular disease without dementia

Frontal test in elderly stroke patients with low education level.

Cognitive impairment in intracerebral hemorrhage and its evolution at three months.

Cognitive status is impaired in apparently recovered stroke survivors.

Neuropsychological Profile of Bilateral Paramedian Thalamus Infarction: 3 Cases.

Lack of self-awareness of deficits: Comparison between patients with stroke located in left and right hemisphere.

\section{Paper Session 19 \\ Memory}

Sala $A \dot{4}$

Prospective Memory is a Sensitive Indicator of Mild Cognitive Impairment.

Contextual Memory and Binding in Hippocampal and Diencephalic Patients.

The Effects of Providing Memory Cues During Verbal and Non-Verbal Learning in Persons with Unilateral Frontal and Temporal Lobe Tumours.

Changes in Verbal Memory Function During Internal Carotid Artery Occlusion by Balloon Angiography.

\section{Paper Session 20}

\section{Adult Traumatic Brain Injury}

Sala $A 3$

Neuropsychological and Functional Recovery after moderate to severe TBI-Support for systematic post-acute rehabilitation.

Is autobiographical Recall compromised by depressive rumination in survivors of Acquired Brain Injury?

Impaired National Adult Reading Test (NART) Performance in Traumatic Brain Injury (TBI)

Impact of Impaired Self-Awareness on Select Outcomes After Traumatic Brain Injury. 
10:30-11:30 AM

1. CHEUNG, M

2. HOWARD, CE

3. MUHLERT, N

4. SANTOS, FH

\section{1:30 AM-12:00 PM}

\section{1:30 AM-1:30 PM}

\section{Paper Session 21 \\ Epilepsy \\ Sala $A 2$}

Post-surgical verbal memory outcome associated with right mesiotemporal functional MRI activation.

Memory and Metamemory in Patients with Temporal and Frontal Lobe Epilepsy.

Evidence for Long Term Forgetting in patients with Temporal Lobe but not Idiopathic Generalised Epilepsy.

Partial Epilepsy in Frontal Right Hemisphere and Mathematical Skills in children.

\section{Coffee Break}

\section{Poster Session 8 \\ Aging, Dementia \\ Hall Exposiciones}

$\begin{array}{ll}\text { 1. } & \text { AVILA, R } \\ \text { 2. } & \text { BALDIVIA, B } \\ \text { 3. } & \text { CHANSON, N } \\ \text { 4. } & \text { CORREIA, R } \\ \text { 5. } & \text { JOANETTE, Y } \\ \text { 6. } & \text { JOANETTE, Y } \\ \text { 7. } & \text { MATHIAS, JL } \\ \text { 8. } & \text { MOZAZ, M } \\ \text { 9. } & \text { RODRÍGUEZ-ARANDA, C } \\ \text { 10. } & \text { SOLE, C }\end{array}$

11. VILLENEUVE, S

12. WATERINK, W

13. ALEGRET, M

14. NANNERY, RA

\section{Aging}

Neuropsychological Correlates of MRI Measures in Elderly Depressed.

Influence of High Education Level in Neuropsychological Assessment: Cognitive Reserve?

Cognitive study of constructional abilities in normal aging.

Qualitative Differences Between Young and Older Adults in Semantic Verbal Fluency.

Maintaining the Ability to Semantically Process Words With Age: A Near-Infrared Spectroscopy Study. Investigating Narrative Discourse Abilities in Successful Aging: A Neuroimaging Study Using NIRS.

Cognitive Predictors of Unsafe Driving in Older Drivers: A Meta-analysis.

Age Related Changes in Postural Knowlege.

Spectrographic evaluation of verbal fluency performance over time in young and healthy elderly subjects.

Functional And Structural Correlates Of Cognitive Reserve In Normal Aging, Mild Cognitive Impairment And Mild Alzheimer's Disease.

The Contribution of Vascular Burden in Persons with Mild Cognitive Impairment.

Implicit learning capability in memory impaired psychogeriatric patients: a controlled study.

\section{Agnosia/Disordered Representations}

The 15-Objects Test: detection of visual agnosia in preclinical and mild Alzheimer's disease. Body Awareness and Balint's Syndrome: An illustrative case study.

\section{Dementia Alzheimer's Disease}

15. ABRISQUETA-GOMEZ, J Applicability of the Abbreviated Neuropsychologic Battery (Neuropsi)in Alzheimer's Disease Patients. Psychobiology Department - Universidade Federal de São Paulo.

16. ABRISQUETA-GOMEZ, J What Do Really Need to Rehabilitate for Demented Seniors? Universidade Federal de São Paulo / Brain Checkup - Consulting and Research in Cognitive Neurosciences.

Driving, Mild Cognitive Impairment and Dementia.

Accumulation of genetic risk factors and cognitive impairment in Alzheimer disease.

Influence of concomitant depression in Alzheimer disease on non verbal memory.

Clustering and Switching in Semantic Fluency: Predictors of the Evolution to Alzheimer Disease?

Improving Clock Drawing Test Assessment in Ageing by Qualitative Analysis of Errors.

Limitations with the active manipulation of the information at early stage of dementia of Alzheimer type.

Discourse Analysis of Seven Bilingual Patients with Alzheimer's Disease.

Measuring MCI awareness using (modified) IQCODE.

Episodic Memory Impairment in Alzheimer's Disease: Encoding or Retrieval Deficits?

Stability and change in picture naming by Spanish Alzheimer's patients: a longitudinal study using photographs.

A three years long study on the semantic impairment in dementia of the Alzheimer type.

Comparison of the results of the Brief Cognitive Screening Battery, the Mini Mental State Examination and the Dementia Rating Scale of Mattis.

Semantic Verbal Fluency in the Preclinical Phase of Alzheimer's Disease: a Longitudinal Analysis of Clustering and Switching Processes from the Paquid Study.

Memory function in older adults with and without Alzheimer's disease employing a kinesiology-movement based intervention.

\section{Dementia Subcortical}

Emotional Processing in PD: Knowing What Are We Doing?

Differential Impairment in Verbal and Visuospatial Memory in Nondemented Parkinson's Disease Patients.

Decision Making and Estimation of Probabilities in Parkinson Disease Dementia.

\section{Dementia: other}

Progressive Loss of Memory about Famous People in Right Temporal Variant of Semantic Dementia.

Frontal Dysfunction in ALS May Be Associated with Loss of Cognitive Insight in the Absence of Behavioral Change. The Psychological Support within Neuropsychological Rehabilitation: About a Case of PCA.

Semantic Dementia (SD): A Longitudinal Case Study of Autobiographical Memory Impairment and of Anatomical and Functional Alterations.

Neuropsychological profiles in Semantic Dementia and Alzheimer disease: 4 presenile cases.

Neuropsychologic profile of the Syndrome of Korsakoff: Presentation of a clinic case. 
40. ADROVER-ROIG, D

41. ANDERSSON, MF

42. ANSALDO, AI

43. GAWRON, N

44. HANNA-PLADDY, B

45. RODRÍGUEZ, R

46. FUCHS, K

47. DIAZ, U

48. JÓDAR VICENTE, M

\section{2:00-1:30 PM}

1. BERNARDO, M

2. PARELLADA, E

3. FERNÁNDEZ-EGEA, E

4. SEGARRA, N

5. PENADÉS, $\mathrm{R}$

12:00-1:30 PM

1. ALHASSOON, O

2. DURAZZO, T

3. DOM, G

4. UEKERMANN, J

\section{2:00-1:30 PM}

1. SPENCER-SMITH, MM

2. CROWE, $\mathrm{L}$

3. HESSEN, E

4. SPENCER-SMITH, M

5. BENJAMIN, C

\section{Normal Aging}

Distraction and Perseverative Scores of the Madrid Card Sorting Test (MCST) as Sensitive Indexes of Cognitive Decline in Healthy Aging.

A neuropsychological and MRI study of response competition and goal maintenance in older age.

Aging and Second Langauge Learning: A Different Way of Being Performant.

Neuropsychological Performance of Healthy Older Persons in Context of Chosen Theories about Brain Aging.

Neuropsychological Profiles of Musicians: Evidence for Cognitive and Motor Maintenance in Aging.

Working memory and aging.

\section{Other}

Medication Management Skills in Individuals with Mild Cognitive Impairment.

Effects of a Cognitive Stimulation Program in Third Age.

Verbal and Spatial Learning in Old Men and Women.

\section{Symposium 7}

Neurocognition and Psychopathology in Schizophrenia: and Integrated Vision

Inside Neuroscience.

Discussant: Miguel Bernardo

Sala A3

Neurocognition and psychopathology in schizophrenia: and integrated vision inside Neuroscience.

Auditory Hallucinations in First Episode Schizophrenic Patients.

Emotion assessment with PET-18F-FDG.

Cognition, Cerebral Structure and Outcome in Schizophrenia.

Cognitive Rehabilitation in Schizophrenia and Functional Recovery.

Invited Symposium

Impact of Psychiatric and Other Co-Morbidities on Neuropsychological

Functioning in Alcoholism

Chair: Omar Alhassoon

Sala $A 2$

Impact of psychiatric and other co-morbidities on neuropsychological functioning in alcoholism.

Comorbid chronic cigarette smoking in alcohol use disorders: The neurobiological and neurocognitive consequences. Decision-making deficits in alcohol-dependent patients with and without comorbid personality disorder.

Depression and cognitive functioning in alcoholism.

\section{Symposium 8}

Childhood Head Injury: Incidence, Timing Effects, and Functional Outcomes. Chair: Megan Spencer-Smith Sala $A 4$

Childhood Head Injury: Incidence, Timing Effects, and Functional Outcomes.

Incidence of Traumatic Brain Injury in a Pediatric Population.

Neuropsychological Function 25 years after Mild Head Injury. A Comparison of Outcome after Pediatric and Adult Head Injuries.

Timing of Brain Insult Effects on Children's Executive Functions.

Childhood Head Injury: Impact on Cognitive and Behavioural Measures of Executive Function. 


\title{
Abstracts Presented at the International Neuropsychological Society, Federation of Spanish Socicties of Neuropsychology, Spanish Neuropsychological Society, Spanish Psychiatry Society Joint Mid-Year Meeting
}

\author{
July 4-7, 2007 \\ Bilbao, Spain
}

\author{
WEDNESDAY AFTERNOON, JULY 4, 2007 \\ Symposium 1 \\ 6:30-8:00 p.m. \\ Obsessive-Compulsive Disorder : From \\ Neurobiology to Neurocognition. \\ Chair: Jose M Menchon \\ J. MENCHON MAGRIÑA \& J. VALLEJO RUILOBA. Obsessive- \\ Compulsive Disorder: From Neurobiology to Neurocognition. \\ The symposium is addressed to examine the links between neurobio- \\ in the ventral part of the putamen and in the anterior cerebellum. All \\ these brain alterations were abnormally correlated in patients with OCD, \\ and age statistically significantly contributed to the relative enlargement \\ observed in the striatal areas. Patients with prominent aggressive ob- \\ sessions and checking compulsions showed reduced amygdala volume \\ in the right hemisphere. The pattern of anatomical features depicted by \\ this voxelwise approach is consistent with data from functional studies. \\ The reported anatomical maps identified the specific parts of the fron- \\ tostriatal system that were altered in patients with OCD and detected \\ changes in anatomically connected distant regions. These data further \\ define the structural brain alterations in OCD and may contribute to \\ constraining the prevailing biological models of this psychiatric process. \\ Correspondence: Carles Soriano-Mas, Carles.Soriano@crccorp.es
} logical data, neuropsychological performance and clinical dimensions in obsessive-compulsive disorder (OCD). Results from several sources, mainly structural and functional neuroimaging, obsessional symptoms in some neurological conditions and treatment procedures such as psychosurgery, have yielded theories on dysfunctional fronto-subcortical circuits in OCD. Neuroanatomical models from neuroimaging research provide a useful framework to understand neuropsychological dysfunctions. On the other hand, family studies suggest a genetic component in OCD. Possible relations between genetic studies and neurocognition in OCD will be examined. In the last years has been proposed to consider different clinical dimensions in OCD which could involve different neurobiological substrates. The relationship between these different clinical dimensions and neuropsychological performance will be analyzed. Finally, the neuropsychological dysfunctions in OCD, in particular verbal and non-verbal memory performance, will be studied. At the conclusion of the symposium the participant should have information about neuroanatomical models in OCD, genetic studies, clinical dimensions and their relationship to neuropsychological performance.

Correspondence: Jose M Menchon,jmenchon@csub.scs.es

C. SORIANO-MAS. Neuroanatomical Models of ObsessiveCompulsive Disorder.

Recent technical developments have made it feasible to describe the structural brain alterations detected in the magnetic resonance images of a large series of patients with obsessive-compulsive disorder (OCD) using imaging procedures that allow the evaluation of volume changes. We will describe findings in a consecutive sample of 72 outpatients with OCD and 72 age- and sex-matched control subjects. Three-dimensional sequences were obtained in all participants. The brains of patients with OCD showed reduced gray matter volume in the medial frontal gyrus, the medial orbitofrontal cortex, and the left insulo-opercular region. A relative increase in gray matter volume was observed bilaterally
M. DEL PINO ALONSO. Genetic Vulnerability and Neurocognition in Obsessive-Compulsive Disorder.

Obsessive-compulsive disorder (OCD) runs in families, but the specific contribution of genetic and environmental factors to its development is not well understood. We will issue whether there are differences in perceived parental child-rearing practices between OCD patients and healthy controls, and whether any relationship exists between parental characteristics, depressive symptoms and the expression of particular OCD symptom dimensions. Severe OCD and mild to moderate depressive symptoms were detected in our sample. Compared with healthy controls, OCD patients perceived higher levels of rejection from their fathers. No differences between the groups with respect to perceived levels of overprotection were detected. The seventy of depressive symptoms could not be predicted by scores on any perceived parental characteristics. Hoarding was the only OCD symptom dimension that could be partially predicted by parental traits, specifically low parental emotional warmth. Social/cultural variables such as parental child-rearing patterns, in interaction with biological and genetic factors, may contribute to the expression of the OCD phenotype.

Correspondence: $M^{a}$ del Pino Alonso mpalonso@csub.scs.es

D. MATAIX-COLS. Symptom Dimensions in Obsessive-Compulsive Disorder and Neuropsychological Performance.

This presentation will reviewed sex differences in obsessive-compulsive disorder (OCD) related to differences in neuropsychological functioning compared to healthy men and women. Participants were 56 consecutive patients (33 male, 23 female) and 40 healthy control participants (20 male, 20 female) of comparable characteristics. Male and female patients had comparable symptom severity, illness duration, comorbidity, in- or out-patient status, and medication usage. An exten- 
sive neuropsychological test battery was administered including tests of general nonverbal intelligence, attention, verbal and nonverbal memory, and executive functions. Male and female OCD patients showed comparable neuropsychological performances on most cognitive domains. However, we found some evidence for cross-sex shifts in verbal fluency tasks (FAS and Category Alternation Test [CAT]), the reading component of the Stroop test, and the Wechsler Adult Intelligence ScaleRevised (WAIS-R) Digit Span-Forward test. Post hoc analyses revealed that female patients showed reduced performance on these tests compared to healthy women, in the male-typical direction. Among OCD women only, there were significant negative correlations between OCD symptom severity and performance on the CAT and the reading Stroop. We conclude that sex does not seem to be a major determinant of neuropsychological function in OCD, but the observed cross-sex shifts on some tasks deserve further examination.

Correspondence: David Mataix-Cols D.Mataix@iop.kcl.ac.uk

C. SEGALÀS. Verbal and Non-Verbal Memory Processing in Obsessive-Compulsive Disorder.

We will reviewed studies investigating biases and deficits in verbal and nonverbal memory related to Obsessive-Compulsive Disorder (OCD). Although the research has been mixed concerning different patterns for these two sensorial memory for verbal information, there is more consistent evidence suggesting impairment for non-verbal information, particularly for complex visual stimuli and the individual's own actions. Further, a number of studies indicate that patients with OCD report less confidence in their judgments about recognition memory. Finally, OCD appears to be associated with an attentional bias favouring threatening information, as well as reduced levels of cognitive inhibition. The review concludes with a number of recommendations for future research. Correspondence: Cinto Segalàs segalas16@hotmail.com

\section{Symposium 2 6:30-8:00 p.m.}

\section{Neuroimaging Studies of Neural Plasticity: New Modalities and Methods in Focal, Generalized, and Developmental Brain Disorders}

\section{Chair: Jack Fletcher}

J.M. FleTCHER, A.C. PAPANicolaOU, E.M. CASTILlo, J.J. JURANEK, J.M. FLETCHER \& M. DENNIS. Neuroimaging studies of neural plasticity: New modalities and methods in focal, generalized, and developmental brain disorders.

Symposium Description: The technology for structural and functional brain imaging is rapidly evolving and has important implications for neural plasticity and reorganization. This symposium presents a series of studies using structural MRI (voxel- based morphometry and diffusion tensor imaging) and magnetoencephalography (MEG) in typically developing children and adults, patients with focal lesions (strokes), epilepsy, generalized disorders (spina bifida), and a neurodevelopmental disorder not associated with specific injury, dyslexia. Each of these studies illustrates the sensitivity of these different neuroimaging methods to variations in neural organization after brain injury with clear implications for understanding impairments and preservation of function in these disorders. The discussion focused on how these methods have evolved to shed light on historical issues involving neural plasticity that are of continued interest to neuropsychology.

Correspondence: Jack M. Fletcher, Ph.D., Psychology, University of Houston, 2251 W Holcombe Blvd., 222 U of Houston TMC Annex, Houston, TX 77204-5053.E-mail:jackfletcher@uh.edu
A.C. PAPanicolaOU, E.M. Castillo \& J.J. JURANEK. Representation of Receptive Language Functions: Relations with Age, Gender, Task, and Mode of Stimulus Presentation.

Objective: Magnetoencephalography (MEG) is a functional neuroimaging method used to assess the neural representation of cognitive and motoric functions. Of particular interest are relations of language functions and factors known to potentially alter the representation of language in the brain, including age, gender, task variations, disordered brain function, and mode of stimulus representation. We used MEG to explore these variations and their impact on the plasticity of language representation. Participants and Methods: The reliability of receptive language brain activation profiles and the robustness of their prominent topographical features were assessed in the context of five experiments involving 97 normal volunteers of both genders ranging in age from seven to 84 years. In addition, these variations were examined in patients with epilepsy, tumors, and dyslexia.

Results: Across all experiments involving normals, profiles were characterized by significant bilateral activity centered in the superior temporal gyrus, and in activity lateralized to the left middle temporal gyrus. These features were invariant across age, gender, variation in task characteristics, and mode of stimulus presentation. Several cases of aberrant profiles will be reviewed exemplifying three basic types of functional reorganization of the brain: (1) Hemispheric dominance shift, mainly in cases of left medial temporal lobe pathology associated with epilepsy; (2) Shifts in topography within the left hemisphere, usually associated with tumors; and (3) a combination of topographical and timing-of- activity onset aberrations associated with dyslexia.

Conclusions: MEG is a reliable assessment of the neural representation of language that is sensitive to variations in brain plasticity associated with different disorders.

Correspondence: Jack M. Fletcher, Ph.D., Psychology, University of Houston, 2251 W Holcombe Blvd., 222 U of Houston TMC Annex, Houston, TX 77204-5053.E-mail:jackfletcher@uh.edu

E.M. CASTILLO, J.I. BREIER, C. BOAKE, J.J. JURANEK, S. SARKARI \& A.C. PAPANICALAOU. Aberrant Cortical Functionality of Sensorimotor and Linguistic Networks in Stroke. Objective: Changes at the anatomical and neurophysiological level can modulate the functional impact of stroke. In a series of studies we tested how function-specific profiles of cortical activity differ in stroke patients from those obtained in healthy volunteers and whether specific profiles of cortical activation are associated with better functional status after stroke. Participants and Methods: In three independent studies, a total of 35 first stroke patients and 20 aged-matched volunteers were evaluated. Participants underwent neurological evaluation, high resolution MRI, diffusion tensor imaging (DTI) and MEG recordings of somatosensory (Study A), motor (Study B) and receptive language functions (Study C). MEG-derived profiles of activation were related to functional status and radiological findings.

Results: In Study A, patients showed aberrant profiles of cortical activation associated with different levels of somatosensory deficit. The presence of focal oscillatory activity (perirolandic delta) in the affected hemisphere correlated with the severity functional measurements of somatosensory deficit. Study B indicated that the structural integrity of the ipsilesional corticospinal tract correlated with measurements of fine motor performance. In study C, aphasic patients exhibited relatively decreased activation in areas known to be involved in receptive language functions (left superior temporal gyrus).

Conclusions: The results suggest that functional deficit after stroke correlates with decreased activation in preexisting networks supporting normal function. Functional recovery seems to require normalization and/or reweighting of the components of preexisting networks, rather than the new engagement of unaffected cortical tissue.

Correspondence: Jack M. Fletcher, Ph.D., Psychology, University of Houston, $2251 \mathrm{~W}$ Holcombe Blvd., 222 U of Houston TMC Annex, Houston, TX 77204-5053.E-mail:jackfletcher@uh.edu 
J.J. JURANEK, E.M. CASTILLO, P.P. ALVAREZ, L. EWING-COBBS, S. SHIRIN \& A.C. PAPANICOLAOU. Anatomical and Functional Differences in Children with Spina Bifida: MRI and MEG Studies. Objective: Few quantitative studies have investigated anomalous structural development of the brain in children with spina bifida meningomyelocele (SBM). Similarly, few neurophysiological studies have investigated quantitative measures of brain function in SBM. The present study investigated group differences between SBM and age- matched controls in anatomical measures (e.g. cortical thickness and corpus callosum area) and neurophysiological responses to tactile stimulation (e.g. MEG somatosensory evoked fields).

Participants and Methods: Cortical thickness measurements were compared in 16 children with SBM and 16 age-matched controls. High resolution MR images acquired on a Philips 3T scanner were automatically segmented into 3 classes: gray matter, white matter, and CSF using Freesurfer v3.0.4 software (www.surfer.nmr.mgh.harvard.edu). Using the Witelson method, the corpus callosum (CC) was subdivided into 3 areas, the genu, body, and splenium. A subset of SB subjects $(n=8)$ also participated in MEG studies of somatosensory evoked fields.

Results: Global analyses indicated group differences in rostrocaudal patterns of cortical thickness. In controls (as expected), Temporal $>$ Frontal $>$ Parietal $>$ Occipital. In SBM, Frontal $>$ Temporal $>$ Parietal>Occipital. Quantitative measures of mean cortical thickness in frontal regions were significantly different between groups (SB $\mathrm{M}=3.07$, Control $\mathrm{M}=2.83, \mathrm{p}<0.01)$. Group comparisons of the body of the $\mathrm{CC}$ demonstrated significant differences: $\mathrm{SB}<$ controls $(\mathrm{p}<0.05)$. Amplitude of ipsilateral SII responses in SB positively correlated with $\mathrm{CC}$ body area measurements $(r=0.79, \mathrm{p}<0.01)$.

Conclusions: The SB brain is significantly reorganized at structural and functional levels after early, congenital brain injury.

Correspondence: Jack M. Fletcher, Ph.D., Psychology, University of Houston, $2251 \mathrm{~W}$ Holcombe Blvd., 222 U of Houston TMC Annex, Houston, TX 77204-5053.E-mail:jackfletcher@uh.edu

J.M. FLETCHER, T.J. ELUVATHINGAL, K.M. HASAN, L. EWINGCOBBS \& M. DENNIS. Neural Reorganization in Spina Bifida: Evidence from Diffusion Tensor Imaging.

Objective: Spina bifida meningomyelocele (SBM) is a congenital neurodevelopmental disorder associated with significant changes to the central nervous system, including spine and brain malformations and hydrocephalus. This study employed MRI diffusion tensor imaging to measure four major association pathways.

Participants and Methods: MRIs with DTI sequences were obtained on 38 children with SBM and a control group of 31 age and sex-matched normally-developing children. Compact white matter fiber tracking was performed to quantitatively assess four association pathways important for cognitive and motoric functions: arcuate fasciculus, inferior longitudinal fasciculus, inferior fronto-occipital fasciculus, and uncinate fasciculus.

Results: Fractional anisotropy was reduced in the association pathways of SBM. Transverse diffusivity was elevated in most of the fiber tracts of the group with SBM, indicating less development of myelin relative to controls. Axial diffusivity was also elevated in the majority of the association pathways of children with SBM, possibly due to a combination of defects in the intrinsic axonal properties and increases in the extracellular/extra axonal space. Although controls showed the expected inverse correlations of age and diffusion metrics indicating increased white matter with age, this relation was not apparent in the group with SBM.

Conclusions: DTI non-invasively detected disrupted development of association pathways and defective myelination in children and adolescents with SBM. The absence of age-related changes in the axial and transverse diffusivities in the group with SBM suggests that continuing myelin loss and deterioration in the axonal properties is a persistent deficit. Correspondence: Jack M. Fletcher, Ph.D., Psychology, University of Houston, 2251 W Holcombe Blvd., 222 U of Houston TMC Annex, Houston, TX 77204-5053.E-mail: jackfletcher@uh.edu

\section{Symposium 3 \\ 6:30-8:00 p.m.}

\section{Literacy and Metalinguistic Awareness in Different Languages}

\section{Chair: Esmeralda Matute}

E. MATUTE \& M. ROSSELLI. Literacy and Metalinguistic Awareness in Different Languages.

Symposium Description: There is a growing interest in studying the relationship between learning to read and metalinguistic awareness in different languages. However, less attention has been paid to the relationship between metalinguistic skills and writing. Different issues underlie this topic; several of them have been related to the study of dyslexia. For this Symposium, we have included studies carried out on three languages with different degrees of orthographic shallowness: English and French, considered to be examples of deep orthographic systems, despite the large differences between them; and Spanish, a language recognized as a shallow system. Our objective is to emphasize how metalinguistic awareness -including phonological, phonemic and morphological awareness- relate to the acquisition of reading and writing skills in these languages, as well as to manifestations of dyslexia and hyperlexia. This Symposium will provide examples of four different approaches to the study of these topics: Dr. Matute will present results on reading performance and phonological awareness among school-age, Spanish-speaking children; Dr. Saldaña will discuss the role of phonological and orthographic word processing in children with hyperlexia; Dr. Defior will report new findings concerning the influence of phonological processing in dyslexic Spanish-speaking children; and, finally, Dr. Nadeau will provide evidence on the differences in morphological awareness and writing in Francophone and Spanish-speaking children.

Correspondence: Esmeralda Matute, PhD, Instituto de Neurociencias, Universidad de Guadalajara, Francisco de Quevedo 180, Guadalajara 44130,Mexico.E-mail: ematute@cencar.udg.mx

\section{SALDAÑA. Phonological and Orthographic Word Processing in Children with Hyperlexia.}

Objective: Children showing a significant discrepancy between their word-reading and text comprehension abilities are often classified as hyperlexic. However, the diagnostic criteria for hyperlexia and the interpretation of processes involved in their word-reading are still widely debated in the English and Spanish literature. The few studies published in Spanish have argued for strong orthographic or visual processing in hyperlexic children (Martos-Perez y Ayuda-Pascual, 2003; Talero-Gutierrez, 2006). However, data from recent studies in English (Newman et al., 2006, Saldaña et al., in prep) seem to indicate that hyperlexic readers use phonological processing in a similar way to typically developing readers. Data is presented from one of these studies (Saldaña et al., in prep.). Fourteen adolescents with hyperlexia and Autism Spectrum Disorders (ASD) and 12 typically developing adolescents, matched on word reading, age and receptive vocabulary, were administered phonological, orthographic and semantic processing tasks. Hyperlexic readers were divided into two groups according to whether word-reading ability was greater that their receptive vocabulary scores and text comprehension ("true hyperlexics", $n=7$ ) or their reading ability matched their receptive vocabulary scores ("poor comprehenders"). No differences were found on accuracy scores on word semantic processing tasks. However, the "true hyperlexics" outperformed the poor comprehenders on orthographic processing and both poor comprehenders and the control group on phonological processing. These data from English-word processing are compared to the published cases of Spanish hyperlexic readers. Implications for the assessment, definition and comprehension of underlying processes of hyperlexia in English and Spanish are discussed. 
Correspondence: Esmeralda Matute, PhD, Instituto de Neurociencias, Universidad de Guadalajara, Francisco de Quevedo 180, Guadalajara 44130, Mexico.E-mail: ematute@cencar:udg.mx

E. MATUTE, T. MONTIEL, M. ROSSELLI \& C. HERNÁNDEZRAMIREZ. Phonological Awareness and Reading Skills in SpanishSpeaking Children.

Objective: The aim of this study was to analyze the relationship between reading ability and phonological awareness in Spanish-speaking children of school age.

Participants and Methods: One hundred and nineteen Spanish-speaking children (61 girls and 58 boys) from 1st to 6th grade were evaluated using ECOFON - Evaluación de Conciencia Fonológica (Phonological Awareness Assessment. This battery includes 10 phonological tasks: syllable decoding, syllable counting, rime and onset detection, phonemic deletion, word phonemic decoding, non-word phonemic decoding, phonemic substitution, word phonemic blending and non-word phonemic blending. In addition, four subtests (Word reading, non-word reading, sentence reading, reading speed and text comprehension) from the Reading domain of the Evaluación Neuropsicológica Infantil - ENI (Child Neuropsychological Assessment) were used to assess reading skills. Results: High correlations were found between all the ECOFON subtests and the reading measures. The type of school (private or public) and the school grade as well as the the interaction between them had a significant effect on all reading tasks. However the effect of the type of school disappeared in children at 4th grade. The scores on the ECOFON were also significantly influenced by the interaction between the child "s grade and the type of school.

Conclusions: Our results suggest that there is a strong association between reading performance and phonological awareness in Spanishspeaking children, even after becoming proficient in reading.

Correspondence: Esmeralda Matute, PhD, Instituto de Neurociencias, Universidad de Guadalajara, Francisco de Quevedo 180, Guadalajara 44130, Mexico.E-mail: ematute@cencar:udg:mx

F. SERRANO \& S. DEFIOR. Playa, blaya, mlaya. The Influence of Item Type in the Spanish Dyslexics' Reading.

Objective: This work aims to study the influence of items with different phonological processing load in dyslexics' reading abilities. These reading abilities were assessed with three tasks using different types of items, namely words, pseudowords and non-words.

Participants and Methods: Using a reading level-matched design, reading performance of thirty-one dyslexic children was compared to that of two control groups, an age-matched control group (CA) and a reading level-matched control group (RL). Each control group also consisted on 31 children. The three reading tasks were composed of eleven items each. Non-words differ from pseudowords because they do not follow the Spanish word building phonotactic rules. Monosyllable, bisyllable, trisyllable and polysyllable items were used. All items have similar syllabic structure, and they are composed of simple graphonemes, and include a consonant cluster.

Results: Results show that dyslexics performed worse in all tasks compared to CA -as expected- and RL children. These results are dramatically increased in non-word reading. It seems that dyslexic children's problems become clearer in this type of item, due to non-word's higher phonological processing load and dyslexics' known phonological deficit. These findings are discussed regarding implications for dyslexia assessment and intervention in Spanish.

Correspondence: Esmeralda Matute, PhD, Instituto de Neurociencias, Universidad de Guadalajara, Francisco de Quevedo 180, Guadalajara 44130, Mexico.E-mail: ematute@cencar.udg.mx

M. NADEAU \& A. GONZALEZ-REYES. Morphological Awareness and Writing: A Comparison of Spanish- and French-Speaking Children.

Objective: This study examines morphological awareness in two languages characterized by different degrees of orthographic shallowness: Spanish and French. While Spanish is considered a shallow system since the phoneme-grapheme-phoneme conversion rules are quite easy to master, French is often used as an example of a deep orthographic system. One feature of French that accounts for its opacity is the conflict between its oral and written morphology, as related to grammatical concordance. A comparison of French and Spanish may provide a better understanding of the role of oral language support and of the implicit and explicit morphological awareness required to master writing codes. Participants and Methods: In this study, eighty-four 6th-grade Quebecois Francophone children matched by age and sex to a group of Mexican Spanish-speaking children were asked to carry out two different tasks: related to explicit, and implicit, morphology awareness, respectively.

Results: Results showed that when explicit grammar awareness is requested, higher scores are obtained by Spanish-speaking children, whereas in the implicit awareness task, French- and Spanish-speaking children experience the same degree of difficulty, as both groups produce more errors of agreement in the implicit awareness task.

Conclusions: Our results have theoretical implications for the role of morphological awareness in reading development and reading disabilities related to the building of learning strategies, whatever the shallowness of the orthographic system may be.

Correspondence: Esmeralda Matute, PhD, Instituto de Neurociencias, Universidad de Guadalajara, Francisco de Quevedo 180, Guadalajara 44130, Mexico.E-mail:ematute@cencar.udg.mx

\section{THURSDAY MORNING, JULY 5, 2007}

\section{Poster Session 1 \\ Assessment, Cross Cultural Issues, Forensics, Emotion 9:00-11:00 a.m.}

\section{Assesment/Psychometricss}

B. APPELS \& E. SCHERDER. The Diagnostic Accuracy of Dementia Screening Instruments with an Administration Time of Ten to Thirty Minutes; a Systematic Review.

Objective: To describe the diagnostic accuracy of dementia screening instruments with an administration time of 10-30 minutes, controlled for possible biases due to methodological shortcomings, differences in methods of data collection or reporting.

Participants and Methods: We searched in Pubmed and included dementia screening instruments that met the criteria: administration time of 10-30 minutes, designed to detect mild dementia, solely based on $\operatorname{cog}$ nitive tests, representing minimally two cognitive domains. Excluded were interviews, observational scales, informant ratings, computer tests and remote screening instruments. The quality of the studies was judged with the Quality Assessment tool for Diagnostic Accuracy Studies (QUADAS). Studies were classified as 'high quality' and 'low quality' according to a weighted scoring scheme, the prerequisite of an adequate reference standard, and the relation between their QUADAS-score and the median. Dementia screening instruments without a study of 'high quality' were not taken into further consideration.

Results: Thirteen dementia screening instruments fulfilled the inclusion criteria. Eight of these instruments had at least 1 diagnostic study of 'high quality': Addenbrooke's Cognitive Examination, CAMCOG, Cognitive Abilities Screening Instrument, Neurobehavioral Cognitive Screening Examination, Prueba Cognitiva de Leganes', Rowland Universal Dementia Assessment Scale, Seven Minute Screen and Syndrom-Kurztest. 
The diagnostic accuracy of each instrument is described in terms of likelihood ratios. Other charateristics, such as cognitive domains represented and vulnerability to demographic variables, were also highlighted. Conclusions: The RUDAS is validated and has proved to be most capable in discriminating demented patients from healthy controls in a community population. The CASI seems suitable for screening subjects with illiteracy and a low level of education. In secondary care, the 7MS has the highest diagnostic accuracy, despite its sensitivity to demographic variables.

Correspondence: Bregje Appels, Slotervaart hospital, Louwesweg 6, Amsterdam 1066 EC, Netherlands.E-mail:psbap@slz.nl

\section{Visuospatial Abilities}

\section{A. BERARDI, C. PAPOT, O. HABERT \& A. PRUSKI.Virtual Reality- related Sickness Symptoms in Neurological Patients.}

Objective: The aim of this study was to determine whether VR can be used safely with minimal VR-related sickness symptoms in neurological patients.

Participants and Methods: Participants were 20 wheelchair users: 10 patients with stroke and 10 with multiple sclerosis (MS). Patients moved in two VR environments on a simulated wheelchair (Berardi et al., 2007; Papot et al. 2007). Patients' performance was compared to that of 10 age, sex and education-matched controls (all $\mathrm{F}<1$ ). After each VR test, a detailed questionnaire on VR-related sickness symptoms was administered. Results were analyzed using ANOVAs.

Results: None of the participants reported any sickness symptoms that caused interruption of the session. In VR experiment 1, participants reported equivalent degrees of presence in the scene and a good correspondence of VR with reality. Symptoms of general discomfort, vertigo, bodily warmth, headaches, sweating, increased salivation, nausea, eve strain, fatigue, difficulty concentrating, pallor, dry mouth, and blurred vision were very low and equivalent in all groups (all p>.22). The degree of immersion was reported to be higher in stroke and MS patients relative to controls (both $\mathrm{p}<.02$ ). MS and controls, but not stroke patients, found it as easy to act in the VR environment as they did in real life $(\mathrm{p}<.02)$. Both patient groups reported a good correspondence between their real and the simulated wheelchair $(p=.14)$. In experiment 2 , stroke patients reported greater immersion than controls $(p=.01)$. Stroke patients reported more symptoms of fatigue than controls $(p=.01)$ and of dry mouth $(\mathrm{p}=.006)$ and sweating, $(\mathrm{p}=.006)$ than MS patients and controls. No other differences were significant.

Conclusions: Results suggest that VR can be used safely for a period of up to 20-30 minutes in neurological patients with minimal VR-related sickness symptoms.

Correspondence: Anna Maria Berardi, Ph.D. in psychology, Department of Psychology, Paul Verlaine University - Metz, UFR SHA, Ile du Saulcy, Metz57006,France.E-mail: berardi@univ-metz.fr

O. BRUNA, M. PUYUELO, N. CUllell, V. VILlalta, A. DERGHAM, G. NIEVES \& E.H. WIIG. Early detection of cognitive impairment. Adaptation of the Alzheimer's Quick Test to the Spanish population.

Objective: Although the Mini Mental State Examination (MMSE) is an established screening test for Alzheimer's Disease (AD) for which extensive research exists, the Alzheimer's Quick Test: Assessment of Parietal Function (AQT), is a relative newcomer to the field of screening tests for early evidence of probable AD. The purpose of the present study was to adapt the AQT to the Spanish population and determine the sensitivity and specificity of the test in our country, in order to have other screening tests for the early detection of cognitive impairment in AD.

Participants and Methods: AQT and MMSE were administered to 369 normal subjects from 20 to 80 years old to obtain normative data in Spain, and to 70 adults in the initial phases of AD to determine the sensitivity and specificity of the test.
Results: We have obtained normative data in the Spanish population for the AQT test, and results show significant differences $(p<.001)$ between normal subjects and people in the early stages of $\mathrm{AD}$, with high levels of specificity and sensitivity. There are significant differences between people younger and older than 60 years, and regression analysis shows that age explains the variance of all tasks of the test.

Conclusions: The results show that AQT can be used in the Spanish population as a screening test for early evidence of probable AD.

Correspondence: Olga Bruna, PhD, Faculty of Psychology Blanquerna, University Ramon Llull, C/ Císter, 34, Barcelona 08022, Spain. E-mail: olgabr@blanquerna.url.edu

M. GIANNAKOU, M.H. KOSMIDIS, V.P. BOZIKAS, E. ARETOULI \& K. FOKAS. Developing a Visual Test Examining Mentalizing Abilities for Psychiatric and Neurological Patients.

Objective: The ability to infer the mental state of others in order to predict and explain their behavior is referred to as mentalizing ability (MA). We sought to test the clinical and research appropriateness of a task exploring MA in psychiatric and neurological patients.

Participants and Methods: We developed a cartoon test (Visual Theory of Mind Test; VToM), comprising 22 cards depicting short stories. After decoding the meaning of each story, participants had to choose which of the alternative cards for each story provided a logical ending. Four stories required first order ToM (how the protagonist will act having a false belief for a situation), six stories required attribution of intention (what the protagonist is thinking of doing) and six stories required attribution of desire (what the protagonist is going to do after perceiving another person's desire). The remaining six stories were included as a control task, to test story comprehension without any MA. We administered the VToM to 35 healthy participants (age: $\mathrm{M}=35.23$, $\mathrm{SD}=13.45$ ) and 12 patients with schizophrenia (age: $\mathrm{M}=39.92$. $\mathrm{SD}=7.08$ ). The ToM stories were also administered to 10 patients with frontotemporal dementia (age: $\mathrm{M}=64.20, \mathrm{SD}=7.62$ ).

Results: Our healthy participants had an accuracy rate, ranging from $63 \%$ to $96 \%$ on 21 story-items. Both patient groups performed more poorly than healthy controls on 1st order ToM. Also, schizophrenic patients' performance pattern differentiated them from controls, as they had the lowest accuracy on ToM stories.

Conclusions: We suggest that the VToM test can be a reliable test for assessing MA in clinical populations.

Correspondence: Maria Giannakou, Psychology, Psychology, Aristotle University of Thessaloniki, Stratigi 14, Thessaloniki 54352, Greece. Email:mariag@psy.auth.gr

G. GUILERA, O. PINO, J. GÓMEZ, J.E. ROJO, J. REJAS, T. DÍEZ, A. MARTÍNEZ-ARÁN, N. SEGARRA, E. VIETA, M. BERNARDO, B. CRESPO-FACORRO, R. TABARES, M.J. CUESTA, M. FRANCO \& S.E. PURDON. Is the SCIP-S a reliable tool for a neuropsychological brief assessment?

Objective: The Screen for Cognitive Impairment in Psychiatry (SCIP; Purdon, 2005) is a brief assessment tool designed for evaluation of neuropsychological impairment in psychiatric disorders. The SCIP requires less than 15 minutes and it has three alternate forms to minimize practice effects over repeated testing. Each form consists of five subscales for the assessment of immediate verbal list learning, working memory, verbal fluency, delayed list learning and visuomotor tracking. Previously we demonstrated very good form equivalence and test-retest stability of both the English and Spanish versions of the SCIP in healthy college students. The present study examined the test-retest reliability of the Spanish version of the SCIP (SCIP-S; Pino et al., 2006) in a large sample of psychiatric patients.

Participants and Methods: The SCIP-S was administered to a total of 203 patients, 126 with schizophrenia and 77 with bipolar disorder type I, between 18 and 55 years of age. The entire sample was evaluated at 
two points in time, baseline and day $7(+/-2)$, with the same form of the SCIP-S (i.e. 1-1, 2-2, 3-3).

Results: Intraclass correlation coefficients between both administrations ranged from a respectable 0.70 on the delayed verbal memory subscale to a high of 0.79 on the working memory subtest.

Conclusions: The SCIP-S demonstrated good test-retest reliability in patients suffering from schizophrenia or bipolar disorder.

Correspondence: Oscar Pino, Psychiatry, Granollers Hospital Foundation - Benito Menni CASM, Francesc Ribas s/n, Granollers Barcelona 08400, Spain.E-mail: opino1@gmail.com

K.M. HIRD, I. DAVIES, N. CICCONE \& K. KIRSNER. Should Aphasia Diagnosis be Shaped for Evidence Based Practise?

Objective: This study was designed to identify diagnostic tools that would facilitate the extension of evidence based practise to aphasia. Since choices for aphasia therapy are constrained by the assessment data that informs them, the underlying models of language processing need to be explicit and critically evaluated. Typically, aphasia assessment batteries are motivated by the theory of modularity. The aim of the present study was to contrast assessment profiles derived from (a) the modular approach and (b) an alternative procedure based on dynamic systems approach to communication. It was hypothesized that, first, measures based on dynamic systems woud align more closely with each participant's description of his or her communication difficulties; second, that the dynamic systems approach would have different implications for treatment (relative to the modular approach) and, third, the treatment golas derived from the dynamic systems approach woudl more closely reflect the goals and ambitions of participants.

Participants and Methods: The study involved three participants with aphasia. Assessments included the BDAE, BNT, SRP stimuli and RTT. All speech samples were recorded digitally. Pause analysis segmentation procedures described in Kirsner, Dunn, Hird \& Parkin (2002) were applied to spontaneous speech samples.

Results: The results revealed that while both assessment batteries identified the presence of deficits in speaking fluency and linguistic processing, the dynamic measures were objective, specific and quantified clinical impressions.

Conclusions: The dynamic systems approach therefore provided detailed baseline data describing changes in successful communication of intentions in a natural speaking context. It is proposed that the dynamic system approach better reflects self-organization of a speaker's intentions. In the case of a speaker with aphasia it reflects the product of the underlying dynamics of spared neural capcity that can be recruited to support language processing (Van Orden, Holden \& Turvey, 2003). Correspondence: Kathryn M. Hird, PhD, Psychology, Curtin University of Technology, GPO Box U1987, Perth, WA 6845, Australia. E-mail: K. Hird@curtin.edu.au

J. LEATHEM \& D.R. BABBAGE. How many know the date and why: A preliminary evaluation.

Objective: Knowing the date has long been included in the formal and informal assessment of memory. Just how many can accurately recall the day, month, year and date however is unclear as the questions are embedded within broader sections e.g., orientation (Mini-Mental Status Exam) and mental control (Wechsler Memory Scale). Further, today, with instant access to the date through mobile phone and computer technology, questions arise as to the validity of using the date as a measure of memory at all. This study sought to clarify these issues.

Participants and Methods: As part of a broader study, 200 participants, 40 in each of 5 consecutive age bands, were asked, "What's the date today?" and, "How did you know what the date was today"?

Results: $82.8 \%$ of participants correctly stated the date, $12.9 \%$ were +or -one day out and the remainder out by up to a year. Most $(24.3 \%)$ knew the date because of its proximity to an important event, $19.6 \%$ because they had used the date already that day, and $16.4 \%$ said they "just knew". Only $6 \%$ initially went to refer to new technology. There was no difference between the age groups in either rates of knowing the date or strategies used.

Conclusions: Current rates for knowing the date were obtained. Cueing from electronic devices occurred rarely suggesting that knowledge of date remains valid within memory assessment. Wide variability observed in response delay suggests that time taken to state correct date, may be a useful assessment adjunct.

Correspondence: Janet Leathem, PhD, Psychology, Massey University, 24 King St, Mt. Cook, Wellington 6005, New Zealand. E-mail: J.M. Leathem@massey.ac.nz

\section{LESUEUR \& K. BEKKEN. CONFUSION IN LEARNING} DYSFUNCTION CLASSIFICATION: TOWARD A TIGHTER THEORY.

Objective: For some time, clinical practitioners have been experiencing considerable confusion when attempting to apply diagnostic criteria to patients. These difficulties expose an abundance of overlapping symptoms and a lack of theoretical clarity to drive decision-making principles. The extensive incertitude currently surrounding the actual practice of diagnosing individuals suffering learning dysfunction suggests that the classification of these disorders is in a state of flux and therefore potentially misled and misleading. Our objective has emerged from this experienced need to rethink the theoretical approach to diagnosing learning dysfunction.

Participants and Methods: We conducted an ad hoc descriptive review of 150 assessment profiles representing a broad range of ages and learning dysfunctions (e.g., ADHD, NLD, LLD, etc.) across 40 specific symptoms and measures. We then tested a number of hypotheses stemming from current diagnostic criteria, and a number prompted by our own observations in the clinical setting.

Results: Demographics and behavior data were generally consistent with what is normally seen in these populations, with one or two intriguing exceptions. For example, data showed an abundance of overlapping symptoms and no evidence for clearly demarcated diagnostic categories with "necessary and sufficient conditions." In fact, many of the current criteria (e.g., VIQ - PIQ) for certain diagnoses (e.g., NLD) proved only weak predictors of the diagnostic category compared to others (e.g., VIQ - Rey), while some diagnoses (e.g., ADHD) virtually never appeared in isolation from other symptom patterns.

Conclusions: The data suggest further analyses of larger groups to tease out real categories. More importantly, they encourage a radical reconceptualization of the classification of learning dysfunction, providing considerable insight into possible ways of rethinking our theoretical understanding of these patterns.

Correspondence: Kaaren Bekken, PhD, CDN, 76 Bedford St, Ste 12, Lexington, MA 02420.E-mail: kbekken@gmail.com

M. LLANERO, J. RUIZ, P. MONTEJO \& M. MONTENEGRO. Preliminary data to standardize an easy-administration Go-NoGo Task in normal aging, mild cognitive impairment, and Alzheimer's disease.

Objective: Cognitive deficits can be detected several years before the clinical diagnosis of dementia when mild cognitive impairment is observed. Go/NoGo decision-making evaluation may be a key factor in early diagnose of mild cognitive impairment and Alzheimer's disease, as a measure of inhibition of automatic responses.

Participants and Methods: In this study, we present data of an easyadministration Go/NoGo Task on old adults without cognitive deficit, mild cognitive impairment patients and early Alzheimer's disease diagnosed patients. In a first part, participants must knock twice on the table when examiner knocks once and they must knock once when examiner knocks twice. In a second part, participants must knock again twice when examiner knocks once (1-2 trials) but they must stand still when 
examiner knocks twice (2-0 or inhibition trials). We set and administered the same sequence in both parts. It consisted in twenty trials (ten of each type of trial).

Results: The results indicate that there are significant differences in number of errors in the first part of the test. No cognitive impairment participants are less prone to errors than mild cognitive impairment patients and Alzheimer's disease patients. In the second part test there are also differences in both types of trial between no cognitive impairment, mild cognitive impairment patients and Alzheimer's disease patients.

Conclusions: These results show how our simple Go/NoGo Task is useful to evaluate inhibition as one of the cognitive process that must be assessed in order to early diagnose cognitive impairment and its severity since it discriminates between mild impairment and Alzheimer's disease. Future directions include standardization for the Spanish of the sequence and the instructions designed for this study.

Correspondence: Jose Maria Ruiz, Universidad Complutense de Madrid, C/ Porto Lagos, 7, Alcorcón, Madrid 28924, Spain. E-mail: jm.ruiz. sdl@gmail.com

G. Llebaria, J. PAgonabarraga, C. garcía, B. PASCUALSEDANO \& J. KULISEVSKY. Utility of the Mattis Dementia Rating Scale for screening dementia in Parkinson's disease. Validation of the instrument in a Spanish population.

Objective: Parkinson's disease (PD) patients exhibit cognitive impairment (PD-ND) which may progress to dementia (PDD). PD-ND and PDD mainly involves dysexecutive-attentional deficits. Most neuropsychological batteries for dementia screening rely in tasks related to cortical dysfunction, while frontal-subcortical deficits may be overlooked. The Mattis-Dementia-Rating-Scale (MDRS) measures the degree of frontal-subcortical defects. Although MDRS has been validated as a PD cognitive functioning screening test, its utility for screening PDD is unknown. We aim to validate MDRS for diagnosis of PDD in a Spanish population-based sample.

Participants and Methods: Performance of MDRS to 90 prospectively recruited Spanish PD patients (60 PD-ND, 30 PDD) fulfilling UK-PDSBB criteria. Dementia was diagnosed according to DSM-IV-TR and Clinical-Dementia-Scale $(\mathrm{CDR})>1$. Univariate, logistic regression and ROC curve analysis were calculated.

Results: Regression analysis showed MDRS total scores to independently differentiate PD-ND from PDD $(p<0.001)$, despite influence of age and education in the univariate analysis. The area under the ROC curve was 0.98 , indicating that the MDRS has an excellent discriminant ability to diagnose PDD. A cut-off score of $<=123$ had to yield high sensitivity (93\%), specificity (93\%), and positive (88\%) and negative $(93 \%)$ predicitive values.

Conclusions: To our knowledge, this is the first study to formally assess the MDRS as a useful screening test in PDD. Our sample shows the MDRS as a good scale to provide an objective measure to distinguish PD-ND from PDD. The cut-off score estimated in our sample is quite similar to that observed in American population samples, which points to the cross-cultural applicability of MDRS.

Correspondence: Gisela Llebaria, Neurology, Hospital de la Santa Creu i Sant Pau, Sant Antoni Maria Claret 167, Barcelona 08025, Spain. Email: GLlebaria@santpau.es

E.C. MACEDO, K. LUKASOVA, A.C. BARBOSA \& C. KURIYAMA. NEUROPSYCHOLOGICAL ASSESSMENT OF DEVELOPMENTAL DYSLEXIA: PSYCHOMETRIC TESTS AND EYE MOVEMENTS.

Objective: Developmental dyslexia, specific impairment of reading ability, has large scale of cognitive and behavior traits. A multiple case study was conducted to assess the cognitive skills frequently reported as impaired in dyslexic population: phonological awareness, automatic naming, vocabulary and motor skills.
Participants and Methods: Ten dyslexic children and 10 control children with normal reading acquisition were assessed in individual evaluation. The two groups were match on age (mean age of 10.63), grade ( 4.42 years of education) and sex ( 8 boys and 2 girls). The inclusion criteria guaranteed a normal intelligence and sustained attention skills. The assessment was divided in two parts. In the first part, all children were administered battery of the following psychometric tests: Phonological Awareness Test, Automatic Object Naming Test, Automatic Category Naming Test, Peabody Picture Verbal Test, Verbal Fluency, Token Test and Perdue Pegboard Test. In the second part of evaluation, eye movements were recorded in a simple task of vertical point pursue.

Results: Between group comparison revealed significant impairment $(p<0.05)$ of dyslexic children in phonological awareness and automatic naming. No significant differences were found for vocabulary and motor skills. Analysis of eye pursue data showed a significant deficit ( $p=0.03)$ of dyslexic for the number of correct pursues to the left side. Results support theories of phonological deficit in developmental dyslexia in our carefully selected and matched dyslexic population. However, the results of eye pursue indicate that even in this pure phenotypic group there is a subtle trait of impairment in motor or executive function.

Conclusions: Overall, this study acknowledges the importance of procedures that integrate neuropsychological evaluation with other assessment tools such as eye movement register in complex developmental disorders.

Correspondence: Elizeu C. Macedo, Mackenzie University, $R$. Consolacao, 896. Sala 62. Amantino Vassao, Sao Paulo 01302-907, Brazil.E-mail:elizeumacedo@uol.com.br

\section{L.F. MALLOY-DINIZ, P.P. MORAIS, D. FUENTES \& A. BECHARA.} Brazilian Adaptation of Iowa Gambling Task.

Objective: Iowa Gambling Task (IGT) is a neuropsychological task developed to assess decision-making in several contexts, taking into account neuropsychological and neuropsychiatric conditions. The task was developed in English. In spite of its easy comprehension, the use by individuals of other mother tongues could be impaired if they have cognitive/intellectual deficits, low educational and social profile. The aim of this work is to adapt IGT to Portuguese language (IGT-br) and compare it with the original English version.

Participants and Methods: The IGT-br of the test was developed in Windows environment. However, it maintains the same visual characteristics and schedule of reinforcement/punishment used in the English version. Fifty Brazilian adults were assessed and divided in two groups: 1) Twenty-five individuals with Proficiency Certificate in English (17 women and 08 men) tested on IGT and 2) twenty-five Brazilian adults who do not speak English (15 women and 10 men) tested on IGT-br. Both groups were paired by age, intellectual level and educational level. We used Pearson Test-T for independent samples to compare the results of the groups.

Results: Differences between the groups mentioned above were not observed, in terms of amount of choices on the different card decks and on the pattern of choices along five successive blocks of 20 trials. Our results are similar to the ones described in literature concerning normal adults.

Conclusions: The equivalence between IGT and IGT-br leads to the conclusion that this last version can be applied for research purposes in the Brazilian context. This suggests that cultural differences do not impair the application/interpretation of the test in Brazil. Information about use and License of any IGT's version can be obtained with Prof. Antoine Bechara (Iowa University).

Correspondence: Leandro F. Malloy-Diniz, PHD, University Human Sciences Faculty, Universidade FUMEC, Rua Gonçalves Dias, 31 Bairro Funcionários, Belo Horizonte 30130090, Brazil. E-mail: leandro. fernandes@terra.com.br 
L.F. MALLOY-DINIZ, E.P. NASSIF, A.M. LEVY, W.B. LEITE \& D. FUENTES. Performance of Brazilian Children on the Tower of London Test: comparison by age, gender and scholar attendance.

Objective: This study aims to obtain norms for the Tower of London (TOL) Test for Brazilian Children between four years and six months and eight years of age. Furthermore, the research evaluates the relationship between environmental factors (type of school), age and gender and the performance on the test.

Participants and Methods: The study assessed 347 Brazilian children (175 girls and 172 boys) from public $(n=161)$ and private $(n=186)$ schools aged between 4 and 8 years. For such, Kricorian version of TOL was used. Children were divided in seven different groups for six months. Children with history of neurological and psychiatric disturbances and with intelligence bellow $25 \%$ tille measured by Raven's Colored Matrices were excluded. General Linear Model Univariate analysis was performed by the use of age group, gender, and school type as independent variables and TOL score as a dependent variable.

Results: Differences related to gender an age at the performance on the TOL were not found $[\mathrm{f}(1.969)=30.673 ; \mathrm{p}=.161$ and $\mathrm{f}(.228)=3.611$; $\mathrm{p}=.967$ respectively]. Nonetheless, we found statistically significant differences between the two types of educational background on TOL performance $[\mathrm{f}(25.682)=374.397 ; \mathrm{p}<.0001]$ with children of from public schools performing worse than the ones from private schools. No interaction was observed between factors (age, gender and school type).

Conclusions: The lack of difference between age groups can be explained by the ceiling effect on this version of TOL. Despite this lack of age differences between age groups, our results point to the importance of environment (type of school) in the development and assessment of planning ability.

Correspondence: Leandro F. Malloy-Diniz, PHD, University Human Sciences Faculty, Universidade FUMEC, Rua Gonçalves Dias, 31 Bairro Funcionários, Belo Horizonte 30130090, Brazil. E-mail: leandro. fernandes@terra.com.br

M. MARTIN ARAGONESES, R. LÓPEZ-HIGES, D. DEL RÍO GRANDE \& S. RUBIO VALDEHITA. A Cognitive Approach to the Assessment of Sentence Comprehension: the ECCO Test.

Objective: The aim of this work is to explain the structure, properties and applications that the ECCO test offers to potential user, both for the assessment of normal subjects and for subjects with language impairments. Participants and Methods: We collected data of 2238 subjects distributed from six to 85 years old. ECCO (Exploración Cognitiva de la Comprensión de Oraciones; Cognitive Assessment of Sentence Comprehension; López-Higes, del Río \& Fernández, 2005) is a computerized test which allows the assessment of sentence comprehension in Spanish using a wide variety of structures and a simple verification sentencepicture task. It also includes subtests to explore subjects' knowledge about the lexical items used in the sentences, and their verbal working memory span.

Results: The analysis of the main subtest (Sentence Comprehension, $\mathrm{SC}$ ) revealed that the most of the items are easy elements -this has a high reliability both global and through the age groups, but that it includes items -lexical and syntactic foils, which are particularly discriminative or informative. The information function showed that the SC subtest is slanted towards middle and lower trait levels.

By dissociating the propositional density and the syntactic complexity effects, the subtest is sensitive to the dissociation between Broca's aphasic and Alzheimer disease patients.

On the other hand, the Lexical Knowledge subtest provides very useful information when it is applied to subjects with language impairment, because it allows determining if the difficulties in sentence comprehension have a lexical or syntactic base.

Conclusions: These results provide only an example of the wide range of possibilities we believe this new test offers for the assessment of sentence comprehension abilities and for research purposes in Spanish language.
Correspondence: Maria Teresa Martin Aragoneses, Dpto. Psicología Básica II (Procesos Cognitivos). Facultad de Psicología, Universidad Complutense de Madrid, Despacho 1105 C. Buzón 25. Campus de Somosaguas., Pozuelo de Alarcón 28223, Spain. E-mail: mt.m. aragoneses@psi.ucm.es

M. MONTENEGRO, P. MONTEJO, J. RUIZ, M. LLANERO, E. DE ANDRES, A. GARCIA, A. REINOSO \& E. GARCIA. Discriminant analysis on screening dementia tests in the Spanish: usefulness to early diagnose mild cognitive impairment and Alzheimer's disease. Objective: Mild cognitive impairment has been suggested as a term for a boundary area between normal aging and dementia, especially Alzheimer's disease. Inherent in the transition from normal aging to dementia is cognitive decline, which can be early diagnosed using multiple assessments. As one of the most important tools when assessing early cognitive changes is neuropsychological evaluation, screening tests were evaluated to determine which test is more useful to diagnose mild cognitive impairment and Alzheimer's disease.

Participants and Methods: Spanish participants were assessed with Mini Mental State Examination (MMSE), 7 Minute Screen (temporal orientation test, enhanced cued recall, clock drawing, and verbal fluency), Word List of Wechsler Memory Scale (WMS-III) and Geriatric Depression Scale (GDS). Epidemiological data including age, sex, education and family status, is also analyzed.

Results: The results indicate that tests (and items) assessing new learning and delayed recall seem to provide valuable information for screening and diagnosis of mild cognitive impairment and Alzheimer's disease. Neither epidemiological data nor depression show statistical differences. Conclusions: Reliable detection of mild cognitive impairment is important in determining, amongst others, the efficacy of emerging treatments. Cognitive assesment must include an acurate memory evaluation considering that learning and delayed recall items are the best predictors of clinical outcome.

Correspondence: Jose Maria Ruiz, Universidad Complutense de Madrid, C/ Porto Lagos, 7, Alcorcón, Madrid 28924, Spain. E-mail: jm.ruiz. sdl@gmail.com

T. OLIVARES, J. BARROSO, G. RAMÍREZ, A. NIETO \& Y. PÉREZ. Verbal fluency: practice effects and use of alternate forms in serial neuropsychological assessment.

Objective: Two alternate versions of phonetic and semantic verbal fluency were examined to evaluate interform equivalence and temporal stability for serial assessment.

Participants and Methods: 92 healthy subjects (34male, 58 female) were evaluated in two sessions (T1-T2) with a six months interval. Two versions of a letter fluency task (FAS vs TER) and semantic fluency (animals vs vegetables) were administered. The order of test presentation was counterbalanced such that 29 participants (11 male, 18 female) with a mean age of 21,9 (sd: 5,22 ) were administered version A first (FAS/Animals), and 27 participants (11 male, 16 female) with a mean age of 22,8 (sd: 4,43) received version B first (TER/Vegetables). Also two groups of 18 participants received the same version at the retest. Results: There were no significant differences between the two versions of phonetic fluency. Form effect: $(F(3,84)=1,63 ; p=0.19)$. A significant effect of the moment of assessment was found with a better performance for the second evaluation (T2): $(F(1,84)=10,6 ; p=0.002)$. Form by Moment interaction was not statistically significant $(\mathrm{F}(3,84)=1,32 ; \mathrm{p}=0,27)$. With regard to semantic fluency, a significant Form by Moment interaction was found $(F(3,84)=8,2 ; p=0.0001)$ indicating a best performance on animals category related to vegetables category when both measures were used first (T1).

Conclusions: Our results indicate the equivalence between the two phonetic triads but a significant practice effect in all measures independ- 
ently of the order of test administration. Performance on semantic fluency calls in to question the interform equivalence. This should be taken into account when used in longitudinal testing.

Correspondence: Olivares Teresa, Doctor, Psychobiology, Faculty of Psychology, Campus de Guajara, La Laguna. Tenerife 38205, Spain. Email:tolivarp@ull.es

O. PINO, G. GUILERA, J.E. ROJO, J. GÓMEZ, T. DÍEZ, J. REJAS, M. FRANCO, M.J. CUESTA, R. TABARES, B. CRESPO-FACORRO, M. BERNARDO, E. VIETA, N. SEGARRA, A. MARTÍNEZ-ARÁN \& S.E. PURDON. Is the SCIP-S an adequate tool for a neuropsychological brief assessment?

Objective: The Screen for Cognitive Impairment in Psychiatry (SCIP; Purdon, 2005) is a brief assessment tool designed for evaluation of neuropsychological impairment in psychiatric disorders. The SCIP requires less than 15 minutes and it has three alternate forms to minimize practice effects over repeated testing. Each form consists of five subscales for the assessment of immediate verbal list learning, working memory, verbal fluency, delayed list learning and visuomotor tracking. The main objective of the present study is to assess the criterion validity of the Spanish version of the SCIP (SCIP-S; Pino et al., 2006) in comparison to a neuropsychological battery.

Participants and Methods: The SCIP-S and neuropsychological battery were administered to a total of 203 patients, 126 with schizophrenia and 77 with bipolar disorder type I. The neuropsychological battery included subscales from the WAIS-III (speed of processing, working memory and vocabulary), WMS-III (list of words I and II), TMT (A and B), and semantic fluency.

Results: Correlations between the SCIP-S and neuropsychological tests were suitable in all subscales of the SCIP, ranging from 0.36 (between the SCIP working memory subtest and the WAIS-III Letter-Number scale) to 0.59 (between the SCIP verbal immediate memory and RAVLT).

Conclusions: The results demonstrate that the SCIP-S is a good instrument to screen the neuropsychological status of psychiatric diseases in terms of verbal memory, working memory and speed of processing. Correspondence: Oscar Pino, Psychiatry, Granollers Hospital Foundation - Benito Menni CASM, Francesc Ribas s/n, Granollers Barcelona 08400, Spain.E-mail: opino1@gmail.com

J. RUIZ, M. LLANERO, M. MONTENEGRO \& P. MONTEJO. Early Alzheimer's disease diagnose and mild cognitive impairment: How useful is the Trail Making Test in the Spanish?

Objective: The Trail Making Test (TMT) is an accessible neuropsychological instrument that provides information on a wide range of cognitive skills, but primarily it is a test of motor speed and visual attention. In Trail Making, Part A, the subject's task is to quickly draw lines on a page connecting 25 consecutive numbers. In Part B, the subject must draw the lines alternating between 12 numbers and 12 letters.

Participants and Methods: Both parts of the standard Trail Making Test time and errors were assessed in a Spanish sample (old adults without cognitive deficit, mild cognitive impairment patients and early Alzheimer's disease diagnosed patients).

Results: The results show that participant's time to finish both parts was significantly different. Pairwise comparisons show that old adults without cognitive impairment are faster in both parts than cognitive impairment patients considering them as a unique group of participants. No differences were found on time between mild cognitive impairment and Alzheimer's disease patients.

Number of errors also shows differences between groups. Pair wise comparisons show that that the no cognitive impairment group is less prone to errors than cognitive impairment patients. Again, no differences were found on number of errors between cognitive impairment groups. Neither was found for time nor errors in age, sex and academic education.
Conclusions: These results indicate that TMT is a useful test to asses cognitive impairment in Spanish patients and that there are no differences between mild cognitive impairment and Alzheimer's disease recently diagnosed patients. We propose that actual scoring (time and errors) does not differentiate between levels of cognitive impairment. That supports the idea that functional assessment is the most accurate way to diagnose Alzheimer's disease.

Correspondence: Jose Maria Ruiz, Universidad Complutense de Madrid, C/ Porto Lagos, 7, Alcorcón, Madrid 28924, Spain. E-mail: jm.ruiz. sdl@gmail.com

I. SANCHEZ-CUBILLO, J.A. PERIAÑEZ, M. RÍOS-LAGo, D. ADROVER, J.M. RODRÍGUEZ-SÁNCHEZ, J.I. QUEMADA, F. BARCELÓ, J. TIRAPU \& B. CRESPO-FACORRO. The validity of Trail Making Test in assessing executive functions: contributions of cognitive and perceptual factors.

Objective: Some criticism surrounds the TMT entail its utility as a measure of executive functioning. Whereas it has been widely accepted that TMT is one of the most sensitive general indicators of the presence of brain dysfunction, there has been controversy about the specificity of the test to address distinct lesional or functional alterations. The main purpose of the present study was to examine whether TMT B or any of the commonly used derived scores provide information about cognitive control relative to motor and perceptual factors.

Participants and Methods: 47 healthy subjects were included in the study. The different weights of switch-cost, motor speed, speed of perceptual processing and working memory were studied in relationship to their performance in TMT and its derived scores proposed elsewhere. Correlation and regression analyses were used.

Results: The results suggest that TMT-A is influenced by perceptual processing and working memory and part B by working memory, perceptual processing and switch-cost. Motor speed did not predict performance in any raw or derived scores. On the other hand, the derived $\mathrm{B} / \mathrm{A}$ score, described as more useful in measuring executive control in part B than any other derived score, did not correlate with switch-cost measures. Possible explanations for these results are discussed and recommendations for its clinical use are proposed.

Conclusions: TMT seems a valid measure of executive functions. The main constructs assessed by TMT are working memory, perceptual processing and task switching. Motor speed does not seem to influence TMT performance. The derived scores seem useful as clinical measures of executive functioning.

Correspondence: Ignacio Sanchez-Cubillo, Brain Damage Unit, Aita Menni Hospital, Egaña 10, Bilbao 48010, Spain.E-mail: sanchez-cubillo@gmx.net

\section{M.A. SEDO'. MULTIPART MODELS PRODUCE VISUAL SEGMENTATIONS AND VERBAL CONFABULATIONS.}

Objective: "Multipart models" (MPM) are unusually confusing to subjects who cannot perceive whole images (right hemisphere dysfunctions, absence of transient macrocellular images in dyslexia); or to subjects who build verbal associations with little spatial support, generating "verbal analogs" and "giving themselves verbal directions for its reproduction" (Koppitz).

Participants and Methods: In an urban school system, we administered the 27-model Beery Developmental Visual-Motor Integration Test and the 40-model "Test of Alternative Processing of Spatial Images", or T.A.P.S.I.: an experimental sequence of MPMs, covering the ages of 3 to 14 . We tested 50 successive needy elementary students referred for special education evaluation.

Results: As expected, there was a very large correlation between both measures (.75); and MPMs triggered a much larger number of spatial "fragmentations" and verbal "confabulations", even at the erliest age levels. 
Conclusions: The answers obtained suggest that in some subjects "language is an organ of perception much more than a means of communication" (Jaynes), and that a test exploring this kind of alternative processing may offer important insights about the alternative ways of processing spatial-motor-conceptual information described by Mishkin \& Ungerleider since 1982 and Paivio since 1986.

Correspondence: Manuel A. Sedo', Ph.D., Test Development, MultiLingual Testing, 9 Ingleside Rd., Natick, MA 01760. E-mail: manuel@sedo.net

M.A. SEDO', A. BILBAO \& M. SUEIRO. "FIVE DIGIT TEST": A DIGITAL VERSION OF THE STROOP TEST APPROPRIATE FOR MULTICULTURAL USE.

Objective: "FIVE DIGIT TEST" is a digital adaptation of the Stroop Test which relies minimally on past learning and experience and language mastery. Based on the concepts from "one" to "five", it alternates between the activities of reading and counting "groups of signs" presented like the signs on playing cards. The test uses reading and counting for the production of serial naming tasks that are either "automatic" or "controlled": that is, casual and effortless, or effortful and self-controlled mental decisions. Parts I and II explore "automatic" responses, and include reading the values of digits, or counting quantities of stars. On Parts III and IV ("controlled" reactions) the groups of digits are presented in non-corresponding quantities (five $4 \mathrm{~s}$, four $3 \mathrm{~s}$ ), and the tasks require the inhibition of reading responses so as to count the "quantities" of digits seen; or impose frequent switching between the activities of reading and counting.

Participants and Methods: Bilbao studied 348 subjects (ages 7 to 17), and Sueiro studied 331 subjects (ages 17 to $80+$ ), representing the spanish population.

Results: "Automatic" scores reflected the acquisition of cognitive resources early in life (7 to 17) and its eventual decline late in life (after $60)$, with very similar scores at all adult ages. "Controlled" situations showed dramatically higher effort and diminished speed.

Conclusions: FDT provides easy objective measures of cognitive performance which should help detect neurological dysfunctions in a reliable, valid, and multiculturally appropriate way.

Correspondence: Manuel A. Sedo', Ph.D., Test Development, MultiLingual Testing, 9 Ingleside Rd., Natick, MA 01760. E-mail: manuel@sedo.net

\section{M.A. SEDO' \& P. MORAGA. “KIDDYWORDS”: FIVE LITTLE STEPS ON THE LEXICAL ROAD.}

Objective: "KIDDY WORDS" was developed from two experimental lists exploring early childhood lexical acquisition (Carroll y White, 1973: Stoddard y Vondergaard, 1980). These lists helped us lower the floor of the "BOSTON NAMING TEST" (Goodglass, Kaplan \& Weintraub, 1991). by providing "chronological markers" for the ages of 2 to 6 and adding density at its lower age levels. "KIDDY WORDS" consists of five 9-item pages corresponding to the lexical expectations for each chronological year

Participants and Methods: This adaptation used a panel of experts to better fit the local language style, and uses "only" the 9 items appropriate to a child's age, which reduces the administration time to some 2 minutes: 85 children were tested at each age level.

Results: Test-retest reliability was .81 to .90 in 4 of the 5 age intervals; and test means were 13 to 17 (of a total possible 18 points) at each age level, showing thus good homogeneity for all age levels. We preserved a "qualitative" scoring based on clinical observations, and on the fresponses cues provided by the examiner in case of failure: 1) an action. use or context: or 2) the initial syllable of the target word.

Conclusions: Even at very early ages, one single page can help screen the lexical development of the child, and observe the lexical access to words through "functional or contextual" associations or through the "compensatory" use of phonological cues.

Correspondence: Manuel A. Sedo', Ph.D., Test Development, MultiLingual Testing, 9 Ingleside Rd., Natick, MA 01760. E-mail: manuel@sedo.net

C. SKILBECK, L. ALLEN, D. BRECHIN \& J. PRICE. The Factor Structure of the Coughlan Adult Memory \& Information Processing Battery (AMIPB).

Objective: Although the AMIPB has been used routinely since 1985, research on its structure is lacking. The current study objective was to provide such research. A 3-factor structure was hypothesised: 'Verbal Learning', Visual Learning', and 'Information Processing'.

Participants and Methods: Scores from 367 neurological patients who attended for routine neuropsychological assessment and completed AMIPB form 1 were entered into a Principal Components Analysis (PCA). with Varimax Rotation. Factors were identified using an initial Eigenvalue $>1.0$. Data on age, sex. HADS, IQ, and NART IQ were also examined in relation to factor scores.

Results: PCA yielded 4 factors (64.3\% of total variance): 'Verbal Learning' (Factor 1; 23.7\%), 'Visuospatial Learning' (Factor 2; 20.6\%), 'Information Processing' (Factor 3; 12.6\%), and 'Intrusions' (Factor 4; $7.4 \%)$. Age correlated significantly with factor scores, from $\mathrm{r}=+.33$ ('Intrusions') to $\mathrm{r}=-.39 /-.42$ (factors 1-3). NART IQ and obtained FSIQ correlated poorly $(<.25)$ with all factors. However, (NART IQ - obtained FSIQ) discrepancy correlated significantly with factors 1-3 (-.39 to $.42)$, with a low correlation (+.18) with 'Intrusions'. Negligible correlations were found between the HADS and the factors.

Conclusions: Results suggest the AMIPB has a 4-factor structure. $\mathrm{Hy}$ pothesised factors were noted, with an unpredicted fourth factor, 'Intrusions'. Weak correlations between the factors and NART IQ should reassure clinicians about the AMIPB's utility, and overall effects of mood also appear to be minimal. Factor sensitivity to acquired cognitive dysfunction is supported by correlations between factors and (NART IQ obtained FSIQ) discrepancy.

Correspondence: Clive Skilbeck, PhD, psychology, university of tasmania, university of tasmania, sandy bay campus, Hobart, TAS 7001, Australia.E-mail: clive.skilbeck@utas.edu.au

E. WALLIS, E. WOLFF, R. SUGARMAN, D.L. ROWE \& E. GORDON. The Neuropsychological profile of Diffuse Traumatic Axonal Injury using an Automated Test Battery: Insights from the Brain Resource International Database.

Objective: To identify the key cognitive markers of injury within the neuropsychological profile of patients who have sustained significant diffuse traumatic axonal injury using an automated test battery. Patients with traumatic brain injury (TBI) present with a wide range of deficits across the core domains of cognitive functioning, including sensorimotor capacity, attention, memory, executive planning, emotional recognition and aspects of language functioning.

Participants and Methods: 29 patients with a history of significant TBI, defined by Loss of Consciousness exceeding 24 hours and the presence of Post Traumatic Amnesia (PTA) were sourced from the only international, standardized database of the human brain, the Brain Resource International Database (BRID). They were compared with matched controls using integrated and converging evidence across core domains of affect, cognition and Event Related Potentials (ERP's). Z scores were computed to delineate parameters outside of normal performance.

Results: A convergent model including measures of affect (increased depression) and cognition (decrease in working memory, decrease in speed of information processing, decrease in planning ability, decrease in language functioning and decrease in attentional domains) was found to reliably identify the key deficits associated with Traumatic Brain Injury at $\mathrm{Z}$ scores $=-1.0$ and worse. 
Conclusions: The use of an automated battery thus demonstrates the potential to reliably identify biomarkers for personalized medicine, with psychophysiological data adding to the specificity of already sensitive markers.

Correspondence: Elizabeth Wallis, MSocSci, MAP, Brain Lab, Sunninghill Hospital, 4 Saxonwold Mews, Waltham Road, Johannesburg 2196, South Africa. E-mail:wallis@wallishardy.co.za

\section{N.J. WILDE \& E. STRAUSS. Effects of Stimulus Repetition and Recall Order on Spatial Span Performance.}

Objective: The WMS-III Spatial Span subtest includes forward (FS) and backward span (BS) trials. BS is thought to be more difficult and more demanding of working memory because of the requirement to reorder the stimuli. However, previous studies have shown that Spatial BS scores are often equal to or greater than FS scores (Wilde et al. 2002. 2004). In contrast, Digit BS scores are as a rule substantially lower than FS scores. However, the design of the Spatial Span subtest confounds the ability to evaluate the cognitive demands of FS vs. BS. For one, the same stimuli are used in the FS and BS trials. In addition, FS always precedes BS. The goal of this study was to disentangle these possible confounds by investigating the effects of stimulus repetition and recall order on FS and BS performance.

Participants and Methods: 150 undergraduates (114 women, 36 men) participated in one of five experimental conditions. Each subject was administered two trials (FS and BS). The following between-subjects parameters were evaluated: Stimulus Repetition (same or different stimuli used in FS and BS), Recall Order (FS then BS, BS then FS), and Stimulus Set (to control for differences in stimulus difficulty).

Results: Results did not differ when stimulus set was controlled for. eliminating the need to discuss this possible confound. There were significant interactions between Order, Repetition and BS. Specifically, when BS followed FS, BS scores were higher when the stimuli were repeated. However, BS scores were significantly higher when they preceded FS than when they followed FS. FS scores were unaffected by the effects of order and repetition.

Conclusions: Results imply a facilitative effect of repeating the FS stimuli on subsequent BS scores. This has implications for clinical interpretation of the WMS-III subtest. Interestingly, BS scores were substantially higher when they were administered before FS, suggesting an interfering effect of FS on subsequent BS.

Correspondence: Nancy J. Wilde, PhD, Psychology, University of Auckland, Tamaki Campus, Private Bag 92019, Auckland 1142, New Zealand.E-mail:n.wilde@auckland.ac.nz

\section{Cross-Cultural Issues}

M. LAUTERBACH, E. MENKE, B. DIAS, C. LOUREIRO \& J. LOPES. Writing Acquisition in a Bilingual Setting: Mutual Interferences of Portuguese and German.

Objective: Children that attend a bilingual school learn simultaneously two different phoneme-grapheme correspondence systems (PGC$\mathrm{S})$. Hence they have to deal with both, intra- and inter-language ambiguities of PGC-S. This additional demand can lead up to to specific interference errors.

The aim of this study is to investigate the interference of the PGC-S of German and Portuguese during writing acquisition.

Participants and Methods: 128 pupils of the German School in Lisbon and 107 monolingual Portuguese pupils, attending 2nd to 4th grade, participated in the study. Children were classified in three language proficiency groups: bilingual, German and Portuguese dominant.

The stimulus material consisted of three lists of 25 words each: German and Portuguese nouns and a list of pseudo-words. Pseudo-words had to be written twice, in German and in Portuguese. Stimuli were digitally played back for dictation.
The dependent variables were the number of correct answers and the type of orthographic error. Results were analyzed statistically with SPSS software.

Results: Generally Portuguese monolinguals performed better in the word $(\mathrm{t}=-6,708$, df 233, $\mathrm{p}<.001)$ and in the pseudo-word $(\mathrm{t}=-5,501$, df $233, \mathrm{p}<.001)$ list than the pupils of the German school. This difference decreases as children become older.

Bilinguals performed in all lists similarly as the Portuguese dominant group of the German School (no significant differences).

Interference errors were observed mainly in the Portuguese word list for all language dominance groups $(t=10,126$, df 127, $\mathrm{p}<.001)$.

All pupils of the German School performed better on the German pseudoword list as compared to the Portuguese one $(t=-2,799$, df 127, $\mathrm{p}=.006)$. Conclusions: The language of instruction exerts a strong effect on the writing proficiency of bilingually taught children. Bilingual children behave on all lists as their Portuguese dominant peers, thus they assimilate the language of the community.

Correspondence: Martin Lauterbach, Faculty of Medicine Lisbon, Hospital Santa Maria, Lisbon 1649-028, Portugal. E-mail: martin. lauterbach@sapo.pt

\section{RAMÍREZ \& F. OSTROSKY-SOLIS. SELF-AWARENESS AND CULTURAL INFLUENCE IN A NORMAL SAMPLE.}

Objective: Impaired self-awareness (ISA) is presented in many psychiatric disorders as well as brain lesions and pathological diseases. Different scales have been developed to measure ISA in populations with brain injury, Alzheimer, or schizophrenia. Patient Competency Rating Scale (PCRS), developed by Prigatano \& Fordyce, is the most used scale to measure ISA in traumatic brain injury patients. Some cross-cultural studies has been realized, however data on a normal Latin-American population no exist. The purpose of the present investigation is standardizing the Patient Competency Rating Scale in a normal Mexican Spanish-speaking population neurological and psychiatric intact.

Participants and Methods: The PCRS was administered individually to 286 volunteers' participants (16-70 years old), with a range of 6-20 years of education, 181 female and 105 male. Sample was divided into a control group (who's answered the patient's scale version) and an informant group (relatives, friends or acquaintances that answered the relative's version scale).

Results: Total PCRS' score obtained by the control group was $116.89 \pm 13.36$ (range $=89-149$ ), for the informant group total score was 18.23 \pm 14.23 (80-149). Cronbach's alpha for PCRS(P) was 0.8845 and for the informant group PCRS(R) was 0.8976. The factorial analysis grouped the data on 4 factors: daily live activities, interpersonal, cognitive and emotional-cognitive. This factors were analyzed with an structural equations modeling.

Conclusions: Despite it is a sample of neurologically intact participants, the total score on PCRS was significantly different of the score previously cited by other authors. The study shows the cultural influence on the estimation of our own abilities.

Correspondence: Maura Ramírez, National Autonomus University of Mexico, Av. Universidad 3004 Col. Copilco-Universidad, Mexico, D.F. 04510, Mexico.E-mail:maujaraf@yahoo.com.mx

J. SALVADOR, G. ACLE \& C. ARMENGOL. Analysis the use or presence of self regulation when otomi children carried out a reading comprehension activity with expositive texts.

Objective: Analyse the use or presence of self regulation when 16 otomi of fifth grade of elementary school carried out a reading comprehension activity with expositive texts; they assisted to a monolingual spanish elementary school.

Participants and Methods: The participants were evaluated with a semiestructured clinical interview and a verbal prtocol in a thinking 
aloud mode, they were trained to verbalize what they were thinking before, during and after the readingactivity. The children's teacher was also interviewed to get information about his own knowledge of reading comprehension and sef regulation and how he used them in daily activities at the class.

Results: The findings were organized in those categories that reflected the different sel regulation components: votion, planning, action proposal, verification and evaluatio. Internal factors as beliefs, previous knowledge activation and situated cognitionas well as external factors: reding conditions and the learning method of the reading activity were considered.

Conclusions: Relevant findings showed that volition was the main component activated of self regulation. The previous knowledge activation and situated cognition were those internal factors that contribute ina a significant way to achieve reading comprehension; they are used by the children to regulate the cognitive activity with their own rural context while they are reading. Otomi children repeated in a consistent manner the reading method in which they learn to read, external valuations are more privileged than the acquisition of self regulation process, from here, the big challengers to propose.

Correspondence: Judith Salvador, Ph D, Posgrado, UNAM, Cda. Gorriòn 17. Col Rinconada de Aragon, Estado de Mexico 55140, Mexico. E-mail: jsc@servidor.unam.mx

L. SAN MIGUEL-MONTES, M. MARGARIDA, M. DIAZ, E. SENIOR, L. DORNA, D. PEREZ \& A. PUENTE. Verbal Memory and Executive Functions of Puerto Ricans with Parkinson's, Traumatic Brain Injury, Epilepsy, Mild Cognitive Impairment, Dementia and Cerebrovascular disease.

Objective: Considering the lack of validated neuropsychological tests for our Puerto Rican population this study aimed to develop empirical data for measures of verbal memory and executive functions.

Participants and Methods: Our cross-sectional study reviewed the records of 264 neurological patients, both male $(64 \%)$ and female $(46 \%)$ with a mean education of 12.4 years $(\mathrm{Sd}=3.2)$. Patients were subdivided into seven groups of Parkinson's (30), Epilepsy (20), TBI (90), MCI (34), Dementia (35), Depression and CVA (30), at the Neurology Clinics- University of Puerto Rico Medical School. Records were reviewed confidentially with IRB approval. Neuropsychological data included: WMS-Logical Immediate, WMS- Delayed, RAVLT-Total, Immediate and Delayed, BNT, FAS, Trails-A and Trails-B. Descriptive analyses used SPSS.

Results: Parkinson's group showed lower scores on FAS ( $\mathrm{m}=26$; $\mathrm{Sd}=7$ ), WMS-Immediate $(\mathrm{M}=8 ; \mathrm{Sd}=6)$, Trails A $(\mathrm{M}=87 ; \mathrm{Sd}=72)$ and (Trails B $(\mathrm{M}=246 ; \mathrm{Sd}=162.8)$. Epilepsy obtained the lowest scores on FAS $(\mathrm{M}=18 ; \mathrm{Sd}=8.3)$, and WMS Logical-Delayed $(\mathrm{M}=9 ; \mathrm{Sd}=6.97)$. TBI scored $(\mathrm{M}=33 ; \mathrm{Sd}=12.5)$ RAVLT-Total and $(\mathrm{M}=6 ; \mathrm{Sd}=4.0)$ WMS-Delayed. Depression patients showed better scores on WMS-Delayed (12.10; $\mathrm{Sd}=6.06)$. MCI revealed RAVLT-Delayed $(\mathrm{M}=6.71 ; \mathrm{Sd}=4.6)$, Dementia obtained (RAVLT-Delayed of 3.8; $\dot{M}=4.3$ ). CVA group scored $(\mathrm{M}=217.16 ; \mathrm{Sd}=65.9)$ on Trail B.

Conclusions: In summary, Puerto Rican Parkinson's patients present difficulties in executive functions, Epilepsy present delayed verbal memory deficits but spared for verbal learning. TBI patients presented difficulties for verbal learning and delayed verbal memory. Depression patients had difficulties in verbal learning but not on delayed verbal memory. MCI group presented verbal learning deficits, however, Dementia patients were the most impaired in verbal learning, delayed verbal memory and executive functions. Further studies should focus on group and cross-cultural comparisons.

Correspondence: LIza San Miguel-Montes, Psy. D., Neurology, University of Puerto Rico Medical School, PMB 200 \#1353 St. 19, Guaynabo 00966, Puerto Rico.E-mail: lesmm28@hotmail.com

\section{Cross-Cultural Test Development}

M. GORISSEN \& J. LEITZ. The Use of Effort Tests in non native speakers: Risk for False Positives with the French WMT?

Objective: The Word Memory Test is a well validated measure for mental effort. The test appears quite robust for effects of age and intelligence: the WMT can be used for children who have attained a 3rd grade reading level. Moreover, mentally retarded adults perform normally on the task. However, in neuropsychological practice, we often test patients from immigrant populations who do have good reading ability in another language and have a normal to high intelligence, but are not native speakers of the language in which they are tested. This might lead to false positives when they perform under the cut-off score of an effort test. These populations might be at risk of being falsely accused of underperforming.

Participants and Methods: We tested undergraduate students with different native tongues and cultural backgrounds on the French WMT. All students were administered a French placement test used to determine the level for their French course. Three groups were discerned: beginners, intermediate, advanced, and native speakers. The computerized WMT was administered.

Results: Results show that participants with an intermediate (and higher) level of French performed well above the clinical cut-off score of the effort test. In the beginners group a few participants scored under the cutoff score. However, the level of French of these participants was so low that testing in French would never be considered in a clinical setting anyway.

Conclusions: Again, the WMT appears to be quite robust against other effects than mental effort.

Correspondence: Marielle Gorissen, PhD, dept of Psychology, american university of Paris, 31 avenue Bosquet, Paris 75007, France. E-mail: mgorissen@aup.fr

L.G. JOHNSON \& T. GUALTIERI. CNS Vital Signs (CNSVS): A Valid and Reliable Computerized Neuropsychological Battery for the Evaluation of Mild Cognitive Impairment.

Objective: Development of a valid and reliable computerized battery appropriate for the evaluation of mild cognitive impairment and medication effects in neuropsychiatric patients (TBI, ADHD, LD, etc.) in cross-cultural populations.

Participants and Methods: Normative data for the English version was developed from over 500 healthy controls (ages 10-89). The battery is comprised of seven common neuropsychological measures (verbal and visual memory, finger tapping, symbol digit coding, shifting attention, stroop and cpt). Most patients can complete the test independently in 30 minutes. It is downloadable via the internet, simple to use and easy to interpret.

Results: Domain standard scores (Mean=100; SD=15) and percentile ranks are provided in five areas: Memory, Psychomotor Speed, Reaction Time, Cognitive Flexibility and Complex Attention. Reliability and validity coefficients are within acceptable ranges and are comparable to those of other computerized tests, as well as traditional Neuropsychological measures. Several studies have shown that the battery is sensitive to various medications (stimulants, antidepressants and antipsychotics). Conclusions: CNSVS is a valid and reliable computerized battery for the evaluation of mild cognitive impairment. It is being used in several international drug trials and has been translated into over 50 different languages. Approximately 100 clinicians and researchers in the US, Canada and UK are using the English version. Researchers in a number of other countries (Taiwan, Sweden, Italy, Brazil, Venezuela, Belgium, Finland and Greece) are using the foreign language versions and efforts are underway to establish norms in these languages.

Correspondence: Lynda G. Johnson, Ph.D., North Carolina Neuropsychiatry, 1829 East Franklin Street, Chapel Hill, NC 27514. E-mail:ljohnson@ncneuropsych.com 
M.C. MIRANDA, L.F. COELHO \& O.F. BUENO. Are the Computerized and Manual Versions of the Wisconsin Card Sorting Test Equivalent? A Comparative Preliminary Study on Samples of North American and Brazilian children.

Objective: The present study investigated the performance of a sample of Brazilian children aged 6-10 years, using the computerized version of the WCST. The results were compared to those of the manual version applied to both Brazilian and American populations.

Participants and Methods: The Brazilian sample in the present study was composed of 206 children from Public schools in Sao Paulo City. The comparison between the data obtained in this study and that from the sample of North American and Brazilian children from the standardization study with the manual version was carried out by age-group. Results: There were age effects in almost all measures, except for four of them. The comparison carried out with the North American children (manual version) showed significant differences in almost all measures in the age groups, the Brazilian children presented a poorer performance. No differences were observed only in the 6-year-old group. The comparison between the Brazilian samples showed differences in some measures; however, the sample of the present study obtained higher scores than that of the manual version.

Conclusions: The sample in the present study was composed of children attending Public schools, from low socio-economic backgrounds, which probably reflects the differences between Brazilian and American sample. However, the differences between the Brazilian samples cannot be explained for that same reason, since the sample in this study showed better performance, the differences are probably due to the nonequivalence of the versions of the test. The present study is currently being expanded, by assessing children from Private schools so more consistent data regarding the differences can be obtained

Correspondence: Miranda Monica, psybiology, federal university of são paulo, R. Embau, 54, São paulo 04039-060, Brazil. E-mail: mcarol@psicobio.epm.br

\section{Emotion}

M. BALCONI \& A. CARRERA. Cross-modal (Voice and Face) Perception of Emotion. ERPs and Behavioral Analyses.

Objective: The study investigated the simultaneous processing of emotional tone of voice and emotional facial expression by event-related potentials (ERPs), through an ample range of different emotions (happiness, sadness, fear, anger, surprise, and disgust).

Participants and Methods: Auditory emotional stimuli (a neutral word pronounced in an affective tone) and visual patterns (an emotional facial expression) were matched in congruous (the same emotion in face and voice) and incongruous (different emotions) pairs. Fourty one subjects were submitted these patterns.

Results: ERPs variations and behavioral data (response time, RT) were submitted to repeated measures analysis of variance. We considered the 150-250 ms poststimulus time-window in order to explore the cognitive processes (long latency ERP variations) in addiction to the perceptive (early ERP variations), fully investigated by previous researches. ANOVA showed two different ERP effects, a negative deflection (N2), more anterior-distributed $(\mathrm{Fz})$, and a positive deflection $(\mathrm{P} 2)$, more posterior-distributed, with different cognitive functions.

Conclusions: P200, which is highly sensible to pattern congruent/incongruent condition (with more intense amplitude for congruent rather then incongruent stimuli) constitutes an intersensory integration marker. On the contrary, N2, more sensible to the type of emotion, may be a marker of the emotional content displayed. Finally, behavioral results indicate that congruence causes a RT reduction for some emotions (sadness) and an inverse effect for other emotions (fear, anger, surprise). This result is discussed with reference to the different adaptive functions of the emotional correlates.

Correspondence: Michela Balconi, PhD, Dep. of Psychology, Catholic University of Milan, Largo gemelli, 1, Milan 20123, Italy. E-mail: michela.balconi@unicatt.it
V.P. BOZIKAS, M.H. KOSMIDIS, M. GIANNAKOU, M. SAITIS, K. FOKAS \& G. GARYFALLOS. Emotion Perception in ObsessiveCompulsive Disorder.

Objective: The purpose of the present study was to investigate the ability to perceive facial and vocal affect in a group of patients with obsessive compulsive disorder (OCD) and to explore the specific emotions that might be troublesome for them.

Participants and Methods: Participants were 25 patients with OCD and 25 healthy controls, matched on age, education, and gender. They were assessed with computerized tests of affect perception, using photographs of faces, sketches of everyday scenarios, and affective prosody, as well as a facial recognition test. The specific emotions examined included happiness, sadness, fear, anger, disgust, surprise, and neutral expressions. Severity of OCD symptoms in the patient group was measured with the Yale-Brown Obsessive Compulsive Scale (Y-BOCS).

Results: Patients with OCD were not impaired in the perception of emotion, as measured with through the use of still photographs, sketches of everyday scenarios, and affective prosody, as compared with age-, sex, and education-matched healthy individuals. Moreover, patients with OCD did not differ from healthy individuals in discriminating facial identity. With regard to each emotion type separately, patients performed equally to healthy individuals in all the different emotions examined.

Conclusions: Emotion processing (through facial expressions and affective prosody), does not appear to be deficient in patients with OCD; therefore, any psychosocial impairment observed in people with OCD may reflect deficits in more basic cognitive functions and/or symptom severity.

Correspondence: Maria Giannakou, Psychology, Psychology, Aristotle University of Thessaloniki, Stratigi 14, Thessaloniki 54352, Greece. Email:mariag@psy:auth.gr

J.J. EVANS, B.A. WILSON, A. CALDER \& A. BATEMAN. The Frequency and Nature of Deficits in Facial Emotion Perception After Brain Injury.

Objective: We examined a consecutive series of patients referred for day-patient neuropsychological rehabilitation to determine the frequency of deficits in facial emotion perception. A detailed analysis of errors was also undertaken. The hypothesis that deficits in identifying certain emotions (e.g. fear) are related to task difficulty rather than to individual emotion-specific processes was investigated.

Participants and Methods: Participants were 104 patients with acquired brain injury and 90 controls. Sixty photographs taken from Ekman and Friesen's Pictures of Facial Affect series (10 each for happiness, sadness, fear, anger, surprise and disgust) were shown. Accuracy and specific errors made were recorded. For patients, additional neuropychological assessment data was recorded.

Results: There was no difference between patients and controls on perception of happiness and surprise. For all other emotions patients were significantly poorer than controls. For fear, $33 \%$ were impaired, with $18 \%$ impaired on sadness, $20 \%$ on anger, $18 \%$ on disgust. Error analysis showed that some emotions are mistaken for just one other alternative, whilst for others a broader range of errors is apparent. Degree of impairment in identifying specific emotions was correlated with task difficulty (defined by control group performance)

Conclusions: Facial emotion perception deficits are relatively common after brain injury, with $53 \%$ our sample impaired on at least one emotion. Perception of fear is most vulnerable to brain injury because accurate fear perception requires greater cognitive resources than other emotions, so making it vulnerable to the broader information processing deficits consequent upon brain injury.

Correspondence: Jonathan J. Evans, PhD, Psychological Medicine, University of Glasgow, Section of Psychological Medicine, Gartnavel Royal Hospital, 1055 Great Western Road, Glasgow G12 OXH, United Kingdom.E-mail:.jje2k@clinmed.gla.ac.uk 
G.P. LEE, C.L. CLASON \& J.R. SMITH. Emotional Neural Networks as Revealed by fMRI and Electrical Stimulation of Implanted Electrodes in the Human Brain.

Objective: Recent investigations using lesion, functional imaging, electrophysiological, and intracarotid amobarbital procedure methods have begun to yield information about the neural substrates underlying emotional expression and internal feeling states.

Participants and Methods: These emotional-neural associations were investigated in sixty-three participants who either underwent fMRI (healthy controls, $\mathrm{N}=10$ ) while viewing 20 positively and 20 negatively valanced emotional photographs from the International Affective Picture System (IAPS, Lang, 1999) or who had an emotional experience after low-level electrical stimulation of intracranially implanted depth electrodes for evaluation of seizure localization $(\mathrm{N}=53)$.

Results: Emotional photographs resulted in significantly increased BOLD responses in the following brain regions during fMRI: 1) amygdala and anterior temporal polar regions, 2) anterior cingulate gyrus, supplemental motor area (SMA), and mesial frontal lobe, 3) dorsolateral frontal lobe, and 4) cerebellum. Affective responses following depth electrode stimulation (e.g., fear, sense of dying, unpleasant feeling, sense of well-being/euphoria) were elicited from anterior and mesial temporal, insular, orbitofrontal, and anterior cingulate gyrus/mesial frontal lobe stimulation sites. Laterality effects were observed during both fMRI and electrode stimulation with negative/dysphoric affective states causing greater right hemisphere activation during fMRI and being observed more frequently after right $(\mathrm{N}=18)$ than left $(\mathrm{N}=3)$ electrode stimulation.

Conclusions: Results suggest a series of interconnected neural sites important for the production and experience of emotion. A model of how each particular brain region makes a unique contribution to the resulting emotional experience will be presented.

Correspondence: Gregory P. Lee, Ph.D., Department of Neurology, Medical College of Georgia, BA 3278, 1120 15th Street, Augusta, GA 30912-3275.E-mail: glee@mcg.edu

L. NAHUM, S. LEHMANN, S. MORAND, R. PTAK \& A. SCHNIDER. Temporally Dissociated Cortical Processing of Inherent Emotional Salience and Behavioural Relevance of Visual Stimuli.

Objective: Both stimuli with high inherent emotional salience (iES; e.g.. threatening stimuli, such as, a spider) and more neutral stimuli of behavioural relevance (bR; e.g., unexpected outcome of an action) may induce rapid behavioural adaptations (Mohr et al., Neuroimage 2007). In the present study, we used high-resolution evoked potentials and spatio-temporal mapping to compare the cortical processing of inherent emotional salience as opposed to the behavioural relevance of visual stimuli during reversal learning.

Participants and Methods: High-density evoked potentials (ERPs) were recorded in 6 participants. Subjects variably had to predict which one of two faces would have a spider (high iES condition) or a circle (low iES) on its nose. The presentation of the other stimulus indicated that the target stimulus had switched to the other face.

Results: Appearance of the spider (high iES) induced strikingly different electrocortical responses (higher amplitudes, earlier peaks) than the neutral stimulus, independent of behavioural relevance, between 100-200ms. Between 220-260ms, map topographies significantly differed. By contrast, behaviourally relevant absence of anticipated outcomes (switch trials) induced specific alterations of map configuration between $400-500 \mathrm{~ms}$, independently of the iES of the stimuli.

Conclusions: Inherent emotional salience of a stimulus appears to be processed much earlier, with vigorously different electrocortical responses, than the learned behavioural relevance of stimuli. (Study supported by Swiss National Science Foundation grant no. 32000-113436 to A.S.) Correspondence: Louis Nahum, Neurorehabilitation, HUG, Avenue de Beau-Séjour 26, Genève 1211, Switzerland. E-mail: nahum. louis@bluewin.ch
K.T. PASSAGLIO, R. BARROS NETO, L. FENSTERSEIFER, B.R. MEIRELES, J.P. CRUZ, L.C. SANTOS \& W.S. LINS. An Empiric-theoretician Assay about the Possible Psychological and Physiological Benefits of the Laugh on the School Environment.

Objective: The present study aims to carry through a research to investigate its influence on different abilities that take care effectively of the purpose of learning.

Participants and Methods: Specific tests had been used in order to evaluate attention, perception, memory, organizations of space and spacetime, capacity of understanding and structure of thought, emotional interpretation of texts, reasoning and development in mathematical tasks. These tests had been performed in a public school with adolescents (1215 years old), in two days and later, during 10 days, the promotion of laugh was made. The tests had been reapplied. Physiological parameters as cardiac frequency, arterial pressure, sensorial threshold of pain and the dosage of the IGA on the saliva were evaluated before and after the students watched a comedy movie.

Results: The result data had been processed statistically using the coefficient of correlation of Spearman and Test t-Student before and after the workshops of laugh. The results were positive showing the improvement in the response of the tests. The physiological parameters had also showed positive results such as increase of the cardiac activity, relief of pain and increase in the IGA.

Conclusions: The results point to a potential action of laugh on the tested abilities, indicating to be an excellent tool for the creation of a healthful school environment, which would result in improvement of the process of learning / teaching.

Correspondence: Kátia T. Passaglio, Doctor, Psychology, PUC Minas University, Waltr Ianne Street, 255, São Gabriel, Psychology Coordenation, Belo Horizonte 31980-110, Brazil. E-mail: ktpassaglio@terra.com.br

\section{Forensic Neuropsychology}

N. ARIAS, F. OSTROSKY-SOLÍS \& M. PÉREZ. Clinical, neuropsychological, and psychophysiological profiles in impulsive and premeditated Mexican inmates.

Objective: Recent neuroimaging studies and neuropsychological test findings support the contention that prefrontal dysfunction is associated with psychopathic personality traits and antisocial behavior. Aggressive behavior has traditionally been classified into two distinct subtypes: an affective, impulsive aggressive display or a planned, predatory aggressive act. While a number of investigations have examined the clinical and physiological correlates in impulsive aggressive individuals few studies have been focus on the predatory aggressive type.

Participants and Methods: We administered a neuropsychological test battery, including measures of frontal lobe dysfunction, attention and memory and a battery of personality questionnaires and clinical scales sensitive to antisocial personality disorder and monitored the psychophysiological activity during the presentation of emotionally charged stimuli, with event related brain potentials (ERPs).A sample of 60 incarcerated criminal offenders, 30 premeditated aggressive and $30 \mathrm{im}-$ pulsive aggressive Mexican inmates were studied.

Results: Premeditated aggressors show a significant personality pathology scoring higher on measures of impulsivity, verbal and physical aggression, anger and hostility respect to impulsive group. Significant differences between impulsive and premeditated were found in the ReyOsterreith figure and executive functions and in the amplitude of ERPs. Conclusions: Results are discussed in terms of distinct neuropsychological profiles for each group.

Correspondence: Nallely Arias, National University of Mexico, Av. Universidad 3004, Mexico D.F. 04510, Mexico. E-mail: naxiillii21@hotmail.com 
S. BERENTSEN \& D.C. OSMON. Attention and Symptom Validity Testing: Surprising Results in a Simulation Study.

Objective: This study investigated attention, as a factor in the Green Word Memory Test performance.

Participants and Methods: Thirty-three undergraduate participants ( 9 males and 24 females, mean age $=23$ ) were divided randomly into simulator $(\mathrm{n}=17)$ and normal $(\mathrm{n}=15)$ control groups. All subjects were classified as good or poor attention based upon performance on the Neuropsychological Assessment Battery (NAB) Attention module.

Results: A one-way ANOVA with post-hoc Scheffe comparisons demonstrated differences between poor attention simulators and all other groups $(\mathrm{F}[3,29]=13.65, \mathrm{p}<.001)$ on a composite WMT score. Significant differences between simulator and control groups were also found on all individual WMT scales. On the modified one-back WMT, performance did not differ between the four simulator/attention groups on either the regular WMT items or the one-back items $(\mathrm{F}[3,26]=2.05, \mathrm{p}>.05$ and $\mathrm{F}[3,26]=0.66, \mathrm{p}>.05$, respectively). All control and simulator groups performed well on both the WMT-regular and WMT-one back items (above 90\%).

Conclusions: Since poor attention simulators performed worse than good attention simulators on the WMT, it is possible that attention plays a role in performance on the test when trying to simulate. Additionally, since simulators performed as well as controls on the one-back WMT, it is likely is that simulators' ability to fake test results may be impaired in a demanding task like the WMT-attn test where they must switch between regular and one-back trials.

Correspondence: David C. Osmon, Ph.D., Psychology, University of Wisconsin-Milwaukee, 2441 E. Hartford Ave, Milwaukee, WI 53211. Email:neuropsy@uwm.edu

\section{A.B. BILBAO, A. BIZE \& I. BOMBIN. Factors influencing the} simulation of memory deficits.

Objective: The aim of this study is to evaluate which factors influence the performance of simulators on neuropsychological tests. Researchers studying malingering often use simulators to investigate new ways to discriminate people with true and feigned memory deficits. However, there are importante differences between simulators and malingerers regarding their degree of motivation or the time for preparation. These differences might compromise the ecological validity and reliability of malingering research.

Participants and Methods: 25 patients with memory deficits secundary to TBI were compared with 100 subjects divided in 4 diferent experimental conditions. Experimental conditions resulted from the combination of 2 binary variables (1)presence/absence of a significant external reward (2) presence/absence of a one week time interval between reception of instructions and evaluation. Test administered included the Hisckock \& Hisckock procedure, Coin in the Hand Test, Rey 15 item Test, HVLT and Digit Span.

Results: Significant differences were observed among 4 experimental groups. Subjects who participated in the condition which combined external reward + one week time interval between reception of instructions and evaluation had a closer performance to brain injured individuals than any other experimental group. In addition, several subjects from this experimental group and none of the other experimental groups were classified as "real patients" in a discriminant analysis.

Conclusions: Experimental conditions of external motivation and preparation time appear to be an important factor influencing the performance of simulators of memory deficits. Malingering research should take these two variables into account in order to obtain the most reliable data on specificity and sensibility of different test procedures designed to assess memory malingering.

Correspondence: Alvaro B. Bilbao, PhD, Neuropsychology, CEADAC, C/ Río Bullaque No 1, Madrid 28034, Spain. E-mail: alvarobilbaobilbao@hotmail.com
R. VILAR-LÓPEZ, M. BELLÓN-GUARDIA, S. SANTIAGORAMAJO, A. CARACUEL-ROMERO, M. GÓMEZ-RÍO \& M. PÉREZGARCÍA. Detection of Malingering in a Spanish Traumatic Brain Injury Population using the Rey Complex Figure Test.

Objective: The present study uses a known groups design to examine the utility of the Rey Complex Figure Test (RCFT) in detecting malingered neurocognitive dysfunction in a Spanish population.

Participants and Methods: Two groups were studied: 44 patients with traumatic brain injury (TBI) and 10 individuals suspected of malingering (SM) based on scores indicative of poor effort on two or more malingering tests (VSVT, TOMM, the b test, Dot Counting Test and Rey 15-item test) that were involved in litigation. All of them underwent an extensive neuropsychological assessment that also included the RCFT. The selected variables of the RCFT were: Copy, Inmediate recall, Recognition and Combination score (according to Lu, Boone, Cozolino \& Mitchell, 2003).

Results: Statistical significant differences among the groups were found in Recognition $[\mathrm{F}(1,52)=5.80 ; \mathrm{p}=0.021]$ and Combination score $[\mathrm{F}(1$, $52)=6.92 ; p=0.012]$ variables.

Conclusions: Opposed to other publication, our results indicate that the RCFT has a limited utility for its use in malingering detection. One possible explanation is that there exist cultural differences between the samples in the two studies that affect the test results. Given our small sample size, further studies are necessary to confirm this hypothesis, but our results do not support the use of the RCFT as a reliable indicator in the detection of malingering in TBI Spanish patients. Thus, in our context, we should relied on specific tools that had been proved in Spanish samples, like the VSVT, the TOMM or The b test.

Correspondence: Raquel Vilar-López, Universidad de Granada, Facultad de Psicología, Departamento de Personalidad, Evaluación y Tratamiento Psicológico, Campus de la Cartuja, sn, Granada 18002, Spain.E-mail: raquelvilarlopez@hotmail.com

\section{Language: Other (e.g., Naming, Fluency, Reading)}

\section{L.E. FONSECA, B. GOTTHEIL, S. DEFIOR CITOLER, A. ALDREY, M. PUJALS, G. ROSA, G. JIMÉNEZ FERNÄNDEZ \& F. SERRANO} CHICA. LEE Test de Lectura y Escritura en Español.

Objective: La lectura es una habilidad compleja que persigue la comprensión del texto escrito. Sus componentes básicos son el reconocimiento de palabras y la comprensión lectora. Ambos son necesarios para el logro de la competencia en esta habilidad. Se presentará un test para niños de primero a cuarto año de escuela primaria integrada por pruebas que evalúan los subprocesos intervinientes. Contempla actividades de lectura de palabras y pseudopalabras seleccionadas teniendo en cuenta la frecuencia, longitud y complejidad ortográfica, lectura de frases y textos para evaluar el reconocimiento de palabras y la comprensión lectora. Igualmente se evalúan procesos de esritura mediante dos subpruebas de escritura de palabras y pseudopalabras. Dos subpruebas complementarias, identificación de letras y segmentación fonémica permiten precisar el diagnóstico de niños con dificultades en la adquisición del código escrito español. También investiga otros aspectos como la fluidez lectora y la prosodia.

Participants and Methods: 395 niños de escuelas de Buenos Aires, Argentina y 400 niños de Granada, España realizándose pruebas estadísticas de validez y confiabilidad.

Conclusions: Se observa que el test LEE es una prueba válida para comparar la competencia general de los niños en lectura y escritura. pudiéndose detectar las dificultades que presentan los niños y los procesos afectados

Correspondence: Liliana E. Fonseca, Licenciada, Psicopedagogía, Universidad Nacional de San Martín, Frías 2235, SAN ISIDRO, Pcia de Buenos Aires 1642, Argentina. E-mail: Ifonseca@psicopedagogica. com.ar 


\section{Other}

E. RIVLIN. Multi-cultural medico-legal issues in the neuropsychological assessment of children/young adults in India and the UK.

Objective: The legal processes and laws vary between India and the UK but share similarities regarding clinical examination/neuropsychological assessment, planning for rehabilitation, a multi-disciplinary team approach of experts and provision of evidence. India's vast population, 14 official languages, public ratio of Courts and Specialists, the paucity of essential administrative machinery impact at both the cultural and humane level. India does not have protection of various contingencies such as part payment into Court or a later settlement only providing for assessment of damages as provided in the Schedule of Compensation for immediate requirement. The Human Rights Protection Act in India 1993 encompasses all human rights violations, the 2006 Act bans child labour. Juvenile Courts operate in both countries; the nature of the crime, intellectual/neuropsychological capacity are considered. The Indian Legal Services Authority pass claims in civil damages by way of alternative dispute resolution systems and is comparable with the British system in its attempts to focus on acceptable end results. The advice of the UK Court of Protection for Clients incapable of managing their own affairs and provisional damages for children under 18 years is presently unavailable in India. Neuropsychological assessment to cater for the multicultural diversity in both countries and the development of new neuropsychological assessment instruments/rehabilitation approaches with reference to complex single case presentations involving personal injury, disability, neuropsychological deficit, murder, development of case and settlement are discussed.

Correspondence: Elise Rivlin, Clinical Psychology, Central Manchester \& Manchester Children's University Hospitals NHS Trust, Booth Hall Children's Hospital, Charlestown Road, Blackley, Manchester M9 7AA, United Kingdom. E-mail: lynda.wilkinson@cmmc.nhs.uk

\section{Paper Session 1 11:15 a.m.-12:15 p.m.}

\section{Memory: Imaging \& Electrophysiology}

A.J. LUNDERVOLD, M. YSTAD, S. ADOLFSDOTTIR \& A. LUNDERVOLD. Hippocampal Volume and Delayed Recall Performance in Older Adults.

Objective: To examine the relationship between performance on a test of delayed recall and a measure of hippocampal volume, and the additional effects of age, gender and the $\varepsilon 4$ allele of the Apolipoprotein E (APOE) gene. Participants and Methods: A group of 80 healthy non-demented subjects ( 54 women) aged 46 to 77 years (mean $=59 \mathrm{yrs}$ ) with a mean $\mathrm{IQ}=115$ were studied using the delayed recalled measure (number of words) from the California Verbal Learning Test (CVLT) and automated segmentation of 3D MRI recordings with estimates of the hippocampal and total intracranial volumes. A hierarchical linear regression analysis was conducted, with performance on the memory test as the dependent variable. Hippocampal volume divided by total intracranial volume (ICV) was included as the initial predictor, followed by age, gender and risk status of APOE $\varepsilon 4$.

Results: The results on the CVLT measure and the hippocampal volume were not different between carriers and non-carriers of $\mathrm{APOE} \varepsilon 4$ and were not significantly correlated with age. Women obtained significantly higher results on both the recall and MRI measure. The hierarchical regression analysis showed that the hippocampal volume explained a significant portion of the variance in the delayed recall measure $\left(r^{2}=.19\right)$. Age did not add to the explained variance, but by including gender, the $\mathrm{r}^{2}$ increased to .28 . Risk status did not convey a significant change, and the full model gave an $\mathrm{r}^{2}=.29$.
Conclusions: The observed patterns of higher performance and larger normalized hippocampal volume in women than men are consistent with earlier studies. Within the age range of the present study, the normalized hippocampal volume and gender seem to be significant predictors of delayed recall performance in healthy older individuals.

Correspondence: Astri J. Lundervold, PhD, Biological and Medical Psychology, University of Bergen, Jonas Lies vei 91, Bergen 5009, Norway.E-mail: astri.lundervold@psych.uib.no

B.P. UZZELL. Memory Loss in Young Adulthood: Too Young for DAT. Objective: The capacity to store and retrieve information is optimal during young adulthood. Well known is the diminution of memory capacity after traumatic brain injury in young adults, and after dementia of the Alzheimer's type (DAT)in the elderly. Less well know is the loss of optimal memory capacity due to systemic pathophysiology, such as prolonged temperature elevations. This study identifies the often unrecognized origin of short-term memory loss in a young adult with sustained temperature elevations accompanied by febrile convulsions.

Participants and Methods: Case Study: A 24-year-old female, college graduate experienced profound hyperthermia (approximately 106 degrees Fahreheit)over a two-week period, before medical treatment controlled the infectious origin.

Results: Neuropsychological assessment four years later revealed a significant short-term memory loss similar to that associated with DAT, slow processing speed contributions to memory deficits (Salthouse, 1996), and mildly impaired word fluency.

Conclusions: A proposed mechanism is hyperthermia and accompanying generalized seizures dramatically increases the cerebral metabolic rate for oxygen. A state of cerebral hypoxia ensures when the circulation is insufficient to meet this increased metabolic demand. Cell death in hypoxic sensitive areas of the medial-temporal brain region (hippocampus) may occur, which could have a profound effect on memory. MRI and EEG findings will be presented.

Correspondence: B. P. Uzzell, PhD, Memorial Neurological Association, 7777 SW Frwy Ste 900, Houston, TX 77074. E-mail: bpuzzell@hal-pc.org

S. LEHMANN, S. MORAND, C. JAMES \& A. SCHNIDER. Cortical Signature of Amnesia: a High-Resolution Event-Related Potential Study. Objective: Amnesic patients have decreased variation of evoked-potential responses in recognition tasks between 300-600 ms. A recent single case study using high-resolution ERPs demonstrated that amnesia may be associated with a failure to rapidly activate distributed cortical networks during encoding (Lehmann et al., Neuropsychologia, in press). In this study, we used electrical spatiotemporal mapping to examine memory processing in a group of amnesic patients.

Participants and Methods: High-density evoked potentials were recorded as 11 amnesic patients with various aetiologies and 12 control subjects performed a continuous recognition task composed of meaningful designs. Results: Presentation of pictures induced precisely the same electrocortical maps in amnesics and controls during the first $200 \mathrm{~ms}$. Beyond this period, processing significantly differed. Between 200-300ms, amnesic patients expressed different topographical maps than controls. Maps specific to amnesic patients correlated with the visual P1 component, suggesting continued activation of posterior visual areas rather than distributed processing. From $350-550 \mathrm{~ms}$, presentation of repeated items induced modulation of the same cortical maps as new items in controls. In amnesics, by contrast, it induced distinct cortical maps, indicating different cortical processing of new and old information.

Conclusions: Amnesia is associated with strikingly different cortical activation than normal memory processing, starting as early as $200 \mathrm{~ms}$ after stimulus presentation. (Study supported by Swiss National Science Foundation grant no. 32000-113436 to A.S.)

Correspondence: Armin Schnider, M.D., HUG Service de Neuroreeducation, 26 Av. de Beau-Sejour, Geneve 14 CH-1211, Switzerland.E-mail: armin.schnider@hcuge.ch 
E. SOLESIO, F. MAESTÚ, P. CAMPO, A. CAPILLA, D. DEL RÍO, J. RUIZ-VARGAS, J. GONZÁLEZ-MARQUÉS \& T. ORTIZ. Retroactive interference modulates brain magnetic activity in normal aging: a magnetoencephalography approach.

Objective: Interference as a cause of forgetting has long been a topic of interest in normal aging. There are two forms of interference, retroactive and proactive. Retroactive interference results when subsequent material disrupts memory for previously learned information.

Our hypothesis was to consider that susceptibility to interfiring information increases when inhibitory mechanisms turns ineffective. Thereby. resistance to interference engages executive control mechanisms and working memory performance. Our aim was to identify differential spatio-temporal patterns of brain magnetic activity tested with Magnetoencephalography by the presentation of a retroactive interference task in two experimental conditions: active and passive interference.

Participants and Methods: The sample consisted of 20 healthy spanish old adults. Subjects performed a speeded working memory task descended from the Sternberg paradigm that contained three phases: encoding, interference and recognition. MEG data analysis was focused on recognition. Results: For the active interference group, results showed a higher brain activity in the left medial regions of the temporal lobe (MRTL) and the left anterior ventral prefrontal region (AVPR) during the first $400 \mathrm{~ms}$. For the passive interference group, there was a higher activity in the left AVPR from 700 to 800 -ms time window.

Conclusions: We found a fronto-temporal network consist of the medial regions of the MRTL and the AVPR that regulates the interference control. Executive functions and working memory mechanisms are implemented by prefrontal circuits in interaction with other posterior regions. In conclusion, the presentation of both active and passive interference tasks, modulates the brain magnetic activity patterns in normal aging, involving a time- modulated control mechanisms.

Correspondence: Elena Solesio, MEG Center of The University Complutense of Madrid (Spain), C/Boixareu Rivera n0112, 30F, Guadalajara 19002, Spain.E-mail: elesolesio@yahoo.es

\section{Paper Session 2 11:15 a.m.-12:15 p.m.}

\section{Childhood Acquired Disorders}

\begin{abstract}
E.M. WESTRUPP, E. NORTHAM, P. ANDERSON \& L. DOYLE. Adult Psychiatric Outcomes of Very Low Birth Weight Survivors.

Objective: Since the 1970 s, survival rates of very low birth weight (VLBW) children have increased dramatically. Recent research indicates that many VLBW individuals have brain abnormalities and that these abnormalities are associated with poor outcome. While an extensive body of research has looked at child outcomes in this group. few studies have examined adult outcomes. We examined the persistence of a number of childhood behaviour problems and psychiatric disorders in a cohort of VLBW survivors, within a longitudinal study design.
\end{abstract}

Participants and Methods: Participants: 210 survivors with birth weight 500-1500 grams and 60 randomly selected normal birth weight controls. This group was followed from birth, at 5, 8 and 14 years, and most recently, aged 25-29 years. Measures: We addressed the limitations of previous research by combining the use of screening assessments; Conners' Adult ADHD Rating Scales (Conners, 1999) and the Symptoms Checklist (SCL-90-R) (Derogatis, 1994), with the Structured Clinical Interview for DSM-IV-TR for Axis I Disorders Research Version, for adult psychiatric assessment.

Results: Discriminate function analysis with binary outcome, and correlations with continuous outcome measure, were used to compare rates of psychopathology across groups. Preliminary findings show that $49 \%$ of the VLBW participants were elevated on one of the subscales of the
SCL-90-R. A further $86 \%$ of these participants were diagnosed with one or more psychiatric diagnosis in the SCID interview.

Conclusions: Findings were consistent with extensive research evidence showing strong associations between birth weight and childhood attention deficits, behaviour problems and psychiatric outcomes. This is the first longitudinal research to show that VLBW adults are more at risk of having several psychiatric disorders and that childhood behaviour problems childhood behaviour problems and psychiatric disorders tend to persist into adulthood in this population. Theoretical implications will be discussed in terms of models of resilience.

Correspondence: Elizabeth M. Westrupp, PhD Candidate, Behavioural Sciences, The University of Melbourne, The University of Melbourne, Parkville, Melbourne, VIC 3010, Australia. E-mail: ewe@unimelb.edu.au

M. RIS, D.W. BEEBE, E. HOLMES, D. ARMSTRONG, J. FONTANESI, R. MULHERN \& J. WISOFF. Neurobehavioral Outcome in Extracerebellar Low Grade Astrocytomas in Children. Objective: To determine whether children treated with surgery for low grade astrocytomas in locations outside the cerebellum evidence neurocognitive, academic, adaptive, or emotional/behavioral sequelae.

Participants and Methods: Ninety three patients from a natural history study of low grade astrocytoma (CCG 9891/POG 9130) were tested an average of 4 months following surgery. Patients with hemispheric tumors (58) were compared to those with supratentorial midline (20) and brain stem (15) tumors. Rates of below normal scores (25th \%ile or below) were calculated for the entire sample and compared across these groups.

Results: Initial analyses indicate a greater than expected rate of below normal IQs $(42 \%)$ in this sample, given a median parent education of 14 years. Fifty four percent were below normal on a measure of adaptive behavior. Participants with cerebral hemisphere tumors were are greatest risk for below normal IQs while those with supratentorial midline tumors were at greatest risk for below normal scores in adaptive behavior. Higher rates of academic, but not emotional/behavioral difficulties were also found. Further multivariate analyses are underway investigating pre-, peri-, and post-surgical predictors of outcome as well as more specific deficits in verbal, nonverbal, processing speed, and working memory abilities.

Conclusions: Following surgery for low grade astrocytoma, a significant number of patients were found to function below normal-results similar to those described by Beebe et al. (2005) for children with low grade cerebellar lesions. Even in the absence of neurotoxic radio- and chemo-therapies, routine neuropsychological follow-up of children following treatment for low grade tumors appears to be warranted. Correspondence: M. Douglas Ris, Ph.D., Behavioral Medicine \& Clinical Psychology, Children's Hospital Medical Center, 3333 Burnet Ave., Cincinnati,OH 45229.E-mail: douglas.ris@cchmc.org

S. MEACHEN, J. PERRY, S. RAZ, A. GUSTAFSON-DEBASTOS, J. BAPP-NEWMAN \& D. BATTON. Extreme Prematurity: Do Two Additional Weeks of Gestation Matter at the Edge of Viability?

Objective: Few studies have investigated the developmental implications of two additional weeks of gestation for infants born at the threshold of viability. In this study we examined differences in neuropsychological outcome between infants born at 23.0 to 24.9 weeks and those born at 25.0 to 26.9 weeks.

Participants and Methods: Preschool aged children born prior to 27 weeks of gestation were recruited. Children were divided into two groups: $23 / 24$ weeks, $n=18 ; 25 / 26$ weeks, $n=16$. The groups were similar on socio-demographic factors as well as ante-, peri-, and post- natal complications, with the exception of oxygen support days. We assessed performance on multiple domains of neuropsychological functioning. including intellectual abilities, language, memory, and gross and fine 
motor skills. The data were analyzed using MANCOVA, with SES and oxygen support days as covariates.

Results: The adjusted mean Performance IQ for the gestationally younger group was significantly lower than that of the gestationally older group $(89.8, \mathrm{SD}=18.5$ vs. $106.3, \mathrm{SD}=19.3 ; \mathrm{F}[1,27]=5.55, \mathrm{p}=$ $.026)$. The two groups did not differ significantly on other outcome measures: Verbal IQ $(\mathrm{F}[1,27]=2.06$, n.s. $)$, fine Motor skills $(\mathrm{F}[1,28]=$ 1.3 , n.s. $)$, gross Motor skills $(\mathrm{F}[1,28]=1.8$, n.s. $)$, receptive language $(\mathrm{F}[1,27]=.9$, n.s. $)$, expressive language $(\mathrm{F}[1,27]=1.9$, n.s. $)$, and long-term retrieval $(\mathrm{F}[1,26]=.1$, n.s. $)$.

Conclusions: Two additional weeks of gestation at the very edge of viability appear to be selectively associated with visuospatial, but not with verbal, motor, or memory performance.

Correspondence: Sarah Raz, Ph.D., Merrill-palmer Institute, Wayne State University, 71 E. Ferry St., Detroit, MI 48202. E-mail: sarahraz@wayne.edu

C. BENJAMIN, V. ANDERSON, R. PINCZOWER, R. LEVENTER, M. RICHARDSON \& M. NASH. Pre- and Post-Encephalitic Neuropsychological Profile of a 7 Year-old Girl.

Objective: Our understanding of the neuropsychological effects of encephalitis has largely come from studies in which the level of pre-morbid functioning is estimated. Moreover, data on the neuropsychological effects of encephalitogenic pathogens other than the herpes simplex virus (HSV) are scant. The objective of this case study was to document the pre- and post-morbid neuropsychological profile of a young girl who suffered a non-HSV encephalitis (possible aetiology: Mycoplasma pneumoniae).

Participants and Methods: We present a 7 year-old girl who had intellectual and language assessment seven months prior to the onset of non-HSV encephalitis, and again post-encephalitis. Neuroimaging (MRI), speech, psychological and neuropsychological assessments were completed.

Results: MRI post-illness demonstrated basal frontal, temporal and (limited) parietal damage. Neuropsychologically, pervasive changes were apparent in the domains of personality, behaviour, emotionality, attention, executive function, speech, language and memory.

Conclusions: This case study suggests that the deficits seen following non-HSV encephalitis, and possibly Mycoplasma pneumoniae encephalitis in particular, reflect both acute basal brain injury and more diffuse insult. The profile of this patient's non-HSV encephalitis appears to differ most from that seen following typical HSV encephalitis with respect to marked executive and attentional difficulties.

Correspondence: Christopher Benjamin, Ba(Hons), Psychology, The University of Melbourne, Swanston Street, Parkville, VIC 3052, Australia.E-mail: cbenj@unimelb.edu.au

\section{Paper Session 3 11:15 a.m.-12:15 p.m.}

\section{Neuropsychology of Depression}

\footnotetext{
G.M. ARZOLA, R.G. GOMEZ, J. KELLER, H.B. SOLVASON \& A.F. SCHATZBERG. Effects of personality on executive functioning in refractory depression.

Objective: Chronically depressed patients typically exhibit rigid and maladaptive behavior patterns. Neuropsychological characteristics such as mental rigidity, inattention, and impulsivity are also observed. However, the link between neuropsychological performance and personality disorder traits in depressed patients is unclear. In this study we examined how personality, specifically Cluster B traits (Histrionic, Narcissistic, Antisocial and Borderline), affects executive functioning in patients with refractory major depression.
}

Participants and Methods: 28 participants were recruited at Stanford University's Depression Research Clinic. Participants met criteria for Major Depressive Disorder, as assessed by Structured Clinical Interview-DSM. As part of a treatment study, subjects were administered baseline measures for depression, personality and neuropsychological functioning. The measures included in this study are: Hamilton Depression Rating Scale, Millon Clinical Multiaxial Inventory-II, Trail Making Test, Stroop Test, and Design Fluency from the Delis-Kaplan Executive Function System.

Results: Correlational analyses were conducted to examine the relationships between depression, executive functioning, and personality disorder traits. Preliminary results indicated no direct link between depression and executive functioning ( $r$ range $=.00$ to -.31 , all ps $>.05$ ); but depression was related to severity of personality disorder traits (rs range $=.44-.54$, all $\mathrm{ps}<.05)$. Severity of Cluster B personality traits was correlated with executive functioning measures (rs range $=.39$ .54 , all ps $>.05)$. Multiple regression analyses will be conducted when more data is collected.

Conclusions: Interestingly, depression only had an indirect relationship with executive functioning through personality traits. These findings suggest that mental health professionals should be aware of relation between Cluster B personality traits and executive dysfunction in chronically depressed patients.

Correspondence: Gladys M. Arzola, M.A., Neuropsychology; Psychiatry and Behavioral Sciences, Pacific Graduate School of Psychology and Stanford University School of Medicine, 1080 Tanland Dr. Apt.\#101, Palo Alto, CA 94303. E-mail: marina.arzola@gmail.com

M. MILDERS, S. BELL, O. RUNCIE, R. SERRANO \& J. PLATT. Emotional Expression Recognition in Affective Disorder: A Longitudinal Study.

Objective: Abnormalities in recognising emotional expressions in patients with affective disorders may be related to abnormal activity in brain areas involved in emotion in affective disorder. To date it is unclear whether these abnormalities are trait or state effects, because of the lack of longitudinal data, and whether abnormal expression recognition is due to response bias or to the patients perceiving emotional expressions differently than healthy controls. This study aimed to address these questions.

Participants and Methods: Patients with affective disorders, unipolar $(n=19)$ and bipolar $(n=12)$, were re-assessed 3 times with threemonth intervals on an expression labelling task and a face-matching task. Both tasks presented the same faces displaying 6 expressions (neutral, sad, anger, fear, disgust and happy) with a range of intensities. Patients' performance was compared to that of matched healthy controls $(\mathrm{n}=27)$.

Results: Unipolar depressed patients showed a tendency to label lowintensity expressions as sad, rather than using any negative emotion. Bipolar patients were more accurate than controls in labelling expressions of fear and disgust. Similar response patterns were found across assessments. Performance on the face matching task was comparable between patients and controls on all 3 assessments, indicating that the patients were as sensitivity as controls in discriminating between the facial expressions. Symptom severity in the patients, measured at each assessment, was not associated with their expression recognition performance.

Conclusions: The results suggest that abnormal expression labelling in the patients reflected response biases rather than abnormal perception of facial expressions and that these abnormalities represented a trait rather than a state effect.

Correspondence: Maarten Milders, University of Aberdeen, School of Psychology, Aberdeen AB24 2UB, United Kingdom. E-mail: $m$. milders@abdn.ac.uk 


\section{S. MARRENECA \& I. MARTINS. Emotional Processing in Major Depression.}

Objective: To study emotion recognition and emotional memory in a mood disorder

Participants and Methods: 26 individuals with major depression and 26 healthy subjects matched for gender, age and education participated in this study. The assessment battery included tests of emotion recognition (presented through facial expressions and sentence prosody) and an emotional memory test, composed of sentences with positive, neutral and negative content. Performance was compared with repeated measures ANOVA. Emotions were the within-subject factor and group (patients or healthy controls) was the between-subject factor.

Results: Patients had a significantly better recognition of expressions of sadness $(t=2.21, p=0.03)$ but were worse in the recognition of fear $(t$ $=-2.69, p=0.01)$ and neutral expressions $(t=-2.18, p=0.03)$, than controls. On the auditory modality, all emotions were significantly worse recognized by the patient group $(\mathrm{F}=6.32, \mathrm{p}=0.01)$. In the emotional memory tasks, it was found that both groups had an enhanced memory for positive information.

Conclusions: These results suggest that depressed patients present a disruption in the recognition of emotions, with a bias towards negative emotions, which may, in turn, reinforce their depressive mood. Although memory processes are known to be affected in depression, the effect of memory enhancement found in healthy individuals is spared in depressive states which may be used as a cognitive strategy to overcome their general memory impairment.

Correspondence: Silvia Marreneca, Biology, Language Laboratory Research, Faculty of Medicine of Lisbon, Av Prof Egas Moniz, Lisbon 1649-028, Portugal.E-mail: smariaf@hotmail.com

J. GRAY, P. GALLAGHER, M. BURT, B. MONTAGNE, L. ROBINSON \& I.N. FERRIER. Mood Congruent Biases in the Perception of Facial Affect in Individuals with Bipolar Disorder while Depressed.

Objective: To test the hypotheses that (1) Individuals with bipolar disorder while depressed will be less accurate than normal controls in their identification of happiness in facial expressions; (2) the degree to which this is true will correlate with measures of anhedonia; (3) individuals with bipolar disorder while depressed will be more accurate in their identification of negative emotions and sadness in particular; and (4)that the degree to which this is true will correlate with measures of psychological distress and sadness in particular

Participants and Methods: 26 outpatients fulfilling DSM IV criteria for Bipolar Disorder in a Current Depressive Episode and 24 healthy comparison subjects underwent the experimental procedure. They viewed dynamic displays of faces showing each of five emotions (happy, fear, sad, anger, disgust. In each 'movie' a face morphed from neutral to one of a set of selected intensities (ranging from 30 to 100\%). The subjects had to select the identity of the emotion being displayed. Subjects also provided a clinical and other data from which measures of anhedonia and psychological distrees were derived. Results: Patients were worse than controls at identifying happiness in low intensity happy faces, confirming hypothesis 1 . Patients were not significantly better at identifying sadness or indeed any negative emotion, failing to confirm hypothesis 3 . The degree to which patients were worse at identifying happiness was related to anhedonia, but was also related to psychological distree.

Conclusions: Individuals with bipolar disorder while depressed show mood congruent bias in the perception of facial expression of emotion. They are worse than healthy comparison subjects at identifying happiness in low intensity happy faces. They may be better at identifying sad expressions, but, in this group, any such effect seems weaker than the effect on the identification of happy faces. Any relationship to potentially seperable components of depression (such as anhedonia and sadness) remains unclear.

Correspondence: John Gray, PhD, Newcastle University, Leazes Wing, RVI, Newcastle upon Tyne NE, United Kingdom. E-mail: gray600@btinternet.com

\author{
Poster Session 2 \\ Medical Disorders, Psychopathology, \\ Psychopharmacology \\ 11:30 a.m.-1:30 p.m.
}

\section{Autoimmune Disorders (e.g., CFS, Lupus, fibromyalgia)}

S. MARTINEZ, C. CACERES, L. CATAlà, A. DAVAlos, E. DOMINGO, P. LATORRE \& M. MATARÓ. IS THERE PROGRESSIVE COGNITIVE DYSFUNCTION IN SJÖGREN SYNDROME?

Objective: The aim of the present study was to examine cognitive functioning in Sjögren syndrome and to evaluate the possible progression of cognitive deficits over time.

Participants and Methods: Twelve subjects with primary Sjögren syndrome and ten control subjects diagnosed of migraine completed a neuropsychological test battery at baseline and at 8 years follow-up. The test battery was designed to be sensitive to fronto-subcortical dysfunction and included measures of visuomotor speed, verbal and visual memory and visuoespatial and executive functions.

Results: At baseline assessment, there were no significant differences between the groups on the different cognitive domains evaluated. Both patients with migraine and patients with Sjögren syndrome showed neuropsychological impairment in memory and frontal lobe functions as measured by scores 1.5 standard deviations below the standardized norms. However, patients with Sjögren syndrome showed significantly higher levels of depression (Beck Depression Inventory; Sjögren: mean $=22.33, \mathrm{SD}=8.95$; migraine $=11.80, \mathrm{SD}=9.6 ; \mathrm{t}=2,73$; $\mathrm{p}=0.013)$ and fatigue (Krupp's Fatigue Severity Scale; Sjögren: mean $=50.58, \mathrm{SD}=14.1 ;$ migraine $=38.80, \mathrm{SD}=12.1 ; \mathrm{t}=2.95 ; \mathrm{p}=0.008)$ than patients with migraine. At follow-up examination, the initial cognitive deficits remained stable in both groups as did emotional and fatigue symptoms.

Conclusions: Cognitive deficits in Sjögren syndrome are minimal and preliminary evidence indicates that there is not evidence of progressive cognitive impairment in this autoinmune disease.

Correspondence: Silvia Martinez, Hospital Can Ruti, CR Canyet s/n, Badalona 08916, Spain. E-mail: silvimar2@hotmail.com

\section{Behavioral Neurology}

C. GARCIA-SANCHEZ, J. KULISEVSKY, B. PASCUAL-SEDANO, J. PAGONABARRAGA, A. GIRONELL \& A. ESTEVEZ-GONZALEZ. Usefulness of the Frontal Systems Behavior Scales in the assessment of decline behavioral disorders associated with Parkinson's Disease.

Objective: To examine cognitive and behavioral (through FrSBE assessment) features associated with advanced PD.The clinical condition of patients with advanced Parkinson's disease (PD) is characterized by cognitive changes and behavioral disorder. Neuropsychological methods for assessing cognitive changes associated with frontal system damage have been a focus of study for many years. The Frontal Systems Behavior Scales (FrSBe) assesses behavior associated with damage to the frontal-subcortical brain circuits including executive dysfunction, apathy and disinhibition

Participants and Methods: 26 patients with idiopathic, non-demented advanced PD and motor fluctuations recruited from surgery candidates with a mean age of $64.19+/-7.95$ years and an average history of $12.88+/-4.27$ were assessed with Mattis's Dementia Rating Scale (MDRS), Mini-Mental State Examination (MMSE) and phonetic and semantic fluency. Behavior was assessed with the FrSBe (Familiar form). 
Results: Comparison with normative data showed a normal cognitive performance $(\mathrm{MDRS}=124.12+/-13.47 ; \mathrm{MMSE}=26.76+/-3.05)$, however some measures of executive function were impaired (phonetic fluency $=9.92+/-5.83$; semantic fluency $11.85+/-5.40)$. FrSBE showed an important and pathological level of apathy (72.04 +/- 16.64).

Conclusions: In patients with advanced PD is important to assess behavioral disorders associated to fronto-subcortical damage. The apathy is a frequent disorder associated to advanced PD and is present through of normal cognitive performance. The Frontal Systems Behavior Scales is a useful tool in the assessment of decline behavioral disorders associated to Parkinson's Disease.

Correspondence: Carmen Garcia-Sanchez, Hospital Sant Pau, S.Antonio $M$ Claret, Barcelona 08017, Spain. E-mail: cgarcias@santpau.es

\section{Endocrine Disorders/Hormones}

T. HIRVIKOSKI, A. NORDENSTRÖM, T. LINDHOLM, F. LINDBLAD, M. RITZÉN, A. WEDELL \& S. LAJIC. Prenatal Dexamethasone Treatment of Children at Risk for Congenital Adrenal Hyperplasia Affects Cognitive Functions.

Objective: In Sweden, during 1985-95, 40 foetuses at risk for congenital adrenal hyperplasia $(\mathrm{CAH})$ were treated with dexamethasone (DEX) from gestational week 6-7, to prevent virilisation of affected females. After chorion villous biopsy at week 10 treatment was stopped when the foetus was either male or unaffected female, ie in 7 out of 8 cases. Affected females were treated till term. We report long-term effects on neuropsychological functions and scholastic performance of this controversial treatment.

Participants and Methods: Prenatally DEX treated children $(n=26)$. as well as sex and age matched controls $(\mathrm{n}=35), 7-17$ years (median 11 yrs), were assessed with standardized neuropsychological tests (NEPSY and WISC-III) and child-completed questionnaires measuring self-perceived scholastic competence (SPPC) and social anxiety (SASC-R). A parent-completed questionnaire (CBCL/4-18 School Scale) was used to evaluate school performance.

Results: There were no between-group differences concerning psychometric intelligence, handedness, memory encoding, and long term memory. Short term treated, CAH unaffected children performed poorer than the control group on a test assessing verbal working memory $(p=0.003)$, and rated lower on a questionnaire assessing self-perception of scholastic competence $(\mathrm{p}=0.003)$. This group also showed increased self-rated social anxiety $(p=.026)$. Pre- and postnatally treated, CAH affected children performed poorer than controls on tests measuring verbal processing speed, although this difference disappeared when controlling for the Full-Scale IQ.

Conclusions: This study indicates that prenatal DEX treatment during the first trimester is associated with previously not described longterm effects on verbal working memory and on certain aspects of selfperception. These findings may thus question future DEX treatment in $\mathrm{CAH}$. Therefore, we encourage additional studies of larger cohorts to either confirm or challenge the present findings.

Correspondence: Tatja Hirvikoski, MSc, Dept of Psychiatry, Karolinska University Hospital, NPE (R5) /KS, Stockholm 17176, Sweden. E-mail: tatja.hirvikoski@sll.se

\section{Epidemiology}

H. WILLIAMS, H. RYLAND, P.J. YATES \& A. HARRIS. A 6 month prospective study of immediate and general risk factors for presenting to an Emergency Department with head versus orthopaedic injury.

Objective: Head injuries are a major cause of lifelong disability. General risk fatcors include age, gender, socio-economic status and type of residential environment. However, little is known about the actual causes of injury. We aimed to identify the rate and causes of all head injuried patients presenting to an Emergency Department.

Participants and Methods: A prospective epidemiological study of head injury (HI) attendees and comparison group of upper limb orthopaedic injured patients over a 6 months period (winter to summer). All participants had proforma completed at admission for: GCS, PTA (HI group), alcohol/drug use, previous injury, place and type of incident, whether accidental or intentional, and (in case of MVA) specific details regarding event. Data from Emergency Department datasets was also accessed regarding, for example, length of stay and discharge.

Results: Around $180 \mathrm{HI}$ attendees per month were assessed, along with a similar group of orthopaedic patients. Analyses of rates and relative risk trends were conducted, in particular regarding age, gender, and nature of injury type.

Conclusions: It is hoped that findings will enable better management of $\mathrm{HI}$ patients through health systems, and that there might be a reduction in Head Injury rates with improved preventative actions.

Correspondence: Huw Williams, Exeter University, Washington WSinger Labs, Exeter EX4 4QG, United Kingdom. E-mail: w.h. williams@ex.ac.uk

\section{Genetic disorders}

R.H. BLOOMER \& P.A. PEZZULO. Hyperekplexia, Genetic abnormal glycene channels: A Review and Case Study.

Objective: Hyperexplexia, "Stiff Man Disease" or "Startle Disease" is a rare autosomal progressive genetic disorder first reported in 1967 by Gastaut \& Villeneuve. It has since been reported by investigators in several countries worldwide. Hyperekplexia is characterized by an exaggerated startle reflex which reverberates with seizures lasting for several minutes. Other neurological symptoms include hypertonicity, both voluntary and involuntary tremors, memory loss and extreme sensitivity to stimuli, including light, sound and touch.

Participants and Methods: We present neuropsychological study of a a 48 year old female presenting with Hyperekplexia. The case history chronicles the progressive nature of this disorder over 40 years. We review the current literature and suggested treatments

Results: Hyperekplexia is caused by a defective glycene chloride ion channels which causes a delay in repolarization of the inhibitory neurons. In turn, this causes an over-compensatory counter musclar response generating both voluntary and involuntary tremor. Neuropsychological findings, include physical motor, memory and cognitive processing deficits and perseveration. There is diminution in both motor and cognitive function related to mild background stimulation (e.g., white noise) The subject details increased seizure activity and hypersensitivity to stimulation and progressive loss of physical activity over life span .

Conclusions: The resulting defensive reclusive life style may interfere with more widespread recognition of Hyperekplexia. Neuropsychological assessment is sensitive to many aspects of Hyperekplexia and may be used to clarify the diagnosis or to track the progression of this disorder or the effects of psychopharmaceutical treatments.

Correspondence: Richard H. Bloomer, ED.D, M.S., Emeritus, The university of Connecticut, 272 Prospect St., Willimantic, CT 02662. E-mail: rhbloomer@pobox.com

M. MACHADO, L. ANDREU, M. SANZ \& M. SERRA. Neuropsychological, linguistic (and genetic) profile of a family with severe language impairment, transmitted as an autosomicdominant trait.

Objective: The present study is centred in the language and cognitive abilities of a family who exhibit a severe Language impairment (LI), with an inheritability autosomic-dominant pattern, similar to the well known KE family in UK.

Participants and Methods: The G family is composed by three generations. Their intelligence data are obtained according to their IQ 
(WISC-R, WAIS III and Raven), Language's analysis has three main sources: (1) the TB-R aphasia profile (2005), Bilingual Aphasia Test (1987), and the LARSP (1989). Other abilities evaluated are: auditory discrimination (non-verbal and verbal), short-term phonological memory, and apraxia of speech.

The genetic study carried out is a conventional and molecular cariotype, and the cribbage of the FOXP2 gene, with analysis of the mutations of this last gene and of the variant R55H and R328X

Results: The results, evidence an important sensory-motor difficulty that could be linked to the family's cognitive difficulties as well as the LI. Significant similarities with the pathological pattern of the KE family have been found. The generalized deficit in all the aspects of language competence, but mainly in syntax and not in morphology, is consistent with the peculiarities of the English and Spanish crosslingusitic results in SLI.

The genetic study is still under process of analysis.

Conclusions: The former linguistic and cognitive data, suggest that LI is a complex developmental disability, and according to our findings, we expect that the link between LI (including its underlying cognitive basis) and genetics is much more weak than the one that is been suggested up to now. The data will be discussed in relation to the novel theories of genetics and Neuro-cognition.

Correspondence: Maria Clara Machado, Department of Language Pathology-Universidad de Barcelona, C/Juliol 21, 10-20, Barcelona 08041,Spain.E-mail:maclamps@yahoo.com

\section{P.M. SARA, M. FERNADO \& A. LUIS. NEUROPSYCHOLOGICAL} EVALUATION IN THE APERT SYNDROME. A CASE REPORT.

Objective: Apert syndrome, also called acrocephalosyndactylia type 1 , is characterized by severe craniosynostosis, midface hypoplasia, symmetric syndactyly (hands and feet) and central nervous system anomalies (malformations of the corpus callosum and limbic structures, gyral abnormalities among others). Other frequent problems are megalencephaly, ocular and otologic manifestations, cervical vertebral anomalies and dermatologic characteristics. Intelligence in patients varies from mental deficiency to normaly, also the neuropsychological profile of patients.

Participants and Methods: We have used data from the "Asociación Nacional Síndrome de Apert" to evaluate, through neuropsychological instruments ( Stroop Color and Word Test, Wisconsin Card Sorting Test, Wechler Adult Intelligence Scale, Rey Osterrieth... among others), a 21 years-old male with Apert syndrome.

Results: This study describes in detail the neuropsychological profile and cognitive processes, such as attention, memory and executive functions. Conclusions: Describe the neuropsychological profile in that king of disease (rare disease) is important for increase the quality of life of patiens and their families

Correspondence: Pérez M. Sara, Universidad Complutense de Madrid, Camarena 10080 c, Madrid 28047, Spain. E-mail: sara.perez. mart@gmail.com

\section{Infectious disease: HIV/AIDS}

\footnotetext{
D. FREED, O. BARAK \& D. TURNER. Depression and Coping in Persons Infected with HIV.

Objective: AIDS is a collection of symptoms and infections resulting from specific damage to the immune system, initiated by HIV. It was found that 60 to $90 \%$ of AIDS patients' brains show some form of pathology. Thus infection with HIV can be considered a form of CNS injury. Similar to other victims of CNS damage, high rates of depression have been reported in persons inflicted with HIV. However, little work was done on the positive coping abilities following CNS injuries, in particular HIV. The purpose of the current study was to examine the correlations between depression and coping (Hope, Optimism and Social support) among persons with HIV.
}

Participants and Methods: 40 male persons diagnosed as HIV positive, who are routinely evaluated at the AIDS center, Sourasky Medical Center. Subjects were asked to fill questionnaires: the Beck Depression Inventory revised (BDI-R), the Life Orientation Test revised (LOT-R), the Adult Hope Scale (AHS) and the Tel-Aviv Social Support questionnaire (TASS). Each evaluation lasted 20 minutes.

Results: BDI-R scores were significantly higher in the HIV positive group, indicating a high level of depression. LOT-R and AHS scores were significantly lower in the HIV positive group, indicating low levels of optimism and hope, respectively. TASS scores were significantly lower in the HIV positive group, indicating a low level of social support. BDI$\mathrm{R}$ scores were negatively correlated with LOT-R and AHS scores and TASS scores. Finally, it was found that LOT-R and AHS scores had a strong negative correlation only in the group of severe depression. Thus, optimism and hope might act as mediators in cases of light, but not severe depression following HIV infection.

Conclusions: The findings of the current research might suggest that coping plays a crucial part in adapting and living with HIV. The various levels of coping that were found within light depression indicate the uniqueness of coping variables as mechanisms that enable adaptation to life with HIV.

Correspondence: Ohr Barak, Behavioural sciences, Tel-Aviv Yafo College, 14 Rabenu Yerucham St., POB 8401, Yaffo 68114, Israel. E-mail: obar@mta.ac.il

L.A. CYSIQUE, M. CHERNER, S. LETENDRE, S. GIBSON, F. VAIDA, C.F. AKE, J. MCCUTCHAN, S.P. WOODS, I. GRANT, R.K. HEATON \& R.J. ELLIS. Predictors of neuropsychological improvement in HIV+ individuals initiating antiretroviral therapy.

Objective: To evaluate the baseline demographic, laboratory, and treatment variables associated with neuropsychological (NP) improvement in a cohort of $\mathrm{HIV}+$ individuals initiating combination antiretroviral therapy (ART).

Participants and Methods: Study participants included $28 \mathrm{HIV}+$ individuals with mild-to-moderate NP impairment who initiated ART, $45.8 \%$ of whom were ART naive. ART was composed of at least 3 agents. Participants were re-tested at week 24 on ART with the same battery of standard NP tests from which a global deficit score was calculated. NP change was assessed using reliable change indexes (RCIs), which were derived from HIV seronegative participants who underwent repeat assessment with the same NP tests as the HIV+ cohort.

Results: RCI analyses at week 24 revealed that $67.8 \%$ of the HIV+ individuals improved, whereas $32.1 \%$ had stable NP impairment and none declined. Univariate analyses showed at least modest associations between NP improvement and both absence of ART exposure $(\mathrm{p}=.05)$ and ART penetration score $(p=.08)$. These two predictors also contributed uniquely to a multivariate model that was significant $(p=.02)$. Baseline plasma nor CSF viral load did not contributed significantly to the model.

Conclusions: NP improvement occurred in 2/3 of a cohort of NP impaired HIV+ participants by 24 weeks after initiating ART and was more likely in those initiating first ART and starting ART with better CNS penetration. Improvement was not associated with differences in pre-treatment viral loads in CSF or plasma. These findings suggest that the first exposure to ART can have important effects on HIV-associated NP impairment; that NP improvement is linked to ART that has greater penetration into the CNS; and that subjects who remain impaired despite ART may do so for reasons unrelated to baseline HIV replication, such as persistent immune activation.

Correspondence: Lucette A. Cysique, Ph.D., Psychiatry, University of California San Diego, HNRC 150 Washington Street, San Diego, CA 92103.E-mail: lcysique@ucsd.edu 


\section{Infectious disease: Other (e.g., hepatitis C, West Nile virus)}

E. LOJEK, M. RADKOWSKI, A. JARMINSKA, J. JAROSZEWSKA, A. KOWALSKA, A. HORBAN, J. HIGERSBERGER \& I. WEBER KANIUK. Neuropsychological Functioning in HIV/HCV Co-infected Subjects Undergoing the Interferon and Ribavirin Therapy.

Objective: The present study shows the preliminary results of a twoyear research program aimed at analyzing the influence of interferon/ribavirin (IFN/RB) treatment on neuropsychological functioning in sixty-one HIV/HCV co-infected (HIV/HCV+) subjects.

Participants and Methods: Thirty-six patients (group E) took part in the IFN/RB treatment program ( $\mathrm{n}=35$ in the follow-up). A control group (C) included $25 \mathrm{HIV} / \mathrm{HCV}+$ persons who did not undergo that program ( $\mathrm{n}=15$ in the follow-up). There were significant differences between the groups on age and education on both assessments. Group E was younger ( 5 years on average) and less educated ( 2 years on average) in comparison with the control subjects. There were no differences on gender and medical data regarding HIV infection. Group E showed significantly worse results on all indexes of HCV infection.

The follow-up examination was conducted in the middle of the treatment program, 3 months after it started. A comprehensive battery of neuropsychological tests was administered for the assessment of neurocognitive functions, quality of life and depression.

Results: At baseline group E showed significant impairment on executive functions (the WCST), memory (the CVLT) and nonverbal fluency (the RFFT), but not on quality of life (the SIP) or depression (the BDIII) compared with group C. At 3 month follow-up neurocognitive abilities significantly improved but there was a marked worsening of quality of life and depression in patients undergoing the IFN/RB treatment program compared with group C.

Conclusions: The study confirms earlier data showing neurocognitive disorders in HIV/HCV+ subjects. It also indicates the necessity of monitoring the negative psychological side-effects of the IFN/RB treatment. Correspondence: Emilia Lojek, Ph.D., Faculty of Psychology, Warsaw University, Faculty of Psychology Warsaw University, Stawki 5/7, Warsaw00-183, Poland. E-mail: emilia@psych.uw.edu.pl

\section{Medical Disorders}

S. GELB, W.J. THORNTON, R.J. SHAPIRO \& H. DUMKE. Kidney Transplantation: Neuropsychological Abilities as a Mediator of the Relationship Between Health Status and Everyday Problem Solving. Objective: Everyday Problem Solving (EPS) is a measure of interest when attempting to identify cognitive tests that accurately reflect one's ability to function in everyday life. In previous work, we have demonstrated that kidney transplant (TX) recipients perform worse than controls on traditional neuropsychological measures. We have also found that for Chronic Kidney Disease (CKD) participants, neuropsychological abilities partially mediate the relationship between health status and EPS performance. In the present study, we assessed whether neuropsychological abilities mediate the relationship between health status (TX recipient versus healthy control) and EPS performance, and hypothesized that findings for kidney TX recipients would be similar to those found for persons with CKD.

Participants and Methods: We used a cross-sectional design in order to compare EPS and neuropsychological performance in 48 kidney TX recipients and 49 control participants. Groups were matched on age and education, and were carefully screen for other conditions (e.g., stroke) with known neuropsychological sequelae.

Results: In a series of regression analyses, TX status predicted worse EPS performance and memory/executive functioning abilities. Hierarchical regression analysis revealed that memory/executive abilities partially mediated the relationship between health status and EPS $(\Delta \beta=$ .11; Sobel's $\mathrm{Z}=-2.31, \mathrm{p}<.05)$, although TX status still remained significantly associated with worse EPS performance.
Conclusions: Although neuropsychological abilities appear to partially underlie EPS declines in chronic illness, TX recipient status remained independently associated with reduced EPS. This suggests that EPS measures may provide additive value beyond traditional neuropsychological measures, thereby highlighting the potential benefits of including EPS measures in neuropsychological assessments.

Correspondence: Shannon Gelb, M.A., Psychology, Simon Fraser University, RCB 5246, 8888 University Drive, Burnaby, BC V5A 1S6, Canada.E-mail:slund@sfu.ca

R.O. HOPKINS, C.W. KEY \& L.K. WEAVER. Predictors of Depression and Anxiety in Acute Respiratory Distress Syndrome Survivors.

Objective: Anxiety and depression commonly follow acute respiratory distress syndrome (ARDS). No studies have investigated risk factors for depression and anxiety in ARDS survivors. We assessed risk factors of depression and anxiety in ARDS survivors.

Participants and Methods: ARDS survivors were administered the Beck Depression and Anxiety Inventories at one and 2 years after hospital discharge. Demographic and medical data were obtained. Stepwise multiple regression determined predictors, including demographic and medical variables, of depression and anxiety. Predictions of 2-year outcomes were performed with and without one-year outcomes as potential predictors. Data are shown as mean \pm SD.

Results: Of 67 ARDS survivors, 41 were female, with a mean age of 46 \pm 16 years, and mean education level of $13 \pm 2.3$ years. The $\mathrm{PaO} 2 / \mathrm{FiO} 2$ ratio, hours of mechanical ventilation, a history of smoking, and APACHE II scores predicted anxiety at one year $(\mathrm{R} 2=.256, \mathrm{p}<.001)$ accounting for $26 \%$ of the variability. Alcohol abuse, female sex, and presence of cognitive sequelae at one year predicted depression at one year $(\mathrm{R} 2=.203$, $\mathrm{p}=.003$ ) accounting for $20 \%$ of the variability. At two years predictions including one-year outcomes accounted for more variability than the individual predictors alone. Anxiety at one year and a history of smoking significantly predicted anxiety at two years $(\mathrm{R} 2=.414, \mathrm{p}<.0001)$, accounting for $41 \%$ of the variability. Depression at one year and the presence of cognitive sequelae at one year significantly predicted depression at two years $(\mathrm{R} 2=.623, \mathrm{p}<.0001)$, accounting for $62 \%$ of the variability. Conclusions: ARDS and its associated treatment are risk factors for the development of depression and anxiety.

Correspondence: Ramona O. Hopkins, Ph.D., Psychology, Brigham Young University, 1082 SWKT, Provo, UT 84602. E-mail: mona_hopkins@byu.edu

A. HUIDOBRO \& R. PEREZ ASENJO. Is there a place for Neuropsychology in the Emergency Room? Gershwin revisited.

Objective: We want to illustrate with a case report the utility of neuropsychological testing for the detection of brain pathology in an Emergency Room, an usually highly technified environment.

Participants and Methods: The patient was a male, 59 years old, without previous psychiatric or medical diseases, who was brought to the Emergency Room of our hospital by his family, due to the development of changes in his behaviour and personality in the previous months.

Results: Initially he was classified as a psychiatric patient, probably afectted by a depressive syndrome, by the Internal Medicine physicians. After a basic neuropsychological tests we detected a severe frontal lobe dysfunction; then we demanded a brain imaging test which demonstrated a big brain tumor, in frontal region. The patient was diagnosed of a multiform gliobastoma and died few weeks later.

Conclusions: Even though most physicians ignore them, the neuropsychological tests have a great utility in an Emergency Room environment, helping us to improve the efficiency of radiological techniques and avoiding unnecessary examinations.

Correspondence: Alvaro Huidobro, MD, Hospital 12 de Octubre, Av.Cordoba s/n, Madrid 28041, Spain. E-mail: alvaro.huidobro@gmail.com 
S. SÁNCHEZ, F. OSTROSKY-SOLÍS \& J. ALBERÚ-GÓMEZ. Cognitive Function In Mexican End Stage Renal Disease Population. Objective: The present work represents the first part of a large study being done in Mexico that pretends to describe Mexican ESRD population in terms of cognitive function and follow them through their different renal replacement therapies.

Participants and Methods: 36 End Stage Renal Disease (ESRD) patients were administered the NEUROPSI ATTENTION AND MEMORY (a neuropsychological battery that assess attention, concentration, memory, motor functions and some executive functions and is standardized in Spanish Speaking population from 6 to 85 years old), the Hospital Anxiety and Depression Scale and the Epworth Sleepness scale. Biochemical markers of renal function were also assessed.

Results: $35 \%$ patients showed an impaired global score of the neuropsychological battery. This global score did not show a correlation with the score of the anxiety and depression or sleepness scales. The main impairment was observed in the Stroop subtest, verbal and visual delay recall, and sustained attention. No differences were found in global functioning between different dialysis treatments.

Conclusions: Our results reveal evidence of specific attention, memory and executive deficits in ESRD patients.

Correspondence: Sofía Sánchez, INCMNSZ, UNAM, Vasco de Quiroga 15, Tlalpan, Mexico 14000, Mexico.E-mail: sofiasan@yahoo.com

\section{Other}

W.N. PACHAS \& K. BEKKEN. The Role of Memantine (Namenda) in the Treatment of Mental Dysfunction in Patients with Fibromyalgia (FM).

Objective: Patients with FM often complain of variable degrees of short term memory deficit, poor organizational skills, slow reaction to visual stimulation such as traffic lights and signs, and other mental changes. All of these symptoms appear to be independent of other symptoms that affect these patients, such as sleep disorder, pain, and fatigue. Because of the favorable effect of memantine on mental functioning in Alzheimer's patients, we investigated the possibility that this drug would have a beneficial effect on mental functioning in FM.

Participants and Methods: A set of 15 patients with FM (aged 20-65), drawn randomly from a larger clinical population, were evaluated with neuropsychological tests before and after treatment with memantine. Results from each testing session were compared both within and across subjects to determine if there positive effects on functioning, and whether there were any trends in such improvement. Patient self-report of functional changes was also evaluated.

Results: Patients reported variable responses overall. While a few patients did not notice any changes, the majority reported significant improvement in their level of mental function, including short term memory. Similarly, the majority showed measurable improvement in functioning on standardized tests with memantine.

Conclusions: The implications of this improvement on the underlying bases of FM are considered. Double-blind, placebo-controlled studies will be necessary to confirm the encouraging favorable effects of memantine. Correspondence: Kaaren Bekken, PhD, CDN, 76 Bedford St, Ste 12, Lexington,MA 02420.E-mail: kbekken@gmail.com

L. CASAS, D. BADENES, S. MUNTAL \& M. AGUILAR. COGNITIVE FUNCTION IN PATIENTS WITH SEVERE CAROTID STENOSISEVALUATION OF COGNITIVE MESURES BEFORE TREATMENT OF REVASCULARATION.

Objective: Neuropsychological outcomes after carotid endarterectomy (CEA) have been investigated extensively. However, cognitive patterns have not been studied. This study is aimed To evaluate the cognitive function in preoperative patients with severe carotid stenosis looking to identify neuropsychological characteristics associated with the side of the stenosis (right or left stenosis).
Participants and Methods: 44 patients with severe carotid stenosis (29 left and 15 rigth) were examined on their cognitive function by MMSE, RBANS, TMT, Token Test, Corsi Block-Tapping Test, Stroop, BNT, FAS and Tower of London before treatment of carotid revascularization (endarterectomy or stenting) and follow-ups. Tests were chosen to assess functioning in both hemispheres. Results were analysed by inter-group comparisons. All the patients had full activities of daily living without any hemiparesis, aphasia, agnosia, and apraxia at the time of the examinations.

Results: There were no statistically significant differences at baseline cognitive performance between the two groups except in attention percentile (RBANS) where right patients performed significantly better than left patients (p.0.002).

Conclusions: We need to make further analysis post carotid revasculation, inter-group and within-group comparisons, to find if is any differences in cognitive hemispheric functioning between both groups. Correspondence: Laura Casas, Neurology, Hospital Mutua Terrassa, C/Castell 25 sotano, Terrassa 08221, Spain. E-mail: lauracasass@gmail.com

E. Poutiainen, E. KIURU, M. TIAINEN \& R.o. ROINE. Does Intensive Reduction in Blood Glucose Levels at the Acute State Improve Cognitive Outcome in Patients Resuscitated from Cardiac Arrest?

Objective: Cardiac arrest (CA) results in global cerebral ischemia and hypoxic-ischemic injury with wide-ranged cognitive deficits. In the early postresuscitation period, a number of pathophysiologic alterations are caused not only by primary ischemia, but also by their combination with reoxygenation and recirculation. High blood glucose concentrations occurring over the first $24 \mathrm{~h}$ after CA have found to have deterious effects on functional recovery. However, no studies exist on the therapeutic reduction in blood glucose levels during an intensive care. The aim of this randomized prospective study was to examine if an intensive reduction in glucose levels has an effect on cognitive outcome in resuscitated CA patients.

Participants and Methods: Patients were randomized to a group of reduced glucose level of 4.0-6.0 mmol/l (14 patients) or 6.1-8.0 mmol/l (14 patients). Time to basic and advanced life support, and restoration of spontaneous circulation (ROSC) was recorded at the acute state. After seven months of the onset Pittsburg outcome scale, IADL sale, neurological status and neuropsychological examination was performed. Neuropsychological examination included measures of memory (WMSR/logical memory, WMS-III/list learning), executive function (TMBTMA, Stroop), visuomotor speed (TMA, WAIS-R/Dsy), visual skills (WAIS-R/BD, VOSP/1,6,7), language skills (naming of ten line drawings), and depression (BDI).

Results: This randomized controlled prospective study found no significant differences (Student's t-test)in background variables or in medical, cognitive, or functional status between the resuscitated CA survivors with glucose levels reduced to $4.0-6.0 \mathrm{mmol} / \mathrm{l}$ and to $6.1-8.0$ $\mathrm{mmol} / \mathrm{l}$ during the acute state.

Conclusions: There is no need for intensive control for blood glucose level, when it ranges from 4 to $8 \mathrm{mmol} / \mathrm{l}$, during an intensive care of resuscitated CA patients.

Correspondence: Erja Poutiainen, PhD, Department of Psychology, University of Helsinki, PoBox 9, Helsinki 00014 UnivHki, Finland. Email:erja.poutiainen@helsinki.fi

G. SAAVEDRA, J. BERNABEU, R. BÉSELER \& C. GRAO. One Case of Heatstroke and Neuropsychological Sequelae.

Objective: Although signs of central nervous system dysfunction (including mental status changes) are considered essential to the diagnosis of heatstroke (HS), there is littled detailed information available concerning the residual neuropsychological effects of HS in previously healthy adults. One case of neuropsychological evaluation of exertional HS is presented here. 
Participants and Methods: We present the case of a 46-year-old male who suffered HS with multiple organ failure (neurologic, hemodinamic, hematologic, hepatic and renal) while working outdoors. We show the neuropsychological evaluation findings at 3 months and at 5 months after HS.

Results: Patient presented with hyperthermia (43 C), GCS 7, SaO2 93\%, hypotension (66/34) and tachycardia (FC166). Inicial CT and MRI findings were within normal limits. He had a history of hypertension, dyslipemia, alcoholism and lacunar infarcts without sequelae. After 6 days of sedation, he woke up bradypsychic and disoriented. He was hospitalized ( 3 months later) in the Brain Injury Unit presenting ataxia and alterations in coordination which prevented him from standing up and walking, disphagia, dysarthria and severe dystonia. On neuropsychological examination he showed temporal disorientation, inattention, alteration in language skills, agnosia, apraxia, alteration in executive functions and behavioural disturbances. Cognitive intervention was started (daily rehabilitation sessions, tasks to perform with his wife and patient and family education). Two months later bradypsichia, apraxia, dysarthria, inattention and prefrontal deficit persisted.

Conclusions: Residual cerebellar deficits are those most frequently described after HS. Our findings are consistent with the literature. The bradypsichia, inattention and the prefrontal difficulties are described less frequently. As other authors point out, there is not just one pattern of cognitive impairment associated with HS. Therefore it is necessary to systematically evaluate all possible neuropsychological sequelae in HS patients.

Correspondence: Gloria Saavedra, Hospital La Magdalena, Cuadra Collet, 32 (Pda. Bovalar), Castellón 12004, Spain. E-mail: saavedra_glo@gva.es

\section{B. SEGURA, M.A. JURADO, N. FREIXENET, C. ALBUIN, C. SOLER, R. MARTíN \& J. MARTÍNEZ. Metabolic Syndrome and Psychomotor Speed Deficit.}

Objective: Metabolic Syndrome is a set of vascular risk factors which increase the risk of vascular pathology and arteriosclerosis. Neuropsychological deficit in processing speed has been described in vascular subcortical pathology and has been related with cerebral white matter hyperintensities. The aim of this study is to explore if this deficit is present in Metabolic Syndrome as a prodromic state of vascular subcortical pathology.

Participants and Methods: We recruited 25 patients diagnosed with Metabolic Syndrome by the National Cholesterol Education Program (10 men and 15 women, mean age 66.48 7.467 , range of age 5080, mean years of education 7.56 \pm 3.917$)$ and 15 controls without any Metabolic Syndrome criteria (9 men and 6 women, mean age $63.93 \pm 7.630$, range of age $52-76$, mean age of education 8.53 \pm 4.207 ). Age, gender, years of education and IQ estimation through Vocabulary subtest of WAIS-III didn't show significant differences between groups. Exclusion criteria were: scores <26 in MMSE, smoking, history of pathology and use of medications affecting CNS. Psychomotor speed, as part of a more extensive examination, was assessed with Grooved Pegboard test.

Results: To test for differences we used non-parametric analysis (U Mann-Whitney statistics) of the Statistical Package for Social Science (SPSS Win.;v.11.0). Metabolic Syndrome group and controls were significantly different in execution time. Patients were slower than controls using the dominant $(\mathrm{U}=92.000 \mathrm{p}=0.008)$ and non-dominant hand ( $\mathrm{U}=96.000 \mathrm{p}=0.01)$.

Conclusions: Metabolic Syndrome patients show a deficit in psychomotor speed. The result could indicate that Metabolic Syndrome patients are in a prodromic state of subcortical vascular pathology. Correspondence: Angeles Jurado, PhD, Psiquiatria i Psicobiologia clínica, University of Barcelona, Passeig de la Vall d'Hebron 171, Barcelona 08035, Spain.E-mail:majurado@ub.edu
K. VIRUDHAGIRINATHAN. Dental and Medical Behaviour Problems and Management in ADHD, Autistic and Learning Disability Children in India.

Objective: Oral health has gained considerable significance among the Indian population in the past 10 years. This has been reflected in the importance given to oral health of children since the age of 2 years. A group of 100 children diagnosed with ADHD, LD, Autism had moderate to severe dental problems. Most of these children were in the age group of 6 - 14 years. The common dental problem seen in Indian children were, Malocclusion, Tooth anomlies, Developmental defects, Dental caries, Nursing bottle caries or Baby bottle Decay, eriodontal or gun disease, Thumb or Finger sucking and Bruxism (night grinding). Most prevalent problems seen in these children was thumb or finger sucking. In most cases this habit of thumbo $r$ finger sucking was due to a behavioural problem associated with ADHD or LD. These children had undergone a complete neuropsychological evaluation and then taken for dental treatment. Inability to chew food and indifference to maintain oral hygiene was commonly seen among these ADHD and LD children. Bruxism or tooth grinding was another common complaint from parents of children with hyperactive behaivour. Behaviour therapy and remedial coaching given to these ADHD and LD children was vital in providing comprehensive dental care. Many cases required medical management for hyperactivity. Parental counseling was one of the most important factors involved in achieving the desired result while treating these children.

Correspondence: Dr. B.S Virudhagirinathan, Clinical neuropsychologist, Kanchi Kamakoti Childs Trust Hospital, B1, Sri Govind Apartment, old \#17, New \#32, shenoy Road, Nungambakkam, Chennai-600 034, India, E-mail Bsv18@Yahoo.Com

\section{WANG. Neuropsychological Aspects of General Anaesthesia: A Western Perspective.}

Objective: It has long been recognised that a general anaesthetic will produce variable degrees of (generally reversible) cognitive impairment during the post-operative period. These impairments tend to be in the way of working memory and encoding difficulties, along with lability of affect which may be a cause, or exacerbating factor. There are suggestions in the literature that specific substances (e.g. anti-cholinergic drugs used intra-operatively, IGF1 insufficiency, etc.) may mediate post-operative cognitive impairment. Studies of these cognitive sequelae and their clinical implications will be reviewed.

Another aspect of neuropsychological function which is less wellknown is the dissociation of implicit from explicit memory as a consequence of light or inadequate anaesthesia. Episodes of full awareness with explicit recall are more common than many anaesthetists realise (around 1 in 500 operations). Awareness with full recall is usually distressing and associated with acute PTSD reactions. Furthermore, often patients will demonstrate high levels of intra-operative consciousness but without post-operative explicit recall. This is because many anaesthetic drugs impair the encoding phase of memory. It is likely that previous studies demonstrating intra-operative implicit (unconscious) learning have involved learning episodes during such states. Implicit memory can be demonstrated in states of intra-operative wakefulness without explicit recall, including implicit emotional memory. There is an intriguing literature in which patients have developed psychological disturbance following operations with GA in which the patient has no explicit recall, but the nature of the disturbance is indicative of inadequate anaesthesia. Experimental studies that attempt to investigate the mechanisms by which this may occur are reviewed.

Correspondence: Michael Wang, Email mw125@le.ac.uk 


\section{Psychopathology/Neuropsychiatry/Other}

V.P. BOZIKAS, M.H. KOSMIDIS, M. GIANNAKOU, M. SAITIS, G. GARYFALLOS \& K. FOKAS. Humor Appreciation of Captionless Cartoons in Obsessive-Compulsive Disorder.

Objective: It would appear that the core neural regions and cognitive processes implicated in obsessive-compulsive disorder (OCD) pathophysiology might overlap with those involved in humor appreciation. There are no studies to date, however, that have explored humor appreciation in OCD. We investigated humor appreciation in a group of remitted patients with OCD.

Participants and Methods: We examined 25 patients $(10$ men) with OCD and 25 healthy controls ( 14 men), matched on age, education, and gender. We administered Penn's Humor Appreciation Test (PHAT), a computerized test comprising 20 pairs of captionless cartoons by Mordillo. His style of humor involved an incongruous detail in the visual depiction, specifically an anomalous detail in an otherwise typical scene or the main character being unaware of a peculiar detail in his/her world to which the observer was privy. Consequently, appreciation of his cartoons required the perception of anomalous information and/or the comprehension of the depicted character's mental state. Each set of stimuli consisted of two almost identical drawings, one of which was funny, due to the alteration of a detail in the cartoon, whereas the other was not funny. Severity of psychopathology was evaluated with the YaleBrown Obsessive Compulsive Scale (Y-BOCS).

Results: Patients with OCD did not differ from healthy controls on the PHAT. Performance on this measure did not significantly correlate with age of onset and duration of illness, as well as with severity of obsessions and compulsions as measured with the Y-BOCS.

Conclusions: Humor appreciation, based on captionless cartoons, does not seem to be deficient in OCD.

Correspondence: Maria Giannakou, Psychology, Psychology, Aristotle University of Thessaloniki, Stratigi 14, Thessaloniki 54352, Greece. Email:mariag@psy.auth.gr

L.C. BUTTERFIELD, L.E. OELKE, C.R. CIMINO, J. SANCHEZRAMOS \& R.A. HAUSER. The Influence of Apathy and Depression on Cognitive Functioning in Parkinson's Disease.

Objective: Apathy, depression, and cognitive impairment are common among patients with Parkinson's disease (PD). Recent research suggests that apathy may account for cognitive deficits over and above that of depression. The present study examines the independent influences of depression and apathy on memory and executive functioning in patients diagnosed with PD. Participants and Methods: Forty-three patients with idiopathic PD completed a self-rating measure of depression (BDI-II) and apathy (AESS), and cognitive tests of immediate and delayed memory (HVLT-R) and executive functioning (WCST-64). In addition, 28 caregivers completed an informant-rating of apathy (AES-I). Data was analyzed using correlation and hierarchical regression.

Results: Immediate memory was correlated with informant-rated apathy $(\mathrm{r}=-.60, \mathrm{p}<.001)$ and self-rated apathy $(\mathrm{r}=-.33, \mathrm{p}<.05)$, with a trend for depression $(\mathrm{r}=-.30, \mathrm{p}=.07)$. Delayed memory was correlated with informant-rated apathy $(\mathrm{r}=-.50, \mathrm{p}<.01)$, with trends for self-rated apathy $(\mathrm{r}=-.30, \mathrm{p}=.06)$ and depression $(\mathrm{r}=-.32, \mathrm{p}=.05)$. Correlations with executive functioning revealed a trend for informant-rated apathy $(\mathrm{r}=-.37, \mathrm{p}=.052)$ only. Hierarchical regression analyses showed that apathy uniquely contributed to memory performance, but not to executive functioning, when controlling for depression, but only when informant ratings of apathy were entered.

Conclusions: Preliminary data suggests that self-rated apathy does not contribute to cognitive performance over and above that of depression. Informant-rated apathy may better predict cognitive performance. We plan to evaluate whether our findings will be supported with an increased sample size.

Correspondence: London C. Butterfield, BA, Psychology, University of South Florida, 4202 E. Fowler Ave., PCD 4118G, Tampa, FL 33620. Email:londonbutterfield@hotmail.com
P. CABALLERO, L. CARRAL FERNANDEZ, A. DUARTE ARMOLEA, P. BARBAS CALVO, J. ARTAL SIMON \& J. VAZQUEZ-BARQUERO. Visual Memory Deficits In The Early Phases Of Bipolar Disorder. Objective: Neuropsychological functioning in bipolar disorder has traditionally been considered to be preserved in the asymptomatic states. Despite this fact, at the moment there is accumulating evidence that $\operatorname{cog}_{-}$nitive deficits are common in patients with bipolar disorder even in euthymic states, including organizational and visual memory impairments. Most of the research has been carried out with chronic patients and it is not known if these deficits are present since the first episodes of the illness. Thus, the main aim of this study is to determine whether there are organizational and visual memory deficits in the early phases of bipolar disorder.

Participants and Methods: Ten early-phase euthymic bipolar patients and sixteen healthy controls were assessed using the Rey-Osterrieth Complex Figure. Testing included copy of the figure, immediate recall and long-term recall. Also, a measure of organization was derive from the strategy the participant used. Groups were comparable on age, sex, education and expected intellectual ability. Data was analyzed using MannWhitney and t tests.

Results: Patients with bipolar disorder were significantly more impaired than healthy controls on immediate recall and long-term recall. There were no differences on copy accuracy, but interestingly, the groups were different on copy organization.

Conclusions: Euthymic bipolar patients at the early phase of their illness show visual memory deficits characterized by recall impairments as well as difficulties in organizational processing. Bipolar patients use a more detail-oriented strategy than controls. These results suggest that cognitive deficits are present at the early stages of bipolar disorder. Correspondence: Patricia Caballero, Hospital Marques de Valdecilla, avda cantabria sn, Santander 39008, Spain. E-mail: patris22@hotmail.com

J. GRAY, J.M. THOMPSON, V. WAGGOTT, L. ROBINSON \& I.N. FERRIER. Subjective organization and impaired verbal learning learning in bipolar disorder while euthymic.

Objective: A recent meta-analysis of studies comparing cognitive function in individuals with bipolar disorder and normal controls (Robinson et al., 2006) confirmed large effect sizes for multi-trial verbal list learning and medium effect sizes for other aspects of verbal learning and memory. The same study reported medium and large effect sizes for different aspects of executive function. Impaired executive function may reduce learning reduce verbal memory performance by affecting encoding or retrieval. Indeed this may be the cause of the reported verbal memory impairment in individuals with bipolar disorder while euthymic. Impaired encoding in a multi-trial list learning task may manifest in reduced subjective organization over the trials (Tulving, 1962).

Participants and Methods: We examined the performance of $63 \mathrm{eu}-$ thymic bipolar patients and 63 matched controls on the Rey AVLT. Alongside the conventional measures of recall and recognition, we measured subjective organization over the five learning trials (Tulving, 1962). Results: Patients' scores were significantly worse for all measures of learning and memory, but not for subjective organization. Subjective organization predicted recall significantly better for the control rather than the patient group.

Conclusions: Euthymic bipolar patients' poor performance on verbal list learning is not due to poor organization at recall. Rather, they do not seem to gain the same advantage as controls from the equivalent level of organization.

Correspondence: John Gray, PhD, Newcastle University, Leazes Wing, RVI, Newcastle upon Tyne NE, United Kingdom. E-mail: gray600@btinternet.com 
V.A. HAALAND \& N.I. LANDRØ. Executive Functions in Patients with Borderline Personality Disorder.

Objective: Borderline personality disorder (BPD) is associated with disturbances in emotionality, behavior control, interpersonal relations and cognition. An increasing number of studies have been devoted to the neuropsychological functions of persons with BPD. As far as executive functions are concerned, some studies have consistently showed dysfunctions in patients with BPD, while others report no such dysfunctions.

In the present study, the executive functioning of patients with BPD was compared to persons without a history of mental disorders.

Participants and Methods: 29 patients with BPD (diagnosed according DSM-IV-TR criteria) age $18-37$, with mean GAF-S $=50$, and mean $\mathrm{IQ}=95$ were compared to healthy age-matched controls $(\mathrm{N}=25)$. Measures of executive functions included the Stroop Color-Word Test, Tower of London, Digit Span, Paced Auditory Serial Addition Test, Letter Number Span, Controlled Oral Word Association Test, Wisconsin Card Sorting Test (WCST), Iowa Gambling Task and a N-back task.

Results: Patients with BPD performed significantly below healthy controls on all the measures except on indicators of basic attention like digit span forward and the 0-back condition of the n-back task, and all the indicators of mental flexibility (WCST).

Conclusions: Patients with BPD seem to show a specific profile of impairments in executive functions. This might shed light on the underlying neurobiological foundation of the disorder and could account for some of the functional difficulties seen in daily living.

Correspondence: Vegard. Haaland, Dipl.psych, Department of Psychiatry, Sorlandet Hospital HF, Serviceboks 416, Kristiansand 4604, Norway. E-mail:vegard.oksendal.haaland@sshf.no

N.M. HALL, R. KUZMINSKYTE \& P. FINK. Neuropsychological Markers of Multisymptomatic Functional Disorder.

Objective: Many patients in various medical settings complain of physical symptoms that are not attributable to any known conventionally defined disease and are not adequately supported by clinical or paraclinical findings. Many of these patients also report problems with memory and concentration but there is very little neuropsychological research on their cognitive deficits. This study investigated the specific neuropsychological deficits of patients with many functional symptoms.

Participants and Methods: The concept of a single functional disorder is still controversial and there is no common agreement about the diagnostic criteria. Nevertheless, there is good reason to consider the various functional syndromes as a single disorder. In this study we refer to this disorder as Multiple Functional Disorder (MFD). Neuropsychological assessment was conducted on 22 MFD-patients selected for having many $(>6)$ medically unexplained symptoms, and 27 controls matched on age and gender. Exclusion criteria were co-morbid psychiatric illness, drug or alcohol abuse, pregnancy, and presence of a well defined somatic disease.

Results: Patients with MFD and controls scored similarly on tests measuring attention and concentration and on structured short- and long term memory tests. However, patients scored relatively lower than controls on tests of processing speed, verbal ability/general knowledge and long term memory as measured with more unstructured tasks.

Conclusions: The performance of patients with MFD on tests of attention and concentration indicates that they were well motivated for testing and suggests that their cognitive symptoms can not be explained solely by distraction due to pain or negative thoughts and emotions. Further research is needed to decide whether the identified cognitive deficits of the patients are a contributory cause or an effect of MFD. An increased knowledge about cognitive deficits may aid diagnosis and treatment of the patient group.

Correspondence: Nicoline M. Hall, Ph.D., Research Unit for Functional Disorders, University of Aarhus, Barthsgade 5, 1., Aarhus 8000 C, Denmark.E-mail:nicolinehall@gmail.com
A.J. LAWRENCE, J. LUTY, N.A. BOGDAN, B.J. SAHAKIAN \& L. CLARK. Neuropsychological Functioning and Varieties of Impulsivity in Problem Gamblers and Alcoholics.

Objective: High levels of impulsivity are commonly associated with substance use disorders. These elevations may predate the onset of addiction, associated with its vulnerability, or occur as a progressive consequence of chronic drug abuse. Problem gambling is a behavioural addiction that is spared from the harmful effects of drug use, and may provide unique insight into mechanisms of addiction vulnerability. In this study we compared neuropsychological functioning in problem gamblers with a group of alcohol-dependent outpatients.

Participants and Methods: Twenty-three problem gamblers (SOGS score $\geq 3$; 19 meeting criteria for pathological gambling; SOGS $\geq 5$ ) and 18 alcohol-dependent subjects were administered a neuropsychological assessment comprising measures of spatial working memory, stop signal response inhibition, affective decision-making (the Cambridge Gamble task) and reflection impulsivity (the Information Sampling task). Self-report questionnaires were used to assess drug, alcohol and gambling behaviour, as well as ADHD, depressive and OCD-related symptoms.

Results: The problem gambling (PG) group did not differ from the alcohol dependent (AD) group on questionnaire or neurocognitive measures of impulsivity or ADHD symptoms. The AD group made more search errors on the Spatial Working Memory task than the PG group and displayed increased response latencies on the Cambridge Gamble and stop signal tasks.

Conclusions: Alcohol dependency was associated with neuropsychological dysfunction in working memory and motor speed, that was not evident in problem gamblers. These changes in alcoholism may be related to progressive (neurotoxic) effects of chronic alcohol administration. The two groups did not differ on neurocognitive or questionnaire measures of impulsivity, consistent with an association between impulsivity and premorbid vulnerability to addiction.

Correspondence: Andrew J. Lawrence, B.A., Department of Experimental Psychology, University of Cambridge, Downing Street, Cambridge CB2 3EB, United Kingdom.E-mail: ajl54@psychol.cam.ac. $u k$

L.F. MALLOY-DINIZ, F. NEVES, S. ABRANTES \& H. CORRÊA. Neuropsychological aspects of bipolar disorder: analyses according history of suicide attempt and category of suicide attempt (violent $\mathrm{X}$ non-violent).

Objective: Neuropsychological deficits are often described in patients with bipolar disorder (BPD). The relationship of cognition and symptomatology on BPD remains unclear. This research aims to compare memory and executive performance of BPD subjects according history of suicide attempt and category of suicide behavior (violent $\mathrm{x}$ non-violent).

Participants and Methods: The study included 36 BPD outpatients (19 had lifetime history suicide attempt; 17 had no suicide attempt; 8 had violent suicide attempt; 11 had non-violent suicide attempt) and 50 health controls (HC). We use Raven Progressive Matrices (RPM), Rey Auditory Verbal Learning Test (RAVLT), Rey Osterreich Complex figure (ROCF) Stroop Color Word (SCWT), Oral Trails Test (OTT) and Five Digits test (FDT). We perform nonparametric test of Mann-Whitney to compare groups.

Results: HC patients had a better performance on all measures of intelligence, executive functions and memory. Suicide attempters had a worse performance on SCWT (amount of errors on part 3), ROCF (copy) and RAVLT (learning over trials) than non-suicide attempters. Violent suicide attempters had worse performance than non-violent on SCWT (amount of errors), ROCF (copy and retrieval) and RAVLT (A3-A7; B1 and learning over trials).

Conclusions: Cognitive impairment exists in BPD despite patient's history of suicide attempt. We found a relationship between history of 
suicide attempt, violent attempt and measures of memory and impulsiveness. Future studies with larger sample and considering another variables (ex.: premorbid cognitive traits, type of disorders and humor state) are needed to explain the relationship of suicide attempt and cognition on BPD.

Correspondence: Leandro F. Malloy-Diniz, PHD, University Human Sciences Faculty, Universidade FUMEC, Rua Gonçalves Dias, , 31 Bairro Funcionários, Belo Horizonte 30130090, Brazil. E-mail: leandro. fernandes@terra.com.br

I. RAMOS, G. GARRIDO, U. GONZALEZ, M. PAJARES, M. SANTACANA, A. BIGORRA, J. RODRIGUEZ \& M. GAROLERA. Neuropsychological subtypes in schizophrenia.

Objective: The aim of the study is to establish different schizophrenia subtypes depending on the neuropsychological outcome of schizophrenia patients.

Participants and Methods: 107 schizophrenia patients diagnosed by DSM-IV were enrolled in the study. All patients were clinically stable under antipsychotic treatment. Neurocognitive assessment consisted in evaluation of episodic memory and executive functions, since these are the cognitive areas of most significance of the adaptive functioning in schizophrenia patients. Therefore, the assessment included the following tests: California Verbal Learning Test, Wisconsin Card Sorting Test. Tower of London and a verbal fluency test (FAS). To study if there were different neuropsychological subtypes a cluster analysis was applied.

Results: After a cluster analysis, three cognitive groups were identified. One group was characterized by deficits in executive functioning (fronto-temporal subtype, with frontal predominance), a second group characterized by deficits in verbal memory (fronto-temporal subtype, with temporal predominance), and finally a third group characterized by a relatively preserved cognition.

Conclusions: It has been found a certain degree of cognitive heterogeneity in schizophrenia patients. Three subtypes of schizophrenia patients can be established using their neuropsychological performance. Therefore, these subtypes may map different etiologies and treatment strategies.

Correspondence: Irene Ramos, Psychiatry, Consorci Sanitari de Terrassa, ctra. Torrebonica s/n, Terrassa 08227, Spain. E-mail: ramos8irene@hotmail.com

M. SANTACANA, G. GARRIDO, M. PAJARES, U. GONZALEZ, I. RAMOS, A. BIGORRA, J. RODRÍGUEZ \& M. GAROLERA. Is it possible to identify a Kraepelian subtype of schizophrenia in the early stages of the illness?

Objective: For a great majority of schizophrenia patients it has been described a stable course of their neurocognitive deficits. However, it has been described a subgroup of schizophrenia patients (Kraepelian) that show a neurodegenerative course. The aim of the study is to explore the possibility of their identification in the first year of schizophrenia diagnosis.

Participants and Methods: 27 schizophrenia outpatients were enrolled in the study. Patients were diagnosed by DSM-IV and clinically stable at the time of neuropsychological testing. Neurocognitive functions were assessed with a cognitive battery during their first episode ( 6 weeks after the instauration of the antipsychotic treatment) and a year after. The neuropsychological battery included the assessment of the following cognitive functions: verbal intelligence, attention, verbal memory, visual memory, psychomotor speed, visoespatial and visoconstructive abilities and executive functions.

Results: Within the sample of schizophrenia patients, a 14.8\% showed in the one-year follow-up assessment a decline in verbal learning. The rest of the patients $(85.2 \%)$ showed a globally mantained neuropsychological outcome.
Conclusions: The decline in verbal memory tests in a $14.8 \%$ of the sample could fit the existence of a Kraepelian subtype of schizophrenics that is characterized with a progressive cognitive impairment. Therefore, the decline in verbal episodic memory in the one-year follow-up could be a sign of poor prognosis. Consequently, special monitoring could be needed for these patients.

Correspondence: Martí Santacana, Psychiatry, Consorci Sanitari de Terrassa, Carretera de Torrebonica S/N, Terrassa 08227, Spain. E-mail: martisantacana@hotmail.com

\section{Psychopathology: Anxiety/Stress}

S. ANDRES, T. RASPALL, E. LOZANO, C. FALCON, T. PRIETO, N. BARGALLO, A. GARCIA, L. LAZARO \& J. CASTRO-FORNIELES. Response Inhibition in adolescents with Obsessive-Compulsive Disorder and Anorexia Nervosa.

Objective: To compare the inhibition of an automatic response in adolescent patients with Obsessive-Compulsive Disorder (OCD) and patients with Anorexia Nervosa (AN) in a Stroop task and to compare the brain activity studied with fMRI during the performance of the task.

Participants and Methods: Thirteen OCD patients and 10 restrictive AN weight recovered patients (mean BMI 18.9 ) were studied. All patients were between 13 and 18 years old. Participants were under treatment and all of them presented partial remission of their disorders. Neuropsychological evaluation, measures of perfectionism (Child and Adolescent Perfectionism Scale, CAPS), and obsessive-compulsive symptomatology (Leyton Obsessive Inventory, LOI) were also evaluated.

Results: Patients with AN showed better performance in the Stroop task than OCD patients $(p=0.001)$, obtaining also higher scores in CAPS $(p=0.001)$. Both groups were similar in LOI severity scores $(p=0.897)$. Functional MRI activation patterns also differ between groups, AN patients showing greater activation in the cingulate cortex and in the left prefrontal cortex.

Perfectionism scores positively correlated with fMRI activation in the cingulate cortex $(p=0.005)$, the most related structure with the Stroop paradigm.

Conclusions: Adolescents with restrictive AN perform better than OCD patients in the Stroop task. Both groups were similar in severity of obsessive-compulsive simptomatology. Level of perfectionism is positively associated with activation of cingulate cortex during the performance of the task.

Correspondence: Susana Andres, PhD, Neurosciences Institut, Hospital Clinic of Barcelona, villarroel, 170, Barcelona 08036, Spain. E-mail: sandres@clinic.ub.es

A.G. CAPOVILLA, J.M. MONTIEL, F.C. CAPOVILLA \& E. MACEDO. Attencional Deficits in Panic Disorder: Brazilian Version of Computerized Emotional Stroop Test.

Objective: Panic disorder may be accompanied by attention problems, which may be assessed by Stroop interference tasks. Such color-naming tasks usually reveal increased reaction time when the color a word is printed differs from the color that word actually names. Observing whether color-naming interference is affected by emotion-arousing words may help identify emotion problems.

Participants and Methods: In this study, Stroop interference effect of emotion-arousing words was examined in 40 Brazilians, 20 panic disorder patients and 20 non-panic disorder participants. Both groups were asked to name the colors of 165 Portuguese words while ignoring word content. Both groups were compared in terms of reaction time while naming 55 neutral words, 55 negative emotion-arousing words, and 55 positive ones.

Results: Results showed that panic-disorder patients took longer to color-name both negative emotion-arousing words and neutral words as compared to non-disorder participants.

Conclusions: Such findings are compatible with the presence of attention and emotion problems in panic disorder, and show validity evidence 
of the Brazilian version of Computerized Emotional Stroop Test for the assessment of people with that disorder.

Correspondence: Alessandra G. Capovilla, Psychology, University of San Francisco, Rua Alexandre Rodrioues Barbosa, 45, Itatiba 13251-900, Brazil.E-mail: acapovil@usp.br

E. WALKENHORST \& S.F. CROWE. An Examination of the Relationship betweeen State Worry, Trait Anxiety and Working Memory Performance in a Non-clinical Sample.

Objective: This study investigated the effects of trait anxiety and state worry on working memory performance in a normal sample. Phase one of the study examined the effects of trait anxiety and state worry on the components of working memory. Phase two investigated the validity of Eysenck and Calvo's (1992) Processing Efficiency Theory. It was predicted that the processing efficiency of verbal working memory would be compromised in individuals who were high in trait anxiety and in individuals induced into a high state of worry.

Participants and Methods: Sixty adult participants ( 40 females and 20 males with a mean age of 26 years) were divided into two groups on the basis of their trait anxiety scores and were then randomly assigned to a high state worry condition or a low state worry condition in a 2 (trait anxiety: low versus high) by 2 (state worry: low versus high) between-subjects design. Their performances were assessed on the independent components of the working memory system (phonological loop, central executive, episodic buffer and the visuospatial sketchpad).

Results: Contrary to prediction, worry did not lead to a decrement in performance on verbal working memory tasks but unexpectedly enhanced performance on visual working memory tasks in participants with low trait anxiety. Results were also in clear opposition to expectation for Phase 2. Individuals in the conditions of high trait anxiety and/or high state worry displayed shorter response latencies than individuals in the condition of low trait anxiety and low state worry on both verbal and spatial working memory tasks.

Conclusions: The results of the two studies suggest that non-pathological worry may actually perform an adaptive role in cognition. Although non-pathological worry is predominately a verbal-linguistic activity, it may also be complemented by the visual processing of imagery which facilitates problem-solving and adaptive functioning.

Correspondence: Simon F. Crowe, PhD, La Trobe University, School of Psychological Science, Bundoora, VIC 3086, Australia. E-mail: s. crowe@latrobe.edu.au

R. JURADO, D. TABOADA, F. DENIA, J. MINGOTE \& S. FERNANDEZ-GUINEA. Attencional Deficits in Posttraumatic Stress Disorder and Panic Disorder.

Objective: Attencional processes are a major way for a correct interaction with the environment and also, for the processing of information. Studies showed that PTSD, when educational level is controlled for, was associated with decreased verbal memory, attention, and processing speed performance.So our objective is study the attentional performance of subjects suffering from anxiety disorders - Posttraumatic Stress Disorder (PTSD) and Panic Disorder (PD) - in order to determine whether there are differences when compare with healthy subjects.

Participants and Methods: The neuropsychological evaluation was performed in 30 patients with PTSD, 26 with PD and 26 controls, diagnosed according to DSM-IV-R clinical criteria. The following instruments were employed in the evaluation of the different attentional components: Stroop Test of Words and Colours (STROOP), Toulousse Pièron Test of Maintained Attention (Toulousse) and Trail Making Test (TMT). Results: Subjects with PTSD show less reading speed $(p=0.0029)$ and less denomination of colors speed $(p=0.0131)$, in comparison with PD subjects and controls. They also tend to make commission errors in maintained attention tasks $(\mathrm{P}=0.006)$. With respect to the alternation of se- quences tasks, PTSD patients' performance was significantly below when compared to controls $(\mathrm{p}=0.013)$.

Conclusions: We have found differences in attentional performance among PTSD subjects, PD subjects and healthy subjects. Specifically, PTSD patients showed a minor capacity of selective and alternating attention in comparison with PD patients and healthy subjects. With respect the PD group, all the scores were similar to those of healthy people. Correspondence: Rosa Jurado, Doctora, Psicología Básica II: procesos cognitivos, Universidad Complutense de Madrid, Facultad de Psicologia . Campus de Somosaguas. Pabellón I, Pozuelo de Alarcón 28223, Spain. E-mail: rjurado@psi.ucm.es

\section{Psychopathology: Depression}

P. GALLAGHER, J.M. GRAY, R.P. KESSELS, S. WATSON \& I.N. FERRIER. Spatial Memory Impairments in Depressed Patients with Bipolar Affective Disorder.

Objective: Recent studies have found evidence for dissociable cognitive processes within object location memory. Studies in patients with neurological damage suggest separable frontal and hippocampal processes. Patients with bipolar disorder (BD) show significant impairments in verbal memory and 'frontal' executive function with the latter persisting in euthymia, specifically the executive control of visuospatial working memory. The purpose of the present study was to examine the performance of patients with $\mathrm{BD}$ and healthy comparison subjects using a computerised memory location task in which positional processing can be separated from object-location binding.

Participants and Methods: Twenty-six outpatients fulfilling DSM-IV criteria for BD in a current depressive episode and 24 healthy comparison subjects completed the Object Relocation task (Kessels RPC, et al. Behavior Research Methods, Instruments, \& Computers 31:423-428; 1999). Following an initial assessment of object identity and visuospatial construction, 3 task conditions were used: positional memory (POM) - requiring the position of identical objects to be remembered and reproduced; object-location binding (OLB) - requiring objects to be remembered and relocated to pre-marked positions; and a combined condition (COM) - integrating both processes.

Results: Patients with BD were impaired in performance on all three conditions, however only the POM and COM conditions remained significant after correction for multiple comparisons. After using ANCOVA to control for an initial difference between the groups in visuospatial construction, the only remaining significant difference was the impairment in POM.

Conclusions: This study demonstrates that bipolar patients when depressed manifest substantial impairment in positional memory. It remains to be seen whether this deficit persists into euthymia.

Correspondence: Peter Gallagher, Newcastle University, Leazes Wing (Psychiatry),, Royal Victoria Infirmary, Newcastle upon Tyne NE1 4LP, United Kingdom.E-mail: peter.gallagher@ncl.ac.uk

\section{Psychopathology: Schizophrenia}

V.G. BOUDREAU, H. KRISTINSSON, G. MOLINA \& A.E. THORNTON. The Effects of Attention on Language Laterality in Schizophrenia Spectrum Disorder.

Objective: Language lateralization is often attenuated in schizophrenia. Nonetheless, it remains unclear whether impaired attentional processes in the disorder moderate this effect. To systematically control for alertness, we investigated whether the right ear advantage (REA) increases with the aid of a tone cue. It was hypothesized that patients would demonstrate a stronger REA with the tone, and would benefit more from the tone than controls.

Participants and Methods: Twenty-nine patients with schizophrenia spectrum disorder and 32 healthy controls matched on age, gender. and parental education, completed a conventional dichotic listening task and a modified dichotic task in which a binaural tone was presented 
prior to dichotic stimuli. Participants were screened for right-handedness, phoneme discrimination, hearing acuity, and mood symptoms.

Results: Accuracy data were subjected to a Mixed-Design ANOVA with Condition (conventional/tone) and Ear (right/left) as within-subjects factors, and Group (patients/controls) and Stimulus Onset Asynchonicity $(\mathrm{SOA} ; 150 \mathrm{~ms} / 500 \mathrm{~ms})$ as between-subjects factors. Results revealed a significant Task x Ear x SOA interaction, indicating a stronger REA in the cued condition relative to the conventional dichotic task when the SOA was $500 \mathrm{~ms}(\mathrm{~F}=5.86, \mathrm{p}<.05)$. This finding did not differ between groups $(p>.05)$. However, the tone decreased accuracy overall $(\mathrm{F}=6.49, \mathrm{p}<.05)$, which appeared to be driven by a trend in performance in controls $(\mathrm{F}=2.87, \mathrm{p}=.096)$.

Conclusions: Although we demonstrated that the REA can be increased with tone cueing, this finding was not specific to patients with schizophrenia. Moreover, the tone cue failed to improve accuracy during dichotic monitoring, and thus it remains unclear whether alertness moderates language lateralization.

Correspondence: Vanessa G. Boudreau, MA, Psychology, Simon Fraser University, 8888 University Drive, Burnaby, BC V5A 1S6, Canada. Email:vgb@sfu.ca

L. CARRAL-FERNÁNDEZ, C. GONZÁLEZ-BLANCH, P. CABALLERO-MARTÍNEZ, A. DUARTE-ARMOLEA, P. BARBASCALVO, J. RODRÍGUEZ-SÁNCHEZ, M. ÁLVAREZ-JIMÉNEZ, B. CRESPO-FACORRO \& J. VÁZQUEZ-BARQUERO. Unimpaired orbitofrontal function in first-episode schizophrenia: Evidence from the Object Alternation Task.

Objective: Neuropsychological literature indicates that performance on Object Alternation Task (OAT) can be linked to orbitofrontal cortex (OFC). Patients with chronic schizophrenia perform poorly on behavioural and neuropsychological tests sensitive to orbitofrontal dysfunction. In this study we aimed to determine whether OFC dysfunction is present in the early phases of schizophrenia.

Participants and Methods: We examined the performance of 70 patients with first-episode schizophrenia and 21 healthy controls on a computerized version of OAT. Data was analyzed using a nonparametric method (Mann-Whitney U-test).

Results: There was no significant difference between patients and control subjects with respect to the OAT $(\mathrm{p}=0.47)$.

Conclusions: This finding suggests that the OFC function is preserved in the early phases of schizophrenia. Alternatively, the sub-group of patients with this impairment may be underrepresented in first-episode samples.

Correspondence: Laura Carral-Fernández, HUV Valdecilla, Av. Valdecilla s/n, Santander39008, Spain.E-mail: lcarral@gmail.com

A. Duarte armolea, P. Caballero, J. Pelayo terán, C. GONZALEZ- BLANCH, P. BARBAS-CALVO, L. CARRAL, T. MORENO, J. RODRIGUEZ, B. CRESPO-FACORRO \& J. VÁZQUEZ- BARQUERO.NEUROPSYCHOLOGICAL EFFECTS OF CANNABIS ABUSE IN FIRST EPISODE PSYCHOSIS PATIENTS.

Objective: Cannabis is the illegal substance most frequently used in first-episode psychosis patients. In accordance with the literature, cannabis produces neuropsychological deficits while patients use actively this substance, but it is not known if these deficits persist in the abstinence period. The purpose of the present study is to look at the relationship between previous use of cannabis and the cognitive performance of patients compared with those that had never used this substance. Participants and Methods: One hundred thirty-one patients diagnosed with first-episode psychosis were administered a comprehensive neuropsychological evaluation. The exploration was carried out when the patients were included in a treatment program for the early phases of psychotic disorders. The evaluation included tests of verbal learning/memory, verbal comprehensive abilities, speed of processing/executive func- tioning, motor dexterity, motor speed, and sustained attention. Cognitive performance of patient's groups was compared using ANCOVA.

Results: There were no significant differences between both groups in the cognitive domains that have been evaluated.

Conclusions: According to the results, it can not be concluded that the use of cannabis is related to the existence of long-term neuropsychological deficits.

Correspondence: Alaitasune Duarte Armolea, Hospital Marqués de Valdecilla, Avenida España n01-3, Castro Urdiales 39700, Spain. Email:alai_du@hotmail.com

C. GONZALEZ-BLANCH, M. ÁLVAREZ-JIMÉNEZ, B. CRESPOFACORRO, J. RODRÍGUEZ-SÁNCHEZ, L. CARRAL-FERNANDEZ, P. BARBAS-CALVO, A. DUARTE-ARMOLEA \& J. VÁZQUEZBARQUERO. Lack of association between clinical and cognitive change in first-episode psychosis: The first six weeks of treatment. Objective: Cognitive deficits can be observed in the early course of firstepisode schizophrenia spectrums disorders. These deficits have been linked to functional impairments. The relationship between cognitive and clinical change may have an impact in determining the importance of cognition as a treatment target. We aimed to determine the relationship between changes in clinical dimensions and changes in cognitive functioning during the first 6 weeks of pharmacological treatment.

Participants and Methods: Forty-two patients, aged $15-50$ years, admitted to a first episode psychosis program (PAFIP) serving to the community of Cantabria (Spain) completed a brief battery of five neurocognitive tests within 72 hour of pharmacological treatment and six weeks after treatment initiation. The cognitive testing comprised five domains: attention, visuomotor speed, declarative memory, working memory and executive function. Forty-three healthy volunteers were recruited to control for practice effects. Ratings of clinical dimensions (positive, negative and disorganized) were based on the Scale for the Assessment of Negative Symptoms (SANS), and the Scale for the Assessment of Positive Symptoms (SAPS).

Results: Spearman correlations showed that negative dimension was significantly correlated to cognitive change in visuomotor tasks Colors Trails (part 2) ( $\mathrm{r}=-0.352 ; \mathrm{p}=0.030)$ and Ruff $2 \& 7$ (accuracy) ( $\mathrm{r}=-0$. 469; $\mathrm{p}=0$. 003), but not with other visuomotor tasks.

Conclusions: Only marginal relationships, probably due to multiple comparisons, can be found between changes in clinical dimensions and cognitive functioning. Clinical remission is not associated to cognitive change in the early course of the treatment of first-episode psychosis. Correspondence: Cesar Gonzalez-Blanch, HU Marques de Valdecilla, Valdecillas/n, Santander39007, Spain.E-mail: cgblanch@gap01. scsalud.es

M. KIM \& Y. NAMGOONG. The effect of organizational strategy on visual memory in schizophrneic patients.

Objective: This study examined the effect of organizational strategy on visual memory in schizophrenic patients using Rey-Osterrieth Complex Figure Test (ROCF).

Participants and Methods: 20 schizophrenic patients and 20 normals participated. ROCF was administered and the performances on ROCF were analyzed by the Boston Qualitative Scoring system (BQSS). The group differences were analyzed with ANCOVA with covariates of IQ, educational level and BDI score. The mediating effect of organization on visual memory was analyzed by multiple regression.

Results: The schizophrenic patients showed lower scores on fragmentation in copy condition $(\mathrm{p}<.05)$.For immediate recall, patients showed worse performance on configural presence $(p<.05)$. And patients showed worse performances on configural presence $(p<.001)$,cluster presence $(\mathrm{p}<.05)$, cluster placement $(\mathrm{p}<.05)$, detail placement $(\mathrm{p}<.01)$, plan$\operatorname{ning}(p<.001)$,neatness $(p<.05)$ in delayed recall condition. For summary scores, patients showed lower scores on immediate presence and accuracy, immediate retention and organization. 
Organization score mediated the difference between the two groups on ROCF immediate recall, however, the direct effect of group on ROCF immediate recall was also significant (beta=.44, $\mathrm{p}<.01$ ) after controlling for the organization.

Conclusions: This study showed that schizophrenic patients have an impairment of visual memory, and organizational deficit seems to be related to the visual memory deficit in patients. Schizophrenic patients seemed to have difficulties in appreciating the gestalt of a complex visual stimuli during copying the figure. These organizational deficit seems to contribute to difficulties of immediate and delayed recall of the figure. However, even after controlling for the organization, group differneces on visual memory were still observed. This result indicates that visual memory deficit is related not only to the organizational deficit during encoding but to other deficits during retention or retrieval.

Correspondence: Myung-Sun Kim, Ph.D, Psychology, Sungshin Women's University, Sungbugu Dongsun 3ga, Seoul 136-742, South Korea. Email:kimms@sungshin.ac.kr

J. PEÑA, N. OJEDA, P. SÁNCHEZ, E. ELIZAGÁRATE \& J. EZCURRA. Processing Speed Mediates The Relationship Between Verbal Fluency And Functional Disability In Schizophrenia.

Objective: Verbal fluency has been reported to be diminished in patients with schizophrenia. This deficit could partially predict functional disability in this pathology (Green, 2003). However, processing speed (PS) often mediates the relation among cognitive processes.

Our goal is to analyse whether the influence of verbal fluency on functional disability is mediated by the PS in patients with chronic schizophrenia.

Participants and Methods: 94 schizophrenic (DSM-IV criteria) hospitalised were assessed in terms of sociodemographics, clinical and cognitive variables.

Results: Mean age was $(X=37,02+11,15)$, education, $(X=9,48+3,21)$, years of illness $(X=15,29,+9,56)$, and mean days of hospitalisation $(S=676,95,+1645,77)$. Total score obtained in the PANNSS, revealed a chronic mean profile in our sample (PANSSTot $X=48,94,+14,97$ PANSSNeg $X=27,81+10,18$, PANSSPost $X=23,51,+10,81)$. Total score measured by DAS-WHO was $(X=13,62,+3,99)$. Digit-symbol and Stroop-C were averaged to create a PS measure. As expected, mean score in semantic fluency was severely impaired $(X=16,88, S D=5,40)$ and significantly related to DAS-WHO $(r=-.283, \mathrm{p}<.001)$ and to PS $(\mathrm{r}=.561$, $\mathrm{p}<.001)$. PS was also related to DAS-WHO $(\mathrm{r}=-.398, \mathrm{p}<.001)$. In the first step, semantic fluency significantly predicted DAS-WHO $(B=-0,210$, $\mathrm{p}<.01)$. In the second step, processing speed was regressed on semantic fluency to estimate the path between semantic fluency and the PS ( $B=$ $.093, \mathrm{p}<.001)$. In the third stage, semantic fluency $(\mathrm{B}=-.033, \mathrm{p}=0,720)$ and $P S(B=-1.689, p<.005)$ were entered simultaneously in the equation as predictor variables.

Conclusions: Sobel's equation result was significant at $\mathrm{p}<.005$ for PS ( $\mathbf{z}=-3.07)$ suggesting full mediation.

Correspondence: Javier Peña, Psychology Degree, Psychology, University of Deusto, Av/ de las Universidades 24, Bilbao 48007, Spain. E-mail: nuberojaes@hotmail.com

N. OJEDA, J. PEÑA, P. SÁNCHEZ, E. ELIZAGÁRATE \& E. JESÚS. Reasoning Is Related To Verbal Fluency And Processing Speed In Chronic Patients With Schizophrenia.

Objective: The relation between verbal fluency (VF) and processing speed (PS) in schizophrenia has been extensively reported, as well as the relation between VF and misleading reasoning and abstract thinking (AT). This study aims to explore if the influence of VF on misleading reasoning and abstract thinking could be moderated by PS.

Participants and Methods: 95 schizophrenic (DSM-IV criteria) hospitalised were assessed in terms of sociodemographics, clinical and $\operatorname{cog}$ nitive variables.

Results: Mean age was $(X=37,02+11,15)$, education, $(X=9,48+3,21)$, years of illness $(X=15,29,+9,56)$, and mean days of hospitalisation
$(\mathrm{S}=676,95,+1645,77)$. Total score obtained in the PANNSS, revealed a chronic mean profile in our sample (PANSSTot $X=48,94,+14,97$ PANSSNeg $X=27,81+10,18$, PANSSPost $X=23,51,+10,81)$. Difficulties with AT, as assessed by PANNS was $X=3,76,+1,89$. Slowness in the sample was severe (TMT-A, $X=69,90+50,34)$. As expected, mean score in VF was also severely impaired $(X=20,00+9,45)$ and predicted significantly misleading reasoning and abstract thinking $(B=-.617$, $\mathrm{p}<.001)$. A series of hierarchical multiple regression analyses indicated that the association between the VF and AT was moderated by PS. The interaction term VFxPS was significant $(B=.466, p<.05)$.

Conclusions: The analysis of this moderation indicated that the association between reasoning and verbal fluency is lower among those patients with low PS

Correspondence: Natalia Ojeda, PhD, Psychology, University of Deusto, Avda Universidades, 24, Bilbao 48007, Spain.E-mail:nojeda@fice. deusto.es

J. PEÑA, N. OJEDA, P. SÁNCHEZ, E. EliZAGÁRATE \& J. EZCURRA. Significant Differences between First Episode Psychosis and Chronic Patients With Schizophrenia in Semantic Fluency, Verbal Memory and Processing Speed.

Objective: Verbal fluency (VF) could be partially predicted by long term verbal memory in pathology. However, Processing speed (PS) often mediates the relation between cognitive processes in schizophrenia. That is to say, the relation between long term verbal memory and VF is mediated by the PS.

Our goal is to analyse whether this mediation is significant also in first episode psychosis (FEP) or is only relevant in long term phases of the illness.

Participants and Methods: 71 chronic schizophrenic and 39 FEP (DSM-IV criteria) hospitalised were assessed in terms of sociodemographics, clinical and cognitive variables. They did not differ in terms of age $(t=1.51, p=.134)$ or education $(t=-.681, p=.497)$. FAS, Weschler Memory Scale and Stroop-C were included in the cognitive assessment as relevant for the present analysis.

Results: In chronic schizophrenia VF was severely impaired (X=18.07 $+5,50)$ and significantly related to WMS2 $(\mathrm{r}=.30, \mathrm{p}<.05)$ and to PS $(\mathrm{r}=.381, \mathrm{p}<.01)$. However, in FEP, this relation was not significant ( $\mathrm{r}=.16)$ and therefore, do not allow mediation.

In chronic patients, PS was also related to WMS2 ( $\mathrm{r}=-.318, \mathrm{p}<.01)$. In the first step, results in WMS2 significantly predicted VF $(B=.32, p<.05)$. Secondly, PS was regressed on WMS2 to estimate the mediation-path between WMS2 and the PS $(\mathrm{B}=.89, \mathrm{p}<.01)$. Thirdly, WMS2 $(\mathrm{B}=.216$, $\mathrm{p}=.088)$ and $\mathrm{PS}(\mathrm{B}=.116, \mathrm{p}<.05)$ were entered simultaneously in the equation as predictor variable

Conclusions: Sobel's equation result was significant at $p<.05$ for PS $(z=1.99)$ suggesting full mediation of PS in VF and memory in chronic schizophrenia but not in first episode patients.

Correspondence: Javier Peña, Psychology Degree, Psychology, University of Deusto, Av/ de las Universidades 24, Bilbao 48007, Spain. E-mail: nuberojaes@hotmail.com

O. PUIG, S. ANDRÉS, L. LÁZARO, I. BAEZA, E. DE LA SERNA, R. CALVO, R. PENADÉS \& J. CASTRO-FORNIELES. Memory Dysfunction In Adolescents With Schizophrenia Spectrum Disorders.

Objective: Memory deficits and its impact in psychosocial functioning are well known in adult schizophrenia and related disorders. There are less neurocognitive studies in young patients with early-onset of the disorder. Attending to the influence of memory deficits in social functioning and to the crucial role of adolescence in social competence skills learning, there is a need of more studies of memory functioning in adolescent-onset schizophrenia spectrum disorders (AOS). The objetive of 
this work was to study memory functioning in a sample of adolescents with AOS.

Participants and Methods: A group of 13 AOS adolescents (11 schizophrenia disorder, 2 psychotic disorder) was compared with a group of healthy controls $(\mathrm{n}=12)$ in a verbal learning task (RAVLT) and a visual memory test (Visual Memory Reproduction and Delayed Recall of WMS-III). All participants had an estimated IQ in the normal rage $(T>70)$ and the groups did not differ in age or in sex. All AOS patients (6 inpatients, 7 outpatients) were taken second-generation antipsychotic treatment. Non-parametric tests were used due to the sample size.

Results: In verbal learning task AOS group did worse both in learning $(p=0.019)$ and delayed retrieval $(p=0.007)$ than controls. In visual memory task, the AOS group did worse than controls in immediate recall $(p=0.004)$ but they reach a similar performance in delayed recall.

Conclusions: AOS adolescent sample showed worse memory performance both in learning and in delayed recall of verbal information. Visual memory performance was also lower than healthy group but only in the codification of visual information.

Correspondence: Olga Puig, Hospital Clínic Universitari de Barcelona, Villaroel 170,Barcelona 08036,Spain.E-mail: olgapuinav@yahoo.es

J. SANZ, M. VARGAS, J. MARÍN, S. LÓPEZ \& P. REYES. Dysexecutive Syndrome and Illness Severity in Patients with Schizophrenia.

Objective: This study aims firstly to prove that the experimental Spanish version of the Behavioral Assessment of the Dysexecutive Syndrome Battery (BADS) has a satisfactory sensitivity, specificity and convergent validity in the diagnosis of patients with schizophrenia, and secondly that the dysexecutive syndrome is present in about the $40 \%$ of schizophrenic patients and is related to the illness severity.

Participants and Methods: We carried out a cross-sectional study. Sixty-one patients with schizophrenia and 30 healthy participants were assessed by means of two tools, on the one hand the BADS and on the other a battery of neuropsychological tests such as WAIS III working memory index, Stroop, Trail Making, and WCST, all of them characterized by considering sensitive to executive functioning. Likewise, PANSS, CGI and DSM-IV TR AxisV scales were administered.

Results: BADS sensitivity was $88 \%$ and specificity $77 \%$. The area under the ROC curve was $0.86(p<0.000)$. Spearman's Rho bivariate correlations between BADS and the neuropsychological tests revealed a strong pattern of association. Moreover, the correlation matrix indicated that a poor performance in the BADS was related to all the measures of the illness severity. Logistic regression model showed that Risk Ratio to be inpatient in dysexecutive schizophrenia was $4.77(\mathrm{p}<0.005)$.

Conclusions: These data suggest that the BADS is a valid test in assessing the executive functioning in patients with schizophrenia. Furthermore, dysexecutive syndrome is connected to illness severity and may have relevant implications on the design of the oncoming treatment strategies for this disorder.

Correspondence: Juan Carlos Sanz, PhD., University of Extremadura, Apartado de correos 362, Mérida 06800,Spain.E-mail: jcsanz@unex.es

M.L. VARGAS, J.C. SANZ, N. JIMENO, S. LÓPEZ, A.M. DEL BRIO \& J.J. MARIN. Cognitive Adverse Effects of Mild Extrapyramidal Symptoms in Schizophrenia.

Objective: Learning and memory deficits in schizophrenia predict a poorer outcome in several psychosocial areas. The current broad use of atypical antipsychotics has reduced the prevalence of severe extrapyramidal effects, but the cognitive effect of mild extrapyramidal symptoms is still incompletely known. The aim is to know the effect of mild extrapyramidal symptoms on memory, implicit learning and speed of cognitive processing in stable schizophrenia outpatients.

Participants and Methods: A cross-sectional study was carried out on a sample of 45 subjects divided into three groups: healthy control (n
$=15)$, schizophrenia without extrapyramidal symptoms $(\mathrm{n}=18)$ and schizophrenia with mild extrapyramidal symptoms $(n=12)$. All the patients, except one, were under antipsychotic treatment, meanly atypical ones. It was used the following instruments: Extrapyramidal symptoms: Simpson-Angus Scale; Schizophrenia symptoms: PANSS; Memory and learning: California Verbal Learning Test, Benton Visual Retention Test, WAIS-III digit symbol incidental learning; Working memory: WAISIII working memory factor; Processing speed: WAIS-III processing speed factor, Trail Making A and B, Colour Trails 1 and 2. One way ANOVA tests were used.

Results: Schizophrenia subjects performed worse than healthy controls in all the studied areas. The presence of mild extrapyramidal symptoms significantly reduced processing speed $(\mathrm{p}<0.05)$, but this was not associated with differences in learning, memory nor working memory. Conclusions: The adverse cognitive effects of extrapyramidal symptoms could depend on its intensity. The mild extrapyramidal effect caused by atypical antipsychotics seems to elicit slowness of cognitive processing but no a more broad learning or memory impairments.

Correspondence: Martin L. Vargas, Psychiatry, Complejo Asistencial de Zamora, Avda Hernan Cortes 40, Zamora 49007, Spain. E-mail: martinvargas@ono.com

\section{Psychopharmacology}

L. VAZ, S. POMPEIA \& O. BUENO. Cortisol-Induced Impairments of Working Memory Planning Task in Healthy Young Men.

Objective: In comparison to physiological basal levels, acute high-levels of cortisol affect learning and memory. Despite reports of cortisolinduced episodic memory effects, no study has used a comprehensive test battery to evaluate glucocorticoid effects on other memory subtypes. Here we report the results of a double-blind, placebo-controlled, between-subjects design study.

Participants and Methods: Sixteen healthy, young men were randomly assigned to either an acute cortisol (30 mg hydrocortisone p.o.) or placebo conditions. The capsules were administered at $2 \mathrm{pm}$. After $60 \mathrm{~min}$, subjects were submitted to an extensive cognitive test battery (working memory, explicit and implicit memory tasks). Blood samples were obtained before treatment and after the test session for analysis of cortisol. All scores were submitted to ANOVA and Tukey post-hoc comparison.

Results: Compared to placebo, cortisol administration increased cortisol blood levels $(p<0.001)$, had no significant effects on explicit $(p>0.17)$ and implicit memory $(p>0.38)$, but showed working memory impairments in the zoo map test $(\mathrm{p}<0.02)$.

Conclusions: Zoo map test is a planning ability task that involves prefrontal-mediated working memory, and the impairment observed may be due by cortisol-mediated GR receptor activation in this area of the brain.

Correspondence: Leonardo Vaz, Psychobiology, UNIFESP, Rua Benjamim Antonio Bisam, 111, Sorocaba 18055093, Brazil. E-mail: leo@psicobio.epm.br

\section{Stroke}

P. KARZMARK, P.D. ZEIFERT, S. TAN, T.E. BELL-STEPHENS \& G.K. STEINBERG. The Effect of Moyamoya Disease on Neuropsychological Functioning.

Objective: Moyamoya disease is a rare cerebrovascular disorder characterized by progressive occlusion of the supraclinoid internal carotid arteries and other vessels making up the circle of Willis, resulting in the formation of collaterals with a cloudy appearance on angiography. Research has focused on pediatric cases where severe intellectual impairment is frequent. Neuropsychological research in adults is limited to several single case studies. The current study involved comprehensive neuropsychological assessment of a large adult series. 
Participants and Methods: Participants were 36 adult cases of neurodiagnostically confirmed moyamoya disease. Based on demographically-adjusted norms, impairment rate was calculated for all measures. Results: Mean demographically - adjusted performance was in the average to low average range for all neuropsychological measures. Impairment rate was greatest for executive abilities (with 40 to $50 \%$ of cases impaired for several measures)and intermediate for intelligence and mental efficiency. Impairment rate was low for memory. Depression was infrequent and mild.
Conclusions: The overall impact of Moyaymoya disease on cognition in adults is relatively modest, in contrast to the effect in children. The results suggest impact on executive capacity and sparing of memory function. This may relate to the location of affected arteries, age of disease onset, or severity of the disease.

Correspondence: Peter Karzmark, Ph.D., Neurology Dept., Stanford University Medical Center, H3109, 300 Pasteur Dr., Stanford, CA 94305-5234.E-mail: pkarzmark@stanfordmed.org

\section{THURSDAY AFTERNOON, JULY 5, 2007}

\section{Paper Session 4 12:30-1:30 p.m.}

\section{Language \& Aphasia}

A.I. ANSALDO, K. MARCOTTE, A.P. DELGADO \& P. VITALI. Adapted Brain Plasticity in Chronic Aphasia: The Neural Correlates of Efficient Naming.

Objective: This study examined the neural substrate of efficient naming, following specific therapy in a participant with chronic and severe aphasia. Participants and Methods: CM is a 66 year-old man who suffered a fronto-temporal left hemisphere stroke. Ten years after discharge, he received brief and intensive therapy with semantic feature analysis (SFA),resulting in the improvement on naming with 20 treated words. SFA boots semantic representations, to activate phonological representations, and achieve oral naming (Coelho et al. 2000) To examine the neural substrate allowing for recovery, SFA was applied to a larger number of words, in a pre-post therapy event-related fMRI study. Contrasts with treated and non treated nouns and verbs were obtained with SPM2. Results: SFA lead to significant improvement across word categories. Posttherapy activation maps showed two networks allowing for improvement with SFA: the left middle frontal gyrus (BA6) and right precentral gyrus (BA6)sustained naming nouns; with verbs the network was completely rightlateralized, and included the medial frontal gyrus, (BA8/10), the postcentral gyrus (BA40), the inferior parietal lobe (BA40) and the cingulate, (BA31) Conclusions: Brain plasticity and language therapy operate despite chronic and severe aphasia. Post therapy activation maps reveal distinct networks allowing CM to benefit from SFA. With nouns, the network included phonological processing areas bilaterally. With verbs, it comprised semantic processing and phonological processing areas homologus to classic language areas, as well as motor processing areas. In line with pervious reports (Crithley, 2003), activations in the cingulate indicate effortful cognitive and motor behaviour, specifically with the most difficult condition for CM, that of verbs. These results indicate therapy induced brain plasticity following aphasia, and open a window onto the clinical applications of fMRI in clinical aphasiology.

Correspondence: Ana I. Ansaldo, Ph.D, Faculté de médecine, Université de Montréal, Centre de recherche de l'Institut universitaire de gériatrie de Montréal, 4545 Queen Mary, Montréal, QC H3W1W5, Canada. Email: ana.ines.ansaldo@umontreal.ca

K.M. HIRD, R. BROWN \& K. KIRSNER. Anomia in Primary Progressive Aphasia: Specific or General Deficit?

Objective: Anomia is the hallmark symptom of Primary Progressive Aphasia(PPA) (Thompson, Ballard, Trait, Weintraub \& Mesulam, 1997). The selective degeneration of the left perisylvian cortex found at autopsy is thought to provide strong support for the theory of modularity and specific deficit of lexical retrieval in aphasia. An alternative account is that lexical level processing deficits reflect a reduction in processing efficiency rather than a loss of linguistic competence ( Silkes, McNeil \& Drton, 2004).
Participants and Methods: Ten control participants and seven participants with PPA produced three spontaneous discourse samples in each of three sessions within a two month period. An objective pause based segmentation procedure (Kirsner, Dunn, Hird \& Parking, 2002) was employed prior to CIU and error analyses (Nicholas \& Brookshire, 1993). Classification of word types ( Garrard, Maloney, Hodges \& Patterson, 2005) and Type Token Ratio analyses were also performed.

Results: A stable reduction of nouns in the connected speech of PPA participants was evident. The sec/CIU score was unstable across the three sessions for 5 of the 6 participants with PPA. t test for small samples (Crawford \& Howell, 1998), revealed reduced communicative efficiency and significantly more discourse errors for 5 or the 6 PPA participants. Conclusions: The results do not provide unequivocal support for an isolated lexical processing deficit in this population. The highly variable measure of secs/CIU coupled with numerous off task comments focusing on performance, false starts and longer speech samples suggest that, in the absence of increased long pauses, the impairments reflect general cognitive disruption as distinct from a specific lexical deficit.

Our interpretation is in keeping with Silkes, McNeil \& Drton (2004); the deficit involves impaired resource allocation where this occurs in the presence of intact self monitoring skills accompanied by an increased effort to compensate for impaired communicative efficiency. Correspondence: Kathryn M. Hird, PhD, Psychology, Curtin University of Technology, GPO Box U1987, Perth, WA 6845, Australia. E-mail: K. Hird@curtin.edu.au

M. LAGANARO, S. MORAND, V. SCHWITTER, C. ZIMMERMANN, C. CAMEN \& A. SCHNIDER. Electrophysiological Correlates of Recovery from Anomia in Aphasic Patients with Semantic or Phonological Impairment.

Objective: In the present study we analyzed the electrophysiological correlates of recovery from anomia in post-acute aphasic patients.

Participants and Methods: Three aphasic subjects participated in the study. They presented severe anomia associated with semantic impairment or phonological impairment and they significantly improved in naming accuracy in a short period of time. Event-related potentials (ERPs) were recorded at baseline and after a therapy period in each anomic speaker during a delayed naming task. A control group of 15 healthy subjects underwent the same task. Waveform analysis and temporal segmentation were carried out on the ERPs of each anomic patient in comparison with the control group

Results: Waveform analysis and temporal segmentation showed a decrease of abnormal electrophysiological correlates in the first $300 \mathrm{~ms}$ from baseline to post-treatment assessment and an increase of deviant ERPs in the time-window from 300 to $500 \mathrm{~ms}$ in the 2 patients with phonological impairment. In the patient with semantic impairment abnormal electrocortical correlates increased after recovery in the first $300 \mathrm{~ms}$. Conclusions: The results indicate that normalisation of ERPs as well as increase of abnormal electrocortical correlates are associated with recovery from anomia in post-acute patients in different time-windows. The time-window of an increasing of deviant ERPs during recovery seems to correspond to the time course of the impaired encoding processes (semantic or phonological). 
Correspondence: Marina Laganaro, PhD, Geneva University Hospitals, 26, av. Beau-Séjour, Geneva 12111, Switzerland. E-mail: marina. laganaro@hcuge.ch

A. CANCELLIERE. The Definition Naming Test (DNT): A WrittenVerbal Alternative to the Boston Naming Test (BNT).

Objective: To develop a test of naming, the DNT, which allows comparison against an individual's word knowledge for more sensitivity to deficits in individuals with higher intellect/education.

Participants and Methods: The DNT consists of part 1 (36 written definitions - the subject must write one word which best fits each) and part 2 (the same definitions with 20 multiple-choice options for each). Administration to 251 controls (CG) and 56 brain-injured individuals (BIG). Interrater reliability (Kappa), reliability (split-half reliability) and validity (t-test, ANOVA, correlations) were investigated.

Results: Excellent interrater agreement and split half reliability was achieved. CG performed significantly better than BIG on both parts $(p<.0001)$. The difference between free recall and recognition of the target word was significantly higher for BIG than CG. Patients were divided into right (RBD), left (LBD) and bilateral brain damage (BIL). The RBD group performed significantly better than the LBD and BIL groups on part 1 but not part 2 .

The correlation between the DNT and BNT was .68 ( $p<.01)$. Correlations between the DNT and vocabulary, VIQ, PIQ, FAS and reading were remarkably similar to those cited for the BNT. The DNT demonstrated a stronger correlation with vocabulary and verbal fluency. Case studies will be described wherein an individual sustained a brain injury which would predict anomia and elicited complaints of anomia yet the BNT was average while the DNT detected anomia.

Conclusions: The DNT provides a valid assessment of word finding ability with more sensitivity in individuals of higher intellect/education. Correspondence: Andy Cancelliere, private practice, 156 Sheppard Ave. W., Toronto,ONM2N1M8, Canada.E-mail:drcan@dellmail.com

\section{Paper Session 5 12:30-1:30 p.m.}

\section{Structural \& Functional Neuroimaging}

L. KAUFMANN, S.E. VOGEL, C. KREMSER, M. SCHOCKE, J.W. KOTEN \& G. WOOD. Is There A Functional Overlap in Numerical and Spatial Processing? A Developmental fMRI Study. Objective: Aim of this fMRI study is to investigate the neural correlates of numerical and spatial processing in children and adults. While some authors propose that intraparietal regions (IPS) might be domain-specific for number magnitude processing (e.g., Dehaene, 1997), others hypothesize a broader magnitude processing domain within IPS incorporating number, space and time (e.g., Walsh, 2003).

Participants and Methods: 24 healthy individuals (5-29 year-old) were asked to make numerical and spatial magnitude (experimental task) as well as colour discriminations (control task). Stimuli were pictures of two horizontally presented hands, each pair being distinguishable according to number of fingers raised (non-symbolic numerical), orientation (spatial) or thumb colours (task). Altogether, 12 experimental (6 stimuli per block; stimulus duration 4000 ms, ISI $1000 \mathrm{~ms}$ ) and 11 rest blocks $(15000 \mathrm{~ms})$ were presented.

Results: Behaviourally, all participants achieved high accuracies. Baseline imaging effects show distributed fronto-parietal-occipital and cerebellar activations. Though non-symbolic numerical and spatial processing yield overlapping activations in IPS (independent of age), activations are distinguishable regarding hemispheric lateralization and anterior/posterior shift. Moreover, age is positively correlated with IPS activations upon numerical (but not spatial) processing.
Conclusions: Our findings revealing a neurofunctional overlap of nonsymbolic numerical and spatial processing in IPS are inconsistent with the view of a domain-specific number processing system within IPS. The positive correlation between age and activation extent in the IPS upon numerical processing suggests that the functional specialization of intraparietal regions for (non-symbolic) number processing increases with age. Finally, our results are suggestive of a link between the cerebellum and magnitude processing.

Correspondence: Liane Kaufmann, PhD, Clinical Department of Pediatrics IV, Innbruck Medical University, Anichstrasse 35, Innsbruck A-6020, Austria.E-mail: liane.kaufmann@i-med.ac.at

R. SÁNCHEZ-CARRIÓN ABASCAL, P. VENDRELL, X. CALDÚ, N. BARgalló, N. PICó AZANZa, A. GARCíA-MOLINA, A. ENSEÑAT CANTALLOPS, M. BERNABEU \& T. ROIG ROVIRA. Working memory activation following severe diffuse traumatic brain injury: A functional MRI study.

Objective: Traumatic brain injury frequently produces working memory deficits. N-back is a working memory paradigm that activates the prefrontal and superior parietal cortex. The objective of this work was to investigate working memory following TBI and their cerebral activation pattern during an n-back task compared to a matched control group. Participants and Methods: The sample was composed by thirteen patients with severe TBI admitted to Institut Guttmann (23,9 $\pm 5,3$ years old; time since injury 7,6 $\pm 4,2$ months; GCS 5,7 $\pm 1,6$; PTA duration 81,9 $\pm 40,2$ days) and 13 healthy control matched for age, gender and estimation of premorbid intelligence. TBI patients were selected according signs of diffuse axonal injury (DAI) without any focal lesions on clinical MRI assessment. Functional activation (fMRI) was obtained in a 1,5T Sigma GE scanner during an n-back processing (0-, 2- and 3-back) in a bloc design task. fMRI was analysed using SPM2 working in MATLAB 7.0.

Results: Compared to controls, TBI group showed significant working memory impairment in Letters and numbers (WMS-III) ( $\mathrm{p}=0,03)$, Rivermead Behavioural Memory Test $(p=0,001) 2$-back $(p=0,016)$ and 3 back $(p=0,037)$ conditions. Control subjects showed the classical activation of fronto-parietal network, while greater fronto-parietal activation and additional activation in other cerebral regions (medial temporal lobe, anterior cingulated, caudate, putamen, globus pallidum, thalamus and cerebellum) was observed in TBI group.

Conclusions: In conclusion, TBI patients have a pattern of increased cortical activation compared to the pattern of controls and also activated more cortical regions. These findings agree with the model of increased functional activity to compensate the cerebral tissue loss.

Correspondence: Teresa Roig Rovira, PhD, Neuropsychology, Institut Guttmann, Cami de Can Ruti s/n, Badalona 08916, Spain. E-mail: troig@guttmann.com

N. IBARRETXE, B. RAMÍREZ-RUIZ, P. VENDRELL, C. JUNQUE, M.J. MARTÍ, N. BARGALLO \& E. TOLOSA. Memory Impairment and Gray Matter Loss in the Hippocampus of Patients with Parkinson's Disease and Visual Hallucinations.

Objective: We aimed to investigate regional hippocampal gray matter atrophy in Parkinson's disease (PD) patients with visual hallucinations $(\mathrm{VH})$ and its correlation with memory performance.

Participants and Methods: Sixteen PD patients with VH, 19 PD patients without VH and 56 controls were included. Memory was assessed using a version of the Rey's Auditory Verbal learning Test (RAVLT). We applied a region-of interest (ROI) to the hippocampus using voxel-based morphometry (VBM) for group comparisons, single-case individual analysis and correlations of hippocampal gray matter concentration with memory performance.

Results: Significant differences in hippocampal head gray matter density were observed between PD patients with VH and controls, while PD patients without $\mathrm{VH}$ and controls did not show significant differences. Individual analysis of each subject compared to the controls' mean gray matter concentration showed that 5 of $16 \mathrm{PD}$ patients with VH and 5 
of the19 PD without VH had hippocampal head gray matter loss at a level of significance of corrected $\mathrm{P}<0.05$. RAVLT mean learning score for the PD patients with VH was 26.5 \pm 7.3 and for PD patients without VH was $39.2 \pm 6.4(t=-5.4 ; p<0.0001)$. In PD patients with $\mathrm{VH}$ a significant correlation was found between gray matter loss in the anterior part of hippocampus bilaterally and learning scores. The relationship between memory and hippocampal gray matter concentration was also found comparing verbal learning performance between patients that showed hippocampal loss and those that did not $(\mathrm{t}=-2.1 ; \mathrm{p}<0.035)$.

Conclusions: PD patients with VH have a pattern of hippocampal atrophy with anterior predominance and memory impairment correlates with this region. Correspondence: Naroa Ibarretxe, PhD student, Psychiatry and Psychobiology Department, University of Barcelona, Faculty of Medicine, Casanova 143.,Barcelona 08036, Spain.E-mail:naroaibarretxe@ub.edu

S.L. REMINGER, T.J. PATTON, S.M. LUNDY, A.L. BARNES, J.W. STUDEBAKER \&. K.L. KAEMINGK. Hippocampal Volume and Memory Function in Breast Cancer Survivors.

Objective: Chemotherapy and radiation therapies are believed to contribute to memory problems experienced by individuals treated for cancer. However, the precise causal factors and neurological mechanisms for such deficits are currently unknown. The present study sought to identify potential relationships between memory performance and the volume of the hippocampus, a brain structure involved in memory function, in individuals treated for breast cancer.

Participants and Methods: We examined 61 breast cancer survivors (mean age 59.2 years), who had received diagnoses of breast cancer 2 23 years prior (mean 5.6 years). Participants completed a battery of standardized neuropsychological tests and received structural brain magnetic resonance imaging (MRI). Using digitized MRI images, quantitative estimates of hippocampal volume ratios (i.e., corrected for whole brain volume) were calculated for each participant.

Results: Analyses of variance did not demonstrate a difference in memory performance or hippocampal volume when individuals treated with chemotherapy were compared to individuals not treated with chemotherapy. Individuals treated with radiation did show marginally significantly smaller left hippocampal volumes compared to individuals not treated with radiation $(\mathrm{p}=.055)$. Inconsistent with study predictions, left, right. and total hippocampal volumes showed inverse correlations with performance on memory measures (r's ranged from -.21 to - .33 ).

Conclusions: Study results suggest that hippocampal volumes may be adversely affected in some breast cancer survivors. However, the identified negative relationship between memory performance and hippocampal volumes underscores the need for further study to determine the neurological mechanisms that may account for weaknesses in neuropsychological performance.

Acknowledgement: Funded by the Susan G. Komen Breast Cancer Foundation.

Correspondence: Sheryl L. Reminger, Ph.D., Psychology, University of Illinois at Springfield, One University Plaza, UHB 3144, Springfield, IL 62704.E-mail:sremi2@uis.edu

\section{Paper Session 6 12:30-1:30 p.m.}

\section{Parkinson's Disease}

C. SANCHEZ-CASTAÑEDA, B. RAMIREZ-RUIZ, R. REÑE, J. GASCON, M. CALOPA, J. CAMPDELACREU, M. JUNCADELLA \&. C. JUNQUE. Differences Between Neuropsychological Profile and Gray Matter Atrophy in Individuals with Dementia with Lewy Bodies and Parkinson Disease with Dementia.

Objective: There is controversy regarding whether Parkinson's disease with dementia (PDD) and Dementia with Lewy Bodies (DLB) are in fact different manifestations of the same disorder. The two diseases share common neuropathological inclusions in some brain structures, but have different clinical evolution. The objective of this study was to investigate neuropsychological and brain structural characteristics in patients with DLB compared to those with PDD.

Participants and Methods: We examined 40 subjects (11 DLB patients, 13 PDD patients and 16 control subjects). All the subjects underwent a neuropsychological assessment consisting of the CERAD battery, Luria's motor coordination and graphic sequences, Visual Form Discrimination, symbolic gesture, Block design of the WAIS-III and an attentional battery (Conner's Continuous Performance Test, Attentional Network Test and Stroop test). Statistical MRI analysis was carried-out using the Voxel-based Morphometry technique.

Results: PDD group performed significantly poorly in CERAD's delayed memory, making more false recalls $(p<0.05)$, perseverations of the Continuous performance Test and close-in in Luria's graphic sequences $(\mathrm{p}<0.001)$. On the other hand, DLB patients performed worse in the CERAD's false recognition task, but the difference was not statiscally significant. When we correlated the neuropsychological data and the MRI results, we found that the gray matter volume of the right amygdala was inversely related to the number of intrusions in learning $(\mathrm{p}<$ 0.05 ) and the volume of the right cingulate to performance in the Continuous Performance Test (commission errors, average of performance and perseverations) in the DLB group but not in the PDD group ( $\mathrm{p}<$ $0.001)$.

Conclusions: PDD patients had more prefrontal impairment while DLB patients show more memory dysfunctions.

The right amygdala volume inversely correlates with intrusions in learning in DLB group.

The right cingulate volume was related to performance in the Continuous Performance Test in DLB group.

Correspondence: Cristina Sanchez-Castañeda, PhD student, Neurology, Bellvitge University Hospital, c/ Feixa Llarga, s/n, L'Hospitalet de LLobregat, Barcelona 08907, Spain.E-mail: crsanchc8@psi.ub.edu

C. MATTHEWS, P.L. SMITH \& M. HORNE. Cognitive Flexibility in Patients with Parkinson's Disease: The Role of the Basal Ganglia.

Objective: Clinically, patients with Parkinson's disease (PD) demonstrate impaired performance on tasks of cognitive flexibility (Dubois \& Pillon, 1997). In an experimental context, PD patients demonstrate an elevation in the switching cost (Cools, Barker, Sahakian, \& Robbins, 2001) - the increase in reaction time (RT) when switching from one task to another. In patients with PD, the underlying basis of this switching deficit remains to be elucidated. Logan and Bundesen (2003) suggest that the task switching deficit in normal participants can be accounted for by a RT benefit on cue repetition trials, rather than a RT cost per se. This study aimed to investigate this hypothesis in patients with PD. Participants and Methods: Meiran's (1996) task switching paradigm was used to investigate the role of stimulus and response characteristics in task switching. Changes in cues were compared to changes in task and cue, to dissociate the contribution of these two processes to the task switching deficit. Participants were 32 patients with PD, and 32 age and IQ matched controls.

Results: A series of ANOVAs were used to compare RTs between these groups. The results show that patients with PD demonstrate an elevated task switching cost compared to controls, which is over and above the cost associated with a change in cue.

Conclusions: The results will be discussed in terms of two competing hypotheses: that PD patients failed to benefit from a repetition in cue; or, that patients with PD demonstrate a specific deficit in task switching. The basis of this task switching deficit in terms of the role of basal ganglia in attention and cognitive flexibility will also be discussed. Correspondence: Christine Matthews, Ma/PhD, Psychology, The University of Melbourne, 125 Curtain St, Carlton North, VIC 3054, Australia.E-mail:cmmatt@unimelb.edu.au 
J.A. OGDEN \& M. GUICHERIT. Does Bradyphrenia Exist in Parkinson's Disease OFF or ON L-dopa?

Objective: Previous publications using inspection time (IT) as a measure of bradyphrenia (slowed thinking) in Parkinson's Disease (PD) suggest that further studies should account for age, visuoperceptual abilities, bradykinesia, and L-dopa medication. We attempted to answer these questions in our IT study.

Participants and Methods: Twenty-eight non-depressed, non-demented, PD participants (mean age 66.9yrs) in Modified Hohn and Yahr Stages 2-4, and with unimpaired visuoperception on the Visual Object and Space Perception Battery, were compared ON and OFF L-dopa, with 28 age, education, ethnicity, Verbal IQ and occupation case-matched Controls. There were 8 women and 20 men in the PD group and 14 women and 14 men in the Control group. The mean Unified Parkinson's Disease Rating Scale for motor symptoms (UPDRSm) for the PD gp was $21.95 \mathrm{ON}$ and $30.25 \mathrm{OFF} \mathrm{L}$-dopa. The experimental task required judging which leg of a Pi symbol ( $\Pi$ ) was the shorter, after it was flashed on a computer screen followed by a mask. There were 20 trials for each of nine time intervals from 20 to 250 millisecs. A participant's IT was the time interval where $80 \%$ accuracy was achieved.

Results: A repeated measures ANOVA with IT as the dependent variable and controlling for gender demonstrated a significant Group effect with the PD group having longer ITs than Controls in both ON and OFF conditions $(p=.002)$. There was a significant status $(\mathrm{ON} / \mathrm{OFF}) \mathrm{x}$ group interaction, with only the PD group showing longer ITs in the OFF versus $O N$ condition $(p=.024)$. No significant correlations were found between the UPDRSm and the ITs ON or OFF L-dopa.

Conclusions: Bradyphrenia was demonstrated by PD participants both $\mathrm{ON}$ and OFF L-dopa, and could not be explained by age, Verbal IQ, education, or impaired visuospatial perception. Bradyphrenia was not correlated with motor impairment, but L-dopa did ameliorate it to some degree. As previous studies have concluded that L-dopa does not influence bradyphrenia, this tentative finding requires further study. Correspondence: Jenni A. Ogden, PhD, Psychology, City campus, University of Auckland, University of Auckland, Private Bag 92019, Auckland 1142, New Zealand. E-mail:ja.ogden@auckland.ac.nz

R. MARIA, T. TORRALVA, G. GONZALO \& M. FACUNDO. Detection of Executive Deficits in patients with early Parkinson Disease.

Objective: The objective of this study is to asses cognitive functions in patients in the early stages of the disease and to examine both the sensitivity of standard cognitive tests as well as more complex and ecological executive battery for it's detection

Participants and Methods: 10 patients of recent diagnosis of PD (3 years or less), underwent a classical cognitive battery and a more specific and complex executive ecological examination. 10 healthy controls matched for sex, age and education level were also examined. The "ecological" executive battery included tests proven to be sensitive to prefrontal dysfunction including test of Theory of Mind (Eyes Recognition), Planning and Organization (the Hotel Task and an adaptation of the Met-hv) and Decision Making (the Iowa Gambling task - IGT).

Results: There were no significant differences detected among patients and controls in age, educational level nor premorbid IQ. There were no significant differences in any of the classical tests of cognitive functions. On the contrary the ecological battery proved to be sensitive enough to cognitive changes in early PD thus revealing differences in theory of mind $(p=0.045)$, Planning and organization (Met $p=0.01)$ and decision making (IGT $5 \mathrm{p}=0.02$ ).

Conclusions: Patients with early PD showed impairment in executive functions despite not having detected anything with standard cognitive tests. This impairment was captured with a more ecological executive battery revealing that this battery is much more sensitive to the cognitive deficits found in early stages of PD than standard cognitive tests. Correspondence: Teresa Torralva, MD, Neuropsychology, INECO, Castex 2356, Buenos Aires 1425, Argentina. E-mail: ttorralva@neurologiacognitiva.org
Symposium 4
3:30-5:00 p.m.

\section{Impulsivity and Decision-Making in Substance Abusers}

\author{
Chair: Karen Bolla
}

\section{K.I. BOLLA. Impulsivity and Decision-Making in Substance} Abusers.

Symposium Description: Intact decision-making (D-M) and cognitive impulsivity are paramount for the development of adaptive behaviors. It involves the evaluation of whether a response or behavior will elicit reward or punishment (i.e., the operation occurring after coding of the stimulus features) (input) and the selection of the behavior determined to be optimum prior to acting on that behavior (output). This process is dependent on emotional and motivational coding, working memory, conflict monitoring and resolution, as well as response activation and inhibition. Good D-M involves primarily a right hemispheric, prefrontal neural network. It has been shown repeatedly that substance users show impulsivity and faulty decision-making. These deficits may result in poor choices that would perpetuate future drug use and other high-risk behaviors. The symposium speakers will cover their work on D-M and impulsivity in diverse substance abusing groups. Geert Dom will discuss his work in D-M in alcohol-dependent patients who may also have comorbid personality disorders. Karen I. Bolla will present her data on the dose-related association between MJ use, pattern of brain activation, and D-M performance. Luke Clark will discuss his recently developed new task of D-M and his findings when used to assess D-M in amphetamine users. Boris Quednow will present results on the cognitive neurotoxicity of MDMA (Ecstasy) with regard to behavioral impulsivity and $\mathrm{D}-\mathrm{M}$ cognition. The role of emotion and motivation on D-M in cocaine and opiate users will be covered by Antonio Verdejo-Garcia. Antoine Bechara, as the discussant, will discuss future directions for investigating impulsivity and decision-making in SA.

Correspondence: Karen I. Bolla, Ph.D., neurology, johns hopkins univ. school of medicine, 4940 eastern ae, Baltimore, MD 21224. E-mail: kbolla@jhmi.edu

K.I. BOLLA. Decision-Making in Chronic, Heavy Marijuana Users. Objective: Decision-making appears to be one area that is hindered by heavy drug use. This is evidenced by the drug users' persistent use of potentially neurotoxic substances despite the potential negative physical, psychological, social, and legal consequences with which they are faced. This continued use may be attributable to damage to specific neural networks (prefrontal cortex) involved in decision-making and performance monitoring. Faulty decision-making has now been reported to be associated with cocaine, MDMA, amphetamine, opiates, and marijuana use. However, few studies have examined the ability of the substance user to learn over two separate testing sessions and within each session.

Participants and Methods: This study utilized the Iowa Gambling Task (IGT), a widely used measure of decision-making, to determine the effects of MJ use on learning this task, in 25-day abstinent MJ users.

Results: Differences were analyzed between and within two consecutive testing sessions. MJ users had lower scores than controls on the total IGT net score. Both the MJ users and control group showed betweensession learning, but the MJ users learned at a slower rate. In addition, the learning curve from the beginning to the end (block $x$ block) of the IGT was very limited in the MJ group. Dose-related measures of MJ use (joints/week) predicted IGT performance (the heavier the drug use the lower the performance). 
Conclusions: The effects of MJ use on decision-making and learning may have important implications for the development of novel treatment interventions.

Correspondence: Karen I. Bolla, Ph.D., neurology, johns hopkins univ. school of medicine, 4940 eastern ae, Baltimore, MD 21224. E-mail: kbolla@jhmi.edu

G. DOM. Decision-making deficits in alcohol-dependent patients with and without comorbid personality disorder.

Objective: Impairments in decision making are a consistent finding in substance use disorder (SUD) populations. However, decision-making deficits are not specific for SUDs and are also reported in the context of other psychiatric

disorders such as antisocial and borderline personality disorders (PDs). Given the frequent comorbidity between SUD and DSM-IV Axis II disorders from the impulse control spectrum (cluster B PD), it might be questioned whether the decision-making impairments typically reported in SUD populations reflect the addictive disorder, the cluster B PD, or a combination of the two.

Participants and Methods: Data will be presented on the relationship between decision-making, alcohol dependence and cluster B comorbidity. We compare the decision-making performance of non-substance-abusing controls on the Iowa Gambling Task (IGT) with that of three abstinent alcohol-dependent samples, (1) alcoholic patients without PD; (2) alcoholic patients with a cluster A or C PD (non-related to impulse control); and (3) alcoholic patients with a cluster B PD (related to impulse control). Results: All three alcohol-dependent subsamples performed below the controls. The alcoholic patients with a cluster A or C PD had the highest IGT score, followed by the alcoholic patients without a PD, while the cluster B alcoholic patients were the most impaired.

Conclusions: These findings suggest that alcoholic patients with a comorbid cluster B PD are particularly impaired in their decision-making. These deficits may underlie the severe problems that characterize cluster B alcoholic patients specifically in inappropriate behaviors (e.g. polysubstance abuse, legal, and professional dysfunction). Clinical implications are discussed.

Correspondence: Karen I. Bolla, Ph.D., neurology, johns hopkins univ. school of medicine, 4940 eastern ae, Baltimore, MD 21224. E-mail: kbolla@jhmi.edu

\section{B. QUEDNOW. Impulse control and decision-making cognition in Ecstasy users.}

Objective: Chronic administration of the common club drug 3,4-methylenedioxymethamphetamine (MDMA, "Ecstasy") is associated with long-term depletion of serotonin (5-HT) and loss of 5-HT axons in the brains of rodents and non-human primates. In addition, accumulating evidence suggests that recreational MDMA consumption may also affect the human serotonergic system. 5-HT is supposed to play an important role in the regulation of impulse control and decision-making cognition (DMC). Thus, we investigated impulsivity and DMC in abstinent MDMA users.

Participants and Methods: Nineteen male, abstinent MDMA users; 19 male, abstinent cannabis users; and 19 male, healthy control subjects were examined with the Matching Familiar Figures Test (MFFT), a Go/No-Go Task (GNG) for impulsivity, and a Gambling Task (GT) for DMC.

Results: MDMA users showed significantly elevated impulsivity in the MFFT I-score, but not in commission errors of the GNG, compared with healthy controls. Cannabis users did not yield altered impulsivity compared with healthy controls. In the GT, MDMA users performed significantly worse than cannabis consumers and healthy controls, whereas cannabis users died not differ from controls. In addition, the I-score as well as the DMC performance were correlated with the amount of MDMA intake.
Conclusions: In line with some recent studies MDMA users have shown elevated impulsivity and impaired DMC. However, it is still unclear whether these deficits are derived from predispositionally decreased 5HT levels, or if they are caused by neurotoxic effects of MDMA. This important question will be discussed in the light of recent findings and our new results on this topic.

Correspondence: Karen I. Bolla, Ph.D., Neurology, Johns Hopkins University School of Medicine, 4940 Eastern Ave., B Building, Rm 123, Baltimore,MD 21224.E-mail: kbolla@jhmi.edu

\section{A. VERDEJO-GARCIA. Emotion processing and decision-making} in cocaine and heroin abusers.

Objective: Substance abuse is associated with widespread cognitive impairments, of which decision-making deficits may play a particularly important role on drug use initiation, response to treatment and relapse. Adaptive decision-making relies on both cognitive and motivational-emotional processes. It has been proposed often that chronic drug use results in heightened response to drug use stimuli (frequently measured as craving), and subsequent reduced valuation of natural reinforcing stimuli. This unbalanced valuation of reinforcing stimuli may bias decision-making towards drug consumption, ignoring other alternative reinforcers. Cocaine and heroin are two widely used drugs that have both common and differential effects on the Central Nervous System. Both drugs equally seem to impair decision-making abilities, but the motivational and emotional substrates underlying these deficits are still unclear. Participants and Methods: We will present a series of experiments addressing the influence of motivational and emotional factors on decision-making performance in cocaine and heroin users.

Results: These experiments have shown: (1) that cocaine and heroin users have altered subjective response to natural reinforcing stimuli-we have defined this altered response as abnormal "experience of emotions"; (2) that abnormal experience of emotions may contribute to decisionmaking impairment in cocaine and heroin users; (3) that there are subtle discrepancies within emotion processing and decision-making performance in users of these two drugs; and (4) that emotion processing and decision-making deficits are not resolved with prolonged abstinence. Conclusions: These results may have important implications for the design of early prevention, treatment, and prevention of relapse interventions. Correspondence: Karen I. Bolla, Ph.D., neurology, johns hopkins univ. school of medicine, 4940 eastern ae, Baltimore, MD 21224. E-mail: kbolla@jhmi.edu

L. CLARK. Neuropsychological mechanisms of impulsivity in chronic amphetamine users.

Objective: Chronic users of stimulants including amphetamines and cocaine display elevated impulsivity on personality measures, impaired inhibitory control on neurocognitive measures, and structural brain changes in the ventral aspects of the frontal cortex. This talk will review the neuropsychological mechanisms of impulsivity in stimulant abusers, with particular attention to the construct of 'reflection impulsivity', referring to the tendency to gather and evaluate information prior to making complex decisions.

Participants and Methods: Chronic stimulant users meeting DSM-IV dependence to amphetamines, and former users of amphetamines (abstinent for at least one year) were assessed on a neurocognitive assessment of executive function and decision-making, including the novel Information Sampling Task to assess reflection impulsivity.

Results: Information sampling on the new task was directly related to decision accuracy, consistent with the defining feature of the construct (Evenden JL 1999 Psychopharmacology 143:111-122). Current and former users of amphetamines displayed reduced information sampling and impaired decision accuracy, in comparison to healthy controls. Delay averse responding and inattention were not able to account for the group differences.

Conclusions: The findings indicate reduced reflection during complex decision-making in current and former users of stimulants. Reduced 
reflection may bias the individual towards risky options, and thus predispose recreational and/or harmful drug taking and relapse from abstinence. Other work suggests these processes are associated with the orbitofrontal cortex. Future research is required to disentangle the extent to which these impulsive effects are related to the pre-existing vulnerability to substance abuse, as opposed to the damaging consequences of long-term administration.

Correspondence: Karen I. Bolla, Ph.D., Neurology, Johns Hopkins University School of Medicine, 4940 Eastern Ave., B Building, Rm 123, Baltimore, MD 21224.E-mail: kbolla@jhmi.edu

\section{Symposium 5 3:30-5:00 p.m.}

\section{Neuropsychological Dysfunction in Early Phases of Psychosis}

\author{
Discussant: Julio Bobes \\ Discussant: Miguel Gutiérrez
}

\section{J. BOBES GARCÍA. Neuropsychological Dysfunction in Early Phases of Psychosis.}

The objective of this symposium is to explore whether the early course of illness including first onset of psychotic symptoms influences neuropsychological functioning and psychopathology in first-episode schizophrenics. Through the different presentations we will review the effect of medication on cognitive deficits at early phases of psychotic symptoms, neuroimaging profiles, correlations with clinical symptoms, and variables which influences follow-up and outcomes. The data suggests that neuropsychological deficits in first-episode schizophrenia are independent of the early course of clinical symptoms in schizophrenia, and although negative symptoms and higher insight are associated with the length of the prodromal period, some early neuroimaging changes are not determinant in the course of the illness and do not necessarily imply greater neuropsychological impairment.

Correspondence: Dr. Julio Bobes García, Dpto de Psiquiatría Universidad de Oviedo, Cl Julian Clavería, 6, 33006 Oviedo * Spain Tel + 34985 103553,E-mailbobes@uniovi.es

M. GARCÍA-PORTILLA, S. ZARAGOZA \& J. BOBES. Cognitive Dysfunction in Schizophrenic Outpatients under Antipsychotic Treatment.

: Novel antipsychotic medications have been reported to have beneficial effects on cognitive functioning in patients with schizophrenia. However, these effects have been assessed in studies with considerable variation in methodology. A large number of investigator-initiated and industry-sponsored clinical trials are currently underway to determine the effect of various novel antipsychotics on cognitive deficits in patients with schizophrenia. The ability to discriminate between high- and lowquality studies will be required to understand the true implications of these studies and their relevance to clinical practice. This research addresses several aspects of research on cognitive enhancement in schizophrenia, emphasizing how the assessment of cognitive function in clinical trials requires certain standards of study design to lead to interpretable results. Novel antipsychotic medications appear to have preliminary promise for the enhancement of cognitive functioning. However, the methodology for assessing the treatment of cognitive deficits is still being developed. Researchers and clinicians alike need to approach publications in this area with a watchful eye toward methodological weaknesses that limit the interpretability of findings. Correspondence: Dr. Julio Bobes García, Dpto de Psiquiatría Universidad de Oviedo, ClJulian Clavería, 6, 33006 Oviedo * Spain Tel + 34985 103553,E-mailbobes@uniovi.es
B. CRESPO-FACORRO, J. RODRÍGUEZ-SANCHEZ, J. PELAYOTERÁN, L. BARBADILLO, A. DUARTE, R. ROIZ \& J. VÁZQUEZBARQUERO. Neuropsychology and Neuroimaging Correlations in First Episode Psychosis.

Authors will review the association between cognitive deficits and brain functional and structure anomalies observed in some patients with a first episode of schizophrenia. Structural brain differences including decreased gray matter and increased cerebrospinal fluid volumes have been observed in the brains of chronically ill patients with schizophrenia. Deficits in gray matter volume is present in patients presenting with a first episode of nonaffective psychosis. These changes are associated to cognitive deficits in attention, memory and executive function. A 2 year follow up reveals that some cognitive deficits as attention and executive function may improve as part of the general improvement by the clinical symptoms, with no significant brain equivalent changes. However, a 5 year follow up of the sample, suggest that initial improvement was temporal with decrease scores in all cognitive measures (from basal assessment) associated to reduction in volumes at ROI such as frontal and parietal lobes.

Correspondence: Julio Bobes García, Dpto de Psiquiatría Universidad de Oviedo, Cl Julian Clavería, 6, 33006 Oviedo * Spain Tel + 34985 103553,E-mailbobes@uniovi.es

R. SEGARRA, J. IGNACIO EGUÍLUZ \& M. GUTIÉRREZ. Course and Outcome in First Schizophrenic Episode. The Role of Insight and Therapeutic Compliance.

Insight impairment is very common in early schizophrenia, and appears to be associated with a broad range of psychopathology, in cognitive domains, and related to poor treatment adherence. The first part of the presentation will review studies which have examined the clinical and neurocognitive correlates of insight in early schizophrenia. Secondly, we will describe this relation in an early course schizophrenia, schizoaffective, and schizophreniform disorder Spanish sample with a 5 year follow-up. The clinical variations in positive and negative symptoms will be related to the lack of insight in the sample. The characterization of this relation over time will be described and implications for treatment compliance, and outcomes will be discussed.

Correspondence: Julio Bobes García, Dpto de Psiquiatría Universidad de Oviedo, Cl Julian Clavería, 6, 33006 Oviedo * Spain Tel +34985 103553,E-mailbobes@uniovi.es

I. BOMBÍN \& C. ARANGO. Cognitive Profile, Neurological Signs and its Evolution in First-Episode Psychotic Patients: A Two-Year Follow-up.

Patients with schizophrenia are characterized by neurological abnormalities, which can be assessed by bedside clinical examination. These abnormalities have been argued to represent core features of the illness. We review studies published since our last review in 1988 that address the validity of neurological signs as a trait feature of schizophrenia. We conducted a literature search in the following computer databases: MEDLINE, PSYCHLIT, EMBASE, and COCHRANE. The search was limited to articles published from January 1988 to May 2005. Neurological signs occur in the majority of patients with schizophrenia. Their occurrence is independent of demographic and most medication variables. Neurological signs are strongly associated with negative symptoms and cognitive impairments. There is also evidence to suggest that their occurrence is under genetic control. There is compelling evidence to suggest the hypothesis that neurological signs represent a trait feature of schizophrenia

Correspondence: Julio Bobes García, Dpto de Psiquiatría Universidad de Oviedo, Cl Julian Clavería, 6, 33006 Oviedo * Spain Tel +34985 103553.E-mailbobes@uniovi.es 


\section{Invited Symposium 3:30-5:00 p.m.}

\section{Studies on Cognition and Emotion Using Neuroimaging}

\section{Chair: Jose Leon-Carrion}

\begin{abstract}
A. PAPANICOLAOU. Magnetoencephalography as a Functional Neuroimaging method.

An overview of the basic concepts underlying the use of Magnetoencephalography (MEG) as a means of constructing spatiotemporal brain activation maps corresponding to basic and higher functions (linguistic, cognitive and affective) will be followed by a series of specific examples of such use. This series will consists, first, of studies establishing the reliably, validity, and the degree of spatiotemporal detail achievable through MEG-mapping; second of studies exploring the brain mechanisms of oral language comprehension; third, studies exploring the mechanisms mediating verbal and non-verbal memory and other cognitive functions in normal children and adults. Finally, a fourth set of examples will be reviewed focusing on alternative approaches to the study of brain mechanisms necessary for generation of affect. Correspondence: José León-Carrión,Ph.D, Human Neuropsychology Laboratory, Facultad de Psicología. University of Seville C/ Camilo Jose Cela s/n, Sevilla-41018 Spain, Phone: +3495 4574137 Fax: +34 954374558
\end{abstract}

E. CASTILLO. Exploring the language comprehension mechanisms in the Normal and Lesioned Brain.

The sensitivity and efficacy on Magnetoencephalography (MEG) in identifying the mechanism of oral language comprehension as well as aspects of its spatiotemporal constituents corresponding to phonological and semantic operations (that constitute the receptive language function), will first be demonstrated. Subsequently data will be presented demonstrating the compatibility between MEG and intraoperative and extraoperative Cortical Stimulation Mapping (CSM) in terms of their accuracy of identification of such mechanisms. Having thus established the validity of language - specific cortical maps, constructed on the basis of MEG, topographically aberrant maps for the same function will be presented, exemplifying the phenomenon of brain plasticity and functional reorganization. Moreover, the relationship of lesion parameters to degree and type of reorganization (intra- or cross-hemispheric) will be outlined, both in cases of tumors and epilepsy.

Correspondence: José León-Carrión,Ph.D, Human Neuropsychology Laboratory, Facultad de Psicología. University of Seville C/ Camilo Jose Cela s/n, Sevilla-41018 Spain, Phone: +34954574137 Fax: +34 954374558ins@osu.edu

J. LEÓN-CARRIÓN. Does Dorsolateral Prefrontal Cortex (DLPFC) Activation Return to Baseline when Sexual Stimuli Cease? The Role of DLPFC in Visual Sexual Stimulation.

A fundamental question in human sexuality regards the neural substrate underlying sexually-arousing representations. Lesion and neuroimaging studies suggest that dorsolateral pre-frontal cortex (DLPFC) plays an important role in regulating the processing of visual sexual stimulation. The aim of this Functional Near-Infrared Spectroscopy (fNIRS) study was to explore DLPFC structures involved in the processing of erotic and non-sexual films. fNIRS was used to image the evoked-cerebral blood oxygenation (CBO) response in 15 male and 15 female subjects. Our hypothesis is that a sexual stimulus would produce DLPFC activation during the period of direct stimulus perception ("on" period), and that this activation would continue after stimulus cessation ("off" period). A new paradigm was used to measure the relative oxygenated hemoglobin $($ oxyHb) concentrations in DLPFC while subjects viewed the two selected stimuli (Roman orgy and a non-sexual film clip), and also immediately following stimulus cessation. Viewing of the non-sexual stimulus produced no overshoot in DLPFC, whereas exposure to the erotic stimulus produced rapidly ascendant overshoot, which became even more pronounced following stimulus cessation. We also report on gender differences in the timing and intensity of DLPFC activation in response to a sexually explicit visual stimulus. We found evidence indicating that men experience greater and more rapid sexual arousal when exposed to erotic stimuli than do women. Our results point out that self-regulation of DLPFC activation is modulated by subjective arousal and that cognitive appraisal of the sexual stimulus (valence) plays a secondary role in this regulation.

Correspondence: José León-Carrión,Ph.D, Human Neuropsychology Laboratory, Facultad de Psicología. University of Seville C/ Camilo Jose Cela s/n, Sevilla-41018 Spain, Phone: +34954574137 Fax: +34 954374558

G. NORTHOFF. Altered Prefrontal Cortical Function during Perception and Judgment of Emotions in Depression.

Background: Recent neuroimaging and therapeutic transcranial magnetic cortex stimulation (TMS) studies suggest imbalance between left and right dorsolateral prefrontal cortex (DLPFC) in major depressive disorder (MDD). However, fundamental neuropsychological characterization of left DLPFC hypoactivity and right DLPFC hyperactivity in MDD remain to be revealed.

Methods: We investigated 20 medication-free patients with a DSM-IV diagnosis of acute severe MDD and compared them with 29 healthy controls. In the functional magnetic resonance imaging paradigm, emotional stimulation consisted of unexpected and expected judgment and perception of negative and positive pictures.

Results: MDD patients showed hypoactivity in the left DLPFC during both unattended and attended emotional judgment whereas they showed hyperactivity in the right DLPFC only during attended (expected) emotional judgement. In contrast to healthy subjects, left DLPFC hypoactivity during emotional judgment in MDD was no longer parametrically modulated by negative emotional valences and abnormally (inversely) modulated by positive emotional valences. Abnormal hyperactivity in the right DLPFC correlated with depression severity.

Conclusion: We demonstrate the neuropsychological correlates of leftright DLPFC imbalance in MDD. Left DLPFC hypoactivity is associated with negative emotional judgment while right DLPFC hyperactivity is linked to attentional modulation. In sum, we characterize left-right DLPFC imbalance in neuropsychological regard which bridges the gap from resting metabolism and therapeutic rTMS effects to functional neuroanatomy of altered emotional-cognitive interaction in MDD.

Correspondence: José León-Carrión,Ph.D, Human Neuropsychology Laboratory, Facultad de Psicología. University of Seville C/ Camilo Jose Cela s/n, Sevilla-41018 Spain, Phone: +34954574137 Fax: +34 954374558

\section{Poster Session 3 \\ Memory, Attention, Visuospatial Functioning 3:30-5:30 p.m.}

\section{Attention}

L. CASHION. The Melbourne Effect: Differences In Sustained Attention In Urban And Regional Australian Children.

Objective: A majority of standardised psychological tests do not discriminate between urban and regional participants in their normative data. While there is evidence to suggest some cognitive differences be- 
tween regional and urban children, the findings have not been consistently replicated. It was hypothesised that results for the sample from the current study, which included regional and urban children from New South Wales and Victoria, would concur with the normative data.

Participants and Methods: In the current study, 198 Australian children in Years 1, 3, and 5, were administered the Score! subtest of the Test of Everyday Attention for Children as part of a larger executive function battery. This task is a measure of simple sustained attention. Normative data was based on a sample of children who resided in Melbourne, Australia.

Results: Despite performance on all other executive function tests being consistent with published norms, the sample demonstrated significantly lower scores on the Score! test compared to normative data. This finding was based on an alpha of $p<.01$ to account for multiple comparisons. A subsequent analysis examined the children in this study from urban areas, Melbourne, Sydney and Newcastle, and compared their performance to the regional participants. It was found that the urban participants were significantly superior to the regional sample, while no differences were found between urban subgroups based on geographical location.

Conclusions: Findings suggest that that even in populations assumed to be largely homogenous it should not be assumed that regional differences do not exist. The implication of selecting a normative sample from a restricted geographical area or socio-economic group means the potential of misidentification of problem functioning and needs to be considered in neuropsychological test development.

Correspondence: Larry Cashion, PhD, School of Health Sciences, Charles Darwin University, Ellengowan Drive, Darwin, NT 0909, Australia.E-mail:larry.cashion@cdu.edu.au

J.J. EVANS, E. GREENFIELD \& B.A. WILSON. Improving Cognitive-Motor Dual Tasking After Brain Injury.

Objective: People who have suffered brain injury frequently complain of difficulties with dual-tasking. We examined whether the ability to walk and carry out additional cognitive tasks (akin to walking and talking) could be improved by a specifically designed training programme. Participants and Methods: The study was a randomised controlled trial of a five-week cognitive-motor dual-tasking training programme. Twenty participants with evidence of dual-tasking difficulties arising from neurological injury or illness were randomly allocated to treatment or control groups. The training programme involved exercises in walking with gradually increasing secondary cognitive demands carried out twice per day, five days per week. Outcome measures included tests of cognitive-cognitive, cognitive-motor and motor-motor task combinations as well as a questionnaire relating to everyday dual-tasking.

Results: The training programme was viewed positively by participants, and there was a good compliance rate. Participants reported increased awareness of dual tasking demands in everyday life. Analysis of variance showed evidence of improvement as a result of training in performance on primary outcome measures where those measures were very close in format to training conditions, but little evidence of generalisation to other measures of cognitive-motor, cognitive-cognitive or motor-motor dual task combinations. There was some evidence that treatment group participants felt that their dual-tasking performance in everyday life was improved after the intervention.

Conclusions: This study suggests that functionally oriented interventions where training is close in form to everyday behaviour might improve cognitive-motor dual-tasking such as walking and talking. Although methodological constraints limit the extent of conclusions that can be drawn, the findings have implications for rehabilitation, particularly in relation to mobility in attentionally demanding and potentially dangerous situations such as busy urban environments.

Correspondence: Jonathan J. Evans, PhD, Psychological Medicine, University of Glasgow, Section of Psychological Medicine, Gartnavel Royal Hospital, 1055 Great Western Road, Glasgow G12 OXH, United Kingdom.E-mail:jje2k@clinmed.gla.ac.uk
H. HALLELAND, S. ADÓLFSDÓTTIR \& A. J. LUNDERVOLD. Characteristics of adults with ADHD on the Attention Network Test. Objective: Several children with ADHD will have persisting symptoms as adults (Biederman \& Faraone, 2005). The diversity in clinical aspects and therapeutic response in ADHD-patients indicates that ADHD represents a heterogeneous condition.

One way to explore this heterogeneity is "endophenotyping". We expect that valid and meaningful endophenotypes will enable bridging the gap between the knowledge of candidate genes and the manifest symptom complex of ADHD.

Attention Network Test provides measures of the efficiency of different attentional networks and may indicate dysfunctions that contribute to attention disorders, for example ADHD (Rodriguez, 1997). It also provides different hits and reaction time measures as well as variability measures, suggested as promising neuropsychological endophenotypes (Nigg, 2005).

Participants and Methods: 500 adult patients with a diagnosis of ADHD and 500 controls are recruited in the ongoing project. They deliver blood- or spit sample and fill out questionnaires. 100 of the participants will be randomly selected to go trough neuropsychological testing and clinical interview.

Results: Preliminary results indicate that the ADHD-group $(n=28)$ is characterised by a trend towards fewer hits $(\mathrm{p}=.086)$ and higher variability in response time $(p=.079)$ than the control group $(n=15)$.

Conclusions: Preliminary results suggest that the ADHD group differs from the control group on several measures of the ANT. The results from a larger sample will be presented at the conference.

Correspondence: Helene Halleland, Psychologist, Department of Biological and Medical Psychology, University of Bergen, Jonas Lies, Bergen 5053, Norway. E-mail: helene.halleland@psybp.uib.no

M. LAVAllée, M. PÉPIN, M. LORANGER, A. OTERO \& P. LAPORTE. How Does Attention Develop? A study of 6 to 12 Year Old Children From Four Different Cultural Backgrounds.

Objective: This study examines the development of attention among boys and girls aged 6 to 12 years from four cultural backgrounds (French-Canadian (from Quebec), English-Canadian (from Alberta), French and Mexican.

Participants and Methods: Nine psychometric tasks assessing phasic alertness, sustained attention, selective attention, divided attention, and attentional control, presented in visual and/or auditory modes, were completed by 1302 children. Anova (sex X 4 cultural backgrounds X 7 age levels) was performed on each task.

Results: Statistical analyses reveal that performance increases with age, but there is no significant sex or cultural background effects.

Conclusions: Findings also suggest that simple attentional functions are almost achieved before or at the age of 12 , while complex attentional processing continues to develop after the age of 12 . These findings will be discussed in the general context of assessment of children with attention deficit disorders (ADD/ADHD).

Correspondence: Marguerite Lavallée, PhD, Psychology, Université Laval, Pav. F.A.Savard, 1524, Université Laval, Québec, QC G1K 7P4, Canada.E-mail:marge@psy.ulaval.ca

E. PEREZ, A. VARGAS, S. FERNANDEZ-GUINEA \& J. GONZÁLEZMARQUÉS. Auditory Sustained Attention Development.

Objective: The aims of the study are to develop an ecological auditory sustained attention task and to define the development of sustained auditory attention in children from 3 to 9 years old.

Participants and Methods: Two hundred children participated in the first stage of the study. The $18 \%$ of them showed any of the exclusion criteria.

Therefore, finally, the sample consisted of 163 children that were divided, based on their age, in 14 groups, with 6 months interval between each. 
Results: The two-way ANOVA show a main significant effect in the course of the task time. In addition, there was significant effect for the interaction between the course of the task time and the age group.

Conclusions: These results suggest that:

Auditory sustained attention changes with age.

There is a significant increase of auditory sustained attention from 5 years to 6 years old.

Therefore, the development of sustained auditory attention seems to follow a step-wise development rather than a linear-wise development.

Correspondence: Elena Perez, Psicologia evolutiva y de la educacion, University Complutense, Rector Royo Villanova $s / n$, Ciudad Universitaria, Madrid 28040, Spain.E-mail: elenaperezhernandez@psi. ucm.es

\section{C.A. REYNOSO. Funciones ejecutivas en niños con TDAH} predominantemente inatentos.

Objective: Evaluar la atencion sostenida en niños con el diagnostico de TDAH y comparar su rendimiento en pruebas que discriminen las funciones ejecutivas.

Participants and Methods: 30 niños de la consulta de neruologia pediatrica del Intituto Nacional de Rehabilitación, México, diagnosticados con TDAH de acuerdo a los criterios del DSM IV. Se hicieron dos grupos, los pacientes con TDAH predominantemente inantentos y los predominantemente Hiperactivos/impulsivos. Se les aplicó la prueba de Caras y la Torre de Londres. Y se comparó entre los grupos, el tiempo de inicio, tiempo de ejecucion y violacion a las reglas de la torre de Londres con los baremos de atención sostenida a los 6 minutos de la prueba de caras.

Results: Se presenta la comparación de los grupos con t de student, y se encuntra una diferencia significativa entre los grupos.

Conclusions: Los pacientes diagnosticados con TDAH segun los criterios del DSM IV que tienen una atencion sostenida labil en la prueba de caras tienen un bajo rendimiento en las fuenciones ejecutivas medidas con la torre de Londres.

Correspondence: Cesar A. Reynoso, Dr., neurologia pediatrica, INR, Prado sur290, MexicoDF 11000, Mexico. E-mail: creynoso@inr.gob.mx

M. RIOS, J. RODRIGUEZ-SANCHEZ, I. SANCHEZ-CUBILLO, J. PERIAÑEZ, D. ADROVER-ROIG, J. TIRAPU, B. CRESPOFACORRO, J. QUEMADA \&. F. BARCELÓ. Attentional Control and Speed of Information Processing: Dissociations in Four Different Samples.

Objective: Attention, a basic cognitive function that uses to be affected after traumatic brain injury (TBI), schizophrenia spectrum disorders, and normal ageing. On the other hand, speed of processing (SIP) seems to play a relevant role in subjects' performance of neuropsychological test of attention (i.e. TMT, Stroop). The aim of this study was to clarify the existence of functional dissociations in attentional performance and SIP between patients with TBI, schizophrenia and normal ageing, and to address the importance of SIP as an independent deficit to be considered when evaluating attentional performance.

Participants and Methods: 440 participants were included in four different samples: patients with schizophrenia diagnosis, TBI, healthy elders and young healthy controls. SIP and attentional control tests (e.g. TMT, Stroop, digit symbol and digit span) were included in the neuropsychological battery. A series of ANOVA and ANCOVA analyses were performed in order to explore the presence of functional dissociations in attentional performance controlling SIP influence.

Results: The results showed a different performance among the four groups. TBI sample was characterized by deficits in SIP compared to both schizophrenia, ageing and young healthy controls. In addition, TBI, Schizophrenia, and ageing samples exhibit dissociations in attentional performance when SIP was statistically controlled. Finally, the results suggest that the pattern of deficits observed in normal ageing cannot be completely explained as general slowing.
Conclusions: SIP and attentional control are functionally dissociated and seem to be relevant issues in several disorders. Therefore, neuropsychological assesssment and rehabilitation procedures should include a comprehensive analysis of these variables.

Correspondence: Marcos Rios, Psicologia Basica II, UNED, C/Juan del Rosal, 10, Fac. Psicologia, Madrid 28045, Spain.E-mail:mrios@psi.uned.es

D. TABOADA, R. JURADO, F. DENIA, L. ESTEVEZ, M. JIMENEZ_ARRIERO \& S. FERNANDEZ-GUINEA. Impulsivity Effect in Attentional Function on Morbid Obese Patients with Binge Eating Disorder.

Objective: Approximately a $30 \%$ of the obese patients who follow treatment refer serious difficulties with episodes of binge eating which are characterized by perception of lack of control on the ingestion and recurrent guilt after each episode. This disorder is commonly identificated with bulimia nervosa (BN). When we eat there is an increase of dopaminergic response in reinforcement mesocortical neural circuits, with consequent increase on directed behaviour, in which cortex prefrontal is involved.

Objective: to evaluate the relationship between impulsiveness, speed processing and selective attention in obese patients with and without binge eating disorder

Participants and Methods: The neuropsychological evaluation was performed in 15 obese patients (BMI>30) with binge eating references (BED) and subtest 13 obese patients (BMI>30) without binge eating references (no BED) matched by age, sex and educational level. The following instruments were employed: WAIS III subtests: Digit Symbol coding, digit span, vocabulary, Trail Making Test (TMT) and Barrat Impulsiveness Scale (BIS) Results: BED patients show better neuropsychological performance; we found statistically significant differences in digit span $\left(p=0^{\prime} 023\right)$ and commission fails in TMT B $\left(\mathrm{p}=0^{\prime} 047\right)$. Also, BED group show increased impulsiveness (BIS: nonplanning impulsiveness), which correlated positively with better performance on digit symbol coding task $\left(p=0^{\prime} 020\right)$. Conclusions: We have found better execution in BED patients in processing speed, increased attentional span and better ability on alternating attention BED patients seem more impulsive than no BED group. These results on attencional function are opposite to deficits found in bulimia nervosa, so it is probably there are different control inhibitory components related to bingeing affected in each disorder. Nevertheless, these are preliminary results based on small sample, so they must be contrasted with a representative sample.

Correspondence: Rosa Jurado, Doctora, Psicología Básica II: procesos cognitivos, Universidad Complutense de Madrid, Facultad de Psicologia . Campus de Somosaguas. Pabellón I, Pozuelo de Alarcón 28223, Spain. E-mail: rjurado@psi.ucm.es

\section{Cognitive Neuroscience}

V. Plaza-AYLlón, M. ANTEQUERA, M. DAZA, C. NOGUERA, E. DEL ÁGUILA, D. ÁLVAREZ, E. CARMONA \& I. FERNÁNDEZAGIS. Case Report: Attentional Deficits in a Pacients with Left Temporo-Frontal Lobe Damage.

Objective: From a cognitive neuroscience view, attention has been thought of as s set of neural networks that perform very specific computations. So far three of these networks have been amply studied: the alert network, the orienting network, and the executive network. Is very interesting use this neuro-cognitive approach to asses the attentional networks functioning in patients. A method that allows this approach is the Attention Network Test (ANT).

Participants and Methods: Case report: JCG, 20 years old, with symptomatic epilepsy to injury gliotica subcortical frontal left diagnostic. Control group: $(n=15)$ aged between 19-22. We used the ANT to assess the attention networks functioning. The procedure combines a "cost and benefit paradigm" with the "flanker task" to obtain a measure of each attention network. The participants must indicate if the central arrow 
is appearing towards the right or the left side. The efficiency of the networks is evaluated measuring how the times of reaction are being influenced by the attentional cues (of alert, spatial or central) and for the flanks (congruent or incongruent).

Results: Scores showed by JCG in the ANT did not differ from those obtained by the control group. This suggests an intact functioning of the three attentional networks in the left temporal-frontal patient. Nevertheless, a more exhaustive analysis shows significant differences in the interaction of three systems.

Conclusions: The analyses will be discussed keeping in mind the influence of an attentional system on other one, though to priori, there was thought that every system was independent.

Correspondence: Vanesa Plaza-Ayllón, Neuroscience and Science of Health, CERNEP, Carretera de Sacramento s/n, Almeria 04120, Spain. E-mail:vayllon@ual.es

\section{Memory}

J. AHONNISKA-ASSA, J. KUSHNIR \& M. LEVAV. A Case of Developmental Amnesia: Dissociation between Impaired Episodic and Unimpaired Semantic Memory.

Objective: Developmental amnesia refers to a specific developmental disorder in memory functions, with no known underlying brain lesion. It may also refer to an early acquired disorder following pre-or perinatal anoxic episode. The memory disorder is evident from the outset of cognitive development, and may show clear dissociation between intact and impaired memory functions.

Participants and Methods: We present a neuropsychological profile of a six year old girl who suffered a severe incident of acute asphyxia at birth. Although she had neonatal seizures, the patient later showed no obvious neurological signs.

Results: Her results in the intelligence test (WPPSI) fell within a high average level, showing intact verbal and visual abilities. Apparently, with a substantial amount of individual tutoring, her abilities in mathematics and reading were within normal range. Her scores in immediate verbal and spatial memory ranged within normal limits. However, she had major problems in delayed verbal and visual memory. Her episodic memory was low, and she had major problems in daily memory functions as reported by her parents. She showed signs of separation anxiety, emotional distress and rapid mood changes, along with behavior problems, probably as a result from confusion due to memory problems. Conclusions: Together with previous reports, this case illustrates that in developmental amnesia, severe problems in episodic memory may be present with relatively intact abilities in semantic memory. Apparently, this dissociation enables acquiring knowledge, although the learning process may be slower than average. We speculate that lack of episodic memory may also result in severe behavioral and emotional problems. Correspondence: Jaana Ahonniska-Assa, Ph.D, Unit of Neuropsychology Child Rehabilitation Department, Shafra Children's Hospital, Sheba Medical Center, Tel haShomer 52621, Israel. E-mail: assa@netvision.net.il

E. DÍEZ \& M. BEATO. Imagery Encoding Reduces False Recognition: An Event-Related Brain Potentials (ERPs) Study.

Objective: Neuroimaging studies have suggested that frontal and parietal lobes may be important for the process by which we remember information. We measured event-related brain potentials (ERPs) in those brain areas to investigate processes underlying false recognition effect using the Deese-Roediger-McDermott paradigm.

Participants and Methods: Participants studied lists of words under two different conditions (shallow and deep processing) for a subsequent recognition memory test. Under the Shallow Condition, participants studied each word while searching for the letter "o". In the Deep Condition, participants were asked to create a visual mental image of each study word. The same stimulus presentation modality (visual) was used during study and test. ERPs were evaluated in true recognition of previously studied words (old words), in false recognition of associatively related never presented words (critical lures) and in new unrelated words.

Results: Behavioral analysis showed a robust false recognition effect, which was significantly larger in the Shallow Condition. In relation to true recognition, results showed that the Deep Condition subjects had better memory for studied words. ERPs analysis showed FN400 old/new effect (300-500 ms) only in the Shallow Condition. However, a parietal old/new effect $(400-800 \mathrm{~ms})$ was found in the Deep Condition, and a late right frontal old/new effect (1000-1500 $\mathrm{ms}$ ) was found in both conditions. No significative differences were observed between true and false recognition in parietal and frontal brain regions.

Conclusions: Those results indicated more positive activity for true recognition than for rejection of new words. Dual process theories of recognition memory could explain the obtained results.

Correspondence: Maria Soledad Beato, Doctor, Ps. Basica, Psicobiologia y Met., Universidad de Salamanca, Avda. de la Merced, 109-131, Salamanca37005,Spain.E-mail:msol@usal.es

M.E. BENASSI-WERKE, O.F. BUENO, M.G. QUEIROZ, R.S. ARAÚJO \& M.G. OLIVEIRA. Memory Span for Tones, Words and Nonwords in Amateurs Singers.

Objective: We intended to verify whether the same short-term loop of working memory Baddeley's model (phonological loop) can equally manage verbal (numbers and nonwords) and non-verbal (tones) sounds.

Participants and Methods: Amateurs singers $(n=13)$ were submitted to forward (FS) and backward (BS) digit span task. Nonwords and melodic spans tasks were criated and applied following the same rules of digit span task.

Results: For data analyses we used a two-way ANOVA for repeated measures to compare digit, nonwords and melodic spans, in FS and BS; a two-way ANOVA to analyses an index (FS-BS/FS), and NewmanKeuls post hoc tests. We also use Pearson correlations to compare the FS in each kind of task as well BS. In both FS and BS, the recall of numbers was higher than the recall of nonwords $(p<0.05)$ and this was higher than the recall of tones $(p<0.05)$. Correlation was significant for digit and nonword spans in FS $(r=0.63, \mathrm{p}<0.05)$, but it wasn't for nonword and melodic spans, neither for digit and melodic spans. There weren't significant correlations in BS and the index analysis showed that digit span and nonword span didn't differ from each other but the melodic span differed from the other two $(p<0.05)$.

Conclusions: It suggests that there's something in common in the shortterm memory for verbal material, that is different for tones. Semantic and phonetic aspects of sounds may influence backward recall. The higher index in melodic span also suggests that melodic and verbal sounds do not share the same phonological loop of the working memory. Correspondence: Mariana E. Benassi-Werke, Psicobiologia, Universidade Federal de São Paulo, Rua Napoleão de Barros, 925, Vila Clementino, São Paulo 04024-002, Brazil. E-mail: marianawerke@yahoo.com.br

A. BERARDI, C. PAPOT, O. HABERT \& A. PRUSKI. Memory Assessment in a Virtual Reality Environment in Patients with Stroke and Multiple Sclerosis.

Objective: The aim of this study was to use Virtual Reality (VR) to assess in situation the memory abilites of wheelchair users.

Participants and Methods: Six patients with stroke and 10 with multiple sclerosis (MS) were studied. Patients moved through a VR vacation center on a simulated wheelchair controlled by the computer arrows. Patients' performance was compared to that of 10 age, sex and education-matched controls $($ all $\mathrm{F}<1$ ). The groups did not differ in their experience with computers and videogames (both 
$\mathrm{p}>.32)$. After freely exploring the environment, participants were shown how to go from a set point to their room and from the room to the kitchen. They then were required to go alone from the set point to the room and then to the kitchen. Once they reached the room (or the kitchen), they were asked to memorize the objects seen. At the end of the test they recalled the objects seen at each target point. Dependent measures were the time needed to reach each target point and the number of items correctly recalled. Results were analyzed using ANOVAs.

Results: The groups took equivalent amounts of time to construct a map of the vacation center $(p=.16)$. Groups did not differ in the time they took to reach the two target points (both $\mathrm{p}>$.10). Stroke patients recalled fewer objects $(p=.003)$ and MS patients tended to recall fewer objects $(\mathrm{p}=.08)$ than controls.

Conclusions: VR results suggest that stroke and MS patients recall fewer objects than controls in a VR environment and that VR may provide useful functional information in neurological patients.

Correspondence: Anna Maria Berardi, Ph.D. in psychology, Department of Psychology, Paul Verlaine University - Metz, UFR SHA, Ile du Saulcy, Metz 57006,France.E-mail: berardi@univ-metz.fr

\section{BRAMÃO, L. FAÍSCA, M. GUERREIRO \& A. REIS. Semantic Memory Organization: Differential Weighting of Perceptual and Functional Features.}

Objective: There is still an ongoing discussion about the organization of semantic memory. In this study we investigated the hypotheses of the unicity of the semantic system (a single semantic system vs. multiple semantic systems) and explored the differential weighting of perceptual and functional-associative features on the semantic representation of living and nonliving things.

Participants and Methods: Two lexical decision tasks were designed: a within-modality priming task - both prime and target were presented as words - and a cross-modality priming task - prime was presented as a picture and target was presented as a word. Forty-eight stimuli were selected (24 living and 24 nonliving) and each stimulus was matched to a perceptual feature and to a functional-associative feature. Altogether, ninety-six prime-target pairs were selected with living and nonliving items being the primes and the perceptual and functional-associative features the targets. Eighty-two graduated students participated in the experiment.

Results: Although our results did not show any task effect (no difference was found for within-modality and cross-modality priming), we observed that the lexical decision times for the perceptual features were faster when the prime was a living item and the lexical decision times for the functional-associative features were faster when the prime was a nonliving item.

Conclusions: In summary, our results suggested the existence of a single semantic system, where the perceptual features are more important for the semantic organization of living things and the functional-associative features are more important for the semantic organization of the nonliving things.

Correspondence: Inês Bramão, Faculdade de Ciências Humanas e Sociais, Universidade do Algarve, Campus de Gambelas, Faro 8000, Portugal.E-mail:mibramao@ualg.pt

\section{M.F. OLIVEIRA, F.H. SANTOS \& O.F. BUENO. Free Recall and Aging: Bridging Short-Term and Long-Term Memory.}

Objective: There is a decline of both episodic and working memory in aging. However, the relationship between episodic and working memory processes remain unclear. It is possible that the episodic buffer, a working memory component, plays a role on manipulation and integration of the information between long and short-term memory. The aim of this research is to observe the relation between episodic and work- ing memory, specially the buffer episodic performance, in a paradigm using free recall with semantically related words in the middle of the list, associated with two different span tasks, varying in terms of presentation modality: visual phonological (VP) and visuospatial (VS).

Participants and Methods: Four lists with 15 words contained semantically related words in the middle positions (7-8-9) were presented followed by VP or VS tasks, before recall of the words. A group of 15 healthy elderly were compared to young adults $(n=35)$.

Results: The ANOVA did not show differences between the groups in primacy effect and in the semantic facilitation effect $[\mathrm{F}(4,188)=1,97$ $p=0,1]$. The recency effect was abolished. The young adults $[\mathrm{F}(1,47)=3.66 \mathrm{p}=0.05](54.57+5.20)$ recalled more items after the VP task than the older adults $(32.61+8.23) \mathrm{p}=0.03$. A qualitative analysis revealed that the elderly had a low performance in both VP and VS tasks.

Conclusions: These results suggest that elderly performance decreased possibly because of an overload in working memory system due to the presentation of the secondary tasks. These findings might be related to the more items than the episodic buffer can chunk into the working memory, despite its binding property of multimodal information from long-term and short-term memory.

Correspondence: Mirian Akiko F. Oliveira, master degree, Psychobiology, University Federal of Sao Paulo, Rua Napoleao de Barros, 489, Vila Clementino, Sao Paulo 04024001, Brazil.E-mail: akiko@psicobio.epm.br

\section{BUNNAGE. Rapid Forgetting and 'Rapid Remembering' in a Heterogenous Adult Clinical Sample.}

Objective: Bowden et al (1999), Tulsky and Price (2003) and Bowden et al (2001) have highlighted the possible limited value of distinguishing between immediate and delayed components of commonly used measures of memory. These researchers argue for a one factor solution to these memory measures. Others, e.g. Delis et al (2003), argue that clinical populations differ from the normal population. Despite poor factor analytic support distinguishing immediate and delayed memory should be considered useful in clinical practice, e.g. for patients with classical amnesia or suspected of suffering from Alzheimer's disease (Butters et al, 1995; Delis and Kramer, 2000, Graf et al, 1984 and Squire, 1987). What remains to be determined, however, is the clinical utility of distinguishing between immediate and delayed recall in other clinical populations.

Participants and Methods: The relationship between immediate and delayed recall for both verbal and visual information was evaluated in a large $(n=76)$ group of neurological patients not suffering from amnesia or AD. The frequency of differences in memory performance at immediate and delayed recall was recorded, both in the direction of rapidforgetting (RF) and 'rapid-remembering' (RR). The relationship between these differences and anxiety and mood was determined, correlating difference scores with self-report anxiety and mood measures.

Results: The results indicate an equal frequency of both RF and RR. Further, no meaningful relationship between differences in memory performance at immediate and delayed recall and anxiety or mood were found.

Conclusions: The results suggest there may be limited value in distinguishing between memory performance at immediate and delayed recall in patients other than those with AD or amnesia. Differences observed between memory performance at immediate and delayed recall in other groups may be the product of measurement unreliability. Clinicians should therefore collapse these measures, as per Tulsky et al, to increase measurement reliability.

Correspondence: Martin Bunnage, M.Psych (Neuro), Neuropsychology, Frenchay Hospital, North Bristol NHS Trust, The Burden Centre, Frenchay Park Road, Bristol BS16 1JB, United Kingdom. E-mail: Martin.Bunnage@nbt.nhs.uk 
V. CHASSÉ \& S. BELLEVILLE. Use of Orthographic Coding in Verbal Short-term Memory : Evidence from a Single Case Study.

Objective: Psycholinguistic views of verbal short-term memory (VSTM) propose that multiple codes contribute to short-term retention of verbal items. While the role of phonological coding in VSTM has been extensively documented, crucial data supporting the influence of other linguistic representations (e.g. semantic) on retention have been found in brain-damaged patients presenting phonological deficits. Indication for the use of orthographic coding by those patients has recently been raised but has not been formally assessed. The aim of this study was to test the influence of orthographic representations on immediate serial recall (ISR) and compare its impact with that of phonological representations.

Participants and Methods: This was tested in I.R., a brain-damaged patient with selective impairment of phonological memory, and in 10 matched controls. ISR of words with several phonological neighbors was first compared to the ISR of words with few phonological neighbors. The orthographic counter-part was then tested by comparing ISR of words with several orthographic neighbors to that of words with few orthographic neighbors. The impact of orthographic similarity was also assessed by comparing the ISR of lists of phonographic neighbors to that of phonological neighbors. In the two latter tasks, presentation was visual and response was by writing.

Results: I.R. showed no phonological effect in the first task. However, she produced more orthographic errors than controls when words with a large orthographic neighborhood were presented. She also exhibited a marked orthographic similarity effect contrary to controls.

Conclusions: Results support a psycholinguistic view whereby different linguistic codes have the potential to contribute to VSTM.

Correspondence: Véronique Chassé, Université de Montréal \& Centre de recherche de l'Institut universitaire de gériatrie de Montréal, 1050 Pratt, Montréal, QCH2V2V2, Canada.E-mail:veronique.chasse@umontreal.ca

R. CÁNOVAS, L. IRIBARNE, M. ESPÍNOLA, I. LEÓN, D. ROLDÁN \& J. CIMADEVILLA. A New Virtual Task to Evaluate Human Place Learning.

Objective: This study assesses the effectiveness of a new virtual task to evaluate human place learning.

Participants and Methods: The task is called Boxes' Room. The virtual room contains 16 boxes homogeneously distributed on the floor ( 4 raw of 4 boxes each). The room also contains a door, a window, and several pictures on the walls. The task consists in discover the position of the rewarded boxes, that is constant during the whole experiment. Subjects are asked to open the minimum amount of boxes necessary to discover all the rewarded positions in ten trials. In the initial trial subjects needed to open almost all the boxes to discover the position of the rewarded boxes. Opening an unrewarded box is considered an error.

Sixty university students completed the task ( 30 men and 30 women). Subjects were distributed into three groups, which had to find 3,5 and 7 rewarded boxes, respectively.

Results: The latency to discover all the rewarded boxes, the distance covered, and the number of errors were measured. Analysis of the data showed that all the subjects can acquire the task but women are slower and less accurate than men.

Conclusions: These results agree with previous results in other virtual tasks, and support the spatial component of this test.

This study was supported by SEJ2005-05067/PSIC from MEC (Spain). Correspondence: Jose Manuel Cimadevilla, Dr, Neuroscience, University of Almeria, Carretera de Sacramento s/n, Almeria 04120, Spain. E-mail: jcimadev@ual.es
C. COSTE, N. AGAR, A. MACÉ, P. AZOUVI \& P. PIOLINO. Retrieval Mechanisms for Autobiographical Memories after Severe Traumatic Brain Injury.

Objective: Traumatic brain injury (TBI) impaired recalling of episodic autobiographical memories. According to Conway's model, autobiographical memories are constructed via different types of long-term memory representations via a strategic retrieval process. Our research examines the processes leading to autobiographical memory (AM) deficits in severe TBI patients by studying relationships between AM, working memory (WM) and executive functions.

Participants and Methods: 16 TBI patients and 22 controls completed a novel verbal autobiographical fluencies (VAF) task that reproduces the retrieval process proposed by Conway's model. The categorical autobiographical fluencies focused on lifetime periods (VAF1), general events (VAF2), specific events (VAF3) and sensori-perceptive details (VAF4). The WM (subsystems, episodic buffer, central executive), executive functions (updating, inhibition and shifting) and speed processing (SP) were also evaluated with a battery of neuropsychological tests.

Results: The ANOVA show that TBI patients gave fewer responses than controls for all fluencies. TBI patients were also significantly impaired for forward and backward visual spans, episodic buffer, updating and SP. Forward stepwise regression analysis indicated that capacity of visuo-spatial manipulation predicted $8.6 \%$ of the VAF1. Episodic buffer and SP predicted respectively $13.4 \%$ and $8.1 \%$ of the VAF2. SP and inhibition predicted respectively $20.3 \%$ and $11.2 \%$ of the VAF3. Finally, shifting predicted $8.3 \%$ of the VAF4.

Conclusions: All these results confirm the impact of WM dysfunction on AM deficits in TBI. They also provide new insight as they highlight the role in general events access of the episodic buffer which allows the retrieval of episodes by binding information from different modalities and sources.

Correspondence: Cecile Coste, Psychology, CNRS, 71 avenue Ed. Vaillant, Boulogne Billancourt 92774, France. E-mail: cecilecoste@hotmail.com

P. COVRE \& O.F. BUENO. Relation of Working Memory Capacity and Free Recall of Semantically Related Words in Alzheimer's Disease.

Objective: Free recall is enhanced by the insertion of semantically related words in the middle of unrelated lists (semantic facilitation). Our aim was to investigate whether this could be observed in patients with mild Alzheimer's disease (AD) and if the effect is related to the working memory.

Participants and Methods: We compared 17 AD patients to 17 controls on working memory capacity (WMC) and free recall tests. The latter contained 7 and 15-word lists divided into two sets: one composed by unrelated words and the other by lists in which the three intermediary words were semantically related.

Results: AD patients performed worse than controls on the WMC test. To analyze the semantic facilitation effect, only the recall of the three intermediary words from both list's set were considered. AD patients showed the facilitation effect only for the 7 word lists but in a lesser extent than controls. This impairment was observed even when the WMC was covaried on the analysis. To investigate if the effect occurred due to the chunking of the related words, we analyzed the situations in which at least two words of the three related words were recalled, once the participant had recalled one of them. AD patients were impaired on the clustering capacity, but the group difference disappeared when the WMC was used as co-variable.

Conclusions: The facilitation effect did not occur for AD patients on the bigger lists, possibly by a limited capacity processing, which is corroborated by the dependence of the clustering capacity on the WMC. Correspondence: Priscila Covre, graduation student, Psicobiologia, Universidade Federal de São Paulo, Rua Napoleão de Barros, 925 - 10 andar, São Paulo04024-002, Brazil.E-mail: priscilacovre@gmail.com 
B.L. DEWAR, K. PATTERSON, K.S. GRAHAM \& B.A. WILSON. Reacquisition of person knowledge in semantic memory disorders. Objective: Investigation of new learning in semantic memory disorders is important for the development of rehabilitation techniques to improve the everyday function of people living with memory disorders. Such studies also allow the opportunity to explore how systems important for learning, such as the hippocampus and temporal neocortex, interact in the acquisition of semantic facts.

Participants and Methods: Three individuals with Herpes Simplex Viral Enecephalitis (HSVE) and one with a diagnosis of semantic dementia were trained with names and semantic facts relating to ten famous people using a mnemonic and the errorless learning paradigms of vanishing cues and expanded rehearsal.

Results: Practice resulted in improved naming from the trained photograph in all participants, with the HSVE individuals showing particular reliance upon experimenter-provided cues. The ability to retrieve a semantic fact was also better after training, although performance was less robust that that seen for naming, except in the case with semantic dementia who showed the quickest and strongest effects of training. Learning was maintained in the absence of home practice, and was found to generalise to accurate naming of a different photograph, and to a lesser extent, naming when provided with a new semantic fact.

Conclusions: This study confirms that errorless learning paradigms can be used to help boost naming and semantic knowledge of famous people in participants with loss of conceptual knowledge, although learning was still abnormally slow and somewhat inflexible. This finding support theoretical accounts in which different temporal structures are capable of supporting acquisition of new semantic facts independently. albeit significantly slower and with less semantic detail than when both systems are available.

Correspondence: Bonnie-Kate L. Dewar, BSc MSc (Clin Neuropsych), Memory Aids Clinic, Institute of Psychiatry, 3rd Floor Adamson Centre, St Thomas's Hospital, London SE1 7EH, United Kingdom. E-mail: bonnie-kate.dewar@iop.kcl.ac.uk

T. EDGINTON, A. JANSARI \& M. SPILLER. An investigation of a non-selective imagery impairment on strategic and autobiographical memory processes following posterior frontal lobe craniotomy.

Objective: Multiple sensory and strategic processes are required for successful autobiographical memory recollection. Impairments of strategic processes have been associated with cholinergic decline, frontal lobe damage and damage to the anterior cingulate cortex (Rusted et al, 1998; Carter et al, 2000). The impairments tend to be highly selective and specific suggesting functional dissociations between the underlying $\operatorname{cog}_{-}$ nitive architecture that supports search and retrieval processes whereas imagery impairments have been associated with damage to the visual and parietal cortex (Rosenbaum et al, 2004).

Participants and Methods: We report the case of a 30 year-old patient with severe post-morbid imagery deficits in the presence of highly efficient strategic use following surgical removal of a posterior frontal lobe tumour. Autobiographical memory was assessed using the standard AMI and the more sophisticated JWALK (Jansari and Ward, 2005) to specifically investigate the visual imagery components of memory recollections associated with personal autobiographical life knowledge. In addition, a Flashbulb Memory test, assessing recollection for public events, was administered which further fractionated recall into factual, visual, emotional and autobiographical components.

Results: Results confirmed a general deficit in autobiographical memory recollection with no sparing of childhood memories linked to difficulties with forming visual images.

Conclusions: We discuss the bearings these findings have on our understanding of autobiographical memory dependence on visual imagery. Correspondence: Trudi Edginton, PhD, Cognitive Science Research Unit,
University of Westminster, Watford Road, Harrow, London HA1 3TP, United Kingdom. E-mail: t.edginton@wmin.ac.uk

A. ESPINOSA, M. ALEGRET, S. VALERO, G. VIÑAS, G. MODINOS, L. TARRAGA \& M. BOADA. The Rivermead Behavioural Memory Test: an ecological assessment of memory in mild cognitive impairment and Alzheimer's disease.

Objective: Traditional cognitive tests are the most commonly used to discriminate between mild cognitive impairment (MCI) and mild Alzheimer's disease (AD). Given these tests lacks of ecological validity, the Rivermead Behavioural Memory Test (RBMT) was created by Wilson et al. (1991) to assess subtle memory impairments in the daily life. The aim of the present study was to determine if the RBMT is useful to discriminate between MCI, mild AD and cognitively normal subjects.

Participants and Methods: The RBMT was administered to 50 mild AD patients, 50 MCI subjects and 50 healthy control subjects.

Results: RBMT global score showed statistically significant differences between control, MCI and AD groups (F2,146=128.98, $p<0.0005)$. The global RBMT explained $64 \%$ of variance between diagnostic groups. The analyses of the RBMT subtests identified that the combination of Pictures and Date significantly discriminated between MCI and AD groups $(\chi 2=57.52 ; \mathrm{p}<0.0005)$. Immediate Route, Second Name, Orientation and Belonging significantly discriminated between control and MCI groups $(\chi 2=59.20 ; p<0.0005)$ and Orientation, Date, Delayed Route and Appointment significantly discriminated between control and AD groups $(\chi 2=113.45 ; p<0.0005)$.

Conclusions: The RBMT has proved to be an appropriate test to discriminate between normal aging, MCI and mild AD subjects. Considering the common difficulty in clinical practice to distinguish between MCI from mild AD and cognitively preserved subjects, the use these 6 subtests with proven efficacy to discriminate between such groups is recommended.

Correspondence: Montse Alegret, Doctor, Neuropsychology, Fundacio ACE, Marques Sentmenat,35-37, Barcelona 08014, Spain. E-mail: malegret@fundacioace.com

A. ESTEVEZ-GONZALEZ, S. CARRERAS, J. KULISEVSKY \& C. GARCIA-SANCHEZ. RELATION BETWEEN SEMANTIC MEMORY AND ACHIEVEMENT OUTCOMES IN A SAMPLE OF ADOLESCENTS.

Objective: To examine the relationship between performance in a semantic memory task and school marks.

The semantic memory is a kind of declarative memory that includes knowledges about the world. School learning is a very important source of these knowledges. The relationship between semantic memory and school achievement seems obvious. However few studies have showed this relationship.

Participants and Methods: Semantic task consisted of a questionnaire of eighty questions about basic school knowledges (for a similar school level of 14 years of age). Twenty unselected late adolescents (10M/10F; aged: $16-18$ years) from a urban secondary school responded to this questionnaire. Achievement school was determined by marks in mathematics, linguistics and history of the art.

Results: Mean of correct responses were 63 (SD: 10.60; range 36-78). No sex differences were found $(\mathrm{F}=.387, \mathrm{p}<.54)$. The relationship between performance in this semantic memory task and school marks was found in 15 of the 20 subjects $(X 2=10.0 ; p<.01)$.

Conclusions: Our results showed relationship between performance in a semantic memory task and school achievement. Semantic memory tasks could be useful tools to make an appropriate school prognosis. Correspondence: Armando Estevez-Gonzalez, Universty Barcelona, Mitre 59, Barcelona 08017, Spain.E-mail: 20389aeg@comb.es 
P. FIGUEIREDO, S. CAVACO, I. ALMEIDA, J. MASSANO, C. CUNHA, E. MACHADO, M. VAN ASSELEN, I. SANTANA \& M. CASTELOBRANCO. Dissociable Memory Impairments after Selective Bilateral Fornix Damage.

Objective: The fornix is the major efferent pathway connecting the hippocampus with the diencephalon and the frontal lobe and it is regarded as a key link in the memory circuit comprising these structures. It remains a topic of debate whether this circuit supports a unique episodic memory system that can be dissociated from other memory systems. The objective of our study was to test the hypothesis of dissociable memory impairments following fornix damage.

Participants and Methods: We have comprehensively investigated memory performance in a patient, J.T., with selective bilateral fornix damage resulting from neurosurgical cyst removal through a transcallosal approach. Standard neuropsychological assessment of all cognitive functions was performed approximately one year after surgery. Further assessment aimed at clarifying the memory impairments of the patient included a large number of both standard and experimental tests of recall and recognition memory, spatial memory and procedural memory. Results: Standard neuropsychological tests revealed severe impairment in episodic memory recall, with relatively intact recognition memory. while all other cognitive functions were normal. The long-term episodic memory deficits were confirmed in all subsequent tests, while patient J.T. consistently showed normal recognition memory in the Warrington Recognition Memory Test (RMT) for faces, a Delayed Matching-to-Sample (DMS) test and a Visual Paired Comparison (VPC) test. Moreover. patient J.T. was severely impaired in an Object - Relocation spatial memory test, when involving object to location binding but not so for positions only memory conditions. Normal perceptual-motor skill learning was observed in two standard tasks, mirror tracing and rotary pursuit. Conclusions: In summary, our results support the hypothesis that the fornix is part of a memory circuit essential for recollection and recall but not familiarity processes. Our study further demonstrates spatial memory impairments and preserved procedural memory after fornix damage.

Correspondence: Patricia Figueiredo, IBILI, University of Coimbra, IBILI, Az. Sta. Comba, Coimbra 3000-354, Portugal. E-mail: pfigueiredo@ibili.uc.pt

J. GARCiA ORZA, S. MUÑOZ, C. ARRABAL \& L.A. MUÑOZ. Disproportionate Retrograde Amnesia in a Traumatic Brain Injury Patient.

Objective: The diagnosis of Focal Retrograde Amnesia (FRA) constitutes a controversial issue(for a review, see Kopelman, 2000). In the present research an apparently case of FRA is presented, however, a deeper assessment with convergent tests suggests that our patient presents disproportionate retrograde amnesia accompanied by moderate anterograde amnesia.

Participants and Methods: Patient JMJ is a 24 years old man with eight years of formal education who suffered traumatic brain injury after falling from $3 \mathrm{~m}$. high during his job as bricklayer. RMN on October 2006 showed minor impairments in right parietal areas and extensive impairments in frontal and temporal areas of the left hemisphere. Results: The evaluation carried out 5 months after the accident revealed border line intellectual level (WAIS, Verbal CI= 73, Manipulative CI= 82; Total CI = 75) showing difficulties in speed of processing and verbal comprehension. Retrograde memory, assessed with a Spanish version of the Autobiographical Memory Interview (AMI) showed that the personal semantic component of retrograde amnesia was more impaired than the autobiographical one. An adaptation of The Questionnaire about Famous People and Events Recall (Baqués, 1998) confirmed the impairment of personal semantic retrograde memory. Anterograde amnesia assessed with WMS-III suggest a near normal pattern of recall with indexes ranging between 76, for immediate visual memory, and 89, for delayed auditive memory.
Conclusions: As no psychogenic symptoms were observed that could explain the pattern described and taking into account his pre-morbid intellectual level and the actual one, the results obtained may suggest the preservation of anterograde amnesia, and hence the diagnosis of FRA. However, against the data obtained with laboratory tests, the analysis including everyday tests like RBMT-E confirmed the presence of anterograde amnesia.

It is concluded that diagnosis of FRA should be established only under the finding of convergent evidences from different assessment tools.

Correspondence: Javier Garcia Orza, Ph D, Psicologia Basica, Universidad de Malaga, Campus Teatinos s/n, Malaga 29071, Spain. Email:jgorza@uma.es

J. GARCIA ORZA, S. MUÑOZ, L.A. MUÑOZ-BECERRA, C. ARRABAL \& O. FERNANDEZ. Severe Anterograde Amnesia after Damage to Hypothalamus.

Objective: There is still poor understanding of the precise damage that causes anterograde amnesia. The role of hypothalamus in memory is even less known. while it is usually not mentioned as one of the neurological structures involved in anterograde memory, some studies suggest that damage restricted to hypothalamic structures could cause anterograde amnesia. The case of a patient with anterograde amnesia caused by damage to hypothalamic structures is reported in this study.

Participants and Methods: ALG, a 25 vears old woman with thirteen years of formal education was diagnosed in October 2004 of an astrocitoma in the hypothalamus that causes weight increase, metabolic changes, disorientation and anterograde amnesia. The tumour was resected in June 2005 with microsurgery. RMN in January 2006 showed damaged circumscribed to the anteroinferior parts of the hypothalamus. Results: The evaluation carried out in January 2006 showed no differences in intellectual level compared to stimated premorbid intellectual level. Language, constructional praxis, visuoperceptual and spatial abilites were normal. ALG showed disorientation and a dense anterograde amnesia (Overall Profile Score $=0 / 4$ : severe amnesia) as measured with RBMT-E (Wilson et al., 1999). ALG showed difficulties both in auditory verbal and non-verbal memories, and both in recognition and retrieval tasks. Retrograde memory, assessed with a Spanish version of the Autobiographical Memory Interview (AMI, Kopelman et al. 1989) was in the acceptable range: ALG only showed minor difficulties in recent life memories, those that involved facts posterior to the appearance of the tumour. No confabulation was noted. Personality change scale showed increased irritability.

Conclusions: Our results suggest that damaged to hypothalamic structures causes severe anterograde amnesia. Consequently, it is argued that hypothalamic structures play a relevant role in memory processes. Correspondence: Javier Garcia Orza, Ph D, Psicologia Basica, Universidad de Malaga, Campus Teatinos s/n, Malaga 29071, Spain. Email:jgorza@uma.es

E. GOMEZ-PEREZ, G. CASTILLO-PARRA \& F. OSTROSKY-SOLIS. Development of Factors Contributing to Age Effects in Memory Tasks. Objective: Improvements in memory during childhood have been well documented; however, the hypothetical factors underlying such improvements are not fully understood. The purpose of this study was to analyze the development of four factors -strategies, metamemory, employed material (drawings or words) and levels of processing - potentially related with performance in memory tasks.

Participants and Methods: A sample of 120 individuals, dividing according to three age groups (7, 12 and 22 years) participated in this study. Four sort-recall tasks requiring individuals to group items according to 1) any strategy they want (spontaneous condition), 2) size (shallow level condition), 3) phonology (intermediate level condition), and 4) semantics (deep level condition) were used. Items were presented either as line drawings or as words. Levels of recall, clustering during recall, and metamemory (accuracy of subject's estimates of their own memory) were assessed. 
Results: Data was examined in a 4 (conditions) X 3 (age groups) X 2 (employed materials) analysis of variance, with repeated measures on the condition factor. Levels of recall, clustering, and metamemory increased with age. Even though children had a poorer performance than their older counterparts, using a semantic strategy equalized levels of recall between adjacent age groups. In adults, the lowest level of recall was observed in the intermediate (phonological) level, instead that in the shallow (size) level, as could be expected. Using line drawings, compared with words, increased level of recall in all age groups.

Conclusions: Metamemory, type of material, and levels of processing can contribute to memory performance. Deliberated use of semantic strategies can minimize differences among age groups.

Correspondence: Maria E. Gomez, Univerity of Guanajuato, Av. Las Rosas \#501, Col. Jardines del Jerez, Leon, Guanajuato 37530, Mexico. E-mail:esthergp@fulbrightweb.org.

J. PYUN, Y. KANG \& Y.J. KIM. A Comparison of the Prospective Memory among College Students, Old Adults, and Parkinson's Disease Patients.

Objective: This study was conducted to examine the effects of normal aging on the prospective memory and the relationship between the prospective memory and the frontal lobe functions.

Participants and Methods: The subjects were 30 college students (CS), 30 normal older adults (OA), and 30 Parkinson's disease patients (PD). There was no significant difference in the mean age or education level between the OA and the PD. The Cambridge Prospective Memory Test and the Prospective and Retrospective Memory Questionnaire were used to evaluate the prospective memory. The Seoul Verbal Learning Test and the Rey Complex Figure Test were administered to assess the verbal and visual episodic memories. The subjects were also given the Iowa Gambling Task, Sorting Test, and the Korean-Color Word Stroop Test to assess the frontal lobe functions.

Results: The results showed that the prospective memory declines with aging. The OA showed significantly lower scores on the time-based prospective memory than the event-based prospective memory, although the CS did not show any differences between them. The significant correlations between the prospective memory and the episodic memory were found in the OA, but not CS. Also, many significant correlations were found between the prospective memory tests and the frontal lobe tests in the $\mathrm{OA}$ and the PD, only a few correlations were found between them in the CS. From the localization of the frontal lobe tests showing significant correlations with the prospective memory, it was inferred that the prospective memory is modulated by the more anterior part of the dorsolateral prefrontal areas in the CS, whereas it is modulated by both the dorsolateral and the medial prefrontal regions in the $\mathrm{OA}$ and the PD. Conclusions: These quantitative and qualitative changes in the prospective memory by aging and/or frontal lobe dysfunction would support the Compensation Hypothesis (Cabeza et al., 2002) or the Dedifferentiation Hypothesis (Baltes \& Lindenberger, 1997).

Correspondence: Yeonwook Kang, Ph.D., Psychology, Hallym University, Okchon-dong, Chuncheon 200-702, South Korea. E-mail: ykang@hallym.ac.kr

H. KIKUCHI, T. FUJI, N. ABE, M. SUZUKI, S. MUGIKURA, S. TAKAHASHI \& E. MORI. Brain Mechanisms Underlying Dissociative Retrograde Amnesia: an fMRI Study.

Objective: Dissociative (psychogenic) amnesia is thought to reflect a reversible retrieval deficit, affecting conscious access to memories that remain available in storage. We investigated a patient using functional magnetic resonance imaging (fMRI) to determine the brain mechanisms underlying this type of memory disorder.

Participants and Methods: The participant was a 27-year-old man who suffered from an isolated retrograde amnesia for the preceding four and a half years. He was diagnosed to be dissociative retrograde amnesia. To recover his memory, we performed interviews using sodium thiopental, and his memories in the beginning 4 years of amnesic period were successfully recovered. In fMRI experiments conducted before and after the treatments, he was visually presented with three categories of face photographs or names: those of "Recognizable" high school friends, "Unrecognizable" colleagues due to his memory impairment, and "Control" distracters with whom the patient was unfamiliar. He was asked to indicate whether the faces or names were familiar to him, and if he judged the person as familiar, he was further asked to recall past events associated with that person.

Results: The results obtained before the treatments showed that increased activation of the right dorsolateral prefrontal cortex (DLPFC) and decreased activation of the left hippocampus were found while the patient was viewing "Unrecognizable" stimuli compared with "Recognizable" ones. Neither of these brain activities was found after the treatments.

Conclusions: These findings indicate that the right DLPFC activation and the left hippocampus deactivation are associated with involuntary memory suppression in dissociative amnesia.

Correspondence: Hirokazu Kikuchi, Behavioral Neurology and Cognitive Neuroscience, Tohoku University, 2-1 Seiryomachi Aobaku, Sendai 9808575, Japan.E-mail:kiku0215@mail.tains.tohoku.ac.jp

F. LUCCHELLI, I. BIZZOZERO, P. FAGLIONI, M.C. SAETTI \& H. SPINNLER. Processes Underlying the Temporal Gradient in Mild Cognitive Impairment and Alzheimer's Disease: A Latent-Variable Stochastic Analysis.

Objective: In a recent study (Bizzozero et al., in press) the issue of Temporal Gradient (TG) for memory of public events was investigated in healthy people. The implementation of a Markov chains stochastic model, a device specifically suited to investigate latent variables underlying observable cognitive processes, proved successful to provide evidence of retrieval inefficiency for recent memories in normal elderly people (over 75 years). The presence and the extent of TG in Alzheimer's disease (AD) is still a matter of controversy. Moreover, the issue has never been systematically investigated in Mild Cognitive Impairment (MCI), a condition deemed at risk for developing $\mathrm{AD}$.

The aims of the present study were (i) to assess the presence of TG, if any, in MCI and AD patients; (ii) to identify the relative contributions of storage and retrieval processes. The prediction was made that storage should be disproportionately affected in $\mathrm{AD}$ as a result of the extension of the pathology to the neocortex, while in MCI, where the pathological process is still confined to the hippocampal complex, TG should be sustained almost entirely by a retrieval defect.

Participants and Methods: Twenty-two MCI patients and $21 \mathrm{AD}$ patients participated in the study. Memory for public events was examined by a recently developed test (Bizzozero et al., 2004; 2005). A Markov chains stochastic analysis was performed to estimate retrieval and storage.

Results: Eighteen (81\%) MCI and 20 (91\%) AD patients showed evidence of pathological TG. The stochastic analysis demonstrated that storage was significantly impaired in $\mathrm{AD}$ with respect to MCI patients, while retrieval did not differ significantly. Furthermore, in AD patients storage was significantly affected for remote events to a much greater extent than for recent events.

Conclusions: These results tie in with our predictions and are interpreted within the framework of current models of memory consolidation and the purported role of the hippocampus and neocortical structures. Correspondence: Federica Lucchelli, MD, PhD, Neurological Rehabilitation, Alzheimer's Disease Center-AO Salvini, Garbagnate M., via Settembrini 1, Passirana di Rho (Milan) 20017, Italy. E-mail:flucch@libero.it

\section{E. MUÑOZ MARRÓN.VISUAL VERSUS VERBAL MEMORY DEFICIT IN SPINA BIFIDA AND HYDROCEPHALUS.}

Objective: Know the differences between the capacity of visual and verbal memory, in immediate and in delayed recoll, as well as the differences in general immediate memory, delayed memory and working memory in persons with spina bifida and hydrocephalus in order to know the areas with major difficulties and to sit the bases for an effective intervention. 
Participants and Methods: The sample is composed by ten subjects with spina bifida and hydrocephalus, with ages between 19 and 41 years ( 2 males and 8 women), users of the Center for Disabled Physicists of the Association of Madrid of Spina Bifida coordinated with the Community of Madrid. All of them have been assessed with WMS-III (Wechsler Memory Scale-III; Wechsler, 1997). From the punctuations obtained in the test there have been calculated the differences between the different indexes of both modalities -visual versus verbal-, as well as differences in general indexes that it offers (immediate memory, delayed memory and working memory)

Results: The results support the hypothesis of the existence of a major deterioration in the tasks that imply the managing of visual material opposite to the verbal one. This effect appears both in the immediate and delayed recall.

Conclusions: This fact agrees with previous finds in the scientific literature, though the comparison becomes difficult since the practical totality of this one has centred on infantile population and in hydrocephalus of different etiology.

Correspondence: Elena Muñoz Marrón, Asociación Madrileña de Espina Bífida, Ronda de Segovia, 18, 30 10, Madrid 28006, Spain. E-mail: elmuma@yahoo.es

M.G. O'CONNOR, B. LEBOWITZ, J. LY, M.S. PANIZZON, S. DEY, S. ELKIN FRANKSON, M. THALL, K. BLOOMINGDALE \& C. PEARLMAN. ECT and Memory Consolidation: A Dissociation between Anterograde and Retrograde Memory.

Objective: The aim of the present study is to investigate the cumulative effects of a clinically determined course of electroconvulsive therapy (ECT) on anterograde and retrograde amnesia. In this study mood and memory were examined in the context of a protocol driven by therapeutic response rather than by preordained research criteria.

Participants and Methods: The memory abilities of 22 ECT and 22 Nondepressed participants were compared. ECT candidates were examined before and after treatment using standard tests of anterograde memory (e.g., story recall) and a novel procedure for the examination of retrograde memory (face/name paired associates). Groups were matched for baseline learning, age and education. Unilateral and bilateral ECT participants were matched for severity of depression. Findings were analyzed with repeated measure analyses of variance.

Results: Treatment with ECT resulted in a dissociation between anterograde and retrograde memory: following treatment, MDD patients demonstrated significant retrograde amnesia whereas there was no change in their anterograde memory. Unilateral and Bilateral ECT patients performed equally well on tasks of anterograde memory and equally poorly on tasks of retrograde memory.

Conclusions: Results of the study suggest that ECT is associated with isolated impairment for information learned prior to treatment (i.e. retrograde memory), while having no impact on post-treatment learning abilities (i.e., anterograde memory). Further, the use of bilateral versus unilateral ECT stimulation as part of a clinical course of ECT did not result in greater memory loss for information acquired prior to treatment. Correspondence: Margaret G. O'Connor, Ph.D., Neurology, Beth Israel Deaconess Medical Center, 330 Brookline Avenue, Boston, MA 02115. E-mail:moconnor@bidmc.harvard.edu

S. PINHO \& M.R. SIMÕES.Verbal Memory Performance of Children with Learning Disabilities on the Coimbra Neuropsychological Assessment Battery.

Objective: To identify which aspects of verbal memory functioning are decreased in children with learning disabilities.

Participants and Methods: Two subtests of Coimbra Neuropsychological Assessment Battery (Logical Memory and Word Lists) were individually ad- ministered to a group of children with learning disabilities (most of them with dyslexia) and controls. Both groups had 37 participants ( 9 females and 28 males) for the Logical Memory subtest and 49 participants (15 females and 34 males) for the Word Lists subtest. The groups' minimum and maximum age were 10 years and 13 years, respectively (average $=10.06$ years). Results: A two-group ANOVA showed significant differences between children with learning disabilities and controls on the following scores of the Word Lists subtest $($ all $\mathrm{p}<.05)$ : second learning trial $[\mathrm{F}(1,96)=8.44]$; third learning trial $[\mathrm{F}(1,96)=20.35]$; fourth learning trial $[\mathrm{F}(1,96)=9.86]$; interference list recall $[\mathrm{F}(1,96)=22.05]$; immediate free recall $[\mathrm{F}(1,96)=11.12]$; delayed free recall $[\mathrm{F}(1,96)=9.29]$; recognition $[F(1,96)=27.80]$; commission errors $[F(1,96)=18.82]$; commission errors repetition $[\mathrm{F}(1,96)=6.03]$; total learning $[\mathrm{F}(1,96)=12.38]$; learning rate $[\mathrm{F}(1,96)=7.06]$; and proactive interference $[\mathrm{F}(1,96)=9.03]$. For Logical Memory subtest (two stories $\mathrm{C}$ and D) an ANOVA revealed that all the results between both groups were significantly different (all $\mathrm{p}<.05)$ : immediate recall-C $[\mathrm{F}(1,72)=7.73]$; immediate recall- $\mathrm{D}[\mathrm{F}(1,72)=15.74]$; delayed recall-C $[\mathrm{F}(1,72)=12.16]$; delayed recall-D $[\mathrm{F}(1,72)=18.70]$; recognition- $\mathrm{C}[\mathrm{F}(1,72)=4.60]$; and recognition $-\mathrm{D}[\mathrm{F}(1,72)=20.65]$.

Conclusions: Children with learning disabilities exhibit a general deficit in memory performance with verbal materials (words and stories). May be this deficit is only concerned with long-term verbal memory since the results don't show a significant difference between the groups for the first learning trial of List Words subtest.

Correspondence: Salome Pinho, PhD, Faculty of Psychology, University of Coimbra, Rua do Colégio Novo, Apartado 6153, Coimbra 300-801, Portugal.E-mail: salome@fpce.uc.pt

Y.T. QUIROZ, K. SCHON \& C.E. STERN. Semantic Context Interference During a Working Memory Task.

Objective: Semantic category interference effect has been repeatedly observed during picture naming. This effect implies people are slower when naming pictures out of a sequence of items from the same semantic category than those from different categories. We tested this effect in the context of working memory using a Sternberg Task.

Participants and Methods: The stimuli consisted of 150 simple line drawings of everyday objects presented in two different conditions. In the first condition, only items from the same category (SCT) were used, and in the second condition items belonging to different semantic categories (RCT) were used. In each trial, 10 subjects viewed a short list of objects that was followed by a brief memory delay. After the delay, a probe was presented and the subjects had to indicate whether it matched one of the list items (retrieval).

Results: Overall, paired-sample t-tests revealed that reaction times (RT) for SCT trials were significantly longer than those of RCT trials. As expected, RTs for SCT nonmatch trials were significantly longer than RTs for RCT nonmatch trials. In addition, when the probe item was from the same category, but not when it was from a different category during SCT nonmatch trials, RTs were significantly longer compared to those of RCT nonmatch trials.

Conclusions: These findings indicated that working memory was modulated by semantic context and that there was semantic competition but not semantic facilitation during working memory retrieval. Our results are consistent with previous studies that have reported semantic interference as a consequence of lexical/semantic competition. Funded by NSF SBE-0354278 Correspondence: Yakeel Quiroz, Psychology, Boston University, 2 Cummington Street, S109, Boston, MA 02215.E-mail:yquiroz@bu.edu

T. RASPALL, N. BARGALló, C. FALCON, T. BOGET, T. PRIETO, A. DONAIRE, M. DONATE, M. SALAMERO, J. RUMIA, X. SETOAIN, L. PINTOR, I. MAESTRO \& M. CARRENO. Reliable Mesial Temporal Lobe Activation Using a Classical Neuropsychological Memory Paradigm. A fMRI Study.

Objective: Memory function assessment is useful for the diagnosis and long term follow up of some neurological diseases such as temporal lobe 
epilepsy or Alzheimer's disease. Declarative memory depends on the integrity of the mesial temporal lobe (MTL) regions. The Rey AuditoryVerbal Learning Test (RAVLT) is a verbal paradigm that has demonstrated to be a useful tool to evaluate verbal learning and memory during routine neuropsychological testing. To demonstrate if the adaptation of a neuropsychological verbal memory test (RAVLT) into a functional Magnetic Resonance Imaging (fMRI) experiment could be useful to get some insight into the neural substrates engaged in verbal episodic memory. Participants and Methods: Forty-one right-handed healthy control subjects ( 23 women and 18 men) underwent a verbal memory fMRI task consisting on an adapted neuropsychological word learning test using a $1.5 \mathrm{~T}$ scanner. Both encoding and immediate recall tasks were evaluated. Hippocampal ROI and whole brain analysis was performed for both individual and group analysis, together with the correlation between outside scan delayed recall performance and hippocampal ROI activation. Results: Individual and group analysis resulted in significant differences in $\mathrm{T}_{2}$ * contrast over regions within the MTL, reaching the maximum value when comparing immediate recall relative to control condition. All subjects presented mainly bilateral hippocampal activation, although with a variable asymmetry ratio. Successful delayed recall was best predicted by left parahippocampal activation during immediate recall. Conclusions: This study demonstrates that a clinical neuropsychological memory task reliably activates MTL structures on both individual and group analyses. Moreover, the degree of activation within MTL structures predicted the subsequent successful delayed recall.

Correspondence: Toni Raspall, Hospital Clínic i Provincial de Barcelona, Rossellón0 140, Barcelona 08022, Spain.E-mail:toni_raspall@yahoo. es

L. FAÍSCA, I. BRAMÃo, C. FORKSTAM, A.I. REIS \& K. PETERSSON. Implicit learning of structured auditory sequences: An advantage for verbal stimulus.

Objective: Implicit artificial grammar learning (AGL) investigates the human capacity to implicitly extract abstract structural regularities from experience. Several studies suggest that this kind of implicit learning mechanisms might also be involved in language acquisition. In this study we investigated the effect of Portuguese syllable- and pure tone auditory stimulus on implicit learning of an artificial syntactic structure.

Participants and Methods: During the acquisition period (five days), forty participants were engaged in a working memory task: subjects were exposed to sequences of sounds generated from a Reber grammar (with no awareness of its existence). Half of the subjects heard sequences of Portuguese syllables and the other half sequences of pure tones. After the acquisition phase, the participants' knowledge of the underlying grammatical structure was assessed by subjective preference classification as well as grammaticality classification with items never heard before $(50 \%$ grammatical, generated from the Reber grammar, and $50 \%$ non-grammatical, derived from grammatical string by switching two non-terminal positions).

Results: Overall correct performance was higher for subjects exposed to syllables compare to pure tones $(\mathrm{P}=.01)$. Both groups performed clearly above chance level during the grammaticality classification on day $5(\mathrm{P}<.001)$. However, during the prior preference classification on day 5 , learning effects were exclusively observed for the syllable group $(\mathrm{P}=.01)$.

Conclusions: These results suggest that both groups acquired knowledge about the underlying generative grammar once informed about the structured nature of the stimulus material (grammaticality classifications). However, during the prior preference classification test, this effect was only observed for syllables, suggesting that verbal stimuli can facilitate implicit acquisition of an artificial grammar.

Correspondence: Alexandra I. Reis, PhD, Departamento de Psicologia, Universidade do Algarve, Universidade do Algarve, Campus de Gambelas, Faro 8000-810, Portugal. E-mail: aireis@ualg.pt
M.R. SIMÕES, S. PINHO \& I. ALBERTO. Assessment of Non-Verbal Memory in Children with Learning Disabilities on the Coimbra Neuropsychological Assessment Battery.

Objective: To assess long-term memory and short-term/working memory for non-verbal information in a group of children with learning disabilities. Participants and Methods: Non-verbal long-term memory and visual short-term/working memory were assessed in a group of children with learning disabilities and controls (each group with $n=49$ ). The average age of both groups was 10.06 years (the minimum and maximum ages were 10 years and 13 years, respectively). The tests administered belong to Coimbra Neuropsychological Assessment Battery and were the following: Faces Recognition, Rey-Osterrieth Complex Figure, Corsi Blocks, and Trail Making (A and B).

Results: The significant results (all $\mathrm{p}<.001$ ) present in ANOVA were observed on the Rey-Osterrieth Complex Figure Test Copy $[\mathrm{F}(1,96)=13.89]$; Corsi blocks $[\mathrm{F}(1,96)=21.90]$; and Trail making time $[\mathrm{F}(1,96)=23.47$ and $\mathrm{F}(1,96)=46.26$ for $\mathrm{A}$ and $\mathrm{B}$, respectively $]$. It were not found significant differences for the other scores.

Conclusions: Significant differences were not registered between children with learning disabilities and controls concerning non-verbal longterm memory (Faces Recognition and Rey-Osterrieth Complex Figure Tests). However, children with learning disabilities has a lesser spatial span and are faster in Trail making test which assesses the central executive component of working memory.

Correspondence: Salome Pinho, PhD, Faculty of Psychology, University of Coimbra, Rua do Colégio Novo, Apartado 6153, Coimbra 300-801, Portugal.E-mail: salome@fpce.uc.pt

F.F. MALERBI, C.I. SCHEUER, L. STIVANIN, C. OLIVEIRA \& E.R. ANDRADE. Comparison of ADHD and Typical Children in Implicit Memory.

Objective: OBJECTIVE: trace a profile of functioning of ADHD children and compare them with typical children in classical task of implicit memory: anagram solution (Srinivas e Roediger, 1990).

Participants and Methods: METHODS: two groups pared by age and sex: the experimental group composed by 21 ADHD male children, medicated, between 8 and 10, 11 years old (116,86 in months) and the control group with 25 typical children. Children of both groups could not present visual, auditory, neurological problems or school impairments. Implicit memory was tested through the anagram solution, composed by 15 words; linguistically balanced according to written and spoken Portuguese of Brazil. The subject had to read the word, and then separate and mixed phonemes of this word were given to the subject in order to reorganize and reconstruct the word. One (1) point was given to the correct reorganization and zero $(0)$ to error.

Results: RESULTS: Results pointed to worse performance of the ADHD group probably due to difficulties to memorize and reorganize graphemes, even when the word had been presented previously $(0,002)$.

Conclusions: CONCLUSION: Implicit memory, using this kind of task. was better in control group because children used both, semantic and phonology strategies to reorganize words.

On the other hand, ADHD children had more difficulties maybe attached to problems to read the entire word and identification and recognition of each of the phonemes that compose these words.

Correspondence: Fernanda F. Malerbi, department of physical therapy, speech-language patology and occupational therapy, University of São Paulo, benjamim ferreira, 179, São Paulo 02433110, Brazil. E-mail: fernandamalerbi@hotmail.com

M. SUZUKI, R. HASHIMOTO, K. HIRAYAMA, N. ABE, A. UENO, M. TASHIRO, E. MORI, M. ITOH \& T. FUJII. The neural network for temporal order memory retrieval: evidencefrom lesion and PET studies. Objective: Previous studies have shown that the lateral prefrontal cortex and basal forebrain (and/or orbitofrontal cortex) play an im- 
portant role in the retrieval of temporal information of experienced events. We combined neuropsychological and positron emission tomography (PET) imaging studies to investigate whether these regions contribute differentially or commonly to the retrieval of temporal order memories.

Participants and Methods: Participants were a 55-year-old male patient with lesion in the left basal forebrain and orbitofrontal cortex and 14 healthy young male volunteers (mean age: 21.3 years). They experienced a various kind of mini-events (ex. sketched a spoon.) on the 1st and 2nd days of the experiment. On the 3rd day, they were presented with two stimuli, each of which were used in one mini-event, and were asked to judge which stimuli they used recently. We prepared three temporal order retrieval tasks: smallscale, medium-scale, and large-scale tasks. Each task differed in the scale of temporal distance between the two events the participants experienced. Results: In the PET study, we found that left prefrontal activity was associated with the small-scale task, whereas right prefrontal activity with the medium-scale task. In addition, the basal forebrain was activated both in the medium-scale and large-scale tasks, but not in the small-scale task. Critically, in the lesion study, the patient showed a deficit only in the large-scale task.

Conclusions: Our results indicate that the retrieval process for temporal order of experienced events varies depending on their temporal distance, and is supported by different brain networks.

Correspondence: Maki Suzuki, Cyclotron and Radioisotope Center, Tohoku University, 6-3, Aoba, Aramaki, Aoba-ku, Sendai 980-8578, Japan.E-mail:smaki@mail.tains.tohoku.ac.jp

E. TRAMONI, S. KHALFA, J. RANJEVA, M. GUYE, E. BARBEAU \& M. CECCALDI. Can Psychogenic Amnesia Occur without Concomitant Brain Damage?

Objective: Psychogenic amnesia is a massive disorder of autobiographical memory that appears suddenly and that is thought to be related to emotional or psychological traumata rather than to organic cause. We explore this hypothesis in a single case study of a patient presenting severe psychogenic autobiographical amnesia.

Participants and Methods: PP, a 34 year-old man consulted for selective alteration of remembering, which started in a severe and brutal way. Electrophysiological and neuropsychological standard assessments were normal $(\mathrm{QI}=115 ; \mathrm{QM}=139)$. Nevertheless, PP showed a complete inability to recall autobiographical memories as well as any fact or event related to himself. Electrodermal (ED) recordings were obtained in PP while he watched and explicitly judged photographs of personal past events and unfamiliar matched photographs as familiar or unfamiliar. His wife and six other control subjects underwent the same protocol.

$\mathrm{PP}$ and 26 controls were imaged with a 1.5-T MRI. Conventional magnetic resonance images (T1 and FLAIR) and magnetization transfer ratio (MTR) maps were obtained.

Results: PP explicitly judged as familiar only $27 \%$ of familiar photographs while controls performed at ceiling. However, like controls, PP showed a significantly enhanced ED response to familiar compared to unfamiliar photographs.

The anatomical image did not reveal any abnormality. However, selective decrease of MTR in right dorsal longitudinal fascicle (within the white matter of the prefrontal lobe, anterior and lateral to the corpus callosum genu) was found (Anova, $\mathrm{p}<0.005$ ).

Conclusions: These results suggest that PP has preserved implicit processing for non recalled personal memories. Therefore, PP's amnesia could be explained by a mnestic block with a psychogenic origin. However a confined brain lesion evident only by MTR imaging was found in lateral prefrontal regions, which are implicated in the retrieval of autobiographical memories. These results thus question the relationship between brain damage and psychogenic amnesia.

Correspondence: Eva Tramoni, INSERM U751, faculté de médecine, 27 bd jean Moulin, Marseille 13385, France. E-mail: eve. tramoni@medecine.univ-mrs.fr
M. VAN ASSELEN, R. ANDRÉ, I. ALMEIDA, A. FREIRE GONÇALVES, C. JANUÁRIO \&. M. CASTELO-BRANCO. Implicit context learning in patients with Parkinson's disease.

Objective: In order to locate a specific object or event in our environment, the human attention mechanism can quickly prioritize important information. Recently, it was shown that attention is not only drawn by specific features in our environment (bottom-up process), but can also be guided by memory (top-down process). That is, a visual search can become more efficient when the specific context is encountered for the second time. Importantly, the underlying neural correlates of this process are largely unknown. Since the nature of this process is implicit, a set of brain regions that might have an important role are the basal ganglia, which are essential for implicit motor and sequence learning. Participants and Methods: Parkinson patients ( $\mathrm{n}=16 ; 4$ excluded) in an early stage of the disease and age and education matched controls $(n=28)$ were examined with a context cueing task. This is a visual search task involving both repeated and new configurations. Response times and eye-movements were measured.

Results: Overall, it was found that Parkinson's patients are slower than the controls $(p<.05)$. In contrast, both groups showed an equal extend of sensorimotor learning $(p<.01)$, as was reflected in a decrease in response times during the experiment. Finally, whereas the control subjects have faster response times in the repeated trials $(p>0.05)$, no such difference was found for the Parkinson's patients.

Conclusions: The current approach allows investigation of higher-order, non-motor functions of the basal ganglia. Results suggest an important role for the basal ganglia in implicit context learning. Correspondence: Marieke van Asselen, PhD, Faculty of Medicine, IBILI - University of Coimbra, Azinhaga de Santa Comba - Celas, Coimbra 3200-354, Portugal.E-mail: masselen@ibili.uc.pt

\section{A.L. ZANINOTTO, C.R. CRUZ \& O.F. BUENO. ARTICULATORY SUPPRESSION EFFECT ON WORKING MEMORY THROUGHT FREE RECALL TEST.}

Objective: Investigate the articulatory suppression on the recency effect using pseudowords and common words lists.

Participants and Methods: Subjects: 60 graduates and undergraduates, both sexes, aged between 20 and 45 years. Twelve lists were prepared with 15 words each. The last three words were pseudowords with high associability with common words (H), low associability (L) or common words (C) presented randomly. In Experiment 1 ( $\mathrm{n}=30)$ the lists were presented visually while the subjects were articulating loudly the syllable "te" articulatory suppression (VIS-AS) or not (control procedure (VIS-N). In Experiment $2(\mathrm{n}=30)$ the lists were auditorily presented and the subjects were also under articulatory suppression (AUD- AS) or not (AUD- N).

Results: Repeated measures ANOVA showed a recency effect in all conditions $(p<0,05)$. Visual groups (VIS-AS and VIS-N) did not show any difference on recency effect between $\mathrm{H}$ and $\mathrm{C}$ lists, but $\mathrm{L}$ lists without suppression were more recorded than $L$ lists with interference by the articulatory suppression $(p<0,05)$. Auditory groups (AUD-AS and AUD$\mathrm{N})$ showed a difference in the recency effect: $\mathrm{C}$ were more recalled than $H$ and $\mathrm{L}(\mathrm{p}<0,05)$.

Conclusions: $\mathrm{C}$ words in the last three serial positions induced a larger recency effect than $\mathrm{L}$ pseudowords, but recency of $\mathrm{H}$ pseudoword can be manipulated in same conditions. It seems that the recency effect depends not only on phonological loop but also on semantic short-term memory. Correspondence: Ana L. Zaninotto, Universidade Federal de São Paulo - Unifesp, Rua Araújo 79 ap 60, São Paulo 01220-020, Brazil. E-mail: ana.lcz@hotmail.com

A. BERARDI, C. PAPOT, O. HABERT \&. A. PRUSKI. Assessment of Spatial and Motor Abilities in Patients with Stroke and Multiple Sclerosis using a Virtual Reality Environment.

Objective: The aim of this study was to use Virtual Reality (VR) to assess spatial and motor abilites in 20 wheelchair patients. 
Participants and Methods: Ten patients with stroke and 10 with multiple sclerosis (MS) were compared to 10 age, sex and education-matched healthy controls (all $\mathrm{F}<1$ ). The groups' experience with computers, joysticks and videogames was comparable (all p >.33). Participants moved in a VR environment on a simulated wheelchair controlled by a joystick; they carried out several paths following a wall to the right or to the left or going through an empty space. Dependent measures were the time needed to carry out each path and the number of errors. Results were analyzed using ANOVAs.

Results: Stroke patients took longer $(p=.01)$ and made more errors $(p=.006)$ than MS patients and controls overall, and were slower when following a wall on the left side $(p=.05)$. All participants were faster moving through an empty space $(\mathrm{p}=.01)$. Stroke patients made more errors than controls and MS patients in crossing out bells, a measure of visual neglect $(\mathrm{p}=.0001)$, but did not make disproportionately more errors in the left visual field $(\mathrm{F}<1)$; MS patients did not differ from controls $(p=.26)$.

Conclusions: VR results suggest that stroke patients have difficulty following a wall in the left-side of space and that for all subjects it is easier to go through an empty space where no external proximal cues are present. In conclusion VR allows for a controlled in situ evaluation of cognitive and motor functions and may provide information complementary to classic neuropsychological assessment.

Correspondence: Anna Maria Berardi, Ph.D. in psychology, Department of Psychology, Paul Verlaine University - Metz, UFR SHA, Ile du Saulcy, Metz 57006,France.E-mail: berardi@univ-metz.fr

E.H. DE HAAN, G.M. NYS, M.J. VAN DER SMAGT \& T.C. NIJBOER. Brightness agnosia: a disorder of light- and darkness recognition. Objective: We report a patient with extensive brain damage involving the right hemisphere who demonstrated a severe impairment in the appreciation of brightness.

Participants and Methods: We evaluated basic visuosensory perception of brightness, colour, shape, and motion. In addition, we tested the recognition of brightness, colour and common objects.

Results: Visuosensory perception was intact for all primary cues tested. Notably, her acuity as well as luminance matching was normal, suggesting her brightness impairment is not a mere consequence of lowlevel sensory impairments. She was not able to indicate the darker or the lighter of two grey squares, even though she was able to see that they differed. In addition, she could not indicate whether the lights in a room were switched on or off, nor was she able to differentiate between normal greyscale images and inverted greyscale images. As the patient recognised objects, colours, and shapes correctly, the impairment is specific for brightness.

Conclusions: This is the first report of a selective deficit in brightness recognition or 'brightness agnosia'.

Correspondence: Edward H. De Haan, PhD, Psychological Laboratory, Utrecht University, Helmholtz Institute, Heidelberglaan 2, Utrecht 3485CS, Netherlands.E-mail: e.dehaan@fss.uu.nl

P. MONTORO, D. LUNA \& G. HUMPHREYS. Effect of density and connectedness of local elements on the perception of hierarchical patterns: evidence from a simultagnosic patient.

Objective: Simultanagnosia is a rare deficit caused by bilateral parietooccipital brain damage that impairs the perception of several objects at the same time. The mechanisms underlying this deficit are not well understood. A previous study (Huberle \&. Karnath, 2006) using hierarchical stimuli (Navon, 1977) showed that the recognition of the global letter in simultagnosics patients improved when the distance between the local elements was reduced. However, a confound between inter-element distance and number of local elements could invalidate the conclusions. The aim of the present study was to examine the performance of a patient with simultanagnosia, G.K., using compound stimuli in order to study effects of grouping on his ability to report relevant levels of form.
Participants and Methods: The participant was G.K., a 67 year-oldpatient with simultanagnosia. We conducted an experiment using Navon paradigm with compound stimuli composed of either many or relatively few local elements under two different conditions: disconnected or connected, making use of the principle of connectedness grouping.

Results: Both density and connectedness had an effect on the identification of global and local stimuli. Dense (connected and disconnected) and sparse connected stimuli caused greater interference on the identification of the local level than sparse and disconnected stimuli. The effects were observed in accuracy, and, under conditions of strong grouping, the normal advantage for perceiving local over global forms could be completely reversed

Conclusions: The results showed that the strength of grouping among local elements determines the explicit recognition of global stimuli in simultanagnosia, and that such patients are not restricted by a limit spatial window of attention. Moreover, the data suggest that simultanagnosia involves a degree of attentional capture by salient stimuli, in addition to any bias to attend to the local level.

Correspondence: Pedro R. Montoro, Universidad Nacional de Educacion a Distancia, Juan del Rosal, 10, Madrid 28040, Spain. E-mail: prmontoro@bec.uned.es

M. O'REILLY, J. O'BRIEN, A. JOHNSTON, H. HILL \& J. SPENCER. Infant's Use of Facial Motion to Discriminate Gender.

Objective: Face perception is fundamental to the development of social interaction skills. Spencer et al. (2006) previously demonstrated that 5-to-8-month old infants can discriminate individuals purely on the basis of their facial motion. We aim to determine if infants can use facial motion to determine an individual's gender.

Participants and Methods: We examined six* infants at 5-, 7- and 9months using computer-generated facial animations created using motion-capture information from real human faces. We familiarised infants to animated faces from either a male or female actor telling a joke. In the test phase infants were presented with two novel stimuli, a sequence from a male actor telling a novel joke and a female actor telling a novel joke. If infants look longer at the novel gender, we can conclude that they can discriminate between male and female motion sequences.

Results: Familiarisation phase: The mean looking time for the first 2.5 trials was compared to the final 2.5 trials. A 2 (trial block) x 3 (age) repeated-measures ANOVA revealed no effect of trials or age (all $p$ 's $<0.05)$ on looking time.

Test phase:

A 2 (stimulus type) x 3 (age) repeated-measures ANOVA showed no effect of stimulus condition i.e. novel/familiar or age (all p's $>0.05$ ) on looking time.

Conclusions: Whilst the present sample size renders interpretation of the data difficult, the results of this study will further our understanding of how babies perceive faces in general, and how they use dynamic facial information to distinguish between individuals.

* Data collection is currently ongoing; at least 30 infants shall comprise the final study sample

Reference:

Spencer, J, O'Brien, J, Johnston, A., Hill, H. (2006). Infant's discrimination of faces by using biological motion cues. Perception, 35, p79-89. Correspondence: Michelle O'Reilly, Centre for Research in Infant Behaviour, Brunel University, School of Social Sciences, Brunel University, Uxbridge UB83PH, United Kingdom.E-mail:Michelle.OReilly@brunel.ac.uk

\section{Neglect}

G.M. NYS, M. STUART \& C. DIJKERMAN. Mechanisms of Perseverative Behaviour Towards Ipsilesional Locations in Patients with Hemi-neglect.

Objective: About $50 \%$ of neglect patients show ipsilesional target reexploration on standard neglect tasks and in daily life. The present study 
aims to examine which of three potential mechanisms may account for the frequent revisitings, i.e. (i) implicit knowledge of the contralesional side (ii) perseveration in general or (iii) disengagement failure.

Participants and Methods: Thirteen patients with left-sided neglect and nine healthy age- and education-matched controls were tested with three versions of the Bells test on touch screen, i.e. one standard cancellation in which targets had to marked (target-mark), one erase cancellation in which targets had to be erased (target-erase), and a third condition in which all items (including distracters) had to be erased to examine the influence of distracters (full-erase). Finally, a vertical touch screen version of the Brixton Spatial Anticipation was administered to assess the relation with executive dysfunction in general.

Results: Whereas omissions decreased in the full-erase condition only (confirming the influence of stimuli in non-neglected space on neglect, see Mark et al., Neurology 1988), revisitings were the most prominent in this condition and correlated with executive dysfunction in general. Conclusions: This is the first study to show that patients revisit ipsilesional target locations even when stimuli are no longer there. This finding in the full-erase condition resembles the daily behaviour in neglect patients, such as repetitive grasping in empty space or deviation of head and eye movements although no ipsilesional stimuli are present. Our results suggest that comorbid executive dysfunction is the underlying mechanism triggering this pathological behaviour.

Correspondence: Gudrun M. Nys, PhD, Laboratory for Neuropsychology, Ghent University, De Pintelaan 185 (4K3), Ghent B9000,Belgium.E-mail: gudrun.nys@ugent.be

\section{STAMENOVA, E. ROY, M. MASELLIS \& S. BLACK. Hemisphere effects in gesture production in patients with Corticobasal Syndrome.}

Objective: Corticobasal Syndrome is a progressive neurodegenerative disorder characterized by asymmetrical akinetic rigidity, cortical sensory loss, alien limb syndrome and apraxia. Our previous work has shown that CBS patients are more impaired on imitation than pantomime and that their imitation performance does not improve when given a verbal cue for the gesture's function. Stroke studies have shown that the right and left hemispheres play complementary roles in the control of movement. Therefore, we were interested in examining whether the performance of CBS patients would differ on these apraxia tasks depending on the brain side more affected by the disease.

Participants and Methods: Nine patients were included in the study, 3 with left and 6 with right hemisphere involvement. Patients were assessed on 3 transitive gesture tasks: pantomime, imitation and imitation with verbal cueing.

Results: No differences in relation to hemisphere affected were observed in overall performance which showed a main effect whereby imitation was worse than pantomime. In addition, an interaction trend $(p=.06)$ showed that verbal cueing affected the 2 groups differently: the verbal cue improved the performance of right hemisphere-predominant group but performance declined in the left hemisphere-predominant group. Conclusions: Consistent with our previous work CBS affects performance more in imitation. Interestingly, this pattern of apraxia appears regardless of the hemisphere affected and verbal cuing during imitation affects the two groups differently. The improvement in the right-hemisphere patients may reflect their accessing the semantic representations of the gestures in the relatively intact left hemisphere. The decline in the lefthemisphere-patients may have been due to the limited capacity of the already damaged left hemisphere for processing both language and praxis. Correspondence: Vessela Stamenova, PhD (C), University of Toronto, 43 BaybrookCres.,Toronto,ONM1H2R7, Canada.E-mail:viussi@gmail.com

\section{Other}

N. ABE, H. ISHII, T. FUJII, A. UENO, E. LEE, T. ISHIOKA \& E. MORI. A peculiar form of delusional misidentification: a neuropsychological case study providing a window on person identification.

Objective: To investigate the nature of a novel form of delusional misidentification syndrome.

Participants and Methods: The patient was a 74-year-old right-handed woman who exhibited a peculiar form of delusional misidentification due to Alzheimer's disease combined with idiopathic normal pressure hydrocephalus. The pronounced symptom of this patient was that she often misidentified her daughters as her sisters. We conducted comprehensive neuropsychological assessments and a series of quantitative experiments on her ability to identify person.

Results: She showed severe atrophy of bilateral medial temporal lobes as well as right-hemisphere-dominant fronto-temporo-parietal hypoperfusion. On neuropsychological testing, she showed profound deficits in executive function and episodic memory. Detailed experiments revealed that she had a selective deficit in retrieving the blood relationships between herself and her daughters/husband (i.e., she misidentified her daughters as sisters and her husband as father), despite being able to retrieve the names, faces, and some person-specific semantic information (e.g., occupation) for her family members.

Conclusions: These results showed evidence for the impairment restricted to the retrieval process of the blood relationships on close relatives, providing a new perspective for the understanding of the cognitive and neural mechanisms of person identification.

Correspondence: Nobuhito Abe, Behavioral Neurology and Cognitive Neuroscience, Tohoku University, 2-1, Seiryo-machi, Aoba-ku, Sendai 980-8575,Japan.E-mail:n-abe@umin.ac.jp

\section{Paper Session 7 5:15-6:15 p.m.}

\section{Cross-cultural Issues}

F. CONSTANTINIDOU, M. KOUSHIOU \& E. CONSTANTINOU. Age and Language effects on Word Fluency tasks: A cross-cultural comparison between Greek-Cypriot and American-English adults. Objective: To determine the influence of age and language on Semantic and Phonemic Word Fluency performance in a sample of young and older Greek-Cypriot and American-English adults.

Participants and Methods: Four groups participated: young Cypriots (mean age $=26.5, \mathrm{SD}=7.6, \mathrm{~N}=34$ ), older Cypriots (mean age $=67.1$, $\mathrm{SD}=8.1, \mathrm{~N}=26$ ), young Americans (mean age $=25.6, \mathrm{SD}=5.8, \mathrm{~N}=$ 16) and older Americans (mean age $=70, \mathrm{SD}=6.4, \mathrm{~N}=32$ ).

Results: T-tests $(\mathrm{a}=.05)$ showed no significant age differences in the Cypriot or in the American sample. Cross-cultural comparisons revealed that older Americans scored significantly higher than older Cypriots on Semantic $(p<.01)$ and Phonemic Word Fluency $(p<.01)$. Significant differences were also found in the young age group with Americans scoring significantly higher than their Cypriot cohorts but only on the Semantic Word Fluency $(p<.01)$.

Conclusions: Age was not found to influence performance on word fluency in both populations. In contrast, language had a significant effect on word fluency performance. The differences found between Cypriots and Americans can be attributed to the multi-syllabic nature of the Greek language and to other cultural factors such as differences in concept organization and task familiarity. Therefore, language and culture specific norms are required for an objective measure of verbal ability processes (semantic storage and organization) as measured by word fluency tasks. Correspondence: Fofi Constantinidou, Ph.D., Psychology, University of Cyprus, 75 Kallipoleos St., P.O. Box 20537, Nicosia 1678, Cyprus. Email:fofic@ucy.ac.cy 
A. LOZANO \& F. OSTROSKY. Years of Education vs Quality of Education in a Spanish Speaking Sample.

Objective: To determine the contribution of years of education, reading comprehension, word accentuation and age to cognitive performance in a Spanish-speaking population.

Participants and Methods: 1614 participants were administered the NEUROPSI Neuropsychological Test Battery (Ostrosky, Ardila \& Rosselli, 1997, 1999), a reading comprehension measure (Ostrosky, 2000) and the Word accentuation test (Del Ser et al., 1997). Sample age ranged from 16 to 96 years $(x=51.01 ; S D=20.58)$ and level of education ranged from $0-24$ years $(x=5.96$ years; $S D=$ 5.82). All participants were functionally independent and any psychiatric or neurological impairment were discarded through a brief clinical history.

Results: A multiple regression analysis was performed to obtain the contribution of years of education, age, reading comprehension and word accentuation, to the NEUROPSI total score and to each subtest. Years of education $(\mathrm{r} 2=.45, \mathrm{~S} . \mathrm{E} .=13.4, \mathrm{p}<.001)$ and reading comprehension $(\mathrm{r} 2=.10, \mathrm{~S} . \mathrm{E} .=12.1, \mathrm{p}<.001)$ were the strongest predictor variables to the total NEUROPSI score and to each subtest, with years of education contributing more than reading in every subtest, and the amount of contribution varies depending on the function evaluated by the subtest.

Conclusions: Years of education and age contributed with more explained variance to cognitive performance than quality of education measures (reading comprehension or accentuation of words). Results are discussed in terms of how confounding variables affect cognitive performance and that among a native Spanish speaking sample other quality of education measures should be developed

Correspondence: Asucena Lozano, Universidad Nacional Autonoma de Mexico, Zaragoza Mz.12 Lt.10 \#10 Los Héroes, Ixtapaluca, Edo. Mex. 56531, Mexico.E-mail:azucena_lozano@hotmail.com

M.M. PADILLA-VELEZ, B. RAKITIN \& J. MANLY. Neuropsychological Tests Predict IADL Ability Differently Among Non-Demented Spanish And English Speakers In A Community Setting.

Objective: We sought to examine whether neuropsychological variables predict the ability to perform instrumental activities of daily living (IADLs) in community-dwelling elderly; particularly whether they predict differently in English and Spanish speaking people.

Participants and Methods: First-visit data was assessed for 1139 English-speaking and 500 Spanish-speaking (mostly Caribbean-born) nondemented elders in a longitudinal study. Those with dementia, stroke, or severe psychiatric disorder were excluded. Neuropsychological tests included: MMSE Orientation, Buschke Selecting Reminding (SRT; immediate \& delayed recall), Color Trails 1 \& 2 (CT; completion time), Benton Visual Retention (BVRT), WAIS-R Similarities, Boston Naming (BNT), Letter \& Animal Fluency, BDAE Repetition \& Comprehension. and the Rosen Drawing (RDT). IADLs were measured by self-reported assistance in medication-management, meal-preparation, bill-paying, and shopping. Age and education were covaried throughout.

Results: Examining the interaction between language and each neuropsychological measure in relation to IADLs revealed four tests with differential predictive power: Naming $(\mathrm{F}=5.302, \mathrm{p}=.021)$, Repetition $(\mathrm{F}=4.525, \mathrm{p}=.034), \operatorname{RDT}(\mathrm{F}=7.989, \mathrm{p}=.005)$, and CT $2(\mathrm{~F}=4.671$, $\mathrm{p}=.031)$. When language groups were separated, predictors of IADLs among English-speakers were: age $(\mathrm{F}=41.021, \mathrm{p}=.000)$, education $(\mathrm{F}=6.407, \mathrm{p}=.012)$, and CT $1(\mathrm{~F}=23.265, \mathrm{p}=.000)$; among Spanishspeakers predictors were age $(\mathrm{F}=18.783, \mathrm{p}=.000)$, RDT $(\mathrm{F}=9.703$, $\mathrm{p}=.002)$ and CT $1(\mathrm{~F}=8.340, \mathrm{p}=.004)$. R-squared for the individual language models were 092 (adjusted R-squared .086) for Englishspeakers, and .098 (adjusted R-squared .95) for Spanish.

Conclusions: These Results indicate that IADL abilities are not well quantified by neuropsychological testing in community dwelling eld- ers. It is further indicated that these abilities are predicted differently in English and Spanish speaking people.

Correspondence: Margarita M. Padilla-Velez, Ph.D., Neuroscience, Columbia University, 630 West 168th Street, Room 18-324, New York, NY 10032. E-mail:mpv@sergievsky.cpmc.columbia.edu

A.B. BILBAO, M. PUNTES ANSÓN, E. APALATEgUi, A. BIZE, A. REYERO, S. ROZAS, M. REHACER \& I. BOMBIN. The Spanish Hopkins Verbal Learning Test: Development, validation and standardization in Spanish population.

Objective: The aim of this paper is to present a Spanish version of the Hopkins Verbal Learning Test (HVLT). The HVLT is a well known widelly used instrument to assess learning and memory. A low time of administration and the avaliability of 6 equivalent forms make of the original HVLT an efficient tool to assess learning and memory for clinical and research purposes with all kinds of population who might become easilly fatigued (TBI, Dementia) or need multiple evaluations over short periods of time (clinical trials, rehabilitation).

Participants and Methods: 72 words that have the exact same meaning and pronunciation in 22 spanish speaking countries were selected from a list of over 300 words in order to develop 3 alternative equivalent forms. 60 patients with TBI, 10 patients with cerebral anoxia and 20 patients with Probable Alzheimer`s Disease were assessed using the HVLT for validation purposes. A sample of 272 normal controls representing educational, sex and age characteristics of Spanish population (according to the last census)participated in the standardization process.

Results: The three alternate forms showed to be equivalent. TBI patients showed significant differences in the HVLT when stratified using the Rancho Los Amigos Scale. In addition diferential profiles distinguished TBI patients from those with cerebral anoxia and probable AD. Conclusions: The Spanihs Version of the Hopkins Verbal Learning Test with its three alternative forms is a valid instrument that will allow a quick and efficient assessment of learning and memory of Spanish Speaking population for different purposes (research, rehabilitation, forensic and differential diagnosis).

Correspondence: Alvaro B. Bilbao, PhD, Neuropsychology, CEADAC, C/ Río Bullaque NO 1, Madrid 28034, Spain. E-mail: alvarobilbaobilbao@hotmail.com

\section{Paper Session 8 5:15-6:15 p.m.}

\section{Cognitive Rehabilitation}

\section{PAVAN KUMAR \& G.W. HUMPHREYS. Remediation of} Dysgraphia in Two Patients with Primary Progressive Aphasia (PPA). Objective: There have been an increasing number of reports describing the phenomenon of PPA in the last two decades. However, very few published studies have reported remediation work carried out with such patients. Our study hopes to describe successful long-term treatment protocols carried out with two patients with Non Fluent Progressive Aphasia (NFPA), thus demonstrating the feasibility and benefits of therapy regimes in such a population.

Participants and Methods: Initial assessment of our patients revealed surface dysgraphia in the absence of other cognitive deficits. Symptoms, in both patients, were localised to an impaired Graphemic Output Buffer (GOB). Two separate treatment protocols were designed and carried out to suit the patients' individual needs. The aim of the remediation program, was to treat written spelling impairment. We modified existing treatment programs that have proved successful in remediating spelling in patients having static lesions. We further added specific exercises aimed at strengthening the GOB. 
Results: Using sets of target and untreated words, we used multiple baselines to chart the progress of the treatment protocols. Results revealed significant improvement not only in the written spelling of target words but also found evidence of generalisation of treatment effects to other untreated word-sets.

Conclusions: Our study demonstrated that patients with progressive language disorders are amenable to remediation work, thus proving that the progressive nature of the disorder need not be seen as a limitation in itself. Both our patients benefited from the therapy they received and were able to maintain the effects of therapy up to two months post-treatment. Clinical and theoretical implications will be discussed.

Correspondence: Viba Pavan Kumar, Behavioural Brain Sciences Centre, School of Psychology, University of Birmingham, BBSC, Hills Building, School of Psychology, University of Birmingham, Edgbaston, Birmingham B15 2TT, United Kingdom. E-mail:vxp242@bham.ac.uk

\section{S. FERNÁNDEZ GUINEA \& M. DELGADO LOSADA. Benefits of a visualization strategy training in elderly people with memory complains.}

Objective: The aim of the study was to analize the long-term benefits of a memory training program. An ecological visualization strategy training was applied to a sample of elderly people with subjective memory complains but without neurologic o psychiatric diseases. The training focus on the abilities to create mental images of the material to be remembered without its explicit presence.

Participants and Methods: 130 elderly subjects were divided randomly in two groups of 65 people (experimental and control). A paired associated learning test and the visual reproduction (VR) subtest of the Wechsler Memory Scale III were applied to all subjects before and after training and six months later.

Results: In the post-training evaluation there were significative statistical differences between two groups in the following variables: Part I of paired associated test $(\mathrm{F}=6,732 ; \mathrm{p}<0,05)$, Part II of paired associated test $(\mathrm{F}=17,283 ; \mathrm{p}<0,05)$, Part III of paired associated test $(\mathrm{F}=12,339 ; \mathrm{p}<0,05)$, Total Amount $(\mathrm{F}=13,480 ; \mathrm{p}<0,001)$, long-term recall paired associated test $(\mathrm{F}=8,211 ; \mathrm{p}<0,05)$, long-term recall of paired difficult of paired associated test $(\mathrm{F}=8,211 ; \mathrm{p}<0,05)$, Figures Copy of VR $(\mathrm{F}=27,580 ; \mathrm{p}<0,05)$, short-term recall VR $(\mathrm{F}=32,773 ; \mathrm{p}<0,001)$, long-term recall VR $(\mathrm{F}=15,542 ; \mathrm{p}<0,001)$. The experimental group got better results than control group in all measures. These benefits maintained in the six months later evaluation in the following variables: Part I of paired associated test $(\mathrm{F}=24,411 ; \mathrm{p}<0,001)$, Part II of paired associated test $(\mathrm{F}=43,480 ; \mathrm{p}<0,001)$, Part III of paired associated test $(\mathrm{F}=42,716 ; \mathrm{p}<0,001)$, Total Amount $(\mathrm{F}=42,071 ; \mathrm{p}<0,001)$, Figures Copy of VR $(\mathrm{F}=44,222 ; \mathrm{p}<0,001)$, short-term recall VR $(\mathrm{F}=25,775$; $\mathrm{p}<0,001)$, long-term recall VR $(\mathrm{F}=16,591 ; \mathrm{p}<0,001)$.

Conclusions: A daily-living visualization technique training was useful for elderly people with subjective memory complains. The benefits maintained at least six months later the training because elderly people keep using this technique in their daily living activities.

Correspondence: Sara Fernández Guinea, Ph.D., Psicología Básica II (Procesos Cognitivos), Universidad Complutense de Madrid, Facultad de Psicología, Campus de Somosaguas, Madrid 28223, Spain. E-mail: sguinea@psi.ucm.es

M. VINK, R. HORST, M. LUIJPEN, K. MOLLEMAN, F. VAN DEN BERG \& A. BOUMA. Changes in Self-image, Coping and Social Participation after Holistic Neuropsychological Rehabilitation.

Objective: Goal of the present study is to investigate changes in perceived self-image, coping style and participation in social activities after participation in a holistic neuropsychological rehabilitation program Participants and Methods: Participants are a group of patients $(n=69)$ with chronic brain injury who participated in a 16-week intensive holistic group program. Patients and their relatives were assessed before participation in the program, after completing the program, and three years later. Assessment involved the measurement of self-image (using the Self-Image Questionnaire, consisting of 47 bipolar personal characteristics, of coping style (using the Utrechtse Coping Lijst, UCL). Social Participation was assessed by a structured interview.

Results: For each (sub)scale of the questionnaires, paired samples ttests were used to compare pre-treatment and post-treatment scores, and to compare the pre-treatment and 3-year follow-up scores. The SPSS-PC program was used for statistical analysis.

The findings suggest that self-image changes in a positive direction after completing the program. The UCL shows significant positive changes in the use of active coping styles. Social participation is improved. These changes were maintained at the 3 -year follow-up measurement.

Conclusions: From the results of the study it can be seen that perceived self-image did change following brain injury, which supports previous findings. The more positive self-image scores, after treatment and three vears later, support the concept that self-image is a dvnamic entity, which may be changed by experience. The increase in social participation after holistic neuropsychological rehabilitation corresponds with findings of other studies. The relation between changes in coping style, self image and social participation needs further investigation.

Correspondence: Martie Vink, Drs, Rehabilitation Center Amsterdam, Overtoom 283, Amsterdam $1054 \mathrm{HW}$, Netherlands. E-mail: m. vink@RCAmsterdam.nl

C. MATEER. It's just one brain: Integrating cognitive, metacognitive and affective interventions.

Objective: The combined impacts of traumatic brain injury on physical, cognitive, and emotional functioning are well known, but we are only beginning to recognize the degree to which these areas intersect with and impact each other.

Results: It is still common to conceptualize and develop rehabilitation strategies that address them separately with the result that important interactions can be missed. There is also related new research suggesting that the beliefs that individuals who have sustained a TBI hold about their cognitive functioning and the effects of their injuries strongly impact mood, adjustment, and engagement.

Conclusions: Treatment approaches that focus on reshaping these beliefs and that provide a more integrated treatment focus on cognition. metacognition, mood, and behaviour, including modified forms of $\operatorname{cog}$ nitive behaviour therapy, can be highly beneficial in an integrated and holistic approach to rehabilitation.

Correspondence: Catherine Mateer, Ph.D., University of Victoria, Department of Psychology, PO Box 3050, Victoria, BC V8W 3P5, Canada.E-mail:cmateer@uvic.ca

\section{Paper Session 9 5:15-6:15 p.m.}

\section{Symptom Validity in Dyslexia \& ADHD}

B.K. SULLIVAN. Symptom Exaggeration by College Adults in ADHD and Learning Disorders Assessments.

Objective: To test the hypothesis that sub-optimal effort detected by one popular symptom validity measure, the Word Memory Test, should be interpreted as symptom exaggeration.

Participants and Methods: The authors examined ADHD and Specific Learning Disability assessment data collected from healthy adult patients over the past four years at one mid-size Southeastern college. They conducted six tests of this hypothesis, drawing upon extant research.

Results: Rates of apparent symptom exaggeration comparable to those found in medicolegal settings (e.g. personal injury cases), particularly 
in the context of ADHD evaluations, were found. Word Memory Test scores were positively correlated with intellectual and neurocognitive test scores, and negatively correlated with self-report symptom inventory scores. Measures of negative response bias embedded in one common self-report measure of psychopathology (the Personality Assessment Inventory) were not correlated with WMT performance. Unattended WMT administrations led to somewhat higher failure rates than were found when the examiners were present in the room during all phases of the test's administration.

Conclusions: In light of considerable secondary gain motives in this population, the authors conclude that poor effort as evidenced by low WMT scores implies symptom exaggeration and not other factors in these assessments. The routine inclusion of empirically-supported symptom validity measures in post-secondary disability evaluations is recommended, and future research directions are suggested

Correspondence: Brian K. Sullivan, PsyD, Licensed Clinical Psychologist, Acting Associate Director \& Training Coordinator Counseling \& Substance Abuse Services College of Charleston, 175 Calhoun Street, Charleston, SC 29424, ph: 843.953 .5640 fax: 843.953 .8283

A.G. HARRISON. Identifying Students Faking either Dyslexia or ADHD: Preliminary Findings and Strategies for Detection.

Objective: When conducting psychological evaluations, clinicians typically assume that the subject being evaluated is putting forth maximal effort and is not exaggerating or magnifying symptom complaints. While the field of neuropsychology has identified that factors such as effort and motivation can significantly interfere with correct interpretation of self-reported symptoms and test scores, evaluation methods for other psychological conditions such as Attention Deficit Hyperactivity Disorder (ADHD) or Dyslexia have not addressed effort and motivation as potential factors influencing accurate diagnosis. This is worrisome, given that Dyslexia and ADHD are now the most-accommodated disabilities in the post-secondary sector of North America, with over half of all students with disabilities having one or both of these diagnoses.

Results: Results from two recent studies analyzing the performance of students simulating either ADHD or Dyslexia, and comparing their performance to students with genuine disabilities, clearly indicate not only how easily the symptoms of ADHD can be fabricated, but also that students motivated to feign Dyslexia or ADHD could easily perform poorly on tests of reading and processing speed, thus allowing them access to academic accommodations.

Conclusions: Experimental symptom validity tests show promise in identifying student motivated to feign symptoms of these disorders. Correspondence: Allyson G. Harrison, Ph.D, Psychology, Queen's University, 186 Barrie St., Kingston, ON K7L 3K1, Canada. E-mail: harrisna@post.queensu.ca

\section{OSMON. The many faces of effort: Specific symptom validity,} attention and anosadiaphoria.

Objective: There are many reasons for failing effort tests, including the most likely causes of malingering and factitious disorder. This presentation examines three aspects of effort that are rarely discussed but may influence performance on symptom validity tests. First, is effort a general phenomenon or a specific one? Differences is detection of a general effort test, the Word Memory Test (WMT), and a specific effort test, The Word Reading Test (WRT) suggest that effort has both general and specific effects. This study showed that the WRT had showed excellent receiver operating characteristics (e.g., $90 \%$ sensitivity and $100 \%$ positive predictive power at $50 \%$ baserate) and outperformed the WMT in detecting both reading and mental speed simulators. Additionally, a double dissociation between reading and speed simulators on WRT errors and reaction time suggested specific effort effects while poor effort of simulators on the WMT suggested general effort effects. Second, results of a study are presented showing the effect of attention on WMT performance, suggesting what many have suspected, namely that some individuals may slacken performance on tests that do not demand intense effort, especially when their attention skills are weak. Third, the case of a student with documented poor effort on the WMT, who passed symptom validity measures on a second evaluation and yet showed nearly identical cognitive test results when re-evaluated with an extensive, different LD battery. This case calls into question the standard feigned effort interpretation of symptom validity results and discusses issues such as poor attention and lack of appropriate concern for test performance that may help to interpret symptom validity results.

Correspondence: David, Osmon, University of Wisconsin-Milwaukee, Psychology, 2441 E. Hartford Ave, Milwaukee, WI, 53211, USA, Phone: 414-229-6751, Fax:414-229-5219,Email:neuropsy@uwm.edu

\section{FRIDAY MORNING, JULY 6, 2007}

\section{Paper Session 10 9:15-10:15 a.m. \\ Schizophrenia}

\begin{abstract}
P. LOPEZ-GARCIA, E. DEL POZO, P. MOLERO, M. GALVEZ \& F. ORTUNO. Impact of COMT Genotype on Cognition in Schizophrenia Spectrum Disorders.

Objective: Dopamine levels in the prefrontal cortex seem to play a crucial role in cognitive functioning in schizophrenia. Whereas high dopamine concentration in striatum may lead to psychotic symptoms, low dopamine levels in the prefrontal cortex are associated to cognitive deficits. The COMT (catechol-o-methiltransferase) enzyme impacts on the catabolism of prefrontal dopamine. The polymorphism val158met is related to the functional properties of the enzyme, with val/val variants leading to lower dopamine levels in the prefrontal cortex and therefore to poorer cognitive performance. We investigated the impact of the val158met polymorphism on several cognitive domains in schizophrenia spectrum disorders.
\end{abstract}

Participants and Methods: We studied a sample of 27 schizophrenia spectrum disorder patients. All subjects performed several tests of the MATRICS Consensus Cognitive Battery to measure the performance in the cognitive domains of attention (measured with CPT), working memory (measured with the Spatial Span test from the Wechsler Memory Scale) and visual learning (measured with the HVLT-R). Genotyping for COMT val158met polymorphism was accomplished by PCR-RFLP analysis. MANOVA analyses were performed to test groups differences in several neuropsychological tests.

Results: The val/val patients $(\mathrm{n}=7)$ performed worse in all the neuropsychological tests than $\mathrm{val} / \mathrm{met}(\mathrm{n}=16)$ or met/met $(\mathrm{n}=5)$ patients. Significant differences were found for visual learning $(F=4,76 ; p=0,018)$ and spatial working memory $(\mathrm{F}=4,06 ; \mathrm{p}=0,03)$.

Conclusions: The COMT genotype impacts on cognitive performance through dopamine availability in the prefrontal cortex. Val/val carriers may be at risk of developing schizophrenia spectrum disorders and exhibit worse cognitive functioning than val/met or met/met carriers.

Correspondence: Pilar Lopez-Garcia, M.D., Ph.D., Psychiatry, University of Navarra, Ave Pio XII 36, Pamplona 31008, Spain. E-mail: plopezgarcia@unav.es 
O. ROBLES ARANDA, A. ZABALA, I. BOMBÍN, M. MAYORAL, M. RAPADO, P. ANDRÉS, C. GONZÁLEZ, J. RODRÍGUEZ, E. DE LA SERNA \& C. ARANGO. Lack Of Cognitive Performance Specificity Among Subdiagnoses Of Early-Onset First Episode Psychosis.

Objective: There is increased interest in the study of neuropsychological deficits in psychosis, given the relationship between cognition and functional outcome. Although several studies have reported cognitive impairments in early-onset schizophrenia, little is known about the generability or specificity of cognitive performance among diagnostic subgroups in psychosis. This study examines the nature and extent of cognitive impairments in first episode early-onset psychosis (EOP) as well as the possible qualitative and quantitative differences for each diagnostic subgroup.

Participants and Methods: This study describes the basal neuropsychological results of the child and adolescent first-episode psychosis study (CAFEPS), a Spanish multicenter longitudinal study. A comprehensive neuropsychological battery was administered to one-hundred first episode adolescents with EOP, and 98 healthy controls. Diagnostic information was collected at baseline using the Kiddie-Sads-Present and Lifetime Version (K-SADS-PL), and was confirmed at 6 months, per DSM-IV criteria. Three diagnostic categories were established in the patient group: schizophrenia spectrum disorders $(n=45)$, affective psychosis $(\mathrm{n}=28)$ and psychosis not otherwise specified $(\mathrm{n}=28)$

Results: Overall, patients' performance ranged between 0.88 and 2 standard deviations below the controls' mean in all cognitive domains: attention $(p<0,001)$, working memory $(p<0,001)$, executive functioning $(p<0,001)$ and learning and memory $(p<0,001)$. No specific patterns of cognitive performance were observed among diagnostic subgroups.

Conclusions: The pattern and degree of cognitive impairment in first episode EOP is similar to that reported in adult-onset first episode patients. Our results failed to identify significant differences among diagnostic subgroups at the onset of the illness. The longitudinal assessment will help clarify the course of cognitive performance in EOP. Correspondence: Olalla Robles Aranda, Psychology, Psychiatry, Fundacion para la Investigacion Biomedica del Hospital Gregorio Marañón, Unidad Adolescentes, Psiquiatría, C/ Ibiza, 43, Madrid 28009,Spain.E-mail: orobles@mce.hggm.es

I. BOMBIN, A. BILBAO, M. MAYORAL, J. BOBES \& C. ARANGO. Relationship between Cognition, Soft Neurological Signs, and Symptoms with Insight into Schizophrenia.

Objective: Lack of insight into illness and its clinical manifestations of the disease is a core symptom of schizophrenia. Explanatory variables of poor insight include cognitive impairment, more severe psychotic symptoms, and coping-attributional style. The goals of this study were to assess the associations of insight with clusters of psychopathological symptoms, and with neurological or neuropsychological impairment.

Participants and Methods: Sixty-seven patients diagnosed of schizophrenia $(n=47)$ or schizophreniform disorder $(n=20)$ were included in the study. Insight was assessed with the SUMD; psychopathological status was assessed by the PANSS; and soft neurological signs by means of the NES. A comprehensive neuropsychological battery that included measures of attention, working memory, learning and memory, parietalrelated functions, and executive functioning was administered.

Results: Poorer insight was associated with more positive symptoms, and global psychopathology, but not with negative, hostility-excitation or disorganization symptoms. Among cognitive variables, only working memory and executive functioning significantly correlated with insight. Soft neurological signs did not show any association with insight. The total variance of insight explained by the combination of symptoms and cognitive functioning ranged from $13 \%$ to $19 \%$ among the different insight dimensions.
Conclusions: According to our results, lack of insight might be partially explained by a conjunction of more prominent positive symptoms, and cognitive impairment of selected higher functions, such as abstraction and cognitive flexibility. Interestingly, different dimensions of insight are selectively explained by different predictive variables. Correspondence: Igor Bombin, PhD, Neuropsychology, Reintegra, Centro de Rehabilitacion Integral, C/ Eduardo de Fraga Torrejon 4, bajo, Oviedo 33011, Spain.E-mail: ibombin@mce.hggm.es

M.I. KRAKOWSKI, P. CZOBOR \& K. NOLAN. Atypical Antipsychotic Agents, Neuropsychological Deficits andViolence in Schizophrenic Patients.

Objective: The purpose of this investigation was to test the efficacy of novel antipsychotic medications in the treatment of cognitive impairments in violent schizophrenic patients and to examine how improvement in these impairments affected the patients' aggressive behavior. Participants and Methods: This was a randomized, double-blind, parallel-group, 12-week trial. Physically assaultive subjects with schizophrenia or schizoaffective disorder who were inpatients in state psychiatric facilities were randomly assigned to treatment with clozapine ( $\mathrm{N}=33$ ), olanzapine ( $\mathrm{N}=34)$ or haloperidol $(\mathrm{N}=33)$.

The primary neuropsychological measure was the general cognitive index; it was derived from a battery of tests assessing psychomotor function, general executive function, visual and verbal memory, and visuospatial ability. The number and severity of all aggressive events was measured by the overall score on the Modified Overt Aggression Scale (MOAS). Measures of psychiatric symptoms and side effects were also included

Results: The treatment groups showed a substantial difference in the amount of change in the general cognitive index from baseline to end point $(\mathrm{F} 2,87=7.02, \mathrm{p}=.001)$ characterized by advantages of olanzapine vs. haloperidol $(p=.001)$ and olanzapine vs. clozapine $(p=.003)$ but no difference between haloperidol and clozapine $(p=.63)$. Further analyses revealed significant improvement with olanzapine in several of the cognitive domains. Furthermore, the improvement in the general $\operatorname{cog}$ nitive index was significantly associated with a decrease in violence in the olanzapine group, but not in the other two groups.

Conclusions: These data suggest that olanzapine has superior cognitive benefits in violent schizophrenic patients and that this improvement is associated with a decrease in aggressive behavior. As there was a greater reduction in violence in the clozapine group, there may be different pathways for the antiaggressive effect of the atypical antipsychotic agents. Correspondence: Menahem I. Krakowski, PhD, MD, Life Sciences, Nathan Kline Institute, 140 Old Orangeburg Road, Orangeburg, NI 10962.E-mail:krakow@nki.rfmh.org.

\section{Paper Session 11 9:15-10:15 a.m.}

\section{Neuropsychology of Medical Disorders}

\section{B. PEIXOTO, L. LOPEZ \& J. ARIAS. Executive Functions in the} Spectre of Hepatic Dysfunction.

Objective: The minimal hepatic encephalopathy (MHE) is one of the clinical dimensions of the hepatic encephalopathy, which is not usually manifested in an evident manner, but reveals a high prevalence rate and severe implications on the quality of life of the patients with hepatic dysfunction. The executive functions are a group of cognitive processesessential to an autonomous and adapted life. However, the presence of executive dysfunctions in the MHE, has not been duly explored. The present research pretends to analyze the existence of possible executive dysfunctions in the MHE and get to know their distribution in the spectre of the hepatic dysfunction. 
Participants and Methods: We applied two tests for evaluation of the EF, the Behavioural Assessment of the Dysexecutive Syndrome and the Wisconsin Card Sorting Test, to a group of subjects with different degrees of hepatic cirrhosis (Child A Group, $n=20$; Child B Group, $n=$ 20; Child C Group, $n=20$ ), to a group of subjects with hepatitis $\mathrm{C}$ virus chronic infection (HCV Group, $n=20$ ) and to a group of frequent blood donors (Control Group, $n=20$ ).

Results: The obtained results showed the presence of disturbances on the abilities to inhibit previous associations, structured planning, conceptualization, abstract reasoning, organized search and maintaining of a non automatic response, in the mild hepatic dysfunction. With the progress of the hepatic dysfunction, not only the previous disturbances aggravate but also other difficulties appear as in cognitive estimation, atentional supervision and control, maintenance and organization of tasks along a certain period of time and the use of feedback in order to correct behaviour.

Correspondence: Bruno Peixoto, PhD, Sciences, Instituto Superior de Ciências da Saúde- Norte, R. Central de Gandra 1317, Gandra PRD 4585-116, Portugal.E-mail: bruno.peixoto@iscsn.cespu.pt

I. BRANDS, R. KESSELS, J. KAPPELLE, G. BIESSELS \& E. DE HAAN. Type 1 and Type 2 Diabetes: Differential Effects on the Brain? Objective: Diabetes Mellitus (DM) may affect cognitive functioning. It has been suggested that cognitive impairments are more pronounced in DM2 than in DM1, but studies that directly compare the effects of these 2 types of DM are lacking.

Participants and Methods: This study assessed cognitive performance on 5 cognitive domains, psychological well-being (SCL-90-R and BDIII) and brain structure (rating of atrophy, white-matter lesions and infarcts on MRI ) in two groups of patients with DM1 and DM2 (age 61 \pm 5 ) and an age and education matched group of control subjects.

Results: DM1 patients had experienced more hypoglycemic episodes than DM2 patients $(\mathrm{p}<0.001)$, but were better "regulated" on vascular risk factors, such as BMI, triglycerides, hypertension (all $\mathrm{p}<0.001$ ), or hypercholesterolemia $(\mathrm{p}<0.05)$. No between-group differences were observed on neuropsychological measures. Deep white-matter lesions and cortical atrophy were significantly more pronounced in DM2 patients $(\mathrm{p}<0.01)$. Other MRI-ratings were similar in the two groups. DM1 patients and DM2 patients showed a similar pattern of psychological complaints that could not be related to cognitive functioning or MRI ratings. Compared to non-diabetic controls, the cognitive deficits and brain abnormalities of DM patients are subtle. The cognitive impairment can best be characterised as mental slowing.

Conclusions: In conclusion, DM1 patients (34 years DM), have a similar cognitive profile and better MRI ratings than age and education matched DM2 patients ( 7 years DM). Risk factors for the development of these impairments are chronic hyperglycemia and atherosclerotic vascular disease. The occurrence of repeated episodes of hypoglycemia probably does not induce neurocognitive dysfunction.

Correspondence: Ineke Brands, Phd, neuropsychology, Zuwe Hofpoort ziekenhuis, Blekerijlaan 3, Woerden 3447 AC, Netherlands. E-mail: ibrands@horizon.nl

E. VAN DEN BERG, G. BIESSELS, G. NIJPELS \& J.M. DEKKER. Cognitive Dysfunction in Type 2 Diabetes Mellitus: Onset in PreDiabetic Stages?

Objective: Type 2 diabetes (DM2) is associated with cognitive impairments and an increased risk of dementia. We hypothesise that cognitive impairments develop in pre-diabetic stages, before the onset of hyperglycaemia, and progresses gradually thereafter, and that risk factors related to the so-called "metabolic syndrome (MS)" (defined as $=$ or $>3$ of the following: hypertension, obesity, high triglycerides, low HDL-cholesterol, impaired glucose metabolism) are the prime determinants of these early impairments.
Participants and Methods: Participants were recruited through the "Hoorn study", a longitudinal cohort study in which detailed metabolic and vascular data were collected since 1989. Three groups were selected according to the risk factor profile at the 2001 survey: control participants (0-1 MS risk factor; $n=85)$, MS without DM2 (n=81), and recent onset DM2 $(n=54)$. Performance on 11 neuropsychological tasks was expressed in standardized z-scores covering 5 cognitive domains.

Results: We observed small between group differences on cognition (abstract reasoning: control $0.004 \pm 0.11$; MS $-0.03 \pm 0.12$; DM2 0.04 \pm 0.15 ; $\mathrm{p}=0.94$; memory: $0.01 \pm 0.05 ; 0.03 \pm 0.06 ;-0.10 \pm 0.07 ; \mathrm{p}=0.32$; information processing speed: $0.17 \pm 0.10 ;-0.13 \pm 0.10 ;-0.20 \pm 0.12 ; p=0.03$; executive functions: $0.08 \pm 0.07 ;-0.06 \pm 0.07 ;-0.12 \pm 0.09$; $\mathrm{p}=0.18$; visuoconstruction: $0.15 \pm 0.11 ;-0.05 \pm 0.11 ;-0.16 \pm 0.14 ; \mathrm{p}=0.19)$. The MS group had an intermediate position between the DM2 and control group. As expected, the effects in the early DM2 group were smaller than in previous studies with advanced DM2.

Conclusions: This interim analysis suggests that the cognitive impairment that is associated with DM2 already develops in pre-diabetic stages and progresses gradually thereafter. Future analyses will address longitudinal associations between vascular and metabolic risk factors and cognition, to identify determinants of cognitive dysfunction.

Correspondence: Esther van den Berg, MSc, Neurology G03.228, University Medical Center Utrecht, Heidelberglaan 100, Utrecht 3584CX, Netherlands. E-mail: e.vandenberg-6@umcutrecht.nl

J.T. BECKER, O.L. LOPEZ, H.J. AIZENSTEIN, N.J. LOBAUGH, P.M. THOMPSON \& S. JUENGST. Brain Functional Alterations in HIV/AIDS Analysed by the Method of Partial Least Squares.

Objective: There are a variety of methods for analyzing functional brain activation data. By far the most common methods utilize t-tests (and associated methods) that are useful for determining regional activity that occurs above a given threshold (functional specialization). However, another group of methods has focused on identifying functionally associated regions that may exist within a dataset and that can be revealed by analyses that emphasize functional integration. The purpose of this study was to invoke Partial Least Squares analysis in a study of HIV/AIDS.

Participants and Methods: fMRI data using the N-back data were preprocessed via SPM2, and the smoothed, normalized individual scan data were processed through PLSGUI. We tested the statistical significance of the latent variables $(\mathrm{LVs}$ ) by running 500 permutations tests, and the standard errors of the saliences were estimated through 100 bootstrap samples.

Results: The analysis identified one significant $\mathrm{LV}$, which accounted for $70.3 \%$ of the crossblock covariance (p.<001), indicating a linear association between the $\mathrm{LV}$ and the task demands of the $\mathrm{N}$-back. The posterior cingulate was more active in the 0 -back condition, and activity decreases as a function of task difficulty. By contrast, the anterior cingulate was positively associated with the $L V$ and shows increases in activity with increasing task demands.

We extracted the three brain scores for each subject (one representing each task condition) and regressed the 0 -back scores on age, brain structural measures, CD4+ cell counts and HIV RNA in $24 \mathrm{HIV}$-infected subjects. The O-back activation was significantly related to the independent contributions of vascular risk and to a measure of WM integrity derived from a separate PLS analysis.

Conclusions: The HIV-infected subjects with greater vascular risk or lower WM integrity had lower activation during the O-back. This leads us to speculate that the background (i.e., resting state) activity in these subjects is also lower.

Correspondence: James T. Becker, Ph.D., Western Psychiatric Institute and Clinic, University of Pittsburgh, Suite 830, 3501 Forbes Avenue, Pittsburgh, PA 15213. E-mail: beckerjt@upmc.edu 


\section{Paper Session 12 \\ 9:15-10:15 a.m.}

\section{Cognitive Neuroscience}

S. BATE, C. HASLAM, T.L. HODGSON \& J.J. TREE. Evidence of an Eye Movement-Based Memory Effect in Congenital Prosopagnosia. Objective: While extensive work has examined the role of covert recognition in acquired prosopagnosia, little attention has been directed to this process in its congenital equivalent. Indeed, evidence of covert recognition has only been demonstrated in one congenital case in which autonomic measures provided evidence of recognition (Jones and Tranel, 2001), whereas two studies using behavioural indicators failed to demonstrate the effect (de Haan and Campbell, 1991; Bentin et al., 1999). In this paper, we use a behavioural indicator, an "eye movement-based memory effect" (Althoff and Cohen, 1999), to provide evidence that covert recognition can be demonstrated in congenital prosopagnosia using behavioural measures.

Participants and Methods: In an initial experiment, we presented 19 healthy adult participants with 20 novel and 20 famous faces. Each stimulus was presented for five seconds, and the viewing strategies elicited to famous and novel faces were examined. In Experiment 2, we examined the same processes in a patient with congenital prosopagnosia. AA. However, in order to provide evidence of covert recognition in this patient we required a larger set of stimuli to obtain an adequate number of recognised and not-recognised faces for analysis. Thus, we carried out a third experiment that presented $\mathrm{AA}$ with additional stimuli for recognition.

Results: In our control participants, the viewing of famous faces was characterised by fewer fixations and reduced regional sampling compared to novel faces. In Experiment 2, we found some evidence of an eye movement-based memory effect in patient AA, regardless of his recognition accuracy. Finally, evidence of reduced sampling for familiar stimuli again emerged for those famous faces AA failed to explicitly recognize in Experiment 3.

Conclusions: We use these findings to (a) provide evidence of intact structural representations in a case of congenital prosopagnosia, and (b) to suggest that covert recognition can be demonstrated using behavioural indicators in this disorder.

Correspondence: Sarah Bate, BA Psychology, MSc Psychological Research Methods, Psychology, University of Exeter, School of Psychology, Washington Singer Laboratories, Perry Road, University of Exeter, Exeter EX44QG, United Kingdom. E-mail: S.Bate@exeter.ac.uk

J. SEPULCRE, J. GOÑI, I. MARTINCORENA, N. VELEZ DE MENDIZABAL, H. PERAITA \& P. VILLOSLADA. Mechanism of lexical access strategies: evidence from lexical networks in neurological diseases.

Objective: Switching and clustering are strategies to access to lexical pool. We propose an iterative model by which frontal lobe supervise the search in the temporal lobe in category fluency tasks. Thus, we predicted the involvement of bidirectional white matter (WM) pathways between frontal and temporal lobes in order to have a rapid access to proto-types words and to change the sub-category when search is exhausted in temporal lobe (switching), and a local search in the temporal grey matter (GM) network to find semantic related words in a given sub-category (clustering).

Participants and Methods: We analyzed the lexical networks derived from category fluency task (animals) of twenty patients with multiple sclerosis (MS), twenty with Alzheimer disease (AD), and forty healthy controls (HC) matched by sex, age and education. We propose MS as WM lesional model and AD as GM lesional model. We obtained the lexical networks for each group by correlating the evoked words between subjects using Matlab 6.5 and Pajek 1.15 software.
Results: We found switching strategy was impaired in the MS network, but clustering was preserved. AD lexical network showed impairment in switching and clustering. However, AD patients have a preserved access to prototypic words.

Conclusions: Our findings indirectly support the hypothesis for an iterative fronto-temporal (FT) system for lexical access, based in our network analysis and the common disruption of the FT pathways in MS, with relative preservation of GM, and in the severe temporal GM atrophy in AD.

Correspondence: Jorge Sepulcre, MD PhD, Neuroscience, University of Navarra, Pío XII 55, Pamplona 31008, Spain. E-mail: jsepulber@unav.es

\section{K. MCFARLAND, B. MURRAY \& M. RADEL. The Many Faces of Prosopagnosia.}

Objective: Despite considerable attention in the literature, the processes underlying face recognition and prosopagnosia are not yet well understood. Argument continues over the specificity of faces as a visual stimulus and the role of perceptual impairments in the traditional taxonomy of apperceptive and associative subtypes of prosopagnosia. These two 'faces' of prosopagnosia form the focus of the current paper. The broad aim is to inform these on-going debates by profiling the impairments of a single patient in terms of the specificity of her deficit, with reference to the traditional apperceptive/associative taxonomy

Participants and Methods: A single patient (CW: listed with www.faceblind.org) and a control group of five matched, unimpaired, participants completed a comprehensive assessment of basic face and object perception, familiar and unfamiliar face and object recognition, and higherorder perceptual processing.

Results: Results showed that CW's impairment was specific to faces. She passed all tests of basic object perception and tests involving discrimination and recognition of visually complex non-facial stimuli. However, CW showed impairments in the recognition and recall of unfamiliar faces, famous faces, real faces (but not photographs), and in general any face-stimuli requiring holistic processing ( $\mathrm{CW}$ showed no face inversion decrement).

Conclusions: The evidence that visual recognition deficits can be restricted to faces without associated difficulties in recognising objects suggests that faces uniquely, or disproportionately, engage a holistic processing mechanism. This questions the usefulness of the associative/apperceptive taxonomy and it is clear that current neuropsychological assessment procedures inadequately assess face recognition and perceptual impairments. A more comprehensive test battery is required, and suggested, for future clinical research Correspondence: Ken McF arland, Psychology, University of Queensland, School of Psychology, University of Queensland, St Lucia, QLD 4072, Australia.E-mail:ken@psy.uq.edu.au

Y. JOANETTE, C. OUELLET-PLAMONDON, N. SENHADJI, J. ANSADO \& O. MONCHI. The Functional Reorganization of the Brain Underlying Cognitive Successful Aging for Language Is Multidimensional and Task Dependent.

Objective: Successful cognitive aging requires a reorganization of the brain's functional organization. The HAROLD phenomenon described by Cabeza (2002) suggests that this reorganization corresponds to some hemispheric dedifferentiation, particularly in the pre-frontal cortex (PFC). However, this dedifferentiation may express a task-determined reorganization limited to the PFC. The goal of this study was to explore the generalization of age-related laterality reduction to the semantic processing of words, an ability which relies on a large network across many different cortical areas.

Participants and Methods: Twelve young and 12 old participants were scanned using fMRI while performing a category judgment task. 
Results: Behavioral results showed that level of performance was generally similar between young and older adults. fMRI results showed significant activation in the left frontal cortex for the young group whereas the activations included the inferior temporal cortex bilaterally, the right inferior parietal and the left inferior temporal for the older adults in the categorical judgment task vs. the control task.

Conclusions: The use of indexes of activated voxels showed that the dedifferentiation component was largely surpassed by a posteriorization component. Furthermore, subcortical peaks such as the ones observed in the caudate nucleus of the younger group, were absent in old participants. These results suggest that the presumed compensatory role of the functional reorganization with age is more than uni-dimensional (dedifferentiation) and appears to encompass left/right, anterior/posterior and even cortical/sub-cortical dimensions. It is proposed that the nature of such reorganization depends on the task and the cognitive functions being solicited.

Correspondence: Vves Joanette, Ph.D., Centre de recherche, Institut universitaire de gériatrie de Montréal, 4565 ch. Queen-Mary, Montréal, QC H3W1W5, Canada.E-mail:yves.joanette@umontreal.ca

\section{Poster Session 4 \\ Traumatic Brain Injury, Epilepsy, CNS Abnormalities 9:15-11:15 a.m.}

\section{Demyelinating Disease/Multiple Sclerosis/ALS}

\section{M.P. SÁNCHEZ, A. NIETO, J. BARROSO, V. MARTÍN, T. OLIVARES \& M.A. HERNÁNDEZ. NEUROPSYCHOLOGICAL ASSESSMENT AND REGIONAL MRI LESION VOLUME IN MULTIPLE SCLEROSIS PATIENTS TREATED WITH IFN BETA.}

Objective: We aimed to investigate the relationship between magnetic resonance imaging regional lesion volume and neuropsychological performance in a group of early relapsing-remitting (RR) multiple sclerosis (MS) Interferon (IFN) beta treated patients during a 1-year period. Participants and Methods: 42 patients with clinically definite RRMS underwent a comprehensive neuropsychological battery interrogating multiple cognitive domains (attention/processing speed, verbal and visual memory/learning, language, visuo-spatial and visuo-perception functions, motor coordination/programation and abstract/conceptual reasoning skills). Cognitive impairment was determined by comparison with demographically matched healthy controls. Brain MR image analysis included the total brain and regional lesion volume on proton density T2-weighted images. The relationship between MRI lesion volume and neuropsychological tests results was examined before the start of treatment and 1-year follow-up.

Results: At baseline evaluation, patients with attention/processing speed. verbal and visual memory/learning, and motor coordination/programation deficits had higher lesion load on PD scans, especially in temporal and parietal regions. The temporal lesion load on PD-weighted MRI is associated with the majority of the administered tests. At 1 year, patient showed a significant improvement on measures of problem solving, flexibility and verbal and visual memory. Comparison of the mean total and regional T2 MRI lesion volume over 1 year with baseline analysis revealed a significant decrease from 0,42 to $0,31 \mathrm{ml}$ in temporal regions $(\mathrm{t}(16)=3,116 ; \mathrm{p}=0,007)$.

Conclusions: Our results suggest a possible beneficial effect of IFN beta treatment on cognitive functions in RRMS. Extension of follow-up and further data analysis are needed to confirm and clarify these findings. Correspondence: María del Pino Sánchez-López, Department of Metodologia de las Ciencias Comportamentales y Psicobiología, University of La Laguna, Campus de Guajara s/n, La Laguna 38205, Spain.E-mail:mpinosl@um.es
E. GIOGKARAKI, C. POTAGAS, G. KOUTSIS, D. MANDELlOS \& C. SFAGOS. A four factor model for executive function in MS.

Objective: The present study was designed to propose a factor model of a brief battery of neuropsychological tests commonly used in multiple sclerosis (MS) studies, in order to investigate that these tests, even in a widespread disease as MS, could distinguish an impairment on different aspects of executive function from a speed processing impairment. Participants and Methods: A Principal Components Analysis (varimax rotation) was performed on the following 9 variables: Symbol Digit Modalities Task (SDMT); Paced Auditory Serial Addition Task, two trials -one of $3 \mathrm{sec} /$ digit interval (PASAT3) and the other of 2sec/digit interval (PASAT2); Stroop including word reading (StR), color naming $(\mathrm{StC})$, the incongruent word-color condition $(\mathrm{StI})$, and the number of errors in the incongruent condition (StERR); phonemic fluency task (FAS) and semantic category fluency task (SFT). The battery was administered to 150 patients with clinically definite MS.

Results: Based on some criteria (eigenvalues greater than 1, scree plot and interpretability of the factors) 4 components were retained and conceptualized as follows: 1) selective attention, inhibition (SDMT, StI, StERR); 2) working memory, mental calculation (PASAT3, 2); 3) automatic speed processing (StR, StC); 4) word fluency (FAS, SFT).

Conclusions: Factor analysis of commonly used neuropsychological tests of executive function in MS patients revealed four factors, supporting the existence of different aspects of executive function and the conceptualization of speed processing as a separate factor. These aspects may be subserved by different distributed systems. So, they may be also affected separately. This model could have important implications in the interpretation of clinical data.

Correspondence: Erasmia Giogkaraki, MSc, Neurology, Eginition Hospital, 22. Rostand Street, Athens 111-41, Greece. E-mail: erasmia. yogaraki@gmail.com

J. MACNIVEN, C. DAVIS, M.Y. HO, C.M. BRADSHAW, E. SZABADI \& C.S. CONSTANTINESCU. Stroop Performance in Multiple Sclerosis: Information Processing, Selective Attention or Executive Functioning?

Objective: The present study aimed to replicate previous research that people with multiple sclerosis (MS) have an enhanced Stroop interference effect as compared with age- and predicted premorbid IQ-matched healthy control participants. The second aim of the study was to evaluate the hypothesis that controlling for information processing speed may eliminate group difference in RT on the Stroop test between people with MS and age- and predicted premorbid IQ-matched healthy controls. Participants and Methods: In this study, 18 patients with MS were assessed with a neuropsychological test battery including a computerised Stroop test and a computerised test of information processing speed, the Graded Conditional Discrimination Tasks (GCDT). The patient group was compared with an age, sex and estimated premorbid IQ-matched healthy control group.

Results: The patient group's reaction times (RTs) were significantly longer than those of the control group on all trial types in the Stroop test (congruent, neutral, incongruent); however neither the absolute (RTincongruent-RTneutral) nor the relative (100. [RTincongruent-RTneutral]/RTneutral) Stroop interference effect differed between the groups. The linear function relating RT to stimulus complexity in the GCDT was significantly steeper in the patient group, indicating slowed information processing. Linear functions relating RTs on the GCDT to corresponding RTs of a healthy 'reference group' were used to predict RTs on the Stroop test. Discrepancies between predicted and obtained RTs did not differ between the two groups.

Conclusions: The results are discussed with reference to the difference engine model, a theory of diversity in speeded cognition; it is suggested that long RTs on the Stroop test seen in patients with MS may be explained in terms of a general slowing of information processing. It is 
concluded that, in the assessment of people with MS, great caution must be used in the interpretation of performance on neuropsychological tests which rely on RT as the primary measure.

Correspondence: Jamie Macniven, Adult Neuropsychology, Nottingham University Hospitals NHS Trust, Queen's Medical Centre Campus, Nottingham NG7 2UH, United Kingdom. E-mail: jamie. macniven@nottingham.ac.uk

S. STERN, I.E. EVANGELOU, F. TOVAR-MOLL, J. OHAYON, M. EHRMANTRAUT, R.L. KANE, H. MCFARLAND \& F. BAGNATO. Cognitive Deficits in Multiple Sclerosis Patients Correlated with Diffusion Tensor MRI Derived Measurements of the Corpus Callosum.

Objective: About fifty percent of all Multiple Sclerosis (MS) patients develop cognitive deficits (CD). The Paced Auditory Serial Addition Test (PASAT) is used to assess global cognitive function in MS, whose pathological substrates of CD are still unknown. This study attempts to evaluate disease-induced microscopic changes in the corpus callosum (CC) and investigate their relationship with CD in MS.

Participants and Methods: Twenty-four MS patients and 18 healthy volunteers $(\mathrm{HVs})$ underwent a $3.0 \mathrm{~T}$ diffusion tensor magnetic resonance imaging (DT-MRI). At present, fractional anisotropy (FA) and mean diffusivity (MD) have been measured in five regions of interest (ROIs) in the midsagittal plane (genu, anterior [AMB] and posterior midbody [PMB], splenium and total CC) of 13 patients and $18 \mathrm{HVs}$. An unpaired t-test and Spearman's correlation were used to quantify FA and MD differences between groups and correlate them with PASAT scores.

Results: The total CC FA ( $p=0.0004)$ and MD $(p=0.0002)$ were different in MS patients compared to HVs. Correlations existed between PASAT and FA of the splenium $(\mathrm{r}=0.64, \mathrm{p}=0.018)$ and total $\mathrm{CC}(\mathrm{r}=$ $0.62, \mathrm{p}=0.02)$, as well as with MD of the AMB $(\mathrm{r}=-0.65, \mathrm{p}=0.017)$ and total CC $(r=-0.68, p=0.0099)$.

Conclusions: DT-MRI quantified microscopic pathological changes in the CC of MS patients. Regional and global DTI changes in CC were correlated also with a lower PASAT score. These preliminary findings highlight the importance of white matter inter-hemispheric bundle integrity for preserving the cognitive skills of MS patients.

Correspondence: Susan Stern, B.S., NINDS/NIB, NIH, 10 Center Drive, Building 10 5B16, Bethesda, MD 20892. E-mail: sternsu@ninds.nih.gov

J. VOLKERT, H. KÖNIG, G.W. ALPERS, H. MEISSNER \& P. FLACHENECKER. Fatigue in Multiple Sclerosis: Objective Assessment of a Subjective Symptom.

Objective: Fatigue is one of the most disabling symptoms in patients with multiple sclerosis (MS) but it remains difficult to assess objectively in clinical settings. Most previous studies have failed to find correlations between self-report data and objective measures of attention. The aim of this study was to objectively measure the behavioural consequences accompanying the subjective feeling of fatigue. First, we used a more specific self-report measure to assess fatigue. Second, we used computerized tests that measure aspects of attention related to its intensity rather than its selectivity.

Participants and Methods: Fifty-seven MS patients (mean age 45 years, Expanded Disability Status Scale Md $=4.5$ ) were recruited during an in-patient rehabilitation program. They completed a standardized fatigue questionnaire (WEIMuS) and participated in an extended neuropsychological session with several attention tests from a computerized battery (TAP). The tonic alertness test was repeated three times to detect an increase of reaction times over the complete test session. Within each test, mean reaction times (RT) and linear regression coefficients (LRC) were calculated.

Results: The WEIMuS score significantly correlated with RT and LRC in alertness and sustained attention tests, but not with parameters of cognitive flexibility and with error rates. RTs steadily increased across repeated testing of alertness.

Conclusions: Contrary to previous research these findings demonstrate that fatigue can be measured objectively by adequate tests, both via selfreport and computerized tests. Therefore, it is possible to verify patients' clinical status and to evaluate therapeutic interventions.

Correspondence: Julia Volkert, Universität Würzburg, Josef-SchmittStr.3, Lauda 97922, Germany.E-mail: julia_volkert@web.de

\section{Epilepsy}

O.M. AGUILAR MEJIA. Protocol of Neuropsychological follow up in childrens during the epilepsy surgery process.

Objective: To propose a protocol of a presurgical, intraoperating and postsurgical child neuropsychological memory and language assesment. To compare this protocol with a protocol of a presurgical, intraoperating and postsurgical (Ojemanns'protocol) neuropsychological memory and language assesment.

To determine the cognitive impairments of these patients, correlating neurological, neuropsychological and brain mapping and imaging studies that they facilitate to make surgical decision.

Participants and Methods: Two Children diagnosed with refractary epilepsy of the Neuropediatric and neurosurgical station of the Hospital La Misericordia of Bogota - Colombia

Method: Case study

Results: Was designed a protocol of follow up in the surgical process adapted for the child population (Ojemanns'protocol) evaluating the different cognitives functions specially the memory and the language. Conclusions: To use strategies of child education where it becomes familiaried to the child with the monitory intraoperating facilitates the development of the protocol and increases the probability of success of the surgical process.

Correspondence: Oscar M. Aguilar Mejia, Master in Cognitive Neuropsychology, Psychology, Pontificia Universidad Javeriana, Cr. 5 \# 39 -00, Bogota 00571, Colombia.E-mail: oaguilar@javeriana.edu.co

J.C. ALVAREZ-CARRILES, J. MENOR \& J. SALAS-PUIG. Dual-task Performance in Frontal and Temporal Lobe Epilepsy.

Objective: According to literature, dual-task performance impairment has been related to frontal lobe damage, but to our knowledge only one study has tried to test this hypothesis in epilepsy. Our objective is to analyze dual-task performance in frontal (FLE) and temporal lobe epilepsy (TLE) patients, considering their possible different profile and the potential influence that different epilepsy-related factors (lateralization, presence of lesion on MRI, age of onset, epilepsy duration, seizures frequency and drugs regime) have over their performance.

Participants and Methods: 19 FLE and 20 TLE patients were compared to an age, gender and education matched control group of 20 healthy participants. All the participants were assessed with the dualtask procedure developed by Baddeley et al. (1997).

Results: A preliminary one-way ANOVA did not show any statistically significant difference among the three groups. In the epilepsy groups, neither age of epilepsy onset nor epilepsy duration were significantly related to dual-task performance. However, a subsequent ANCOVA controlling for seizures frequency showed that dual-task performance was significantly impaired in right hemisphere epilepsy patients with a structural lesion on MRI. Furthermore, those patients on polytherapy had a worse dual-task performance, especially when the EEG focus was on the right hemisphere. Conclusions: Contrary to our hypothesis, dual-task performance was not differentially decreased on frontal lobe epilepsy patients as a group. However, this does not mean that all the epilepsy patients have a similar dual task performance, since we have observed a selective involvement of right hemisphere on this sort of task, especially when the epilepsy is associated to a structural damage or the patient is under polytherapy. Correspondence: Juan C. Alvarez-Carriles, PhD, Neurology Service, 
Hospital Universitario Central de Asturias (HUCA), Julian Claveria, $s / n$, Oviedo 33006, Spain. E-mail: juancarriles@hotmail.com

P.A. KLAAS, M. DULAY, R. BUSCH, J. HAUT \& E. WYLLIE, Predictors of Post-Surgical Behavioral Outcome in Children with Intractable Epilepsy.

Objective: Epilepsy surgery is an effective option for a subset of children with intractable epilepsy. Cognitive and behavioral problems may persist after surgery, even when there is good seizure control. This study examined what pre-surgical factors predict the presence of post-surgical behavioral concerns.

Participants and Methods: The sample consisted of 47 children (mean age $=11.2$, range $6-15$ years, $60 \%$ female) who underwent surgical resection for seizure control. Pre-surgical measures of behavior (parent report Child Behavior Checklist [CBCL]), seizure-related factors (age at seizure onset, duration of epilepsy), and cognitive factors (IQ, cognitive speed/attention) were used to predict the presence of psychological and behavioral problems evaluated an average of 8 months after surgery (defined by post-surgical CBCL).

Results: Thirty percent of the sample had significant behavioral problems after surgery. Multiple regression analyses revealed that the best predictors of post-surgical behavior problems were pre-surgical behavior problems $(p<0.001)$. For example, the presence of aggressive behavior before surgery predicted continued aggressive behavior after surgery. This was also found for post-surgical mood state, social isolation, and delinquent behavior. Lower pre-surgical Full Scale IQ was a significant predictor of social isolation after surgery $(p<0.05)$.

Conclusions: Results demonstrate that a significant number of children continue to have behavioral problems after surgery. This finding highlights the need for behavioral assessment and intervention in children following epilepsy surgery.

Correspondence: Patricia A. Klaas, Ph.D., Psychiatry and Psychology, Cleveland Clinic Foundation, 9500 Euclid Ave., P5-7, Cleveland, OH 44195.E-mail:klaasp@ccf.org

\section{A. LOPEZ-ROLON, E. TRINKA, T. BENKE \& M. DELAZER. Numerical Cognition and Temporal Lobe Epilepsy: Dot Size Congruency in Large Numerosity Discrimination.}

Objective: The only previous study so far on the effect of temporal lobe epilepsy (TLE) on numerical cognition suggested that TLE patients are in general either unimpaired or only slightly so on tasks based on quantity processing (with Arabic-digit input) because these tasks receive bihemispherical support (Delazer et al, 2004).

We examined further whether similar results would be observed in TLE patients on a quantity based task that employs exclusively non-symbolic stimuli that varied on interstimulus size congruency. This type of stimuli allowed us to examine the extent to which TLE patients may exhibit deficits not only on quantity discrimination, but also on interference as well as visual scanning processes within the context of a quantity based task.

Participants and Methods: Participants: Healthy Controls ( $N=39$ ), TLE patients(Right TLE N=11, Left TLE N=7).

Subjects indicated with a key press whether a given dot array was more or less numerous that another one presented shortly before. The first array contained invariably 50 dots, and the subsequent arrays were either less or more numerous than fifty. Arrays had either only small or only large size dots and were presented in random configurations. Dot size congruency/incongruency between arrays was manipulated for each numerosity comparison.

Results: Compared to controls, patients were considerably more inaccurate, but did not differ significantly on their reaction times. Accuracy rates for controls and patients reflected both the numerical separation between arrays and their dot size congruency. Numerically closer arrays generated lower accuracy rates than numerically farther ones, while arrays with incongruent dot sizes induced higher error rates than congruent ones.
Conclusions: On this multi-componential task, TLE patients presented a general reduction in performance unrelated to impairments specific to quantity processing, presumably due to sustained attention deficits. Correspondence: Alex Lopez-Rolon, M. A., Clinical Department of Neurology, Innsbruck Medical University, Anichstr. 35, Innsbruck A6020,Austria.E-mail: alex.lopez@uki.at

A. LUNA RODRIGUEZ, I. GARAMENDI, M. FORCADAS, A. MOLANO, J. PIJOÁN \& J.ZARRANZ. Changes in Neuropsychological Scores after Anterior Temporal Lobectomy plus Amygdalohippocampectomy.

Objective: To compare the results of Weschler test (IQ), Boston test, Rey figure copy and test of verbal memory of Rey before and after the resection of anterior temporal lobe plus amidalohyppocampectomy, depending on which side was the resection made.

Participants and Methods: We passed all tests to the patients who underwent anterior temporal lobectomy plus amygdalohyppocampectomy $(\mathrm{n}=94)$ and compared to the results obtained 6 months among a year after the surgery. Two tests were used, $T$ test in the intelligence quotient and Wilcoxon sign rang test in all other tests.

Results: Improving of all aspects of IQ was observed when we passed that test to the patients. There were significant statistical differences in global and manipulative parts of the test. The larger differences were observed with manipulative task. When adjusted with the side of resection the results persisted. Left side surgery's patients always had poorer results in every task, in contrast to right side surgery's who obtained better values. Only global and manipulative tasks were statistically significant. In the rest of tests, there were poorer results after surgery but this difference was not statistically significant.

Conclusions: Anterior temporal lobectomy plus amydalohippocampectomy has good results in controlling number of epileptic crisis. Also it has a statistical significant effect in the cognitive status of the patients. There is an improving in all aspects of Weschler test, although only global and manipulative parts of it has a significant $\mathrm{p}$.

Correspondence: Alain Luna Rodriguez, Resident, Neurology, Hospital de Cruces, Plaza de Cruces s/n, Cruces 48903, Spain. E-mail: alunar@arrakis.es

K. RICHARDSON, M. SAY, Z. THAYER, S. LAH \& L. MILLER. Memory Training for Epilepsy Patients: A Preliminary Investigation. Objective: Epilepsy is often associated with impaired memory functioning, but there has been little research into the effectiveness of memory training for these patients.

Participants and Methods: Nine epilepsy outpatients participated in a weekly, six-session, group memory training program, focusing on memory strategies. All participants had either undergone surgical resection or had evidence of a structural lesion, the majority of which affected the temporal lobes $(n=6)$. Four participants continued to experience seizures during the training period. Performance was measured one month before and one month after training using alternate forms of the Rey Auditory Verbal Learning Test (RAVLT) and prospective memory tasks, as well as questionnaires assessing everyday memory and mood. Results: Of these measures, there was a significant improvement in RAVLT percent delayed recall, with greatest improvements in patients with temporal lobe lesions $(p<.05)$. There were no significant changes on other outcomes, although most were in the positive direction. Higher levels of depression and poorer divided attention at baseline were associated with greater improvements on RAVLT total learning $(p<.05)$ and on prospective memory tasks $(p=.01)$, respectively. No significant effects of current seizure activity were found on the individual outcome change scores, although inspection of the data suggests that ongoing seizures tended to attenuate training gains. 
Conclusions: Overall, these preliminary results suggest that epilepsy patients can benefit from memory training groups, particularly those with temporal lobe abnormalities. The effect of current seizure activity on training outcomes is being investigated further.

Correspondence: Kylie Richardson, University of Sydney, 1/26 Albert Street, Petersham, NSW 2049, Australia.E-mail:kylier@psych.usyd. edu.au

\section{HUYTON, I. WRIGHT \& R. NICOLSON. Sleep Problems In Childhood Epilepsy.}

Objective: To explore the prevalence and nature of sleep problems in a paediatric epilepsy sample, and to investigate variables related to sleep problems.

Participants and Methods: A parental report questionnaire was used to assess sleep problems in 76 children with epilepsy for comparison with 115 healthy control children. The questionnaire grouped sleep problems into five factors: parent/child interactions during the night, sleep fragmentation, parasomnias, daytime drowsiness and bedtime difficulties. Items relating to epilepsy were also included in the questionnaire. Results: Children with epilepsy, as a group, showed significantly more sleep problems than healthy controls on all five sleep factors $(p<0.001)$. Daytime drowsiness was the most common problem in epilepsy, with $74.6 \%$ of the epilepsy group showing evidence of significant problems in this area $(z>1.0)$. Within the epilepsy group, age, polytherapy, seizure type, presence of learning difficulties and AED type were all associated with an increased likelihood of sleep problems.

Conclusions: The results of this study indicate that sleep problems are prevalent in childhood epilepsy. The diversity of the sample recruited points to a non-specific vulnerability to sleep problems, common to all types of epilepsy. The presence of sleep problems in epilepsy may have far reaching implications, above and beyond the negative physiological effects that may be associated with sleep deprivation. Cognitive impairment, and subsequent educational underachievement, has long been associated with epilepsy. There is further evidence to show that appropriate sleep structure and a sufficient quantity of sleep are vital for consolidation in certain types of learning. When investigating the cognitive impairment common in epilepsy, it may be important to consider the role of sleep, and the potentially damaging effects of sleep disturbances on learning and cognitive function.

Correspondence: Clare Huyton, BSc (hons) psychology, Department of Psychology, University of Sheffield, Western Bank, Sheffield S10 2TP, United Kingdom. E-mail: c.huyton@sheffield.ac.uk

\section{Genetic disorders}

\section{K.E. THOMASON, I. WRIGHT, R. NICOLSON, C. RITTEY, R. KANDLER \& A. CHATTOPADHYAY. Cognitive Variability in Children with Epilepsy.}

Objective: Cognitive impairment is frequently reported in children with epilepsy. Aside from the direct effects of seizures on cognition, interictal epileptiform discharges (IED), such as spikes and sharp waves, can also have a detrimental effect on transient aspects of cognition. Two studies are presented, investigating the extent to which IEDs are associated with variability in cognition in a sample of children with relatively well-controlled epilepsy.

Participants and Methods: A questionnaire was developed to assess parental reported variability in children's behaviour. 86 parents of children with epilepsy and 77 without epilepsy returned the questionnaires (return rate $56 \%$ and $31 \%$ respectively). In addition, 10 children with epilepsy completed computerised cognitive tasks alongside an ambulatory electroencephalogram at two time points $\sim 22$ hours apart. The tasks comprised continuous visuo-spatial planning, psychomotor speed, spatial and recognition memory.

Results: In Study 1, children with epilepsy were rated as more behaviourally variable than children without epilepsy $(p<.001)$. In Study 2 , children with a higher frequency of IEDs ( $\geq 1$ spike per min) performed poorer on the tasks and were more variable compared to controls (matched for level of performance). Within-participant analyses regarding accuracy and variability around specific IED events are ongoing and will be reported.

Conclusions: Frequent IEDs are associated with variability in cognitive functioning. Although the effects on cognition may be transient, they may accumulate to have a long-lasting effect. However, caution must be taken when deciding whether to directly treat IEDs, especially in children who may have a general learning problem rather than a transient deficit in function.

Correspondence: Kate E. Thomason, BA (Hons), MSc, Psychology, University of Sheffield, Western Bank, Sheffield S10 2TP, United Kingdom. E-mail: k.thomason@shef.ac.uk

\section{Hemispheric Asymmetry/Laterality/ Callosal Studies}

\section{E. DE NÓBREGA, A. NIETO, J. BARROSO \& F. MONTÓN. Verb} Generation in Patients with Hereditary Cerebellar Ataxia.

Objective: It has been proposed that the cerebellum may be involved in verb generation tasks, but results are controversial. The aim of the present study was to examine verb generation in a semantic association task in a group of patients with cerebellar degeneration.

Participants and Methods: Twenty-five patients and thirty-six matched control participants were examined. Patients presented the molecular genotype of Friedreichs Ataxia (FRDA). The task consists on generating a verb in relation to a given noun. Nouns were selected from a normative study. There were two types of nouns: concrete (i.e. telephone, soap) and abstract (i.e. illness, idea) nouns. All nouns had a plausible related verb that sounds similar to it (i.e. teléfono-telefonear/telephonetelephone). The subjects were required to say a verb that sounds different from the given noun (i.e. teléfono-llamar/ telephone-call). So the task can not be performed by phonological association. Instead, it would require a semantic association. An ANOVA with group (patients vs. healthy control individuals) as the between-groups variable and noun (concrete vs. abstract) as the within-group variable was conducted.

Results: There was a significant main effect of group ( $p=0.03)$. FRDA patients produced less correct responses on the generation of verbs relative to healthy control subjects. There also was a significant main effect of noun $(p=0.00)$. Namely, participants produced more correct verbs with concrete nouns than with abstract nouns. Finally, there was no interaction between group $x$ type of noun $(p=0.38)$.

Conclusions: The present results support a role of the cerebellum in the generation of verbs in a semantic association task.

Correspondence: Érika De Nóbrega Hernández, Bachelor, Psychobiology and Methodology of behavioural sciences, University of La Laguna, Campus de Guajara, La Laguna 38205, Spain.E-mail: enobrega@ull.es

S. KAZANDJIAN, C. PEREZ, B. GRANDJACQUES, M. DE AGOSTINI \&. S. CHOKRON. Hemispheric Specialization in Aesthetic Preference in Homonymous Hemianopia.

Objective: Visual aesthetic preference has been studied since Levy (1976) hypothesized that right-handed individuals prefer pictorial arrangements possessing left-to-right directionality. Since then researchers have implicated center of interest within an image (McLaughlin, 1992), reading direction (Chokron \& De Agostini, 2000), and hemispheric specialization (Kinsbourne, 1997) to aesthetic preference. The current study is a preliminary investigation of left (LBD) or right (RBD) occipital lesioned patients (suffering from a right or left homonymous hemianopia-HH) in an attempt to shed light on the role of each visual cortex on aesthetic preference performance.

Participants and Methods: Four RBD/left HH patients (Mean Age $=71.0, \mathrm{SD}=12.73$ ), three LBD/right HH patients (Mean Age $=67.3$. $\mathrm{SD}=9.29$ ), and 13 healthy subjects (Mean Age=34.4, $\mathrm{SD}=15$ ) were presented with 40 pairs of mirror-images, 10 each of the following four cat- 
egories: oriented mobile objects, oriented stationary objects, lateralized landscape images, and centered landscape images with lateralized details. Preference for the top or bottom image of a pair was elicited. Results: Independent t-tests confirmed that healthy subjects showed a significant left-to-right directionality preference for stationary objects. LBD patients only showed a preference for landscape images with details on the right side of space. On the contrary, RBD patients showed a preference for left-to-right directionality for mobile objects, and a rightward preference for landscapes as previously described in healthy leftto-right readers (Chokron \& De Agostini, 2000).

Conclusions: These findings indicate that each occipital lobe may play a specific role in visual directionality and aesthetic preference, also suggesting an interaction between hemispheric specialization and cultural factors, such as reading habits.

Correspondence: Seta Kazandjian, Ph.D., 2ERT TREAT Vision and Equipe Psychologie et NeuroCognition des systèmes perceptivo-moteurs, Laboratoire de Psychologie et NeuroCognition CNRS 5105, Fondation Ophtalmologique Rothschild / Universite Pierre Mendes France, 2 Rue D'Alencon, Paris 75015, France.E-mail: setakaz@gmail.com

A. NARBERHAUS, R. PUEYO, D. SEgarRa, X. CALDÚ, M. GIMÉNEZ, F. BOTET \& C. JUNQUÉ. Verbal Fluency Impairment in Very Preterm Subjects Correlates with the Corpus Callosum Genu. Objective: Brain damage in premature newborns predominantly involves white matter. Thinning of the corpus callosum, using MRI techniques, reflects diffuse white matter damage. It has been reported reductions in the posterior (splenium) and anterior part (genu). Since the anterior part of the corpus callosum connects frontal cortices, the aim of this study is to explore the possible relationship between verbal fluency and this cerebral region.

Participants and Methods: Forty-nine preterm (gestational age $<33$ ) subjects aged 11-18 years, and 52 matched controls (gestational age > 36) were recruited. Corpus callosum total area was segmented in seven parts and corrected by mid-sagittal area. Phonetic and semantic verbal fluencies were assessed.

Results: Premature subjects showed significant phonetic $(t=-2.138 ; p=$ $0.035)$ and semantic $(\mathrm{t}=-2.885 ; \mathrm{p}=0.005)$ verbal fluency impairment, and a reduction of the corpus callosum genu $(\mathrm{t}=-2.824 ; \mathrm{p}=0.006)$, isthmus $(t=-2.852 ; p=0.005)$ and splenium $(t=-4.546 ; p<0.001)$. The correlation analysis in the preterm group indicated that low phonetic verbal fluency is significantly related to the small genu and splenium areas, but additional regression analysis showed that phonetic verbal fluency performance was mainly explained by the genu $(\mathrm{r}=0.318$; $\mathrm{p}=$ 0.026). In controls we did not observe such relationships.

Conclusions: In conclusion, in the very preterm born subjects aging between 11-18 years the low performance in phonetic verbal fluency seem to be more related to the reduction of the anterior part of the corpus callosum than the posterior part.

Correspondence: Ana Narberhaus, University of Barcelona, Passeig. Vall d'Hebron, 171, Barcelona 08035, Spain. E-mail: anarberhaus@ub.edu

\section{Lobar Degeneration}

R. NISHIMURA \& K. YOSHIZAKI. Selective Attention is Modulated by Independent Resource of Each Hemisphere.

Objective: Lavie (2005) explored the load theory of selective attention, where selective attention was modulated by the perceptual load of the relevant stimuli, using a response-competition paradigm. Her theory assumed a single limited attentional resource. The goal of our study was to investigate whether each hemisphere had an independent resource or not, using the response-competition paradigm.

Participants and Methods: Twenty-four right-handed students participated in this experiment, which had two within-factors: Load of the target (High/Low), Congruency between target and distracter (Compatible/Incompatible). In the High condition, a target was presented with the three nontargets in either a left $(\mathrm{LVF})$ or a right visual-field $(\mathrm{RVF})$ and a nontarget was presented at the opposite visual-field. In the Low condition, only a target was presented at either LVF or RVF and four nontargets were presented at the opposite. Participants made speeded responses, indicating whether a peripheral target is one of two pre-specified letters ("N" or "K") while attempting to ignore a central distracter.

Results: A two-way ANOVA (Load X Congruency) on error rates showed a significant interaction, suggesting that the compatibility effect was obtained in the Low-load, not in the High-load condition.

Conclusions: In both the load conditions, the task-relevant stimulus consisted of a target and four nontargets. If a single resource is assumed, the compatibility effect would not vary irrespective of the load conditions. The present results, however, demonstrated that the compatibility effect was found only under the Low-load condition. These results suggest that each hemisphere independently processes the stimuli with its own resource.

Correspondence: Ritsuko Nishimura, Psychology and Communication, Aichi Shukutoku University, 1-68, Namazu-Takitsubo, Mizuho 5010212, Japan.E-mail:06003cpd@asu-nc.aasa.ac.jp

\section{Other}

M.I. GOMEZ-RUIZ, A. AVILA, J. BELLO, P. MAHO, M.A. ESPASA \& G. ESPEL-TRIAS. Primary Progressive Aphasia in a Bilingual Patient: A Case Report.

Objective: Primary progressive aphasia (PPA) is a neurodegenerative disease characterized by focal cortical degeneration of left perisylvian structures. It mainly affects language abilities while other cognitive functions remain preserved for at least two years. Nevertheless, PPA in bilingual patients has not been fully investigated. The purpose of this study is to compare language performance in a bilingual patient diagnosed with PPA.

Participants and Methods: We present a 74 year-old-right-handed man, fluent in Catalan (L1) and Spanish (L2), who developed progressive language impairment with no other cognitive deficits. His native and dominant language is Catalan; L2 was learned between 5 and 7 years old. He completed his education in Spanish ( 5 years). Language assessment was performed with the Catalan and Spanish versions of the Bilingual Aphasia Test (BAT,Michel Paradis). The BAT consists of 32 subtests that can be grouped by linguistic level (phonology, morphology, syntax, lexicon, semantics) and linguistic skill (comprehension, repetition, judgment, lexical access, propositioning, reading, writing). Results: SPECT: Left temporoparietal and frontal hipoperfusion. Language performance was compatible with an anomic-like aphasia in the two languages. Language performance was similar in the two languages although in some sub-tests Spanish was slightly better (Verbal Auditory Discrimination, Syntactic Comprehension, Synonyms, Antonyms, Verbal Fluency, Mental Arithmetic). During spontaneous speech and description of a short story, the patient was more fluent in Catalan.

Conclusions: The languages of our patient showed a comparable pattern of impairment compatible with an anomic aphasia. Nevertheless, L2 results were slightly better than $\mathrm{L} 1$. These results may be influenced by sociolinguistic factors and educational training.

Correspondence: Maria I. Gomez-Ruiz, Neurology, Hospital General Hospitalet, Av. Josep Molins 29-41, Hospitalet de Llobregat 08906 , Spain.E-mail:misabelgr@gmail.com

\section{Sex Differences/Sex Hormones}

\section{J.D. GOMEZ. Language monitoring during surgery in brain} eloquent areas.

Objective: From a case study some modifications to the protocol of Ojemann appear, as well as a proposal of improvement of functional / structural criteria of selection of candidates to surgeries of epilepsy and gliomas of low grad. 
Participants and Methods: Case study

Results: By means of the direct stimulation ("brain mapping") on the exposed cerebral cortex it is possible to determine an individual cartography of the superior functions integrating, sensorial and motor. During the operation of tumors it is possible to verify the integrity of the functional system in study by means of repeated stimulations and - as in the case of the monitoring of the language by means of its continuous evaluation. The introduction of Neuropsychological Brain Monitoring for illiterate people in Colombia has lead to a considerable improvement of the results of the operations.

Conclusions: The proposed protocol facilitates the selection of candidates to surgery of epilepsy, when the structural criteria are not clear to detect the focus. It is possible to do a monitoreo of the language to illiterate people during the surgery of epilepsy doing small modifications to the ojemanns'protocol.

Correspondence: Juan D. Gómez, Dr. Phil., Psicología, Pontificia Universidad Javeriana, Carrera 5 Nr. 39 - 00, Bogotá D.C. 001, Colombia.E-mail:judago5@hotmail.com

\section{J. JUÁREZ \& M. VIRGEN-ENCISO. Sex Differences in the Effects} of Opioid Antagonist Treatment in Alcoholic Patients.

Objective: Clinical studies have supported reduction in alcohol consumption among alcoholic patients during the treatment with opioid antagonists. However, there are not systematic studies concerning the possible sex differences with this treatment. The analyses of these differences was the objective of the present study.

Participants and Methods: Men and women alcoholic patients were treated with naltrexone (an opioid antagonist) $50 \mathrm{mg} /$ day during 12 weeks and they were supervised during the following 12 weeks after this treatment.

Results: Brief Psychiatric Rating Scale (BPRS) scores were higher in women than in men before the naltrexone treatment (NtxT). The hepatic enzyme, gamma glutamyl transpeptidase was higher in men than in women before NtxT and decrease significantly after NtxT in both sexes. Alcohol consumption decreases during NtxT in both sexes, but increase in higher proportion after treatment in men than in women. Craving for alcohol decreases during NtxT and increases again after NtxT in both sexes; however, men showed higher craving for alcohol than women after NtxT. Complaint of side symptoms was significantly more frequent in women than in men during NtxT.

Conclusions: These results show a differential effect of NtxT that depends on the sex of patients. NtxT has beneficial effects in both sexes, but apparently is more successful in women than men after this treatment is interrupted. These sex differences during and after opioid antagonist treatment could depend on social, neurophysiologic and metabolic differences between men and women.

Correspondence: Jorge Juárez, PhD, Instituto de Neurociencias, Universidad de Guadalajara, Francisco de Quevedo \# 180, col. Arcos Vallarta, Guadalajara 44130, Mexico.E-mail:jjuarez@cencar.udg.mx

\section{OTERO DADÍN \& D. RODRÍGUEZ SALGADO. Verbal Memory} in Healthy Young Men and Women and Sex Hormone Cycles.

Objective: The joint influence of sex and variations in sex hormones on verbal memory has not been studied yet. The aim of this study was to assess sex differences in verbal memory and the influence of male and female sex hormone cycles on it.

Participants and Methods: Twenty-four healthy young subjects, 12 men and 12 women with regular menstrual cycles, matched in age, laterality and education level were assessed using verbal memory tests: Rey Auditory-Verbal Learning Test, and Digit Span and Letter-Number Sequencing subtests. Each subject was tested twice coinciding with the high and the low hormonal phases of its cycle: in the morning or in the afternoon for men, and during luteal or menstrual phase for women. Order of phases was counterbalanced across subjects.

Results: Repeated measures ANOVA showed that men scored higher than women in Digit Forward total $(F=7,769 ; p=0,011)$ and $\operatorname{span}(F=$ 6,838; $p=0,016)$ scores, and Digit Backward span score $(F=4,945 ; p=$ $0,037)$, regardless of their hormonal phase. Men also scored higher than women in Letter-Number Sequencing span score $(F=9,103 ; p=0,006)$, but only when they both were in a high hormonal level phase (men in the morning and women in luteal phase).

Conclusions: These results suggest that there is an influence of natural hormonal variations on sex differences in verbal working memory. The results obtained point out that the adult cycles of sex hormones could be an important factor when sex differences in neuropsychological functioning are assessed, and future studies should control or manipulate it.

Correspondence: Dolores Rodríguez Salgado, Clinical Psychology and Psychobiology, University of Santiago de Compostela, Campus Sur, Santiago de Compostela 15782, Spain.E-mail:pcdrs028@usc.es

M. SABUCEDO, J. BARROSO, A. NIETO, M. RODRIGUEZ \& R. CORREIA. Sex Differences in Frontal Measures.

Objective: The existence of sex differences in frontal functions remains quite controversial. Our aim is to study sex differences in several processes associated with frontal functioning: cognitive flexibility/conceptualization and selective attention/inhibition as prefrontal functions, and motor learning and inhibition as premotor functions. For this purpose we selected four tests traditionally used in clinical and neuropsychological research to evaluate these processes: Motor Series and Conflicting Instructions were employed to assess premotor function, while Wisconsin Card Sorting Test (WCST) and Stroop test were used to evaluate prefrontal functioning.

Participants and Methods: Fifty neurologically normal university students ( 25 women, 25 men) were examined. All subjects were assessed with Motor Series, Conflicting Instructions, WCST and Stroop tests.

Results: Data showed sex differences in three of the four measures employed. Within prefrontal functioning, female outperformed men in WCST as they completed more categories, made less errors, perseverative responses, failures to maintain the set and as they used less number of cards. For the Stroop test, male outscored female in the Interference Score while no differences were found for word reading or colour naming. Attending to premotor functioning, female outperformed men in Motor Series, while no sex differences were found in Conflicting Instructions.

Conclusions: Results obtained are in favour of those studies that find sex differences in frontal lobe. Data showed that the two prefrontal tests and one premotor turned out to be performed differentially by men and women. Thus, we suggest that sex differences are not only in prefrontal, but also in premotor functioning.

Correspondence: María Sabucedo Seoane, Psicoloxía Clínica e Psicobioloxía, University of Santiago de Compostela, Facultade de Psicoloxía.Campus Sur, Santiago de Compostela. 15782, Spain. E-mail: msabuce@usc.es

\section{Traumatic Brain Injury}

K. BEKKEN \& W.N. PACHAS. Development of Fibromyalgia (FM) Following Mild Traumatic Brain Injury (TBI).

Objective: Fibromyalgia is a complex disorder characterized by widespread body pain, fatigue, sleep disorders, "fibromyalgia fog", and an array of associated disorders, such as irritable bowel syndrome, Raynaud's phenomenon, reactive depression, and others. The etiology of FM is unknown, although recent studies have shown significant abnormalities in the central and autonomic nervous systems in these patients. We have previously reported similarities in mental changes between FM and mild TBI patients in 22 of 26 enuropsychological measures. Our objec- 
tive here was to use information from neuropsychological evaluation of FM patients who had suffered previous TBI to contribute to our understanding of the mechanisms of FM.

Participants and Methods: We now report a cohort of 15 patients that developed FM following mild-moderate TBI. The symptoms of FM occurred either shortly after or up to two years following the TBI. The clinical course of these patients were consistent with the diagnostic criteria for FM established by the American College of Rheumatology. Standard neuropsychological evaluations were conducted, and results were compared within and between patients to detect trends. Measures included gender, severity of head injury, and severity of FM symptoms. Results: Results show what appears to be a cumulative effect of FM and TBI on deficits in cognitive functioning in these patients overall. Severity of TBI and of FM also exerted effects. Future directions are discussed. Conclusions: We suggest that TBI is a significant risk factor for the development of FM, and discuss the implications of this association on our understanding of FM.

Correspondence: Kaaren Bekken, PhD, CDN, 76 Bedford St, Ste 12, Lexington,MA 02420.E-mail: kbekken@gmail.com

S. BOLOGNANI, D. LANDUCCI-MOREIRA, A. FABRICIO, P. GOUVEIA \& O. BUENO. The Dysexecutive Syndrome Questionnaire as a Measure of Self Awareness and Rehabilitation Outcome.

Objective: Dysexecutive Syndrome (DS) is a group of cognitive impairments usually seen after a traumatic brain injury (TBI). DS have been reported as the main cause of poor outcome and adjustment, even several years after injury. It is very important to develop and test rehabilitation approaches and tools addressing DS. We will report a case of TBI rehabilitation, describing changes in patient's and relative's perception of executive problems.

Participants and Methods: B.C., a 21 year-old man, sustained TBI after a gunshot, resulting in bilateral frontal lobe damage. Four months after injury, his only complaints were loss of olfaction and scalp pain. His parents reported he was slow, distractible, talkative and irritable, with periods of apathy and lack of initiative. The rehabilitation process lasted 36 months, with weekly meetings for B.C. (education, cognitive therapy), and family (education, support sessions). Patient and mother completed the Dysexecutive Syndrome Questionnaire (DEX) at treatment start and after 24 and 36 months.

Results: Relative's scores at DEX showed B.C. had progressive improvement in executive functioning. Patient's scores showed improvement in perception of deficits, increasing accordance rates among his and his mother's scores through time. Changes in DEX scores corroborated gradual return to previous activities and social life.

Conclusions: Cognitive and educational approaches helped improving patient's self awareness, leading to behavioral changes and functional gains. Long term therapy seemed to be helpful, since the deficits were long-lasting and needed to be addressed in real life, as the patient returned to previous activities. DEX was helpful for symptom assessment and individual outcome measurement.

Correspondence: Silvia Bolognani, BA Specialist, Psicobiologia, UNIFESP Universidade Federal de Sao Paulo, Alameda Santos 545 ap 102, Sao Paulo01419-000, Brazil.E-mail: sbolognani@psicobio.epm.br

E. DE GUISE, J. LEBLANC, H. THOMAS, N. GOSSELIN \& M. FEYZ. Should TBI Patients with Premorbid Psychosocial Issues be Excluded from Neuropsychological Neurotrauma Studies?

Objective: Many patients with traumatic brain injured (TBI) admitted to neurotrauma centres suffer from long-term histories of alcohol or drug abuse as well as psychiatric issues. Some studies have shown that these factors affect the cognitive functioning of TBI patients. The goal of this study was to explore the effect of this co-morbidity on the duration of post-traumatic amnesia (PTA) and on the outcome measured by the Glasgow Outcome Scale (GOS) score.
Participants and Methods: Thirty patients who presented with a premorbid history of alcohol or drug abuse and/or psychiatric problems and thirty patients without these issues were compared.

Results: The results showed a significant between-group difference where the patients with co-morbidity had a longer PTA than the group without co-morbidity $(35.6 \pm 23.0$ and $16.4 \pm 8.4$ days respectively, $p=0.005)$. Worse scores were also observed in patients with co-morbidity for the GOS scores $(p=0.04)$, while no group difference was found for Glasgow Coma Scale score upon admission. These differences were not explained by the patient's age.

Conclusions: In conclusion, it seems that these two groups of TBI patients have a different recovery process and mechanisms of cognitive compensation after a cerebral insult. Thus, we believe that the exclusion of patients with premorbid psychosocial issues in neuropsychological studies is relevant within the context of acute neurotrauma, that during the initial days and weeks post TBI. Finally, special consideration of these more vulnerable patients is required in order to put into place specific medical, pharmacological, rehabilitation and psychological protocols of treatment.

Correspondence: Elaine de Guise, Ph.D., Psychology, McGill University Health Center, 1650 Cedar Ave., local D13-124, Montreal, QCH3G 1A4, Canada.E-mail: elaine.deguise@muhc.mcgill.ca

M. DIAZ, L. SAN MIGUEL-MONTES, E. SENIOR \& M.T. MARGARIDA. Demographic Characteristics and Neurocognitive Performance among Puerto Rican Traumatic Brain Injury Patients from Car-Related Accidents.

Objective: The State Administration for Compensation to Automobile Accidents (ACAA) in Puerto Rico reported 30,132 cases in 2005-06, of which $91 \%$ ranged between the ages 15 to 59 . Several of these individuals completed a neuropsychological assessment to evaluate cognitive damages indicating Traumatic Brain Injury (TBI) diagnoses. Our aim was to evaluate demographic characteristics of TBI patients referred by ACAA and analyze neurocognitive changes resulting from car accidents.

Participants and Methods: We reviewed the neuropsychological records of 15 patients, from the Neurology Clinics-University of Puerto Rico School of Medicine, in which 53.3\% were males within the ages of 19 to $56(\mathrm{X}=31.27 ; \mathrm{SD}=11.61)$. Analysis included demographic variables of age, gender and years of education, a cognitive assessment of intelligence (WAIS III) and MRI findings.

Results: Descriptive statistics showed that $26.7 \%$ had a high school diploma at the time of the accident, followed by $20.0 \%$ from recent graduate students. MRI findings showed that $60 \%$ suffered frontal lesions, $20 \%$ had temporo-parietal damages and $20 \%$ presented changes in both hemispheres. Analysis from WAIS III indicated low average scores for Verbal Scale $(X=82.33 ; \mathrm{SD}=13.04)$, Performance Scale $(X=83.53$; $\mathrm{SD}=11.98)$, and Total IQ $(X=81.47 ; \mathrm{SD}=11.56)$. However, a borderline performance was evident in the Processing Speed Index ( $X=77.21$; $\mathrm{SD}=10.63)$.

Conclusions: In summary, our study suggests that the youngest population and less educated engages in risk behaviors while driving a vehicle that can lead to brain damages and consequently to cognitive abnormalities.It is of interest that Processing Speed was the most compromised area, suggesting the effect of the defuse of axonal injuries observed in TBI.

Correspondence: LIza San Miguel-Montes, Psy: D., Neurology, University of Puerto Rico Medical School, PMB 200 \#1353 St. 19, Guaynabo 00966, Puerto Rico. E-mail: lesmm28@hotmail.com

S. DIKMEN, J. MACHAMER \& N. TEMKIN. Retrospectively Assessed Post-Traumatic Amnesia: Is it of any Value?

Objective: Post-traumatic amnesia (PTA) is a useful index of traumatic brain injury severity. However, much concern has been raised about its 
reliability and validity when assessed retrospectively. The present study examined its reliability, and its predictive and concurrent validity with respect to neuropsychological and functional status measures, and how well it performs compared to other measures of brain injury severity.

Participants and Methods: Subjects were 286 consecutive adult hospital admissions with complicated mild to severe non-penetrating traumatic brain injury. They were examined at 1, 6, and 12 months post injury on neuropsychological and functional status measures. At each time point their PTA was also assessed

Results: The correlation between PTA assessed at 1 month with that obtained at 6 and 12 months was .74 and .72 respectively. The correlation between 6 and 12 months was .79. The correlation between PTA and neuropsychological and functional status measures assessed at 1 month were in the mid $.60 \mathrm{~s}$ and above. This relationship declined to the $.40 \mathrm{~s}$ at 6 months and $.30 \mathrm{~s}$ to mid $.40 \mathrm{~s}$ at 12 months. Both time to follow simple commands and GCS showed the same pattern but with weaker relationships to outcome

Conclusions: Retrospectively assessed PTA appears to be a practical and useful measure of severity of brain injury. Its reliability and validity are better than what is thought and certainly as good if not better than TFC or GCS in non-select cases with complicated mild to severe TBI. Correspondence: Sureyya Dikmen, PhD, Rehabilitation Medicine, University of Washington, Box 356490, Seattle, WA 98195. E-mail: dikmen@u.washington.edu

J.M. DOUGLAS. 'I don't like being alone...': Quality of life and Social Support after Severe Traumatic Brain Injury (TBI).

Objective: The aim of this study was to explore the experience of social support and it's impact on life after TBI from the perspective of adults living with the long-term consequences of injury.

Participants and Methods: Participants were 39 adults who had sustained severe brain injuries (posttraumatic amnesia $>4$ weeks) more than 4 years ago. No participant had successfully returned to competitive, paid employment and all were living in the community with family or paid support. Combined quantitative-qualitative methodology was applied. Data collection involved a semi-structured interview with completion of a subjective QOL rating scale and a social support questionnaire. Transcribed interviews were coded and analysed using principles of grounded theory. Results: Regression analysis revealed that social support shared a significant $(\mathrm{F}(1,38)=11.7, \mathrm{p}=.002)$ and substantial proportion of variance with QOL $(\mathrm{R}=.49)$. The roles of instrumental and expressive social support were then evaluated. Only strong-tie support made a significant unique contribution to the prediction of QOL $(p=.02)$. Qualitative analysis revealed that life after injury was dominated by two major themes: 'having friends / people who care and understand' and 'being involved' in activities.

Conclusions: It is not surprising that having friends and participating in activities emerged as highly valued aspects of life for people with TBI - they are valued by us all. More importantly however, these findings emphasise the need to focus rehabilitation efforts on developing skills and systems that promote positive outcomes in these areas.

Correspondence: Jacinta M. Douglas, PhD, Human Communication Sciences, La Trobe University, Kingsbury Drive, Bundoora, VIC 3086, Australia.E-mail: J.Douglas@latrobe.edu.au

S.E. FAIRJONES, J. BATCHELOR, S. MEARES, E. SHORES, J. CHAPMAN, I. BAGULEY, J. GURKA \& J. MAROSSZEKY. Predicting Cognitive Outcome Following Mild Traumatic Brain Injury: Applying Reserve Theory.

Objective: Brain reserve capacity, outlined by Paul Satz in 1993, refers to the brain's ability to maintain function when confronted by degenerative processes or insult. Research investigating acute outcome following mild traumatic brain injury in terms of brain reserve is sparse. The current research involves assessing individuals who have sustained a mild traumatic brain injury (MTBI) at 3 months post-injury to de- termine whether the reserve theory is applicable and of interest in determining outcome and enabling appropriate treatment and strategy implementation.

Participants and Methods: 50 subjects were recruited from an ongoing study at Westmead Hospital, Sydney. Participants were recruited for the original study upon admittance to Westmead Hospital following trauma if aged between 18-65. The first 50 MTBI participants recruited to the study were included in the analyses.

Results: Individuals with a higher cognitive or brain reserve (ie. Higher IQ and education, no history of neurological damage or drug/alcohol abuse) performed better on cognitive measures after sustaining an MTBI than those with a lower reserve.

Conclusions: It is important to assess all the possible premorbid factors which may contribute to outcome following MTBI. Attempting to determine which factors influence cognitive outcome following MTBI is essential in predicting recovery to allow for proper interventions, implementation of recovery strategies and managing the best outcome for patients.

Correspondence: Sarah E. Fairjones, Psychology \& Linguistics, Macquarie University, 19/118 Elizabeth St, Ashfield, Sydney, NSW 2131, Australia.E-mail: s.fairjones@unsw.edu.au

D. FERNÁNDEZ-ESPEJO, R. SÁNCHEZ-CARRIÓN, S. SORIAPASTOR, L. ILANKOVIC, C. FALCÓN, A. GARCÍA-MOLINA, T. ROIG, N. BARGALLÓ \& C. JUNQUÉ. Working Memory correlates with White Matter decrease in diffuse Traumatic Brain Injury.

Objective: The aim of this study was to evaluate changes in white and grey matter concentration and volume following traumatic brain injury by means of the voxel-based morphometry approach. In addition, we tried to identify the cerebral substrate of working memory deficits in these patients.

Participants and Methods: Fifteen patients with severe diffuse traumatic brain injury (TBI) were compared with fifteen healthy controls matched by age, sex, and estimated premorbid intelligence. T1 weighted magnetic-resonance images (MRI) were acquired and analysed with statistical parametric mapping software (SPM2). Working memory was assessed by means of n-back test and working memory index of WAIS-III. Results: Patients differed from controls in all working memory tests. Voxel-Based Morphometry results showed significant white matter (WM) concentration reductions in several brain regions in the TBI group involving both hemispheres and cerebellum, and volume reductions in some pontine tracts. We did not detect any significant gray matter (GM) abnormality in the whole brain analysis. Nevertheless, ROI analysis showed hippocampus, thalamus and caudate nucleus GM significant density reductions. Decreased WM concentration correlated with lower scores on working memory tests, speed processing and with a larger post traumatic amnesia.

Conclusions: These findings suggest that white matter changes in TBI are more important than grey matter changes and partially explain working memory deficits.

Correspondence: Davinia Fernández-Espejo, Psiquiatria i Psicobiologia Clínica, Universitat de Barcelona, Casanova, 143, Barcelona 08036, Spain.E-mail: daviniafernandez@ub.es

A. GARCIA-MOLINA, T. ROIG ROVIRA, R. SANCHEZ-CARRIÓN ABASCAL, A. ENSEÑAT CANTALLOPS, N. PICÓ AZANZA, M. YUGUERO RUEDA \& M. BERNABEU GUITART. Community integration as an outcome of traumatic brain injured rehabilitation. Objective: Traumatic brain injury (TBI) is associated with lifelong impairment across multiple domains. The main objective of rehabilitation is to enable traumatic brain injury patients to return to their life roles and become active members of the community as near as possible to pre-injury levels of participation. The objective was to describe community integration of rehabilitation patients with moderate to severe TBI 
Participants and Methods: Ninety-one patients with moderate or severe TBI divided into two groups (group A: patients followed up 2 years after injury; group B: patients followed up 5 years after injury). Community integration after TBI was assessed using the Sander's version of the Community Integration Questionnaire.

Results: Of the group A, 78.4\% were independent for basic activities of daily living, $45 \%$ participate in leisure activities, and 39.3\% returned to work at follow-up. Group B was more disabled in basic activities of daily living than group A. Both groups show similar levels of integration on Social Integration Scale and Productivity Activities Scale. Both had higher scores on the Social Integration Scale than on Productivity Activities Scale.

Conclusions: Community integration is a useful measure of outcome after TBI rehabilitation, not only for evaluating the clinical effects of TBI rehabilitation but also because of its potential implications for health policy decisions. Our findings in community integration of rehabilitation patients with TBI and implications for rehabilitation are discussed. Correspondence: Teresa Roig Rovira, PhD, Neuropsychology, Institut Guttmann, Cami de Can Ruti s/n, Badalona 08916, Spain. E-mail: troig@guttmann.com

B. GARZÓN, R. SÁNCHEZ-CARRIÓN, C. FALCÓN, T. ROIGROVIRA, N. PICÓ-AZANZA, N. BARGALLÓ \& C. JUNQUÉ. VOXEL BASED DIFFUSION TENSOR ANALYSIS SHOWS WHITE MATTER ALTERATIONS IN SEVERE TRAUMATIC BRAIN INJURY PATIENTS WITHOUT NEURORADIOLOGICAL LESIONS.

Objective: To identify abnormal neural fibres in diffuse traumatic brain injury patients with cognitive impairments using diffusion tensor imaging (DTI), a promising method for the non-invasive detection of the degree of fibre damage by measuring anisotropy of tissular water motion. Participants and Methods: Sixteen normal subjects and 10 patients with severe diffuse traumatic brain injury without manifest neuroradiological lesions who went into a coma (Glasgow Coma Scale less or equal to 8 in all cases) following road accidents and showed cognitive impairment after recovering conciousness. DTI analysis consisted of voxelbased fractional anisotropy comparison between controls and patients performed by SPM5 software.

Results: Voxel-based analysis of fractional anisotropy images revealed statistically significant differences between patients and controls at corpus callosum, superior longitudinal and occipito frontal fasciculi. The splenium of the corpus callosum was the most affected region.

Conclusions: DTI offers a sensitive enough technique to detect abnormal white matter projections associated with diffuse traumatic brain injury leading to cognitive deficit even in absence of neuroradiological pathological manifestations.

Correspondence: Benjamín Garzón, M. Sc., Psicobiologia clínica i psiquiatria, Universitat de Barcelona, CASANOVA, 143, Barcelona 08036, Spain.E-mail: benjamin_garzon@ub.edu

C. HUNT, S. MCDONALD \& J. HENRY. An Experimental Examination Of Disorders Of Control And Drive Following Traumatic Brain Injury.

Objective: People with Traumatic Brain Injuries (TBI) often have difficulties with social and emotional processing. One of the major problems they face relates to the regulation of emotion and behaviour. They often experience either disorders of control, characterised by impulsivity and aggressive behaviours or disorders of drive, characterised by apathy, rigidity and lack of initiative. This study attempted to link neuropsychological deficits suggestive of these disorders and responses towards emotional evocative films. It also attempted to examine any differences between individuals with a TBI and those without in their use of emotion regulation strategies.

Participants and Methods: 20 people who had sustained a severe TBI and 20 matched control participants were shown video clips designed to induce anger. Participants were given instructions designed to en- gender the use of specific emotion regulation strategies whilst watching these stimuli. Participants were also given various neuropsychological tasks designed to examine cognitive markers of disorders of drive and of control.

Results: Individuals who performed poorly on neuropsychological tasks examining impulsivity were also showed an increased angry response to emotional movie clips. Conversely, those who exhibit deficits on a task of cognitive flexibility displayed a decreased sad response to the emotional stimuli.

Conclusions: These findings complement and extend previous clinical work that examined disorders of control and drive through the use of questionnaires. With regards to questions about the use of specific emotion regulation strategies, the current findings were inconclusive. Correspondence: Skye McDonald, PhD, School of Psychology, University of NSW, UNSW, Sydney, NSW 2052, Australia. E-mail: s. mcdonald@unsw.edu.au

R. KNIGHT \& K. O'HAGAN. Recall of Autobiographically Significant Episodes Involving Famous Names by Persons with Traumatic Brain Injury.

Objective: The aim of this study was investigate the effect of traumatic brain injury on knowing and remembering information about famous people.

Participants and Methods: A group of 19 persons (15 men, 4 women: mean age 45) who had experienced a severe TBI an average of 13 years previously were recruited. Each person with TBI was matched with a healthy individual of the same age, gender, and occupation. Participants were administered a randomly-ordered list of 115 famous names, each of whom were famous within a particular decade, and 25 non-famous distracter names. Each participant was first asked to decide which were names of famous people and to state the reason for their fame. The names of each of the persons identified as famous were then readministered, and participants were asked to recall a personal memory of the person. These memories were then divided into those where the participant recalled a specific autobiographical incident associated with the famous names and those where the memory was more general.

Results: The TBI group identified fewer of the famous names but the pattern of the results across decades was equivalent for the two groups. The TBI group had fewer specific memories than the controls, and their deficit was most marked for recent decades. Analysis of the probability of recalling specific memories revealed that recall was poorer for names famous after, rather than before, the TBI.

Conclusions: The results show that persons with TBI have fewer recent personal memories available to contribute in interactions with others. Correspondence: Robert Knight, PhD, Psychology, University of Otago, Box 56, Dunedin 9013, New Zealand. E-mail: rknight@psy.otago.ac.nz

K.J. KWAPIL, A. HINTON-BAYRE, G.M. GEFFEN \& K. MCFARLAND. Neuropsychological Outcome following Mild Traumatic Brain Injury: Clinical Significance and Reliable Change Indices.

Objective: The duration of symptomatology and cognitive impairment following mild traumatic brain injury (mTBI) remains controversial. The present study sought to prospectively and serially assess these outcome measures following such trauma. Particular focus was given to the application of group- versus individual-based analytic techniques.

Participants and Methods: Thirty mTBI cases completed a brief battery of neurocognitive functioning and symptom reporting within hours of injury and serially at one-week, one-month and three-months. Thirty uninjured controls were assessed at identical intervals.

Results: The mTBI group reported more symptoms than controls after one-week. However diagnostic accuracy using symptoms was no greater than chance at one- and three-months. Participants with mTBI performed more poorly than controls on measures of verbal memory and speed of information processing acutely, at one-week and one-month. 
with group differences still evident after three-months. Approximately $70 \%$ of mTBI patients were impaired on one or more tests acutely, oneweek, and one-month. By three-months 52\% demonstrated ongoing impairment. Using reliable change methodology significant recovery was demonstrated by $20 \%$ of individuals by three months, with gains particularly from acute to one-week and from one-month to three-months. However for half of the mTBI participants this recovery was incomplete by three months.

Conclusions: The current results suggest neurocognitive impairment persisted longer than symptoms in an uncomplicated, non-litigating mTBI sample. The results also highlight the importance of an individual approach to the assessment of mTBI and support the notion that a proportion of mTBI cases may have protracted difficulties. Correspondence: Anton Hinton-Bayre, MBBS (Hons), PhD, Department of Emergency Medicine, Royal Brisbane and Women's Hospital, Butterfield Street, Herston, Brisbane, QLD 4006, Australia. E-mail: anton.hb@hotmail.com

A. MCLINDEN, S.R. MILLIS \& R. HANKS. Using the London Handicap Scale to Measure Quality of Life Following TBI.

Objective: To describe health-related quality of life (QOL) at 1 and 2 years post-TBI using the London Handicap Scale (LHS), which has yet to be well-validated in TBI. The association between the LHS, several neuropsychological measures, and two well-validated QOL measures (SF-36 and Satisfaction with Life Scale [SWLS]) was examined, as was the underlying factor structure. It was expected that performance on the neuropsychological measures would predict the LHS score and that the LHS would load onto the same factor as the SF-36 and SWLS. Participants and Methods: Participants were 70 adults aged 16-76 years who sustained a significant enough brain injury to be admitted to an inpatient brain injury rehabilitation service, and were subsequently enrolled in a TBI Model System Project. They were administered a neuropsychological battery (Symbol Digit Modalities Test, Trail Making Test, and Rey Auditory Verbal Learning Test) during inpatient treatment, and then the QOL measures at 1 and 2 years post-TBI.

Results: Contrary to expectations, neuropsychological test performance was not strongly predictive of the LHS score. The LHS correlated positively with both SF-36 indices but not with the SWLS. Also, as hypothesized, a principal components analysis revealed that the LHS loaded onto the same component as the other QOL measures, whereas the neuropsychological measures loaded onto a separate component.

Conclusions: The findings demonstrate good discriminant validity between the LHS and measures of neuropsychological outcome, as well as good convergent validity with the SF-36, a measure that has been wellvalidated for use with a TBI population.

Correspondence: Angela McLinden, M.A., Psychology, University of Windsor, 318 Curry Ave, Windsor, ON N9B 2B7, Canada. E-mail: amclinden@gmail.com

S.J. MEACHEN, R. HANKS, L. RAPPORT \& S. MILLIS. Internal Consistency and Test-Retest Reliability of the Brief Symptom Index 18 (BSI-18) in a Sample of Traumatic Brain Injury Survivors.

Objective: To investigate the reliability of a new self-report screen of psychological distress, the BSI-18 (Derogatis, 2001), among TBI survivors.

Participants and Methods: Participants were 257 adults who sustained moderate to severe TBI, and who completed the BSI-18 during their inpatient rehabilitation stay after clearing posttraumatic confusion $(\mathrm{n}=81)$ or during a follow-up appointment $(\mathrm{n}=176)$. Time post injury for this follow-up group was 6 months to 15 years. Internal consistency and retest reliabilities were examined for inpatients $(77.8 \%$ men) and outpatients (83.5\% men) separately. Thirty-four inpatients and 27 outpatients had two BSI-18 administrations available for testretest analyses.
Results: Internal consistency estimates for the Global Severity Index (GSI) were high in both inpatients and outpatients (Outpatients, $\alpha=.91$; Inpatients, $\alpha=.84)$. Among outpatients, internal consistency estimates of the Somatization (SOM), Depression (DEP) and Anxiety (ANX) indices were $\alpha=.75, .84$, and .83 , respectively. Internal consistency estimates were lower for inpatients $(\alpha=.61, .64$, and .74 , respectively). Retest reliability estimates (rho) for the inpatient participants tested 6 months to 2 years later were: GSI .66, SOM .67, DEP .63, and ANX .57 $(p<.01)$. For outpatients retested after 1 year, test-retest estimates were: GSI .78, SOM .85, DEP .79, and ANX .45 ( $<<.01)$.

Conclusions: Modest reliability estimates may place an upper bound on the validity of the BSI-18 clinical indices in TBI populations. By contrast, the internal consistency and test-retest reliabilities of the 18-item GSI were excellent. The GSI appears to be appropriate for use both during inpatient rehabilitation and at outpatient follow up.

Correspondence: Sarah J. Meachen, B.A.(hon), Psychology, Wayne State University, 5057 Woodward - 7th floor, Detroit, MI 48202. E-mail: smeachen@wayne.edu

S. MILLIS, S.J. MEACHEN, R. HANKS \& L. RAPPORT. Rasch Analysis of the BSI-18 in Traumatic Brain Injury.

Objective: Respondents to the BSI-18 (Derogatis, 2001) rate distress on a scale of 0 (not at all) to 4 (extremely). The aim of this study was to investigate the psychometric properties of the BSI-18 in a sample of TBI survivors via Rasch analysis.

Participants and Methods: 258 people with TBI completed the BSI18 as part of the Southeastern Michigan Traumatic Brain Injury Model Systems, of whom 81 were inpatients $(78 \%$ men) and 176 were outpatients $(84 \%$ men). Rasch analysis provides psychometric information that is not provided by traditional analyses. It compares the response patterns of persons to the entire sample to estimate person "ability" and item "difficulty."

Results: Rasch analysis determined that the 5-category rating scheme used in the standard BSI-18 administration was unsatisfactory. This Likert scaling produced disordered Rasch-Andrich thresholds, suggesting that participants were not able to reliably distinguish the symptom rating categories. There was also poor person-item mapping in which only the most distressed participants were detected. Item categories were then reduced to a dichotomous scales (i.e., symptom present or absent) and the model was refitted. This revised scale had good psychometric properties: a person separation ratio of 1.96 with a person reliability of .79 and an item separation ratio of 5.59 with an item reliability of .97 All outfit mean-squares were less than 1.54 and all infit mean-squares were less than 1.26. Principal component analysis of standardized item residuals supported the existence of a unidimensional scale: the first factor extracted $63.2 \%$ of item residual variance. Person-item mapping was substantially improved.

Conclusions: A binary scoring system for the BSI-18 appears to improve the psychometric properties of the scale when used to assess people with TBI. There is good evidence that the BSI-18 measures one construct of general psychiatric distress in this population, rather than three distinct constructs of somatization, depression, and anxiety Correspondence: Sarah J. Meachen, B.A.(hon), Psychology, Wayne State University, 5057 Woodward - 7th floor, Detroit, MI 48202. E-mail: smeachen@wayne.edu

H. MILLER, R.J. MCCARTER, L. HUNT \& P.M. SHARPLES. Duration of Post-Traumatic Amnesia (PTA) is a Better Predictor of Cognitive Outcome After Paediatric Traumatic Brain Injury (TBI) than Admission Glasgow Coma Score (GCS).

Objective: To investigate the relationship between PTA duration and cognitive outcome at 1 and 12 months after paediatric traumatic brain injury (TBI) and compare the relationship between duration of posttraumatic amnesia (PTA) and cognitive outcome with another severity of injury measure, the Glasgow Coma Score (GCS). 
Participants and Methods: Prospective cohort study of 52 TBI children (mean age 12.5 years) admitted to a Regional Neuroscience Centre. Level of consciousness was assessed by GCS: PTA by Westmead PTA Scale. TBI severity was classified by admission GCS: severe (GCS 3-8), moderate (GCS 9-12) mild (GCS 13-15) and PTA duration: mild (5-60 minutes), moderate (1-24 hours), severe (1-7 days), very severe (1-4 weeks), extremely severe ( $>4$ weeks). Cognitive outcome was assessed using Wechsler Intelligence Scale for Children (WISC-III-short form), Children's Memory Scale (CMS) and Test of Everyday Attention in Children (Tea-Ch). The Wechsler Quicktest assessed academic ability.

Results: At one month post injury, significant negative correlations were observed between PTA duration and VIQ ( $p=0.002)$, PIQ $(p<0.001)$, processing speed $(p=0.029)$, selective attention $(p=0.009)$, attentional switching/control $(p=0.012)$, reading $(p=0.04)$, spelling $(p=0.045)$, and mathematical ability $(p=0.001)$. Admission GCS correlated with VIQ $(p=0.025)$ and mathematical ability $(p=0.048)$ only. At 12 months PTA duration correlated with PIQ $(p=0.004)$, processing speed $(p=0.006)$, General Memory Index $(p=0.001)$ and mathematical ability $(p=0.036)$. Admission GCS correlated with General Memory Index ( $\mathrm{p}=0.031)$

Conclusions: Duration of PTA is a significantly better predictor of cognitive outcome than GCS. Results suggest routine use of a PTA scale would assist clinicians identify patients most at risk of longer-term cognitive sequelae following TBI.

Correspondence: Helen Miller, Neuropsychology, Frenchay Hospital, Room 1, Academic Centre, Frenchay Hospital, Bristol BS16 1LE, United Kingdom.E-mail: helen.miller@nbt.nhs.uk

M.R. NEWSOME, R.S. SCHEIBEL, J.L. STEINBERG, M. TROYANSKAYA, R.A. RAUCH, X. LI \& H.S. LEVIN. Working Memory Brain Activation following Severe Traumatic Brain Injury.

Objective: Because severe traumatic brain injury (TBI) typically impairs and potentially reorganizes cognitive control as a compensatory mechanism mediating recovery, we hypothesized that working memory brain activation during an N-back task would be altered in TBI patients relative to orthopedically injured $(\mathrm{OI})$ patients when memory load was raised from 1- to 2-back.

Participants and Methods: Ten adult patients who had sustained severe TBI and 6 adult patients who had sustained extracranial injury underwent fMRI 3 months post-injury while performing a block design N-back working memory task that used faces as stimuli. The OI patients were chosen to control for predisposing risk factors that might affect cognition. One-back and 2-back conditions were presented in separate scanning runs in which each condition alternated with a 0 -back condition.

Results: Both groups obtained performance A' values of $>.90$ during scanning. In random effects group comparisons between 0-back and 1back conditions, the OI group activated lateral frontal regions to a greater extent than the TBI group, while the TBI group displayed greater nonfrontal activation compared to the OI group. Group comparisons revealed an activation increase between the 1-back and 2-back conditions in TBI compared to OI patients in medial frontal areas, while OI patients did not activate any areas greater than TBI.

Conclusions: These findings suggest that different neural processes accompany similar accuracy rates in TBI and OI patients. Plasticity associated with severe TBI is reflected by under-recruitment of frontal regions during light memory loads, while more challenging working memory conditions result in increased recruitment of working memory associated frontal areas.

Correspondence: Mary R. Newsome, PhD, Physical Medicine \& Rehabilitation, Baylor College of Medicine, 1709 Dryden Ave., Ste 725, Cognitive Neuroscience Laboratory, Houston, TX 77030. E-mail: mnewsome@bcm.edu
P. NOGUERA, M. GARCÍA-BLAZQUEZ, M. PUERTAS, J. CHIRIVELLA, J. FERRI, O. RENAU, N. FERRI, C. COLOMER \& E. NOE. Recovering Verbal Memory after Post-Traumatic Amnesia: a 6-Month Prospective Study.

Objective: To examine memory functions immediately after post-traumatic amnesia (PTA) resolution and to analyze the recovery of learning and memory capacities over a 6 -month follow up.

Participants and Methods: The initial sample included 200 consecutive patients aged $>16$ years, who had sustained a traumatic brain injury (TBI), referred to our hospital since January 2000. Exclusion criteria included: being out of PTA at admission $(n=77)$, being discharged prior to PTA resolution $(n=55)$ and presence of aphasia/anarthria $(n=10)$. Fifty-five patients in PTA at admission were administered the GOAT every day until they were considered to be out of PTA. Verbal memory assessment with the Spanish version of the California Verbal Learning Test, was conducted 2-4 weeks after PTA resolution and 6-months after inclusion in a multidisciplinary rehabilitation program. Memory change over time was correlated to demographic and clinical relevant variables. Results: Most of our patients presented deficits in verbal learning $(87 \%)$, immediate memory $(96.4 \%)$ and delayed memory $(96.4 \%)$ at baseline, with an important effect of retroactive interference (87.2\%). At 6-month follow-up, $70 \%$ showed learning difficulties, $72 \%$ immediate memory deficits, and $79 \%$ delayed memory problems, with still $51 \%$ of the patients showing retroactive interference. Chronicity and PTA duration correlated to the degree of memory change over time.

Conclusions: Prominent deficits in learning and verbal memory remain even many months after resolution of PTA. Most of these problems seem to be related to an impaired consolidation of information associated with an intense retroactive interference. These data should be considered when developing memory rehabilitation strategies.

Correspondence: Enrique Noe, MD, Ph.D, Servicio Daño Cerebral de Hospitales NISA, C/ Río Tajo s/n, Valencia 46011, Spain. E-mail: enoe@comv.es

\section{B. PLOWRIGHT, G. KINSELLA, B. ONG, J. OLVER \& T. KOSSMANN.} Early Cognitive Outcome from Trauma in Older Adults.

Objective: This study reports on the first wave of assessment in a longitudinal study of cognitive outcome in older adults (65 years and over) who have suffered a traumatic accident and sustained a mild or moderate traumatic brain injury (TBI). While the effect of TBI on cognition in younger adults has been well researched, our understanding of the differential impact of trauma on the aging brain is limited.

Participants and Methods: Fifty TBI participants recruited from The Alfred Trauma Center in Melbourne completed standardized neuropsychological measures of new learning and memory, executive attention of working memory, and speed of information processing between two and four weeks post-injury. Experimental prospective memory tasks were also administered to provide a behavioral index of everyday working memory. These findings were compared to 25 age-matched trauma patients without brain injury (trauma control group) and 25 healthy adults living in the community (healthy control group).

Results: All trauma groups (mild TBI, moderate TBI, and trauma control) showed significantly impaired aspects of new learning and memory (initial acquisition, delayed recall) and executive attention (set-shifting, focused attention and inhibition), in addition to significantly slowed speed of information processing relative to the healthy control group. However, only the prospective memory tasks were successful in distinguishing the TBI groups from the trauma control group.

Conclusions: These results are discussed in relation to trauma and aging, and the utility of measures that require an interaction of cognitive domains rather than indexing efficiency in single domains of cognition. Correspondence: Bethan Plowright, School of Psychological Science, La Trobe University, Kingsbury Drive, Bundoora, VIC 3083, Australia. Email: bcplowright@students.latrobe.edu.au 


\section{J.L. PONSFORD \& R.E. WHELAN-GOODINSON. Psychiatric Disorders Following Traumatic Brain Injury.}

Objective: Previous studies of psychiatric disorders following traumatic brain injury (TBI) have used differing methodology, resulting in variable reported frequency of disorders. There is also limited understanding of their causes. The present study aimed to examine, using structured clinical interviews based on DSM criteria, the frequency and association between psychiatric disorders prior to and following TBI, their predictors and impact on outcome.

Participants and Methods: One hundred participants with mild to severe TBI were recruited. Average age was 37.18 years (range 19-74), average time post-injury was 2.98 years (range $0.5-5.5$ years). Measures included the Structured Clinical Interview for DSM-IV diagnoses, Hospital Anxiety and Depression Scale, Alcohol Use Disorders Identification Test, Drug Abuse Screening Test and Glasgow Outcome Scale Extended.

Results: The frequency of many disorders rose from pre-injury to postinjury: depression from $17 \%$ to $46 \%$; anxiety from $13 \%$ to $38 \%$ and psychotic disorders from $1 \%$ to $3 \%$. There were many new cases. There was limited resolution of disorders, with current rates of depression and anxiety being $31 \%$ and $35 \%$ respectively. Rates of substance use disorders fell from $41 \%$ pre-injury to $21 \%$ post-injury, but this is significantly higher than the general population. Co-morbidity of disorders was high.

Higher frequency of novel psychiatric disorders was associated with increased time post-injury and with pre-injury employment. Lower education predicted depression, along with co-morbid anxiety and older age was associated with anxiety, along with co-morbid depression. Pre-injury substance use predicted post-injury substance use.

Conclusions: Findings highlight the importance of identifying those at risk and implementing strategies to address psychiatric disorders post-TBI.

Correspondence: Jennie L. Ponsford, PhD, Department of Psychology, Monash University, Wellington Road, Clayton (Melbourne), VIC 3800, Australia.E-mail:jennie.ponsford@med.monash.edu.au

C. SKILBECK, M. SLATYER, P. CANNAN \& T. BELL. The Factor Structure of the Rivermead Post-Concussion Symptom Questionnaire (RPCSQ) and Relationships with Demographic and Clinical Variables.

Objective: The RPCSQ is very popular, although lacking research on its structure. This was the main aim of the study, using Exploratory (EFA) and Confirmatory (CFA) Factor Analysis. A second aim was to examine factor relationships with demographic and clinical variables to predict immediate post-TBI symptomatology: it was hypothesised that greater symptomatology would be reported by women, older participants, and those with longer PTAs.

Participants and Methods: 508 individuals presenting with TBI at a Department of Emergency Medicine participated. Data were collected on the RPCSQ within 1 week of injury, and at other points post-injury. The sample was divided in half. Sub-sample 1's RPCSQ item scores at 1 month post-TBI underwent EFA (Principal Axis Factoring, with Varimax Rotation): one-month data were selected as that point provided a typical population severity mix. CFA was performed on sub-sample 2 . ANOVAs \& t-tests were conducted on initial RPCSQ factor scores using sex, age group, NART IQ and PTA as independent variables.

Results: EFA and CFA identified four factors: emotional, cognitive, physical, and visual. Women reported more symptoms on factors 1-3. as did those aged 41-60 years. Lower symptomatology was reported by participants with PTAs $<=5$ minutes, with above average NART IQs, and those aged $60+$.

Conclusions: Results for sex and PTA were expected although finding more symptoms in middle-aged participants was surprising (perhaps attributable to the compounding effects of other life stresses). This research should help clinicians interpret RPCSQ ratings and highlights the influence of demographic and clinical variables in determining initial symptoms.
Correspondence: Clive Skilbeck, PhD, psychology, university of tasmania, university of tasmania, sandy bay campus, Hobart, TAS 7001, Australia.E-mail: clive.skilbeck@utas.edu.au

C. SKILBECK, M. SLATYER, K. HOLM \& T. BELL. The Factor Structure of the Hospital Anxiety \& Depression Scale (HADS) in TBI, and Relationships with Demographic and Clinical Variables.

Objective: Research has suggested 2- and 3-factor HADS models, although its structure has not been investigated in TBI. The present study undertook this, using Factor Analysis. It also examined relationships of demographic and clinical variables to the factors identified, aiming to predict immediate post-TBI mood: it was predicted that women would report more problems, as would older participants and those with longer PTAs.

Participants and Methods: Exploratory (EFA) and Confirmatory (CFA) Factor Analyses were conducted with a sample of 370 individuals presenting post-TBI at a Department of Emergency Medicine. HADS data were collected within 1 week post-injury, and at 1, 6, 12 and 24 months. Half of the sample's HADS item scores at 1 month post-TBI underwent EFA (Principal Axis Factoring, with Varimax Rotation): onemonth data were selected as that point provided a typical population severity mix. CFA was performed on the remaining participants' scores. ANOVAs \& t-tests were conducted on 1-week HADS factor scores using sex, age, and PTA as independent variables.

Results: EFA and CFA indicated a 3-factor HADS structure: anxiety, depression, psychomotor. Women produced higher factor scores within 1 week post-TBI, as did participants aged 41-60 years, with fewer mood problems being associated with brief PTAs.

Conclusions: Discussion: Sex and PTA results were predicted, though finding higher factor scores in middle-aged participants was unexpected (perhaps resulting from other life stresses). The findings should assist clinicians understand and predict the effects of age, sex and PTA on post-TBI mood factors, and help in the planning of appropriate treatment interventions.

Correspondence: Clive Skilbeck, PhD, psychology, university of tasmania, university of tasmania, sandy bay campus, Hobart, TAS 7001, Australia.E-mail: clive.skilbeck@utas.edu.au

R.L. TATE, A. PFAFF \& L. JURJEVIC.Patterns of Recovery of Memory Function in the Post-acute Stages after Traumatic Brain Injury.

Objective: An intriguing memory disturbance after traumatic brain injury (TBI) is post-traumatic amnesia (PTA), which follows emergence from coma. PTA is transient, but of variable length, ranging from minutes to many months. In spite of the frequency of PTA, very little is known about the recovery process of memory function during emergence from PTA. Aim: To prospectively examine variations in memory function during the peri-PTA period.

Participants and Methods: Parallel forms of the Rivermead Behavioural Memory Test (RBMT) were administered to 30 inpatients with severe TBI: at rehabilitation admission (Time1), in the early stage of PTA when they demonstrated the capacity to reliably encode simple new information (Time2), and one month after emergence from PTA (Time3). Results: Significant improvement on RBMT scores occurred between all test occasions, although scores remained impaired for $60 \%$ at Time 3 . Memory function showed extreme heterogeneity at Time2 and Time3, from normal to severe impairment. Cluster analysis identified three recovery subgroups: Quick (n=8), Poor $(n=7)$ and Late $(n=15)$. At Time3 the Late group was no different from the Quick group, highlighting their potential for good cognitive recovery. At Time2, however, their scores were comparable to the Poor group, with the risk of overlooking their potential. Five RBMT memory domains were examined in detail, with prospective and visual memory having the greatest prospects for the early identification of patients in the Late group.

Conclusions: These data have important clinical implications and also provide direction for furthering our understanding of the nature of memory disturbance during emergence from PTA. 
Correspondence: Robyn L. Tate, PhD, Rehabilitation Studies Unit, Faculty of Medicine, University of Sydney, PO Box 6, Ryde, QLD 1680, Australia.E-mail:rtate@med.usyd.edu.au

K. WATERLOO, K. MÜLLER, A. JAKOLA, M. LARSEN, B. ROMNER $\&$ T. INGEBRIGTSEN. Five-year outcome after mild traumatic brain injury: a prospective controlled study.

Objective: To study the prevalence of post concussion symptoms 5 to 7 years after mild head injury (MHI)and to investigate whether patients suffer from more symptoms than the normal population.

Participants and Methods: We conducted a 5 to 7 years follow-up of patients $(n=89)$ with MHI. Post concussion symptoms were quantified with the Rivermead Post Concussion symptoms Questionnaire (RPQ) and health related quality of life (HRQL) was measured with the EuroQol-5D. We also quantified subjective general health state with the EuroQol Visual Analogue Scale (EQ-VAS). A age- and sex-matched, but otherwise randomly chosen control group of 89 persons was recruited from the National Population Registry for a cross-sectional comparison. Results: Patiens reported significantly $(p=0.017)$ more post concussion symptoms (median RPQ score 10,95\% C.I. 2-20) than controls (median $2,95 \%$ C.I. $0-4)$. They also reported significantly $(p=0.008)$ lower HRQL (median EQ-5D score $0.866,95 \%$ C.I. $0,79-1,00$ ) than controls (1,00, 95\% C.I. 1.00-1.00), but there was no difference between the groups in their subjective ratings of general health state.

Conclusions: Patients reported significantly more post concussion symptoms and lower HRQL 5 to 7 years after MHI than age- and sex-matched controls from the normal population.

Correspondence: Knut Waterloo, PhD, Department of Neurology, University Hospital of North Norway, Tromso, Tromsø 9038, Norway. E-mail:knut.waterloo@unn.no

V.J. WHITEFIELD, A.B. SHUTTLEWORTH-EDWARDS \& S. RADLOFF. Neuropsychological Profile of Contact Versus Noncontact Sports Players at Grade 12 Level.

Objective: The objective of this study was to establish whether there were indications of permanent neuropsychological sequelae in respect of (i) cognitive test performance and (ii) self-reported postconcussive symptoms (PCS) amongst Grade 12 male students, due to repetitive concussive episodes sustained during participation in a contact sport throughout their school years.

Participants and Methods: Over a two year period all Grade 12 sports players in a South African high school were targeted. Following exclusions for prior neurological or psychiatric disorder, learning disability and substance abuse, the final sample included 189 Grade 12 boys at pre-season, and 154 Grade 12 boys at post-season (age range 16-18 years). The samples were divided into comparative groups of contact (predominantly rugby) and noncontact sport, with equivalence for age and WAIS-III Vocabulary score (a control for premorbid IQ). There was a significantly higher reported incidence of prior concussions amongst the contact compared with the noncontact sports groups. Measures included the ImPACT computerized test, Digit Symbol Substitution and Recall, and two PCS scales. Independent t-test analyses at pre- and post-season, and dependent t-test comparisons pre- versus postseason, were conducted.

Results: All significant effects and predominant trends were in the direction of contact sports players performing more poorly, or presenting with more symptoms, than noncontact sports players.

Conclusions: It is suggested that these effects implicate deleterious neuropsychological consequences of cumulative concussive events due to sustained participation in a contact sport in a significant proportion of high school boys by the end of their high school years.

Correspondence: Ann B. Edwards, PhD, Psychology, Rhodes University, Psychology Clinic, Rhodes University, Grahamstown 6139, South Africa. E-mail: a.edwards@ru.ac.za

\section{Paper Session 13 10:30-11:30 a.m.}

\section{Numerical Representations}

L. KAUFMANN, F. KOPPELSTAETTER, C. SIEDENTOPF, T. GOTWALD \& G. WOOD. Developmental trajectories of magnitude and interference control: an fMRI study.

Objective: Aim of this fMRI study was to assess neurodevelopmental changes in magnitude and interference processing across age by employing a numerical Stroop paradigm. Importantly, our study is the first that implemented a numerical Stroop task a wide age range thus allowing us to study the development of number magnitude processing and interference (irrespective of reading proficiency).

Participants and Methods: 32 healthy individuals aged 8 to 81 (children $n=12$; young adults $n=11$; elderly $n=9$ ) were asked to make numerical and physical magnitude judgments while ignoring the irrelevant stimulus characteristic (physical/font-size and numerical magnitude, respectively). Digit pairs were either incongruent (3 4) or neutral (numerical comparison: 3 4; physical comparison 3 3). Within each task, distance and congruity were manipulated experimentally.

Results: Behaviourally, robust congruity and distance effects were found in all age groups. Conjunction analysis showed common activation sites in response to congruity (prefrontal) and distance (intraparietal) in all groups. Furthermore, an interaction between distance and congruity for children and young adults was observed (but not for old adults): while the distance effect showed a non-linear trend, the congruity effect revealed an almost age-linear trend.

Conclusions: Results reveal that interference and magnitude processing are supported by common brain regions in all age groups. However, the neural correlates of magnitude processing and interference control follow dissociable developmental courses. Specifically, (non-linear) distance effects suggest that relative to children and old adults, young adults had to recruit the smallest network upon processing magnitude, while (age-linear) congruity/interference effects implicate that with increasing age interference control requires increasing effort.

Correspondence: Guilherme Wood, Psychologist, Department of Psychology, University of Salzburg, Hellbrunnerstrasse 34, Salzburg. 5020, Austria.E-mail: guilherme.wood@sbg.ac.at

H. SINZ, T. BENCKE, G. WENNING, H. STOCKNER \& M. DELAZER. Decision Making and Processing of Probability in Mild DAT.

Objective: Despite their cognitive impairment, patients in the mild stages of Alzheimer's disease (DAT) often have to make important decisions regarding their medical treatment, living arrangements and financial issues. While decision-making has been assessed in patients affected by degenerative conditions such as Huntington's disease (Stout et al., 2001) or Parkinson's disease (e.g. Brand et al., 2004; Czernecki et al., 2002), little is known about decision-making in mild DAT patients. A recent study (Delazer et al., 2007, in press) suggests decision making deficits in mild DAT in situations with explicit and stable rules.

Participants and Methods: In the present study we investigate different types of decision making in mild Alzheimer Disease (DAT) using 1) a task with implicit rules for gains and losses (Iowa Gambling task; IGT), and 2) a risk task with explicit rules and emphasis on the estimation of probabilities based on proportions. Furthermore, patients perform tests of verbal and visual memory, object naming, numerical processing and fronto-executive functions.

Results: Compared to healthy controls, DAT patients did not acquire a preference for advantageous choices over 100 trials in the IGT and showed an inconsistent and disadvantageous response pattern. Their performance in the IGT was correlated with impulsivity, but not with 
numerical processing. While the implicit gambling task showed marked differences between patient and control group, DAT patients showed only slightly different response pattern than controls in the explicit task based on the estimation of numerical proportions and probabilities.

Conclusions: The present study highlights that different types of decision making tasks put different demands on cognitive processes.

Correspondence: Hermann Sinz, Klinik für Neurologie, Medizinische Universität Innsbruck; Anichstrasse 35, A 6020 IBK, Hermann.Sinz@student.uibk.ac.at

M.G. BAFFALUY, M. FAYOL \& M. NOEL. consequences of playing a musical instrument in finger gnosis and arithmetic in children. Objective: Impaired finger gnosis and calculation are two frequently co-occurring symptoms constituting the Gerstmann syndrome (Gerstmann, 1940). Interestingly, several recent studies (Fayol, Barrouillet \& Marinthe, 1998; Noël, 2005) revealed that finger gnosis is significantly correlated to numerical performance in children. Indeed, perceptivetactile abilities in children predicted numerical performances better than an intellectual factor, up to 3 years after their first evaluation (Marinthe. Fayol \& Barrouillet, 2001). Based on that, we hypothesized that training finger differentiation would increase finger gnosis and might therefore improve numerical skills. Thus, playing a musical instrument might be an effective finger intervention because of the specificity of finger involvement (Peretz \& Zatorre, 2005).

Participants and Methods: We compared groups of 7 and 10 year-old children - musicians and non-musicians - from the same school. 7 yearolds started to play a musical instrument some months before our testing, while 10 year-olds had a three years training experience. Measures of finger gnosis and of numerical abilities (calculation, problems solving, mathematical understanding...) were taken.

Results: In 7 year-olds, performance levels between musicians and nonmusicians were comparable, both regarding finger gnosis and numerical abilities. On the contrary, 10 year-old musicians excelled over nonmusicians in finger gnosis and in most numerical tasks (even after controlling for intelligence).

Conclusions: Our results suggest that playing a musical instrument might have beneficial effects on numerical skills. Specifically, playing an instrument continuously (i.e., over three years) may improve finger gnosis, and this long-term finger training could be related to the numerical superiority in musicians relative to non-musicians.

Correspondence: Maria Gracia-Bafalluy, Unité Cognition et Développement (CODE), Université Catholique de Louvain 10, Place Cardinal Mercier, Louvain la Neuve, Belgium

maria.gracia-bafalluy@psp.ucl.ac.be

G. WOOD, K. DRESSEL, E. KLEIN, F. DOMAHS, H. NUERK \& K. WILLMES. Effects of problem size and carry-over on fMRI signal in an addition task.

Objective: Problem size and procedural complexity (e.g. the carryover operation) are determinants of performance in mental addition tasks. Previous studies have associated them with the activation in the intraparietal cortex. In the present study we developed a set of items in order to separate experimentally the effects of problem size and carry over on brain activation.

Participants and Methods: Sixteen male right-handed volunteers (mean age $=28$ years; $\mathrm{SD}=5$; range 20 -40 years) participated in the study. In a rapid event-related fMRI design, participants selected the correct response for different addition problems (96 experimental trials +32 null events, presented each 4.5 sec.). In a $2 X 2$ factorial design problem size (large vs. small) and carry over (carry-over vs. no carry-over) were manipulated.

Results: Behaviorally, problem size and carry over effects were observed as well as the interaction between them. The contrasts large > small problem size and carry-over $>$ no carry-over led to activation in the anterior and posterior intraparietal cortex, prefrontal areas and extrastri- ate cortex bilaterally. Coherence analysis revealed interaction between the anterior and posterior portions of the left intraparietal cortex and between the posterior intraparietal cortex and the left angular gyrus associated with the carry-over effect. Interregional coherence was not predictive of the problem size effect.

Conclusions: Although the similar neuronal network is activated by problem size and carry over, the neural processes associated with these two determinants of mental addition are probably different, since the pattern of intraregional coherence in relevant only for the effect of carry over. Correspondence: Guilherme Wood, Psychologist, Department of Psychology, University of Salzburg, Hellbrunnerstrasse 34, Salzburg 5020, Austria.E-mail: guilherme.wood@sbg.ac.at

C. KUTRZEBA, P. MARCHL, H. ZAUNER, G. WOOD, H. NUERK, A. GASSNER \& K. WALLNER. Exercise training improves executive functions of patients with heart failure.

Objective: Patients with heart failure have limited functioning of the left ventricular of the heart which consequently leads to reduced cerebral perfusion and in turn to cognitive deficits. The aim of the present study was to evaluate the outcome of physical exercise training on specific cognitive domains among patients with heart failure compared to controls not undergoing such training.

Participants and Methods: 30 heart failure patients, NYHA classes IIIII (mean ejection fraction=34, sd=8.4), mean age ( $62.8 \pm 8.9$ years) took part in this study. The training group $(n=20)$ underwent a supervised resistance training on a bicycle 20 minutes a day, five times a week, for four weeks. The control group $(n=10)$ patients did not undergo training. After initial measurement of echocardiogram, patients' cognitive functions were assessed in the domains of memory, general intelligence, attention, executive functions, quality of life and numerical cognition. After the training program, all measurements were repeated.

Results: Scores in the cognitive measures did not differ between control and training group in the pre-test. In the post-test, in contrast, the training group showed significant better scores in tests of numerical abilities, alertness, fluid intelligence as well as nonverbal memory, while the control group did not improve in any measure of cognitive functioning. Comparisons between pre- and post-test confirmed that only the training group improved significantly in these cognitive tasks.

Conclusions: Regular exercise training in heart failure patients is associated with an improvement in cognitive functions in the domains of numerical abilities, alertness, fluid intelligence as well as nonverbal memory. Correspondence: Guilherme Wood, Psychologist, Department of Psychology, University of Salzburg, Hellbrunnerstrasse 34, Salzburg 5020, Austria.E-mail: guilherme.wood@sbg.ac.at

\section{Paper Session 14 10:30-11:30 a.m.}

\section{Attention}

\section{J. EGELAND. Differentiating attention deficit in adult ADHD and schizophrenia.}

Objective: It is difficult to differentiate types of attention deficit in subjects with ADHD and schizophrenia. Most previous studies have used overall measures of inattention that may disguise differences in underlying mechanisms. The present study investigated types of inattentive errors and applied a process view of attention.

Participants and Methods: Conners' Continuous Performance Test protocols from subjects with ADHD-combined type (51), ADHD-inattentive type (19) and schizophrenia spectrum disorders (36) were analyzed. Results: Subjects with ADHD-I had more omission errors while subjects with ADHD-C had more errors of commission. This error pattern 
increased during the test. In contrast, subjects with schizophrenia showed a decrease in omissions, indicating a training effect. There were no differences in overall attentiveness between the groups.

Conclusions: Although they performed at the same overall level, the three groups displayed three distinct patterns of inattention. The ADHDI group displayed a lethargic inattention characterized by high fatigue. The ADHD-C group showed a hyperactive-impulsive pattern of inattention, while the schizophrenia group showed an inability to initially focus attention.

Correspondence: Jens Egeland, Ph.D., Department of research,-, Vestfold Mental Health Care Trust, P.o.box 2267, Tonsberg 3103, Norway: Email:jens.egeland@piv.no

\section{J. BUCHHOLZ \& A. AIMOLA DAVIES. An Attentional Orienting Deficit in Adults with Dyslexia.}

Objective: Evidence suggests that a key characteristic of dyslexia is a deficit in the magnocellular (M) pathway of the visual system. These impairments have been disputed because they are often slight, and not always found in all individuals with dyslexia. One alternative proposal is that the visual deficits shown in dyslexia arise from a dysfunction in higher visual areas that receive predominantly magnocellular input, such as the parietal cortex. Since visual attention is not only a major function of the parietal cortex, but is also necessary for the development of the reading system (and subsequent competence in reading), the present study examined covert visual attention in dyslexia.

Participants and Methods: Attentional performance of five adults with dyslexia, on a variety of spatial cueing tasks, was compared against a control group on both a group and individual case basis.

Results: The adults with dyslexia demonstrated normal attentional facilitation of performance when targets were validly cued. However, difficulties related to visual orienting in both visual fields were evident, specifically when adjusting and maintaining attentional focus. Although increasing cue size improved orienting performance, increasing stimulus onset asynchrony had a negative effect on this improvement.

Conclusions: The results suggest that in adults with dyslexia, the attentional system is both diffusely distributed and at the same time sluggish. Reading ability of these adults with dyslexia may be compromised since a difficulty in automatic orienting may affect the planning of eye movements, while a difficulty in maintaining attention may hinder decoding due to increased distraction from nearby text.

Correspondence: Judy Buchholz, PhD, School of Psychology, Ausralian National University, Building 39, ANU, Canberra, ACT 0200, Australia. E-mail:judy.buchholz@anu.edu.au

\section{I.A. HEBER, M. WOLTER, T. KUHLEN \& B. FIMM. Pseudoneglect in virtual space.}

Objective: Healthy subjects demonstrate subtle attentional asymmetries in favor of the left visual field, the so-called pseudoneglect. Peripersonal and extrapersonal space processing are anatomically dissociated by dorsal and ventral visual pathway processing respectively, and the upper and lower visual fields are supposed to be biased towards the extrapersonal and peripersonal space. To investigate attentional processing within virtual 3Dspace, we adressed whether: 1) pseudoneglect effects also occur in virtual space. 2) potential asymmetries depend on the spatial height and depth of the targets and 3) potential biases do occur in virtual space as well.

Participants and Methods: 15 subjects were examined by means of virtual reality (VR) techniques. The VR-paradigm required covert shifts of attention to the left and right hemifield within the upper, center and lower visual field $(\mathrm{FOV})$, within peripersonal $(30 \mathrm{~cm})$ and extrapersonal $(270 \mathrm{~cm})$ space. Reaction times (RT's) towards validly and invalidly cued targets were logged.

Results: The results of the repeated measures analysis of variance show highly significant effects of validity, side and height. Pseudoneglect was present for invalidly cued targets in peripersonal and extrapersonal space.
For validly cued targets, pseudoneglect was restricted to peripersonal space. Additionally, there wasn't any evidence for the proposed biases of peripersonal and extrapersonal space towards the lower and upper visual field, RT's were faster in the central and the lower FOV than in the upper FOV.

Conclusions: The results indicate that pseudoneglect also occurs in virtual space. Furthermore it indicates unequal distribution and differential attentional processing across the three dimensions of virtual 3D-space. Correspondence: Ines A. Heber, Diploma, Neuropsychology, Neurological Clinic, University Hospital RWTH Aachen, Pauwelsstr. 30, Aachen 52074, Germany. E-mail: heber@neuropsych.rwth-aachen.de

P. PERAKAKIS, L. ANLLO-VENTO, P. GUERRA, A. FARIA, I. DEPAULA ANTUNEZ \& J. VILA. Distinguishing Visual Search and Working Memory via Autonomic and Cortical Responses.

Objective: This study was designed to investigate the spatio-temporal dynamics of visual search (VS) and visual working memory (WM) with cortical potentials (ERPs), and to relate ERP measures to the cardiac defense response (CDR). Previous studies demonstrated CDR modulation by these two cognitive operations.

Participants and Methods: Twenty volunteers engaged in VS or WM tasks in counterbalanced order. Each trial included two visual displays separated by $900 \mathrm{~ms}$. In the WM task, subjects held in working memory the colors of 4 squares in the visual field signaled by a central arrow cue. During VS, subjects searched the cued visual field in order to detect a line crossing one of the squares. Following the second display, subjects reported either a color change in one of the squares $(\mathrm{WM})$, or a change in line orientation (VS). A $105-\mathrm{dB}$ white noise was administered at the beginning of each of three CDR trials, while subjects engaged in the VS or WM task. Continuous EEG, horizontal EOG, and ECG were recorded throughout the experiment. RTs and percentage of correct responses were obtained following change and no-change trials. Results: Behavioral responses did not differ across tasks. The amplitude of the N2pc component, a neural index of VS and filtering of irrelevant stimuli, was greater in the VS task, while the CDA, a contralateral parietal wave reflecting WM load, was similar under both conditions. The temporal course of the CDR also distinguished between VS and WM.

Conclusions: These findings demonstrate that, though tightly coupled, VS and WM constitute distinct cognitive and neural operations.

Correspondence: Lourdes Anllo-Vento, Ph.D., Neurosciences, University of California, San Diego, 9500 Gilman Drive, MC 0608, La Jolla, CA 92093-0608.E-mail: lanllo@ucsd.edu

\section{Paper Session 15 10:30-11:30 a.m.}

\section{Childhood Developmental Disorders}

\section{KORKMAN, J. KOSKINEN \& P. PITKÄNIITTY.Social Perception in Children with Asperger Syndrome.}

Objective: Among the core characteristics of Asperger Syndrome (AS) are deficits related to perception of social cues. These include perception of emotional expressions, face recognition, and capacities related to the awareness of other peoples feelings and thoughts, that is, to a theory of mind. The first aim of this study was to assess these capacities in children with AS. AS children were expected to perform more poorly than control children. A second aim was to test the validity of new tasks constructed to reflect these functions.

Participants and Methods: Participants were 20 children aged 8 to 12 years and diagnosed with AS, and 20 normal children. The AS group 
was recruited from special classes for children with AS. The control group was recruited from normal classes.

Two new tests were constructed as parts of the NEPSY II: the Affect Recognition subtest and the Theory of Mind subtest. These subtests, the Memory for Faces subtest from the previously published NEPSY, and the WISC III were administered.

Results: Distributions of age, gender and IQ scores were similar in the AS group and the control group. The reliabilities of the new subtests were adequate. The AS group performed significantly poorer than the control group on the Affect Recognition, Theory of Mind, and Memory for Faces subtests. Correlations with IQ were low (-.05-.11).

Conclusions: The results confirm earlier findings and views of impaired social perception in people with AS syndrome. They also demonstrate the validity and clinical utility of the two new NEPSY II subtests.

Correspondence: Marit Korkman, Psychology, Abo Akademi University, Fabriksgatan 2, Turku20500,Finland.E-mail:marit.korkman@abo.fi

\section{S. HUIJBREGTS \& A. WARREN. Maternal Prenatal Smoking, Inhibitory Control, and Externalizing Behavior in 7- to 9-year-old Offspring.}

Objective: To study different forms of inhibitory control and externalizing behavior in children whose mothers smoked during pregnancy.

Participants and Methods: Forty children (mean age $=7.98 ; 15$ exposed, 25 non-exposed) performed two computer tasks. The Sustained Attention Task requires 400 non-dominant hand and 200 dominant hand responses. Inhibitory control of the prepotent response is required for dominant hand responses. The Delay Frustration Task consists of 45 simple maths exercises. On a number of trials delays are introduced before the next question appears on the screen. The product of the number and duration of button presses during these delays is a measure of frustration-induced inhibitory control. Behavior was assessed using the teacher-rated Strengths and Difficulties Questionnaire.

Results: Prenatally exposed children had more conduct problems (MannWhitney $\mathrm{U}=134.5, \mathrm{z}=-2.1, \mathrm{p}=.038)$ and higher hyperactivity $(\mathrm{U}=$ $124.5, \mathrm{z}=-2.0, \mathrm{p}=.041)$ than non-exposed children. In the Delay Frustration Task, exposed children showed poorer inhibitory control than non-exposed children $(\mathrm{U}=90, \mathrm{z}=-2.6, \mathrm{p}=.009)$. A dose-response relation was also observed (Kruskal-Wallis $\chi 2(2)=7.5, p=.023$ ). There were no significant differences between exposed and non-exposed offspring in inhibitory control scores from the Sustained Attention Task. Only inhibitory control scores from the Delay Frustration Task were related to conduct problems (Spearman's rho: .39, $\mathrm{p}=.022$ ) and hyperactivity (Spearman's rho: .36, $\mathrm{p}=.033$ ).

Conclusions: These results support a distinction between inhibitory control required for handling abstract, decontextualized problems and for the regulation of affect and motivation.

Correspondence: Stephan Huijbregts, PhD, Clinical Child and Adolescent Studies, Leiden University, Wassenaarseweg 52, P.O. Box 9555, Leiden 2300 RB, Netherlands. E-mail: SHuijbregts@fsw.leidenuniv.nl

M. CHEUNG, S.L. SZE \& A.S. CHAN. External organization reduces hyper intrahemispheric theta coherence in children with Autistic Spectrum Disorder (ASD).

Objective: Previous studies suggest that impaired memory functioning of individuals with Autistic Spectrum Disorder (ASD) may be related to their problem in organizing strategies. The present study aims to explore the electrophysiological basis of memory processing in children with ASD under disorganized and organized learning conditions.

Participants and Methods: Twenty high-functioning ASD children and twenty-two age-matched normal children were recruited. They were ad- ministered the non-verbal memory task, which consisted of two sets of arrays, each with 24 figures (grouped into the 4 categories, namely food, animals, clothing, and tools). The children were asked to memorize these figures either arranged randomly or in an organized manner, which were displayed on the computer screen for three minutes. Quantitative EEG data with 32 channels were recorded during three-minute learning trial for disorganized and organized conditions. After each condition, a recognition task, which consisted of 12 previously shown figures and 12 new distractors, was delivered to the children for discrimination. Intrahemispheric coherence pairs were computed for within- and between-group comparison. Results: ASD group yielded significantly higher theta coherence, particularly on the left hemisphere, than normal controls when they encoded the information which was presented randomly $(p<0.05)$. However, when the information was organized for the children to remember, theta hyper-coherence was reduced in the ASD children $(p<0.05)$ although behaviorally they discriminated similar amount of figures during recognition trial for either condition.

Conclusions: The present study found that although children with ASD demonstrated higher theta coherence than normal children when they encoded information presented randomly, reduction in theta hyper-coherence was observed when external organization was utilized for encoding. Therefore, provision of organizational strategies may facilitate children with ASD utilize less effort to remember the information.

Correspondence: Mei-chun Cheung, Ph.D., Institute of Textiles and Clothing, The Hong Kong Polytechnic University, Institute of Textiles and Clothing, The Hong Kong Polytechnic University, Hung Hom, Hong Kong. N.A., Hong Kong. E-mail: tccmchun@inet.polyu.edu.hk

T. BARBOSA, T.C. BUJOKAS SOARES, C.C. RODRIGUES, C. TOLEDO-PIZA, A.C. ROSSETTO \& O.F. AMODEO BUENO. Performance of Dyslexic Children in Pseudoword Repetition.

Objective: The main function of the phonological loop (component of working memory) is to provide temporary storage of sound structures playing important role in language skills, including learning to read and writing. According to many studies, children with learning disabilities present alteration in phonological working memory. One of the tasks used to evaluate this component is the pseudowords repetition task, in which the phonological forms of the stimuli are unfamiliar and so require children to code new phonological sequences and maintain them in phonological memory without degradation for a short time until speaking them. Therefore, the aim was analyze the performance of dyslexic children in this task.

Participants and Methods: Forty-two dyslexic children aged 8 to 13 years were evaluated with the Brazilian Children's of Pseudoword Repetition Test (BCPR), a Portuguese language version of the Children's Test of Nonword Repetition (CNRep). The children were divided into 3 groups according to their ages: G1 (8 to 9 years), G2 (10 to 11 years) and G3 (12 to 13 years).

Results: Data analysis revealed a significant difference between groups in 3 (G1 and G3) and 5 (G2 and G3) syllable words and in the total amount of correct responses (G2 and G3), showing that the number of correct responses decreased as age increased. Substitution errors were the most common, especially in G2 and G3 groups, as well as total errors.

Conclusions: Those data suggest that dyslexic children's performance is worse as they grow older, contrary to normal children's performance that improves throughout the years.

Correspondence: Thais Barbosa, Universidade Federal de São Paulo, R. Embaú, 54, São Paulo 04039-060, Brazil. E-mail: barbosa_thais@hotmail.com 


\section{Poster Session 5 \\ Imaging, Electrophysiology, Cognitive Neuroscience, Executive Functioning 11:30 a.m.-1:30 p.m.}

\section{Cognitive Neuroscience}

D. ÁlVAREZ, C. NOGUERA, M. DAZA, V. PLAZA-AYLLÓN, E. DEL ÁGUILA, E. CARMONA \& I. FERNÁNDEZ-AGÍS.A Study about the Efficiency of Alerting, Orienting and Executive Control Functions in a Nine-Year-Old Boy with Landau-Kleffner Syndrome.

Objective: Landau-Kleffner syndrome (LSK) is a rare childhood neurological disorder characterized by the sudden or gradual loss of the ability to understand and use spoken language. Sometimes LSK may be misdiagnosed as attention deficit disorder. On the other hand, previous studies of ADHD children show some evidence of abnormalities in alerting and/or executive control (Bush et al., 1999). We decide to explore the efficiency of three networks that carry out the functions of alerting, orienting and executive control in a boy with LSK.

Participants and Methods: Nine-year-old boy affected by Landau-KIeffner syndrome, being recruited on the Center of Neuropsychological Assessment and Rehabilitation -CERNEP-“. He shows an abnormal electro-encephalogram and an inability to understand and express language after each epileptic seizure. Also, he shows hyperactivity behavioural disorder and a moderated risk of attention deficit. We evaluated his attentional networks by using a child version of the ANT (attention network test) developed by Rueda et al. (2004).

Results: There were no significant interactions among three attentional functions and the mean of reaction times for each condition was much higher than that observed in normal children.

Conclusions: Although the alerting, orienting and executive functions work well relatively somewhat independently, we have observed a less efficiency of the executive control network. Our results are in agreement with previous studies of ADHD children that show some problems in executive control functioning. Neuroimaging studies about different anatomies of these attentional networks are also crucial for use in diagnostic and treatment studies of childhood psychopathology.

Correspondence: Dolores Álvarez, Neuroscience and Sciences of Health, University of Almería, Ctra. Sacramento s/n, Almería 04120, Spain. Email:dalvarez@ual.es

S.J. RYAN \& T.M. ATKINSON. Gender and Sport Differences in High School Athletes in Phonemic Switching on the Written COWA-FAS. Objective: Research on phonemic fluency indicates that clustering of words develops in early adolescence and is a posterior temporal lobe function, while switching is associated with the frontal lobes and reaches maturity in late adolescence. In this study, switching and clustering patterns in the written version of COWA-FAS were examined in high school athletes who participated in contact-collision and non contact-collision sports. It was predicted that sport-related differences would be reflected in the switching patterns of athletes in contact-collision sports because of the vulnerability of the frontal lobes.

Participants and Methods: A total of 137 male $(n=63)$ and female $(\mathrm{n}=74)$ athletes aged 13-19 $(\mathrm{M}=15.5)$ were administered the written version of the COWA-FAS. This version permits three minutes each for the participant to write down as many words as they can recall that begin with the letters "F", "A", and "S," respectively.

Results: Confirmatory factor analysis techniques yielded that the data represented the latent constructs of switching and clustering; results were invariant across gender and sport type. A latent means analysis demonstrated that females participating in contact-collision sports had significantly fewer switches, on average, when compared to females from non contact-collision sports.
Conclusions: The results suggest that when administering the COWAFAS, switching and clustering may be a more sensitive measure of neurocognitive functioning than the traditionally used total number of words. The study raises concern about the cognitive development of high school female athletes who may be more vulnerable than males to mild head trauma associated with their sport.

Correspondence: Thomas M. Atkinson, MA, Curry School of Education, University of Virginia, 2021 Ivy Rd. Apt. D-4, Charlottesville, VA 22903. E-mail:tma9j@virginia.edu

M. BALCONI \& S. TUTINO. An ERP Analysis of Iconic Language and Iconic Thinking. The Case of Metaphor.

Objective: The present study intends to compare the comprehension of iconic language and the visualization strategy by and ERP analysis applied to metaphor. Several studies investigated metaphor from semantic, cognitive and communicative points of view, but only few were interested in explaining the representational form of figurative language. Participants and Methods: 320 sentences (equally shared in literal and familiar metaphorical endings), were presented to 36 subjects, grouped into three different categories: low-, medium and high-visualizers. An ERPs analysis was applied to the comprehension process, taking into account the temporal window of 250-350 ms post-stimulus.

Results: ERP analysis showed a negative peak around $340 \mathrm{msec}$ (N3) for both the literal and metaphorical endings, with an increased amplitude for the last one. The methaphorical endings revealed the presence of an higher negative deflection in comparison with the literal endings. Moreover, high-visualizers showed an ampler N3 than the medium- and low- ones for the metaphorical endings. Finally, the occipital area were more activated than the others as a function of the metaphorical sentences and the visualization strategy.

Conclusions: These results suggest that metaphor involves an iconic representation, more posteriorly distribuited on the brain, than the literal condition. Moreover, visualizers may increase the iconic way of representation, by using images to comprehend the metaphorical meaning of a sentence.

Correspondence: Michela Balconi, PhD, Dep. of Psychology, Catholic University of Milan, Largo gemelli, 1, Milan 20123, Italy. E-mail: michela.balconi@unicatt.it

A. GONZÁlEZ-PINTO, M. CASADO, P. VEGA, S. BARBEITO, C. GONZÁLEZ, F. MOSQUERA \& A. CABRERA. Neuropsychological functioning in stabilized patients after a first psychotic episode: Bipolar versus Schizophrenia.

Objective: To assess the cognitive yield of patients with first psychotic episode (FPE) and compare neuropsychological profile among patients diagnosed of bipolar disorder (BD),schizophrenia (SCHZ)and control $\operatorname{group}(\mathrm{CG})$.

Participants and Methods: 42 in-patients were included with FPE (DSM-IV), defined as the first time a patient displayed positive psychotic symptoms (delusions/hallucinations). All subjects were evaluated with neuropsychological battery, including measures of attention (Trail Making-A, Stroop), memory (WMS-III) and executive functioning (Trail Making-B, WSCT). Cognitive evaluation was delayed until patients were stabilised enough and clinical diagnosis was made 6 months later from the index episode with SCID-I (DSM- IV). T-test for independent samples were used in the statistical analyses $(p<0.05)$.

Results: 2 patients (not stabilised) were excluded at 6 months. $55 \%$ patients met DSM- IV criteria for BD and $45 \%$ for SCHZ and other psychosis. Compared to CG, BD exhibit significative differences in attention (ST-C: $\mathrm{t}=-3.09 ; \mathrm{p}=.003)(\mathrm{ST}-\mathrm{WC}: \mathrm{t}=-3.14 ; \mathrm{p}=.003)$ and memory (Digit: $t=-4.39 ; p=<.001)$.SCHZ compared with $C G$, shown significative impairments in attention (ST-C: $\mathrm{t}=-3.82 ; \mathrm{p}=<.001)$ (ST-WC: $\mathrm{t}=-4.22 ; \mathrm{p}=<.001$ ), executive function (WCST Number categories: $\mathrm{t}=$ 3.11; $\mathrm{p}=.004)$ and memory (Memory texts: $\mathrm{t}=-3.50 ; \mathrm{p}=.001)$. Compared 
with SCHZ, BD has worse significantly scores in Digit (WMS-III) ( $t=$ $2.62 ; \mathrm{p}=0.01)$.

Conclusions: Neurocognitive impairments in attention, memory and executive function appeared to be common from the onset of psychotic disorder, regardless of diagnosis. A distinct neuropsychological profile between SCHZ and BD has not been identifie.

Correspondence: Ana González-Pinto, PhD MD, Psychiatry, Santiago Apostol Hospital vitoria, Olaguibel, 29. 80 B, Vitoria 01004, Spain. Email:sbarbeito@hsan.osakidetza.net

M. DAZA, V. PLAZA-AYLLÓN, C. NOGUERA, D. ÁLVAREZ, E. DEL ÁGUILA, E. CARMONA \& I. FERNÁNDEZ-AGIS. Attentional Networks Functioning in School-Age Children with ADHD: Differences between ADHD Subtypes.

Objective: Recent researchs have indicated that the subtypes of ADHD in children (Combined type; Inattentive type and Hyperactive-Impulsive type) are associated with characteristic deficits in attention. From a cognitive neuroscience view, attention has been thought of as a set of neural networks that perform very specific computations. So far three of these networks have been amply studied: the alert network, the orienting network, and the executive network. In the present study we used this neuro-cognitive approach to assess the attentional networks functioning in a group of children with a possible ADHD diagnosis (combined type " $\mathrm{AD}+\mathrm{H}$ "; inattentive type "AD" or hyperactive-impulsive type "H").

Participants and Methods: Participants were recruited on the "Center of Neuropsychological Assessment and Rehabilitation CERNEP". Using a spanish version of the Conners' Rating Scales for the assessment of $\mathrm{ADHD}$, participants were classified as "AD+H", "AD" or "H". We used a child-friendly version of the the Attentional Network Test to assess the attention networks functioning. The procedure combines a cost and benefit paradigm with the flanker task to obtain a measure of each attention network. We also used a single-trial computerized version of the Stroop task, certain subtests of the WISC-R and the Trail Making Tests. Results: Significant differences between the three groups were found. Scores obtained from ANT task revealed differences between the groups. The results obtained with the single-trial version of the Stroop task also showed a characteristic pattern of interference.

Conclusions: These data suggest that for a differential diagnosis of ADHD in school-age children, assessment should include measures of attentional networks functioning.

Correspondence: María Teresa Daza, Neurociencia y Ciencias de la Salud, Universidad de Almería, Carretera de Sacramento s/n. La Cañada., Almería 04120, Spain.E-mail:tdaza@ual.es

\section{A.A. GONZÁLEZ GARRIDO \& L.A. ALVELAIS GONZÁLEZ. Early} ERP Components Index Emotional Contexts Preceding A Detectable Change In Auditory Stimulus Stream.

Objective: The main objective of the study was to evaluate the effect of different emotional contexts on the early auditory processing

Participants and Methods: Regarding the emotional feeling they evoked, several five-minutes segments of different commercial movies were evaluated by a group of universitary students, who judged their inclusion in one of three categories: pleasant, unpleasant and neutral. Highly emotion-inducer segments were selected from the three prior categories in order to be displayed to another ten matching healthy, righthanded subjects. Auditory event-related potentials mismatch negativity MMN and N100 were recorded immediately following the stimulation with the emotion-laden fragments.

Results: ERP showed an early positive component peaking around 50 ms that exhibited statistical significant differences related with the emotional content of the task-preceding stimulation. The N100 was stabile during all conditions. The latency of the MMN showed a tendency to be shorter during the condition preceded by a pleasant movie but did not reach statistical significance.

Conclusions: Early ERP components reflect the influence of the emotional context on the first steps of cognitive processing. The results are interpreted as electrophysiological evidences of an early neural evaluation of the suitability between the emotional context and novel stimuli, probably leading the system to modulate the ensuing behavioral output. Correspondence: Andrés A. González Garrido, PhD, Instituto de Neurociencias, Universidad de Guadalajara, Francisco de Quevedo 180, Arcos Vallarta, Guadalajara 44130, Mexico. E-mail: gonzalezgarrido@gmail.com

B.A. KING \& L. BUCHANAN. Semantic Priming in Deep Dyslexia: Investigating the Integrity of the Semantic System.

Objective: Deep dyslexia is an acquired reading disorder characterized by the production of semantic errors during oral reading. Despite the presence of these paralexias, failure of inhibition theory (FIT; Buchanan, McEwen, Westbury, \& Libben, 2003) posits an intact semantic system in deep dyslexia. Given that the extant empirical data are not sufficient to effectively adjudicate between FIT and a proposed alternate logically possible model that incorporates a functional impairment within the semantic network, the current study aimed to empirically assay the central tenet of the alternate account, that of compromised implicit semantic access in deep dyslexia secondary to exaggerated spread of activation within the semantic system.

Participants and Methods: One individual with deep dyslexia and 60 control participants were recruited. Direct and mediated semantic priming was assessed via a continuous lexical decision task.

Results: Modified independent samples t test results indicated that deep dyslexic direct and indirect semantic priming difference scores corrected for overall performance level fell well within the normative range.

Conclusions: The findings of normal deep dyslexic semantic priming effects provide support for the position of preserved implicit semantic access in deep dyslexia, serving, in turn, to offset a threat posed to FIT by an alternate logically possible model. The results are used to frame a novel argument that failure of inhibition confined to the phonological output lexicon underlies not only those aspects of deep dyslexia traditionally attributed to semantic system impairment, namely the concreteness and part-of-speech effects, but also the often overlooked deep dyslexic reading comprehension deficit.

Correspondence: Bruce A. King, Psychology, University of Windsor, 5055 Riverside Drive East, Unit 802, Windsor, ON N8Y 5A6, Canada. E-mail: king1e@uwindsor.ca

F. MAMELI, R. FERRUCCI, S. MARCEGLIA, F. COGIAMANIAN, M. VERGARI, S. MRAKIC-SPOSTA, S. ZAGO, R. DILENA, A. LEONE, D. POLEZZI, G. SARTORI \& A. PRIORI. Prefrontal Cortex Stimulation Interferes with Lying: Evidence for Multiple Neural Mechanisms of Deception.

Objective: Deceiving is a complex social and cognitive functions. No experimental data are available on manipulating the individual attitude to lying.

Participants and Methods: To address these issues we stimulated the dorsolateral prefrontal cortex (DLPFC) using transcranial direct current stimulation (tDCS). Nine healthy volunteers were tested before and after tDCS (sham, anodal, cathodal). Two types of truthful (truthful selected: TS; truthful unselected: TU) and deceptive (lie selected: LS; lie unselected: LU) responses were evaluated using a computer-controlled task. Reaction times (RTs) and accuracy were collected and used as dependent variables.

Results: In the baseline task, the RT was significantly longer for lie responses than for true responses (1002 $\pm 137 \mathrm{~ms}$ vs $1079 \pm 155 \mathrm{~ms} ; \mathrm{F}(1,8)$ $=9.66 ; \mathrm{p}=<0.05)$. At baseline, RT for LS was significantly shorter than RT for LU $(1039 \pm 151$ vs $1117 \pm 160 ; \mathrm{p}<0.001)$. Whereas after 
cathodal and sham stimulation lie responses remained unchanged (cathodal $p=0.24$; sham $p=0.462)$, after anodal tDCS RTs significantly increased but did so only for LS responses $(p=0.037)$.

Conclusions: These findings show that focal manipulation of brain function with tDCS specifically influences lie production and that distinctive neural mechanisms underlie different types of lies.

Correspondence: Alberto Priori, Neurological Sciences, Fondazione IRCCS Ospedale Maggiore Policlinico, Mangiagalli e Regina Elena, via F.-Sforza, 35, Milano 20122, Italy.E-mail: alberto.priori@unimi.it

S. MEMBRIVES, J. SERRA-GRABULOSA, X. CALDÚ, M. JURADO, I.C. CLEMENTE, N. BARGALLó, P. VENDRELL \& C. JUNQUÉ. Relationship between anterior cingulate gray matter density and arithmetic performance: a VBM study.

Objective: Arithmetic processing depends on an extensive cerebral network, including cortical and subcortical regions. One challenge is to demarcate the brain areas that contribute uniquely to numeric computation, as in some of these areas considerable overlap exists between numeric and other cognitive processes. Another challenge is to evaluate how structural brain characteristics could contribute to explain performance differences in arithmetic tasks. The main objective of this study was to evaluate the relationship between arithmetic performance and gray matter density by a whole-brain analysis.

Participants and Methods: The subjects sample was composed of 96 undergraduate students aged between 18 and 24 years. The Arithmetic subtest of the Wechsler scale was used to evaluate mathematical ability. Voxel-based morphometry was used to evaluate the degree of the relationship between gray matter density and arithmetic performance.

Results: Results showed a positive correlation between anterior cingulate $(-2,46,8)$ gray matter density and arithmetic performance $(\mathrm{T}=3.93$ at voxel-level; corrected $P$ value $=0.035$ at cluster-level, $K=267$ voxels), indicating that subjects with lower performance had lower gray matter density in the anterior cingulate gyrus, a region related to calculation, to attentional processes and to working memory.

Conclusions: Anterior cingulate is one region of the cerebral network regulating numeric computation. Although not uniquely related to number and arithmetic processing, our results seem to indicate that its integrity is needed to achieve a normal arithmetic function. More studies are needed to evaluate more precisely the role of this area in arithmetic processing, and the relationship with other regions, by using more specific number and arithmetic tasks.

Correspondence: Josep M Serra-Grabulosa, Psychiatry and Psychobiology, University of Barcelona, Fac. Psycology, Pg. Vall Hebron, Barcelona 08035, Spain. E-mail:jmserra@ub.edu

G. NAVARRETE, R. CORREIA, J. BARROSO \& A. NIETO. Use of Neural Networks and Feature Selection in Neuropsychological Research: Identifying Relevant Variables on MCI.

Objective: As a proof of concept, we discuss a system based on Neural Networks (NN) using Feature Selection (FS) to extract a meaningful group of variables from a massive database that could be used to identify Mild Cognitive Impairment (MCI) and to guide the neuropsychological research in the field.

Participants and Methods: We bring into play a Feed-forward Neural Network (FNN) using a database of 225 neuropsychological variables with 33 subjects. Through an automatic forward FS procedure, we select a small subset of variables that can predict MCI with a high degree of accuracy. The database is composed of 20 normal subjects (n), 5 Amnestic MCI (aMCI; previously diagnosed following Petersen's criteria) and 8 miscellanea cognitive impairment $(\mathrm{m})$.

Results: Results are the average of 5 runs of a leave-one-out procedure. With a subset of 5 variables we can discriminate among groups with a mean accuracy of $95.15 \% \pm 1.68 \%$ (n: $100 \%$, aMCI: $94.74 \%$, m: $85.11 \%$ ). Selected variables in this case are two measures of verbal flu- ency (letter \& action), designs immediate recall, last trial of a procedural memory learning test and premotor functioning.

Conclusions: Although the intrinsic interest of former results, our primary aim with this work is to show the usefulness of non-linear FS techniques and not to claim this particular set of variables as the most relevant MCI predictors. Current findings suggest this approach can be extraordinary helpful dealing with massive amounts of data, which is pretty common in neuropsychological research, pointing to meaningful theoretical paths to further explore.

Correspondence: Gorka Navarrete, Psychology, Cognitive Psychology, Universidad de La Laguna, Campus de Guajara s/n, La Laguna 38205 , Spain.E-mail: gnavarga@ull.es

W. PERRY, R. MOLDONADO, A. MINASSIAN, E. FERGUSON, M. PAULUS \& M. GEYER. The relationship between neuropsychological and psychophysiological measures in the assessment of inhibition among people with Bipolar Disorder.

Objective: Inhibition is referred to as the ability to withhold or attenuate an action or thought and is a critical aspect of executive functioning and of central importance in the regulation of behavior. Inhibitory deficits are key features of the mania of Bipolar Disorder (BD) and provide a behavioral target for research. The focus of this project is to assess the relationship between neuropsychological and psychophysiological measures of inhibitory and executive functioning in BD patients.

Participants and Methods: 13 DSM-IV Bipolar Disorder patients were tested on the following neuropsychological measures: The Go-No-Go, the Flanker CPT, the Wisconsin Card Sorting Test, and the Tower of London test. Patients were also tested on acoustic startle using prepulse inhibition (PPI) of the human startle response, a psychophysiological measure. PPI has been described as an indicator of one's ability to selectively allocate attentional resources to salient stimuli while ignoring redundant and trivial information.

Results: Our preliminary results show that BD patients evidence impaired performance on all measure of inhibitory and executive functioning and a strong association between neuropsychological measures of inhibition and PPI with correlation coefficients ranging between 0.61 to 0.84 .

Conclusions: These results offer construct validation for our psychophysiological measures of inhibitory functioning and may help provide a means of characterizing the attentional deficits that are prominent among BD patients. PPI is a reliable measure and offers an innovative approach to assessing inhibition since there are existing models for understanding the neural substrate of PPI and since intact PPI has been shown to be associated with intact neuropsychological abilities and adaptive functioning.

Correspondence: William Perry, Ph.D., Psychiatry, UCSD, UCSD Medical Center, 200 West Arbor Drive, San Diego, CA 92103-8218. Email:wperry@ucsd.edu

M. VAN GALEN \& P. REITSMA. Developing Automatic Access to Number Magnitude Information.

Objective: When reading Arabic numerals, adult humans will automatically activate semantic information about number magnitude. This association has developed under the influence of exposure to numbers throughout primary school. The current study is an attempt to determine the age at which access to number magnitude becomes automatic. Participants and Methods: The development of automatic access to number magnitude was investigated using two tasks that measure the SNARC effect (Spatial-Numerical Association of Response Codes). The SNARC effect refers to the finding that small numbers are associated with the left side of space, while larger numbers are associated with the right side of space. About 25 children of Grade 1, 2, and 3 (age 7.0, 8.0, and 9.2 respectively) and adults were tested under two conditions: 
1) when number magnitude was part of the task requirements, and 2) when number magnitude was irrelevant.

Results: The SNARC effect was used as an indication that information about number magnitude had been activated; the presence of the effect was evaluated using a regression analysis. In the condition where number magnitude was essential to perform the task, a SNARC effect was found in all age groups. But when number magnitude was irrelevant, a SNARC effect was present only in Grade 3 children and in adults.

Conclusions: The results show that at the age of 9 , children have formed a direct association between Arabic numerals and the corresponding magnitudes. Information about the magnitude of a number is activated even when this is not necessary to perform a task.

Correspondence: Mirte van Galen, Leerstoornissen, PI Research, PO BOX 366, Duivendrecht 1115 ZH, Netherlands. E-mail: M.van. Galen@psy.vu.nl

P.E. VAN VUGT, P. PAQUIER, W. CRETEN, J. DEPREZ, P. PARIZEL \&. J. MARTIN. Tactile Agnosia without Disturbed Tactile Imagery, a Case Study.

Objective: How important are sensorimotor integration and tactile imagery in tactile object identification (TOI)?

Participants and Methods: A 19 year old, right handed woman presented with a disturbed TOI. Data were obtained during clinical evaluation (including EPs and neuroimaging) and experimental assessment of tactile abilities. Performances in the left and right hand were compared with respect to hylognosis, bathygnosis, morfognosis, ipsilateral drawing, oral description, object recognition, object related pantomime, and object naming. X2 and (M)ANOVA were used for the analysis of raw scores and reaction time data.

Results: Blood, liquor, EMG, BAEPs and VEPs were normal. SEPs were pathological in the left arm and leg. CT and MR images disclosed an old CVA in the right parieto-occipital area. A slightly disturbed proprioception and vibration sense in the left fingers and slightly enlarged Weber circles as well as a disturbed two-point-finger discrimination were the major clinical findings. Texture and size evaluation showed no significant difference between the left and right hand. The form discrimination of spheroids and cuboids was significantly worse in the left hand. No significant difference was found during the unilateral drawing and oral description of previously unknown toy construction elements. However, a significant difference was observed during the tactile exploratory recognition task, the unilateral pantomime, and when the patient tried to name every day objects placed in her hand.

Conclusions: Our patient experienced a few "primary" sensory difficulties, but far less important than in previously reported cases of tactile agnosia. TOI seemed to be relatively independent from tactile imagery.

Correspondence: Peter E. van Vugt, PhD, CNO, Antwerp University, Universiteitsplein 1, Campus Drie Eiken, Wilrijk B-2610, Belgium. Email:peter.vanvugt@ua.ac.be

\section{Electrophysiology/EEG/ERP}

M. BALCONI \& S. AMENTA. Neuropsychological Processes in Verbal Irony Comprehension: an Event-related Potentials (ERPs) Investigation.

Objective: Irony is part of our daily experience, that is probably the reason why a lot of studies have been trying to define its nature and the way we are able to understand the pragmatic intentions lying behind ironic communication. This study addresses the issue of pragmatic comprehension of language by analysing the differences or similarities in processing literal and non-literal (ironic) language. The aim of the study is specifically to investigate neuropsychological correlates (ERPs) of irony decoding. Previous outcomes of RT (Reading Times) responses studies showed that non-conventional ironies took longer to process than literal interpretations of the same sentences. That seems to indicate irony comprehension requires more complicated processes. ERPs studies on non-literal language processing revealed the presence of N400, a negative component associated to semantic integration of the discourse $\left(\mathrm{Ku}^{-}\right.$ tas \& Federmeier, 2002).

Participants and Methods: In our study, 120 sentences, equally shared in literal and ironic (with true or false content) trials, were presented to 10 subjects.

Results: ERPs morphological analyses showed, for any trials, two negative peaks at $300 \mathrm{~ms}$ (N3) and $400 \mathrm{~ms}$ (N4) latencies, with an amplitude increase for both components in the ironic condition. These findings suggest that literal and ironic decoding is qualitatively similar, but irony comprehension requires extra inferential processes thus resulting in an increasing of the demand for the cognitive system.

Conclusions: Implications on the opportunity of a distinction between literal and figurative language, as far as comprehension processes are concerned, are discussed.

Correspondence: Michela Balconi, PhD, Dep. of Psychology, Catholic University of Milan, Largo gemelli, 1, Milan 20123, Italy. E-mail: michela.balconi@unicatt.it

D. BRITO, F. OSTROSKY-SOLIS, G. CASTILLO, A. LOZANO, C. MAURICIO \& M. PEREZ. Psychobiology of Art: An Exploratory Study with Event Related Potentials.

Objective: Our purpose was to observe the immediate response of specific brain areas while the subjects were viewing images classified as neutral, pleasant, figurative art paintings and abstract art paintings and to know if this response is different in each condition through the features of the electrophysiology components.

Participants and Methods: We evaluated 10 normal subjects with the Event Related Potential (ERP) technique. Age average was 24.3 years and none of them had received professional training in the fine arts.

Results: The results show that there are significant statistical differences between the pleasant and figurative art painting images in the fronto-central and temporal areas in the right hemisphere $(\mathrm{Fz}, \mathrm{F} 4, \mathrm{~F} 8$, Fe4, Ft8 y C4) on 550 and $600 \mathrm{~ms}$.

Conclusions: We concluded that art perception is related with frontocentral and fronto-temporal activity.

Correspondence: Diana Brito, Licenciatura, Universidad Nacional Autonoma de Mexico, Av. Universidad 3004 col. Copilco Universidad, Distrito Federal 04510, Mexico.E-mail: dianabritonava@hotmail.com

\section{CIVAI, D. POLEZZI, A. BAGLIERI \& G. SARTORI. General and} Specific Knowledge in the Brain.

Objective: How semantic memory is represented in the brain is still a highly debated issue (Martin et al., 2001). In one view, every concept belonging semantic knowledge has super-ordinate/general features as well as sub-ordinate/specific features. Hence, features are stored in the brain based on these two levels (Cree et al., 2005). Neuropsychological evidences seem to support this view; however, while Hodges et al. (1995) find a better performance for general attributes, Humphreys et al.(2005) find the reverse pattern, with an advantage of specific attributes. In an alternative view, features are stored in the brain based on their contribution to the core meaning of the concepts, named Semantic Relevance. Participants and Methods: A group of 16 healthy participants performs a features verification task in which relevance of every feature has been controlled. Every concept presented was preceded by a feature that could be: General or Specific, Highly or Lowly relevant and Congruent or Incongruent with the concept itself. ERPs have been recorded during the task.

Results: Analysis on N400 amplitude, an ERPs that reflects semantic processing, does not show any difference between general and specific features when relevance is matched. Instead, a difference has been found between high and low relevance descriptions as well as a difference has been found between congruent and incongruent descriptions. In addi- 
tion, source analysis (LORETA) does not reveal any difference between general and specific knowledge in activation of brain areas. By contrast, congruent descriptions produce a greater activation of the left medial temporal lobe.

Conclusions: Concluding, our data seem to point in favour of an organization of concepts based on the informativness of features rather than on their specificity.

Correspondence: David Polezzi, Graduate, Department of General Psychology, University of Padua, via Venezia 8, Padova 35100, Italy: Email: david.polezzi@unipd.it

\section{J.S. LITTLE \& F.H. MARTIN. Investigations of Memory Impairment} Following Mild Head Injury Using Event Related Potentials.

Objective: Recent research has shown Event-Related Potentials (ERPs) recorded during attention paradigms distinguish adults who have suffered mild head injury (MHI) from controls. The purpose of this study was to investigate the effects of MHI on ERPs within a word recognition memory paradigm.

Participants and Methods: In an ongoing study with the Neurotrauma Register in Tasmania, two groups of MHI adults were tested at either one month post injury $(\mathrm{n}=10)$ or at 12 months post injury $(\mathrm{n}=8)$. A third group were included as matched controls $(n=10)$. ERPs were recorded during the STUDY phase of a word recognition memory paradigm and averaged as a function of whether they were, or were not, subsequently recognised from a larger list of words during the TEST phase.

Results: Preliminary results show that when the STUDY phase was accompanied by a secondary orthographic task (Does the word contain the letter 'a'?) a significantly larger N400 component distinguished the control group from both MHI groups for words subsequently recognised compared to words subsequently not recognised. Furthermore a significantly larger late positive component (500-650 ms) distinguished the control group from both the MHI groups for words subsequently remembered regardless of whether STUDY was accompanied by the orthographic task or by a word semantic judgement task (interesting/not interesting). No memory performance differences were revealed between the groups.

Conclusions: Results suggest long term differences in electrophysiological correlates of recognition memory following mild head injury that were not evident in recognition memory performance.

Correspondence: Little S. Jason, Bachelor of Arts (Hons.), Psychology, University of Tasmania, Private Bag 30, Hobart, TAS 7001, Australia. E-mail: jslittle@utas.edu.au

A. VELEZ, F. OSTROSKY-SOLÍS \& M. PÉREZ. ELECTROPHYSIOLOGICAL STUDY OF MORAL EMOTIONS.

Objective: Although the brain correlates of basic emotions have been explored, the neural organization of moral emotions in the human brain remains poorly understood

Participants and Methods: In the present study we recorded Event Related Potential (ERP) on 23 normal subjects with an average age of 25.7 years. Subjects were registered while viewing pictures of emotionally charged scenes with and without moral content, as well as emotionally neutral pictures. All the stimuli were previously standardized in our laboratory according to the dimension of unpleasant with moral content (i.e. physical assaults, war scenes) neutral (i.e. household objects) and unpleasant pictures without moral content (i.e. body mutilation, dangerous animals) Results: Unpleasant pictures with and without moral content prompt a marked negative-going slow wave around $500 \mathrm{~ms}$ with higher amplitude at frontal, parietal and temporal sites of the left hemisphere Conclusions: The possible relationships between morality and other prominent human experiences, such religious and esthetic ones, is ful area for research. Another critical issue is the role of environmental influences, such as early experiences formal education and ordinary interpersonal transactions, in shiping the neural architectures underlying moral emotions and effortful moral processing
Correspondence: Alicia Velez, Dr, National University of Mexico, Cenzontle 1-13 Unidad Independencia, C.P. 10100, Del. Magdalena Contreras, Mexico. D.F., Mexico City 10100, Mexico. E-mail: alvelezg@yahoo.com.mx

\section{Executive Abilities/Frontal System}

J. BESNARD, P. ALLAIN, G. AUBIN, F. ETCHARRY-BOUYX, F. DUBAS \& D. LE GALL. A study of utilisation behaviour in patients with frontal lobe lesions.

Objective: Patients with "Utilisation Behaviour" (UB) are described as automatically using objects in the environment in an "object appropriate" manner that is inappropriate for the particular context (Archibald et al., 2001). Lhermitte (1982) first clearly described UB in patients with frontal lobe lesions. Shallice et al. (1989) criticized Lhermitte's clinical procedure for electing UB and reported an experimental investigation of UB in a patient with bi-frontal lesion. No direct comparison of these two procedures and no other task exists to elicit UB.

Participants and Methods: In the current study, we compared these two procedures (Lhermitte, 1983; Shallice et al., 1989) with a new experimental task which was presumed to be more sensitive to elicit UB. In this task, patients were asked to enumerate actions in the presence of unrelated or related objects that could act as triggers for UB. Ten dysexecutive patients with lesions involving the frontal cortex (FP) and 10 normal controls $(\mathrm{NC})$ were tested.

Results: No UB appeared in NC, whatever the protocol used. Observations of the FP with the procedures developed by Lhermitte (1983) and Shallice et al. (1989) respectively revealed 1 and 10 occurrences of UB. Our task revealed 69 occurences of $\mathrm{UB}$ in $\mathrm{FP}$ ( 57 when actions were enumerated in the presence of related objects, 12 occurrences of UB with unrelated objects). Conclusions: These results confirm that our procedure is more sensitive to elicit UB and that the occurence of UB is dependent on the relation between the objects presented and the content of the neuropsychological tests.

Correspondence: Philippe Allain, PhD, Département de Neurologie, Unité de Neutropsychologie,, rue Larrey, Angers 49033, France. E-mail: PhAllain@chu-angers.fr

\section{N. CADAVID \& P. DEL Río. Assessing Executive Functioning in} Colombian Children.

Objective: Information about the executive functioning in normal developing children from Colombia is limited. The aim of this research was to establish the cognitive profile of EF of children from Bogotá-Colombia, to finally have an idea of how EF is developing in this population. Participants and Methods: A cross-sectional exploratory study was conducted using 100 rural and urban children, grouped according to two variables: age ( 4,6 and 8 years old) and socioeconomic status - SES (low, middle, high). The measures used were chosen because they are developmentally relevant in the scientific literature of EF and had appropriate statistical values within Spanish population. These measures included a go/no go task, a digit span task, a planning task, a hand movement task, a spatial orientation task, a motor task, and a verbal regulation task.

Results: Once the reliability and validity of the tests was proven with this population, age and SES effect were found. The cognitive trajectory of EF in Colombian children showed an age-dependent increase and a SES-dependent capability. This last means that depending on the educational, cultural and family resources with which children count with may vary their opportunity to develop successfully their EF to meet daily and academic requirements.

Conclusions: Future research should be focused to study carefully these socioeconomic differences in the development of the EF and in the comparison between different speaking Spanish populations.

Correspondence: Natalia Cadavid, Ps, Basic Psychology, Psychobiology and Methodology, Universidad de Salamanca, Calle Transportistas 711,2F, Salamanca 37006, Spain.E-mail:ncadavid@usal.es 
A.G. CAPOVILla, E.S. ASSEF, F.C. CAPOVILla \& R. PRIMI. Executive Functions and Attention Deficit Hyperactivity Disorder in children.

Objective: The executive functions are involved in the setting of goals and objectives, and their neurological basis is located in the prefrontal cortex. Disturbances in this area, as observed by techniques of functional neuroimaging, are related to psychiatric and cognitive disorders like the Attention Deficit Hyperactivity Disorder (ADHD). Researches indicate that the executive functions can be divided in cognitive components such as aspects of working memory, selective attention, planning, inhibitory control and cognitive flexibility.

Participants and Methods: This study searched evidences of the validity of the instruments by which the components of the executive functions are evaluated, through a correlation between the performance and an external criterion, psychiatric diagnosis of ADHD. The study compared performance of $31 \mathrm{ADHD}$ children from psychiatric units, to those of 31 non-ADHD children from regular schools. The 62 participants were aged from 8 to 12 years old. All 31 ADHD children satisfied DSM-IV criteria and were evaluated using Conners questionnaire, abbreviated version for parents and teachers.

Results: There were used Computerized Semantic Generation Test, Computerized Stroop Test, Trail Making Test B, Visual and Auditory Working Memory Tests, and Tower of London.

ADHD children presented lower performances in Semantic Generation Test, Stroop Test and Trail Making Test B. In the Pearson's correlation analyses, all the performances in the different tests were correlated with some other measurement.

Conclusions: Such findings corroborate the hypothesis that ADHD children present executive deficits. The significant correlations were low, suggesting that such tests evaluate different, although related, constructs, what corroborates the dissociation hypothesis among components of executive functions.

Correspondence: Alessandra G. Capovilla, Psychology, University of San Francisco, Rua Alexandre Rodrigues Barbosa, 45, Itatiba 13251-900, Brazil.E-mail: acapovil@usp.br

D. COSTARDI, M. ISOM \& D. HEAD. Training of Task Switching Ability in Young and Older Adults.

Objective: Advancing age is associated with decreased ability to coordinate multiple tasks, as observed in the task switching paradigm. Previous research suggests that extensive practice can result in ageequivalence under random switching conditions, but not memorybased conditions. The goal of the current study was to explore methods for improving performance in memory-based task switching in young and older adults; and the degree of transfer of training-related improvements.

Participants and Methods: We examined the effects of instructional set and shaping procedures on training in task switching across 3 experimental conditions: explicit memory-based switching with a shaping procedure to increase memory load (EMS), explicit memory-based switching with a constant memory load (EMC), and implicit switching with a shaping procedure (IMS). All tasks required simple judgments about geometrical shapes. A pretest-posttest design with short-term training was used to examine training-related benefits and transfer of training. Thirty-two young and 70 healthy older adults participated in a between-group design.

Results: Significant age differences in global switch costs were observed across all conditions. Both young and older adults significantly benefited from training and evidenced significant transfer of training-related gains in all conditions. Older adults evidenced greater training-related gains in the EMS condition as compared to the EMC. Moreover, there was greater transfer in the IMS than in the EMS condition.

Conclusions: Current results suggest training on coordinating multiple tasks may be an effective intervention to enhance executive control processing in older adults. There appears to be particular benefit of spe- cific methods of training and longer-term training may be necessary to achieve age-equivalence.

Correspondence: Daniela Costardi, FBF S.Giovanni di Dio, via Pilastroni,4,Brescia 25125, Italy.E-mail:daniela@costardi.it

R.D. CREAN, K.R. BUFFKINS \& B.J. MASON. The Effects of LongTerm Cannabis Use on Executive Functioning in Treatment-Seeking Adults.

Objective: Cannabis is the most widely used illicit drug among adolescents and young adults. Studies in adult cannabis users have found cognitive impairments in learning and memory that typically resolve with a month of abstinence; however, neuroimaging abnormalities appear to persist, particularly in the prefrontal cortex, an area that mediates executive functioning. Sensitive and specific neuropsychological measures of executive functioning may elucidate the relationship between neuroimaging and cognitive abnormalities in marijuana users. The objective is to determine if long-term marijuana use impairs performance on tasks assessing specific aspects of executive functioning.

Participants and Methods: Long-term marijuana users seeking treatment completed three subtests (Trail Making, Verbal Fluency and ColorWord Interference) of the Dellis-Kaplan Executive Function System (DKEFS) at baseline evaluation. Mood and sleep quality were also assessed. Results: Significant impairments in executive functioning were observed when compared to normative data. Specifically, when compared with their above-average performance on visual scanning, attention and motor speed, their performance was significantly impaired on measures of visual and motor cognitive flexibility on the Trail Making task. In addition, cognitive flexibility, as measured by switching accuracy from the Verbal Fluency task, was significantly impaired.

Conclusions: Long-term cannabis use impairs executive functioning performance, which is consistent with recent neuroimaging findings. Specifically, cannabis users have difficulty with complex tasks requiring cognitive flexibility in both verbal and non-verbal domains, which are not due to attentional or motor speed problems. The results further our understanding of the connection between long-term cannabis use and cognitive impairments in executive functioning.

Correspondence: Rebecca D. Crean, Ph.D., Committee on the Neurobiology of Addictive Disorders, The Scripps Research Institute, 10550 N. Torrey Pines Rd., Mail code: TPC-5, La Jolla, CA 92037. Email: rcrean@scripps.edu

D. FERREIRA, A. NIETO, J. BARROSO, P. SÁNCHEZ \& J. BUENO. Qualitative Analysis of Phonemic and Semantic Fluency Performance in MCI Patients.

Objective: Verbal Fluency tests are extensively used in clinical neuropsychological assessments because of its high sensitivity to the brain injury. The score most commonly used is the number of words generated. However, since it is a multifactorial task, a qualitative performance analysis can provide information about the different processes involved. We studied the production and the strategies used in Verbal Fluency task in MCI patients.

Participants and Methods: 14 MCI patients and 13 healthy comparable subjects were assessed. They were given a Phonemic Fluency task (FAS) and a Semantic Fluency task (animals). We used Troyer's (2000) method to define cluster (two or more consecutive words belonging to the same category) and switches (number of transitions between cluster, including single words). In addition, we used the number of clusters as a complementary measure of cognitive flexibility. Results: MCI generated less correct words than healthy subjects in both tasks. On the Phonemic Fluency task, there were no significant group differences on neither cluster size nor cluster number, but MCI generated less switches. On the Semantic Fluency Task MCI patients had a normal cluster size and produced similar switches than controls subjects, but they produced a fewer number of clusters. 
Conclusions: MCI patients showed a reduced capacity to generate words on both Phonemic and Semantic Fluency tests. The analysis of strategic processes suggests that this decreased production is caused by a deficit in the frontal component of both fluency tasks (cognitive flexibility and shifting), while the temporal component (semantic memory) remains intact in this sample of MCI patients.

Correspondence: Daniel Ferreira, Psicobiología, Universidad de La Laguna, Facultad de Psicología, campus de Guajara, La Laguna 38205, Spain. E-mail: danifer@ull.es

M. GOMEZ BELDARRAIN, A. GONZALEZ ASTORGANO, A. BILBAO GONZALEZ \& J. GARCIA-MONCO. Sleep Improves Sequential Motor Learning and Performance in Patients with Frontal Lobe Lesions. A pilot study.

Objective: To investigate the influence of sleep on the consolidation pocess of a sequential motor task in patients with acquired focal frontal lesions.

Practice is essential to learn specific motor tasks. Learning a motor skill involves a latent process of consolidation that develops after training to enhance the skill in the absence of any practice. This "off-line" learning depends on sleep. F-MRI shows that this latent consolidation during sleep changes the brain representation of the motor skill by reducing overall the neocortical contributions to the representation, specifically this sleep-dependent improvement was linked to greatly reduced brain activation in prefrontal areas, along with a stronger involvement of parietal regions. We anticipated that patients with frontal lesions who are unable to learn a motor sequence task could benefit from sleep greater than healthy people or patients with parietal lesions, as consolidation of motor skills does not depend on prefrontal regions.

Participants and Methods: We evaluated a group of 14 patients with focal frontal lesions, 15 healthy age-matched controls, and 5 patients with parietal lesions on a serial reaction time task (SRTT) before and after a night of monitored sleep. We also tested verbal (Rey Auditory Verbal Learning Task), visual (Rey Complex Figure) and working memory (N-back). Task.

Results: Frontal patients showed an erratic learning curve at night, with great inter- and intrasubject variability that normalized after sleep. They also showed higher overnight learning of the motor skill and improvement on speed performance on the SRTT. No differences in the other memory tests were found between sessions. Sleep patterns did not difer between groups.

Conclusions: Frontal-injured patients show benefit from night sleep in terms of motor task performance, likely related to an advantageous off-line learning. Sleep, therefore, should be borne in mind as a therapy in motor rehabilitation programs in patients with frontal lesions Correspondence: Marian Gomez Beldarrain, MD, Neurology, Hospital de Galdakao, Barrio Labeaga s/n, Galdakao 48960, Spain. E-mail: mgomezab@sarenet.es

D. HOEROLD, N.P. PENDER \& I.H. ROBERTSON. SelfAwareness,Sustained Attention and Error Processing in Focal Lesion Patients: Neuropsychological and Electrophysiological Correlates. Objective: The current project constitutes an in-depth investigation of self-awareness patterns, sustained attention performance and error monitoring following acquired focal brain injury. We investigate the proposal that both self-awareness and sustained attention may be dependent on background levels of arousal.

Participants and Methods: Participants included 4 types of focal lesion patients: right \& left frontal, as well as right \& left non-frontal lesions.All participants underwent complete neuropsychological assessment. Further, participants performed the Sustained Attention to Response Task (SART, Robertson et al, 1997) and the Error Awareness Task (EAT, Hester et al, 2005). Electrodermal activity was recorded during both of these tasks. Participants' self-awareness was assessed on 3 further levels: online emergent (error awareness during task performance itself), online anticipatory ( through prediction of neuropsychological task performance), and metacognitive (through comparison of patients' symptom ratings with those of significant others). In a second experiment patients were recalled for a specific error-monitoring study using the Error Awareness Task (EAT), during which event-related potentials (ERPs) were recorded. Few studies have investigated error awareness as a specifical component of error processing ( $\mathrm{O}^{\prime}$ Connell et al, in press).

Results: We explore how specific cortical lesions affect error monitoring behaviour and how this is reflected in the ERP components typically associated with error processing.

Conclusions: Through a comprehensive neuropsychological and electrophysiological characterization of self-monitoring behaviours in distinct focal lesion groups, we hope to shed light on the relationship between arousal/alertness levels, sustained attention performance, and self-awareness processes.

Correspondence: Doreen Hoerold, Institute of Neuroscience \& School of Psychology, Trinity College Institute of Neuroscience, Lloyd Building, Trinity College Dublin, Dublin 2, Ireland.E-mail: hoeroldd@tcd.ie

M. LUETHI. Selective Planning Impairment After Theta Burst rTMS Over the Right Dorsolateral Prefrontal Cortex: An Eye Movement Study.

Objective: This study is aimed at comparing the effects of theta burst repetitive transcranial magnetic stimulation (rTMS) over the right dorsolateral prefrontal cortex (rDLPFC) on planning vs. monitoring functions.

Participants and Methods: 17 right handed subjects completed a computerised trail making task. They were instructed to click on numbers in an ascending order while eye movements and mouse clicks were recorded. In a more difficult condition, target stimuli consisted of numbers and letters which had to be clicked in an ascending, alternating order. Monitoring functions were defined as fixations on a target stimulus immediately before the mouse click. Anticipatory fixations on subsequent stimuli were considered as evidence for planning, e.g. fixations on target number 4 before a click on target number 3 was made.

Results: Task difficulty had a strong and reliable effect on these parameters. With greater task difficulty, subjects improved their monitoring behaviour, while anticipatory fixations decreased. After stimulation, subjects showed the same monitoring behaviour compared to the control condition. Also, the increase in monitoring to account for task difficulty was not influenced by rTMS. However, rTMS did significantly decrease anticipatory fixations. Moreover, with respect to planning, rTMS interacted with task difficulty. Subjects were able to perform more anticipatory fixations during the easy condition, and this benefit was more pronounced in the control condition compared to rTMS.

Conclusions: These preliminary within - subjects comparisons suggests that rTMS over the rDLPFC impairs planning, but not monitoring functions.

Correspondence: Mathias Luethi, Neuropsychological Rehabilitation, Inselspital, Berne 3011, Switzerland. E-mail: mathias.luethi@gmail.com

L.F. MALLOY-DINIZ, W.B. LEITE, D. FUENTES, H. CORRÊA, S.S. ABRANTES, D.N. MALLOY-DINIZ \& M.A. SEDÓ.APLICABILITY OF FIVE DIGITS AND ORAL TRAIL TESTS ON THE ASSESSMENT OF EXECUTIVE FUNCTIONS OF ADULTS WITH ADHD.

Objective: Five Digit Test (5DT) and Oral trails test (OTT) are alternative forms of Stroop Collor Word and Trail Making Test respectively. Both 5DT and OTT measure process speed, response inhibition, visual scan and mental flexibility and are very useful on assessment of executive functions on subjects with low academic level, reading disabilities and/or motor fine impairments. The aim of this study was to examine 
the performance of individuals with Attention Deficit Hyperactivity Disorders (ADHD) on these two measures of executive functions.

Participants and Methods: We administered OTT and 5DT, in a sample of 85 ADHD and 105 healthy controls (HC). ADHD and HC individuals have intellectual level above 25 th percentile measured by Raven Progressive Matrices, were aged 18 trough 65 years and have from 8 trough 18 years of formal education. We used T-test for independent samples to examine differences between ADHD and HC on time and number of errors on each condition 5DT and on OTT.

Results: ADHD patients has worse performance than HC subjects both on time to complete tasks and number of error on parts 3 and 4 of 5DT and on parts 2 , B e 3 of OTT. No correlations were found between years of formal education and performance on the tests.

Conclusions: These results reinforce the idea of executive functions impairments on ADHD adults. They also show that 5DT and OTT are valid instruments on the assessment of speed process, response inhibition, visual scanning and set shifting on ADHD patients regardless its educational background.

Correspondence: Leandro F. Malloy-Diniz, PHD, University Human Sciences Faculty, Universidade FUMEC, Rua Gonçalves Dias, 31 Bairro Funcionários, Belo Horizonte 30130090, Brazil. E-mail: leandro. fernandes@terra.com.br

D. MARCHADOUR, P. ALLAIN, C. VERNY, F. ETCHARRY-BOUYX, F. DUBAS \& D. LE GALL. A study of spontaneous and reactive flexibility in patients with frontal lobe lesions and Parkinson's disease.

Objective: According to Eslinger and Grattan (1993), spontaneous flexibility calls upon subjects to generate diverse creative solutions by mounting strategies to move among classes and category of knowledge in response to a question. Spontaneous flexibility impairments are frequent in patients with frontal lobe lesions. These authors suggested that reactive flexibility requires subjects to shift response set in relation to external cues. Reactive flexibility is mediated by the frontal-striatal system. The aim of this work was to study spontaneous and reactive flexibility in patients with frontal and basal ganglia lesions, in order to replicate Eslinger and Grattan's (1993) findings with classical neuropsychological tasks.

Participants and Methods: Ten patients with circumscribed frontal lobe lesions (FL), 10 patients with Parkinson's disease (PD) and 10 normal controls (NC) were given neuropsychological tests assessing spontaneous (phonemic and semantic fluency tasks) and reactive (Modified Card Sorting Test and Trail Making Test) flexibility. All subjects were nondemented and nondepressed and were matched for sex, age and education duration.

Results: Results indicated that FL and PD patients performed at a significantly lower level than NC in both reactive and spontaneous flexibility measures (all p'< .05). FL patients tended to have a higher degree of spontaneous flexibility impairment than PD patients.

Conclusions: Our findings are inconsistent with Eslinger and Grattan's (1993) proposition, indicating that basal ganglia also appears to mediate spontaneous flexibility. This suggests that the production of diverse ideas may not only require direct cortical-cortical interactions by the frontal lobe in order to access knowledge systems but also cortical-subcortical interactions.

Correspondence: Philippe Allain, PhD, Département de Neurologie, Unité de Neutropsychologie,, rue Larrey, Angers 49033, France. E-mail: PhAllain@chu-angers.fr

J.E. MARTIN \& C.E. SKILBECK.Card Sorting Test Performance in a Closed Head-Injured Population:The Role ofVisualWorking Memory. Objective: Poor performance on the Wisconsin Card Sorting Test (WCST: Heaton, Chelune, Talley, Kay, \& Curtis, 1993) has been attributed to deficits in working memory. However, most studies have overlooked the role of the visuospatial sketchpad and many have used WCST versions in which the previous response cards did not remain in view. Based on the findings of a preliminary study it was hypothesised that more errors would be made and fewer categories achieved in the presence of impaired visual working memory ability and without the benefit of visual feedback from previous card sorts.

Participants and Methods: Closed head-injured patients (N=59) differentially impaired on tests of executive function (FAS/Trails) and auditory working memory (digits) or visual working memory (Visual Patterns Test) participated in the study. The control group were those CHI patients with no noted cognitive deficits. A 4 [group: head-injured controls, executive dysfunction only, auditory dysfunction, visual dysfunction] x 2 (visual feedback: with, without) mixed factorial design was used. Participants completed the card sorting tests, on average, within 4 weeks post-injury.

Results: The results showed that patients with executive dysfunction plus a visual working memory deficit made significantly more total errors and failure to establish set scores than head-injured controls, patients with executive dysfunction alone, or patients with executive dysfunction plus an auditory working memory deficit. Perseverative errors contributed less than one third of the total errors made in any of the executive dysfunction groups.

Conclusions: These results suggest that the visuospatial sketchpad contributes significantly to card sorting test performance. The pattern of errors were interpreted as reflecting an inability to inhibit competing responses that affected problem solving and conceptual abilities.

Correspondence: Janine E. Martin, PhD, Royal Hobart Hospital, Dwyer Ward, 48 Liverpool St, Hobart, TAS 7000, Australia. E-mail: Janine. Martin@utas.edu.au

\section{MARTIN ARAGONESES, R. LÓPEZ-HIGES, S. RUBIO VALDEHITA \& D. DEL RÍO GRANDE. Working Memory Span: A Comparison between Measures.}

Objective: Many tasks have been developed to measure WM (Daneman and Carpenter, 1980; MacDonald et al., 2001). Our goals were: 1) to explore the psychometric properties of three WM span tests; 2) to provide information about whether different WM tests measure a single cognitive function; and 3) to verify the validity of the processing-storage independence assumption (PSIA; Towse, Hitch \& Hutton, 2000).

Participants and Methods: Eighty-four Psychology students of Complutense University (Madrid) participated in this study. We used two WM span tests (reading span [RST] and arithmetic span [AST] tests) that included a secondary verification task. Verification time serves as the measure to examine the PSIA. We also used a digit reordering task. To explore the differences between low- and high-span subjects in the verification task, we used the maximum span and computed the lower and upper quartiles to divide the total sample.

Results: The results showed that RST and AST measures formed a cluster not related to digit reordering measures. Regarding the PSIA and in RST, the difference in verification times between the last and the first sentence was clear for the high-span group. Only under these conditions the resource sharing hypothesis seems to be correct. Results in AST were not conclusive.

Conclusions: Taking in consideration their psychometric properties, AST seems to be the most appropriate measure of WM. RST and AST provide a measure of the same construct, whereas digit reordering measures another one. Our results suggest that resource sharing or task switching might be considered strategies associated with an individual variable, the WM-span.

Correspondence: Maria Teresa Martin Aragoneses, Dpto. Psicología Básica II (Procesos Cognitivos). Facultad de Psicología, Universidad Complutense de Madrid, Despacho 1105 C. Buzón 25. Campus de Somosaguas., Pozuelo de Alarcón 28223, Spain. E-mail: mt.m. aragoneses@psi.ucm.es 


\section{J. MCLACHLAN. Normative Five Digit Test Data on Adult Clinical Examinees.}

Objective: Normative adult data on Sedó's Five Digit Test (5DT) were developed on examinees who passed Symptom Validity Testing (SVT). Participants and Methods: Of 135 adults completing both the 5DT and SVT (Computerized Assessment of Response Bias or Word Memory Test) during a psychological disability examination in private practice, $88(65 \%)$ passed SVT. These patients ranged in age from 17 to 68 years (Mean 37.10, SD 11.83), had received from 5 to 19 years of formal education (Mean 12.07, SD 2.37) and consisted of 57 women and 31 men, $57 \%$ of whom were not native English speakers. There were 36 Asian. 14 Black and 30 Caucasian patients with 8 of other background.

Results: Better performance was found on all parts of the test by those who passed SVT compared to those who failed it with t-ratios of $p<.001$ for all the traditional part scores. Further analysis was performed on only the well-motivated examinees. High reliability for all test parts was found with Spearman-Brown coefficients between .93 and .96. Tabled norms were made for the well-motivated persons. These included standard scores, scaled scores and percentiles. When converted into scaled scores, correlation coefficients were significant for education $(p<.01$ to $\mathrm{p}<.001)$ as expected but not with age, gender or English usage. Regression formulas were provided for predicting later part scores from earlier parts.

Conclusions: Use of normed scores helps control for distribution anomalies in the 5DT and should assist in the analysis of the relationship of the 5DT to other cognitive measures.

Correspondence: John McLachlan, Ph.D., Private Practice, 2 Carlton Street, Suite 1405, Toronto, ON M5B 1J3, Canada. E-mail: johnmclachlan@sympatico.ca

F. ORSATI, E.C. MACEDO, D. BRUNONI \& J. SCHWARTZMAN. Executive Deficits in Pervasive Developmental Disorders.

Objective: Autism is part of a spectrum of the Pervasive Developmental Disorders (PDD). It is characterized by deficits in social interaction, communication and behavior. The diagnosis is based on clinical assessment and behavior observation. New methods have been developed and studied for more precise diagnosis. Among these methods neuropsychological assessment can establish profiles and so contribute to more precise interventions. The objective is to assess IQ and executive functions in PDD participants and correlate to typical development.

Participants and Methods: 10 PDD children and adolescents with mean age 11,9 (SD=3,22) matched with 10 children and adolescents with typical development were assessed. The WISC III was used to assess intelligence, Trail Making, Rey Complex Figure and Hanoi Tower were used to assess the executive abilities.

Results: Significant differences were found between General IQ, Executive IQ, part B of Trail Making Test, memory drawing after 3 and 30 minutes in the Complex Figure Test and the Hanoi Tower.

Conclusions: A profile of neuropsychological deficits is characterized by: difficulties on voluntary attention regulation, response inhibition, planning, response initiation and cognitive flexibility; and preserved viso-construction ability. The executive function impairments reflect on the social behavior regulation. The description of the executive profile can contribute to more efficient interventions.

Correspondence: Fernanda Orsati, Master student, Universidade Mackenzie, Rua Joaquim Távora 827 ap. 61, São Paulo 04015001, Brazil.E-mail:feorsati@terra.com.br

J. ORTIZ-GIL, J. GOMAR, B. SANS-SANSA, A. AGUILERA, A. GUERRERO, S. SARRÓ \& E. POMAROL-CLOTET. The Memory and Dysexecutive Deficits in Schizophrenia Are Neuropsychologically Specific.

Objective: The studies that have attempted to disentangle specific impairment in executive, mnesic and attentional functions (Perry, 2001) from generalized intellectual impairment (Blanchard, 1994) in schizophrenia have offered conflicting findings.
We replicated Evans et al. (1997) study with al larger sample of chronic schizophrenic patients. Our objective was to examine whether there are specific executive, working memory and immediate memory deficits in patients with chronic schizophrenia and preserved IQ (>85), in order to avoid the confussional factor implied in generalized intellectual impairment.

Participants and Methods: Forty-three schizophrenic patients and 36 healthy volunteers matched in age $(45.62$, s.d. 10.90 vs. 44.13 , s.d.14.01; $\mathrm{p}=0.597)$, gender ( 30 male and 13 female vs. 23 male and 13 female; $\mathrm{p}=0.580)$ and IQ $(100.42$, s.d.8.88 vs. 103.06 , s.d. $8.72 ; p=0.189)$. We compared their performance on the BADS, an ecologically valid battery for assessing dysexecutive functioning, and on some subtests of WMSIII assessing working memory, immediate memory and orientation.

Results: Schizophrenic patients showed impairment on the 'BADS profile score' $(p<0.001)$ and on working memory ('Digit Span', $p=0.036$; 'Letter-Number Sequencing', $p=0.002$ ), immediate memory ('Logical memory', $\mathrm{p}<0.001$; 'Faces', $\mathrm{p}=0.001$ ) and 'Information and Orientation' $(p=0.003)$ from WMS-III, compared to healthy controls.

Conclusions: These results support the hypothesis that chronic schizophrenic patients might suffer from selective deficits on executive function, working memory and immediate memory. Since the chronic schizophrenic patients had a preserved IQ and were matched with the control subjects in gender, age and IQ, none of these variables could account for the differences in executive function, working memory and immediate memory. Correspondence: Jordi Ortiz Gil, Unit of Research in Psychosis, Benito Menni CASM, C/Antoni Pujadas 38, Sant Boi del Llobregat 08830, Spain.E-mail: jordi.ortiz.gil@gmail.com

G. PLUCK, K. LEE, T. LAMB, K. GEE, R. DAVID \& R.W. PARKS. Frontal Systems Behavior and Psychiatric Symptoms among Homeless Adults.

Objective: Homeless adults tend to perform poorly on assessments of frontal lobe function and many also have psychiatric problems. We wished to examine the relationship, among the homeless, between psychiatric problems and apathy, disinhibition and executive dysfunction as measured by the Frontal Systems Behavior scale (FrSBe) of Grace \& Malloy (2001). This is a self report assessment of behaviors associated with frontal lobe impairment.

Participants and Methods: Seventy one homeless adults (mean age 34.1) were interviewed concerning the presence of the following problems over the past year: depression, attempted suicide, anxiety, psychotic symptoms, daily alcohol use, daily heroin/crack use, and unconsciousness through head injury. They were also asked if they had ever been admitted to a psychiatric hospital. They completed the FrSBe scale concerning their current status. Results: The mean total $\mathrm{T}$ score on the FrSBe was 77.1 (SD=20.9), which is clearly above the normative mean of 50. Indeed, $39(54.9 \%)$ of the participants scored more than two standard deviations above the normal mean. High scores on the disinhibition subscale were significantly associated with the presence of psychotic symptoms and with alcohol abuse. High scores on the dysexecutive subscale were significantly associated with alcohol abuse and having a past psychiatric hospital admission. No psychiatric variables were associated with the apathy subscale. Examining the total FrSBe score, it was found that alcohol abuse and psychotic symptoms were significantly associated with high scores. Conclusions: When frontal lobe impairments are reported in homeless people, these may be associated with co-morbid psychiatric problems, in particular, alcoholism and psychotic symptoms.

Correspondence: Graham Pluck, PhD, Academic Clinical Psychiatry, University of Sheffield, The Longley Centre, Norwood Grange Drive, Sheffield S5 7JT, United Kingdom. E-mail: g.pluck@sheffield.ac.uk

S. ROJAS, R.D. LOREnZANA, G. YAÑEZ \& E. RUIZ. Neuropsychological Post Surgical Rehabilitation in a Patient with a Cerebral Frontal Lobe Tumour.

Objective: The objective of the present study was to report the results that we obtained by an intervention program. 
Objectives about neuropsychological rehabilitation were to increase the patient's Verbal Fluency and to stimulate the capacity to planning, to modify, to execute, and to verify activities.

Participants and Methods: 59 years old male, who had the diagnosis of frontal meningioma; and showed deficits in executive functions and verbal fluency.

We made a neuropsychological evaluation pre and post surgery and one after the neuropsychological rehabilitation. The sessions lasted six months, one and half hour weekly. We worked on EF, stimulating planning ability, to carry out, to modify and to verify his activities. About verbal fluency we worked through conversations and he described pictorial scenes. Results: We observed an increment in the number of words per minute during spontaneous talk. After the intervention in relation with EF we observed a better capacity in planning activities and diminished the time to carry out them. He also was able to make new alternatives in problem solving.

Conclusions: Neuropsychological intervention was beneficial for the patient reflected in better daily activities, for example take a shower, eating and going out. The program increased verbal fluency and improved his communication abilities. Moreover, the program improved his familiar relations

Correspondence: Sulema Rojas, Psychologist, National Autonomy University of México, Esperanza 1012-301 Col. Narvarte Del. Benito Juarez, Mexico 03020, Mexico.E-mail: sulemarojas@yahoo.com.mx

T. BEKINSCHTEIN, J. CARDOZO, T. TORRALVA, M. ROCA \& F. MANES. BUENOS AIRES WAITERS: STRATEGIES TO ENHANCE MEMORY CAPACITY.

Objective: To study how typical Buenos Aires waiters (who have no need to make written notes) memorise all the orders and the respective clients. in particular to study their spatial working memory strategies in a reallife setting.

Participants and Methods: Waiters' ( $N=9,>10$ years of experience) spatial working memory was assessed while working, and were unaware of the experiment. The researcher, who performed as table coordinator, and other seven participants were randomly arranged around the table, ready to make different, previously stated, orders. The assessment was achieved in two different conditions. In the first condition the waiter was asked for eight different drinks and the customers remained seated in their original locations (OL) at the time of the delivery of the orders. The other condition consisted in asking for eight different meals and changing the client's locations (CL) to new, previously established ones, before the waiter arrived to deliver the orders.

Results: We found that the waiters use a bound (feature- location) strategy since all but one presented location errors.

Conclusions: Results were consistent with the strategy reported by the waiters. Perhaps this strategy is commonly used by people and it is necessary to develop (more reliable) tests to demonstrate this hypothesis in "ecological" way

Correspondence: Teresa Torralva, MD, Neuropsychology, INECO, Castex 2356, Buenos Aires 1425, Argentina. E-mail: ttorralva@neurologiacognitiva.org

A.J. VERDEJO-GARCÍA, L. CLARK, C. RIVAS-PÉREZ \& M. PÉREZGARCÍA. EMOTIONAL FEEDBACK MODULATES DECISIONMAKING PERFORMANCE IN THE IOWA GAMBLING TASK.

Objective: To investigate the effects of congruent or incongruent emotional feedback to facilitate advantageous decision-making on the Iowa Gambling Task.

Participants and Methods: 42 healthy undergraduates volunteered in this study. We adapted the Iowa Gambling Task (IGT, Bechara et al., 1994) by inserting affective images from the IAPS picture series, after the financial outcome of each card selection was revealed. There were two experimental conditions as a between-subjects variable. In the emotionally congruent condition, the valence of the images was congruent with the monetary outcome, such that wins were paired with pleasant images and losses were coupled with unpleasant images. In the emo- tionally incongruent condition, the valence of the images was incongruent with the monetary outcomes. Participants were randomly assigned to the congruent (23) or incongruent (19) condition. We explained to participants that during task performance they were going to see some images, but asked them to focus on the decision-making task. We compensated them with $10 \%$ of the play money won at the end of the task. We ran a 2 (emotional condition) x 4 (task blocks) ANOVA to examine the effects of emotional feedback on task performance.

Results: There was a significant condition $\mathrm{x}$ block interaction. Post-hoc repeated-measures ANOVAs for each emotional condition showed that participants in the congruent condition significantly improved their performance across blocks, $\mathrm{F}(3,20)=4.09$, $\mathrm{p}<0.05$, showing a learning curve; whereas participants in the incongruent condition failed to show improvement across blocks, $\mathrm{F}(3,16)=0.65$, $\mathrm{p}=0.58$.

Conclusions: The addition of emotional feedback to the IGT modulates the acquisition of an advantageous or disadvantageous strategy on the task, consistent with a role for somatic-markers in mediating optimal decision-making.

Correspondence: Antonio J. Verdejo-García, Pharmacology, Institut Municipal d'Investigació Mèdica, Dr.Aiguader 80, Barcelona 08002, Spain.E-mail: averdejo@imim.es

\section{Hydrocephalus}

D. BARTRÉS-FAZ, M. MATARó, M. POCA, M. MATARín, J. SAHUQUILLO \& C. JUNQUÉ. Voxel-based Morphometry Analysis of CSF before and after Shunting in Normal Pressure Hydrocephalus. Relation with Clinical and Cognitive Measures.

Objective: Previous studies in normal pressure hydrocephalus (NPH) have found a poor relationship between the reduction in ventricular size following surgery and clinical outcome. The purpose of this study is to use an automated optimized voxel-based morphometry approach (Good et al., 2001) to compare CSF images before and after shunting and determine its relationship with neuropsychological measures.

Participants and Methods: Ten patients with idiopathic NPH were included in the study. Structural MRI and clinical/neuropsychological measures were acquired before surgery and 6 months post shunting.

Results: CSF volume was increased bilaterally in the posterior horns of the lateral (peak max. left: $\mathrm{x}, \mathrm{y}, \mathrm{z}=-30-45-12, \mathrm{t}=9.96, \mathrm{p}<0.001$; peak max. right: $30-44-10, \mathrm{t}=4.29, \mathrm{p}<0.001)$ as well as in the third (peak max.: 12 $-201, \mathrm{t}=6.98, \mathrm{p}<0.001$ ) and fourth ventricles (peak max: $\mathrm{x}, \mathrm{y}, \mathrm{z}=8-51-$ $39, \mathrm{t}=9.13, \mathrm{p}<0.001$ ) before shunting relative to postsurgical intervention. CSF was displaced from the ventricular system to the subarachnoideus space all over the convexity as well as in the interhemispheric fissure after the shunting (peak max: $33663, \mathrm{t}=1204, \mathrm{p}<0.001$ ). Positive correlations were observed between CSF volume in the subaracnoidal temporal lobe space and the total NPH scale (peak max.: $x, y, z=57-4920, t=13.76$, $\mathrm{p}<0.001)$ before surgical intervention, and a negative covariation between COWAT test score and CSF bilaterally in the anterior horns of the lateral ventricles (peak max.: $\mathrm{x}, \mathrm{y}, \mathrm{z}=16241, \mathrm{t}=10.68, \mathrm{p}<0.001$ ) after shunting. Conclusions: Our findings indicate that using a VBM approach is possible to map the CSF distribution pre and post shunting in NPH patients. CSF displaces from intraventricular regions to subarachoideus space following shunting.

Correspondence: David Bartrés-Faz, PhD, Psiquiatria i Psicobiologia Clinica, Facultat de Medicina, Universitat de Barcelona, Casanova, 143, Barcelona 08036, Spain.E-mail: dbartres@ub.edu

\section{Imaging: Functional}

M. GARCÍA-BLAZQUEZ, P. NOGUERA, J. CHIRIVELLA, J. FERRI, N. FERRI, C. COLOMER, O. RENAU, L. DE LA CUEVA \& E. NOE. Structural and Functional Neuroimaging Correlates of Clinical Findings in Four Cases of Infrequent Encephalopathies.

Objective: To describe the clinical findings of four rare cases of infrequent encephalopathies and to describe the relationship between neuro- 
logical, cognitive, psychopathological and functional data and structural (CT/MRI) and functional neuroimaging (18F-FDG-PET) findings.

Participants and Methods: Four comatose patients affected by rare encephalopathies (neurosyphilis, hypoglycemic shock, cerebral anoxia after electric shock, and one case of cerebral fat embolism) who were attending our facility were included in this study. All patients were prospectively assessed with different scales according to their neurological situation during at least one year (Loewenstein Communication Scale, Severe Impairment Battery, Mini-Mental State Examination, WAIS-III, California Verbal Learning Test, Color-Trail-Making Test, Wisconsin Card Sorting test, and Rey Complex Figure). Functional assessment included the Barthel and the FIM-FAM. We also examined the relationship between clinical findings and both structural and functional imaging.

Results: Two patients were still in a minimally conscious state at the end of follow-up. One of the patients showed a severe cognitive deficit, with a profound behavioural alteration (agitation, aggressiveness and disinhibition), which generated a moderate dependency in daily life. The last patient presented moderate cognitive deficits in attentional, learning/memory and executive functions with a minimum dependency in functional activities. Structural neuroimaging were normal or showed subcortical abnormalities whereas 18-F-FDG-PET showed a predominant cortical hypometabolism in all patients.

Conclusions: Our findings provide an interpretation of the brain response to rare encephalopathies revealed in vivo by structural and functional neuroimaging. Specifically, functional neuroimaging techniques can helps us to understand the consequences of those diseases, which by their etiology, evolution, and low incidence are more atypical.

Correspondence: Enrique Noe, MD, Ph.D, Servicio Daño Cerebral de Hospitales NISA, C/ Río Tajo s/n, Valencia 46011, Spain. E-mail: enoe@comv.es

\section{A.P. HALEY, L.H. SWEET, J. GUNSTAD, A. POPPAS \& R.A. COHEN. Family History of Hypertension is Associated with Reduced fMRI Response to a Visuospatial Working Memory Task in Healthy Young Adults.}

Objective: Midlife hypertension (HTN) is associated with increased risk for dementia. However, early identification of vulnerable individuals is complicated by decades of separation between onset of HTN and $\operatorname{cog}_{-}$ nitive impairment, variable cognitive outcomes, and evidence that onset of dementia is often preceded by a decline in blood pressure. Research suggests that family history of hypertension (FH+) may identify individuals at particularly high risk for future cognitive decline. In this study, we employed fMRI to test for differences in hemodynamic response to a cognitive challenge that has been previously related to genetic risk for HTN.

Participants and Methods: Fourteen healthy adults (ages 18 to 39 years) participated in an fMRI study employing a 2 -Back visuospatial working memory task. Seven of the participants were FH+ and seven were FH-. Age, education, and verbal working memory performance were equal between the groups. Successful performance of the visuospatial 2-Back was associated with brain activation in expected areas.

Results: Relative to FH- controls, FH+ participants exhibited lower VS 2-Back related activation in the posterior cingulate gyrus $(t=4.14$, $\mathrm{p}<.05)$, right inferior parietal lobule $(\mathrm{t}=2.42, \mathrm{p}<.05)$, and right inferior temporal gyrus $(\mathrm{t}=2.33, \mathrm{p}<.05)$.

Conclusions: These results indicate that $\mathrm{FH}+$ is associated with subtle deficits in integration of visual-spatial processing with higher cognitive processes even in healthy young adults. They also indicate that fMRI is a sensitive tool for detecting differences in brain function associated genetic risk for HTN. Therefore, fMRI may be a useful tool for developing early markers of risk for vascular cognitive impairment.
Correspondence: Andreana P. Haley, Ph.D., Psychiatry and Human Behavior, Brown University, Butler Hospital, 345 Blackstone Blvd, Providence, RI 02906.E-mail: Andreana_Haley@Brown.edu

M. HAYASHI, T. HASHIMOTO, M. KATO, S. UMEDA, M. MIMURA \&. S. OGAWA. Top-down Processing in Face Perception Detected by Seeing-as-face Task in fMRI Study.

Objective: A previous MEG study showed that a component at $~ 120$ ms (M100) was identified as the detection of face category, earlier than the component of face identification (M170) (Kato et al., 2004). Our aim is to investigate the neural substrate for face perception, using fMRI. Participants and Methods: Participants were 13 healthy adults ( 5 males and 8 females) aged 20-27 (mean age; 22.6 years). They performed a seeing-as-face task in which visual inputs were identical across two conditions, but individual perceptions differed. Stimuli consisted of four ovals and four rectangles, and participants performed a 1-back repetition detection task. In the first (rectangle) condition, participants were instructed to only look at rectangles, which were arranged to form an abstract pattern. In the second (face) condition, participants were instructed to focus on ovals and told they were arranged to form a face pattern.

Results: Behavioural data showed that mean reaction time in face condition (493.1 $\pm 172.4 \mathrm{~ms}$ ) was faster than in rectangle condition $(561.2 \pm 265.8 \mathrm{~ms})$ and that the mean correct rate in face condition $(80.9 \%)$ was significantly higher than in rectangle condition $(71.2 \%)$ $(\mathrm{p}<.05)$. Compared with rectangle condition, we observed significant activation in the right inferior frontal gyrus, the right temporal-parietal junction and the right thalamus in face condition.

Conclusions: The nature of our task, i.e. identical visual inputs with different subjective perceptions, strongly suggests that the right ventrolateral prefrontal area and the subcortical region play an important role for face perception as a top-down process.

Correspondence: Mika Hayashi, Keio University, 35 Shinanomachi, Shinjuku-ku, Tokyo 160-8582, Japan.E-mail: di055010@sc.itc.keio.ac.jp

H. TOMIOKA, Т. TAKAHASHI, В. YAMAGATA, M. YANO, H. KOBAYASHI, M. HAYASHI, A.J. ISOMURA \& M. MIMURA. Driving evaluation for older adult drives using NIRS and driving simulator.

Objective: Police Department of Japan has reported that one third of older adults hold a driver's license. It is essential to appropriately evaluate capability of driving of older adults because of their own safety as well as safety of the public. The present study aimed to evaluate activities of medial and lateral prefrontal cortex of healthy older adults and people with dementia while conducting driving simulation.

Participants and Methods: Participants consisted of 30 healthy older adults who hold driver's license and 8 individuals with dementia. First, oxy-Hb values were monitored by using near-infrared spectroscopy (NIRS)(52 channels, Hitachi ETG4000) while performing on a driving simulator, TEDDY (TOYOTA Central R\&D Labs.) as compared to resting state, i.e. passive viewing. Second, oxy$\mathrm{Hb}$ values between non-eventful and high risk driving situations were also compared.

Results: Participants as a group displayed a mild elevation of oxy-Hb on lateral prefrontal cortex while driving as compared to passive viewing. There was no change or even deactivation observed in the medial prefrontal cortex while driving. Moreover, healthy older adults showed a consistent increase in oxy- $\mathrm{Hb}$ under the high risk situations where one needed to make brake responses against rushing pedestrians or bikes. They exhibited elevated oxy-Hb values on broader areas of bilateral medial and lateral prefrontal cortex in the high risk situations. Reaction time of people with dementia was significantly delayed as com- 
pared to healthy individuals although their patterns of oxy-Hb changes were variable.

Conclusions: Multi-channel NIRS is useful to easily assess and monitor one's activities of prefrontal cortex while conducting driving simulation. Correspondence: Hiroi Tomioka, M.D., Dept. of Neuropsychiatry, Showa University School of Medicine, 1-5-8 Hatanodai, Shinagawa-ku, Tokyo 142-8666, Japan.E-mail: hiroitt@beach.ocn.ne.jp

A. ZAbala, J. SÁNChez, O. ROBles, M. PARELlada, D. MORENO, A. RUIZ SANCHO, M. BURDALO, M. DESCO \& C. ARANGO. Cognitive Correlates of N-Acetyl-Aspartate Alterations in Dorsolateral Prefrontal Cortex in Adolescents with First-Episode Schizophrenia.

Objective: To determine the presence of cognitive deficits in adolescents with first-episode psychosis, to study the specificity of alterations through different psychotic diagnoses and to assess associations between prefrontal cognition and N-acetyl-aspartate (NAA) concentrations in the dorsolateral prefrontal cortex (DLPFC). We hypothesized that the schizophrenia group of patients would present more marked cognitive deficits and reduced levels of $\mathrm{NAA}$ /water in the DLPF region, being both alterations related.

Participants and Methods: Fifty-one adolescents with first-episode psychosis and 45 matched controls were evaluated. Cognition was assessed with a comprehensive neuropsychological battery. The singlevoxel proton spectra were obtained from the right and left DLPFC areas using a PRESS sequence (TE=136 ms, TR=1500 ms, NEX=128) in a 1.5 T Philips Gyroscan ACS.

Results: Deficits in attention, working memory, executive functioning and memory were present in all patient groups (schizophrenia, bipolar disorder and other psychosis) with no specificity detected. In the left DLPFC, differences in NAA/water concentrations were found between the schizophrenia group and controls $(\mathrm{p}=0.004)$. These differences were not affected by sociodemographic factors or partial volume effects. No correlations were detected for the patient or control group between prefrontal cognition and NAA/water measures. However, when taken together all participants, a significant correlation were detected in the left hemisphere $(\mathrm{r}=0.24, \mathrm{p}=0.02)$.

Conclusions: Adolescent onset schizophrenia presents a specific pattern of neural damage at the onset of the illness. Although cognitive deficits are also present, constitute a nonspecific marker of psychosis at its onset. However, cognitive deficits were more marked in the schizophrenia group and may be related to neural damage.

Correspondence: Arantzazu Zabala, Unidad Adolescentes, Hospital General Universitario Gregorio Marañón, c/Ibiza 43, Madrid 28009, Spain.E-mail:azabala@mce.hggm.es

\section{Imaging: Structural}

G. RAMETTI, C. JUNQUÉ, N. BARGALló, C. FALCón, R. CATALÁN, R. PENADÉS \& M. BERNARDO. Correlations between Verbal Memory and Hippocampal Anisotropy in Schizophrenic Patients.

Objective: The hippocampus is one of the most involved structures in schizophrenia. Several MRI studies reported a global reduction of the hippocampus in patients respect to controls. Diffusor tensor imaging (DTI) is a new modality for quantifying the connectivity of white matter fibbers in cerebral structure. In the present study we investigated fractional anisotropy (FA) in the hippocampus using DTI.

Participants and Methods: Participants in this study comprised 24 subjects (mean age \pm standard deviation $(\mathrm{SD})=32.71 \pm 6.5$ ) who met DSM-IV criteria for schizophrenia and 21 healthy subjects (mean age $\pm \mathrm{SD}=31.29 \pm 6.2$ years). MRI data was obtained on a GE Signa $1.5 \mathrm{~T}$ scanner. FA images were analysed using the VBM approach by SPM2 software. We conducted 2 separated analyses of data: we performed two one-sided comparison groups (controls $>$ patients and patients $>$ controls) and a simple regression (correlation) through the FA maps and the scores of the schizophrenics subjects on the memory tests. These analyses were also thresholded at $\mathrm{P}<0.001$ (uncorrected).

Results: Group comparison demonstrated reductions in fractional anisotropy in the left hippocampus of patients ( $\mathrm{t}$ statistic $=3.96$. cluster size 130). There was a significant correlation between delayed recall from Rey's Auditory Verbal Learning Test and hippocampal fractional anisotropy ( $\mathrm{t}$ statistic $=4.24$; uncorrected $\mathrm{P}<0.001$ ).

Conclusions: These results indicate that memory impairment is partially explained by altered connectivity of the hippocampus.

Correspondence: Giuseppina Rametti, psychiatry and psychobiology, University of Barcelona, c/ Casanova, 143, Barcelona 08024, Spain. Email:grametti@ub.edu

A.I. REIS, C. SILVA, L. FAÍSCA, I. BRAMÃO, A. MENDONÇA, P. MIRANDA, A. ANDRADE, P. TINOCO, L. TACKENBERG \& K.M. PETERSSON. The impact of reading and writing skills on brain morphometry: A voxel-based morphometry study.

Objective: Previous research has shown that acquisition of reading and writing skills has repercussions in different cognitive domains, in particular with respect to visuospatial and phonological capacities. In this study we compare groups with and without reading and writing skills, in order to characterize the impact of reading and writing skills on brain morphometry.

Participants and Methods: Voxel-based morphometry was used to compare resonance images from a group of 22 illiterate and 26 matched literate controls.

Results: Our results showed subtle grey matter density differences between literacy groups, mainly located in the left occipital cortex (BA 18/19; literate $>$ illiterate), while the white matter density differences were much more prominent and related to the occipito-temporal - ("what") pathway (in the vicinity of right BA 18/19/20 and left BA 20/37; literate > illiterate).

Conclusions: These results suggest that neuronal connectivity is relatively more affected by literacy and education compared to the neuronal density as such. Similar morphometric findings in dyslexic subjects have been reported, suggesting reduced grey- and white matter densities in different brain regions known to be engage in reading tasks. In short, we observed differences in white matter related to brain regions that are dedicated to visual information processing, suggesting that learning to read and write modifies the density of white matter adjacent to brain regions involved in the processing of written language. The fact that the difference between literacy groups was mainly located in the white matter as opposed to the grey matter suggests that the acquisition and maintenance of reading and writing skills promote the communication in dedicated neocortical networks. Correspondence: Alexandra I. Reis, PhD, Departamento de Psicologia, Universidade do Algarve, Universidade do Algarve, Campus de Gambelas, Faro 8000-810, Portugal.E-mail: aireis@ualg.pt

S. SORIA-PASTOR, M. GIMÉNEZ, A. NARBERHAUS, C. FALCÓN, N. BARGALló, D. SEgARRA, F. BOTET \& C. JUNQUÉ. Neuroanatomical Basis of Performance IQ Deficits in Adolescents with History of Prematurity.

Objective: Several white matter (WM) diseases involve a pattern of discrepancies between verbal and performance intelligence quotients (IQ), being the performance IQ (PIQ) inferior to verbal IQ. There is increasing evidence about the presence of WM damage associated with prematurity. We investigated the neuroanatomical basis of PIQ decrease in adolescents with antecedents of prematurity.

Participants and Methods: Forty-four adolescents (mean age \pm SD: $14.4 \pm 1.6$ years) born before 32 weeks of gestational age and 43 termborn adolescents (14.5 \pm 2.1 years) were submitted to an MRI examination and an IQ evaluation. 
Results: Twenty-six preterm subjects had a PIQ below 100, and 18 had a difference in the relationship VIQ>PIQ equal or more than 15 scaled points. In preterm children, PIQ correlated with the whole brain WM volume $(r=0.32 ; \mathrm{P}=0.036)$ but not with gray matter volume. In contrast, in normal controls PIQ positively correlated only with the whole brain gray matter volume $(\mathrm{r}=0.301 ; \mathrm{P}=0.05)$. Group comparison by voxelbased morphometry (VBM) analysis showed that preterm subjects had diffuse bilateral WM density anomalies compared to controls; complementary, individual VBM analyses demonstrated that $40 / 44$ preterm subjects had WM abnormalities. VBM analysis also showed that subjects with PIQ below 100 had WM abnormalities compared to preterm subjects with PIQ over 100 and normal control subjects. In addition, the 18 preterm subjects with the pattern of PIQ below VIQ more than 15 scaled points had significantly WM concentration anomalies compared to 21 control adolescents without IQ discrepancies.

Conclusions: These results suggest that diffuse WM loss in preterm children may be responsible for long term sequelae in PIQ.

Correspondence: Sara Soria-Pastor, MSc, Psiquiatria i Psicobiologia Clínica, Universitat de Barcelona, C/Casanova 143, 08036 Barcelona, Barcelona 08036, Spain.E-mail: sarasoria@ub.edu

\section{Other}

P.S. BOGGIO, M.A. SILVA, S.P. RIGONNATTI, M. NITSCHE, A. PASCUAL-LEONE \& F. FREGNI. Effects of transcranial direct current stimulation on working memory.

Objective: Transcranial direct current stimulation (tDCS) is a capable technique to modulate cortical excitability in a safety, painless and noninvasive manner. Therefore, this technique of brain stimulation can be used as a research tool of neuropsychological functions. We aimed to investigate the effects promoted by tDCS on working memory (WM) in healthy volunteers and Parkinson's disease (PD) patients.

Participants and Methods: 15 healthy volunteers and 18 PD patients underwent a 3-back letter WM paradigm during sham, anodal or cathodal stimulation ( 1 or $2 \mathrm{~mA}$ ) applied over the left dorsolateral prefrontal cortex (DLPFC) and primary motor cortex (M1).

Results: Results of both groups indicate that only anodal stimulation of the left DLPFC increases the accuracy of the task performance when compared to sham stimulation. However, the improvement of PD patients was just achieved after $2 \mathrm{~mA}$ tDCS.

Conclusions: Results showed that the effect of tDCS depends on the stimulation polarity and is specific to the site of stimulation. The ob- served improvement might be explained by the local increase in the excitability of the DLPFC. With PD patients, tDCS exerted a beneficial effect with a current strength of $2 \mathrm{~mA}$, but not $1 \mathrm{~mA}$. A possible explanation is the dopamine depletion and its effects on WM. It could be speculated that an increase in the local excitability of the DLPFC resulted in an increase in the dopamine release. In such scenario, perhaps $1 \mathrm{~mA}$ would not be as strong as $2 \mathrm{~mA}$ to induce this change in the dopaminergic system. In conclusion, tDCS was able to modulate the performance on a specific WM task.

Correspondence: Paulo S. Boggio, PhD, Mackenzie University, Rua da Consolação, 930, São Paulo 01302-907, Brazil. E-mail: psboggio@gmail.com

M. SALGUERO, J. ALAMEDA \& J. LORCA. Neuroanatomic and functional differences between numerical reasoning and calculation.

Objective: First, to determine the relations that exist between the achievement of operations of calculation of an outlying way and his execution in the context of a problem of numerical reasoning. And to determine the bases neuroanatomic differences for solving an arithmetical operation, so much if it is in a context as if it is in abstract. Participants and Methods: The numerical reasoning and the calculation is evaluated in two types of patients with cerebral acquired damage: On one hand, frontal injured persons who present executive disfunction and on the other hand, patients with injuries perisilvianas of the left hemisphere with alterations in the production of the language.

Results: the patients with linguistic alterations are capable of solving problems and of reasoning with numbers, nevertheless they cannot realize arithmetical simple operations if they present them to him in an outlying way, not in oral form not written. On the other hand, in those of cases the inverse boss observes frontal damage. These patients realize the tasks of calculation of an outlying or abstract way, both in oral and written form, even realizing procedures of taking, but it cannot solve simple problems of numerical reasoning.

Conclusions: the numerical reasoning, that is, the resolution of problems by means of the use of numerical symbols, is independent from the achievement of arithmetical operations of outlying form or out of context, in abstract. These results in spite of novel, they can be explained from the anatomical functional model of numerical prosecution and calculation

Correspondence: María Pilar Salguero, Psychology, Universidad de Huelva, Avda. Fuerzas Armadas, n0 7, Huelva 21071, Spain. E-mail: pilar.salguero@dpsi.uhu.es 
FRIDAY AFTERNOON, JULY 6, 2007

\section{Invited Symposium 12:00-1:30 p.m.}

\section{Developmental Cognitive Neuroscience of the Executive Functions Dependent on the Frontal Lobe: Challenging Long-held Beliefs}

\section{Chair: Adele Diamond}

\section{A. DIAMOND. Developmental Cognitive Neuroscience of the} Executive Functions dependent on the Frontal Lobe: Challenging Long-held Beliefs.

The 4 papers in this symposium present results that challenge long-held assumptions about the neural basis, developmental progressions, \& modifiability of executive functions (EF) including self-regulation, attention, response inhibition, working memory, \& performance monitoring. In the first talk, Amir Raz will present data showing that just by using suggestion EF can be powerfully augmented especially in children. His data show that suggestion can completely abolish the Stroop effect, that the neural change accompanying that is evident in visual cortex before it is evident in frontal cortex (shown by combining fMRI with ERP), \& that suggestion can abolish tics in adolescents with severe Tourette's effortlessly for hours with no excessive tics afterward. The second speaker, Adele Diamond, will present data on a practical method to improve EF skills in 3-5 year olds that is theorydriven, evidence-based, easy to implement using low-cost, simple materials, \& readily transferable across cultures. Even severely at-risk children show marked academic gains \& their EF gains appear to predict the academic ones. Diamond will also present data on best practices for assessing $\mathrm{EF}$ in young children \& throughout life. E.g., she will present data on an improved version of the Flanker task that produces a far larger Flanker effect (6-10x larger), is robust in the face of changes in stimulus size or layout, $\&$ on which dramatic changes are seen over age. The third speaker, Alexandra Hogan, has studied a critical group of adolescents and young adults, those with damage to white matter connecting the anterior cingulate (ACC) to anterior frontal lobe structures but sparing the structures themselves. While researchers have long thought that the most likely source of error-related negativity (ERN) is the ACC. Hogan's data suggest that it is the connections between dorsolateral PFC, pre-SMA, \&/or SMA and the ACC that is critical for generating the ERN. The final speaker, Charo Rueda, will present data on the development of executive attention during childhood, the brain mechanisms underlying that development, genetic contributions to individual differences in that, $\&$ computer-based games that can improve EF even in very young children, including those with autism. Correspondence: Adele Diamond, Canada Research Chair Tier 1 Professor of Developmental Cognitive Neuroscience, Department of Psychiatry, University of British Columbia (UBC), \& Division of Child \& Adolescent Psychiatry, BC Children's Hospital, Vancouver, Canada, email: adele.diamond@ubc.ca

\footnotetext{
A. RAZ.The Developmental Cognitive Neuroscience of Suggestion and its Relation to Improving Attention and Self-Regulation.

Although suggestion can influence many psychological processes, including cognition \& emotion, only sparse evidence exists on its neural basis or rigorously demonstrating its effects. In this talk, I will try to begin to fill that void \& will present findings showing how suggestion can be used as a powerful intervention to improve executive control especially with children \& adolescents who are far more suggestible than adults (85\% of children are highly suggestible but only $15 \%$ of adults).
}

The Stroop effect is remarkably robust \& the Stroop test is considered a "gold standard" task for showing how hard it is to inhibit automatic responses such as reading in proficient readers. While investigators usually study how a process becomes automatic, they typically neglect the reverse question: Whether \& how can an automatic process be deautomatized? Despite its theoretical \& clinical importance, the latter question is usually not only unanswered, but unasked. Is possible to regain control over an automatic process? I will provide compelling evidence that suggestion reduces \& even completely abolishes the Stroop effect, at least in highly suggestible people. My work has shown that suggestion can modulate executive functions such as attention \& inhibitory control, and combining fMRI. EEG \& genetics, I have been able to elucidate the neural basis \& time course for these processes. Preliminary findings from children diagnosed with impulse-control syndromes indicate that suggestion shows great potential for ameliorating involuntary symptomatologies, including tics in children with Tourette's svndrome.

Thus, in this talk I will present recent converging findings from multiple methodologies that focus on a developmental cognitive neuroscience of suggestion \& its relation to improving attention \& self-regulation. Exploring the power of suggestion will likely pave the way to a more scientific understanding of motivation \& expectation, \& of the placebo effect, across development in old as well as young. Correspondence: Amir Raz, Ph.D., ABPH; McGill University; Institute of Community and Family Psychiatry; Sir Mortimer B. Davis-Jewish General Hospital; Montreal, Quebec, Canada email: dramirraz@gmail.com

\section{A. DIAMOND. Novel Methods for Improving and Assessing Executive Functions in Young Children.}

I will present results of direct, practical application for helping at-risk youngsters with low-cost, simple materials \& procedures that classroom teachers can employ. Our data indicate that executive functions (EF) can be taught even to preschoolers \& that such training improves academic performance. I predict it will also reduce the incidence of diagnoses of ADHD \& conduct disorder.

Although EF predicts early school performance better than does IQ \& kindergarten teachers rate EF as THE most important set of abilities needed in their students, over half the children (as many as $90 \%$ of atrisk children) begin school lacking these skills. Two educators (Deborah Leong \& Elena Bodrova) working from a Vygotsky perspective spent over 12 years fine-tuning a preschool curriculum that embeds exercises to improve EF in all classroom activities throughout the day, \& emphasizes interactive, imaginative social plav to help train EF. It has won awards \& I will show data that children in it show extraordinary academic gains, gains on EF skills that generalize to EF activities very different from those ever done in class, \& EF gains appear to predict academic gains. By targeting the 3-6 age range, the goal is to develop positive feedback loops early, versus trying to fight established negative feedback loops at older ages or to remediate later deficits.

I will also present data on best practices for assessing $\mathrm{EF}$ in young children \& throughout life. E.g., selective attention (the ability to inhibit visual distractors) is often assessed with the Flanker task. While the Flanker effect has been widely replicated. it is small \& can easily be lost if the stimuli are too large or too far apart. I will present data on an improved version of the task that produces a far more robust effect (6-10 fold increase in the size of the Flanker effect), that is essentially unaffected by changes in stimulus size or layout, \& that shows far more dramatic changes over age than does the traditional task.

Correspondence: Adele Diamond, Canada Research Chair Tier 1 Professor of Developmental Cognitive Neuroscience, Department of Psychiatry, University of British Columbia (UBC), \& Division of Child \& Adolescent Psychiatry, BC Children's Hospital, Vancouver, Canada, email: adele.diamond@ubc.ca 
A. HOGAN. Impact of Frontal Lobe Disconnection on PerformanceMonitoring in Adolescents and Young Adults.

Goal directed behaviour requires the continuous evaluation of performance and the initiation of appropriate self-correcting actions in the event of an error. It has been suggested that medial frontal cortex (the anterior cingulate cortex [ACC]) serves as a monitor of ongoing performance while the dorsolateral prefrontal cortex acts in a more executive capacity, integrating information about task demands and accuracy of performance. The implication is that the close interaction of these two regions is critical for adaptive behaviour, and that this is facilitated via tracts traversing the deep frontal lobe white matter. Empirical evidence obtained from young people (11-23 years) with unilateral or bilateral lesions of frontal lobe white matter, sparing the gray matter, will be presented to address the hypothesis that it is the connectivity between the ACC and more anterior regions that is necessary for one aspect of performance monitoring, namely error-processing. Specifically, eventrelated potential data (e.g. error-related negativity [ERN]) will be presented along with MRI evidence of disconnection of SMA, pre-SMA, \&/or dorsolateral prefrontal cortex from the ACC. Such frontal lobe disconnection likely also contributes to the deficits in executive function tasks observed in these patients. Disruption to error-processing in the absence of lateral or medial frontal cortex damage suggests that the functional connection between these areas is critical for facilitating performance-monitoring. Correspondence: Dr Alexandra M. Hogan, Developmental Brain-Behaviour Unit, University of Southampton, UK, email: A.Hogan@soton.ac.uk

\section{RUEDA. Development of Attentional Aspects of Executive} Functions during Childhood.

The effortful control of thoughts and responses has been linked to attention by theoretical models. The term executive attention has been coined to describe this attentional function. Within a neurocognitive framework, executive attention has been related to a network of brain areas involved in detecting and resolving conflict situations. Using tasks adapted from the adult literature, we have traced the development of executive attention during childhood and have explored the brain mechanisms underlying such development using event-related potentials. We have found that executive attention development is subject to important constitutionally-based individual differences such as those rooted in genetic and temperamental variability.

The efficiency of the executive attention network also seems to be influenced by experience. We have recently developed a series of computer exercises designed to train children's attention. Data from several studies show that both normal children and children suffering from attention-related deficits (such as autism) show signs of greater attentional efficiency after training as measured by both behavioral assessments and patterns of brain activation. Furthermore, our data suggest that individual differences in the efficiency of the executive attention network are related to selfregulatory behavior as observed in tasks involving affect regulation as well as measures of school achievement and social adjustment.

Correspondence: Dr. Charo Rueda, Grupo de Neurociencia Cognitiva, Dpto. Psicología Experimental y Fisiología del Comportamiento, Universidad de Granada Campus, Universitario de Cartuja s/n 18071 Granada, Spain email: rorueda@ugr.es

\section{Invited Symposium 12:00-1:30 p.m.}

\section{Early Signs in the Identification, Diagnosis and Intervention in Dementia}

\section{Discussant: Natalia Ojeda Del Pozo}

\section{N. OJEDA DEL POZO. Early Signs in the Identification, Diagnosis and Intervention in Dementia.}

The symposia is address to examine the early cognitive symptoms and structural brain changes in various clinical subtypes of dementia, the actual ways to the effective recognition of these signs in both, clinical and community settings, and the identification of early risk factors. Characterization of the early signs and cognitive profiles in Huntington's disease, Alzheimer's disease, and Vascular dementia vs Mild cognitive impairment will be issued. Special interest will be focused on the memory and language profiles. The relevance of early accuracy diagnosis at the preclinical phases of the disease and implications on the management, treatment effectiveness and course will be discussed.

Correspondence: Natalia Ojeda Del Pozo, PhD, Dpto Psicología. Universidad de Deusto, Avda. De las Universidades, 24, 48007 Bilbao Spain Email:nojeda@fice.deusto.es

\section{J. BRANDT. Dementia of Huntington's Disease, and Subtle Cognitive} Impairment in People at Risk.

The first part of presentation will describe the cognitive impairments of patients with Huntington's disease (HD), and how they differ from those seen in other dementias (e.g..

Alzheimer's disease) and other movement disorders (e.g.. Parkinson's disease and spinocerebellar ataxia). These processing impairments will be related to the known striatal and cortical pathology seen in HD. The second part of the presentation will summarize neuropsychological and brain imaging studies of clinically normal offspring of HD patients who carry the genetic mutation (i.e.. presymptomatic HD). Most recent studies reveal changes in brain morphology and function several years prior to the development of diagnosable svmptoms. The implications of these findings for patient management and for prevention trials will be discussed.

Correspondence: Jason Brandt, Ph.D. The Johns Hopkins University School of Medicine, jbrandt@jhmi.edu

\section{J. BECKER. Earliest Manifestations of Dementia in a Community Cohort.}

The treatment and management of Alzheimer's disease and related dementias has moved past the purely symptomatic phase, and there is promise of disease modifying and disease preventing therapies in the relatively near future. Consequently, it is becoming increasingly important to study the preclinical phase of the disease - when pathology is present but has not manifested itself. Data from the Cardiovascular Health Study Cognition Study makes several important points in this regard. First, among individuals with demonstrably normal $\operatorname{cog}$ nition, there are abnormalities of brain structure that predict development of $\mathrm{AD}$; second, mildly impaired cognition, in the absence of significant comorbidities, is the first representation of $\mathrm{AD}$; third, vascular risk factors play an important role in modifying the risk, or time to develop AD.

Correspondence: James Becker. Alzheimer's Disease Research Center, University of Pittsburgh., beckerjt@upmc.edu

\section{BERTHIER. Language Dissolution in Alzheimer's Disease and} Primary Progressive Aphasia.

Patients with Alzheimer's disease (AD) often present with episodic memory loss, but as a rule, they develop mild anomic or paraphasic speech with abnormal comprehension and variable deficits in repetition (transcortical sensory aphasia, Wernicke's aphasia). On the other hand, patients with fluent primary progressive aphasia (fPPA) have a history of language disorder chiefly characterized by word finding difficulties without early deficits in memory. In spite of the striking differences in the pattern of presentation, the differential diagnosis between cases with atypical AD presentation and APPA may be difficult to establish. AD is heterogeneous and cases with atypical presentation due to focal accentuation of pathology in the language cortex can mimic fPPA. In addition, $20 \%$ of PPA cases have pathological abnormalities consistent with $\mathrm{AD}$. While differential diagnosis can be based upon 
clinical grounds, genetic testing, and neuroimaging, progress in language testing further improves the ability to distinguish between AD and fPPA. Moreover, the profile of language performance and the type of errors are better that numerical performance to differentiate patients. For example, in AD associated with mutations in the presenilin-1 intron 4 mutation naming is preserved until late stages, whereas impaired naming is a very early feature of "semantic dementia" due to PPA. The recognition of cases with atypical $\mathrm{AD}$ presenting with features of fPPA is of paramount importance, since language deficits in AD are amenable to pharmacological intervention and cognitive rehabilitation. whereas cases with fPPA due to non-AD pathology can only be treated with cognitive therapy.

Correspondence: Marcelo Berthier. Unidad de Neurologia Cognitiva y Lenguaje. Centro de Investigaciones Medico-Sanitarias (CIMES), UniversidaddeMalaga.,marberthier@yahoo.es; mbt@uma.es

\section{Symposium 6 12:00-1:30 p.m.}

\section{Neurobiology Of The Self: How The Brain Creates A,"Self,", And What Happens When Things Go Wrong}

\section{Speaker: Feggy Ostrosky}

F. OSTROSKY, C. MATEER, B. WILSON, L. CLARE, S. FERNANDEZ-GUINEA \& O. FEGGY. Neurobiology Of The Self: How The Brain Creates A “Self”, And What Happens When Things Go Wrong.

Symposium Description: Philosophical theories have tried to advance in what moment in human life the human consciousness emerges and which are its distinctive characteristics. This framework suggests a hierarchical organization of consciousness, where the basic level corresponds to the simple perception of the external and the internal stimuli (the purpose is maintaining an organism alive), and the complexity of consciousness grows until reaching the maximum level that corresponds to the "meta-self-awareness" where the organism is aware that he/she is self-aware (Morin, 2006). It has been suggested that particular brain structures intervene in the organization of awareness. While the highest and most abstract aspects of the self are made possible by the hierarchically highest and most phylogenetically recent neural structures, all levels of the neural hierarchy may make a contribution to the self (Feinberg 2005). Impairment of self-awareness can be due to different causes, including dementia, amnesia, stroke, schizophrenia, and traumatic brain injury. The purpose of this symposium is to increase our understanding between the self and the brain and how the self is transformed in cases of brain lesions including amnesia (Wilson) dementia

( Clare, Rowlands, Bruce \& Downs) traumatic brain injury (Fernandez de Guinea), and cross-cultural studies using quantitative scales to measure the intensity of impaired self-awareness ( Ramirez \&. Ostrosky-Solís).

Correspondence: Feggy Ostrosky, Ph.D., National University of Mexico, Rivera de Cupia 110, Mexico City 11900, Mexico. E-mail: feggy@servidor.unam.mx

\section{B. WILSON. The Man Who Believes He Has Been Unconscious Or Dead For Twenty Years.}

Objective: CW, a British musician, developed Herpes Simplex Encephalitis in 1985. He has lost most of his hippocampal areas and is densely amnesic. His memory appears to have shown no real improvement over a twenty one year period. He has an extremely dense amnesia with normal immediate recall, severely impaired recall after a delay or distraction, a long period of retrograde amnesia and some evidence of implicit memory. For over 20 years $\mathrm{CW}$ has believed that he has just woken up and frequently says that the period before awakening is like being dead. He sometimes talks as if he has died. For example, he once said "when I was young there was only one millionaire but when I died there were millions". He has developed auditory hallucinations and delusional beliefs. This paper a) considers the relationship between awareness and consciousness b) describes CW's anatomical damage and $c$ ) considers to what extent the brain lesions have affected his sense of identity and to what extent the changes are due to the sequelae of a very severe amnesia.

Correspondence: Feggy Ostrosky, Ph.D., National University of Mexico, Rivera de Cupia 110, Mexico City 11900, Mexico. E-mail: feggy@servidor:unam.mx

L. CLARE, J. ROWLANDS, E. BRUCE \& M. DOWNS. Awareness in people with moderate to severe dementia living in residential care. Objective: Dementia provides a useful framework for exploring the hierarchical organization of conscious awareness and the impact of cognitive impairment on the experience of self. Most studies, however, have focused on identifying deficits in awareness in the early stages of dementia. We examined transcripts of conversations with people living in residential homes who had moderate to severe dementia in order to identify aspects of retained awareness and characterize the nature of subjective experience in this context. Researchers visited the homes at 3 month intervals and recorded 307 conversations with 84 individuals, together with contextual information. Transcripts were analyzed using grounded theory methods. Open coding identified categories reflecting aspects of retained or compromised awareness and themes expressing the nature of subjective experience. Subsequent focused coding explored how the data related to current theoretical models of awareness.

Correspondence: Feggy Ostrosky, Ph.D., National University of Mexico, Rivera de Cupia 110, Mexico City 11900, Mexico. E-mail: feggy@servidor.unam.mx

\section{S. FÉRNANDEZ GUINEA. Self-Awareness in Brain Injury: Decision-} Making and Activities of Daily Living.

Objective: A common sequelae of acquired brain injury (ABI) is impaired self-awareness of deficits. These deficits have been reported as ranging from $45 \%$ to $97 \%$ in patients with traumatic brain injury, depending on severity of injury and the assessment measures employed. Self-awareness deficits in brain injury can be observed in relation to a wide variety of impairments and phenomena, such as physical/motor problems, cognitive deficits and behavioral and social disturbance. Overall there is little evidence to support an association between self-awareness and general intellectual functioning or with specific cognitive processes, in spite of the commonly held view that deficits in executive function are associated with reduced behavioral/social self-awareness. There are reported deficits on experimental paradigms assumed to be sensitive to decision-making, one the areas which may be fruitful for investigating awareness deficits. The main ain of our research was to explore the relationship among self-awareness deficit, decisionmaking, executive functions and the ability to success in the activities of daily living in a large sample of ABI patients. Data obtained will be discuss in relation with the theoretical models have been proposed to explain impairments of self-awareness in brain injury patients and their implications for neuropsychological rehabilitation outcome.

Correspondence: Feggy Ostrosky, Ph.D., National University of Mexico, Rivera de Cupia 110, Mexico City 11900, Mexico. E-mail: feggy@servidor.unam.mx 
M. RAMÍREZ \& F. OSTROSKY-SOLÍS. Assessment of awareness: A Cross-Cultural Study.

Objective: Self-awareness is altered by several factors such as impaired self-awareness (ISA), lack of awareness of cognitive deficits, altered ability to monitor own behaviors, as well as poor awareness and insight of altered interpersonal skills, and impact on others. The Patient Competency Rating Scale (PCRS) developed by Prigatano and Fordyce (1986) provides a quantitative assessment of the ISA extent in patients with traumatic brain injury (TBI). Despite the existence of cross-cultural studies with the PCRS scale, there are no indications as to what happens in the Latin-American population without TBI. The purpose of the study herein is to standardize the PCRS scale in cognitive intact Mexican subjects, with no neurological disorders and functionally independent.

Participants and Methods: A sample of 274 subjects was studied (137 informers and 134 respondents) Procedure: the Spanish translation of PCRS was analyzed.

Results: The total PCRS for the control group was 118.29 \pm 13.70 , and $120.29 \pm 13.83$ for the informant group. The Cronbach alpha coefficient was 0.9005 and 0.9037 , respectively. The $t$ test for independent groups showed statistically significant differences in five items.

Conclusions: The standardization of the PCRS in the Mexican population has shown contradictory findings as control subjects and their informers tend to score themselves positively (overestimation of their skills) and negatively (underestimation of their skills): in healthy individuals it is not expected to see both trends. The importance of considering cultural factors when applying and interpreting the scale is emphasized.

Correspondence: Feggy Ostrosky, Ph.D., National University of Mexico, Rivera de Cupia 110, Mexico City 11900, Mexico. E-mail: feggy@servidor.unam.mx

\section{Poster Session 6 \\ Childhood \& Developmental Disorders, Substance Exposure 3:30-5:30 p.m.}

\section{Child - Acquired Disorder: other}

J. BERNABEU, A. CAÑETE, C. FOURNIER, G. ALMERICH, J. SUAREZ, G. SAAVEDRA \& V. CASTEL. Neurocognitive sequelae in pediatric oncology: neuropsychological profiles and rehabilitation.

Objective: To study treatment effects (cranial radiotherapy, methotrexate, etc.) on CNS cognitive and behavioral functioning, to find out differences among CNS tumors, leukemia and healthy children in neuropsychological profiles in order to design rehabilitation strategies.

Participants and Methods: 150 children were studied: benign brain tumor, malign brain tumor, leukemia, healthy-control.

Assessment was performed with our neuropsychological protocol, about 5 hour testing per child. It includes 25 tests to obtain 54 neuropsychological processes in 9 cognitive areas (general cognitive abilities, motricity, visual perception, non verbal abilities, language, memory, attention, executive functions and academic abilities). Achenbach scales for parents, teachers and self-report were used for psychopathological assessment. We also measured reaction times, vigilance, impulsivity and inattention with Conners' CPT-II.

Statistical analyses (parametric, non-parametric and ANOVA) were carried out by using SPSS 14.0 package.

Results: All clinical patients showed deficit in neuropsychological assessment.

Significant results: CNS treatment appears to be related with ADHD inattentive subtype, white matter damage is suggested. Malignant brain tumor obtained the worst scores.
The longer time from diagnosis, the worst scores in cognitive general abilities, visual perception, memory and attention areas. Leukemia patients present mild deficit in all areas excluding non-verbal abilities, motor skills and visual perception. Language processes are more affected (receptive vocabulary, denomination, semantic fluency, VIQ).

When prescribed, methylphenidate improves neuropsychological functioning.

Conclusions: Neuropsychological assessment offers cortical and subcortical cognitive and behavioral functioning.

Knowing specific diagnose-related neuropsychological late effects allow us to design target-oriented rehabilitation procedures (drugs, training, advising, follow-up, etc.) to improve/compensate/palliate them.

Correspondence: Jordi Bernabeu, Master, Hospital La Fe, Pediatric Oncology Unit, Avgda. Campanar, 21, Valencia 46009, Spain. E-mail: bernabeu_jor@gva.es

M. GROGAN, P. MINTON, R. BRUNSDON, P. JOY, S. KELLIE, H. SOMERVILLE \& E.A. SHORES. Long-term Neuropsychological Effects of Low-grade Brain Tumours in Children: A Specific Focus on Attention, Executive Functioning and Quality of Life.

Objective: Over the past 20 years, an increase in the survival rates for children diagnosed with a brain tumour has lead to a greater focus on the neuropsychological late-effects, educational implications and quality of life in this clinical population. Research suggests that many areas of cognitive, social and emotional functioning are affected which need to considered for prognosis, rehabilitation and educational intervention. This study investigated the long-term neuropsychological effects of low grade brain tumours diagnosed and treated during childhood, with a specific focus on attention, executive functioning and quality of life.

Participants and Methods: Subjects were recruited through The Children's Hospital at Westmead, Australia. Clinical participants were lowgrade brain tumour patients diagnosed and treated during childhood and at least 5 years post treatment. The control group consisted of noncerebral solid tumour patients (Wilm's tumour) and siblings. The test battery included tests of intellectual functioning, attention, executive functioning and investigated current quality of life.

Results: Preliminary findings suggest that children diagnosed with a brain tumour demonstrate reduced non-verbal intellectual functioning and poorer quality of life when they are at least 5 years post treatment.

Conclusions: It is anticipated that the findings from the present study will assist with the clinical management of children with low-grade tumours, and lead to increased confidence when making decisions regarding prognosis, rehabilitation and educational intervention.

Correspondence: Melissa Grogan, Doctor of Psychology (Clinical Neuropsychology), Psychology, Macquarie University, Balaclava Road, North Ryde, NSW 2109, Australia.E-mail:mjgrogan@bigpond. com

K. VELLA, A. CAMPBELL, N. LINCOLN, W. WHITEHOUSE, J. WILLIAMS \& D. WALKER. Child Brain Injury Research Programme: Preliminary data on predictive validity of the Health Utilities Index (HUI) in children with acquired brain injury.

Objective: This study aimed to determine the predictive validity of a recently developed health-related quality of life measure, the Health Utilities Index Mark 3 (HUI-3) in intelligence, memory and behavioural outcomes in a population of children who have suffered an ABI.

Participants and Methods: This study considered a subset of data from a longitudinal prospective design looking at outcome of neuropsychological function after childhood ABI. 42 children who had been recruited to the study were assessed at various stages between admission to hospital and 12 months post injury. The outcome measures were the Conners Parents and Teachers Rating Scales - Revised (CPRS-R-R, CTRSR), the Children's Memory Scale (CMS), the Wechsler Intelligence Scale 
for Children Version III (WISC-III) and the Strengths and Difficulties Questionnaire (Parents and Teachers) (SDQ). The HUI-3 as measured at baseline and 3 months was correlated with these outcome measures, as well as more traditional demographic measures to determine whether there were any significant associations between the HUI measurements and outcome.

Results: Correlations between the HUI- 3 both at baseline and at 3 months were significant with some subsets of the CMS, WISC-III and the SADQ. However, there were only limited correlations with behavioural measures, as measured on the CPRS-R-R and CTRS-R. Baseline characteristics such as age at injury, severity of injury, diagnosis and family composition were also found to be significant and this may have been the mediating effect for the associations found with the HUI.

Conclusions: Measures of intelligence, memory and some limited measures of behaviour are related to the HUI-3 which suggests that there might be some role for the use of the HUI in predicting outcome. However, the sample size was small and there were some significant ceiling effects which may have distorted the data. Future research should focus on obtaining a larger and more representative sample to ensure both internal and external validity.

Correspondence: Kristina Vella, B.Psy(hons), Msc, Institute of Work, Health and Organisations, 8, William Lee builidings, Science and Technology Park, University Boulevard, Nottingham NG7 2RD, United Kingdom.E-mail: kristina.vella@nottingham.ac.uk

\section{Child - Acquired Disorder: TBI}

\section{A. EMOND, V. MICHELL, I. WRIGHT, H. MILLER \& P. SHARPLES. Performance of Primary School Children on Measures of Post- Traumatic Amnesia (PTA).}

Objective: Scales specifically equipped to assess PTA in children include the COAT and Westmead PTA scale. Assessment of young children is problematic due to increased population variance in attentional and memory performance, compounded by limited normative data for children and those with pre-morbid cognitive difficulties. A number of adapted scales have been proposed (Ruijs et al 1992; Starship PTA scale, Fernando et al 2002) but these necessarily cover only narrow age ranges and are based on a traditional 'adult' construct of PTA, which does not have established validity in young children. Criticisms of the construct validity of PTA scales include failure to include measures of prospective memory functioning (COAT) and/or measures of attention (Westmead).

This poster described a normative study comparing the developmental datasets for candidate measures of PTA.

Participants and Methods: Children aged 6 - 16 years completed a composite assessment including attention measures alongside, PTA scales as appropriate (COAT, Westmead \& Starship). IQ data from schoolbased tests is also reported.

Results: Correlations between PTA scales and age and IQ data give evidence for the validity of these scales for us in young children or those with learning difficulties.

Conclusions: Suggestions for clinical use of such scales are made with reference to our findings.

Correspondence: Alice Emond, BSc, Neuropsychology, North Bristol NHS Trust, Neurology Unit, Frenchay Hospital, Bristol BS16 1BR, United Kingdom.E-mail: alice.emond@nbt.nhs.uk

R.D. NAIR, E. TALBOT, G. HUGHES \& A. STARZA-SMITH. Sexuality in Adolescents following Traumatic Brain Injury: A Systematic Review of Literature and Research Proposal.

Objective: Developing sexuality is an important aspect of adolescence, which may be affected following traumatic brain injury (TBI). Most research has focussed on adult TBI brain-behaviour correlates, with minimal involvement of adolescents. It has often been observational, and not considered the "intent" of behaviour. Such research should address ongoing concerns, and pre-empt and prevent problems in adulthood. The objectives of this paper are to review the literature on adolescents' sexuality following TBI, and present a proposal which addresses some aspects of the deficits observed in research.

Participants and Methods: A search strategy was developed for MEDLINE and modified for other databases. Cross-references and book chapters were also accessed. Inclusion/exclusion criteria were developed, and all papers identified were independently evaluated for inclusion by two reviewers.

Results: A total of 857 abstracts were obtained from the search strategy, and cross-references. Of these, only 33 were included for initial screening; and a total of 16 papers satisfied the inclusion criteria. Four papers were excluded as they only had adult samples. Nine papers examined sexuality following TBI, but only three of these were specific to adolescents. The foci of latter papers were on incidence and prevalence of sexual dysfunctions, and one examined interview methodology.

Conclusions: There is paucity in knowledge of adolescents' sexuality following TBI. Research needs to examine adolescents' skills and knowledge, and deal with issues related to sexuality directly. A research proposal that addresses this lacuna observed in the literature, and the potential use of internet-based interviewing to address issues of sex with this population will be presented.

Correspondence: Roshan D. Nair, Dept of Clinical Psychology \& Neuropsychology, Nottingham University Hospitals NHS Trust, IWHO, \& William Lee Buildings, Science \& Technology Park, Nottingham NGT 2RQ, United Kingdom. E-mail: roshan. nair@nottingham.ac.uk

L. SANTOS, M.R. SIMÕES \& L. BORGES. Attention and Executive Outcomes after Traumatic Brain Injury in Childhood: A Prospective Study with Coimbra Neuropsychological Assessment Battery.

Objective: Testing the following hypotheses:

Severe TBI children present poorer performance on cognitive, attentional and executive measures when compared with children without history of TBI.

Increased severity is associated with lower results on cognitive, attentional and executive measures.

Younger children at the time of the lesion have poorer results than their peers on cognitive, attentional and executive measures.

Participants and Methods: The clinical group was compared with 30 children of normal population, controlling for age, gender, residence and degrees of education of the parents.

Results: The mean WISC-III scores of the severe TBI were, without exception, statistically inferior to the normative mean.

The two groups significantly differed in several measures of attention such as sustained and divided attention.

The children of the clinical group performed poorer in Verbal Fluency (Phonemic) task when compared with the control group as they gave fewer valid answers $(\mathrm{F}=7,991 ; \mathrm{p}<0.01)$.

In Trail Making Test B, severe TBI children showed performed poorly in two ways: they needed more time to finish the task $(\mathrm{U}=152,000$; $\mathrm{p}=0.005)$ and also made more errors $(\mathrm{U}=172,500 ; \mathrm{p}=0.006)$.

In Tower of London test, errors type 1 were more frequent with the severe TBI group ( $\mathrm{U}=314,000 ; \mathrm{p}=0.024)$.

Conclusions: The paediatric TBI can have (besides others) important consequences in attention and executive functioning susceptible of interfering not only with normal development of these areas, but also with the development in general, and by this we mean language, socialization, memory, academic progress and professional carrier.

Correspondence: Salome Pinho, PhD, Faculty of Psychology, University of Coimbra, Rua do Colégio Novo, Apartado 6153, Coimbra 300-801, Portugal.E-mail: salome@fpce.uc.pt 
C.B. MELLO, G.F, XAVIER \& O.F. BUENO. GENDER AND SOCIOCULTURAL DIFFERENCES IN CATEGORIZATION AND MEMORY PERFORMANCE: A STUDY IN BRAZILIAN CHILDREN.

Objective: It has been demonstrated that children's performance in categorization and in some verbal memory tasks increases substantially in school age period. Since these skills are verbally mediated perhaps as a function of formal education, we can expect differences in performance according to subject's gender and sociocultural environments. The present study examined age, gender and rural/urban effects in categorization and memory tasks. Participants and Methods: A sample of 127 children aged 7 to 10, of both sexes and from rural and urban districts of two states in Brazil was submitted to tasks of categorization, immediate and delayed free recall of a set of 30 semantic related figures, and also to digit span (forward and backward) and verbal fluency (semantic and phonological) tests. For the categorization tasks it was carried out a logistic regression analysis and ANOVAs for the remaining tests.

Results: The results confirmed an increase with age of conceptual stimuli organization $(\mathrm{F} 2=172 ; \mathrm{p}<0,001)$ and of number of items recalled $(\mathrm{F} 4,120=2,5 ; \mathrm{p}<0,004)$. On the other hand, rural and urban children did not differ in the free recall or in the categorization tasks; a rural/urban effect was found in semantic $(p<0,001)$ and phonological verbal fluency $(p<0.05)$, as well as in forward digit span $(p<0.05)$; urban children performed better than the rural. Gender differences were also found: girls performed better than boys only in categorization task $(p<0,03)$ and in the delayed free recall $(p<0,02)$.

Conclusions: Results suggest that performance in categorization and recall tasks is not influenced by sociocultural and gender variables, as happens to other neuropsychological functions.

Correspondence: Claudia B. Mello, PhD, Psychobiology, Universidade Federal de Sao Paulo, Rua Embau, 54, Sao Paulo 04039-060, Brazil. E-mail:cberlim@ib.usp.br

\section{Child - Assessment}

G. CASTILLO-PARRA, E. GOMEZ-PEREZ, M. GONZALEZVILLANUEVA \& F. OSTROSKY-SOLIS. Performance in Attention and Memory Neuropsychological Tests and its Relation with Academic Achievement in Children.

Objective: The process of education requires an adequate functioning of the cognitive processes that allow to attend and to memorize the information enough time to be learned. Nevertheless, current studies are insufficient to bring over the relation that exists between school performance and the functioning of attention and memory. The aim of the present study was to explore if performance in attention and memory neuropsychological tests is related to academic performance.

Participants and Methods: A total sample of 49 normal school-age children of the second grade of primary school, with an mean age of 7.78 years, was divided in two groups according to their school performance: group 1 was conformed by 27 children with low school performance and group 2 was integrated by 22 children with high school performance. Cognitive functions were evaluated using the neuropsychological battery Neuropsi Attention and Memory [Neuropsi Atención y Memoria] (Ostrosky-Solis et al., 2003).

Results: We carry out an one-way anova to compare the total score of attention and executive functions, the total score of memory and the total score of attention and memory between both groups of academic performance. The results showed significant differences between groups in the total score of memory and the total score of attention and memory. Children with high academic performance also obtained higher scores in the cognitive tests, than children with low academic performance. The analysis of Pearson correlation showed that only the processes of memory were narrowly related to the academic performance of the evaluated children.

Conclusions: These results suggest that the deficits of memory, not in attention, might underlie learning problems that could be reflected in a low academic performance.
Correspondence: Gabriela Castillo-Parra, Doctor, Faculty of Psychology, University of Guanajuato, Av. Las Rosas \# 501, Jardines del Jerez, Leon, Guanajuato 37530, Mexico.E-mail: gcastillo_parra@yahoo.com.mx

G. ESPINOSA, I. GALTIER, A. NIETO \& J. BARROSO. Developmental Aspect of Verbal Fluency: an Analysis of Clustering and Switching of School-Aged Children.

Objective: Verbal fluency is dependent upon a number of processes which may develop during the elementary school vears. Analysis of cluster formation can inform us about the different components involved in this task. "Cluster size" has been proposed as associated with temporal lobe functions, while "switching" reflects the necessary cognitive flexibility for new subcategory search and is associated with frontal functions. Very few investigations have analyzed clustering process in children. In this study we aimed to examine quantitative and qualitative age-related differences in verbal fluency during childhood.

Participants and Methods: Participants were 79 school-aged children divided into three groups: G1: 6-7 years $(n=27)$; 62 : 8-9 years $(\mathrm{n}=28)$ and G3: $10-11$ years $(\mathrm{n}=28)$. All participants were native Spanish speakers without learning disabilities. They performed a phonemic fluency task (F, A, and M). Two scoring modalities were obtained in each letter: quantitative (number of words produced) and qualitative (number of clusters. switches and mean cluster size).

Results: Results revealed a year-group effect on total words generated, with a significant increase between G1 and older groups. Children from G1 produced less number of clusters and switches than children from G2 y G3. No differences were found among groups for mean cluster size.

Conclusions: The increase in fluency in older children and concomitant increase in switching measures but not in cluster size, suggests that the age difference in performance can be accounted for mainly by changes in cognitive flexibility related to frontal lobe maturation. Thus, the use of qualitative analyses can detect executive problems in children with developmental neurological deficits.

Correspondence: Iván Galtier Hernández, Psicobiología y Metodología de las Ciencias del Comportamiento, Universidad de La Laguna, Campus de Guajara, La Laguna 38205, Spain.E-mail: igaltier@ull.es

P. JANSEN \& K. GREENOP. Cognitive Processing Patterns and Reading Outcomes in a Sample of South African Children.

Objective: School failure is an area of concern in South Africa. Since little is known regarding cognitive developmental patterns and reading acquisition, this logitudinal study presented an opportunity to follow the group of children, evaluate their base line at 5 years and re-evaluate the same group at 10 years.

Participants and Methods: The children (n=199) were participants of a larger group who were seen initially at 5 years to determine their base line scores for verbal fluency, pegboard speed and cognitive abilities using the $\mathrm{K}-\mathrm{ABC}$ mental processing scales.Subgroups of children were identified based on the 5-year-old scores;those whose scores were below average on the mental processing scales(n=59-group 1$)$; a subgroup who scored below average on either one of the scales $(n=70$-groups 2 and 3);a group whose scores were average but uneven with high average to above average scores ( $n=26$-groups 4 and 5$)$ and a group who scored average on both scales ( $n=43$-group 6 ).

Results: At ten years the mental processing profiles had altered although groups 1 and 2 showed below average scores on one of the MP scales but no significant differences for verbal fluency. Although the basic skills in reading were present in the sample,children whose scores were below average at 5 years scored significantly lower on rhyme production $(\mathrm{p}<05)$. letter knowledge,spelling and decoding $(\mathrm{p}<01)$. Reading comprehension scores were below average for the group but significantly lower for Group $1(p<05)$.

Conclusions: Although initial low performance scores did not seem to restrict reading acquisition,reading comprehension was below average 
for all groups but especially for Group 1. Mental processing patterns were not significantly related to outcome.

Correspondence: Patricia Jansen, Ph D, nursing and midwifery, trinity college, d'olier street, Dublin, Dublin Dublin 2, Ireland. E-mail: jansenp@tcd.ie

M.C. MIRANDA, E.G. SINNES, S. POMPÉIA \& O.F. BUENO. A Comparative Study of Performance in ihe Conner's Continuous Performance Test (CPT-II) between Brazilian and North American Children.

Objective: The present study investigated the performance of Brazilian children in the continuous performance test CPT-II, and compared results to those of the norms obtained in the USA children

Participants and Methods: The Brazilian sample was composed of 384 children (half boys) aged 6-11 years, which represented the socioeconomic class distribution of São Paulo city and who were pre-screened for attention deficit disorders (ADHD).

Results: Age and gender effects in the Brazilian sample were similar to those previously described confirming that this population responded to the task in a similar manner as children from other cultures. There was age effect in almost all measures, such as percentage of omission and commission, hit reaction time, preserverations $(p s<0,01)$, and performance increased as children aged. There was gender effect. Female subjects presented higher performance in the measures of percentage of omissions, hit reaction time, signal detectability ( $\left.\mathrm{d}^{\prime}\right)$ and response bias (ps<0,0001). The comparison with the sample of American children was made based on gender and age. In the 6-7-year-old age group and 8-9year age group, the samples only did not differ in the percentage of omission; in the 10-12-year-old age group all measures differed ( $p s<0,0001)$; the Brazilian sample showed higher performance in all measures.

Conclusions: It is discussed that differences between samples probably reflect lack of ADHD screening of the USA children. More studies are necessary to determine if the CPT-II is a cross-cultural test with subjects from different samples matched for age, gender, and socioeconomic status screened for ADHD

Correspondence: Miranda Monica, psybiology, federal university of são paulo, R. Embau, 54, São paulo 04039-060, Brazil. E-mail: mcarol@psicobio.epm.br

M. MUSZKAT, C.B. MELLO, L.R. BRUNONI, A.L. PILLA LUCE, T. BARBOSA, E.G. SINNES \& O.F. BUENO. CLINICAL AND NEUROPSYCHOLOGICAL FINDINGS IN CHILDREN WITH LOW ACADEMIC ACHIEVEMENT: PRELIMINARY RESULTS FROM A BRAZILIAN STUDY.

Objective: Brazilian epidemiological data refers that around $30 \%$ of children in initial years of elementary school may present problems in academic achievement. This study was designed to (a) analyze different diagnoses related to low academic achievement in elementary school children and (b) analyze the value of qualitative neuropsychological procedures for detecting differences in neuropsychological profiles in specific diagnoses.

Participants and Methods: A sample of 75 children with poor academic achievement, in the rage age of 7 to 12 (23 girls and 52 boys), was referred by their teachers to clinical and neuropsychological evaluation in a rehabilitation program in Sao Paulo, Brazil. Children were diagnosed clinically and assessed using neuropsychological procedures, which included perceptual, praxis, visual-constructive, language, verbal and visual working memory, visual memory and selective attention skills.

Results: According to descriptive analysis, mental retardation was detected in 34 children, 22 met criteria for learning disabilities and 10 for oppositional defiant or conduct disorders. A comparison between the children with conduct disorders and learning disabilities showed similar neuropsychological profile in terms of perceptual and visual-constructive skills. On the other hand, children with conduct disorders were better in terms of perceptual, praxis, verbal working memory, visual memory and expressive language skills. A better performance of learn- ing disabilities in comparison to conduct disorder was observed only in visual working memory and in comprehensive language performance. Conclusions: In sum, our study revealed different diagnoses in children with low academic achievement and suggest differences in neuropsychological profile according to diagnosis, resulting in relevant information for intervention programs.

Correspondence: Mauro Muszkat, PhD, Psychobiology, Universidade Federal de Sao Paulo, Rua Embau, 54, Sao Paulo 04039-060, Brazil. E-mail:mauromuszkat@uol.com.br

G. SHERESHEVSKY. Examination of the Internal Structure of the DSS-ROCF Organization Scoring System in a Clinical Sample.

Objective: Current study examined the internal structure of the DSSROCF organization scoring system. Hypotheses were: structure would vary across copy and recall conditions; structure within conditions would reflect individual differences in visual-spatial processing of complex figure.

Participants and Methods: 109 children, aged 7 to 14, with several neurological conditions, from a number of clinical and educational settings in St. Petersburg, Russia, were assessed. For study purposes scoring protocol was modified so as to yield 25 identical items for each condition. This allowed comparing the structure of organization scoring system across copy and recall conditions.

Results: Correlation matrices for each condition and condition pairs were examined. Consequently, factor analysis, using principal axis factoring, was performed, yielding factor structure for every condition. Items in copy condition, present in $95 \%$ or more of cases, were omitted. Factor structure was different for copy and two recall conditions, the latter being similar. For copy condition 3 -factor model was best fitting. with factors: figure center intersections, middle vertical line, elements of the left center box. For immediate and delaved recall conditions 4factor model was best fitting, with factors: left part of the contour, right part of the contour, figure center intersections, elements of the left center box.

Conclusions: Results indicate that copy and recall of the Rey-Osterreith compex figure in children may recruit different skills, with recall probably being more sensitive to visual-spatial processing and organization of information. Differences within conditions may reflect individual differences in functional systems, supporting processing of complex visual-spatial information, which should be further investigated with clinical subgroups.

Correspondence: Gary Shereshevsky, St.Petersburg State University, V.M. Bekhterev Psychoneurological State Research Institute. St. Petersburg, Russia, 14 Exeter Street, Brooklyn, NY 11235. E-mail: shgary@yandex.ru

\section{Child - Developmental Disorders}

J.C. ALVAREZ-CARRILES, J. SALAS-PUIG, C. ANTÓN, L. BENAVENTE \& A. GARCÍA-MARTÍNEZ. Long-term Verbal and Non-verbal Functioning in Landau-Kleffner Syndrome: A Case Report.

Objective: Landau-Kleffner syndrome (LKS) is a rare childhood epilepsy syndrome involving progressive auditory agnosia related to the appearance of paroxysmal EEG activity over the temporo-parietal regions. Neuropsychological studies have almost exclusively focused on the auditoryverbal impairment that appears in the acute phase (childhood and adolescence) of the syndrome. Our objective is to describe the long-term evolution (adulthood) of not only verbal but also non-verbal functions in one case of LKS.

Participants and Methods: DF is a 27- year-old right-handed male. that after a normal psychomotor and verbal development was diagnosed with LKS at the age of $41 / 2$ years. In order to establish his current $\operatorname{cog}$ nitive profile, a broad neuropsychological battery covering different aspects of verbal and non-verbal processing (30 different measures) was administered. 
Results: Currently, although our patient has developed basic reading and writing skills, he still shows a clear auditory agnosia and a marked deficit in grammatical processing that interferes with complex written sentences comprehension. His verbal IQ is 76, with special difficulties in Information and Comprehension subtests. On the other hand, nonverbal tasks performance and calculating abilities were all in the normal range (performance $I Q=110$ ), showing in some cases a high-level of performance (i.e. TONI-2 IQ=126).

Conclusions: Although auditory agnosia may be a stable feature of LKS from its early beginning, that does not prevent, as this case exemplifies, from developing other verbal (reading and writing) and non-verbal abilities. Neuropsychological rehabilitation and vocational programs should focus on these potentially preserved or recoverable functions in order to achieve a normal personal and professional adjustment.

Correspondence: Juan C. Alvarez-Carriles, PhD, Neurology Service, Hospital Universitario Central de Asturias (HUCA), Julian Claveria, $s / n$, Oviedo33006,Spain.E-mail: juancarriles@hotmail.com

M. ANTEQUERA TORRES, P. FERNÁNDEZ OLIVARES, C. SANTOPOLO HITA, L. VIVANCOS MOREAU, B. MARTINEZ HERRADA, B. GARCÍA TORRES \& J. MARÍN MUÑOZ. DEMENTIA IN A PATIENT WITH DIAGNOSIS OF A GENERALIZED DISORDER OF THE DEVELOPMENT.

Objective: Description of the clinic and neuropsichological exploration of a diagnosed patient of Disorder Generalized of the Development without specification (F84.9; CIE-10) in the adult age and Mixed Dementia. Participants and Methods: Data of clinical history, such as: personal and familiar antecedents, explorations (general medical, neurological, neuropsychological and psychopatologic), analytical and MRN. Furthermore it is explained not only the pharmacological intervention but also the psychological one.

Scales of the daily life and psychiatric were administered as well as a complete neuropsychological protocol that includes in the computer science program Experia.dem (Minimental, Germcide, Cerad, Clock Drawing Test, Trail Making Test).

Results: The neuropsychological exploration shows an alteration of the attentional functions, motor speed of processing, fluencies,, concept formation and immediate and delayed memory.

Conclusions: After analyzing the collected data we observed a compatible neuropsichological profile with a fronto-temporal dysfunction with implication of the white substance and suggestive clinical of Mixed Dementia.

Correspondence: Martirio Antequera Torres, Unidad de Demencias, Hospital Universitario Virgen de la Arrixaca, Carretera Madrid Cartagena; s/n, El Palmar 30120, Spain.E-mail:manteque1@hotmail. com

N. BOTTING \& G. CONTI-RAMSDEN. The relationship between language and affective disorder in individuals with and without specific language impairment (SLI).

Objective: This study examined the risk of affective disorders in adolescents with specific language impairment (SLI).

Participants and Methods: 139 adolescents with a history of SLI (15;10 years) and a peer group of 124 typically developing (TD) adolescents (15;11 years) participated, who were in their final year of compulsory schooling. The risk of affective disorders was assessed using the Moods and feelings Questionnaire (MFQ) and the Child Manifest Anxiety ScaleR (CMAS-R). Comprehensive language and cognition data was available for all participants (TD and SLI) concurrently and also longitudinally for those with SLI. Parents of the SLI group also completed a Family History Interview (FHI) regarding their own experience of affective disorders in childhood and in adulthood.

Results: A clear increased risk in affective symptoms was found for the SLI group on both self- and parental-report. Girls scored less favourably than boys when groups were combined, but these were due to the effect of the TD group, with no gender differences found in the SLI group. No relationships between affective disorder and concurrent language were found. A few modest associations with earlier receptive language were noted. Parental history of affective difficulties in childhood was the most predictive factor for adolescent affective symptoms in the SLI group.

Conclusions: There is a marked higher rate of anxiety and depression in adolescents with SLI which does not appear directly or simply associated with the language difficulties per se.

Correspondence: Nicola Botting, PhD, Language and Communication Science, City University, London, Northampton Square, London EC1VOHB, United Kingdom. E-mail: nicola.botting.1@city.ac.uk

S. KISELEV \& Y. LAPSHINA. Investigation of Visuospatial Abilities in SLI Children with Poor Grammar Understanding.

Objective: The goal of study is to answer the question - are there visuospatial difficulties in Russian-speaking children with specific language impairment who have poor grammar understanding (Grammatical (G) - SLI children)?

Participants and Methods: Seventeen G-SLI children aged between 6:9 to 8:3 participated in the study. The age control group consisted of seventeen normally developing children. We used the adapted for children subtests from Luria's neuropsychological assessment battery that were developed to assess the visuospatial abilities. It includes the copy of three-dimensional table, the constructive praxis subtest, the Head's subtest, and visual gnosis subtest.

Results: One-way ANOVA revealed a significant main effect of group on level of performance of all used visuospatial subtests.

Conclusions: The obtained results imply that G-SLI children have weakness in visuospatial abilities. We assume that G-SLI children have the alteration of a particular cerebral mechanism responsible for the function of "simultaneous and spatial synthesis" that plays important role both in grammar understanding and visuospatial functions

Correspondence: Sergey Kiselev, Ph.D., clinical psychology, Ural State University, Lenina 51, Yekaterinburg 620083, Russian Federation. Email:eskisa@rambler.ru

A. LOPEZ-SAlA, A. SANS, C. BOIX, R. COLOMÉ \& M. MARISTANY. Neuropsychological profile of 35 children with Asperger's Syndrome.

Objective: Asperger's syndrome was described by Hans Asperger seventy years ago. It's characterized by impairment in social interaction. peculiarities in language and in nonverbal communication, as well as restricted set of interests.

Objective. To analyse the neuropsychological profile.

Participants and Methods: Criteria derived from Gillberg and Gillberg (1989) were used to identify 35 children with Asperger's Syndrome diagnosed in our neurological department form January 2000 since December 2006. A retrospective study of the medical report and neuropsychological assessment has been performed.

Results: Familiar antecedents, motor coordination deficits, good speech abilities, social relation problems and restrictive interests are data to suspect this entity. The neuropsychological assessment showed a good verbal performance except in pragmatic aspects of language and better mechanical reading than comprehension, as well as good constructional praxis. On the other hand, they had troughs on attentional tasks and social behaviour understanding, and showed graphomotor dyspraxia.

Conclusions: This study underlines the alarm signs to suspect a possible Asperger's syndrome. We emphasize the importance of analysing the cognitive profile to understand the pathofisiology of this entity, to achieve a differential diagnosis with other similar entities and to establish a treatment program.

Correspondence: Anna Lopez-Sala, Neurology, St Joan de Déu Hospital, Passeig Sant Joan de Déu,2, Esplugues de Llobregat (Barcelona) 08950, Spain.E-mail:annalopez@hsjdbcn.org 
A. LOPEZ-SALA, A. SANS, R. COLOMÉ, C. BOIX, A. SANGUINETTI \& R. GASSIÓ. Neuropsychological profile of dyslexia: a sample of 150 Spanish children.

Objective: Dyslexia is characterized by an unexpected difficulty in reading in children who otherwise possess the intelligence, classroom instruction and motivation considered necessary for accurate and fluent reading. It is perhaps the most common neurobehavioural disorder affecting children, with prevalence rates rating from 5 to 17.5 percent. It is a persistent, chronic condition and in most of the cases it is familial and heritable. The neurological disorder underlying dyslexia is the same across the cultures reported by cross-language studies. Functional neuroimaging studies disclose a different activation pattern in dyslexic's brains when reading. A posterior dysfunction in the dominant hemisphere in and around the angular gyrus is postulated (Shaywitz 2002). Objective: To analyze the neuropsychological profile of 150 dyslexic children.

Participants and Methods: We have reviewed 150 dyslexic children diagnosed in our neurological department from January 2000 since December 2006. A retrospective study of the medical report and neuropsychological assessment has been performed.

Results: Besides the main mechanical reading problem and the deficits on orthography, dyslexic children show problems memorizing automatic verbal sequences, fast naming retrieval, phonetic fluency, phonological short term memory, expressive language, attention difficulties and handwriting.

Conclusions: Our results support de idea of a left posterior brain dysfunction because the neuropsychological profile correlates with the functional brain model. We postulate that the extension of the brain dysfunction may produce a different degree of cognitive symptoms. Correspondence: Anna Lopez-Sala, Neurology, St Joan de Déu Hospital, Passeig Sant Joan de Déu,2, Esplugues de Llobregat (Barcelona) 08950, Spain.E-mail: annalopez@hsjdbcn.org

M.D. ROLDÁN-TAPIA, J. RAMOS-LIZANA, L. MARTINEZCAZORLA \& M.M. SÁNCHEZ-JOYA. Study of the visuoperceptive, executive and motor functions of a sample of children born with great prematurity.

Objective: The main objective of the study is to explore the difficulties in perceptive tasks and executive functions in children with great prematurity, relating them with the presence or absence of dysfunctions motors, taking in account level of education and weight of the child and weeks of pregnancy at the moment of childbirth.

Participants and Methods: We have designed a study with a sample of 20 children of less than 32 weeks of gestation and/or less than $1500 \mathrm{gr}$, with ages understood between the 4 and 7 years; comparing it with a sample of non premature children and of characteristic demographic and educational similar.The neuropsychological evaluation consists on a battery specific standardized test in the Spanish population to evaluate mainly visuo-perceptive, visuo-constructive, social cognition, language and executive functions. Measures of intelligence are taken by means of the Kaufman Intelligence Battery.

Results: Analyses of variance were done using the motor disturbances, age, sex and educational level as covariates. Significant differences in the Rey-Osterrieh figure, face recognition (K-BIT), Fluency (MSCA) and visoperceptive subtest (Cumanin battery) were found. The outcomes were lower in premature children with low weight (less than $1500 \mathrm{gr}$ ) and with psychomotor disturbances.

Conclusions: We conclude that a central nervous system immature and motor dysfunction is widely related with the cognitive deficit showed in childbirth prematurity.

Correspondence: Maria D. Roldán-Tapia, University of Almería, Fac HH yCC Educacion II, Almería 04120, Spain.E-mail: mdroldan@ual.es
S. VAN HOOREN, N. RULAND, P. VERBOON \& I. GOOSSENS. Inhibition, attention and simple reaction time of children with attention deficit hyperactivity disorder.

Objective: According to Barkley's (1997) theoretical framework on attention deficit hyperactivity disorder (ADHD), there are differences in cognitive test results between ADHD subtypes. Children with elevations of hyperactivity/impulsivity symptoms would perform less well on tasks that measure inhibition and cognitive flexibility. Children of the inattentive subtype would get lower results on simple reaction time tasks. This framework predicts that the subtypes do not show any differences in measures of attention. The objective of this study was to determine whether subtypes of ADHD show a different neuropsychological profile.

Participants and Methods: This study compared medication free children (ages 7 to 12 ) with hyperactive type $(n=11)$, inattentive type $(n=15)$, and the combined type $(n=34)$ of attention deficit hyperactivity disorder. The subtypes were compared on tasks measuring inhibition, cognitive flexibility, attention and simple reaction time. The three tests used were the Amsterdam Neuropsychological Test, the Wisconsin Card Sorting Test and the Stroop Colour-Word Test.

Results: A multivariate analysis of variance revealed that there were no significant differences between the three subtypes on inhibition, $c 0$ nitive flexibility, attention and simple reaction time. Post hoc univariate analyses showed that children who were diagnosed with the hyperactivity subtype performed poorer on tasks measuring inhibition compared to children with the inattentive subtype. However, no differences were seen on the other cognitive domains using post hoc univariate analyses.

Conclusions: Overall, these results do not support the hypothesis of different neuropsychological profiles of impairment in subtypes of ADHD. Correspondence: Susan Van Hooren, Open University of the Netherlands, P.O. box 2960, Heerlen 6401 DL, Netherlands. E-mail: susan. vanhooren@ou.nl

L. VAN ITERSON. Kaufman Factors in three groups of Special school children: Learning Disabilities, Childhood Psychiatry, and Epilepsy. Objective: Traditionally, three Kaufman factors have been distinguished in the WISC-R: the Verbal Conceptual Factor (VC), the Perceptual Organization Factor (PO) and the Freedom from Distractibility Factor (FD). We studied the hypothesis that samples of children with developmental disorders differ in factor scores and factor-profiles.

Participants and Methods: Using special school eligibility as independent variable for group membership, Dutch WISG-Rs of $\mathrm{N}=307$ children with FSIQ $>75$ (mean=94), aged 6-13 (mean=10) years were collected: Learning Disabilities $(n=78)$, Psychiatric Disorders $(n=129)$ or Epilepsy $(\mathrm{n}=100)$. With Mancova, controlling for age and sex, we tested differences between samples in terms of (A) means of the factor scores and (B) range between highest and lowest factor. With chi-square statistics, we contrasted $(\mathrm{C})$ the frequencies in which specific factor-profiles emerge.

Results: (A) Adjusted means showed no differences for PO; Epilepsy>Learning Disabilities for VC; Learning Disabilities and Psychiatry $>$ Epilepsy for FD. (B) Factor ranges show no between-group differences. (C) Rates of profiles differ $(p<.001)$. In Learning Disabilities, $\mathrm{PO}$ is highest in the profile in $59 \%$ of children; while $\mathrm{VC}$ is lowest in $58 \%$. In Epilepsy, $\mathrm{VC}$ is highest in $47 \%$; whereas FD is lowest in $50 \%$. In Psychiatry, factors are evenly distributed.

Conclusions: Kaufman factors reveal that school problems in epilepsy and in learning disabilities are of different origin, supporting the notions that in learning disabilities, a verbal/phonological weakness is a core problem, whereas in epilepsy, processing speed and working memory difficulties are characteristic. In psychiatric disorders, no notorious pattern appears, possibly due to the diagnostic heterogeneity of the sample.

Correspondence: Loretta van Iterson, drs, psychology, De Waterlelie, Spieringweg801, Cruquius2142 ED, Netherlands.E-mail:itersonl@dds.nl 
S. ZOTTER, J. MARKSTEINER, E. WEISS, U. WEISS, M. STEINMAYR-GENSLUCKNER, E. HABERLANDT \& L. KAUFMANN. Frontoexecutive Functions in Five Adolescentes with Asperger Syndrome: Preliminary Findings.

Objective: Main aim of this study is to investigate fronto-executive functions in individuals with Asperger Syndrome (AS). Specifically, we are interested in assessing the predictive value of parent ratings for actual performance levels on fronto-executive tasks. Executive dysfunctions (e.g., flexibility, planning) are considered to be neurocognitive core deficits in individuals with autism spectrum disorder (including AS).

Participants and Methods: 5 adolescents with AS (13 to 20 years old) are compared to 5 healthy matched controls. AS diagnosis conformed to DSM-IV criteria (APA, 1994) and was corroborated by ADI$\mathrm{R}$ and ADOS. Intelligence was estimated by using German versions of the Wechsler Scales (children and adult form). Executive functions were assessed experimentally by subjecting participants to a PC-administered test series (CANTAB, Cambridge Cognition Ltd) and indirectly, by collecting parent ratings of executive functions relevant for everyday-lifeactivities (BRIEF).

Results: Results reveal that relative to healthy controls, patients display specific performance profiles being characterized by deficient planning (CANTAB subtest: stockings of cambridge) and cognitive flexibility (CANTAB subtest: intra-/extradimensional shift) while performance on working memory and inhibition tasks was comparable to healthy controls. Importantly, these performance differences remain after controlling for intellectual level and moreover, closely correspond to parent behaviour ratings.

Conclusions: Overall, our findings are suggestive of specific performance profiles regarding executive functions in individuals affected with AS (Ozonoff \& Jensen, 1999). Interestingly, AS patient's pronounced difficulties in planning and flexibility (as measured by experimental tasks) are also reflected in parents rating of every-life-activities. Thus, our results suggest good predicitive value of parent ratings regarding executive functions in individuals affected with AS.

Correspondence: Liane Kaufmann, PhD, Clinical Department of Pediatrics IV, Innbruck Medical University, Anichstrasse 35, Innsbruck A-6020, Austria.E-mail: liane.kaufmann@i-med.ac.at

\section{Drug/Toxin Exposure (incl. alcoholism)}

\section{CABEZUDO, R. SABORIT, J. MARTINEZ \& J. BERNABEU. Multidisciplinary treatment of Wernicke-Korsakoff encephalopathy: a case study.}

Objective: To describe a woman affected by a Wernicke-Korsakoff syndrome comorbid with symptoms of histrionic personality.

Participants and Methods: A 55 years old woman with a WernickeKorsakoff syndrome induced after morbid obesity gastroplasty was studied. Physical, cognitive and behavioral assessment was done.

Neuropsychological assessment was performed with BNI, behavior and cognitive function questionnaire, WMS III, BADS and FIM-FAM. Neurological, physical and psychiatric assessment were also performed.

Results: We registered deficit in memory, conspiracy, and alterations in walking and manipulation due to dysaesthesias. Dependence in daily instrumental abilities and problems with behavior were also registered.

Conclusions: A multidisciplinary treatment in rehabilitation (physiotherapy and occupational therapy, neuropsychology and psychiatry) prove that the Wernicke-Korsakoff symptoms have developed favorably in a short period of time ( 2 months), but this improvement has been disguised by other depressive sings and symptoms of histrionic personality disorder and dependence.

Correspondence: Jordi Bernabeu, Master, Hospital La Fe, Pediatric Oncology Unit, Avgda. Campanar, 21, Valencia 46009, Spain. E-mail: bernabeu_jor@gva.es
S.F. CROWE \& M.J. BARKER. The Long Term Neurocognitive Consequences of Benzodiazepine Use.

Objective: Most investigations into the effects of the long-term use of benzodiazepines tend to focus upon only one or two specific areas of cognition rather than assessing the full range of neuropsychological functioning, making it unclear whether the effects of these drug are generalized rather than specific.

Participants and Methods: This paper presents the results of three meta-analyses which address the range of cognitive effects of the drug. A total of thirteen research studies met the strict inclusion criteria. The neuropsychological tests employed in the studies were each categorized as measuring one of 12 cognitive domains. The specific questions addressed were: 1) Are there cognitive effects of long-term benzodiazepine use? 2) Does the cognitive function of long-term benzodiazepine users improve following withdrawal? and, 3) Are previous long-term benzodiazepine users still impaired in neuropsychological functioning at follow-up as compared to controls or normative data?

Results: Taken together, the results provided strong support for the hypothesis that long-term benzodiazepine use did lead to cognitive deficit across all 12 domains of functioning. While the results indicated that long-term benzodiazepine users did show recovery of function in many areas after withdrawal, significant impairment in most areas of cognition in comparison to controls remained after discontinuation.

Conclusions: The findings indicate that benzodiazepine use causes global compromise in cognition. While previous benzodiazepine users are likely to experience improved cognitive functioning after withdrawal, the data did not support the notion of full restitution of function, at least in the first six months following cessation. This suggests that there may be some permanent deficits or deficits that take periods longer than six months to completely recover following prolonged use of the drug.

Correspondence: Simon F. Crowe, PhD, La Trobe University, School of Psychological Science, Bundoora, VIC 3086, Australia. E-mail: s. crowe@latrobe.edu.au

H.K. NILSEN, N.I. LANDRO \& P.C. BORCHGREVINK. Effects of Chronic non-malignant Pain and of Long-term Opioid Therapy on Neuropsychological Functioning.

Objective: Chronic pain is associated with neuropsychological impairments, particularly in the areas of attention and working memory. However, the literature is not entirely consistent. Another unsettled issue is the possible detrimental effect of opioid medication.

Participants and Methods: Twenty chronic non-malignant pain patients on long-term codeine therapy, twenty pain patients not using opioids, and twenty healthy controls were tested with Stroop color- naming test (selective attention), Letter-Number Span test (working memory capacity), and PASAT (working memory executive control). The three groups were comparable in age (mean 40.1 years), gender ( $58 \%$ women), and years of education (mean 13.0 years). The two pain groups were also comparable with respect to pain level (NRS mean 5.7), health-related quality of life (EORTC-Q30) and personality traits (BFI). The subjects were tested two times (T1 and T2) the same day with 5 hours interval, the codeine group both at therapeutic and subtherapeutic levels.

Results: Both pain groups showed a tendency towards reduced functioning as compared to healthy controls on the PASAT and the Stroop, but not significant in the pain group. The pain codeine group performed significantly below controls on the overall PASAT at T2 and in the last part of PASAT at both T1 and T2. Codeine level at test did not influence the results.

Conclusions: While there was no statistical evidence for reduced functioning in chronic pain, the results indicate that the executive aspect of working memory in patients with chronic pain on long-term codeine therapy was redused. This could imply important consequenses in work and daily life. Correspondence: Halvard K. Nilsen, MD, Faculty of Medicine, The Norwegian University of Science and Technology, Trondheim, Lerstadtoppen 34, Aalesund 6014, Norway.E-mail: halvardnilsen@yahoo.no 


\section{SCHEUER, C.C. OLIVEIRA, S. SCIVOLETTO, F. MALERBI \& L. STIVANIN.AUTOBIOGRAPHICAL AND SEMANTIC MEMORY OF DRUG USERS AND NON USERS ADOLESCENTS: GENDER COMPARISON.}

Objective: Psychiatric disorders in childhood and adolescence may impair the access to the memory of facts and events occurred during these periods. The main purpose of this study was to compare the autobiographical and semantic memory of a group of adolescent drug users and a control group of adolescent non-users as well as compare both genders. Participants and Methods: 37 adolescents users of alcohol and/or drugs, in outpatient treatment for chemical dependence ( 25 males and 12 females) and 40 adolescents non-users (25 males and 15 females) participated in this study. Memories of the childhood of all the subjects were collected through two questionnaires: Kihlstrom \& Schacter (1995) for semantic memory and Borrini et al. (1989) for autobiographical memory.

Results: Comparison between both groups pointed to more difficulties to access autobiographical $(p=0.0305)$ and semantic memory $(p=0.042)$ among drug users. Gender comparison showed that boys drug users remembered less autobiographical and semantic events and facts when compared to girls drug users $(p=0,018 ; p<0,001)$. The same was observed among non-users boys and girls $(p=0,0212 ; p=0,0235)$.

Conclusions: Results pointed that boys, regardless of drug use, have more difficulties to access autobiographical and semantic memory. These difficulties are in part related to the use of drugs, but also seems to be linked to gender differences.

Correspondence: Christian C. Oliveira, Ambulatory of Adolescents and Drugs of the Childhood and Adolescence Psychiatry Service, School of Medicine of University of São Paulo, Av Jabaquara 1469 apto 112 Bloco A3, Sao Paulo 04045002, Brazil. E-mail: christianfonoaudio@yahoo.com.br

M. PARADA, M. CORRAL, N. MOTA-MIRANDA, A. CREgO \& F. CADAVEIRA. Effects of Alcohol Binge Drinking on College Students' Memory: Preliminary Results.

Objective: Recent neuropsychological studies suggest that alcohol binge drinking (drinking four or more drinks per occasion for female and five drinks or more for male in a short period) is associated with cognitive difficulties, mainly in memory function. The objective of this study was to determine the effect of this consumption pattern on declarative verbal memory.

Participants and Methods: The sample consisted of 40 college students (ranging 18 to 20 vears old) selected according their scores on the AUDIT: 22 binge drinkers ( 9 men and 13 women) and 18 non-binge drinkers ( 5 men and 13 women). Exclusion criteria were previous clinically significant brain disorders, a history of substance abuse and family history of brain disorders. The declarative verbal memory was examined with the Logical Memory subtests of the Wechsler Memory Scale-third edition (WMS-III). Student t tests were used for statistical analysis.

Results: The binge drinkers obtained significantly lower scaled scores that non-binge drinkers in total immediate recall $(p=.04)$, total delayed recall $(p=.01)$ and idea delayed recall $(p=.003)$.

Conclusions: The binge-drinking pattern is associated to lower immediate and delayed verbal memory performance. Further studies should be to determine if these memory differences are reversible with abstinence and if have negative effects on the academic achievement. Correspondence: María Parada, Psicología Clínica y Psicobiología, Psychology University of Santiago de Compostela Spain, Facultad de Psicología campus sur $s / n$, Santiago de Compostela 15782, Spain. Email:mparadaiglesias@yahoo.es

M. PARADA, M. CORRAL, M. FERNÁNDEZ-EIRE \& F. CADAVEIRA. Performance of Alcoholic Patients on Behavioral Assessment of the Dysexecutive Syndrome (BADS).

Objective: To determine the neuropsychological profile of chronic male alcoholic patients in the Behavioural Assessment of Dysexecutive Syndrome (BADS)
Participants and Methods: The sample consisted of 29 subjects (ranging 28 to 50 years old): 19 recently detoxified alcoholic patients (range 15-90 days of detoxification) and 10 healthy controls. Data were analyzed with the Mann-Whitney U test.

Results: Groups showed significant differences in the profile scores of Rule Shift Cards $(p=.02)$, Key Search $(p=.001)$ and Modified Six Elements Test $(p=.02)$ and the BADS total score $(p=.001)$. Further analysis showed differences in total time $(p=.001)$ in first trial of Rule Shift Cards and in total time $(p=.02)$ and number of errors $(p=.001)$ of second trial; the pattern of search $(p=.001)$ in Keys Search ; the direct score $(p=.001)$ of Action Programme; the planning time $(p=.01)$ and total time $(p=.03)$ in first trial of Zoo Map and planning time $(p=.001)$, total time $(p=.001)$ and the number of errors $(p=.03)$ in second trial and in number of subtask attempted $(\mathrm{p}=.02)$ and number of rule breaks $(p=.03)$ in Modified Six Elements Test.

Conclusions: These results suggest that alcoholic patients have difficulty in cognitive flexibility, strategy formation, planning and organizing. These problems must be taken account of in planning the treatment, because alcoholic patients with executive deficits tend to have a worse prognosis, associated with a higher number of relapses.

Correspondence: María Parada, Psicología Clínica y Psicobiología, Psychology University of Santiago de Compostela Spain, Facultad de Psicología campus sur s/n, Santiago de Compostela 15782, Spain. Email:mparadaiglesias@yahoo.es

S. REMY \& A. BERARDI. Relations Between Sensation Seeking, Cannabis and Tobacco Use in Young Adults.

Objective: The aim of this study was to determine whether young adults with cannabis and/or tobacco use differed on the subscales of the Sensation Seeking Scale (SSS, Zuckerman, 1978)

Participants and Methods: Four groups of females were studied: 10 current users of both cannabis and tobacco, 10 current cannabis usersonly. 10 current tobacco users-only and 10 nonusers of either tobacco or cannabis. All groups were matched on age, education, and depressive symptoms (all $p>.24$ ). Because it has been previously demonstrated that females suicide attempters and females with suicidal ideation are high sensations seekers (Remy \& Berardi, 2003, 2006), participants in this study never made a suicide attempt and did not have any suicidal ideation.

Results were analyzed using one-way ANOVAs comparing the four groups. Results: The main effect for the Disinhibition subscale of SSS was significant $(F=5,9 ; p=.002)$. Bonferroni post-hoc t-tests corrected for multiple comparisons suggested that cannabis users-only had higher scores than nonusers on the Disinhibion subscale of SSS $(p=.0004)$; cannabis users-only also tended to have higher scores on the Disinhibition subscale of SSS than tobacco users-only ( $p=.03$, corrected for multiple comparisons). Users of both cannabis and tobacco had higher scores on the Disinhibition subscale of SSS relative to nonusers $(p=.005)$. Main effects for the Thrill and Adventure, Experience Seeking and Boredom susceptibility subscales were not significant $($ all $\mathrm{F}<1)$

Conclusions: These results show that cannabis users-only have higher disinhibition than tobacco users-only and that cannabis users (with or without tobacco use) have higher disinhibition relative to nonusers. Correspondence: Sébastien Remy, PhD Student, Psychology, University Paul Verlaine, Metz, Ile du Saulcy, BP 30309, Metz 57006 cedex 01, France.E-mail:contact@sebastienremy:com

M. RODRÍGUEZ-ÁLVAREZ, M. CORRAL, D. RODRÍGUEZSALGADO \& C. FERNÁNDEZ-EIRE. Wisconsin Card Sorting Test (WCST) Performance in Substance Abusers.

Objective: Different research has suggested that substance abuse is associated with a poor performance on WCST, but little is known about different pattern of performance taking into account patient groups. The aim of this study was to identify the pattern of executive function im- 
pairment in chronic alcoholism and intravenous drug abuse male patients, which have been in abstinence a minimum of fifteen davs.

Participants and Methods: We compared WCST performance in 30 recently detoxified alcoholics (ALCH) (mean age =38,97), 15 AIDS former intravenous drug users (AIDS-IVDUs) (mean age $=33,93), 27$ former intravenous drug users (IVDUs) (mean age $=26,19)$ and 19 individually matched healthy controls (mean age $=29,45$ ).

Results: Analyses of variance were carried out between groups on different measures of WCST. The performance by ALCH and AIDS-IV DUs patients was poor compared with control subjects on number of categories completed, percent of perseverative responses, and percent of perseverative errors. Only AIDS-IVDUs patients made less percent of conceptual level responses than controls and IVDUs. The percent of nonperseverative errors, failure to maintain set, and learning to learn measures showed no significant group differences.

Conclusions: These results suggest that ALCH and AIDS-IVDUs patients may show a similar pattern of deficits on WCST when compared with IVDUs and controls. The "frontal lobe hypothesis", according to which substance abusers are impaired on function tests related to the frontal lobe, was partially confirmed in our sample.

Correspondence: Montserrat Corral, Universidad de Santiago de Compostela, Facultad de Psicología, campus sur s/n, Santiago de Compostela 15782, Spain.E-mail:mcorral@usc.es

\section{J.N. WILKINS, S.S. WILKINS, R.M. CORNNER, A.M. QUIRING \& M. HRYMOC. Neuropsychological Findings and Work Function in Alcohol Dependent Subjects.}

Objective: Neuropsychological performance is often decreased in alcohol dependent subjects. This study assessed whether neuropsychological measures were related to work function.

Participants and Methods: Thirty subjects were recruited as part of a larger protocol. All subjects met DSM-IV criteria for substance dependence. Mean age was 35.5 years(+/- 7.57), and mean education was 14.6 years (+/- 2.9). Eighty percent of subjects were male; $83 \%$ Caucasian, 10\% Hispanic, and 3.3\% African American and 3.3\% American Indian. Eighty seven percent of the group worked, 3\% were students, and $10 \%$ were unemployed. Subjects all received a battery of tests at baseline, including; The Wechsler Test of Adult Reading, WAIS-3 digit span, letter-number sequencing and block design, Rey-Osterreith Complex figure, Wisconsin Card Sorting Test, Addiction Severity Index, and Endicott. Pearson correlations (2 tailed) were run between the neuropsychological findings and measures of work and family functioning. Results: A significant negative correlation was found between the ASI employment scale and digit span (both forwards and backwards), $\mathrm{p}=.02$ and .006 respectively. There was also a significant negative correlation between the ASI employment scale and perseverative errors on the WCST, $p=.002$. The Endicott employment scale trended toward significance with Block Design, $\mathrm{p}=.094$.

Conclusions: Alcohol dependent subjects have been found to have lower than average neuropsychological performance on tasks of attention, memory, visual-spatial skills and frontal functioning. In this study, attention (as measured by the Digit Span) and executive functioning (perseverative errors on the WCST) were related to poorer performance on the employment scale of the Addiction Severity Index. In addition, there was a trend toward significance for the Endicott employment scale and Block Design. Neuropsychological measures appear ecologically valid regarding work functioning in alcohol dependence.

Correspondence: Jeffery N. Wilkins, MD, Psychiatry, Cedars-Sinai Medical Center, 8730 Alden Dr., Suite C301, Los Angeles, CA 90048. Email:wilkinsj@cshs.org

\section{Genetic disorders}

O. INOZEMTSEVA \& E. MATUTE. Learning Disabilities Spectrum in Congenital Adrenal Hyperplasia (CAH) girls.

Objective: Although evidence of an increased risk of learning disabilities (LD) in CAH patients exists, data are not conclusive. The use of standardized tests and international criteria for diagnosing LD in CAH patients would clarify this issue. Therefore, the aim of this study was to assess the presence and type of LD in 11 CAH girls (CAHG) with normal intellectual level (IQ > 85 points).

Participants and Methods: Specific tasks from reading, writing and mathematic domains of The Child Neuropsychological Assessment ENI (Matute, Rosselli, Ardila \& Ostrosky, 2007) for Spanish-speaking children were used according to the DSM-IV-TR criteria for LD identification. CAH subjects were matched with a control group (CG), oneby-one, by age (range 7-16 years), school grade and school type.

Results: No IQ differences between groups were found. A qualitative analysis, subject-by-subject of standard scores on the reading, writing and mathematics measures in both groups was done in order to identify those subjects who scored 2 SDs below the mean levels on at least 2 measures in the same domain. Our results showed that six CAH patients and only one CG girl pointed below their age mean. Five out of these six CAH patients had reading disabilities and one girl exhibited a mathematics disability. In three cases, reading disability was associated with writing and mathematics difficulties. Conclusions: Our results confirmed the high incidence of LD in CAH population, and they indicate that the LDs of CAH girls are basically, though not exclusively, of the reading type.

Correspondence: Olga Inozemtseva, PhD, Universidad de Guadalajara, Francisco de Quevedo 180, Guadalajara 44130, Mexico. E-mail: oinozem@yahoo.com

\section{Learning Disabilities/ADHD}

C. BOIX LLUCH. REVIEW OF NEUROPSYCHOLOGYCAL PROFILE IN A SAMPLE OF 125 ADHD CHILDREN C Boix, A Sans, R Colomé, A López- Sala, A Sanguinetti, R Gassió, M Maristany, M Pámias UTAE. Servei de Neurología. Hospital Sant Joan de Déu. Barcelona, Spain. Objective: Attention-deficit/hyperactivity disorder (ADHD) consists of pervasive behaviour and cognitive symptoms beginning in childhood. It has a neurobiological basis and affects 3- $8 \%$ of school- aged children and 3-4 \% of adults.

Nowadays ADHD is understood as a disorder of behavioural inhibition and self-regulation due to deficits in the prefrontal cortex and related subcortical systems.

Neurocognitive deficits associated with ADHD are likely to have deleterious effects on social functions and school performance.

Analyze the neuropsychological profile in a large sample of ADHD children.

Participants and Methods: We have reviewed 125 ADHD children, age ranging between 6 - 17 years old. All children met DSM-IV-R criteria for $\mathrm{ADHD}$ and were recruited out of a specific learning disabilities unit in a Neurology Hospital Department, throughout the year 2006.

We analyze retrospectively neuropsychological assessments.

Results: Of the entire sample, combined ADHD subtype occurred in 76 children $(60 \% \%)$, ADHD predominantly inattentive in $42(33 \% \%)$ of the cases and $7(5,6 \%)$ had ADHD predominantly hyperactive/impulsive subtype. There were 104 boys and 21 girls. Comorbid dyslexia was diagnosed in $24 \%$ ADHD children. No other specific learning diagnoses were made. Neuropsychological profile was heterogeneous. Nevertheless, we have seen that there are some cognitive domains that are persistently altered in relation to executive functions.

High percentage of the ADHD children had reading and writing difficulties, which can be differentiated clearly from ADHD children with comorbid dyslexia.

Conclusions: We consider that cognitive deficits in ADHD, especially the impairment in executive functions, are responsible for the difficulties in school performance and learning disabilities.

Complete neuropsychological examinations are necessary to distinguish cognitive performance in ADHD population for a correct diagnosis and course of treatment.

Correspondence: Cristina Boix Lluch, Learning disabilities Unit, Neurology Department, Hospital Sant Joan Deu, C/ santa rosa, 39-37,3a pl, Esplugues del Llobregat 08950, Spain.E-mail: cboix@hsjdbcn.org. 
A.G. CAPOVILLA \& F.C. CAPOVILLA. Nature of Reading Difficulties in Brazilian Children with Developmental Dyslexia.

Objective: According to an information processing approach, different reading strategies prevail at different stages and depending on print characteristics. The first stage, logographic, is marked by the prevalence of the logographic strategy in which recognition is limited to a few familiar words. The second stage, alphabetical, is marked by the prevalence of grapheme-phoneme decoding. The third stage, orthographic, is marked by the prevalence of a lexical strategy, with visual recognition.

Participants and Methods: Using Reading Competence Test (RTC), this study analyzed reading strategies used by 13 Brazilian dyslexic children and compared them to those used by 2196 1st-7th grade normolexic students. RCT analyzes the ability of using each strategy and is made of seven types of print-picture pairs (a print associated with a picture). The task is to circle correct print-picture pairs (either regularly-spelled or irregularlyspelled words associated with their corresponding pictures) and to cross out incorrect ones (either words associated with unrelated pictures, or nonwords of four types: that sound like words, that look similar to words, that sound similar to words, that neither sound nor look similar to words).

Results: Results revealed that the difficulty presented by dyslexic children is eminently phonological: In word reading, dyslexics performed equivalent to normolexic children, whereas in nonword reading, they performed significantly worse, especially for nonwords that look or sound similar to words.

Conclusions: The study corroborated the notion that phonological processing is a major component of alphabetical reading and spelling, and that phonological processing disorders are mainly responsible for the specific reading difficulties that characterize developmental dyslexia. Correspondence: Alessandra G. Capovilla, Psychology, University of San Francisco, Rua Alexandre Rodrigues Barbosa, 45, Itatiba 13251-900, Brazil. E-mail: acapovil@usp.br

R. COLOME, A. SANS, A. LÓPEZ-SALA, C. BOIX, R. GASSIÓ \& M. ALQUEZAR. Non Verbal Learning Disorder: Neuropsychological Assessment of 40 Children. R Colome MSC, A Sans MD, A LopezSala MSC, C Boix MSC, R Gassio M Alquezar MSC Department of Neurology Sant Joan de Déu Hospital. Barcelona. Spain.

Objective: Non verbal learning disorder (NVLD) is a neurological condition which is considered to be a developmental disability. It is characterised by a specific dysfunction in motor, visuospatial and social skills in patients with normal intellect and regular language development. Warning signs at school are poor arithmetic and drawing skills as well as deficient psychomotor coordination. Social judgment and social problem solving are also typically impaired. Furthermore, these patients seem to have a higher risk of emotional disorders. Current evidence and theories suggest that dysfunction of white matter in the right hemisphere could be the cause. The clinical characteristics and the diagnostic criteria are not fully agreed.

The aim of this study is to analyse the neuropsychological profile

Participants and Methods: The sample is composed of 40 children from 7 to 15 years, diagnosed in our neuropsychological unit. The main complain of their parents attending our unit was the learning difficulties at school.

Results: The motor clumsiness and the difficulties in maths, drawing and handwriting, should lead us to the diagnosis. Soft signs in neurological examination are very marked and constant.

The neuropsychological assessment reveals visuospatial and visuoconstructive difficulties, dyscalculia, dysgraphia, poor reading comprehension as well as attentional and motor skills deficits. Most of them had also affective and social relation problems.

Conclusions: Non verbal learning disorder (NVLD) is a developmental disability which too often goes undiagnosed. We emphasize the importance of an interdisciplinary team in order to reach a diagnosis.

Furthermore the neuropsychological examination reveals specific profiles for each child that would make possible to address and to accommodate an educational program.
Correspondence: Roser Colome, Hospital Sant Joan de Déu, Passeig Sant Joan de Déu, 2., Esplugues de Llobregat 08950, Spain. E-mail: rcolome@hsjdbcn.org

\section{A.D. HUTCHINSON, J.L. MATHIAS \& M.T. BANICH. Corpus Callosum Morphology in Children and Adolescents with Attention Deficit Hyperactivity Disorder.}

Objective: Several studies have examined corpus callosum morphology in children and adolescents with ADHD. A recent meta-analysis of atypical brain morphology in children and adolescents with ADHD by Valera et al (2006) reported that there appears to be a reduction in the splenium of the corpus callosum in children and adolescents with ADHD compared with healthy controls. The current study examined callosal morphology in more detail by also considering possible gender differences and comorbid conditions in children and adolescents with ADHD.

Participants and Methods: A meta-analysis of 13 studies was conducted on studies in which corpus callosum measurements were obtained in children and adolescents with ADHD and healthy controls. In addition, corpus callosum size in children and adolescents with ADHD and comorbid conditions were compared with controls who had the comorbid conditions but without ADHD. Finally, gender differences in corpus callosum morphology were explored.

Results: Consistent with Valera et al. (2006), our meta-analysis indicated that the splenium was smaller in children and adolescents with ADHD compared with healthy controls. In addition, there was some evidence for a gender difference with boys exhibiting a smaller rostral body. There were no significant differences in corpus callosum size in the studies of children and adolescents with ADHD and comorbid conditions.

Conclusions: The smaller splenium may contribute to the attention problems of children and adolescents with ADHD and the smaller rostral body found in boys with ADHD may be related to some of the executive problems that are associated with ADHD.

Correspondence: Amanda D. Hutchinson, Psychology, University of Adelaide, 505 Manhattan Drv, Apt \#202, Boulder, CO 80303. E-mail: amanda.hutchinson@adelaide.edu.au

C.C. RODRIGUES, T. BARBOSA, C. TOLEDO-PIZA, A.C. ROSSETTO, T.B. SOARES NUNES \& O.A. BUENO. The influence of verbal and performance abilities in IQ measures in dyslexic children.

Objective: Students with dyslexia usually do not read and express themselves in writing nearly as much as their peers without dyslexia. A logical consequence of this is a drop in their verbal capacity. Looking at stability of IQ in individual with dyslexia, there is not much empirical research available, and the results are contradictory. The purpose of this study was to compare verbal and performance intelligence measures of dyslexic children.

Participants and Methods: Forty-two children aged 8 to 15 years were evaluated. The children were tested in a private room in 2 sessions. The test employed was WISC-III, using the block design, picture arrangement, picture completion and coding subtests to obtain the Performance Scale IQ (PIQ) and vocabulary, similarities, digit span and arithmetic subtests for the Verbal Scale IQ (VIQ). The sum of the weighted scores were converted to achieve the IQ estimated measures.

Results: The analysis of the results, according to ANOVA, indicate discrepancy between VIQ and PIQ $(\mathrm{p}<0,01)$, with VIQ average 95.6 and PIQ average 101.69. IQ and students' age in VIQ $(-0,63)$ and PIQ $(-0,43)$ are correlated $(p<0.05)$, showing an IQ decline throughout the years. Conclusions: These results suggest that dyslexic students present higher abilities in performance tasks than in verbal tasks and more deficits may occur, if dyslexia diagnosis is delayed, especially in verbal abilities. Correspondence: Camila C. Rodrigues, Psychobiology, UNIFESP/ EPM, Rua Embaú, 54, São Paulo 04039060, Brazil. E-mail: camilacruzrodrigues@hotmail.com 
L. STIVANIN, C.C. OLIVEIRA, F.F. MALERBI \& C.I. SCHEUER. Processing time and characteristics of picture naming from Brazilian children with reading disorders.

Objective: Studies related to children with specific reading disorders (SRD) indicate to a deficit in picture naming. The aim of this study was to verify the processing time for the picture naming of Portuguese-speaking Brazilian children with SRD.

Participants and Methods: The sample was composed by 25 readers (average age $=8,6 y$ ) and 20 children with reading disorders (average age $=8,3 y$ ), 2 nd grade, from both genders. Children with general development and language disorders and learning difficulties were excluded from the research. The picture naming test was composed by 96 pictures, divided into stimuli groups, with equivalent balance to written words of the Brazilian Portuguese. Pictures were presented in a computer screen; children were asked to name them. The processing time (latency time and time of duration of the verbal emission) were registered in a program specially designed for this research and the naming was analyzed according the criteria of Kohn and Goodglass (1985) by phonological, semantic and perceptual errors.

Results: Into what respects to the processing time no statistical differences between children with SRD and their controls were observed ( $p>0$, 05). However, results pointed too more correct naming of the control group $(p<0.05)$ and more phonological errors of children with SRD.

Conclusions: In conclusion, these children with SRD process pictures in similar time as their controls, but have difficulties to access phonological representations of the picture names.

Correspondence: Luciene Stivanin, University of São Paulo, Teodoro Sampaio, 632/52, São Paulo 05406-000,Brazil.E-mail: stivanin@usp.br

M.E. TAPIA YEO \& G. YAÑEZ TELLEZ. Effects of Phonological Awareness and Visuospatial Distribution Training in a Child with Reading, Writing and Calculation Learning Disabilities.

Objective: The learning disabilities are the most frequently neuropsychological problems in school age children. This work describes the assessment and training program in a case of a ten years old boy with normal intelligence but with learning disabilities in reading, writing and calculation areas.

Participants and Methods: A 10 years old boy with normal intelligence and learning disabilities was the only subject.

The neuropsychological evaluation shows that the patient had a problem with the acquisition of the phonological awareness and the visuospatial distribution.

We designed a specific training program in those areas using insight activities and specific phonological awareness program

Results: The patient acquired the ability to divide syllables correctly in a hearing word, that was reflected in an increase in the quantity and the quality of words writing and reading.

He also was able to carry of correctly arithmetic problems. The writing and drawing distribution improved considerably.

Conclusions: This findings may suggest that if problems related to learning disabilities have a basic abilities problem foundations, it should be treated at first to show a better performance in school.

Correspondence: Martha E. Tapia Yeo, Master, Neuropsychology, UNAM, Amores 229-16 Col.Del Valle, México City. 03100, Mexico. Email:marthapia@hotmail.com

C. TOLEDO PIZA, C.C. RODRIGUES, T. BARBOSA, A.C. ROSSETTO, T.C. BUJOKAS SOARES \& O.F. AMODEO BUENO.Verbal andVisualSpatial Working Memory Abilities in Dyslexic Children.

Objective: Studies comparing the relationship between reading and writing difficulties in children, and their associated neuropsychological profiles, indicate a possible deficit or delay in the overall development. This study investigates whether children with dyslexia present distinctive working memory profiles for visual-spatial and verbal tasks.
Participants and Methods: A sample of 41 Brazilian dyslexic children, of both sexes (27 boys and 16 girls), aged 8 to 15 years, participated in this study. Two types of working memory tasks were administered: the Digit Span Recall test (forward and backward recall), to investigate verbal memory, and the CORSI Block Recall test (forward and backward recall), to investigate visual-spatial memory. Each child was tested individually and data were analyzed using the Wilcoxon test.

Results: Findings suggest significant differences in verbal and visuospatial memory span tasks, both for forward and backward recall. The mean value for both forward and backward Block recall (4.88 and 4.23, respectively) is higher than the forward and backward Digit Span recall (3.90 and 2.95 respectively). Such results indicate deficits in verbal working memory performance were more observed. Other findings show that memory span was significantly greater when recalled forward than backward, for both span tasks.

Conclusions: The results are consistent with the idea that backwards memory span performance requires more attentional resources, which can be disrupted in dyslexics. Such findings suggest that working memory skills play an important role on the acquisition of reading and writing, and are important to consider possible mechanisms for the contribution of working memory to the learning process.

Correspondence: Carolina Toledo Piza, Psychobiology, UNIFESP EPM, R. Embau, 54, São Paulo 04039060, Brazil. E-mail: carolatp@terra.com.br

\section{K. VIRUDHAGIRINATHAN \& V. BABOO SANKAR. Behavioural Approach In The Management Of Dental Problems In Children With ADHD And ASD.}

Objective: To Emphasise the role of Behaviour Management in ADHD and Autism Spectrum Disorders Children during management of dental problems.

Participants and Methods: 80 children diagnosed with ADHD or ASD were given routine dental examination. Most of them were found to be having moderate to severe dental problems. 65 children were brought to the dental department for subsequent follow up by their parents. Among these 65 children 40 children came for regular periodic dental reviews. One to one talk with these children and the parents regarding the importance of oral hygiene and the approach to good oral health yielded excellent results. Parental counselling formed a part of every dental visit. These children were called for Dental review every 2 months. A remarkable improvement was seen at the end of 2 years.

Results: 40 children who came for regular dental reviews over a period of 2years showed remarkable improvement in their Oral hygiene. Behaviour modification proved to be of great help in improving oral health of these children.

Conclusions: Long term Behaviour therapy, medication for certain children with hyperactivity, occupational therapy for certain children and regular parental counselling during dental management showed positive results in improvment of oral health status of these ADHD and ASD children.

Correspondence: Keerthivasan Virudhagirinathan, BDS, DENTAL SURGERY, KUMARAN HOSPITALS PVT LTD, 214(869) EVR PERIYAR SALAI(P.H.ROAD), KILPAUK, Chennai 600010, India. E-mail: keerthiv@yahoo.com

K. WOLFSON, K. BEKKEN \& J. STASIOR. ADVANCES IN DISTINGUISHING CLINICAL PROFILES USING TWO SCORING SYSTEMS FOR THE REY-OSTERRIETH COMPLEX FIGURE.

Objective: The Rey-Osterrieth Complex Figure is one of the most widely used neuropsychological assessment tools both in the U.S. and abroad. There are a number of different scoring systems that have been developed over the years, including the Taylor 36-point system and the Waber and Bernstein Developmental Scoring System (DSS).

Participants and Methods: This study examines a sample of 40 children drawn at random from a clinical population of children with learn- 
ing disabilities, aged 6-16. Within-subject comparisons were made using these two scoring systems to determine how each of these systems characterized the children's neurocognitive profile. Variables of interest included age, gender, and diagnosis.

Results: Results are discussed in light of these two scoring systems' ability to describe the children's specific information processing styles. Strengths and weaknesses of each system are discussed based on the data.

Conclusions: We have begun to develop an internationally applicable scoring system that integrates and extends elements from both systems to better delineate important processing components, including style, configuration, and approach. (The poster is presented in English and Spanish.)

Correspondence: Kaaren Bekken, PhD, CDN, 76 Bedford St, Ste 12, Lexington, MA 02420.E-mail: kbekken@gmail.com

\section{Other}

R. BARROS NETO, K.T. PASSAGLIO \& M.C. ANDRADE.Theorical and Practical Inquiry of Motor Development in 2 to 11 Years Old Children.

Objective: This research work is based on concepts brought from Psychomotricity that considers that the motor, mental and emotional potentialities of an individual are in constant interactions and that the body is the place of these manifestations of the being. The tonic modifications, the positions and the movements are the exponents of all the personal history of the individual, of its representations, its experiences and its imaginary. The objective of this study is to reevaluate an instrument of inquiry of the motor development of children, built by Louis Picq and Pierre Vayer, to help professionals and people that work in this area.

Participants and Methods: The research was carried through in nine institutions of Belo Horizonte, Brazil, grouped in different economic levels, low, average and high. With, approximately, twenty children (2-11 years old) in each institution. The evaluated parameters had been: eyemotor coordination, dynamics of the hands, general dynamics, position and balance, corporal project and percipient organizations, of space and space-time.

Results: Through the studies of accuracy and validity it can be confirmed that the tested instrument really measures what it is expected to measure. For the study of the psychomotor answers, it was not observed consistent differences in relation to the sorts, however some variations of answers between the social levels had been observed.

Conclusions: The high economic level group was inferior to the low group, mainly in the parameters related with the motor coordination and activities, possibly reflecting the space limitation of universe where they are differently inserted.

Correspondence: Raul Barros Neto, Master of Science, Biology, PUC Minas University, Dom José Gaspar Avenue, 500, Coração Eucarístico, ICBS, Belo Horizonte 30535-901, Brazil. E-mail: raulbneto@gmail.com.br

P. VALDEZ, T. HERNÁNDEZ, H. TIRADO, A. GARCÍA \& C. RAMÍREZ. Developmental Changes in Performance of a Stroop Task.

Objective: The objective of this study was to determine developmental changes in behavioral inhibition using a modified Stroop task.

Participants and Methods: 540 participants were assessed (234 males and 306 females), ranging from 8 to 21 years of age. The test required the participants to read 48 words written in an incongruent color ink, and then they had to name the color of the ink of the same 48 words. In both conditions they were required to proceed as fast as possible.

Results: Time required to name the color of the ink was taken as a measure of behavioral inhibition. This variable attained adult level of performance at the group 16-17 years of age. There were no gender differences in performance.
Conclusions: The capability to inhibit behavior, as measured with this test, attains adult level of performance during adolescence. This is only one dimension of executive functions, so it is important to further analyze other components of these functions during development.

Correspondence: Pablo Valdez, Ph.D., School of Psychology, Universidad Autonoma de Nuevo Leon, Mutualismo 110, Col Mitras Centro, Monterrey64460, Mexico. E-mail: valdez.pa@gmail.com

F. LECKY. The incidence of school instability in 13-22 year olds in full time education following traumatic brain injury.

Objective: This study identified 13-22 year old patients on presentation to the Emergency Department after varying severity of head injury, including those who did not lose consciousness or did not require a CT brain scan. Those patients were approached two weeks after the brain injury by letter, to inform them that they would be contacted at one month and have a questionnaire administered by telephone. Consent was obtained from the parents of those patients who were minors. The questionnaire was administered after one month post head injury. using the KOSHI Questionnaire, which is a validated children's duplication of the Glasgow Outcome Score. The resulting disability in school instability will be quanitfied and present.

Correspondence: Doctor Fiona Lecky, Department of Emergency Medicine, Clinical Sciences Building Hope Hospital, Eccles Old Road, Salford M6 8HD, E mail Fiona.E.Lecky@manchester.ac.uk

\section{C.A. TÁRNOKY. A SERVICE FOR CHILDREN RECOVERING FROM ABI.}

Objective: This describes a local service set up in Manchester to facilitate placement and service delivery for children following severe brain injury. The service is introduced to the family and liaison of services is facilitated and the child returned to appropriate education.

Correspondence: Catherine Tarnoky, Dept. of Clinical Psychology, Booth Hall Hospita, Charlestown Rd., Manchester, M9 7AA, UK, Tel. 0161 9185032

\section{B. VIRUDHAGIRINATHAN \& F. JAHAN. NEUROPSYCHOLOGICAL ASSESSMENT AND MANAGEMENT OF LEARNING DISABILITY IN INDIA.}

Objective: Learning Disability (LD) is one of the most alarming problems in school going children in India, which has least awareness. The concept and definition of Learning Disability (LD) in India is quite different from the West, because we have 14 official languages, multi-cultural and different educational patterns, which vary from one state to another. Even though Hindi is the National language, English is the medium of instruction in most schools. We do not have a common educational system and teaching pattern like the West, hence the diagnosis and management of LD is different. To see the prevalence, pattern and effect of LD in learning and behaviour, we have done a study in different schools at Chennai. A systematic evaluation of Learning Disability was done at The Help Child - Centre for Learning Disability. We have assessed 700 normal school going children between 6 to 16 years. It was alarming that about $50 \%$ of these children suffer from LD in writing, spelling, reading, arithmetic or in combination. The analysis showed that most common form of disability was in writing and spelling. A small proportion of children exhibited difficulty in arithmetic. Another interesting observation is that some percentage of LD children have co-morbid Attention Deficit Hyperactivity Disorder as screened by DSM - IV. We have evolved different patterns of errors in writing, reading and spelling besides quantifying the level of Learning Disability, which were the basis for the management of these children.

Correspondence: Dr. B.S Virudhagirinathan, Clinical neuropsychologist, Kanchi Kamakoti Childs Trust Hospital, B1, Sri Govind Apartment, old \#17, New \#32, shenoy Road, Nungambakkam, Chennai-600034, India,E-mailBsv18@Yahoo.Com 


\section{Psychopharmacology}

L. OLIVEIRA, L. VAZ, Z. SANCHEZ \& S. NAPPO. Effects of Current and Past Crack Cocaine Use on Neurocognitive Functioning.

Objective: Although there is abundant evidence of changes in brain structure and metabolism due to crack-cocaine abuse, its effects on $\operatorname{cog}$ nitive functioning remain unclear. Considering that this information would be relevant in planning effective treatment interventions, clarify some of the crack-cocaine effects on cognition was the main aim of this research.

Participants and Methods: A sample of 59 subjects was assigned to a comparison group (non-drug users) $(\mathrm{N}=18)$ and crack-cocaine lifetime users, subdivided into 2 groups: users (subjects under a 1 to 30 days of crack-cocaine abstinence) ( $\mathrm{N}=17)$ and ex-users (subjects who had a period up to 6 months of crack-cocaine abstinence) $(\mathrm{N}=24)$. Crack users and ex-users were matched for crack-cocaine years of consumption. Socio-demographic data concerning on age, gender, level of education and social economic status (SES) were similar among groups. Subjects were submitted to Mini-Mental State Examination (MMSE) and tasks evalu- ating executive functions, verbal memory and attention. Mood state was also measured through Beck Depression Inventory (BDI) and IDATE. All scores were submitted to ANCOVA and Duncan post-hoc comparison. Results: Non-drug and crack-cocaine ex-users performed better on BDI than users $(p<0.05)$. Compared to non-drug user performance on DSST (attentional measure), ex-users $(p<0.05)$ and users $(p=0.08)$ performed worse. Verbal memory performance on Logical Memory (immediate and delayed recall) was impaired among users, relative both to control group and ex-users $(\mathrm{p}<0.05)$. Finally, control group performance on MMSE was better than users and ex-users ones $(\mathrm{p}<0.05)$.

Conclusions: Chronic crack-cocaine use seems to improve BDI score and disrupt general cognitive functioning (MMSE), memory and attentional resources. On the other hand, the worst crack-cocaine ex-users performance was on attentional measures, suggesting that it would persist after years of abstinence, in despite of a "recovering" on verbal memory measures.

Correspondence: Lucio Oliveira, CEBRID, UNIFESP, Rua Botucatu, 862, Edifício de Ciências Biomédicas, 1o. andar, São Paulo 04023062, Brazil. E-mail:lucio@psicobio.epm.br

\section{SATURDAY MORNING, JULY 7, 2007}

\section{Paper Session 16 9:15-10:15 a.m.}

\section{Alzheimer's Disease}

\section{A. BAHAR-FUCHS, G. SAVAGE, C. ROWE \& S. MOSS. Decline of Olfactory Identification in MCI and AD: Evidence for the Breakdown of Olfactory "Semantics"}

Objective: Olfactory decline represents one of the earliest signs of AD, and deficits in olfactory identification have been shown to occur in MCI, especially on unirhinal testing. However, The nature of errors that AD and MCI patients commit on olfactory identification tasks has not been explored to date and the organisation of olfactory knowledge remains largely unknown. The aim of the current study was to replicate recent findings regarding the lateralisation of early olfactory decline in MCI, and to determine whether AD and MCI patients commit more errors when the target smell is either semantically related or unrelated to the distracters.

Participants and Methods: 15 AD, 16 MCI and 11 control participants were recruited and tested for their olfactory identification and olfactory memory unirhinally using a modified version of the University of Pennsylvania Smell Identification Test.

Results: MCI patients $(M=4.7, S . D=2.1)$ performed no better than $\mathrm{AD}$ patients $(\mathrm{M}=3.6, \mathrm{~S} . \mathrm{D}=1.8)$ and both groups performed worse than controls $(M=6.7, \mathrm{~S} . \mathrm{D}=2)$ on overall identification scores. There was no interaction between nostril and group.

A moderate correlation between left and right nostril performance was found among the control and MCI groups, but not among the $\mathrm{AD}$ group. Examination of error types showed that odours which were semantically unrelated to the target odour were selected as frequently as semantically-related odours by the $\mathrm{AD}$ group on both nostrils and by the MCI group on the right nostril, while the control group predominantly selected the semantic distracter when making errors. Similiar trends were observed on olfactory memory Conclusions: Asymmetrical performance on olfactory identification is a feature of AD but not of MCI. This may be a result of more general inconsistencies in performance with disease progression. The nature of olfactory decline may take the form of weakening of associations between semantically related odours, as has been suggested for semantic knowledge in other modalities.
Correspondence: Alex Bahar-Fuchs, Monash University, Melbourne, Wellington Rd., Clayton, Melbourne, VIC 3800, Australia. E-mail: bfalex2003@yahoo.com

E.J. BARBEAU, M. DIDIC, E. TRAMONI, O. FELICIAN, J. RANJEVA, P. COZZONE, M. PONCET \& M. CECCALDI. Early Diagnosis of Alzheimer's Disease Using a Visual Recognition Memory Task.

Objective: To evaluate if a newly designed visual recognition memory test could be useful in early diagnosis of Alzheimer's disease (AD). Rationale: Neurofibrillary tangles, responsible for the early signs of AD have been shown to begin in a subregion of the perirhinal cortex before spreading to other portions of the medial temporal lobe. In the monkey, damage to the perirhinal cortex severely impairs performance on visual recognition memory tasks. Studies in humans corroborate these findings. We thus hypothesised that, within a population with an amnestic mild cognitive impairment (aMCI), those with incipient $\mathrm{AD}$ would perform poorly on such a task Participants and Methods: We developed a visual delayed matchingto-sample task called DMS48, consisting of 48 pictures that have to be recognized after an hour delay when each picture is presented with a distracter. Twenty-three patients fulfilling the criteria of aMCI (mean MMSE: 26.6. SD: 1.6) were recruited. All underwent a full neuropsychological evaluation. Their performance was compared with that of 10 patients with mild $\mathrm{AD}, 20$ patients with moderate $\mathrm{AD}, 20$ patients with Parkinson disease (PD), and 40 age-matched controls.

Results: Control subjects and patients with PD performed close to ceiling. Patients with mild AD had very low scores, while patients with moderate $\mathrm{AD}$ answered at random. MCI patients obtained scores that were between those of control subjects and patients with mild AD (78\%, SD: $16 \%)$. Of the MCI patients, 78\% (18/23) had impaired scores (1.5 SD below the mean). Two neuroimaging studies measuring grey matter loss (VBM) and perfusion (SPECT) showed that patients failing on the DMS48 had abnormalities characteristic of early AD.

Conclusions: These results indicate that most aMCI patients show pathological results on a visual recognition memory task. Furthermore, they indicate that the patients failing on the DMS48 are at high risk of AD. These results question the nature of the memory impairment at the predementia stage of $\mathrm{AD}$

Correspondence: Emmanuel J. Barbeau, PhD, Université Toulouse 3 , CNRS, CerCo, Faculté de Médecine de Rangueil, Toulouse Cedex 9 31062, France.E-mail:emmanuel.barbeau@cerco.ups-tlse.fr 
T. MOORTHY \& M. CROSSLEY. Predicting Alzheimer Disease in English- and French- Speaking Canadians.

Objective: Individuals with Alzheimer disease (AD) exhibit deficits across multiple cognitive domains years before clinical diagnosis. Past research suggests that language-of-origin can influence cognitive performance. Consequently, results from the study of English-speaking participants may not be directly generalized to seniors from other language backgrounds when making diagnostic decisions. This study investigated the predictive value of neuropsychological and demographic measures included in the Canadian Study of Health and Aging (CSHA) in determining diagnostic outcome for French-speaking and English-speaking participants over a five-year period.

Participants and Methods: Participants ( $\mathrm{n}=141$ French; $\mathrm{n}=225$ English) completed episodic memory (Buschke Cued Recall Test), letter fluency (FAS), category fluency (Animal Naming), and visuomotor processing (Digit Symbol) measures in their native languages.

Results: Logistic regression analyses revealed advanced age, poor delayed free recall, and reduced visuomotor processing speed as significant predictors of AD for English-speaking participants (sensitivity: 88.5\%; specificity: $93.9 \%$ ). Advanced age and poor delayed recall emerged as significant predictors of AD for French-speaking participants (sensitivity: $80.0 \%$; specificity: $85.6 \%$ ). Poorer performance on category relative to letter fluency, a pattern deemed to be sensitive in predicting $\mathrm{AD}$, was apparent only for the French-speaking group. However, language-based fluency tasks did not emerge as significant predictors of AD for either group.

Conclusions: The present findings confirm that age and episodic memory performance are among the best predictors of $\mathrm{AD}$ and can contribute to diagnostic decisions involving participants from different linguistic backgrounds.

Correspondence: Thamarai Moorthy, Ph.D, Psychology, Misericordia Community Hospital, Rm 216 Mother Rosalie Health Services Centre, 16930 - 87 Avenue, Edmonton, AB T5R 4H5, Canada. E-mail: ThamaraiMoorthy@caritas.cha.ab.ca

K. BAYNES, V. CHAND, L. BONNICI \& S. TOMASZEWSKI FARIAS. Idea Density as a Measure of Communicative Skill in Alzheimer's Disease.

Objective: Low idea density in early life is a risk for the development of Alzheimer's Dementia (AD) in late life. Unlike grammatical complexity, idea density is associated with vocabulary skills and does not decline precipitously with aging, although decline accelerates with AD (Snowden et al, 1996; Kemper et al, 1989; Kemper et al, 1993). Idea density may be sensitive enough to track decline in language use after diagnosis with $\mathrm{AD}$ and help determine if language decline contributes to the level of functional impairment. The goal of this study is to compare idea density in $\mathrm{AD}$ and control subjects to determine if idea density can predict communicative and functional decline.

Participants and Methods: Narrative samples of oral speech were collected from 11 patients from the pool of probable $\mathrm{AD}$ patients at the $\mathrm{UC}$ Davis Alzheimer's Disease Research Center and 13 normal control subjects of similar age and education. Idea density was analyzed using a manual that was developed by the authors building on methods described by Kemper (1993). A battery of standard neuropsychological measures and a newly developed informant-based measure of everyday cognition (ECog, Farias et al, 2006) were collected for all participants. Informants for ECog were persons with frequent contact with participants. Data collection and analysis were done blind.

Results: Initial analysis indicates that, after adjusting for age, the idea density in the narratives of $\mathrm{AD}$ patients is lower than in healthy elders ( 3.715 vs. $4.025, \mathrm{~F}=7.6, \mathrm{p}=.01)$. Moreover, idea density correlates with ECog-based ratings of everyday language $(\mathrm{r}=-.50, \mathrm{Z}=-2.3, \mathrm{p}=.01)$ and category fluency $(\mathrm{r}=.55, \mathrm{Z}=2.7, \mathrm{p}=.007)$ but not ECog measures of everyday memory or episodic memory $(r=-.230$ and .376 , respectively, ns).
Conclusions: Idea density appears to be a sensitive measure of language decline in $\mathrm{AD}$ compared to normal control subjects. Importantly, declines in idea density are also associated with informant ratings of everyday language.

Correspondence: Kathleen Baynes, Ph.D., Center for Neuroscience, University of California, 1544 Newton Court, Davis, CA 95616. E-mail: kbaynes@ucdavis.edu

\section{Paper Session 17 9:15-10:15 a.m.}

\section{Frontal Systems \& Executive Functioning}

A. SISTIAGA, I. URRETA, M. JODAR, C. DOMÍNGUEZ, I. BEITIA,
J. EMPARANZA, A. COBO, J. MARTÍ-MASSÓ \& A. LÓPEZ DE MUNAIN. Neuropsychological Profile in Myotonic Dystrophy (DM1). Objective: DM1 is a slowly progressive miopathy, characterized by a variable multisystemic affectation. Molecular basis of this autosomal dominant disease is an unstable and pathological (CTG)n repeat located on chromosome 19. Central nervous system involvement in DM1 includes cell loss, atrophy, focal white matter lesions and reduced blood flow, affecting particularly frontal, parietal and subcortical areas. Previous reports in DM1 have described main deficit on executive functions and visuocosntructive abilities. The objective of this study is to describe cognitive impairment in DM1, and to value the weight of variables such as patient age, molecular defect or progenitors sex on cognitive function.

Participants and Methods: 104 molecularly confirmed DM1 patients and 41 control subjects were assessed using a comprehensive neuropsychological examination.

Results: Patients performed significantly worse than controls in all test measuring executive function, excepting verbal fluency. Difficulties on attention, response inhibition, abstract reasoning and planning were detected. Patients also performed significantly worse on processing speed and visuospatial abilities. Significant negative correlation with CTG expansion size was found on most of the neuropsychological assessment and patients who had maternal inheritance showed lower Intelligence Quotient (IQ) than those who inherited DM1 from their father. In DM1, 34\% of the IQ can be explained by the expansion size and the educational level.

Conclusions: We confirm dysexecutive, visuospatial and processing speed impairment in DM1 suggesting fronto-dorsolateral and subcortical involvement. Patient expansion size and educational level play an important role on DM1s cognitive function outcome.

Correspondence: Andone Sistiaga, Hospital Donostia, Unidad Experimental, Hospital donostia; P0 Dr. Beguiristain s/n, San Sebastian 20.014, Spain.E-mail: asistiaga@chdo.osakidetza.net

J.C. FLORES LAZARO \& F. OSTROSKY-SOLIS. Development of Neuropsychologycal Functions related to theAnterior Prefrontal Cortex. Objective: Anterior prefrontal cortex is the most recent frontal lobes area that appeared in human brain evolution. Cognitive functions depending of this frontal lobes region represents the highest hierarchy in cognitive processing.

Objective: to know the developmental characteristics of three executive functions related to the anterior prefrontal cortex: abstraction, metacognition and proverb comprehension, between 6 and 30 years old.

Participants and Methods: 200 normal subjects ranging from 6 to 30 years old, divided in 5 groups: 6-8,9-11,12-14, 15-17 and 18-30 years old. The Frontal lobes and Executive function battery (Flores and Ostrosky 2005) was used to evaluate this processes. To analyze and compare the results one way ANOVA has been applied.

Results: We found that Abstraction measured by the number of spontaneous abstract categories produced, presents an extended and differentiated development: a slow development during childhood, accelerating its development during adolescence and reaching maximum performance 
until the 18-30 years old range. Metacognition indicate differential stages according to the different age-range, overestimation in the 6-8 and 9-11 age range, executive performance in the 12-15-age range and metacognitive performance in the 15-17 and 18-30 age ranges. Indicating that different frontal lobes areas participate during development of this capacity. Proverbs comprehension presents a very slow development during the firsts age ranges reaching maximum performance until the 18-30-age range.

Conclusions: Results indicate that these functions presents different characteristics and a larger developmental span than other executive functions previous reported. Results are discussed according to neurobiological data on the sequential development of APFC.

Correspondence: Julio Cesar C. Flores Lazaro, Candidate to PhD, Psychology, National Autonomous University of Mexico, UNAM, Ave. Universidad 1953, edif 6, depto 302, Mexico City 04510, Mexico. E-mail: j_neuro@yahoo.com.mx

\section{A.R. GIOVAGNOLI.The Characteristics of Theory of Mind in Patients} with Frontal Variant Frontotemporal Dementia.

Objective: Recent studies suggest that theory of mind (ToM) deficits may be an hallmark in the early course of frontal variant frontotemporal dementia (fvFTD). This study was thought to make a qualitative analysis of ToM impairment in this condition.

Participants and Methods: Fourteen patients with fvFTD, selected according to clinical and neuroimaging criteria, were compared with 14 healthy subjects with the same age and education. ToM was tested using the Faux Pas task that requires the recognition of social faux pas in 20 stories. Analysis of performance included: (i) identification of the person making a fax pas; (ii) ability to appreciate the appropriateness of behaviour; (iii) comprehension of the intentionality; (iv) identification of feelings; and (v) story comprehension. Other neuropsychological tests assessed executive functions, language, memory, praxis, and visuospatial abilities.

Results: One-way ANOVAs, with verbal comprehension, abstract reasoning, episodic and working memory as covariates, revealed that fvFTD patients were significantly impaired in the non-faux pas stories, showing false positive errors, while they obtained adequate total scores in the faux pas stories. Qualitative analysis of single aspects showed significant deficits except for story comprehension.

Conclusions: The impairment of fvFTD patients in ToM is characterized by deficits in understanding others' intentions and feelings and in judging the appropriateness of others' behaviour, although they can guess social faux pas. False recognition errors may reflect poor ToM as well as impulsivity. These results indicate that different intrinsic facets contribute to ToM impairment in fvFTD, explaining loss of empathy and altered interpersonal conducts.

Correspondence: Anna R. Giovagnoli, MD, Clinical Neuroscience, "C. Besta" National Neurological Institute, Via Celoria 11, Milano 20133 , Italy.E-mail: rgiovagnoli@istituto-besta.it

I. BOMBIN, A. BILBAO \& T. LEGIDO. Dysexecutive versus Frontal Syndromes: Clinical Implications and Outcome Relevancy.

Objective: Executive functioning (EF) has been attributed a fundamental role in self-regulation and monitoring of intentional behavior, as well as in sustaining functional independence. (Pre)frontal areas have been postulated as the neuroanatomical substrate of EF (frontal syndrome). However, there is growing evidence that the localization of the brain insult is not such relevant as the clinical-cognitive profile (dysexecutive syndrome) in predicting functional outcome.

Participants and Methods: Seventy-eight patients with acquired brain injury $(\mathrm{ABI})$ were assessed at discharge of a comprehensive rehabilitation program by means of a neuropsychological battery that included measures of EF, attention, memory, working memory, and overall cognitive functioning. The sample was divided in three subgroups attending to the localization of the brain impairment: a) (pre)frontal areas alone $(\mathrm{n}=37) ; \mathrm{b})$ mixed areas $(\mathrm{n}=24)$; and c) non-frontal impairment ( $\mathrm{n}=17)$. Six months after discharge, functional independence was measured by the CHART. EF impairment and functional independence among the three subgroups was compared by means of an
ANOVA. Stepwise lineal regression and general lineal models were performed to determine the extent of variance of functional independence explained by either EF measures or anatomical localization.

Results: There were no major differences in the degree of EF impairment and post-discharge functional independence among the three subgroups. The variance of post-discharge functional independence explained by EF impairment was by far higher $(43 \%-75 \%)$ than that explained by the neuroanatomical localization of impairment $(0 \%-5 \%)$.

Conclusions: According to our results, a clinical description (dysexecutive syndrome) is closer to the real-life EF construct than a localizationistic one (frontal syndrome).

Correspondence: Igor Bombin, PhD, Neuropsychology, Reintegra, Centro de Rehabilitacion Integral, C/ Eduardo de Fraga Torrejon 4, bajo, Oviedo 33011,Spain.E-mail: ibombin@mce.hggm.es

\section{Paper Session 18 9:15-10:15 a.m.}

\section{Pediatric Traumatic Brain Injury}

S.S. LAH, W. LEVICK, A. EPPS, L. PARRY \& L. AZIZI. Implicit memory in children who have sustained severe traumatic brain injury: Preliminary findings.

Objective: Deficits in explicit memory have been well recognised in children who have sustained traumatic brain injury (TBI). Implicit memory outcome, however, has remained largely unknown. Implicit memory is believed to be supported by different neural structures and to be fully mature at an early age. Our study aimed to examine implicit memory in children with TBI using a developmentally sensitive task.

Participants and Methods: The study included children who had sustained TBI $(n=11)$ and control subjects $(N C, n=6)$. Inclusion criteria for all children were: (i) age at assessment 6 to 16 (ii) fluent in English (iii) IQ $>70$ (iii) no pre-existing difficulties. For the TBI group, only children who had sustained severe TBI over 24 months prior to testing were included. The groups were matched on age at assessment and family socio-economic status. All subjects were administered a battery of neuropsychological tests, including a developmentally sensitive implicit memory task that contained two sets of fragmented pictures and two conditions (study and test).

Results: We found a significant interaction on the implicit memory task $(p=.046)$. There was no difference in the identification thresholds of the two groups in the study condition. Both groups showed lower identification thresholds for the previously studied items in the test condition, but the improvement was much greater for the NC group. This indicates implicit memory deficits were present in children with TBI, as measured by the picture fragment completion task.

Conclusions: Our study is the first to find evidence of implicit memory deficits in children who have sustained severe TBI.

Correspondence: Suncica S. Lah, PhD, MSc, BA(Hon), Psychology, Univerisity of Sydney, Mungo Mac Callum Building (A19), Sydney, NSW 2006, Australia.E-mail: sunnyl@psych.usyd.edu.au

S.E. WALL, W.H. WILLIAMS \& I. FRAMPTON. The Assessment of Empathy and Theory of Mind Following Childhood Brain Injury and the Implications for Neurorehabilitation.

Objective: Impairments in empathising and constructing a theory of mind are well documented in the adult acquired brain injury (ABI) literature. However, there is a paucity of such research in children and adolescents, despite the critical developments in social-emotional functioning that occur across this period. In the current study we explored the effects of childhood ABI on empathising and theory of mind seen in early adolescence.

Participants and Methods: Twenty-five adolescents with ABI of varying aetiologies were assessed. Fifty typically developing adolescents matched on age and gender acted as controls. Each child completed a series of assessments, including naturalistic and parent- and self-report measures of social-emotional function. 
Results: The ABI sample showed global social-emotional impairments relative to the controls. The ABI sample was less able to generate appropriate empathic responses to a character in auditory vignettes. They demonstrated less accurate theory of mind responses to an audio-visual conversation between two adolescents. Those injured between the ages of 2- and 6-years had a greater percentage of borderline or impaired performance on the social-emotional measures than those injured before the age of 2-years or after the ages of 6-years, particularly on the Empathy task. Associations between these social-emotional skills, executive functioning and emotion recognition were also investigated. Individually designed intervention strategies were explored with a small subset of the ABI sample with an $n=1$ design, to further explore these associations. Conclusions: Impairments in key components of social-emotional functioning are evident after childhood brain injury, which have implications for daily functioning.

Correspondence: Sarah E. Wall, School of Psychology, University of Exeter, Washington Singer Laboratories, Perry Road, Exeter EX4 4QG, United Kingdom. E-mail: s.e.wall@ex.ac.uk

H. MILLER, S. MATTYS, R. MCCARTER \& P.M. SHARPLES. Relationship Between Linguistic and Cognitive Functioning in Children Who Have Sustained Traumatic Brain Injury (TBI) Compared With that Seen in Non-injured Controls.

Objective: There is increasing evidence that language competence is compromised by paediatric traumatic brain injury (TBI). Psychologists differ concerning whether the language faculty is autonomous or derives from cognition. By relating linguistic outcome to contemporaneous cognitive data we may begin to identify the key mechanisms that underlie such sequelae. The aim was to investigate relationships between language and cognition in TBI and non-injured children.

Participants and Methods: Longitudinal prospective cohort study. 42 children (26 TBI; 16 controls) were studied. 29 (69\%) were boys. Mean age was 12.15 years (SD 3.5). Language was assessed by the Wechsler Objective Language Dimensions, Test for Reception of Grammar and the Diagnostic Analysis of Non-Verbal Accuracy > 12 months post-TBI. Cognition was assessed by the WISC-III. Test of Everyday Attention in Children and Children's Memory Scale.

Results: Analyses show significant differences between TBI and controls with respect to semantic processing $(p=0.025)$ and auditory decoding ability $(p=0.017)$, but not grammatical ability $(p=0.329)$, and visual decoding $(p=0.712)$. In TBI children, further analyses showed semantic processing was positively correlated with age $(\mathrm{p}=<0.001)$, verbal IQ $(\mathrm{p}=0.001)$, verbal immediate and delayed memory $(\mathrm{p}=0.03 ; \mathrm{p}=0.01)$; auditory decoding was positively correlated with performance IQ $(p=0.019)$, verbal immediate memory $(p=0.01)$, attention $(p=0.023)$. In controls, semantic processing correlated with verbal IQ $(p=0.03 ; p=0.005)$.

Conclusions: TBI children had poorer semantic processing skills and auditory decoding abilities. Results show a significant relationship between semantic processing, auditory decoding and aspects of cognition. More efficient language skills associated with better cognition suggests linguistic imparments after TBI are at least inpart due to cognitive dysfunction. Understanding the underlying mechanisms of this relationship is essential for effective neuro-rehabilitation.

Correspondence: Helen Miller, Neuropsychology, Frenchay Hospital, Room 1, Academic Centre, Frenchay Hospital, Bristol BS16 1LE, United Kingdom.E-mail:helen.miller@nbt.nhs.uk

M.R. NEWSOME, R.S. SCHEIBEL, J.V. HUNTER, Z.J. WANG, Z. CHU, X. LI \& H.S. LEVIN. Brain Activation during Working Memory after Traumatic Brain Injury in Children.

Objective: We hypothesized that moderate to severe traumatic brain injury (TBI) would impact developing frontal neural circuitry mediating working memory and be reflected by diminished prefrontal brain activation and working memory capacity during an N-back task in children with TBI relative to typically developing (TD) children. In contrast, we postulated that children who developed age-appropriate working mem- ory despite having sustained TBI would exhibit activation comparable to TD children.

Participants and Methods: Eight children (11-15 years) with moderate to severe TBI were individually matched with 8 TD children on age, handedness, gender, and highest $\mathrm{N}$-back level obtained at 70\% criterion. One to three runs of a block design N-back task (0- vs. 1-back, 0- vs. 2back, and 0-vs 3-back) were administered in the scanner, depending on which levels of the task the child performed at criterion during training, and changes in BOLD signal in 6 regions of interest were measured: lateral frontal, medial frontal, and nonfrontal, bilaterally.

Results: Despite no group difference in extent of brain activation, patients whose criterion performance was reached at lower memory loads than their matched TD control counterparts demonstrated less extensive frontal and extrafrontal brain activation than controls. Patients who performed the same memory load (3-back) as controls demonstrated more frontal and extrafrontal activation than controls.

Conclusions: Results may be explained by differential diffuse axonal injury to prefrontally-guided, distributed networks and encourage longitudinal fMRI investigation of patterns of brain activation and associated cognitive performance in larger samples of children sustaining TBI.

Correspondence: Mary R. Newsome, PhD, Physical Medicine \& Rehabilitation, Baylor College of Medicine, 1709 Dryden Ave., Ste 725, Cognitive Neuroscience Laboratory, Houston, TX 77030. E-mail: mnewsome@bcm.edu

\section{Poster Session 7 Stroke, Cognitive Rehabilitation, Language 9:15-11:15 a.m.}

\section{Agnosia/Disordered Representations}

T. NIJBOER, G.M. NYS, M.J. VAN DER SMAGT \& E.H. DE HAAN. Brightness Agnosia: a Disorder of Bright- and Darkness Recognition. Objective: We report a patient with a selective impairment in recognising bright- and darkness in absence of low-level sensory-perceptual deficits. To the best of our knowledge, no case of "brightness agnosia" has been reported in literature so far. The aim of the present study is to describe this patient and to examine several aspects of bright- and darkness recognition and matching in more detail.

Participants and Methods: Patient LZ is a 66-year old right-handed woman, who suffered an acute subarachnoid haemorrhage. The ruptured aneurysm was succcesfully clipped, but she developed severe vasospasms which resulted in a large ischemic infraction of the right hemisphere involving the parietal and temporal lobes with extensions into the occipital and frontal regions. LZ shows signs of left-sided neglect and hemiparesis. Her husband told us that she has additional problems with indicating whether lights in a room were switched on or off. We investigated her basic visuo-sensory functions (shape, luminance, colour, and motion), and subsequently her higher-order visual skills, specifically her ability to appreciate or recognise brightness.

Results: Acuity as well as luminance matching was normal, suggesting that her brightness impairment is not a mere consequence of low-level sensory impairments. She was not able to indicate the darker or the lighter of two squares, even though she was able to see that they differed. In addition, she could not indicate whether the lights in a room were on or off, nor was she able to differentiate between normal greyscale images and inverted greyscale images. As the patient recognised objects, colours, and shapes correctly, the impairment appears to be specific for brightness. Conclusions: Our findings suggest that recognition deficits can occur for a single visual primitive in isolation, even in the absence of low-level visuo-sensory deficits. So far, this is the first case of "brightness agnosia" reported in literature.

Correspondence: Tanja Nijboer, Psychological Laboratory, Utrecht University, Heidelberglaan 2, Utrecht 3584CS, Netherlands. E-mail: t.c.w.nijboer@fss.uu.nl 


\section{Apraxia/Motor Sequencing}

M. VAN ZANDVOORT \& E. DE HAAN. Aphemia after a Right Hemisphere Stroke Due to Generalised Serial Order Impairment. Objective: This case study reports a patient who, after a large right hemisphere stroke, presented with muteness followed by severe persistent speech deficits.

Participants and Methods: A 40 old year right-handed male suffered from a large ischaemic stroke in the right hemisphere in the distal supply area of the MCA resulting in extended superficial cortical damage. After the stroke, he presented with a left hemiplegia. More surprising was his complete muteness the first days, whereas he was alert and perfectly able to act on verbal instruction. He seemed to comprehend what he was told, and demonstrated no signs of apathy or (buccofacial) apraxia. He underwent extensive neuropsychological examination and was followed in the first year.

Results: Neuropsychological evaluation on the ward demonstrated neglect and memory impairments. He had no spontaneous speech, but when questioned he was able to reliably respond yes and no. On a confrontation naming task he named 20 out of 20 items correctly, but he had severe difficulties in pronouncing words that contained more than 2 syllables. After the second syllable, he abruptly stopped and fell silent, unable to finish the word. The same behaviour was found when he had to read out loud, repeat words, or imitate sounds and non-words; he started off all-right but stopped after the second syllable or (monosyllabic) word. The suggestion of a serial order deficit (for sequences exceeding 2 items) was tested in the non-verbal domain. The results showed that the serial order problem was equally present when he was asked to repeat motor sequences with his (right) hand. Again the impairment was strikingly present on series entailing more than 2 items.

Conclusions: Right (frontal) cortical damage may give rise to severe generalised serial order impairments resulting in profound communication disturbances as well as motor sequencing problems. These findings will be discussed in the light of working memory, mental representation buffers and motor initiation functions of the (pre)SMA

Correspondence: Martine van Zandvoort, PhD, Utrecht University, Heidelberglaan 2, Utrecht NL-3584 CS, Netherlands. E-mail: $m$. vanzandvoort@fss.uu.nl

\section{Cognitive Intervention/Rehabilitation}

J.H. BULTITUDE \& R.D. RAFAL. Can Adaptation to Vertically Shifting Prisms Ameliorate Gait Disturbance? A Preliminary Investigation using Healthy Participants.

Objective: Adaptation to laterally displacing prisms can improve the performance of patients with hemispatial neglect on tasks such as horizontal line bisection (Rossetti et al., 1998), and ameliorate their postural imbalance (Tilikete et al., 2001). As yet, there has been no investigation of the effects of adaptation to vertically shifting prisms on spatial or postural impairments. We report preliminary research aimed at investigating whether such vertical adaptation could be applied to the treatment of gait disturbance stemming from postural imbalance.

Participants and Methods: Twenty healthy participants bisected 20 long vertical lines (195mm to $392 \mathrm{~mm}$ ) from a standing position before and after prism adaptation. During the adaptation phase participants walked for 15 minutes while wearing prisms that shifted the visual field upwards or downwards by $15^{\circ}$.

Results: A repeated-measures ANOVA revealed differential effects of upwards and downwards shifting prisms: Adaptation to upwards shifting prisms resulted in a downwards shift in bisection error, while there was no change in bisection performance for downwards shifting prisms.

Conclusions: This research is the first to demonstrate changes in vertical line bisection performance following adaptation to vertically shifting prisms. These results have potential implications for understanding both the effects of prism adaptation on spatial attention and on gait disturbance.
Correspondence: Janet H. Bultitude, University of Wales, Bangor, Brigantia Building, Penrallt Rd, Bangor, Gwynedd LL57 2AS, United Kingdom.E-mail:psp230@bangor.ac.uk

S.M. ENCINAS, G. TOMÉ, N. CERULla, D. BADENES \& M. AGUILAR. Formative Groups: a formative and therapeutic resource for caregivers of mild dementia patients.

Objective: Caregivers of mild-dementied patients always needs information. Months after diagnostic this necessity increase. Formative groups are a resource, help, and therapeutic mesure.

We want to demonstrate that a formative groups for caregivers of patients with dementia diagnosed recently can improve the knowledge about dementia, burden, anxiety and their quality of life.

Participants and Methods: 84 caregivers of dementied patients participated in formative groups (four sessions, $2 \mathrm{~h}$, monthly form). Formative groups were designed according to the "Programa Europeo Formación Promoción Salud 1997" methodology and directed by a neuropsychologist or a social worker. The 84 caregivers were evaluated before and after their participation on the formative groups using the following test batteries: Zarit Scale (burden of caregiver), STAI (anxiety), EuroQuol (health state) and DSQoL (quality of life) and we also evaluated the self-perception of their knowledge about dementia and care (knowledge test). Finally, we compared the results by statistic analysis.

Results: Burden of caregiver decrease from 32,16 to 30,69 ( $p<0,001$ ): reduce the anxiety mesured by STAI (Trait: from 68,24 to 53,$29 ; \mathrm{p}=0,002$; State: from 52,68 to 41, 97; $p<0,001)$. However, general health state evaluated with Euro-Quol decrease: from 6,67 to 6,26; $<<0,0001$. Quality of life mesured with DSQoL improve from 10,88 to 9,$53 ; p<0,0001$. Knowledge level about dementia: increase in accordance to the course, specially the non pharmacological treatment of symptoms, but keep existing comprehension doubts about pharmacological treatment.

Conclusions: Formative groups for caregivers of mild dementia patients give a satisfactory information and formation. Reduce the anxiety and the burden of caregivers improving the quality of life and the health state. This formative resource must to be an obliged proposal in dementia treatment.

Correspondence: Silvia M. Encinas, neurology, Hospital Mútua de Terrassa, c/castell 25, Terrassa 08221, Spain.E-mail: silmunt@hotmail.com

G. ESPEL-TRIAS, A. JARNE, F. SASTRE, X. CARDONA \& M.I. GOMEZ-RUIZ. Application of a Neuropsychological Rehabilitation Program of the Executive Function in Patients with Schizophrenia.

Objective: In schizophrenics, the appearance of several cognitive deficits has been ascertained by numerous valid studies. These deficits affect different cognitive domains (e.g. attention, language, memory and, especially, executive function) and are associated with poor functioning in daily life. The objectives of this study are two: 1) to evaluate the efficiency of a executive training program (ETP) applied to people with schizophrenia. and 2) to evaluate the maintenance of the results after the program.

Participants and Methods: Nineteen schizophrenic patients were divided into two groups: the cognitive training group (CTG) (12 patients) and the control group (CG) (7 patients). All subjects underwent three cognitive evaluations (initial, 6 months, 12 months) that assessed symptoms, quality of life, functional performance and executive functions. The ETP lasted 6 months and included exercises of planning, problem solving and abstract reasoning.

Results: The CTG obtained better scores immediately after the ETP. The patients of this group partially maintained these results in the evaluation done 6 months after the finishing of the program. These positive results were mainly observed in the capacity of planning, mental flexibility, abstract reasoning and problem solving. An improvement was also observed in the rest of parameters that were evaluated. 
Conclusions: The results show the effectiveness of the cognitive training program that not only appeared immediately after the program, but with the 12-months evaluation. The effects of the ETP may have had a positive influence in the decreased of the schizophrenic symptomatology and, therefore, an increase of quality of life and functional performance.

Correspondence: Gemma Espel-Trias, Hospital General de L'Hospitalet, Avd. Josep Molins, 29-41, L'Hospitalet de Llobregat 08909, Spain. Email:gemma.ps@copc.es

A. GARCIA-MOLINA, R. SÁNCHEZ-CARRIÓN ABASCAL, N. PICÓ AZANZA, A. GARCÍA RUDOLPH, E. OPISSO SALLERAS, M. BERNABEU, J. TORMOS \& T. ROIG ROVIRA. Therapeutic effectiveness of a Computerized Cognitive Rehabilitation Program in neurorehabilitation of traumatic brain injury.

Objective: The main objective of neurorehabilitation is to enable patients with brain injury to return to their life roles and become active members of the community as near as possible to pre-injury levels of participation. The purpose of this study was to examine the therapeutic effectiveness of a Computerized Cognitive Rehabilitation Program (PREVIRNEC) in persons with traumatic brain injury (TBI).

Participants and Methods: Fourteen patients with moderate or severe TBI admitted to a Brain Injury Rehabilitation Unit. Mean age: $27 \pm 8.4$ years; time since injury: $11.7 \pm 5.4$ months. Patients participate in PREVIRNEC during 24 sessions. The difference between pre- and post-treatment neuropsychological test scores was used to measure improvements in the domains of attention, memory and executive functions. The impact of patients' cognitive impairment on activities of daily living was rated by their families.

Results: PREVIRNEC was found to be effective in improving attention. memory and executive functions. After the application of PREVIRNEC. significant performance improvements were found on Stroop Test $(p<0.016)$, sustained attention $(p<0.026)$, backward digits span $(p<0.025)$, verbal learning $(p<0.002)$, TMT B $(p<0.003)$, and WCST $(\mathrm{P}<0.011)$. Eleven families reported patient improvement in activities of daily living.

Conclusions: These preliminary results show that PREVIRNEC improves attention, memory, and executive functions. This cognitive recuperation has a positive influence on their functional outcome on daily life activities.

Correspondence: Teresa Roig Rovira, PhD, Neuropsychology, Institut Guttmann, Cami de Can Ruti s/n, Badalona 08916, Spain. E-mail: troig@guttmann.com

R. HARDY \& E. WALLIS. An Innovative Approach to the Cognitive Rehabilitation of Traumatic Brain Injury in Previously High Functioning Individuals.

Objective: To discuss through the use of detailed case studies, innovative approaches towards the cognitive rehabilitation of Traumatic Brain Injury (TBI) within previously high functioning individuals. Clinical experience has highlighted that traditional rehabilitation tools are ineffective, insufficient and lacking in depth of content when addressing the needs of this population.

Participants and Methods: A qualitative case study approach was used to evaluate the cognitive rehabilitation programs of eight previously high functioning patients with history of severe TBI. Severity of injury was classified by Glascow Coma Scale scores below eight and Post traumatic Amnesia exceeding four days. Innovative, specific and client-centred rehabilitation programs were developed to enhance the treatment efficacy of these patients.

Results: Context specific rehabilitation tools which were directly applicable to the patient's previous work environment and areas of interest proved more effective than traditional methods. Interest, motivation and enthusiasm was sustained which resulted in the patient's being more committed and engaged to the rehabilitation program. The specific and individualised nature of the tools allowed the patients to translate the treatment program into workplace efficiencies. Despite functioning at lower levels, these patients have successfully continued with gainful employment though not necessarily in the same areas as they were previously employed.

Conclusions: The use of an innovative, client-centred approach in the cognitive rehabilitation of previously high functioning individuals, demonstrates the potential of a personalised rehabilitation program which moves away from the traditional 'one size fits all' methodology.

Correspondence: Elizabeth Wallis, MSocSci, MAP, Brain Lab, Sunninghill Hospital, 4 Saxonwold Mews, Waltham Road, Johannesburg 2196, South Africa.E-mail:wallis@wallishardy.co.za

K.I. HODDER, C. HASLAM \& P.J. YATES. The Method of Choice in Memory Rehabilitation: Contributions of Errorless Learning, Vanishing Cues and Spaced Retrieval.

Objective: Three promising techniques used in memory rehabilitation are errorless learning, vanishing cues, and spaced retrieval. However their effectiveness has rarely been compared and not in a sufficiently controlled manner to draw meaningful conclusions. This research directly compared these techniques, both in isolation and in combination, under controlled conditions.

Participants and Methods: Healthy adults took part in a series of studies comparing the three methods for learning face-name associations. These participants learned information under standard and dual task conditions: the latter to facilitate comparison with patient performance. The effectiveness of the most promising techniques was subsequently confirmed with thirty patients with acquired brain injury.

Results: Analysis of recall performance showed that whilst errorless learning was effective to some extent, learning under conditions of spaced retrieval was most effective for both healthy adults and memory-impaired patients. Importantly, a combination of techniques proved no better than isolated techniques in any of the studies.

Conclusions: Spaced retrieval may be the method of choice in memory rehabilitation and deserves further attention. This research also challenges the assumption that memory performance is improved by combining several techniques and encourages a more targeted approach to memory rehabilitation.

Correspondence: Kathryn I. Hodder, School of Psychology, University of Exeter, Washington Singer Laboratories, Perry Road, Exeter EX4 4QG, United Kingdom. E-mail:K.I.Hodder@Exeter.ac.uk

\section{A. MACKAY. Dual-Task Training in Older Adults.}

Objective: One of the most challenging everyday cognitive tasks is to juggle more than one task at a time. Research focused on the effects of training to improve performance on multiple tasks in young and older adults (Bhrer, et al., 2006; Kramer, et al., 1999) has demonstrated training-related gains that also transfer to novel tasks. Thus, training was not specific to the task at hand, but generalized to new tasks. There is evidence that the form of training can influence the magnitude of the improvement. In a study described by Kramer (1999), older adults' performance improved to a level similar to that of younger adults when the training required them to vary the prioritization of the two tasks (2080, 40-60, 50-50 vs. simply 50-50).

Participants and Methods: Our aim was to replicate these findings in an independent study using conceptually similar tasks. Preliminary results indicate improved performance for 7 older adults (aged 62 to 85 years) in both speed of response and accuracy over 5 days of training. Results: Speed for task A: F $(4,24)=5.03, \mathrm{p}<.01, \mathrm{n} 2=.46$; Speed for task B: $\mathrm{F}(4,24)=3.37, \mathrm{p}<.05, \mathrm{n} 2=.36$. Accuracy for task $\mathrm{A}: \mathrm{F}(4,24)=$ 7.80, $\mathrm{p}<.05, \mathrm{n} 2=.57$; Accuracy for task $\mathrm{B}: \mathrm{F}(4,24)=8.30, \mathrm{p}<.001$, 
$\mathrm{n} 2=.58$. Prioritization condition $(20-80,40-60,50-50,60-40,80-20)$ did not correlate with time spent on each task across individuals.

Conclusions: Older adults demonstrated substantial gains with training. Priority may not be modulating performance as expected. Further study is needed to determine the key elements required for training and transfer of cognitive skills.

Correspondence: Anna MacKay, M.A., Psychology, Washington University in St Louis, One Brookings Drive, MB 1125, St Louis, MO 63130. E-mail: ajmackay@artsci.wustl.edu

A. MORERA, O. GELONCH, N. CERULLA, E. TALLANT, S. RAMOS, G. CHICO \& M. AGUILAR. Traumatic Brain injury by precipitation. Benefits of a neuropsychological rehabilitation associated to a program of social integration.

Objective: An individual long-lasting treatment based on a cognitive and social approach can help to recover the focal cerebral injury caused by traumatic lesions.

Participants and Methods: The patient: male, 57 years old, professional climber, right-handed, high level of education. He suffered cerebral damage after fall in 1980 with apparent total clinical recovery. In 2000 , he fell down again.

In 2004, the patient still had serious sequels: personality disorder, functional dependency to third and inpatient care. In the cognitive area, the patient showed a serious anosognosia, failures in immediate and delayed verbal and visual memory, attention capacity impairment, and a slowing of the speed of information processing, language and executive functions.

During three years, the patient has received a personal rehabilitation program that includes weekly one-hour sessions of cognitive stimulation. He has also participated in a working group whose three most common areas of activity are social, cultural and recreative activities.

Results: At present, the patient has partial anosognosia, reduced of the speed of information processing, inmediate memory impairment and both attentional capacity and language expression deficit.

Conclusions: The patient, as well as his family and medical team, took a turn for the better. The program had important benefits, as we saw several improvements in behavioral disorder. This program has also had an influence over recovering functional capacity and independence, the cognitives capacities of delayed verbal memory, visual and spatial capacities and executive functions. He continues taking part in a reinsertion and participation program.

Correspondence: Anna Morera, AVAN, C/Antoni Maura 3, 1-4, Terrassa 08225, Spain. E-mail: annamorera@yahoo.es

A. OTERO, F. OTERO, M. LAVALLÉE, M. PÉPIN \& M. LORANGER. Development of Cognitive Abilities: An Educational Experience.

Objective: This project aims at increasing cognitive and learning abilities in primary school children through their participation to a cognitive training program (REEDUCA) in which some meta-cognitive exercises are added.

Participants and Methods: Following a Pre-test-Intervention-Post-test type of design, 160 Mexican children from 1st to 6th grade of primary school have participated to the Reeduca program, exercising five cognitive abilities: Attention, Memory, Logical Reasoning, Visual-space Representation, and Mental Calculus, along with some metacognitive exercises.

Results: Significant positive changes are observed between initial and final evaluations in the five cognitive abilities examined, at all six grade levels. The improvement is in large part due to the application of the structured and systematized intervention program. Besides, important attitudinal changes such as diminution of impulsiveness, increase of reflection and strengthening of self-conception have been registered.

Conclusions: At the primary school level, cognitive and learning abilities are likely to be improved and enriched with suitable application of structured and systematized intervention programs. To add metacogni- tive activities to such programs, also brings along other effects such as attitudinal changes toward all learning process.

Correspondence: Araceli Otero, Psychology, Universidad Nacional Autonoma de México, Corregidora 143, México 1200 D.F., Mexico. Email:aoteroalva@att.net.mx

\section{TRINIDAD. Neuroconstructivistic Intervention for Attention Deficit Hyperactive Disorder.}

Objective: The Neuroconstructivistic Intervention for Attention Deficit Hyperactive Disorder (ADHD) considers the complex and dynamic interaction between internals dispositions or cognitive functions of the human being and his environment to the understanding of the disorder. The key aspects of the intervention are the neuropsychological functions within the context of its manifestations. The model's interventions are presented according to the child needs and interest considering the interaction with the environment and combining the participation of parents and teachers. The modules of intervention are: "Self awareness and Our awareness" (child and child-family-school interactions working on metacognition and selfregulation of affect and behavior), "Bending" (flexibility, persistence, and initiation/inhibition), "Focus" (attention and working memory), Strategy (planning).

Participants and Methods: 25 children from 8-10 yrs participated in the program. They were previously assessed. Inclusion criteria: ADHD any type. Exclusion criteria: any pervasive disorder or conduct disorder, and child in ADHD medication. The child's need expressed in the assessment set the starting module. The modules begin with individual and family/teacher's interventions then peers interventions. Always considering the child context or environment where the skills will be manifested. This intervention consists approximately: 12-15 sessions.

Results: Cognitive functions such as: attention working memory, planning mental flexibility, self regulation and metacognition were significantly improved in an ecological way. Strategies of intervention and case example will be addressed.

Correspondence: Laura Trinidad, PhD, Trinidad Institute for Neuropsychological Studies, Calle 11 I-29, Flamboyan Gardens, Bayamon 00959, Puerto Rico. E-mail: lauratrinidadphd@gmail.com

M. VINK, R. HORST, M. LUIJPEN, K. MOLLEMAN, A. ZWOLS \& A. BOUMA. Changes in Executive functions after Holistic Neuropsychological Rehabilitation.

Objective: Goal of the study is to investigate changes in executive functions after participation in a holistic neuropsychological rehabilitation program.

Participants and Methods: Participants are a group of patients $(\mathrm{N}=61)$ with chronic brain injury who participated in a 16-week intensive holistic group program. Patiens and their relatives were assessed before participation in the program, after completing the program, one year later and three year later. Assessment involved neuropsychological testing and questionnaires on executive functioning.

Results: Paired samples t-tests were used to compare pre- and post-treatment scores, pre-treatment scores and one-year follow-up scores as well as pre-treatment and three-year follow-up scores. The SPSS-PC program was used for statistical analysis.

There were no significant changes in the scores of neuropsychological testing. Concerning the questionnaires, significant changes were found in cognitive and socio-emotional aspects of executive functioning (subscales initiative, independant behaviour, self-control and interpersonal behaviour.

Conclusions: After participation in a holistic neuropsychological rehabiliation program, there seem to be positive changes in executive functioning, as perceived by patients and their relatives. The perceived changes, as judged by the patients, are more positive, compared to their relatives. One and three year later, these changes are maintained.

Correspondence: Martie Vink, Drs, Rehabilitation Center Amsterdam, Overtoom 283, Amsterdam 1054 HW, Netherlands. E-mail: m.vink@RCAmsterdam.nl 


\section{Language: Aphasia}

V. BALASUbRAMANiAN, L. MAX \& M. COSTEllo. Auditory Comprehension of Lexical versus Deep Structure Ambiguities in Three Groups of Brain Damaged Adults.

Objective: This study verifies the prediction that damage to anterior or posterior areas of the left hemisphere (ALH, PLH) or damage to the right hemisphere (RH) will not result in significant differences among the brain damaged groups in auditory comprehension of sentences with lexical, or deep structure ambiguities (LA \& DSA).

Participants and Methods: Eight normal controls (NO), seven right hemisphere damaged (RH), eleven anterior left hemisphere damaged (ALH) and eight posterior left hemisphere damaged patients served as subjects in this study. A set of 20 ambiguous sentences, 10 in each type was pooled from previous studies. A set of four pictures was drawn for each sentence (two of them truly represented the two meanings of the target sentence, one shared some aspects of the meaning and the other was quite unrelated to the target sentence. On hearing each stimulus sentence the subject was to point to the two pictures from among the four that represented the two meanings of the sentence. Each correct response (pointing to two appropriate pictures for each sentence) was given one point.

Results: Multiple comparison tests (Games-Howell) at the alpha $=0.05$ level of significance indicate that on test DSA the normal scores were significantly higher than those of the other groups, but the other three groups were not significantly different from each other. On the LA test, however, the NO scores wre not significantly different from the RH, PLH. and ALH scores.

Conclusions: The performance of the experimental groups on the DSA task supports the prediction based on reviews of research.

Correspondence: Venu Balasubramanian, Ph.D, Speech-Language Pathology \& Audiology, Seton Hall University, McQuaid Hall, 400 South Orange Ave, South Orange, New Jersey, NJ 07079. E-mail: balasuve@shu.edu

S.F. CASTRO, I. GOMES \& S. CALÓ. Portuguese Version of Psycholinguistic Assessments of Language Processing in Aphasia, PALPA-P.

Objective: The test battery PALPA, from Psycholinguistic Assessments of Language Processing in Aphasia (Kay, Lesser, \& Coltheart, 1992), provides a set of 60 tasks designed to closely examine four aspects of language function: phonological processing, reading and spelling, sentence comprehension, and word / picture semantics. Since it was originally published in English, it has been adapted to other languages, such as Hebrew and Spanish. The goal of the present work was to adapt it to Portuguese (European variant).

Participants and Methods: The characteristics of Portuguese, with a special emphasis on phonotactics and orthography, were taken into account to select words, pseudowords, and sentences that varied according to the same psycholinguistic variables as the original stimulus materials, and that were analogously controlled for frequency, imageability, length, and semantic content. Pictures used as stimuli (for rhyme judgement and picture naming) were created anew, since they had to correspond to the selected Portuguese words. Preliminary versions of the tasks were presented to college students to evaluate task instructions and materials. Then, the revised versions of the tasks were presented to selected groups of healthy subjects - young adults, older adults, and children, $\mathrm{N}$ per group $=$ at least 40 .

Results: The 60 tasks composing the original PALPA are now available in the Portuguese language, including descriptive statistics for each task and healthy control group.

Conclusions: Since it is based on the same principles as PALPAs in other languages, and in the strict sense adapted to Portuguese, PALPA-P may be a valuable tool for cross-language research on language and aphasia. Correspondence: São Luís F. Castro, PhD, University of Porto, Faculdade Psicologia e CE - UP, Porto 4200 - 392 Porto, Portugal. E-mail: slcastro@fpce.up.pt
J. EL KADIB \& J. GARCIA ORZA. Cognitive Linguistic Assessment for Arabic-Speaking Patients in Morocco: Presentation of a Battery. Objective: The need to develop assessment tools based in our knowledge about the cognitive processes involved in the evaluated behavior has been evident in the nineties. This conducted to the development of tests like EPLA, BORB, VOSP and others. The evaluation of language impairments in Arabic speaking patients in Morocco is difficult because the batteries commonly used are non-standardized direct translations of classical test and are not based on cognitive theories. This study sought to develop a standardized battery aimed to assess the linguistic alterations of neurological origin in Arabic speaking patients from Morocco.

Participants and Methods: Following a cognitive point of view of linguistic processing the battery comprises 13 tasks aimed to evaluate oral language both at the word and sentence level. Comprehension processes are assessed through minimal pair discrimination with words and nonwords, auditory lexical decision, associative matching, word-picture matching and sentence-picture matching tasks. Production is evaluated by means of object and action naming, repetition with words and nonwords and sentence production tasks. Verbal working memory and praxias are assessed with complementary tasks. The application of the test takes about forty five minutes. Data from 42 healthy adults are provided as reference group. The case of $\mathrm{N}$, a Moroccan 27 years old aphasic patient, who suffered damage to the left frontal and parietal areas, is also reported.

Results: The evaluation demonstrated that $\mathrm{N}$ shows alterations of the speech output processes restricted to the phonological output lexicon and articulatory difficulties confined to some fricative and velar phonemes. Conclusions: The data suggest the usefulness of the battery in diagnosing aphasic impairments from a cognitive point of view.

Correspondence: Javier Garcia Orza, Ph D, Psicologia Basica, Universidad de Malaga, Campus Teatinos s/n, Malaga 29071, Spain. E-mail:jgorza@uma.es

G. LEAL, M. AGUIAR, I. FONSECA, L. FARRAJOTA, J. FONSECA, L. GONCALVES, M. LAUTERBACH \& I. PAVÃO MARTINS. Intensive vs Conventional speech therapy in aphasia due to ischaemic stroke: a randomized controlled trail.

Objective: There are few randomized controlled trails on the efficacy of speech therapy for aphasia rehabilitation. As a consequence of that the evidence on the efficacy of different modalities of therapy is lacking or insufficient.

Participants and Methods: We conducted a randomized controlled trial comparing intensive speech therapy versus conventional therapy for an total equal number of hours of therapy. The study was conducted in two centres and the outcome measures were evaluated by an examiner blinded for the therapeutic intervention. Inclusion criteria were: a single ischaemic stroke of the middle cerebral artery, in the first 12 weeks post onset. ages between 40 and 80 years, a minimum of four years of literacy and an aphasia quotient (AQ) between 10 and $75 \%$. Patients were evaluated with the Aachen aphasia battery and the Lisbon aphasia battery. Outcome measures were: the $A Q$ and the functional communication profile (Sarno, MT., 1969).

Results: From a total of 500 patients with aphasia had been observed in both centres in two years of study only 15 patients fulfilled the inclusion criteria and agreed to participate ( 4 females and 11 males. with an age average of 57,13 years who observed, on average 5,93 weeks after stroke)., Reasons for exclusion were haemorrhagic stroke, long evolution time at first assessment, and low or high AQ. There were no differences between the two therapeutic groups on first evaluation either on clinical or demographic variables. Patients submitted to intensive therapy had higher scores on all intermediate assessments than those on conventional therapy. Although these were not significant. At the present moment, some patients have not had their last assessment yet.

Conclusions: Preliminary evidence suggests that intensive therapy is better than conventional therapy to promote an earlier communication but its long term efficacy remains to be investigated. 
Correspondence: José Fonseca, IMM Faculty of Medicine of Lisbon, LEL CEEM FML Av Prof Egas Moniz, Lisbon 1699-028, Portugal. E-mail: jfonseca@fm.ul.pt

\section{Language: Other (e.g., Naming, Fluency, Reading)}

E. ANDRESEN \& D.C. OSMON. “Semantic” Priming:A Meta-Analysis. Objective: Research into semantic priming has provided a plethora of research and knowledge concerning the organization of the mental lexicon. Despite the breadth and number of published semantic priming studies, few qualitative or quantitative reviews have been conducted recently. To our knowledge, the only quantitative review of any section of this vast literature is Lucas' (2000) meta-analysis of semantic priming without association. In order to further investigate semantic priming effects, a meta-analysis of semantic priming articles with and without association was conducted.

Participants and Methods: After performing a search of online databases and perusing the references of key articles, 17 studies were included in the meta-analysis. 17 effect sizes were computed and tested for heterogeneity, representing a total of 1,013 research participants.

Results: The average effect (Hedges g) was small: -0.35 (95\% confidence interval $=-0.43$ to $-0.26 ; Q(16)=7.44)$. In order to investigate potential differences between different types of semantic priming (pure semantic, pure associative, and mixed), a moderator analysis was run, yielding small effects for semantic and associative priming, and a moderate effect for mixed. All analyses were homogeneous.

Conclusions: The results of the current meta-analysis clearly differ from Lucas (2000). Potential variables that could account for the difference include the differing inclusion criteria and the different foci of interests between these analyses. Nevertheless, this meta-analysis further clarifies this confusing literature, and highlights the need for additional highquality studies in this area.

Correspondence: Elizabeth Andresen, B.A., Psychology, University of Wisconsin-Milwaukee, 3016 N Oakland Ave \#117, Shorewood, WI 53211.E-mail: andrese2@uwm.edu

\section{A. CANCELLIERE. The Effects of Aging and Education upon Word Finding Ability: Data from a New Test.}

Objective: To investigate changes in word finding with aging (16-82) and education using the Definition Naming Test (DNT). Gradual improvement in naming with aging (and increasing education) was expected until age 60 and up.

Participants and Methods: The DNT allows comparison of an individual's word finding ability against their word knowledge. The DNT consists of part 1 (36 written definitions - the subject must write one word which best fits each) and part 2 (the same definitions with 20 multiple-choice options for each). The DNT was administered to 251 controls $(\mathrm{CG})$ and statistical analyses performed.

Results: The age groups (and n) included 16 -24 (45), 25-39 (58), 40$54(62), 55-69(59)$ and 70-82 (25). Scores on part 1 and part 2 were utilized and the ANOVA was highly significant for both parts. The 1624 group performed significantly lower than all other groups. There was virtually no difference between the 25-39 and 40-54 group with improved performance seen in the 55-69 group. A substantial decline was then seen in the 70-82 age group.

Education groupings were some high school, high school diploma, some college, college diploma, some university, university degree and postgraduate degree. An ANOVA indicated significant differences in word finding ability across the seven education groups.

Conclusions: Word finding ability as measured by the DNT showed continuing improvement into the 55-69 age range and only with entry into the 70-82 age range did evidence for a decline appear. Education predicted word finding test performance with the some university, university degree and postgraduate degree groups demonstrating the highest scores.
Correspondence: Andy Cancelliere, private practice, 156 Sheppard Ave. W.,Toronto,ONM2N1M8,Canada.E-mail:drcan@dellmail.com

L. FAÍSCA. The role of working memory in the interpretation of predicative metaphors.

Objective: Theoretical models for metaphor comprehension usually specify mental operations which involve the activation on semantic networks and the manipulation of conceptual features in memory. However, individual cognitive resources have been systematically neglected by psychological research on metaphor comprehension. To further understand the role of individual differences on metaphor interpretation, we investigated the association between working memory capacities (storage and processing) and the interpretation of predicative metaphors.

Participants and Methods: Working memory capacities (storage and processing components) were characterized on 36 participants who also interpreted twenty predicative metaphors (10 previously evaluated as easy to interpret, 10 evaluated as difficult); comprehension times were recorded and paraphrase quality was evaluated by two independent judges.

Results: Comprehension times showed a stronger correlation with processing components of working memory when compared to storage components $(\mathrm{r}=-.56$ versus $\mathrm{r}=-.31)$; correlations were weaker for easy metaphors than for difficult ones. However, the quality of interpretation did not show any significant association with working memory capacities.

Conclusions: Our findings suggested that individual differences on working memory processing capacities might account for the variation observed in the interpretation of predicative metaphors. Storage capacities seem to play a smaller direct role in metaphor processing. Additionally, we have found that difficult metaphors seem to be more dependent of on working memory processing resources than familiar metaphors; these results suggest that the interpretation of easy metaphors may involve cognitive resources different from those used in the interpretation of difficult, unfamiliar metaphors.

Correspondence: Inês Bramão, Faculdade de Ciências Humanas e Sociais, Universidade do Algarve, Campus de Gambelas, Faro 8000, Portugal.E-mail:mibramao@ualg.pt

F.R. GÓMEZ VELÁZQUEZ, A.A. GONZÁLEZ GARRIDO, M. AMANO FLORES \& D. ZARABOZO. Longitudinal Study Demonstrates that Naming Speed is the Best Early Predictor of Reading Abilities in Spanish-speaking Children.

Objective: It has been stated that Rapid automatized naming letter task can predict reading difficulties, but there are no developmental evidences supporting this notion in Spanish, which is an orthographic sallow language. The aim of the present investigation was to prospectively evaluate the accuracy of naming speed and phonological awareness tasks to characterize the efficiency in the reading acquisition process in Spanish-speaking children.

Participants and Methods: We studied one hundred and twenty nine right-handed, healthy Spanish-speaking children at the beginning of their first year in one elementary school, with yearly follow-up evaluation at the second and third scholar grades. The examination included the performance of naming speed tasks for objects, letters, numbers and colors, as well as the completion of seven tasks evaluating the phonological awareness during the first and the second grades. In the third grade, text-reading and spelling tasks were also evaluated.

Results: The present results showed that naming speed predicts the reading abilities better than phonological awareness. A stepwise linear regression analysis showed that letter naming speed predicts the reading speed -words per minute- in a $17 \%$, the errors in $13 \%$, reading comprehension in $8 \%$ and the production of homophone-errors while writing real-words at $13 \%$. Only sound-blending and deletion of the initial phoneme tasks contributed to explain reading fluency and reading comprehension performances.

Conclusions: As in other languages, Spanish-speaking readers impaired in naming speed tasks are slow but acute decoders, while the children 
impaired in phonological sensitivity are poorer decoders. Naming speed assessment at the beginning of the reading acquisition could be crucial in Spanish-speaking children due to the proven efficiency predicting the appearance of reading difficulties.

Correspondence: Fabiola R. Gómez Velázquez, PhD, Instituto de Neurociencias, Universidad de Guadalajara, Francisco de Quevedo 180, Arcos Vallarta, Guadalajara 44130, Mexico. E-mail: fabiolargomez@gmail.com

\section{T. MONTIEL \& E. MATUTE. Metacognition, Reading ability and} Schooling.

Objective: This study focused on basic reading ability and schooling effects in the performance on phonological awareness and visuo-postperceptual analysis tasks.

Participants and Methods: We compared the achievement between three groups: Illiterate Group ( 8 women and 2 men, age mean $=38.20$ years old), Literate non-schooled Group ( 8 women and 2 men, age mean $=37.20$ years old $)$ and Schooled Group ( 8 women and 1 man, age mean $=35.22,6$ years schooling) on phonological awareness tasks (syllabic, intrasyllabic and phonemic levels) and the Palmer part-probe task adaptation (Kolinsky, Morais \& Content, 1987).

Results: A non-parametric variance test found a group effect on the phonological awareness tasks, except syllabic segmentation task. Schooling subjects showed significantly higher scores than illiterate in the rest of phonemic awareness tasks, and than literate participants in the rime detection, phonemic segmentation and phonemic blending tasks. Literate participants had significantly higher scores than illiterate in onset detection and initial phoneme suppression tasks. The achievement between groups was similar in the Palmer part-probe task.

Conclusions: The results suggest that syllabic segmentation is acquired before learning to read or school attendance. Learning to read facilitate the initial phoneme identification, but it is schooling associated with reading acquisition that help to master phonemic awareness ability. Finally, the visuo-postperceptual analysis is not associated with schooling or reading acquisition.

Correspondence: Teresita Montiel, PhD, Instituto de Neurociencias, Universidad de Guadalajara, Francisco de Quevedo 180, Col. Arcos Vallarta, Guadalajara 44130,Mexico.E-mail:tere_montiel@yahoo.es

A. OTERO, R. FLORES, F. OTERO \& M. LAVALLÉE. Lectura Inteligente: An educational software that favors speed, understanding and efficient reading in Mexican secondary school students.

Objective: This study is part of a broader research on cognitive and affective resources of students of the secondary level in relation to generalized reading difficulty. In this paper, we present the results of a reading training program by means of an educational software exercising reading speed, understanding and efficient reading (integration of speed and strategies for understanding).

Participants and Methods: An experimental group of 160 students attending the secondary school (13 to 16 years of age) were invited to work with the software "Lectura Inteligente" (Intelligent Reading) in the school's computer room. Following a pre post test type of experimental design, they were first submitted to an initial diagnostic evaluation at the beginning of the year; each week afterwards, they worked during an hour with the software (approximately 35 to 40 hours) to end up with a final evaluation. A control group comprising the same number of students of the same school level was submitted only to the initial and final evaluations.

Results: Quantitative Results: Results show a significant positive effect of reading training on speed, understanding and efficient reading in the experimental group as compared to the control group.

A qualitative analysis of the students' comments gathered during their training reveals an important modification in the students' perception and attitude regarding reading.

Conclusions: The use of educative software to promote speed, reading understanding and efficient reading turns out to be a useful tool to help solve the generalized problem of slow reading in Mexican secondary school students, as was indicated in recent international evaluations.

Correspondence: Araceli Otero, Psychology, Universidad Nacional Autonoma de México, Corregidora 143, México 1200 D.F., Mexico. Email:aoteroalva@att.net.mx

L. STIVANIN, C.C. OLIVEIRA, F.F. MALERBI \& C.I. SCHEUER. Correlation between Latency Time and Reading Efficiency of 2nd, 3rd and 4th Grades Children.

Objective: Efficiency of performance in reading depends of precision and processing time. The aim of this study was to verify the correlation between latency time and efficiency of word reading of Portuguese-speaking Brazilian children.

Participants and Methods: Seventy-four children of 2nd, 3rd and 4th grades, both genders, attending regular public school in the city of São Paulo, composed the sample. Data about the development and language were provided by parents and information regarding the school performance, by teachers. Children with general development and language disorders were excluded from the research.The reading test was composed by 96 words, divided into stimuli groups: frequency of occurrence of the written word, extension and complexity for the Portuguese spoken in Brazil. Stimuli were presented in a computer screen and children were asked to read them. Latency time (precision of 8 decimals) and responses were registered in a program specially designed for this research. Results: Negative correlations between latency time and precision were observed, just for low frequency and complex words $((p<0,05)$, especially in 3rd and 4th grades.

Conclusions: These results pointed that low frequency words were read slower and with more errors, pointing that correlation between latency time and precision depend of the physical properties of the written word and the level of scholarization.

Correspondence: Luciene Stivanin, University of São Paulo, Teodoro Sampaio, 632/52, São Paulo 05406-000, Brazil. E-mail: stivanin@usp.br

D. ZARABOZO, F.R. GÓMEZ-VELÁZQUEZ, A.A. GONZÁLEZGARRIDO \& O.L. VEGA GUTIÉRREZ. Evaluating PseudoHomophones With Event Related Brain Potentials In Spanishspeaking Children.

Objective: Reading acquisition difficulties in children have been related with phonological awareness disturbances and slow naming speed. Naming speed seems to correlate with reading difficulties and orthographic recognition problems but their intrinsic relationships are still uncertain. Regarding theses antecedents, our main objective was to evaluate the recognition process of visual objects and the nature of orthographic lexical access in Spanishspeaking children with average or low naming speed scores.

Participants and Methods: One hundred forty -five healthy, righthanded, normal IQ second-grade children were evaluated to select two comparable groups of fifteen subjects, depending upon their performance in a rapid naming battery of tasks: slow namers (SN) and average namers $(\mathrm{AN})$. Children performed a task in which two successive stimuli were presented for $500 \mathrm{~ms}$ (ISI= $1500 \mathrm{~ms}$ ) in a PC screen: an easy-naming draw and a word that matched (control condition) or not (a: semantic violation, b: pseudo-homophones, the word is phonologicaly equal but is an orthographic violation) the draw. Subjects were instructed to judge, as soon as possible, if the pair of stimuli matched or not, while ERP were simultaneously recorded.

Results: Behavioral results showed that AN had a significantly higher number of correct responses detecting the pseudo-homophones or orthographic violations, with shorter reaction times. SN showed poorer reading performance than $\mathrm{AN}$. Salient ERPs components were parietal negativities with maxima at 320 and $340 \mathrm{~ms}$ for pseudo-homophones and semantic violations respectively in AN. ERPs showed higher amplitudes and slightly later latencies for SN children in all conditions. 
Conclusions: Results evidence that orthographic processing automation seems to represent a later stage in reading acquisition that is hardly reached by slow naming children and that can be functionally explored by ERP methods.

Correspondence: Daniel Zarabozo, PhD, Instituto de Neurociencias, Universidad de Guadalajara, Francisco de Quevedo 180, Arcos Vallarta, Guadalajara44130,Mexico.E-mail:dzaraboz@cencar.udg.mx

\section{Neglect}

D. ÁLVAREZ, V. PLAZA-AYLLÓN, E. DEL ÁGUILA, M.T. DAZA, I. FERNÁNDEZ-AGIS, E. CARMONA \& C. NOGUERA. A Study about Diagnosis and Evolution in a Patient with Balint's Syndrome by Encephalitis.

Objective: Balint's syndrome is an uncommon neuropsychological disorder characterized by a triad of symptom: optic ataxia/optic dysmetria, optic apraxia and simultagnosia. Two conditions make our case special: presence of neglect and the origin of syndrome, because it was caused by encephalitis. We summarize in this work diagnosis and successful treatment strategies applied to this case. To the best our knowledge, there are not previous descriptions of encephalitis as an etiological cause and effective therapy for this condition.

Participants and Methods: A fifty-nine-years-old woman affected by balint's syndrome, being recruited on the Center of Neuropsychological Assessment and Rehabilitation -CERNEP-. She shows bilateral occipitoparietal lesion in MRI and bilateral temporoparietal hypoperfusion in SPECT related to vasculitis probably caused by encephalitis. Patient presented optic ataxia and dysmetria, optic apraxia, simultagnosia and left neglect, and no memory difficulties except orientation and spatial memory. As treatment we used training in spatial orientation by imagination, in visual search by ocular scanning to compensate simultagnosia, neglect and optic ataxia, in organization and planning tasks by imagination and verbal guide and in emotion and auditive attention by musicotherapy.

Results: There were a significant advance in execution and comprehension of everyday life tasks, in emotional consequences of disorder and in neuropsychological disorders.

Conclusions: Using cognitive strategies and emotional praecox rehabilitation is possible ameliorate neuropsychological consequences of a disorder intriguing as balint's syndrome. Musicotherapy can be an exceptional strategy to work with emotional consequences of neuropsychological disorders and can be used to rehabilitate cognitive capabilities too. Our patient is better oriented know in personal and spatial plane, and can be independent in some everyday tasks.

Correspondence: Dolores Álvarez, Neuroscience and Sciences of Health, University of Almería, Ctra. Sacramento s/n, Almería 04120, Spain. Email:dalvarez@ual.es

C. DIJKERMAN, A. ZEDLITZ \& G. NYS. The effects of prism adaptation on the landmark task and the mental number line in healthy subjects.

Objective: Previous studies have shown that patients with unilateral hemispatial neglect exhibit specific defects on tasks in which the mean of two numbers was asked (Zorzi et al., 2002, Rossetti et al., 2004). Patients made mistakes in the direction of the higher numbers. This finding is consistent with a systematic rightward impairment on line bisection. Other studies (Rossetti et al., 1998; 2004) observed a reduced bias on both line and number bisections tasks after prism adaptation, suggesting common functional mechanisms.

In this study we assessed whether prism adaptation would result in alterations in the mental number line in healthy subjects.

Participants and Methods: Thirty-three healthy volunteers participated. They were divided into three groups (a leftward, a rightward and a control prism adaptation group). All groups performed both a number bisection task and the landmark task (in which participants were asked to judge whether a line had been pre-bisected left or right of the centre).
Results: The results showed a bias in the landmark task after adaptation to leftward and rightward deviating prism goggles, compared to a control adaptation. On the number bisection task, left and right deviating prism goggles did not result in such a bias.

Conclusions: These findings suggest that prism adaptation can have different effects on a number bisection task compared to a line bisection task. Possible mechanisms will be discussed.

Correspondence: Chris Dijkerman, PhD, Helmholtz Institute, Utrecht University, Heidelberglaan 2, Utrecht 3584 CS, Netherlands. E-mail: c. dijkerman@fs.uu.nl

T. LOETSCHER \& P. BRUGGER. Stars in the Sky: Assessing Inattention and Hyperattention in Spatial Neglect.

Objective: At this symposium we introduce a novel method (visuo-spatial randomization) for detecting a deficit of "disengagement", i.e. a contralesional inattention as a consequence of an inability to move the spotlight of attention away from ipsilesional stimuli

Participants and Methods: The visuo-spatial randomization task requires repeated pointing to random locations on a touch screen. The patient has to conceive of the black screen as the night sky and to indicate the location of the stars "as they are randomly distributed over the sky". This is done by repeated touches onto the screen with the right hand index finger. Importantly, there were two conditions. In the first condition (baseline), no sensory feedback was given to the patient, i.e., the stars put onto the sky remained invisible. In the second condition, any touch at a certain location would immediately "illuminate" a star at the respective location.

We report the performance of 3 patients with left-sided hemispatial neglect assessed by standard tests such as line bisection and copving.

Results: Despite all 3 reported patients made similar omissions when copying a drawing, their performance in the visuo-spatial randomization task was widely different: While one patient benefited from the visual feedback condition, neglect was unchanged in a second patient and even enhanced in the third one.

Conclusions: With the novel method dissociations between individual patients can be detected, that are not easily uncovered by traditional testing. So far, the method has proven to be practicable in clinical settings.

The next step will be to relate these patterns to the performances in neuropsychological tests, to lesion anatomy and to the rehabilitation outcome in a larger patient sample.

Correspondence: Tobias Loetscher, Department of Neurology, University HospitalZurich,Zurich 8091, Switzerland.E-mail:tobias.loetscher@usz.ch

M. THIMM, G.R. FINK \& W. STURM. Effects of a New Computerised Exploration Training on Neural Recovery from Spatial Neglect.

Objective: In previous studies we have shown that chronic neglect resulting from right hemisphere lesions can be ameliorated by alertness training or optokinetic stimulation. The present study investigated the behavioural and neural effects of a new developed computerised training that combines elements of both trainings. In this training patients have to observe lively scenes in the role as a photographer. They have to follow a view finder with their eyes and press a button ("shutter release") to "take a photo" as fast as possible whenever an object appears in the finder.

Participants and Methods: Six right-hemisphere stroke patients presenting with stable hemineglect performed the training, 45 minutes daily for three weeks. Neuropsychological tests and fMRI were assessed two times before (baseline) and two times after (long term effects) the training period. The fMRI paradigm was a stimulus detection task derived from the neglect task of the "Test Battery for Attention Performance" (TAP).

Results: Short term improvements of neglect appeared in the form of less omissions and shorter reaction times of left sided stimuli in the scanner task. This was accompanied by increased neural activity in the left superior frontal gyrus (BA 9) and the left superior parietal lobule 
(BA 7). By contrast, there was no increased neural activity at the baseline and at the long term fMRI.

Conclusions: The results suggest that short term amelioration of neglect can be mediated by enhanced neural activity in homologous brain areas contralateral to the lesioned frontoparietal network for spatial attention.

Correspondence: Markus Thimm, Neurology, University Hospital RWTH Aachen, Germany, Bismarckstr. 98, Aachen D-52066, Germany: E-mail: mthimm@ukaachen.de

\section{Other}

\section{A. FEDOROV, A. CHIBISOVA \& J. CHIBISOVA. LOW VISION PATIENTS REHABILITATION. NEUROPHYSIOLOGICAL BASIS OF THE RECOVERY FROM OPTIC NERVE LESION.}

Objective: Given that bilateral optic nerves (ON) lesion does not only causes the vision loss, but also affect to functional activity of subcortical and cortical visual centers. Applying of weak alternating current can restore functional activity at all levels of the visual system include increasing of optic nerve conductivity.

Participants and Methods: We describe outcomes of 812 patients with total and partial optic nerves lesion, vascular, posttumor origin. They were treated 10 days with electrical currents applying to the eyeball. where parameters correspond to a striate cortex and ON functional state. Ophthalmologic examination and EEG power spectra were performed. Results: We noted an improvement of visual acuity (VA) and enlargements of visible field area from $49.2 \%$ to $64.5 \%$. These results were stable within 18 months after treatment. We found a correlation $(r=0.78$; $\mathrm{P}<0.035$ ) between alpha rhythm power spectra level (in a projection of striate cortex) and improved visual acuity or visual field size.

Electrical stimulation produced in EEG a two alpha-activity focuses - in projection of striate and somatosensory cortex.

Conclusions: Electrical stimulation of the visual field improves VA and reduces the size of the visual field defect. In addition, increased EEG alpha activity suggests a new interaction between both visual flow processing systems of the geniculostriate and pulvinartemporal systems. These attributes together with the phenomenon of activation of nonspecific associative systems (thalamofrontal and thalamoparietal) suggests that electrical stimulation might affect the entire visual system on all levels which underlie recovery process

Correspondence: Anton Fedorov, MD, Center of Clinical Electrical Stimulation, Grazhdansky pr. 114, Saint-Petersburg 195267, Russian Federation.E-mail:antfedorov@inbox.ru

\section{SOBANSKA. The Preserved Multiplication Fact Retrieval in an Alexic Patient: A Case Report.}

Objective: According to the triple-code model (Dehaene\&Cohen, 1995) multiplication problems and its results are stored in the LTM as the phonological sequences of words. The correct transcoding of the input format of the operands into the corresponding phonological representation is the condition for the successful arithmetic fact retrieval, as it allows completing the memorized word sequence, e.g., $7 \times 4 \longrightarrow$ "seven times four, twenty eight". According to the phonological storage hypothesis, if the transcoding processes fails, the retrieval of the arithmetic facts becomes impossible.

The purpose of the study was to investigate calculation abilities in a severely alexic patient in order to explore the role of the phonological representation of numbers in multiplication fact retrieval.

Participants and Methods: This study presents the patient MF, a 76-yearold chemistry teacher, who has had a left hemisphere infarction. She suffered from mixed aphasia with moderate speaking and mild comprehension impairments, and severe alexia including alexia for Arabic digits.

Eighty-one multiplication problems (from $1 \times 1$ to $9 \mathrm{x} 9$ ) were presented to MF on two sheets of paper. Her task consisted of reading each problem aloud and giving the result in two ways: in written and orally.
Results: Although MF made 35 reading errors (43\%) she gave the correct answers to 77 out of 81 problems (95\%) in the written modality and to 70 out of 81 problems $(86 \%)$ in the spoken modality. Only once she gave the right answer to her incorrect reading of the problem $(9 \mathrm{x} 1$ read as "eight times one, eight" the written answer: 9).

Conclusions: The fact that MF would give the correct answer, despite reading the problem erroneously suggests that non-phonological representation may be involved in the storage and retrieval of multiplication facts, assumption which does not match the phonological storage hypothesis. Implications of these findings for the debate about the representation(s) underlying the storage and retrieval of arithmetic facts are discussed.

Correspondence: Marta Sobanska, Master of Science, Neuropsychology, Warsaw University, Stawki 5/7 Str., Warsaw 00-183, Poland. E-mail: marta@psych.uw.edu.pl

\section{VUKOVIC, J. VUKSANOVIC \& I. VUKOVIC. TYPES AND} EVOLUTION OF APHASIA FOLLOWING CLOSED HEAD INJURY.

Objective: Although the association between language disorders and brain injury has been known for a long time, there are still few detailed descriptions of aphasia after closed head injury. Types and evolution of posttraumatic aphasia are presented in this paper.

Participants and Methods: 40 brain injury patients are included in the sample. Patients were initially seen within the first 7 days after brain injury. They were subsequently interviewed and tested with Boston Diagnostic Aphasia Examination (Goodglass and Kaplan, 1983) one month and six months post onset.

Results: The results have shown that in the case of brain trauma all types of classical aphasic syndromes appear. Six months later, the changes of aphasia type in all patients are noticed. In 14 cases total recovery has occurred

Conclusions: In brain-injured patients same types of aphasa exist as in patients following other etiology (stroke, for example. Different evolutionary paths of aphasic syndromes are pointed out.

Correspondence: Mile Vukovic, University of Belgrade, Visokog Stevana 2,Belgrade 11000,Vugoslavia.E-mail:mvukovic@yubc.net

\section{Stroke}

C. ARRABAL, L.A. MUÑOZ, C. GUTIERREZ, R. VICENT, L. VERGARA \& O. FERNANDEZ. Patients' Clinical Evolution in the Stroke Unit.

Objective: The following study is aimed to evaluate the cognitive and functional evolution of patients admitted at the Stroke Unit of our Hospital. Patients and relatives were also asked about their satisfaction with the services provided by the Unit. The Stroke Unit provides early assessment and intensive care of people with symptoms of acute stroke by a multidisciplinary team. The aim of the Unit is to diminish the deficits associated to this pathology.

Participants and Methods: 50 patients were randomly selected to take part in this study. They were evaluated at admission and discharge and no age or other selection policy restrictions were applied for inclusion in this study. Improvements in functional capacity were measured with Barthel Index and cognitive evolution was assessed with Pfeiffer's Short Portable Mental State Questionnaire (SPMSQ). Patient global evolution was assessed with Rankin Scale. Finally indexes of welfare quality were obtained from patients and their relatives through questionnaires.

Results: Results showed clear improvements in functional as well as cognitive status when admission and discharge data were statistically compared. Patients and relatives also manifested high levels of satisfaction regarding welfare quality.

Conclusions: Our study suggests that the specialization of hospital units provides, at least in the case of our Stroke Unit, a more efficient attention to patients and relatives. This seems to improve outcome in patients functional and cognitive status, and patients' and relatives' subjective satisfaction. 
Correspondence: Carlos Arrabal, Graduated, Servicio de Neurología, Hospital Regional Universitario Carlos Haya., Avda Carlos Haya s/n, Malaga 29010,Spain.E-mail: carlosarrabal@hotmail.com

\section{N. CHE DIN, M. ARIFIN, S. ALIAS, S. GHAZALI, M. AHMAD \& P. SUBRAMANIAM. Factors Influencing Depression among Stroke Patients.}

Objective: The study examined the influence of duration of stroke, social support, coping styles, activities of daily living, acceptance of illness, and cognitive function on the mood of the stroke patients.

Participants and Methods: This cross-sectional survey study recruited a total of 67 stroke patients who attended the Out Patient Neurology Clinic. They were interviewed and assessed for cognitive functions using Mini Mental Status Examination, coping styles with Adult Coping Scales, social support using Provision of Social Relations, activities of daily living with the Blessed Rating Scale, acceptance of illness with the Acceptance of Illness Rating Scale and symptoms of depression with Beck Depression Inventory.

Results: Depression was significantly related to cognitive function $(\mathrm{r}=$ $-.296 *)$, coping styles $(\mathrm{r}=-.243 *$ to $-.521 * *)$, social support $(\mathrm{r}=.405$ to $\left..501^{* *}\right)$, cognitive function $\left(\mathrm{r}=-.296^{*}\right)$, acceptance of illness $(\mathrm{r}=-$. $\left.361^{*}\right)$, activities of daily living $(r=-.405)$ but not with any sociodemographic variables. Regression analysis showed that significant predictors of depression include activities of daily living $(16 \%)$, cognitive function $(8.8 \%)$, acceptance of illness (13\%), friend support (16\%), family support $(23 \%)$, total support $(25 \%)$, problem-oriented coping $(25 \%)$, nonproductive coping (25\%), optimism (11.6\%), and sharing $(2.9 \%)$. Conclusions: The results were similar to previous research in post-stroke depression whereby social support and positive coping were important factors in reducing stroke while cognitive impairment and poor activities of daily living worsened the depression. Treatment of post-stroke depression will speed up the progress of stroke recovery.

Correspondence: Normah Che Din, Master of Arts in Clinical Psychology, Health Psychology Unit, Universiti Kebangsaan Malaysia, Faculty of Allied Health Science, Universiti Kebangsaan Malaysia, Jalan Raja Muda Abdul Aziz, Kuala Lumpur 50300, Malaysia.E-mail:normah@medic. ukm.my

G. DESMARAIS, C. STRIEMER, E.A. ROY \& M.J. DIXON. Using Structure to Improve Memory for Visual Material: Linking Encoding Approaches to Memory Deficits for Visual Information in a Patient with a Focal Lesion to the Right Frontal Lobe.

Objective: We investigated the link between encoding approaches and memory deficits in RF, a 34 year old man who sustained a small, localized right frontal lobe ischemic stroke. Although he generally successfully recovered from the acute effects of the stroke, RF reported lingering problems with attention and memory. An initial evaluation revealed normal memory for verbal material but impaired memory for visual information. Despite normal copying of the Rey-Osterreith Complex Figure, RF's immediate and delayed recalls of the figure were impaired. RF's initial figure copying revealed a disorganized strategy, which led to poor recall.

Participants and Methods: We attempted to improve RF's performance using a structured presentation of the Rey-Osterreith Complex Figure (showing the figure in a succession of pieces as it is drawn), and contrasted that to his performance on an alternative figure presented traditionally. RF's abilities were also investigated using the Sustained Attention Response Task, Attention-Related Cognitive Errors, Visual Memory Span, and the Grooved Pegboard.

Results: RF showed no attentional deficits, and a normal visual memory span. Structuring the Rey Complex Figure improved RF's performance on both immediate and delayed recall compared to the initial assessment. Furthermore, once presented with the strategy, and left to copy an alternative figure without imposed structure, RF's immediate recall further improved.

Conclusions: This suggests that though using a structured presentation can enhance performance on such a task, once patients are presented with a strategy, they may fare better on their own.

Correspondence: Genevieve Desmarais, Ph.D., Kinesiology, University of Waterloo, BMH Building, 200 University Avenue West, Waterloo, ON N2L 3G1,Canada.E-mail: gdesmara@ahsmail.uwaterloo.ca

A. DUMBRAVA \& C. BALUT. Apathy More Than Depression Seems to Interfere with Early Motor Recovery After Stroke.

Objective: Several studies proved the negative impact of depression on recovery from stroke; less (if anything) is known concerning the influence of apathy on the same process.

The present study is aimed to look at this neglected aspect.

Participants and Methods: During hospitalization, the evolution of the neurological recovery after mildly to moderately severe ischemic stroke (assessed with NIHSS) has been compared in equivalent (in respect to usual demographic and clinical parameters) groups of patients corresponding to each combination of depression (major or minor, according to DSM-IV criteria) and apathy (estimated with "The Apathy Evaluation Scale" of Marin, Biedrzycki and Firinciogullari, 1991): with depression but no apathy $(n=25)$, with apathy but no depression $(n=19)$, with depression and apathy $(n=23)$, without any of the two $(n=27)$.

Results: At discharge, the improvement in NIHSS score was similar in patients with depression and without depression or apathy but was significantly lower in the presence as compared with the absence of apathy (either alone or associated with depression).

Conclusions: So, it seems that during the early period after stroke, spontaneous recovery is more negatively influenced by apathy rather than by depression.

The impact of apathy versus depression in the long run evolution and rehabilitation of stroke patients waits further examination.

Correspondence: Andrei Dumbrava, "Al.I.Cuza" University, B-dul Copou, Iasi6600,Romania.E-mail: rodumb@yahoo.com

\section{A. DUMBRAVA \& C. BALUT. Motor Symptoms of Post-Stroke Depression.}

Objective: Motor dysfunction has been viewed as central to the symptomatology of depression since Kraepelin. However, despite being an overt and relatively objective component, it remains usually assessed just by the impression of the clinician and not by more rigorously psychometric procedure. The aim of the present study is to overcome this aspect.

Participants and Methods: Motor symptoms has been recorded (distinctly for each component) using "The Motor Agitation and Retardation Scale" (Sobin, Mayer, Endicott, 1998), during the first and the last week of hospitalization for first ever ischemic stroke, in two groups of patients with $(\mathbf{n}=53)$ and without $(\mathbf{n}=57)$ depression (major or minor, according to DSM-IV criteria), equivalent to each other in respect to age, sex ratio, length of education and level of neurological symptoms (mild to moderate, based on NIHSS).

Results: At the first moment of investigation, the two groups were significantly indistinguishable on each of the motor symptoms explored; however, before discharge from the hospital, the depressed patients showed a significantly higher degree of motor retardation compared with the nondepressed.

Conclusions: Motor retardation seems to be associated with depression after stroke beyond the restriction induced by the cerebro-vascular accident. The significance of the present data is discussed in the context of the current debate over psychological versus neurological views of poststroke depression.

Correspondence: Andrei Dumbrava, "Al.I.Cuza" University, B-dul Copou, Iasi 6600,Romania.E-mail: rodumb@yahoo.com 


\section{GUTIÉRREZ. SUBCORTICAL ISCHAEMIC VASCULAR DISEASE WITHOUT DEMENTIA.}

Objective: To examine the relationship between raw scores in affective, behavioural and cognitive dysfunction scales

in patients with subcortical stroke lesions in the acute phase and after three months.

Participants and Methods: 74 patients with subcortical stroke lesions were studied. Mean age: $60 \pm 13.6,74.3 \%(n=55)$ male and, $25.7(n=19)$ were female. CAT and MRI examinations were performed in the first 48 hours. Recurrent stroke lesions were observed in $28 \%(n=21)$ of subject, $73 \%(n=54)$ had hemorrhagic infarct and $27 \%(n=20)$ ischemic infarct. The following scales were administered on all subjects: learning and memory, attention, language, executive functions, affective and behavioural.

Results: Significant differences were observed at the acute phase and three months after in the different scales: depression scale (HRS-D) ( $p=$ $.006)$, MMSE $(p=.000)$, obsessive compulsive disorders (MOCI) $(p=$ $.003)$, semantic fluency $(p=.017)$, Trail Making Test $(p=.002)$, and Boston Naming Test $(\mathrm{p}=.003)$. Patients with recurrent stroke lesions had lower raw scores than patients without recurrent stroke lesions in digits WAIS $(p=.027)$, memory (RAVLT $p=.007)$ and TMT-A $(p=.0 .22)$. Significant differences were observed between two age groups (Age-1(2360) Age-2 (61-82)) in the neuropsychological test: DW ( $p=.021)$; RAVLT $(p=.001)$; phonetic and semantic fluency $(p=.0 .39 ; p=.040)$; Naming $(\mathrm{BNT})(\mathrm{p}=.008) ;$ TMT A/B (p: .004) and visuospatial functions (p: .026). Conclusions: The performance of patients with subcortical stroke lesions in the affective and behavioural scales improves after three months, but they have low raw scores in executive functions, language and, attention at three months. Patients with recurrent stroke have worse performance in attention, memory and executive functions. Oldest patients with subcortical stroke lesions obtain worse raw scores in learning and memory, attention, language, executive functions and visuospatial functions.

Correspondence: Luis Gutiérrez, student doctorate, Department of Personality, University of barcelona, Psg/ Vall d'Hebron, 171, Barcelona 08035, Spain. E-mail: lgcabe@hotmail.com

\section{JIMENEZ, M. ZURRON, M. CORRAL, M. RODRIGUEZ-YANEZ, M. BLANCO, R. LEIRA \& J. CASTILLO. FRONTALTEST IN ELDERLY STROKE PATIENTS WITH LOW EDUCATION LEVEL.}

Objective: Most of patients with stroke in our population are older than 55 years, and have a low education level. Our objective is to study the sensibility and specificity of frontal test in elderly stroke patients with low education level. The present study shows the preliminary results. Participants and Methods: We included 20 patients with a first episode of ischemic stroke and low education level ( $<7$ years of education). $\mathrm{Pa}-$ tients were classified in two groups according to neuroimagen findings: patients with frontal lesions ( $\mathrm{n}=12$, mean age 70 years) and patients with pure non-frontal cortical lesions (temporal, parietal or occipital lesions without subcortical lesions) ( $\mathrm{n}=8$, mean age 70 years). We compared the score of both groups in this different frontal test: digit and inverse digit test, Stroop test, Zoo subtest in the BADS Battery, Hanoi Tower, Frontal Assesment Battery (FAB), Wisconsin Card Sorting Test (WCST), Boston Naming Test (BNT), verbal fluency ("p") and semantic fluency (animals).

Results: Patients with frontal lesion showed worse score in digit and inverse digit test, Stroop test, Hanoi Tower and BNT, more errors (total and persevere) in WCST and lower verbal and semantic fluency. This differences was only statistically significant in verbal fluency $(p=0,039)$ and in the Hanoi Tower $(p=0,035)$.

Conclusions: Verbal fluency and Hanoi Tower performance are the most specific frontal test in elderly patients with low education level. However, more patients must be included to confirm these results.

Correspondence: Isabel Jiménez, Neurology, Hospital Clinico Universitario, Travesa da Choupana s/n, Santiago de Compostela 15011, Spain.E-mail: isajmartin@yahoo.es
I. JIMÉNEZ, B. MIGUEL, M. RODRÍGUEZ-YÁÑEZ, L. ROGELIO, Z. MONTSERRAT, C. MONTSERRAT \& J. CASTILLO. COGNITIVE IMPAIRMENT IN INTRACEREBRAL HEMORRHAGE AND ITS EVOLUTION AT THREE MONTHS.

Objective: Intracerebral hemorrhage are nearly $20 \%$ of strokes, however its cognitive impairment has not been well studied. Our objective is to study the cognitive impairment caused by intracerebral hemorrhage and its evolution during the first three months.

Participants and Methods: Twenty five patients with first episode of intracerebral hemorrhage were prospectively studied, excluding patients with severe aphasia. A cognitive evaluation were performed at hospital discharge and at three months, including the following test: Mini-Mental Screening Test (MMSE), semantic fluency (animals), phonemic fluency ("p"), the Spanish version of California Verbal Learning Test, Complex Figure of Rey, Boston Naming Test, Command comprehension test, digit and inverse digit, and Praxis test, Frontal Assessment Battery (FAB), Geriatric Depression scale of Yesavage (GDS) and the NeuroPsiquiatric inventory (NPI).

Results: At discharge, $72 \%$ of patients showed impairment in memory, $71 \%$ in executive function, $48 \%$ in praxis, $8 \%$ in sustained attention and $8 \%$ in speech. At three months, a global improvement were shown in relation with discharge (MMSE: $25.40 \pm 4.5$ vs. $23.12 \pm 5.3$, $\mathrm{p}=0.001$ ), as well as in memory test (short-term memory: $-0.96 \pm 1.17$ vs. $-1.64 \pm 1.31, p=0.001$ ), executive functions (inhibitory control: $1.92 \pm 0.97$ vs. $1.50 \pm 1.02, p=0.022)$ and praxis $(8.7 \pm 1.79$ vs. $7.74 \pm 2.59$. $\mathrm{p}=0.033)$.

Conclusions: Most of patients with intracerebral hemorrhage shown memory, executive functions and praxis impairment at discharge, observing improvement in these areas at three months.

Correspondence: Isabel Jiménez, Neurology, Hospital Clinico Universitario, Travesa da Choupana s/n, Santiago de Compostela 15011, Spain.E-mail: isajmartin@yahoo.es

G.O. LINARES \& G.G. ORTEGA. Cognitive status is impaired in apparently recovered stroke survivors.

Objective: Cognitive status of stroke patients with total functional recovery remains unknown. We aim to determine different cognitive functions in "recovered" stroke patients at six months

Participants and Methods: Ortega.G, Quintana.M, Ribó.M, Santamarina.E,Delgado.P, Rubiera.M, Maisterra.O, Molina.CA, Alvarez-Sabin. J. We prospectively studied consecutive stroke patients with a modified Rankin Scale score $(\mathrm{mRS})<=1$ at six months. All patients underwent a complete neuropsychological evaluation including: attentional, information processing speed, visuospatial, learning and memory, language and executive functions. All scores were adjusted by age, education and were standardized (0 to100). Global cognitive status was defined as the mean score of all individual functions. Scores $<40$ determined impaired functions. Clinical and demographic data were also collected

Results: 47 patients were studied, mean age was 65 and $28 \%$ were female. Mean time of education was 9 years. $38(81 \%)$ patients had an ischemic stroke, of them 4 had a TIA. On admission median NIHSS score was 3 , at discharge 1 and $23 \%$ had a mRS of 1 at 6 months. Most subjects $(59.6 \%)$ had a global cognitive impairment (GCI). Language was affected only in $4 \%$ of patients. The remaining cognitive functions were impaired in more than $50 \%$ of subjects being executive ability the most affected $(72 \%)$. GCI was higher among patients with intracerebral hemorrhage $\left(100 \% \mathrm{Vs}_{\mathrm{s}} 50 \%, \mathrm{p}=0.006\right)$. Other variables associated with GCI were: previous stroke $(p=0.027)$ and no-smoking $(p=0.032)$. Initial stroke severity was not associated with GCI $(p=0.57)$

Conclusions: In most self-sufficient stroke survivors with apparently no limitations in daily activities a neuropsychological evaluation prove an important cognitive impairment. Commonly used outcome measures may be insufficient to evaluate full recovery. 
Correspondence: Gemma O. Linares, Psychologist, Stroke Unit, Hospital de la Vall d'Herbon, Passeig de la Vall d'Herbon 119-129, Barcelona 08035, Spain. E-mail: gemmaortega@yahoo.es

P. MARTOS, S. FERNÁNDEZ, D. CÁNOVAS, I. DELGADO, A. ROVIRA \& M. JODAR. Neuropsychological Profile of Bilateral Paramedian Thalamus Infarction: 3 Cases.

Objective: The artery of Percheron is a single rare artery that is involved in the paramedian thalamic-mesencephalic arterial supply. The paramedian artery syndrome includes acute loss of consciousness frequently following by neuropsychological disturbances. The objective of the present study was to assess the role of paramedian structures in all cognitive domains, and specially executive functions and memory.

Participants and Methods: We reported 3 patients, (2 men and a woman, 45, 67, and 71 aged respectively). All three, began with a coma followed by fluctuating levels of consciousness and several memory and behavioural manifestations. A MRI with nuclei delimitation was done in all of them, and we assessed cognitive functions with an extensive neuropsychological battery, when patients were neurologically stabilized.

Results: Neuroimaging findings showed the same bilateral lesion of the dorsomedial nuclei in all three patients. Neuropsychological assessment showed only an impairment in executive functions in all three patients, while verbal memory was affected in only one case. No other cognitive deficits were found in our patients.

Conclusions: Any of the three patients of our study presented severe amnesia syndrome. In two patients, neuropsychological deficits affected only executive functions. Only one patient showed a recall memory deficit characterized by confabulations and tendency to improve with recognition. In this patient, the deficit in executive functions was more severe. These results support the hypothesis that memory impairment after bilateral dorsomedial infarct is secondary to a severe executive dysfunction.

Correspondence: Patricia Martos, Neuropsychology, hospital Tauli, Pintor Juan Angel Saez 14 3A, Vitoria 01008, Spain. E-mail: bjpatri@hotmail.com

M. PASCUAL, N. PAÚL LAPEDRIZA. B. GONZÁLEZ RODRÍGUEZ, J. BLÁZQUEZ ALISENTE, M. RIOS LAGO \& D. DE NOREÑA MARTÍNEZ. Lack of self-awareness of deficits: Comparison between patients with stroke located in left and right hemisphere.

Objective: The aim of the study was to assess the relationship between hemispherical locations the injury in the stroke and the lack of self-awareness of deficits

Participants and Methods: In this study we analyzed the frequency the disorder self-awareness of deficit in patients with located hemispherical vascular pathology. We used the Spanish version of Patient Competency Rating Scale, "Índice de Competencia del Paciente", administrated to patients and their familiar members. The sample included 30 outpatients recruited from Brain Injury Unity belong to Beata María Ana Hospital, in Madrid.

Results: The individuals with lesions located on the right hemisphere showed more differences on the questionnaire Patient Competency Rating Scale, compared with their family members, in opposition to individuals with lesions located on the left hemisphere.

Conclusions: Lesion localization is a relevant factor associated to lack of awareness in stroke patients.

Correspondence: Margarita Pascual, Ph D, Brain Injury Unity, Hospital Beata Maria Ana, c/ Vaquerías 7 Bis, Madrid 28007, Spain. E-mail: margapasdarling@yahoo.es

\section{Paper Session 19 10:30-11:30 a.m.}

\section{Memory}

A. BLANCO-CAMPAL, T. BURKE, R. COEN, B. LAWLOR \& J. WALSH. Prospective Memory is a Sensitive Indicator of Mild Cognitive Impairment.

Objective: Prospective Memory (PM) is the ability to remember to perform an intended action in the future, and has been argued to be a more sensitive indicator of early dementia, compared to retrograde episodic memory. Relatively little is known about PM functioning in Mild Cognitive Impairment (MCI). The study aimed to: 1. Investigate the frequency, based on self-report, of PM deficits in MCI ; 2. Establish the discriminatory capacity of a PM test for detecting MCI.

Participants and Methods: Three groups participated in the study. A group of MCI-suspected Alzheimer's disease patients (MCI-AD; $n=20$ ), a group of Vascular Cognitive Impairment-No Dementia patients (VCIND; $\mathrm{n}=19$ ), and a group of normal controls (NC: $\mathrm{n}=21)$. A newly developed PM test (Silly Sentences (SS)), The Prospective and Retrospective Memory Self-Report Questionnaire, and other widely used neuropsychological measures tapping into overall cognitive functioning, attention, episodic memory, and executive functioning were used.

Results: Both MCI subgroups reported a higher frequency of retrospective and PM failures compared to NC. However, MCI subgroups did not report a disproportionately higher frequency of PM failures compared to retrospective memory failures. Receiver Operating Characteristics Curve analysis showed excellent discriminatory capacity of the PM test for detecting MCI. With a cut-off score of $<8$, the SS-NonSpecific condition achieved: Sensitivity $=81 \%$, Specificity $=95 \%$, and Overall Accuracy $(\mathrm{OA})=86 \%$, with Positive Predictive Value $(\mathrm{PPV})=97 \%$, and Negative Predictive Value $(\mathrm{NPV})=74 \%$. What's more, only the SS-Specific-Non-Salient test discriminated between MCI subgroups $($ Sensitivity $=68 \%$, Specificity $=84 \%, \mathrm{OA}=76 \%, \mathrm{PPV}=81 \%$, $\mathrm{NPV}=71 \%)$. Although correct classification rates for MCI-AD's was low $(56.3 \%)$, up to $80 \%$ of VCIND patients and $100 \%$ of controls were correctly classified.

Conclusions: Based on its high discriminatory capacity, the present study argues that PM tests are a potential useful adjunctive tool to detect MCI. Correspondence: Alberto Blanco-Campal, Dr Clinical Psychology, University College Dublin, 27, The Spires, Termonfeckin, Drogheda 000, Ireland.E-mail: campalblanco@gmail.com

R.P. KESSELS, C. PIEKEMA, A. POSTMA, M.P. HENDRIKS, A.J. WESTER \& G. FERNÁNDEZ. Contextual Memory and Binding in Hippocampal and Diencephalic Patients.

Objective: The hippocampal-diencephalic circuit plays an important role in long-term contextual memory formation. However, its role in working memory is less clear. Previous fMRI studies have shown hippocampal activation during working-memory tasks that rely on the binding or integration of multiple features (e.g., spatial context or colour). However, patients with lesions in the hippocampus or diencephalon typically have intact working memory function, but most studies have focused on context-free working-memory paradigms.

Participants and Methods: Twenty-seven patients with hippocampal lesions following epilepsy surgery or diencephalic dysfunction due to Korsakoff's syndrome were examined using a contextual Sternberg working-memory paradigm, in which single items (colour, location) or combinations of features (item-colour, item-location) have to be maintained for a short period of time. Complexity of the task conditions was comparable. Results were compared with a matched control group ( $\mathrm{N}=30)$. Results: ANOVA indicated that the task performance in the patients was worse than the controls for the hippocampal $(p<0.05)$ and the diencephalic $(p<0.0005)$ group, indicating that working memory is impaired 
in these patients. However, there was neither a significant main effect of binding nor a binding $\mathrm{x}$ group interaction.

Conclusions: These findings indicate that the hippocampal-diencephalic memory circuit is clearly implicated in working-memory processing, i.e. online maintenance of information during a short time period. We did not find evidence for disproportionate deficits in binding multiple items compared to single items within working memory. This is in contrast with previous results indicating binding deficits in long-term memory in similar patient groups.

Correspondence: Roy P. Kessels, PhD, Geriatrics \& Medical Psychology, Radboud University Nijmegen Medical Centre, Huispost 925, PO Box 9101, Nijmegen6500 HB, Netherlands.E-mail: r.kessels@ger.umcn.nl

M. MAITZ \& M. PERDICES. The Effects of Providing Memory Cues During Verbal and Non-Verbal Learning in Persons with Unilateral Frontal and Temporal Lobe Tumours.

Objective: Tulving et al's (1994) HERA model suggests encoding and retrieval processes in episodic memory are differentially mediated by the left and right frontal lobes respectively. This study investigated the effects of cueing on verbal and non-verbal memory performance of patients with unilateral frontal or temporal tumours. We hypothesised that cues provided at either the acquisition or recall stage would differentially improve memory performance of patients with left or right frontal lesions, respectively, but not that of patients with temporal lesions.

Participants and Methods: Subjects were patients with unilateral cerebral tumours (Left Frontal=17, Right Frontal=20, Left Temporal=10, Right Temporal=11). Cues (supraordinate categories for classifying tobe-remembered information) were provided either at the encoding stage, the retrieval stage, or at both encoding/retrieval stages of verbal and nonverbal 'list-learning' tasks. Performance in each condition was compared with baseline performance (no cues provided).

Results: For both Frontal groups, recall consistency, total recall, and delayed recall improved under any cueing condition on the verbal task. In the Left Temporal group, cueing at both encoding/retrieval improved total and short delayed recall. In the Right Temporal group, cueing at either encoding or retrieval improved recall consistency, while cueing at both encoding/retrieval improved total recall. On non-verbal tasks, only cueing at both encoding/retrieval improved recall consistency in the Right Frontal group.

Conclusions: Results did not support our hypotheses or the HERA model. Verbal recall improvements in both Temporal groups suggest that memory impairment associated with temporal lobe lesions does not simply reflect modality-specific encoding deficits.

Correspondence: Michael Perdices, PhD, Neurology, Royal North Shore Hospital, Pacific Highway, St Leonards, Sydney 2065, Australia. E-mail: mperdices@nsccahs.health.nsw.gov.au

M. PERDICES, M. MORGAN, T. HARRINGTON, W. SORBY, V. GRINELL \& N. ASSAAD. Changes in Verbal Memory Function During Internal Carotid Artery Occlusion by Balloon Angiography. Objective: One option for surgical management of internal carotid artery (ICA) aneurysms is to ligate the artery proximal to the aneurysm. This can, however, cause ischaemia and impair cognitive function unless there is adequate collateral perfusion to the area normally supplied by the ligated vessel. This study presents an innovative technique that has been developed to pre-operatively estimate the cognitive risk associated with this procedure.

Participants and Methods: Balloon angiography is used to occlude the artery for 20 minutes and a brief neuropsychological assessment is performed during the occlusion. The Reliable Change Index method is used to determine if there is a significant change between baseline (pre-occlusion) and intra-occlusion cognitive performance. Data for 28 right handed female patients undergoing occlusion of the ICA
(Left=15, Right=13) are presented. Only results for verbal memory (Rey Auditory Verbal Learning test: Sum of learning trials score) are reported.

Results: There was no difference between groups for either age (Left=51.5,SD=18.2; Right=56.2,SD=12.9) or years of education (Left=12.1,SD=5.5; Right=10.8,SD=2.1). The proportion of individuals demonstrating significant memory deterioration during occlusion did not differ between groups ( $\mathrm{Left}=40 \%$; Right $=46 \%$ ). After controlling for age and education, baseline verbal memory performance was a significant predictor of memory deterioration during occlusion $(\mathrm{X} 2(1)=5.536$, $\mathrm{p}=0.019$; Nagelkerke R2=0.27). Individuals showing memory deterioration during occlusion had significantly higher memory performance $(\mathrm{t}(26)=2.54, \mathrm{p}=0.017)$ at baseline $(47.3, \mathrm{SD}=9.3)$ than those who did not $(37.7, \mathrm{SD}=10.4)$.

Conclusions: Unilateral occlusion of the ICA may impair verbal memory. Occurrence of impairment is related to premorbid level of memory, but not exclusively associated with left ICA occlusion.

Correspondence: Michael Perdices, PhD, Neurology, Royal North Shore Hospital, Pacific Highway, St Leonards, Sydney 2065, Australia. E-mail: mperdices@nsccahs.health.nsw.gov.au

\section{Paper Session 20 10:30-11:30 a.m.}

\section{Adult Traumatic Brain Injury}

\section{F. CONSTANTINIDOU.Neuropsychological and Functional Recovery} after moderate to severe TBI-Support for systematic post-acute rehabilitation.

Objective: To determine the effects of post-acute cognitive rehabilitation on neuropsychological and functional performance in patients with traumatic brain injury (TBI).

Participants and Methods: Two groups of ss with moderate-severe TBI (experimental $n=19$, and control $n=13$ ) and one group of non-injured normal ss participated, $n=16$. TBI ss were randomly assigned into the experimental or the control group and were matched on critical variables and severity indices. There was no difference between TBI participants in pre-tx performance on dependent measures such as the MPAI-3, CIQ, or any of the neuropsychological measures.

The experimental TBI group received systematic categorization training as part of a clinical trials project testing the effects of the Categorization Program. TBI control group subjects did not receive the CP training; instead they received cognitive tasks traditionally used in their facility. All ss were monitored in order to receive similar amounts of cognitive treatment. Subjects received about 12 weeks of post-acute rehabilitation. Ss were assessed before rehabilitation and at the end of their treatment.

Results: MANOVA yielded significant gains $(\mathrm{p}<.05)$ on 12 neuropsychological measures for the TBI experimental group in the areas of processing speed, reasoning, concept formation, visual memory, and executive functioning. The control group improved on 6 of the neuropsychological measures. In terms of functional gains, MANOVA yielded significant gains on total post CIQ for both groups $(\mathrm{p}=.0001)$. Pairwise t-tests showed significant changes on the 3 subscales for the experimental group. TBI controls only improved in Productivity subscale. MANOVA on the MPAI-3 resulted in significant improvement for both groups on all subscales.

Conclusions: Post acute rehabilitation is beneficial to patients with TBI. Patients enrolled in systematic categorization training demonstrate a greater degree of neuropsychological and functional improvement than patients who receive conventional cognitive rehabilitation.

Correspondence: Fofi Constantinidou, Ph.D., Psychology, University of Cyprus, 75 Kallipoleos St., P.O. Box 20537, Nicosia 1678, Cyprus. Email:fofic@ucy.ac.cy 
A.L. BESSELL, E.R. WATKINS \& H. WILLIAMS. Is autobiographical Recall compromised by depressive rumination in survivors of Acquired Brain Injury?

Objective: Previous studies with non-brain injured have identified that rumination is prevalent in both the development and maintenance of depression. We aimed to explore how depressive rumination may contribute to overgeneral memory recall in ABI patients, by assessing the effects of manipulating ruminative self-focus on autobiographical memory performance across levels of brain injury.

Participants and Methods: 58 ABI individuals with mild (28) and moderate/severe (30) brain impairments were given baseline measures of executive function, autobiographical memory, mood and rumination. They were randomly assigned as matched pairs into one of 2 condutions - distraction or a rumination task. Follow up measures were taken of autobiograpchial recall and rumination.

Results: Findings indicate that ruminative self-focus reduces the specificity of autobiographical memory in individuals with ABI. This occured for both levels of injury severity - mild and moderate-severe.

Conclusions: We conclude that depressive rumination plays a role in the reduced access to autobiographical memories. These findings indicate the value of identifying and treating depression, and targetting ruminative self-focus, amongst this population, such as by Cognitive Behaviour Therapy.

Correspondence: Huw Williams, Exeter University, Washington WSinger Labs, ExeterEX44QG, United Kingdom.E-mail:w.h.williams@ex.ac.uk

T.A. DEAN \& C. SKILBECK. Impaired National Adult Reading Test (NART) Performance in Traumatic Brain Injury (TBI).

Objective: Effects of time post-injury and TBI severity on NART performance were examined. The hypothesis was that the NART can be impaired post-TBI and, therefore, that scores will recover over time. NART performance in mild TBI was predicted to significantly improve by 6 months post-TBI, and that moderate and severe TBI groups would show significant improvement by the 12-month assessment.

Participants and Methods: A prospective longitudinal design was employed and 122 Australian TBI participants completed the NART soon after injury and at 6-and 12-months post-injury. The group was divided into mild $(\mathrm{n}=60)$, moderate $(\mathrm{n}=40)$ and severe TBI $(\mathrm{n}=22)$ subgroups (PTA being the index of severity). Data were examined using Repeated Measures ANOVAs for participants completing a NART at all three time points, at initial and at 6-months follow-ups $(\mathrm{n}=177)$, and at 6 and 12 -months post injury $(\mathrm{n}=141)$. Demographic data were also obtained (gender, education).

Results: NART performance improved significantly over time $(p<.001)$. Mild and moderate TBI groups showed significant improvement by 6 month post-injury and by 12 -months for the severe group (both $p<.05$ ). The effects of demographic variables were negligible for gender, although participants with at least 11 years education obtained significantly lower mean NART error scores.

Conclusions: NART administration soon after TBI under-estimates premorbid IQ, even in those with mild TBI. Whilst NART assessment of premorbid IQ at 6 months follow-up appears appropriate for those with brief PTA, for those experiencing severe TBI 12 months post-trauma is more likely to provide a valid estimate.

Correspondence: Tracey A. Dean, Masters Clinical Psychology (student), Psychology, University of Tasmania, 135 King Street, Sandy Bay, Hobart, TAS 7005, Australia.E-mail:traceys@utas.edu.au

K.D. CICERONE, T. MOTT, J. AZULAY \& J.F. MALEC. Impact of Impaired Self-Awareness on Select Outcomes After Traumatic Brain Injury.

Objective: Impaired self-awareness (ISA)is common after TBI and considered to impact clinical outcomes and response to rehabilitation. We evaluated the impact of ISA on outcomes of patients receiving neuropsychological rehabilitation. We hypothesized that (1) patients with ISA would exhibit greater neuropsychological impairment but report bet- ter functioning, and (2) patients with ISA would show less response to treatment.

Participants and Methods: Sixty-two TBI patients completing a controlled 4 month trial of neuropsychological rehabilitation. Clinical ratings of ISA were made with the Mayo-Portland Adaptability Inventory(MPAI4), and two groups derived from dichotomous classification of ISA. Outcome measures included neuropsvchological functioning (NP). Community Integration (CIQ),perceived self-efficacy for management of symptoms(SE), and subjective life satisfaction (PQOL). Between-group differences and response to rehabilitation were examined using repeated measures ANOVAs, and post-hoc analyses conducted using independent t-tests.

Results: Twenty-three participants with ISA were slightly earlier postinjury and less educated. There were no differences on overall NP or CIQ measures, and no differences in response to treatment. Participants with ISA reported greater $\operatorname{PQOL}(p=.006)$ although there was no effect of ISA on treatment response. Participants with ISA also reported greater SE prior to treatment $(p<.01)$ but this did not change, while participants without ISA showed increased SE after treatment.

Conclusions: Impaired self awareness has a differential impact on outcomes after TBI, with the primary effect of ISA evident on self-report measures such as perceived self-efficacy and life satisfaction. Contrary to expectation, there was little effect of ISA on response to neuropsychological rehabilitation. The relationships among ISA, time post-injury, and self-appraisal over the course of rehabilitation merit additional investigation. Correspondence: Keith D. Cicerone, PhD, JFK-Johnson Rehabilitation Institute, 2048 Oak Tree Road, Edison, NJ 08820. E-mail: kcicerone@solarishs.org.

\section{Paper Session 21 10:30-11:30 a.m.}

\section{Epilepsy}

M. CHEUNG, A.S. CHAN, Y. CHAN \& J. LAM. Post-surgical verbal memory outcome associated with right mesiotemporal functional MRI activation.

Objective: To examine the verbal memory processing in patients with left temporal lobe epilepsy before and after temporal lobectomy by functional magnetic resonance imaging (fMRI)

Participants and Methods: Ten right-handed pre-operative patients with left temporal lobe epilepsy (left-TLE), aged 14 to 40 years, were recruited from Prince of Wales Hospital in Hong Kong. The mean duration of illness was 11 years (range, 2 to 30 years) and mean age at epilepsy onset was 14 years (range, 4 to 25 years). Before surgery, they performed a complex visual scene-encoding task during fMRI to determine the memory activation in the mesial temporal lobe and their verbal memory performance was evaluated by Hong Kong List Learning Test (HKLLT) outside the scanner. One year after temporal lobectomy, same fMRI paradigm was delivered and their verbal memory performance was assessed by the HKLLT again. Region-of-interest (ROI) analyses were used to quantity functional activation in the mesial temporal lobe and analyses included correlation between functional activation and verbal memory performance.

Results: Consistent with our previous findings, patients' verbal memory performance as assessed by the HKLLT was positively correlated with functional activation in both mesial temporal lobes before surgery (left. $r(10)=0.736 ; p<0.05$; right, $r(10)=0.646 ; p<0.05)$. After temporal lobectomy, there was a slight decline in verbal memory as assessed by the 30-minute delayed recall trial of the HKLLT (before: $8.90 \pm 3.25$ words; after: $7.50 \pm 3.69$ words; $\mathrm{t}(9)=-2.33, \mathrm{p}<0.05)$ and their performance in verbal memory was significantly correlated with functional activation in the mesial temporal lobe contralateral to temporal lobectomy (right. $\mathrm{r}(10)=0.748 ; \mathrm{p}<0.05)$.

Conclusions: The present study demonstrated that change in verbal memory performance was found in the left-TLE patients after temporal 
lobectomy and their post-surgical verbal memory outcome was associated with functional activation contralateral to temporal lobectomy. Correspondence: Mei-chun Cheung, Ph.D., Institute of Textiles and Clothing, The Hong Kong Polytechnic University, Institute of Textiles and Clothing, The Hong Kong Polytechnic University, Hung Hom, Hong Kong N.A., Hong Kong. E-mail: tccmchun@inet.polyu.edu.hk

C.E. HOWARD, P. ANDRES, P. BROKS, G. MAZZONI \& R. NOAD. Memory and Metamemory in Patients with Temporal and Frontal Lobe Epilepsy.

Objective: Research has shown that the relationship between subjective complaints and actual memory deficits is usually rather weak in patients with epilepsy (Blake et al., 2000; Gleissner et al., 1998). The aim of the current study was to investigate whether metacognitive knowledge and cognitive performance were differentially disturbed in patients with temporal and frontal-lobe epilepsy.

Participants and Methods: Twelve patients with temporal-lobe epilepsy. twelve patients with frontal-lobe epilepsy and twelve matched controls participated in a combined Judgement-of-Learning and Feeling-of-Knowing computerised episodic memory task to investigate metamemory performance. Recall was tested after a 30-minute retention delay and at four weeks.

Results: Preliminary findings show lower recall performance in patients with epilepsy. In terms of their metamemory, they tend to over-estimate their performance, as compared to controls, who tend to under-estimate it. Conclusions: The finding of an inability to monitor one's own memory performance could be crucial for memory rehabilitation in patients with epilepsy. These results will be discussed in terms of their association with executive and memory deficits in frontal and temporal lobe epilepsy respectively.

Correspondence: Pilar Andres, University of Plymouth, Portland Square, Plymouth Pl4 8aa, United Kingdom. E-mail:pandres@plymouth.ac.uk

N. MUHLERT, C.L. ISAAC, R.A. GRÜNEWALD, M. REUBER, N. HUNKIN \& S. HOWELL. Evidence for Long Term Forgetting in patients with Temporal Lobe but not Idiopathic Generalised Epilepsy. Objective: Studies have shown that memory is impaired in TLE but that patients tend to overestimate memory problems in comparison with performance on objective tests. This may be due to increased forgetting over several weeks (e.g. Blake et al., 2000), a deficit not tapped by standardised tests of memory. The causes of this long term forgetting (LTF) are unclear but could relate to neuropathology or to seizure activity. The purpose of this study was to compare performance of patients with TLE with identified neuropathology in medial temporal lobes and patients with IGE which is associated with generalised seizures with no focal neuropathology.

Participants and Methods: Participants included patients with TLE $(\mathrm{n}=12)$, IGE $(\mathrm{n}=12)$ and a healthy control group $(\mathrm{n}=12)$. Memory for visual and verbal stimuli was matched across groups at a delay of 20 seconds. Retention was assessed at further delays of 30 minutes and 3 Weeks.

Results: Recall and recognition across groups was similar at the 30 minute delay. In contrast, TLE, but not IGE, patients showed significantly increased forgetting compared to controls at 3 weeks on recall of visual scenes $(\mathrm{t}=-2.9, \mathrm{p}=0.009)$ and verbal recognition $(\mathrm{t}=-2.6, \mathrm{p}=$ $0.019)$. There was no effect of seizures during the 3 week delay on retention.

Conclusions: Our findings suggest that LTF occurs in patients with TLE, but not IGE. This provides some evidence that LTF may not result solely from seizure activity.

Correspondence: Nils Muhlert, B.Sc (Hons), M.Sc., Psychology, University of Sheffield, Western Bank, Sheffield S10 2TP, United Kingdom. E-mail: n.muhlert@shef.ac.uk
F.H. SANTOS, J. MOLINA, E. GARZON \& O. BUENO. Partial Epilepsy in Frontal Right Hemisphere and Mathematical Skills in children.

Objective: Neuropsychological impairment in executive functions has been abundantly reported in children with Partial Epilepsy in Frontal Right Hemisphere (PEFRH). In the other hand, deficit in mathematics in these children are rarely studied. We present a detailed investigation of number processing and calculation for four children with PEFRH.

Participants and Methods: The participants were 4 boys with Partial Epilepsy in the Right Hemisphere: GF (6 years-old, frontal seizures, $I Q=78)$, VS (6 years-old, fronto-temporal seizures, IQ=81), FM (11 years-old, frontal, $\mathrm{IQ}=70$ ), and $\mathrm{CF}$ (12 years-old, fronto-central, $\mathrm{IQ}=50$ ) in mathematical skills (Battery for Number Processing and Calculation in Children - Zareki-K ou Zareki-R, respectively for kindergarten or revised version, and Arithmetic from WISC III) and visuospatial memory (Block Span task). The scores were compared to normative data.

Results: CF was impaired in almost all measures, except for forward block span, and Zareki-R subtests: counting dots, verbal comparison of numbers, and visual estimative of quantities. FM showed normal scores for arithmetic, block span, visual and qualitative estimative. He was impaired in counting backwards, dictate, mental calculation, reading numbers and problem solving. VS was affected only in two subtests of Zareki-K while GF presented difficulties with Arithmetic and 7 of 9 tests of Zareki-k. The results are discussed considering the IQ scores, the specific seizures location and evidences for executive dysfunctions.

Conclusions: In conclusion, children with PEFRH may have problems in number processing and calculation. The Zareki batteries were more sensitive to detect mathematical difficulties then Arithmetic WISC III subtest, visuospatial working memory was not affected in these children with right frontal lobe epilepsy.

Correspondence: Flavia H. Santos, Ph.D. in Science, Experimental Psychology, UNESP, Universidade Estadual Paulista, Avenida Dom Antonio 2100, Parque Universitario, Assis 19806900, Brazil. E-mail: flaviahs@psicobio.epm.br

\section{Poster Session 8 \\ Aging, Dementia 11:30 a.m.-1:30 p.m.}

\section{Aging}

R. AVILA, M.A. MOScoso, S.R. RIBEIZ, D.M. BEZERRA, F.L. DURAN, G.F. BUSATTO \& C.M. BOTTINO. Neuropsychological Correlates of MRI Measures in Elderly Depressed.

Objective: Depression, especially in elderly, is associated with structural abnormalities of cortical and subcortical regions, as well as cognitive deficts. Several voxel-based-morphometry (VBM) studies have often detected gray and white matter abnormalities in patients with late life depression. However, VBM studies that investigated correlations between gray and white matter abnormalities and scores of cognitive measurements have been scarce.

The purpose of this study was to determine the correlation between VBM brain volumes and scores in neuropsychological tests and psychiatric scales in elderly depressed patients and health controls.

Participants and Methods: This study examined differences in clinical, demographic, and gray and white matter volumes on brain MRI between 29 elderly depressed subjects and 11 health control. Analysis of covariance for age and education were performed to assess the relationship between neuropsychological and clinical scores, and brain atrophy.

Results: Depressed patients with more atrophy in the brain white matter demonstrated worse performance on Visual Reproduction Test; and when the atrophy was in the gray matter, more alteration was observed 
in Activities of Daily Living Scale. The control group demonstrated less depressive symptoms when they had less atrophy in the brain white matter; and less atrophy in the gray matter showed better performance in semantic verbal fluency test, CAMCOG. Cancellation Test and FOME. Conclusions: These preliminary results corroborate that elderly depressed present neuropsychological deficits and suggest possible neuroanatomical correlations for these findings.

Correspondence: Renata A. Thomas, Master, Psychiatry, University of Sao Paulo, Rua antonio de Gouveia Giudice 1406, Sao Paulo 05460001, Brazil.E-mail:avilarenata@uol.com.br

B. BALDIVIA, O.F. BUENO, V. ANDRADE, L.J. VAZ \& L. OLIVEIRA. Influence of High Education Level in Neuropsychological Assessment: Cognitive Reserve?

Objective: The Cognitive Reserve concept posits that plenty of stimulation throughout the lifespan (like occupational and educational attainment) supply a set of skills or repertories that can modify the Alzheimer disease course and protect people from cognitive impairment in the healthy elderly. However, it is unclear if higher education can attenuate these cognitive modifications, as a decline in episodic memory, working memory and processing speed. The purpose of this research is to observe the protective effect of high education before the neuropsychological profile of healthy aged.

Participants and Methods: Thirteen healthy elders (69.15 \pm 4.99$)$ was submitted to attentional, memory, language, reasoning, conceptualization and executive function tasks. They had their performances compared to thirteen healthy young group (33.46 \pm 8.62$)$. Both groups had higher educational level $(M=14.5)$.

Results: The t-test results showed better performance of the older group in Vocabulary $(p=0,004)$ and Comprehension $(p=0,045)$ tasks in comparison to the younger group, but lower performance in Picture Arrangement $(p=0,004)$. However ANOVA showed lower performance to older group than younger group for Stroop $(p=0,0001)$ and delayed recall of Associated Pairs $(\mathrm{p}=0,024)$ tests.

Conclusions: These results suggest that the stimulation provided by of high educational level induces a reduction of the negative impact in the neuropsychological profile of aged people, although cognitive domains are more vulnerable than others.

Correspondence: Beatriz Baldivia, master, UNIFESP, Rua Napoleão de Barros, 925, Sao Paulo 04024-002, Brazil. E-mail: biabaldivia@yahoo. com.br

N. CHANSON, P. ALLAIN, G. BERRUT, F. DUBAS \& D. LE GALL. Cognitive study of constructional abilities in normal aging.

Objective: Some data of the literature relative to the study of the effects of normal aging on constructional abilities state a degradation of performances with age, in particular during the spontaneous drawing or copy of simple or complex designs (Ska et al., 1986 ; Nasr-Wyler et al., 1998). In this study, we wished to complete these data and to analyze the impairments met by healthy old subjects by using the cognitive drawing production model of Van Sommers (1989).

Participants and Methods: Thirty nine subjects divided into four age groups (50-56, 60-69, 70-78, 81-88 years) were asked to produce, on oral order or in copy, meaningful and meaningless abstract designs in 2 and 2.5 dimensions, with or without help. These tasks were designed to test the different modules distinguished by Van Sommers (1989). We also tested motor, visuoperceptual and visuospatial abilities and semantic knowledge on drawings in each participant.

Results: Our results revealed that the oldest subjects (70 years and more) were more impaired than the subjects of the two youngest groups (50/56 years and 60/69 years) in all conditions. The copy improved performance in all group. Performance in additional specific tests was comparable in the four groups.

Conclusions: These results suggest that beyond the age of 70 years, the constructional difficulties of old subjects become more marked. Deficit does not seem connected to motor, visuoperceptual, visuospatial or semantic impairment. In regards to Van Sommers's model (1989), the system of visual representations seems preserved while the system of graphic production seems impaired, apparently because of planning difficulties.

Correspondence: Philippe Allain, PhD, Département de Neurologie, Unité de Neutropsychologie,, rue Larrey, Angers 49033, France. E-mail: PhAllain@chu-angers.fr

R. CORREIA, J. BARROSO, A. NIETO \& M. SABUCEDO. Qualitative Differences Between Young and Older Adults in Semantic Verbal Fluency.

Objective: Although no differences in total number of words generated have been reported before, a more qualitative approach was conducted in order to further examine differences between young and older adults when performing a semantic cued fluency task. Since Clustering and Switching have been proposed as two major cognitive components of verbal fluency with respectively dissociable neural substrates, we follow Troyer et al. (1997) guidelines with the aim of getting more information about normal aging cognition and brain changes associated.

Participants and Methods: Two groups of ten participants each [young adults age $X(S d)=41.10$ (5.705); old adults age $X(S d)=67.60(3.062)]$ were compared on a semantic verbal fluency task (animals). All participants were neurologically normal and right-handed, and both groups were equally distributed by sex.

Results: Our findings point that older adults produced larger clusters than young adults $(\mathrm{F} 1,18=6.996 \mathrm{p}=0.016)$ even when no significant differences were found between groups in total number of clusters generated $(F 1,18=1.465 p=0.242)$. However, young adults generated more isolated words $(\mathrm{F} 1,18=9.615 \mathrm{p}=0.008)$ and switched more often $(\mathrm{F} 1,18=16.729 \mathrm{p}=0.001)$ than older adults.

Conclusions: Despite previous results did not show differences in total number of words produced, qualitative differences between young and older adults in semantic verbal fluency become evident when deeper analysis are carried out. Taking together, these findings suggest that older adults experience some difficulties regarding the switching component of verbal fluency tasks. Since switching has been proposed to rely upon frontal lobe functioning, these results are congruent with brain aging studies where mild frontal lobe impairment is usually reported in normal aging.

Correspondence: Rut Correia, Facultad de Psicología, Universidad de La Laguna, Campus de Guajara s/n, La Laguna 38205, Spain. E-mail: rcorreia@ull.es

K. KaHlaoui, V. Vlasblom, F. LeSage, N. SENHADJI, H. BENALI \& Y. JOANETTE. Maintaining the Ability to Semantically Process Words With Age: A Near-Infrared Spectroscopy Study.

Objective: Neuroimaging studies have reported the presence of age-related reduction in hemispheric asymmetry on various cognitive tasks, a phenomenon captured by Cabeza in his HAROLD model. Studied by reference to memory, inhibitory, and perceptual processes, such a phenomenon has still never been described for the semantic processing of words. The aim of this study was to examine a possible reduction with age of the pattern of brain activation underlying the semantic processing of words using Near-Infrared Spectroscopy (NIRS), a non-invasive method which allows measurements of the concentration of oxygenated (oxy$\mathrm{Hb})$, and deoxygenated (deoxy-Hb) hemoglobin in the cortex.

Participants and Methods: Twelve young (25-35) and 12 old adults (67-84) were submitted to a NIRS acquisition while completing a lexical decision task (words/pseudowords) using lateralized stimuli presentation. Both sources and optodes of NIRS (TechEn CW32) were placed bilaterally over frontal and temporal regions, including Broca and Wernicke's areas. NIRS measures (oxy-Hb and deoxy-Hb) were analyzed in an event-related paradigm.

Results: While behavioural results showed relatively comparable performance between the two groups, NIRS results showed that older adults 
activated a larger bilateral network, involving both frontal and parietal regions, showing a clear change with age of the pattern of brain activation for semantic processing of words.

Conclusions: These findings suggest that the involvement of both hemispheres is part of a brain's compensatory mechanism subtending the preservation of language abilities in healthy aging. This study also confirms the relevancy of NIRS as a convivial and efficient neuroimaging technique for the study of cognitive healthy aging.

Correspondence: Yves Joanette, Ph.D., Centre de recherche, Institut universitaire de gériatrie de Montréal, $4565 \mathrm{ch}$. Queen-Mary, Montréal, $Q \mathrm{CO}$ H3W1W5,Canada.E-mail:yves.joanette@umontreal.ca

L.C. SCHERER, B. SKA, F. GIROUX, F. LESAGE, N. SENHADJI, K. MARCOTTE, H. BENALI \& Y. JOANETTE. Investigating Narrative Discourse Abilities in Successful Aging: A Neuroimaging Study Using NIRS.

Objective: Understanding the mechanisms underlying successful cognitive aging is important given the aging population. Though studies have been done for memory or attention abilities, few studies have considered language, and even fewer on narrative abilities. Discourse represents a complex cognitive ability relying on a unique conjunction of several $\operatorname{cog}$ nitive processes. By reference to the HAROLD phenomenon, it is expected that the neural bases of many of those abilities might dedifferentiate. The goal of this study was to explore the neurofunctional changes associated with successful aging for the comprehension of narratives (micro, macro-structure and situational level) using Near-Infrared Spectroscopy (NIRS). This technique allows to acquire sophisticated cortical measures while being in a relatively naturalistic environment.

Participants and Methods: Neuroimaging and behavioral data of 10 younger adults and of 10 elderly participants looking at micro- and macrostructures, as well as on the situational model were collected during the processing of short narratives. Ten younger adults under a low-proficiency L2 (second language) condition were also included in order to explore the possibility that the age-related neurofunctional reorganization would partly express a high-demanding resource allocation.

Results: Results show that there are definitely within- and across hemispheric differences in the neurofunctional pattern of activation in the older participants by reference to the younger ones, part of it being shared with younger adults in a low-proficiency condition.

Conclusions: This study allows for description of a more global functional reorganization with age, which goes beyond the expected dedifferentiation process. It stresses the relevance of the NIRS approach to study complex cognitive abilities.

Correspondence: Yves Joanette, Ph.D., Centre de recherche, Institut universitaire de gériatrie de Montréal, $4565 \mathrm{ch}$. Queen-Mary, Montréal, QC H3W 1W5, Canada.E-mail:yves.joanette@umontreal.ca

\section{J.L. MATHIAS \& L. EZIS. Cognitive Predictors of Unsafe Driving in Older Drivers: A Meta-analysis.}

Objective: Age-related changes in cognitive functioning, combined with a higher rate of motor vehicle accidents in older drivers, have led to a heightened interest in the driving abilities of older individuals. At present, there is no uniform approach to license re-assessment in older drivers, nor are existing practices evidence-based. Moreover, chronological age alone is a poor indicator of diminished driving ability and mandatory on-road assessments of driving ability in older drivers are impractical, highlighting the need for screening tests. The current study therefore undertook a meta-analysis of research published between 1980 and 2006 that compared the cognitive performance of older drivers in order to identify the cognitive tests that best discriminated between older drivers who either passed or failed a driving assessment.

Participants and Methods: A comprehensive search of the PubMed, PsycINFO, and CINAHL electronic databases was undertaken. All studies were screened using detailed inclusion and exclusion criteria.
Results: Cohen's d effect sizes were calculated to measure differences in the cognitive performance of older drivers who either passed or failed a formal driving assessment (i.e., on-road, closed-circuit, or driving simulator). Percentage overlap statistics measured the extent to which the cognitive performance of the pass and fail drivers overlapped, 95\% confidence intervals determined statistical significance, and Fail-safe N's assessed the potential impact of the bias toward publishing significant results.

Conclusions: The findings are discussed in terms of the most discriminating cognitive domains and the tests that provide cost-effective and evidence-based cognitive assessments that identify potentially unsafe drivers who are most in need of a full driving assessment.

Correspondence: Jane L. Mathias, PhD, Psychology, University of Adelaide, North Terrace, Adelaide, SA 5005, Australia. E-mail: jane. mathias@adelaide.edu.au

M. MOZAZ, M. GARAIGORDOBIL, G.P. CRUCIAN \& K.M. HEILMAN. Age Related Changes in Postural Knowlege.

Objective: Cognitive changes have been reported during healthy aging, but to our knowledge, age induced alterations of purposeful learned motor skills (praxis) of the upper limb has not been investigated.

Participants and Methods: Eighteen healthy elderly (66 to 88 years participants), who performed normally on the MMSE, were divided into two age groups, younger old (66-76) and older old (76-88). These subjects were administered a discrimination test that assessed forelimb transitive and intransitive postural knowledge.

Results: A repeated measures analyses of variance revealed significant main effects for age and task. There were no significant differences between the two groups in education or performance on the MMSE, but the older group did perform worse on posture recognition. While there were more errors in the recognition of transitive versus intransitive postures in the two groups, results showed that the recognition of both transitive and intransitive postures decline with age at similar rate.

Conclusions: These results support the postulate that healthy aging adversely influences both transitive and intransitive praxis knowledge. Correspondence: Maria Mozaz, PhD, Procesos Basicos, Facultad de Psicologia UPV/EHU, Avda. Tolosa, 70, San ebastian 20080, Spain. Email:MJ.Mozaz@ehu.es

C. RODRÍGUEZ-ARANDA \& R.B. ARNESEN. Spectrographic evaluation of verbal fluency performance over time in young and healthy elderly subjects.

Objective: It has been demonstrated that performance on different verbal fluency tests (VFTs) decreases over testing time. Studies addressing this issue have evaluated rate of word production across consecutive time intervals either in young healthy subjects or in varied patient populations. The present study assessed word production in VFT as a function of time in healthy elderly subjects in order to evaluate the impact of normal age-related psychomotor decline in VFTs.

Participants and Methods: A precise analysis of word generation was performed by the use of sound spectrographic techniques, which allowed measurement of utterances and pauses with millisecond accuracy. In order to evaluate age-related differences in VFTs performance two groups of subjects were tested: twenty young adults under 40 years and twenty healthy elderly over 65 years. Proper cognitive screening for dementia was carried out prior to testing. Both phonemic and semantic fluency tests were employed in the study. Only the first 30 seconds were analyzed. Subsequently, spectrographic analyses were done. Initial reaction time (time to generate the first word after instructions were given), total speech time (total time of word production) total speech rest time (total duration of silence between each word), and in-between word latencies were analyzed.

Results: Results were analyzed by 10 -second periods and they showed a significant decline in the latter parameters in healthy elderly compared to young adults. 
Conclusions: We conclude that additionally to pure decline in high cognitive functions in the elderly, psychomotor slowing in word production is an important element causing the age-related differences observed in VFTs performance.

Correspondence: Claudia Rodríguez-Aranda, Department of psychology, University of Tromsoe, Huginbakken 32, Tromsoe 9037, Norway: E-mail: claudia@psyk.uit.no

C. SOLE, D. BARTRÉS, C. JUNQUÉ, L. RAMI, B. BOSCH, A. VILLAR, N. BARGALLÓ \& J. MOLINUEVO. Functional And Structural Correlates Of Cognitive Reserve In Normal Aging, Mild Cognitive Impairment And Mild Alzheimer's Disease.

Objective: Cognitive reserve (CR) relates to aspects of brain structure and function able to sustain neuropathology such that the greater the reserve, the more severe the brain damage to cause clinical impairment. Objective: To study how grey and white matter (GM/WM) tissues and brain function were related to $\mathrm{CR}$ in normal aging, mild cognitive impairment (MCI) and mild Alzheimer's disease (AD).

Participants and Methods: Structural (voxel-based morphometry) and functional images (recognition memory task) were analyzed using statistical parametrical mapping (SPM2) in 16 controls, 12 MCI and 16 AD. CR proxies were educational and occupational attainment, WAIS vocabulary score and a tailored questionnaire of CR. Multiple regression analyses and simple correlations were carried out.

Results: fMRI: Among controls, increased CR was associated with decreased brain activity in the postcentral $(\mathrm{x}, \mathrm{y}, \mathrm{z}=-30,-18,50 ; \mathrm{t}=7.70$; $\mathrm{p}<0.001)$ and precentral gyrus $(\mathrm{x}, \mathrm{y}, \mathrm{z}=38,-4,30 ; \mathrm{t}=7.06 ; \mathrm{p}<0.001)$ with significant effects for educational/occupational attainment in this latter region. For $\mathrm{AD}$, there was a positive correlation between cognitive and mental stimulating leisure activities and brain activity in the cuneus $(\mathrm{x}, \mathrm{y}, \mathrm{z}=12,-60,8 ; \mathrm{t}=5.97 ; \mathrm{p}=0.007)$. VBM: Higher vocabulary correlated with increased GM in the mid frontal gyrus for controls $(\mathrm{x}, \mathrm{y}, \mathrm{z}: 25,40$,$20 ; \mathrm{t}=6.91 ; \mathrm{p}<0.001)$. MCI with higher literacy presented reduced WM in the posterior cingulate $(\mathrm{x}, \mathrm{y}, \mathrm{z}:-12,-64,15 ; \mathrm{t}=6.29 ; \mathrm{p}<0.001)$. More educated $\mathrm{AD}$ cases showed decreased GM in the anterior cingulate $(\mathrm{x}, \mathrm{y}, \mathrm{z}$ :$1,18,41 ; \mathrm{t}=5.9 ; \mathrm{p}<0.001)$.

Conclusions: Inverse associations between CR and structural and functional data were evidenced for controls and pathological aging. Increased brain atrophy among MCI and $\mathrm{AD}$ with increased CR supports the $\mathrm{CR}$ theory, indicating that those more educated patients can tolerate more brain damage.

Correspondence: Cristina Sole, University of Barcelona, Casanova, 143, Barcelona 08036, Spain.E-mail: ssolepadulles@yahoo.es

S. VILLENEUVE, S. BELLEVILLE, S. GAUTHIER \& J. LACOMBE. The Contribution of Vascular Burden in Persons with Mild Cognitive Impairment.

Objective: The presence of vascular diseases in older persons is associated with a higher risk of Alzheimer's disease and with larger executive deficits. Vascular risk factors and diseases have thus been suggested to represent a vascular burden that decreases brain reserve in older adults. The goal of this study was to assess the impact of vascular burden on the cognition of persons with mild cognitive impairment (MCI). Vascular burden in MCI may be associated with executive impairment, similar to what was found in healthy older adults. By reducing cognitive reserve, it may also increase the memory deficits that are characteristic of MCI. Participants and Methods: This was tested in seventy-two persons with amnesic single-domain or multiple-domain MCI and 72 matched controls. Neuropsychological tasks were used to assess four domains: executive functions, episodic memory, speed and general cognitive status. For each domain, a composite score was derived by combining individual Zscores for target measures. A vascular burden score was determined by computing each participant's number of vascular diseases.
Results: Correlations between the vascular burden score and the composite scores indicated that larger burden scores among MCI persons, were associated with lowest executive performance. An ANOVA was used to compare participants having at least one vascular disease with those with no cognitive burden. MCI persons having at least one vascular disease were found to be more impaired in the executive domain than MCI persons with no burden.

Conclusions: This study indicates that vascular burden increases the amount of executive impairment in persons with MCI.

Correspondence: Sylvia Villeneuve, Université de Montréal, 1050 Bernard, Montreal, QC H2V 1T8, Canada. E-mail: sylvia. villeneuve@umontreal.ca

S. VAN HOOREN, H. VAN DAMMEN \& W. WATERINK. Implicit learning capability in memory impaired psychogeriatric patients: a controlled study.

Objective: Implicit learning strategies may have the potential to positively influence memory functioning of memory impaired patients. The goal of this study was to investigate whether psychogeriatric patients were able to support their memory by implicit learning strategies that rely on visual, motor or verbal tasks.

Participants and Methods: The performance of 47 psychogeriatric patients with memory deficits was compared with a control group of 54 individuals. Both groups have been exposed to a number of tests on explicit and implicit memory. The explicit memory task was a verbal learning test. In order to measure implicit learning, three tests were used: a visuomotor learning test, a visuoconstructive learning test, and an errorless learning test (i.e. a test that focus on the prevention of errors during acquisition of information).

Results: As expected, the psychogeriatric patients performed worse on the explicit memory test compared to the control group. Both groups have shown significant learning effects on all implicit memory tests. In addition, an errorless learning paradigm enhanced the retention process in both groups.

Conclusions: Thus, psychogeriatric patients benefit from implicit learning on visuomotor, visuoconstructive and verbal tasks. The fact that psychogeriatric patients can learn via implicit learning on several domains may have important consequences for their treatment.

Correspondence: Susan Van Hooren, Open University of the Netherlands, P.O. box 2960, Heerlen 6401 DL, Netherlands. E-mail: susan. vanhooren@ou.nl

\section{Agnosia/Disordered Representations}

M. ALEGRET, G. MODINOS, A. ESPINOSA, S. VALERO, I. HERNÁNDEZ, A. MAULEÓN, M. ROSENDE, M. SÁNCHEZ, L. TÁRRAGA \& M. BOADA.The 15-Objects Test: detection of visual agnosia in preclinical and mild Alzheimer's disease.

Objective: The 15-Objects test (15OT), a visual discrimination task, was created by Pillon et al. (1989) to assess the slowing of cognitive processing in Parkinson's disease (PD). This test, based on the Poppelreuter test, consists of two figures with 15 over imposed images of objects. Poppelreuter type tests are frequently included in neuropsychological batteries to detect visual agnosia. However, more complex tests are required to detect visual agnosic signs in those patients that perform correctly on simple visual tests. The aim of the present study was to analyse the usefulness of the 15OT to assess visual agnosia in preclinical and mild Alzheimer's disease (AD).

Participants and Methods: The 15OT (form A or B) (Poppelreuter test and Luria's clock), and two tests sensitive to simple visual gnosias, were administered to 14 mild AD patients, 11 mild cognitive impairment (MCI) patients and 11 healthy control subjects.

Results: The analysis of variance (ANOVA) showed statistically significant differences between groups on the $150 \mathrm{~T}$ total performance 
$(F(2,63)=20.33 ; \mathrm{p}<0.001)$ and errors $(\mathrm{F}(2,63)=7.90 ; \mathrm{P}=0.001)$. Bonferroni's post-hoc analyses showed that $\mathrm{AD}$ patients performed significantly worse than MCI patients $(p=0.005)$ and controls $(p<0.001)$. A better performance on the 150T was related to a better performance on the two classical simple gnosia tests, that is, the Poppelreuter test $(r=0,498$, $\mathrm{p}<0,001)$ and Luria's clock $(\mathrm{r}=0,345, \mathrm{p}=0,005)$.

Conclusions: Results of the present study suggest that the 15OT is a useful tool for the detection of visual agnosia in MCI and AD patients when their performance on simple visual gnosia tests is still relatively preserved.

Correspondence: Montse Alegret, Doctor, Neuropsychology, Fundacio ACE, Marques Sentmenat,35-37, Barcelona 08014, Spain. E-mail: malegret@fundacioace.com

R.A. NANNERY \& B.A. WILSON. Body Awareness and Balint's Syndrome: An illustrative case study.

Objective: Balint's syndrome results from a difficulty in representing and interacting with external space, following bilateral parietal lobe damage. In cases such as autotopagnosia damage to the parietal lobes can also lead to deficits in the representation of internal space or body awareness.

This study looks at the integrity of body awareness in a patient with Balint's, despite deficits in representing external space.

Participants and Methods: MU, a 50-year-old male, with extensive bilateral occipito-parietal damage, following an anoxic brain damage, presented with Balint's syndrome.

MU's body awareness was assessed as follows. He was cued verbally to point to parts of his own body and body parts on a mannequin. He was then requested to mirror the pointing of body parts on himself and on a mannequin. Finally, his semantic understanding of body parts was assessed.

Results: In the absence of any semantic difficulty in understanding body parts, there is a clear significant difference in his performance between pointing to body parts on himself compared to pointing to external stimuli $(X 2=47.89, p<0.001)$. This suggests that MU has preserved personal body awareness despite Balint's syndrome.

Conclusions: The neuropathology underlying Balint's syndrome involves the posterior parietal lobe (Rafal, 1997), which programs movement to external objects (Connolly et al., 2001). Single cell studies in monkeys and neuroimaging in humans suggest that it is the superior parietal lobule, however, that processes the positions of body parts relative to each other. It is likely that the sparing of this region preserves body awareness in Balint's syndrome.

Correspondence: Rory A. Nannery, BSc, MSc, Cognition and Brain Sciences Unit, Medical Research Council, 15 Chaucer Road, Cambridge CB2 7EF, United Kingdom.E-mail: rory.nannery@mrc-cbu.cam.ac.uk

\section{Dementia Alzheimer's Disease}

J. ABRISQUETA-GOMEZ, F. OSTROSKY-SOLIS, P. BERTOLUCCI \& O. BUENO. Applicability of the Abbreviated Neuropsychologic Battery (Neuropsi)in Alzheimer's Disease Patients. Psychobiology Department - Universidade Federal de São Paulo.

Objective: The NEUROPSI is a brief neuropsychological battery developed to briefly assess a wide spectrum of cognitive functions. The aim of this study was to examine the applicability of a Portuguese version of this battery and verify the efficacy to detect cognitive impairment in Alzheimer's disease (AD) patients.

Participants and Methods: The NEUROPSI was applied to 75 elderly persons, 25 patients with a probable AD in mild-stage (AD1), 25 patients in moderate-stage (AD2) and 25 healthy elderly persons (control group), matched with the AD patients for age and schooling. Before testing all participants were applied the Mini-Mental State Exam (MMSE).

Results: Results showed significant differences in total scores on the tests: NEUROPSI $(p<0.001)$ and MMSE $(p<0.001)$, the control group scored highest in both of the tests followed by group AD1 and then group AD2. When cutoff scores for each test were determined, we noted that NEUROPSI was more sensitive and specific than MMSE in differentiating AD1 patients and control group. Differences were also found between the initial phase and the moderate phase

Conclusions: The results indicate that the NEUROPSI may be more efficient than the MMSE for detecting AD patients in the initial stage of the disease.

Correspondence: Jacqueline Abrisqueta-Gomez, PhD, Psychobiolohy, Universidade Federal de Sao Paulo, Rua Embaú,54, Vila Clementino, Sao Paulo 04039-060, Brazil.E-mail: jacky_ag@hotmail.com

J. ABRISQUETA-GOMEZ, V.D. VIEIRA, F. CANALI, C.C. PONCE, A.D. MARQUES MARTINS, B.G. SCHLECHT \& O.A. BUENO. What Do Really Need to Rehabilitate for Demented Seniors? Universidade Federal de São Paulo / Brain Checkup - Consulting and Research in Cognitive Neurosciences.

Objective: The benefits of non-pharmacological interventions are now accepted within the practical parameters for treating dementia in seniors. However, there is still a need to identify the real needs of patients and care givers in order to implement more effective interventions.

Objective: Investigate and prioritize patient needs to be focused in neuropsychological rehabilitation (NR).

Participants and Methods: Thirty Alzheimer patients in initial to moderate phase of the disease were selected for NR. In order to implement the intervention, they were assessed using neuropsychological instruments to examine their cognitive, functional and behavioral performance. The Revised Memory and Behavior Problem Checklist (RMBPC) was used to analyze the frequency of memory problems (MP), mood alterations (MA) and disruptive behavior (DB) presented by patients, and caregivers' negative responses to problems.

Results: There were declines in all cognitive functions and particularly memory. Scores on functional scales showed decline rated slight in $50 \%$, partial in $25 \%$ and total in $25 \%$ of the patients. RMBPC indicated predominance of MP (57\%) followed by MA (28\%) and DB (15\%). Caregivers' stress responses were not proportional to the frequency of problems; $48 \%$ (MP), $38 \%$ (MA) and $14 \%$ (DB), there was greater concern for MA and DB although it presented less frequently.

Conclusions: Activities for this group should seek to control MA and DB as the most stressful problems for members of patients' families and the ones that most affect functionality. NR for dementia patients ought to reflect a genuinely biopsychosocial model expressing the wishes and values of patients and their relatives.

Supported by AFIP

Correspondence: Jacqueline Abrisqueta-Gomez, PhD, Psychobiolohy, Universidade Federal de Sao Paulo, Rua Embaú,54, Vila Clementino, Sao Paulo 04039-060, Brazil.E-mail: jacky_ag@hotmail.com

D. BADENES, L. CASAS, J. CEJUDO \& M. AGUILAR. Driving, Mild Cognitive Impairment and Dementia.

Objective: Our objectives are first, to study if the tests used for the renovation of driving licence are appropriated and second, to set which neuropsychological tests may be useful for the decision about driving capacity on MCI and Mild Dementia patients. Furthermore we will evaluate the relationship between the routine neuropsychological tests used in a dementia unit and driving tests for licence renewal.

Participants and Methods: One hundred eighty four people were included distributed among three groups: 92 MCI, 55 MD and 40 healthy control. Sociodemografic and clinical data was recorded and cognitive functioning was by administering the RBANS, Trail Making Test (TMT), Block Design. Driving capacity was assessed both by the ASDE standard battery and UFOV test.

Results: We establised the cut off point of ASDE and UFOV tests for the prediction of clinical group. For MD group the sensitivity of ASDE was $86 \%$, specificity $95 \%$ (for subtest "concentrated attention and monot- 
ony resistence"; UFOV test sensitivity was $88 \%$, specificity $81 \%$. For MCI Group both tests showed a lower level of sensitivity and specificity (lower than $70 \%$ ). According to the performance on the UFOV only a half of MCI group could drive and patients showed different driving profiles according to MCI subtype. Neuropsychological tests results that showing best correlations with performance on driving test were: Block Design. Immediate Memory, Delaved Memory and TMT A.

Conclusions: 1.People with Mild Dementia mustn't drive.

2. ASDE can detect people suffering from dementia at mild stages in basis of the performance on this test.

3. According to the UFOV the half of people with a diagnosis of MCI should not drive.

4. The administration of the complete ASDE battery or UFOV allows the detection of those patients that are at risk of unsafe driving.

5.- The neuropsychological tests who to correlate by driving test was: Constructional praxis, memory and psychomotor performance are correlated to driving habilities on the studied samples.

Correspondence: Dolors Badenes, Neurology, Hospital Mutua de Terrassa, Castell 25, Terrassa 08221, Spain. E-mail: dolors. badenes@terra.es

L. CATALÀ, K. BEYER, C. CÁCERES, I. CLEMENTE, A. DÁVALOS, P. LATORRE, S. MARTÍNEZ \& M. MATARÓ. ACCUMULATION OF GENETIC RISK FACTORS AND COGNITIVE IMPAIRMENT IN ALZHEIMER DISEASE.

Objective: The study of genetic risk factors in Alzheimer disease has been a focus of interest in recent studies which highlight the possible involvement of multiple genes in the etiology of the disease. The objective of the present study is to assess the relationship between the accumulation of genetic risk factors and cognitive functioning in a sample of 120 Alzheimer patients.

Participants and Methods: Seven different genes were selected that. according to the literature, have a role in the development of the disease: Apolipoprotein E (ApoE), 2 ApoE promotors (-491AT and TH1/E47), Presenilin 1 (PS1), Butyrylcholinesterase (BchE), Cathepsin D (CatD) and Cystatin C (CST3). The sample was divided in two groups, a group of low genetic risk (LGR, $n=56$ ) with the presence of a maximum of 1 risk genotype and a group of high genetic risk (HGR, n=64) with more than 1 risk genotype. Both groups were comparable with regard to sex. age, years of disease duration [LHR: 2,67(1,53) vs, HGR: 2,55(1,36)] and levels of depression according to the Hamilton Depression Scale [LGR: 13,14(7,28) vs, HGR: 11,89(6,03)].

Results: The high genetic risk group obtained significantly lower scores in memory (Remote memory, $p=0,02$; RAVLT total learning, $p<0,01$ ), language (Boston Naming Test, $\mathrm{p}<0,01$ ), visual perception (Poppelreutter, $\mathrm{p}=0,01)$, ideomotor praxis $(\mathrm{p}<0,01)$ and executive functions (similarities WAIS, $\mathrm{p}<0,01)$.

Conclusions: These results suggest a relationship between the accumulation of genetic risk factors and the severity cognitive impairment in Alzheimer disease.

Correspondence: Laia Català, Neurology, Hospital Universitary Germans Trias i Pujol, CR. Canyet S/N, Badalona 08916, Spain. E-mail: laiacatala@gmail.com

I. CONTADOR, B. FERNÁNDEZ, J. CACHO, I. BURÓN \& I. ARRANZ. Influence of concomitant depression in Alzheimer disease on non verbal memory.

Objective: To research the influence of clinical depression (D) in non verbal memory task (NVM) of patients with Alzheimer disease (AD).

Participants and Methods: The sample was composed by 51 participants. Of these, 10 were patients with NINCDS-ADRDA criterion of

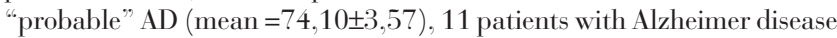
and provisional criteria of depression in AD (Olin et al., 2002; mean = $76,00 \pm 3,79$ ), and 30 healthy subjects (HS; mean $=72,90 \pm 5,84$ ). The groups have been compared on Dementia Rating Scale (DRS-2; Jurica et al., 1988), Cornell Depression Scale (CDS), and specific battery of NVM task: Corsi test (C), Block Test (BTT), visuo-spatial learning test (VLT), Visual Pattern Test (VPT), Abstract Design Test (ADT) and Complex Figure Test (CFT). The groups no differ in terms of age, sex or years of schooling. AD groups showed similar level of general cognitive impairment $(\mathrm{t}=-1,63, \mathrm{~g} . \mathrm{l}=19, \mathrm{p}=.119)$.

Results: Statistical significant differences were found between $\mathrm{AD}$ and HS groups on DRS-2 $(p<.001)$ and all NVM measures: Corsi test $(p<.001)$, BTT $(p<.001)$, VLT $(p<.001)$, VPT $(p<.001)$, ADT $(p<.001)$ and CFT $(\mathrm{p}<.001)$. However, no statistical significant differences were found between AD and ADD in NVM tasks, which only differs in CDS. Conclusions: There were not differences in non verbal memory task between AD and ADD groups. The neuropsychological consequences of depression on NVM in AD may be overshadowed by the influence of neurodegenerative disease.

Correspondence: Israel Contador, Psychology, University of Salamanca, Avda Merceds/n, Salamanca370005,Spain.E-mail:icontador@usal.es

A.B. FAGUNDO, S. LOPEZ, M. ROMERO, J. GUARCH, T. MARCOS, R. BLESA \& M. SALAMERO. Clustering and Switching in Semantic Fluency: Predictors of the Evolution to Alzheimer Disease?

Objective: Alzheimer Disease (AD) patients frequently exhibit a deficit in semantic fluency. Observation of clustering (words produced inside the same subcategory) and switching (ability to change the subcategory) reveal that two different but complementary cognitive strategies are used in the fluency task.

Purpose:(1)To compared semantic fluency performance and clustering and switching performance among patients with $\mathrm{AD}$, subjects with memory complaints and healthy controls.

(2) To examine the clinical utility of this verbal fluency scoring system in the prediction of incident $\mathrm{AD}$, in a sample of memory complaints subjects. Participants and Methods: $42 \mathrm{AD}$ patients, 38 subjects with memory complaints, and 25 healthy controls were compared in in semantic fluency performance (total generated words, cluster size and number of switches) The 38 subjects with memory complaints were followed-up for a maximum period of two years. They were re-evaluated and either remained in the initial group (memory complaints group) or defined as probable $\mathrm{AD}$.

Results: Group differences were conducted with ANOVA. AD patients generated significantly fewer correct words $(p<.001)$ and showed a reduction in cluster size $(\mathrm{p}=.008)$ and switching $(\mathrm{p}<.001)$. Memory complaints group showed a significant reduction of corrected words $(\mathrm{p}<.001)$ and clustering $(\mathrm{p}=.008)$ compared to healthy controls. After 2 years, the subgroup of patients who were diagnoses as AD showed a reduction in semantic fluency $(p<.01)$ and smaller clusters $(p=.007)$ compared to no change in diagnostic subgroup. There were no significant differences on switching scoring. A logistic regression to determine which variable would better predict the evolution to $\mathrm{AD}$ was performed. The cluster size was better predictor to AD evolution than total words generated and switching.

Conclusions: Alzheimer Disease and memory complaints patients have an alteration in both qualitative and quantitative aspects of semantic fluency. A clustering analysis would enhance the early $\mathrm{AD}$ diagnostic reliability.

Correspondence: Ana B. Fagundo, Psychology, Institut Municipal d'Investigació Médica, Caspe 190, entresuelo 1a, Barcelona 08013, Spain.E-mail:bettyart2@hotmail.com

Y. GAESTEL, N. RAOUX, H. AMIEVA, C. FABRIGOULE \& J. DARTIGUES. Improving Clock Drawing Test Assessment in Ageing by Qualitative Analysis of Errors.

Objective: The Clock Drawing Test (CDT) is widely used in assessing cognitive impairments, especially in the case of the detection of dementia. Yet, more than 20 descriptions of methods to assess and analyse CDT results can be found in litterature. This study was designed to qualita- 
tively describe the errors displayed in this task by non-demented and demented subjects with two main goals: to better understand how CDT results are influenced by socio-demographic variables and to specify the nature of the underlying cognitive processes giving raise to errors.

Participants and Methods: The data used were recorded on a sample of 1695 subjects issued from a French prospective cohort (3 City study). We examined subjects' drawings and listed all the errors occuring. Multiple regression analyses were conducted, taking into account the demented status, age, education and gender. Then we qualitatively described the errors to better specify the underlying cognitive impairments.

Results: Results confirmed that age, gender and educational level significantly influence CDT performance. Moreover, certain errors, common in normal ageing, were distinguished from those more specific in dementia. Conclusions: Three specific cognitive domains were impaired in CDT: semantic, executive and visuo-spatial. Visuo-spatial errors were very frequent in normal ageing and did not characterize demented subjects. Semantic and executive errors occurred in both populations, but with deeper deficits in demented subjects. Thus, taking into account the nature of errors occurring in CDT may help to determine which cognitive domains are impaired and to increase the discriminating power of this test. Correspondence: Helene Amieva, PhD, INSERM U.593, Université Bordeaux 2, 146 Rue Léo Saignat, Bordeaux 33076, France. E-mail: Helene.Amieva@isped.u-bordeaux2.fr

\section{GARCÍA-VIEDMA, S. FERNÁNDEZ \& R. MARTOS. LIMITATIONS WITH THE ACTIVE MANIPULATION OF THE INFORMATION AT EARLY STAGE OF DEMENTIA OF ALZHEIMER TYPE.}

Objective: The attentional control deficits could explain the presence of alterations in the complex attentional components and executive functions at the early stages of dementia of Alzheimer type (DAT). We explored the attentional control operations related to: a) the coordination of different tasks, b) the inhibition of irrelevant information, c) the change of information recovery strategies or action plans, and d) activation of the information from the long term memory (Baddeley, 1996; Miyake et. al., 2005).

Participants and Methods: Fourteen patients with initial DAT and 14 healthy older adults were applied the following tasks: a dual-task condition, a Stroop-like test, a task-switching, a random number generation activity and a subtest of ECCO battery (López-Higes, del Río and Fernández-Guinea, 2005).

Results: The results showed significant differences between both groups in reaction time and percentage of errors in the Stroop-like test $(\mathrm{F}(1,26)=$ 11.150, $\mathrm{p}=.003 ; \mathrm{F}(1,26)=11.719, \mathrm{p}=.002)$ and task-switching $(\mathrm{F}(1,26)=7.774, \mathrm{p}=.010 ; \mathrm{F}(1,26)=5.601, \mathrm{p}=.026))$. The DAT patients presented a worse performance in all the tasks. In addition, the patients showed a more stereotyped performance in the random number generation $(\mathrm{U}=49.5, \mathrm{p}=.023)$. They also got an inferior score in the subtest of ECCO battery $(\mathrm{F}(1,26)=5.476, \mathrm{p}=.027)$, and in the dual task condition $(\mathrm{F}(1,25)=4,818 ; \mathrm{p}=.038)$.

Conclusions: The patients at the early stages of DAT would present a generalized deterioration of the attentional control. These deficits imply difficulties with the active manipulation of the information. These data also would support the utility of the attentional control as a cognitive marker of the beginning of the DAT.

Correspondence: Ma Rosario García-Viedma, Doctor, Psicología, Universidad de Jaén, Paraje Las Lagunillas s/n, Jaén 23071, Spain. Email:mrgarcia@ujaen.es

M.I. GOMEZ-RUIZ, A. AVILA, J. BELLO, P. MAHO, M.A. ESPASA \& A. AGUILAR. Discourse Analysis of Seven Bilingual Patients with Alzheimer's Disease.

Objective: Studies in bilingual patients with Alzheimer's disease (AD) are consistent with preferential preservation of the first acquired language (Ribot's law) and with problems in language choice and language mixing during spontaneous speech. The purpose of this study is to compare Spanish and Catalan discourse abilities of seven bilingual patients diagnosed with $\mathrm{AD}$.

Participants and Methods: We compare 7 patients with early AD according to NINCDS-ADRDA criteria. For all of them, their native and dominant language was Catalan (L1), and L2 (second language) was learned between 5 and 7 years old. They all completed their education in Spanish (mean years: 5 ). Spontaneous speech in each language was analysed following a neurolinguistic approach based on the post-test analysis of the Bilingual Aphasia Test (BAT) (Michel Paradis, 1987). We also included items regarding linguistic interferences (that is, code-switching and borrowing of words and morphosyntactic structures).

Results: Discourse analysis showed a slightly better fluency when patients spoke in their first language (Catalan). Nevertheless, the number of linguistic interferences from L2 to L1 were much higher than from L1 to L2 and this difference was statistically significant $(p<0,05)$.

Conclusions: Although fluency was slightly better in patients first language, the influence from one language to the other was asymmetrical. It would be expected that more words and utterances from L1 should appear in L2, such previous studies have shown. However, in healthy subjects, this pattern of linguistic interferences is common in Catalonia. These results indicate the importance of being aware of the sociolinguistic differences that exist among states.

Correspondence: Maria I. Gomez-Ruiz, Neurology, Hospital General Hospitalet, Av. Josep Molins 29-41, Hospitalet de Llobregat 08906, Spain.E-mail:misabelgr@gmail.com

\section{JOOSTEN \& M. DEKKERS. Measuring MCI awareness using (modified) IQCODE.}

Objective: To investigate awareness MCI patients and its possible correlation with personality characteristics and disease variables.

Participants and Methods: 66 MCI patients and their close relatives from 4 hospitals in the Netherlands participated in this study and were compared to 20 control couples. Differences in memory problem evaluations between MCI patients and relatives were compared to the evaluations of the control couples using the IQCODE. This analysis provides a measure for MCI awareness. MCI awareness measurement was correlated with cognitive impairment, gender, age, education, mood, neuroticism and coping.

Results: IQCODE measurements showed a significant difference between the MCI/relative group and the control couples $(p<0.001)$

IQCODE measurement showed significant correlation with mood and neuroticism $(p<0.01$ and $p<0.05$ resp.) and with memory performance $(\mathrm{p}<0.001$ MMSE, $\mathrm{p}<0.05$ AVLT $)$

Conclusions: (modified)IQCODE can measure MCI awareness and analysis of this patient group shows that awareness is correlated with mood, neuroticism and memory performance.

Correspondence: Liesbeth Joosten, Msc, medical psychology/memory clinic, university medical centre, Reinier Postlaan 4, Nijmegen $6500 \mathrm{HB}$, Netherlands.E-mail: l.joosten@ger.umcn.nl

X. MILleT, J. DARTIGUES, C. FABRIGOUle \& H. AMIEVA. Episodic Memory Impairment in Alzheimer's Disease: Encoding or Retrieval Deficits?

Objective: Even though perceptual priming persists following short delays, patients suffering Alzheimer's disease (AD) fail to exhibit perceptual priming effect when study-test delay is incremented. Nonetheless, multiple exposures with the material appeared to strengthen long-term memory trace during incidental retrieval in $\mathrm{AD}$ patients. The present study investigates the presence of a long-term priming effect in AD through a procedure involving material repeated exposures.

Participants and Methods: The procedure consisted first in administering the Free and Cued Selective Reminding test (FCSRT) to 20 patients suffering mild to moderate AD and 20 matched elderly adults. The FCRST is a word-list recall test involving semantic encoding at the ini- 
tial phase of the test, followed by free and cued recall trials and a recognition task. After a 30-minute delay, participants were finally submitted to a word identification priming task consisting in identifying the words studied during the FCSRT among new words lexically matched. Because the FCSRT involves repeated exposures with the material, we hypothesise that AD individuals will produce long-term repetition priming similar to controls.

Results: The AD patients were massively impaired in explicit measures of the FCRST while they exhibited a long-term priming effect similar to the elderly control group.

Conclusions: Whereas explicit retrieval of information is definitely impaired in AD, more implicit processes allowing the retrieval of that same information seem to be still efficient. Spared priming abilities in a delayed implicit memory task should lead to question the common statement that memory deficits in $\mathrm{AD}$ are solely due to encoding deficiency. Correspondence: Helene Amieva, PhD, INSERM U.593, Université Bordeaux 2, 146 Rue Léo Saignat, Bordeaux 33076, France. E-mail: Helene.Amieva@isped.u-bordeaux2.fr

F. MORENO-MARTÍNEZ, R. DAVIES \& F. CUETOS. Stability and change in picture naming by Spanish Alzheimer's patients: a longitudinal study using photographs.

Objective: Picture naming is an important task for the assessment of cognitive deterioration in dementia of the Alzheimer's type (DAT) because naming performance has been found to decline gradually throughout the course of the illness. Current theories ascribe the declining accuracy evidenced by DAT patients to a progressive loss of more specific semantic features pertaining to pictured concept representations, linked to a rise over time in the production of semantic errors that name the category of the pictured object, or a category coordinate.

Participants and Methods: We report a new study of naming in DAT conducted with Spanish-speaking patients. Nine patients presenting evidence of mild-to-moderate DAT, and neurologically intact age- and education-matched controls, were asked to name 112 photographs depicting a range of objects, both living or natural things and artefacts. The novel contribution of our study, in which development in naming performance was studied at three time points, was the use of colour photographs to mitigate against the possibility that superordinate errors are made because the line drawings in previous studies did not readily evoke basic level concepts.

Results: Age-of-acquisition and name agreement were found to be important influences both on the likelihood that patients or controls would name a picture correctly and on the likelihood that the type of response made by patients would change over time.

Conclusions: Patients made increasing numbers of superordinate errors as the illness progressed and performance in both the naming task and a non-verbal semantic task deteriorated over time.

Correspondence: F. Javier Moreno-Martínez, Psicología Básica I, U.N.E.D., C/ Juan del Rosal, 10, Madrid 28040, Spain. E-mail: fjmoreno@psi.uned.es

F. MORENO-MARTÍNEZ, K.R. LAWS, M. GOÑI-IMÍZCOZ \& S. MORIANO-LEÓN. A three years long study on the semantic impairment in dementia of the Alzheimer type.

Objective: The study of category-specific effects or categorial dissociations of living and nonliving domains in dementia of the Alzheimer's type (DAT) is a matter of ongoing and permanent debate among researchers. In this way, researching on category-specific effects could provide us a better understanding on the organization of conceptual knowledge in human brain. In spite there have been a large number of transversal studies on this topic, no many longitudinal ones have been carried out. While more complicated to execute, longitudinal studies are especially suitable for investigating pathologies like DAT, which is known to be diffuse and -usually- slowly progressive.
Participants and Methods: We present longitudinal data, gathered through a three years period, of nine (five females) DAT patients -from an initial cohort of fourteen- and 34 (17 females) healthy elderly volunteers with similar demographic characteristics. All the participants were assessed with several tasks of the Nombela semantic battery (Moreno \& Cañamón, 2005): 1) Category fluency, 2) naming of color photographs without cueing, 3) naming in response to verbal description, 4) sorting task, and 5) word-photographs matching.

Results: While, respect to controls, DAT patients showed a profound semantic impairment in every semantic task, it was not observed a differential impairment of living and nonliving domains.

Conclusions: Our findings are discussed in relation to recent theories of semantic representation, particularly in relation with correlated structure models, since they propose a relationship between level of brain impairment and deficit of living and nonliving domains.

Correspondence: F. Javier Moreno-Martínez, Psicología Básica I, U.N.E.D., C/ Juan del Rosal, 10, Madrid 28040, Spain. E-mail: fjmoreno@psi.uned.es

G.E. PEREIRA, R. NITRINI \& P. CARAMELLI. COMPARISON OF THE RESULTS OF THE BRIEF COGNITIVE SCREENING BATTERY, THE MINI MENTAL STATE EXAMINATION AND THE DEMENTIA RATING SCALE OF MATTIS.

Objective: To investigate the correlation between the performance at the (BCSB), Mini Mental State Examination (MMSE) and the Mattis Dementia Rating Scale (DRS-2) of a Portuguese sample of healthy elderly, as well as the influence of the educational level, age and gender in the results.

Participants and Methods: We evaluated 64 healthy elderly, with selection based on strict inclusion and exclusion criteria (median age $=79.8$ years; $31 \mathrm{~F} / 23 \mathrm{M}$; median schooling $=4$ years). The sample was divided into three groups of educational level (2-4 years, 5 -8 years and 9-12 years) and in three age groups (65-75 years, $76-80$ and $>80$ years. All the individuals had been submitted to the BCSB (consisting by the following tests: incidental memory, immediate memory, learning, delayed recall and recognition of ten figures, verbal fluency (animals) and clock drawing), MMSE and DRS-2.

Results: The scores in all items of the BCSB, MMSE and DRS-2 were not influenced by the educational level, age and gender. A perfect positive correlation was verified between the results of the individual items of the BCSB, BCSB (total) and the results of the DRS-2 (total) $(\mathrm{p}=0.01)$. No correlation was verified between the results of the BCSB (total), Verbal Fluency, Clock Drawing and the results of the MMSE. Positive correlations emerged between the MMSE and incidental Memory, Learning and Delayed Recall $(p=0.05)$, as well as immediate Memory and Recognition $(p=0.01)$.No significant differences had been observed in relation to gender in none of the results of the three tests.

Conclusions: Age, educational level and gender did not influence the performance of this sample of healthy elderly in three neuropsychological batteries. There was a perfect correlation between individual items of the BCSB and total DRS-2 scores.

Correspondence: Gilberto E. Pereira, Master, ISMAI, rua António Soares Pereira, no 57, 3.4, Maia, Porto 4470, Portugal. E-mail: gilbertopereira2@clix.pt

N. RAOUX, J. DARTIGUES \& H. AMIEVA. Semantic Verbal Fluency in the Preclinical Phase of Alzheimer's Disease: a Longitudinal Analysis of Clustering and Switching Processes from the Paquid Study.

Objective: To investigate the cognitive processes underlying the deficit in semantic verbal fluency observed in the preclinical phase of Alzheimer's Disease (AD), we qualitatively analyzed subjects' verbal production in the light of Troyer's model (1997). According to this model, verbal fluency performance depends on two fundamental processes: clustering (referring to the ability to generate words belonging to a specific subcategory of semantic network) and switching (referring to the ability to shift from one subcategory to another). 
Participants and Methods: The results are gathered from the longitudinal PAQUID study. 51 future AD subjects having completed the animal verbal fluency task at dementia incidence, 2 years and 5 years before clinical diagnosis were included. Each case was matched to two control subjects (total sample $=153$ subjects). The number of switches (transition between clusters) and the mean cluster size (number of words within a cluster) were calculated.

Results: The total word production was significantly reduced 5 years before the dementia incidence. Whereas the mean cluster size was not different between the two groups at any time of the follow-up, the switching index was lower in the future $\mathrm{AD}$ subjects including 5 years before dementia incidence. A significant decline of this parameter was found all along the prodromal phase until the clinical dementia diagnosis. Conclusions: Our findings suggest a dissociation between clustering and switching processes, with a very early decline for switching processes. Therefore, the diminished fluency performances of future AD patients would be better explained by shifting ability impairment rather than by degraded semantic storages.

Correspondence: Helene Amieva, PhD, INSERM U.593, Université Bordeaux 2, 146 Rue Léo Saignat, Bordeaux 33076, France. E-mail: Helene.Amieva@isped.u-bordeaux2.fr

K. SHAW, L. COONAN, L. ENNEW, L. YAGUEZ \& D. MATTHEWS. Memory function in older adults with and without Alzheimer's disease employing a kinesiology-movement based intervention.

Objective: To explore the effect of a non-aerobic kinesiology-movement based activity on memory in older adults with and without Alzheimer's disease $(\mathrm{AD})$.

Participants and Methods: The study employed a repeated measures design and included 3 groups; dementia movement group, dementia control group, healthy older adult movement group. All members of the dementia groups met the DSM-IV diagnostic criteria for AD. Participants engaged in a 6-weekly group of either non-aerobic kinesiology-movement based activity or a socialisation- memory skills training control group. Cognitive functioning (assessed using subtests from the CANTAB), mood, and participant's subjective reports of memory function were measured at baseline and post-intervention.

Results: The healthy older adults performed significantly better on all measures of cognitive functioning relative to the dementia groups both before and following intervention. The dementia groups did not differ at baseline in any variable, with the exception of a learning test. Following intervention both movement groups showed a significant improvement in attention relative to baseline. The dementia movement group also performed significantly better on a visual recognition memory test following intervention compared to the dementia control group. No differences were found on the subjective measures found between groups.

Conclusions: The results suggest that non-aerobic kinesiology-movement based activity may facilitate attention in older adults with or without AD. Such movements may also have a beneficial effect on visual recognition memory in people with $\mathrm{AD}$. The results from this study suggest a novel movement based activity that may be useful for older adults with AD. Correspondence: Kendra Shaw, PhD DClinPsyc, Oliver Zangwill Centre, Princess of Wales Hospital, Lynn Road, Ely, Cambridgeshire CB6 1DN, United Kingdom.E-mail:kendra.shaw@nhs.net

\section{Dementia Subcortical}

Y. HIGUERAS \& J. MUÑIZ-CASADO. Emotional Processing in PD: Knowing What Are We Doing?

Objective: To comment studies which assess emotional processing in Parkinson Disease (PD). The differences in outcomes may be due to the methodology because they use to develop face processing (FP) tasks to assess emotional processing (EmP).

Participants and Methods: In a sample of 24 patients with idiopathic PD and 24 controls, we administered the Benton Facial Recognition Test-Ab- breviated (BFRT-A). This test evaluate the visual discrimination capacity and the ability to match unfamiliar faces without the interference of the mnemonic component. We perceive no DSM-IV criteria of dementia nor depression according to DSM-IV criteria and Yesavage Depression Scale score. We also check if task the authors used involved FP in order to assess EmP. Results: we compared PD and control groups in the correct responses, omissions and mistakes in the BFRT-A. We found statistically significant differences in all measures ( $p$ 0.001) between groups so visuoperceptive processing of faces is impaired in first stages of the disease. The authors mainly used emotional recognition and perception of facial expressions, emotional prosody tasks and facial expression tasks. We found indeed other studies in which they used recognition tasks of emotional intonation in reading sentences, to read sentences in a emotional manner or to identify emotional meaning in some sketches.

Conclusions: dopaminergic system is related very closely and complexly with EmP. Different studies used face emotional processing to assess this fact and reach contradictory outcomes. We propose that, as other authors begins to do, we should experiment with different tasks in which FP were suppressed, because this ability is impaired, as well as visoperception and executive function. Making cognitive neuropsychology needs the knowledge of cognitive processes to prevent wrong conclusions. Correspondence: Yolanda Higueras, Neurology, HGUGM, Doctor Esquerdo 46, Madrid 28007, Spain.E-mail:yolandahigueras@hotmail.com

I. GALTIER, A. NIETO, J. BARROSO \& N. LORENZO. Differential Impairment in Verbal and Visuospatial Memory in Nondemented Parkinson's Disease Patients.

Objective: Memory problems are frequently reported in PD patients. Verbal learning and memory are usually examined, but less is known about visoespatial learning. In this study we aimed to examine verbal and visuospatial learning and memory in nondemented PD patients using a similar procedure that allows comparing the performance on both memory tasks. Participants and Methods: We studied sixteen patients (age range: 46-75; MMSE mean: 26.56) and fifteen matched controls (age range: 49-78; MMSE mean: 28.43). Illness severity was assessed by UPDRSMotor Examination (mean: 32.50) and Hoehn \& Yahr Scale (II-III). CVLT was used to assess verbal learning. To examine visuospatial learning we used the 8/30 Spatial Recall Test (an adaptation of 10/36 SRT; Rao et al, 1991) that includes five learning trials as well as free and cued recall, similarly to CVLT. In addition visuospatial function was evaluated with Judgment of Line Orientation Test.

Results: No significant differences were found between both groups for any of the CVLT measures. However, for 8/30 SRT, PD patients showed worse performance on several learning trials as well as in total learning. No differences were found in visuospatial free and cued recall. Performance on JLOT was impaired in PD patients and correlated with visuospatial learning capacity.

Conclusions: Our results suggest that visoespatial learning impairment is an early problem in PD that precedes verbal memory deficits. These deficits can be explained as a consequence of early affectation of visoespatial functions. We propose the 8/30 SRT as a useful test for early detection of visuospatial memory deficits in Parkinson disease.

Correspondence: Iván Galtier Hernández, Psicobiología y Metodología de las Ciencias del Comportamiento, Universidad de La Laguna, Campus de Guajara, La Laguna 38205, Spain.E-mail: igaltier@ull.es

H. SINZ, T. BENKE, G.K. WENNING, H. STOCKNER, W. POEWE \& M. DELAZER. Decision Making and Estimation of Probabilities in Parkinson Disease Dementia.

Objective: While decision making has been examined in non demented patients with Parkinson's disease,indicating a potential key role of dopamine in taking risky decisions, (e.g. Brand et al., 2004; Czernecki et al., 2002) and Huntington's disease (Stout et al., 2001) little is known about decision making in patients with Parkinson disease dementia (PDD). 
Participants and Methods: In the present study different types of decision making processes were investigated using 1) a task with implicit rules for gains and losses, the Iowa Gambling Task (IGT), and 2) a risk task based on explicit rules and emphasis on the estimation of probabilities based on proportions. Additionally, patients performed tests of verbal and visual memory, object naming, numerical processing and frontoexecutive functions.

Results: Though as a group PDD patients differed from healthy controls in neuropsychological background tests (memory, fronto-executive functions, numerical processing) they showed a similar response pattern in the explicit gambling task which requires the estimation of numerical proportions and probabilities. By contrast, in the implicit gambling task PDD patients showed an inconsistent response pattern and made more disadvantageous and risky decisions than controls.

Conclusions: The present study points out that in different types of decision making tasks different types of cognitive processes are demanded. It further suggests that PDD patients have more difficulties in emotionally driven, implicit decision making tasks, than in explicit tasks with stable rules.

Correspondence: Hermann Sinz, Cognitive Neurology and Neuropsychology, Innsbruck Medical University, Anichstrasse 35, Innsbruck6020,Austria.E-mail: Hermann.Sinz@student.uibk.ac.at

\section{Dementia: other}

O. FELICIAN, S. JOUBERT, E.J. BARBEAU, E. TRAMONI, J. RANJEVA, M. PONCET \& M. CECCALDI. Progressive Loss of Memory about Famous People in Right Temporal Variant of Semantic Dementia.

Objective: To report the detailed neuropsychological examination of a case of right temporal variant of semantic dementia

Participants and Methods: Single case study of CM, a 61-year-old righthanded woman, presenting with a slowly progressive deterioration in the identification of familiar and famous persons, contrasting with the relative preservation of other cognitive domains.

Results: Full-scale IQ was 94 (verbal: 96, performance 92). Primary face perception skills were intact. Evaluation of semantic memory revealed a severe loss of knowledge concerning famous people (assessed through presentation of their face, voice and name) and famous monuments. In contrast. knowledge related to other domains of semantic memory was preserved. Picture naming (Snodgrass and Vanderwart battery),as well as performance on the verbal and visual PPTT were preserved. Knowledge about famous melodies (humming, singing and naming) was very good. Quantitative volumetric measurements of temporal lobe structures showed a prevalent atrophy of temporopolar regions, greater in the right hemisphere.

Conclusions: The results of the present study suggest that right temporal variant of frontotemporal lobar degeneration can be characterized by a selective semantic impairment involving certain kinds of exemplars such as people and monuments, while other domains of semantic memory remain intact. This study offers interesting insights regarding the contribution of the right temporopolar cortex to semantic memory. Correspondence: Emmanuel J. Barbeau, PhD, Université Toulouse 3, CNRS, CerCo, Faculté de Médecine de Rangueil, Toulouse Cedex 9 31062, France.E-mail: emmanuel.barbeau@cerco.ups-tlse.fr

C.V. FLAHERTY-CRAIG, A. BROTHERS, B. DEARMAN, D. WAGNER \& Z. SIMMONS. Frontal Dysfunction in ALS May Be Associated with Loss of Cognitive Insight in the Absence of Behavioral Change.

Objective: To determine the relationship between cognitive insight and behavioral change in ALS patients with frontal dysfunction.

Participants and Methods: Eight ALS patients with poor verbal problem solving ability to screen exam (Neurobehavioral Cognitive Status Exam) underwent more extensive evaluation to characterize their capacities for verbal and non-verbal problem solving, as well as ability to discern Eckman emotional facial expressions. Problem solving measures included Guilford Alternate Uses, Missing Cartoons and Expression Grouping. Each measure was preceded and followed by patient ratings of how well they would do and how well they did. Correlations between test findings and ratings of performance were made by Spearman rho. Behavioral change was evaluated by completion of self-report and family report measures created for this study, which included behaviors of concern to ALS patients; e.g., driving, eating and use of BiPap. The Mann Whitney $\mathrm{U}$ test was applied to evaluate mean differences.

Results: All patients evidenced difficulties with most or all of the problem solving measures, as well as emotional expressions. All insight measures demonstrated negative correlations to cognitive test findings $(p<.046$ - .018). Behavior rating scale findings did not differ significantly between patient and family reports $(\mathrm{p}=.240)$.

Conclusions: Executive functioning change in ALS may be characterized by diminished reasoning across a broad spectrum in the presence of lost insight, while in the absence of any evident behavioral change. An understanding of this in the clinical setting is vital to treatment planning, with greater emphasis needed on support of both patient and caregivers in the process of decision making throughout the course of the disease process.

Correspondence: Claire V. Flaherty-Craig, Ph.D., Neurology, Penn State Hershey Med, 500 University Drive, H037, Hershey, PA 17033. E-mail: ccraig@psu.edu

O. GELONCH, A. LÓPEZ, E. TALLANT, A. MORERA, S. RAMOS, S. MEZA, G. CHICO \& M. AGUILAR. The Psychological Support within Neuropsychological Rehabilitation: About a Case of PCA.

Objective: To expose the importance of making at the same time emotional support and neuropsychological rehabilitation in the cases of mild dementia with preservation of insight.

Participants and Methods: PCA is a subtype of AD characterised by an unusual repercussion of the disease on the cortical visual associative areas, although with a clinical profile clearly different from the typical AD.

We presented a patient of 57 years old who has presented a progressive course characterised by a precocious and disproportionate affectation of the complex visual processing of 5 years' duration. At the moment she presents apperceptive visual agnosia, simultanagnosia and deficits located in the posterior areas of language (alexia), as well as important difficulties in the attentional ability. She presents less important impairment in other cognitive areas (executive and memory functions) and reactive depressive simptomatology. She is independent in the B-ADL and the I-ADL, although the visual impairment begins causing mistakes to her.

Results: She participates in a program of non-pharmacological treatment that includes cognitive stimulation and occupational therapy to help her to compensate her limitations and to stimulate the preserved functions. The cognitive impairment has progressed but the insight has not been reduced, causing an important frustration to her, faced with the evidences of this impairment.

Conclusions: The neuropsychologist must add the emotional support to the neuropsychological training program, with the objective to assure that the cognitive, emotional and behavioural disturbances have the minimum repercussions on her global performance, and to assure therefore one more global and effective intervention.

Correspondence: Olga Gelonch, AVAN, Joan Amades, 1, Sabadell 08206 , Spain.E-mail:ogelonch@copc.es

V. MatuszeWki, P. PIOLINo, A. PÉlerin, F. MEZENGe, C. LALEVÉE, V. DE LA SAYETTE, F. EUSTACHE \& B. DESGRANGES. Semantic Dementia (SD): A Longitudinal Case Study of Autobiographical Memory Impairment and of Anatomical and Functional Alterations.

Objective: Autobiographical memory plays a crucial role as it allows constructing a feeling of identity and continuity. The aim of this study was 
to emphasize patterns of autobiographical memory impairment, of anatomical and functional alterations in one SD patient with disease worsening.

Participants and Methods: We studies patient PG in two testing sessions held 18 months apart. Autobiographical memory was assessed using the TEMPau task designed to gauge autobiographical memory across 5 time periods covering the entire life-span [1]. The patients underwent a MRI examination and a resting PET scan using [18F]. Results: Results (statistical significance according to z-scores) of the first testing session revealed that PG's performances, compared to control subjects were lower regardless of the time period. Qualitative analysis of these data highlighted decreased performances with increasing time interval. Performances on the second assessment also showed impaired performances for every time period and qualitative analysis of z-scores showed decreased performances compared to the first testing session for nearly every time period. Morphological and functional abnormalities $(p=0.001$ uncorrected) concerned at the first assessment bilateral temporal lobe predominant on the left, which spread out with disease worsening. Conclusions: Results of the first autobiographical memory assessment suggest a reversed temporal gradient and are in keeping with previous studies [2]. The breakdown of autobiographical memories with advancing pathology and the neuroimaging results are in line with Conway's model according to which the personal semantic knowledge base (stored in the temporal cortex) plays a crucial role in the retrieval of autobiographical memories [3].

References: [1] Piolino et al., 2003, Brain, 22,1-30, [2] Ivanoiu et al., 2006, Neuropsychologia, 44, 1936-55, [3] Conway M.A., 1996. In D.C. Rubin, Cambridge University Press.

Correspondence: Vanessa Matuszewki, Post- doctorate, Inserm E 0218Université de Caen, Laboratoire de Neuropsychologie, CHU de Caen, Avenue de la Côte de Nacre, Caen cedex 14033, France. E-mail: vanessamatuszewski@hotmail.com

M. PINO. Neuropsychological profiles in Semantic Dementia and Alzheimer disease: 4 presenile cases.

Objective: To show the usefulness of neuropsychological assesment in differential diagnostic in 4 presenil dementia cases and to compare them with neuroimaging

Participants and Methods: Neuropsychological assesment was administered to 3 men and 1 woman with suspected dementia but still not classified. It consists in: family interview and the administration of test of these cognitive domains: mini-mental statuts, attention, language, visual gnosis, writting habilities, memory and constructional praxis. Table1 Results: The results and neuroimage for each subject is shown in table 2 Conclusions: From all the administered tests, the constructional praxis was the the most especific to distinguish the cognitive impairment due to left fronto-temporal degeneration from the parieto-temporal one, in clinical terms: Semantic Dementia from dementia of Alzheimer type. The results are coincident with the bibliografy found

Correspondence: Maria Pino, Xarxa Sanitaria i Social Santa Tecla, Cl Joan Fuster, s/n, Tarragona 43007, Spain.E-mail:mpino73@gmail.com

\footnotetext{
C. SANTOPOLO HITA, M. ANTEQUERA TORRES, L. VIVANCOS MOREAU, B. MARTINEZ HERRADA, P. FERNÁNDEZ OLIVARES, B. GARCÍA TORRES \& J. MARÍN MUÑOZ. Neuropsychologic profile of the Syndrome of Korsakoff: Presentation of a clinic case.

Objective: Describe the clinical of a patient with syndrome of Korsakoff with an Alcoholic Dementia evolution by means of his neuropsychological valoration.

Participants and Methods: It has been fundamentally based on the clinical history, being emphasized his personal antecedent and explorations (general medical, neurological, neuropsychological and psichopatologic), analitic and MRN. Moreover the prescribed treatment was contributed. Scales of the daily life, psiquiatrycs, neuropsychologicals
}

protocols; including the informatic program Experia.dem (Minimental, Germcide, Cerad, Clock Drawing Test, Trail Making Test), and other tests related to the fronto-temporal alterations were administered.

Results: The results obtained in the neuropsychological exploration showed an alteration of the fluencies, as much immediate visual and verbal memory as delayed, with affectation of the recognition. With a minimental of $23 / 30$.

Conclusions: The analisys of the neuropsychologicals data confirms one bilateral temporal dysfunction on which the definitive diagnostic is based. Correspondence: Carmen María Santopolo Hita, Hospital, Carretera Cartagena-Murcia, El Palmar 30120, Spain. E-mail: santopolo24@hotmail.com

\section{Normal Aging}

D. ADROVER-ROIG, J. PERIANEZ \& F. BARCELÓ. Distraction and Perseverative Scores of the Madrid Card Sorting Test (MCST) as Sensitive Indexes of Cognitive Decline in Healthy Aging.

Objective: Healthy ageing is accompanied by neurofunctional changes that allegedly lead to a selective decline in executive functions compared to other general cognitive functions. However, the specificity of such alterations is still a matter of debate. We used a new measure of cognitive flexibility, the Madrid Card Sorting Test (MCST; Barceló, 2003), in order to explore (1) its relationship with some well-known indexes of executive functions, and (2) its relative sensitivity for assessing normal cognitive decline.

Participants and Methods: One hundred healthy elderly people (mean age $=62.4, \mathrm{SD}=8.6 ; 48-88$ years) took part in the study. Exclusion criteria were MMSE $<24$ and Geriatric Depression Scale scores $>14$. Two age groups (cut-off 59 years) were compared using a neuropsychological battery to assess general cognitive and executive function, including the Brixton test (Shallice \& Burgess, 1999), Paired Associates Learning (PAL, Sahakian \& Robbins, 2000), Zoo Map (Alderman, Evans, Emslie, Wilson \& Burgess, 1996) and the MCST (Barceló, 2003), as well as other classical tests such as Trail Making Test, Stroop and Digit Symbol. A linear combination of executive function indexes was used to estimate general cognitive status, which showed an interaction with age.

Results: The most age-sensitive measures were MCST distraction and perseveration errors and, marginally, Stroop interference. These age-related differences were even more reliable after partialling out the speed of processing.

Conclusions: Multivariate analyses suggest that "it is important to recognize both the unity and diversity of executive functions" (Miyake et al $2000)$ and the relative role of speed of processing in age-related cognitive changes.

Correspondence: Daniel Adrover-Roig, Phd Student, Psychology, University of the Balearic Islands, Ctra. Valldemossa Km 7.5 Edifici Beatriu de Pinós, despatx 25, Palma de Mallorca 07122, Spain. E-mail: daniel.adrover@uib.es

M.F. ANDERSSON, M. YSTAD, A. LUNDERVOLD \& A. J. LUNDERVOLD. A neuropsychological and MRI study of response competition and goal maintenance in older age.

Objective: We investigated the effect of age on measures of responsecompetition and goal maintenance, where these are measured by time to complete and number of errors in the Delis, Kaplan and Kramer's (2001) version of the Color-Word Interference Test (CWIT). In a sub-sample, we also examined the association between selected CWIT- and morphometric MRI measures.

Participants and Methods: Participants were recruited from an ongoing study of "Cognitive aging, brain functions and genetic markers". A sample of 285 healthy individuals, divided into age groups: $46-54$ yrs (young), 55-64 (middle) and 65-79 (old), completed the CWIT. In a subgroup of 90 participants ( 64 women, mean age $=59 \mathrm{yrs}$ ) we estimated right 
and left frontal lobe grey matter volumes from 3D MRI recordings using FreeSurfer. Correlation analyses between completion time and error measures in the four CWIT conditions versus frontal lobe grey matter volumes were performed.

Results: We found significantly longer completion-time in the Color naming, Inhibition and Switching conditions and significantly more errors in the Switching condition for older adults than younger adults. There was a weak negative correlation between frontal lobe grey matter volumes and the Inhibition and Switching conditions.

Conclusions: The results indicate that age is associated with slowness in response-time and reduced ability to keep task goals available when inhibiting and switching between tasks. Thus, older adults seem more vulnerable to effects of context. The correlations between the selected MRI and CWIT measures were weak. A next step will be inclusion of demographic and fine-grained MRI variables in the analysis.

Correspondence: Martin F. Andersson, Department of Biological and Medical Psychology, University of Bergen, Jonas Liers vei 91,, Bergen 5053, Norway. E-mail: Martin.Andersson@psybp.uib.no

\section{G. RABOYEAU \& A.I. ANSALDO. Aging and Second Langauge Learning: A Different Way of Being Performant.}

Objective: The aim of this study was to compare brain plasticity sustaining second language (L2) learning, in elderly and young subjects.

Participants and Methods: Highly-performant elderly and young French-speaking adults learnt 80 Spanish(L2)words: 40 cognate/easy words, and 40 non-cognate/difficult words. The lexical learning phase consisted in a first 5-day shallow-learning period, and a second two-week deep-learning period. Naming in L2 during er-fMRI scanning was performed after each learning period. Functional data were analysed with SPM2.

Results: Reaction times and error rates decreased significantly between T1 and T2, with both age groups. Networks differed across groups, being significantly larger with the elderly. Thus, with cognates, significant activations were observed the left inferior frontal lobe (BA46/10), the medial frontal lobe (BA9/10), the cingulate, the visual cortex, and the cerebellum bilaterally; with non-cognates, the network comprised the same areas, and also the inferior frontal lobe (BA11/46), and the thalamus bilaterally. At T2, young participants significantly activated the caudate nucleus, bilaterally, both with $\operatorname{cog}$ nate and non-cognate naming. The elderly showed two distinct patterns: with cognates, there was significant activation in the right inferior frontal lobe (BA47), whereas with non-cognates, activation maps included the right orbital frontal gyrus (BA11/47), and the left inferior frontal gyrus (BA44/45).

Conclusions: These results suggest that the encoding and/or retrieval of new phonological material requires the recruitment of larger and bilateral networks in the elderly. Consolidation brings a concentration of the network, regardless of age; however, with the elderly, there is an increased reliance on structures sustaining attentional processing. In summary, equivalent behavioral performances in L2-learning engage different neural structures across groups, thus reflecting age dependent brain plasticity mechanisms

Correspondence: Ana I. Ansaldo, Ph.D, Faculté de médecine, Université de Montréal, Centre de recherche de l'Institut universitaire de gériatrie de Montréal, 4545 Queen Mary, Montréal, QC H3W1W5, Canada. Email: ana.ines.ansaldo@umontreal.ca

\section{N. GAWRON. Neuropsychological Performance of Healthy Older Persons in Context of Chosen Theories about Brain Aging.}

Objective: The presented results are part of an ongoing research on neuropsychological validation of three theories about brain aging: a) the hemi-aging model, b) the frontal lobe hypothesis and c) the neurodegenerative hypothesis. The purpose of this fragment of the study was to determine neuropsychological similarities and differences between $\operatorname{cog}_{-}$ nitive functioning of healthy elderly and clinical groups with a) right hemisphere damage b) frontal lobe dysfunction, or c)mild neurodegenerative disease.

Participants and Methods: The study was run on four groups: 17 neurologically intact elderly (79.8 $\pm 6,7$ years old, MMSE $28.7 \pm 1.2), 15$ right hemisphere damage patients (RHD) with infarctions in different RH areas (63,2 $\pm 15,5$ years old, MMSE $26.4 \pm 2.13), 15$ patients with mild subcortical vascular dementia $(\mathrm{SVaD})$ (who display frontal dysfunction, 77.1 \pm 4.9 years old, MMSE $26.2 \pm 2.2$ ), and 11 persons with

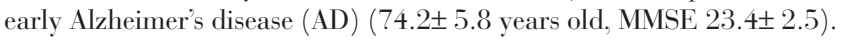
The presented outcomes concern: attention (Bourdon Cancellation Test), reasoning (WAIS-R (PL) Similarities), executive functioning (Wisconsin Card Sorting Test), memory(WAIS-R (PL) Digit Span, California Verbal Learning Test) and narrative discourse (story retelling, comprehension of metaphors and inferred meanings from the Polish version of Right Hemisphere Language Battery).

Results: One-way ANOVA and Tukey's post-hoc test on raw scores revealed few statistically significant differences between the performance of healthy elderly and mild SVaD group, as well as RHD group. AD group performed significantly lower than healthy elderly in almost all trials.

Conclusions: These preliminary results confirm previous evidence that neurologically intact cognitive aging has similar pattern to mild SVaD's. Therefore, they support the frontal lobe hypothesis. However, these findings also suggest existence of some similarities between healthy cognitive aging and right hemisphere damage symptoms.

Correspondence: Natalia Gawron, Faculty of Psychology, Warsaw University, ul. Stawki 5/7, Warsaw 00-183, Poland. E-mail: ngawron@o2.pl

B. HANNA-PLADDY \& E. DOUGLASS. Neuropsychological Profiles of Musicians: Evidence for Cognitive and Motor Maintenance in Aging.

Objective: Playing a musical instrument is a complex cognitive process involving reading musical notation, memorizing musical passages, and translating musical symbols into sequential and precise fine motor skills. Previous studies have suggested that musicians have distinct cerebral characteristics reflective of cortical plasticity, and that functional differences are related to the age of acquisition. However, it is unclear if playing a musical instrument is protective against age-associated cognitive and motor decline, and if this is related to extent of training.

Participants and Methods: Forty healthy participants (ages 50-80) were classified into groups based on their level of musical training (none; intermediate; advanced), and details of their age of acquisition, duration, and continued participation were obtained. All subjects received a neuropsychological evaluation and performed skilled motor tasks. Advanced and intermediate musicians did not differ in age of acquisition, but advanced subjects were more likely to continue playing late in life.

Results: Advanced subjects recalled more items on a verbal memory task (CVLT-II) when compared to the intermediate $(\mathrm{p}<.03)$ and non-musical subjects $(p<.02)$. They also had better non-verbal recall (VR-II, WMSIII) than the non-musical group $(p<.02)$, and were faster on Trails B than non-musicians $(\mathrm{p}<.04)$. On skilled motor tasks, the advanced group displayed more pronounced manual asymmetries and faster speed on the grooved pegboard task and a novel tweezer dexterity task $(\mathrm{p}<.01)$, when compared to the other groups.

Conclusions: Extended musical training appears to provide maintenance of cognitive and motor skills in advanced age. Memory recall, visuomotor speed, motor dexterity and precision are better preserved in instrumental musicians with more advanced musical training. These findings support the premise that musicians experience cortical plasticity, and that functional differences in musicians are a result of years of musical training.

Correspondence: Brenda Hanna-Pladdy, PhD, Landon Center on Aging/ Neurology, University of Kansas Medical Center, 3901 Rainbow Blvd, Mailstop 1005, Kansas City, KS 66160.E-mail: bhpladdy@kumc.edu 


\section{R. RODRÍGUEZ \& J. GONZÁLEZ MARQUÉS. WORKING MEMORY AND AGING.}

Objective: The age-related deficits on memory performance are not uniform. The substantial age-related decrements existed in working memory tasks. The aim of the present study was to analyse alterations produced in normal aging in different working memory tasks.

Participants and Methods: 71 individuals were divided in two age groups: (a) 55-65 years old and (b) 66-75 years old. Every subject was pretested on Spanish version of Mini Mental State Examination (MMSE) to rule out any evidence of cognitive decline or dementia. Also we evaluated possible depression signs by Yesavage Geriatric Depression Scale. We used the following tasks: Digit span (WMS-III), Spatial span (WMSIII), Letters and numbers (WMS-III), Mental control (WMS-III), Stroop test, D2 test, TMT (A and B), a task of searching in a map, a switching task, a dual task and a task based on the Daneman and Carpenter's task (1980).

Results: We found statistical significant differences in Spatial span, Mental control, part of Stroop test (colour, word-colour and pc', but not in interference), in the concentration index of D2 test, in A and B TMT, in the map searching task, in time used in switching task, in number of errors in dual task, and in number of remembered word and interferences in Daneman and Carpenter's task.

Conclusions: Our experiment provides evidence that age is a crucial factor in working memory, especially from 65 years old. Now we are concerned with trying to explain if they are due to deficit in inhibitory control, to reduced processing resources or to retrieval speed reduction. Correspondence: Raquel Rodríguez, Metodología de las CC. del Comportamiento, UNED, C/ Juna del Rosal, 10, Madrid 28040, Spain. E-mail: rrodriguez@psi.uned.es

\section{Other}

\section{K. FUCHS \& C. MANNING. Medication Management Skills in} Individuals with Mild Cognitive Impairment.

Objective: Individuals with Mild Cognitive Impairment (MCI) have less cognitive decline than those diagnosed with dementia and do not exhibit impairment in activities of daily living. This is often determined by self or family report rather than direct assessment of self-care tasks. We sought to determine how individuals with MCI perform on a medication management task and to assess performance in individuals with amnestic versus nonamnestic patterns of MCI.

Participants and Methods: Six participants met the Petersen criteria for amnestic MCI. Eight were diagnosed with nonamnestic MCI. Participants were given tests of learning and memory, working memory, and medication management. In the latter task, subjects were asked to fill a weekly pillbox from mock prescription bottles with dosage instructions. Results: The two groups were not significantly different in age $(p=.46$; range 61-80 yrs), education ( $\mathrm{p}=.22$; range 12-20 yrs), MMSE score ( $\mathrm{p}$ $=.62$; range $24-28)$ or on a working memory task $(\mathrm{p}=.28)$. As expected, the groups were significantly different on delayed recall for a 10-item memory test $(p<.01)$. Performance did not differ on the number of errors on the medication management test $(p=.48)$. However, there was a significant correlation between MMSE score and the number of errors $(p<.035)$. Further analysis indicated that only two participants performed this task without error, and nine made at least two errors.

Conclusions: Individuals with amnestic and nonamnestic MCI may have greater difficulty on complex tasks of self-care than is apparent to family members.

Correspondence: Kathleen Fuchs, PhD, Neurology, University of Virginia, PO Box 800394, Charlottesville, VA 22908-0394.E-mail:klf2n@virginia.edu
U. DIAZ, B. MATELLANES \& J. MONTERO. EFFECTS OF A COGNITIVE STIMULATION PROGRAM IN THIRD AGE.

Symposium Description: Increase of life expectancy in our culture has implied an increase in prevalence of pathologies associated to the ageing process. Thus, proposals of multidisciplinary intervention are increasingly necessary for an integral approach of these pathologies.

In this sense, the main goal of the current research has been the creation, development and implementation of a cognitive stimulation program. Hypothesis for this research states that people with cognitive decline, treated by means of this cognitive stimulation program, maintain and/or improve their cognitive performance in both dependent variables of language and visual motor coordination. Independent variable is the cognitive program known as "Gimnasia de la Memoria" [Memory Gym]. With this purpose, it has been developed a clinical design with pre and post treatment measures, and an analysis of changes, in a sample of 10 elderly people ( 3 men and 7 women)

Statistical analyses, by means of the Wilcoxon test, show that we obtain an improvement for language and visual motor coordination variables, with the following results: in the one hand, for language function, our sample, assessed with the language subscale from CAMCOG test, experimented an increase of their performance from a mean score of 17,50 (with a standard deviation of 6,819 ) to 19,90 (s.d.= 7,047), $\mathrm{z}=-2,579$, $\mathrm{p}=.01(\mathrm{p}<.05)$; in the other hand, for visual motor coordination, our sample, assessed with ideational motor praxis subscale from CAMCOG test, improved their performance from a mean score of 4,70 (s.d.=1,567) to $5,50($ s.d. $=1,581), \mathrm{z}=-2,333, \mathrm{p}=.02(\mathrm{p}>.05)$.

Correspondence: Unai Diaz, Universidad de Deusto, Avda. Universidades,24,Bilbao 48007, Spain.E-mail: undiaz@yahoo.com

\section{SEGALÀ CASTRO \& M. JÓDAR VICENTE. Verbal and Spatial Learning in Old Men and Women.}

Objective: To examine whether there are any sexual differences in verbal and spatial memory in old age. Several studies have found differences between men and women in spatial and verbal cognitive tasks. The present study compared spatial span and verbal learning between old men and women to determine if differences between sexes are also found in learning and how this might be influenced by different patterns of aging in both sexes. Unlike other studies, the present one has controlled cardiovascular risk factors.

Participants and Methods: Sixty healthy subjects (25 men and 35 women) participated in the present cross-sectional study where cardiovascular risk factors were included as criteria of exclusion. Spatial learning was measured using the Corsi Block tapping test whereas verbal learning was measured with the Rey Auditory Verbal Learning Test (RAVLT). Results: Level of education differed between the two groups. Therefore, ANOVA with sex as the independent variable was conducted together with an analysis of covariance being the education level the variable to covariate. A female superiority was found in RAVLT track $4 \mathrm{p}=0.04$, RAVLT track $5 p=0.03$ and RAVLT delayed recall $p=0.01$ while no differences were found in visual learning.

Conclusions: Women maintain superiority in verbal learning throughout their life span. Our group of old men did not perform significatively better in spatial memory. More research is needed to clarify if men generally outperform women in tasks of spatial memory and whether $\operatorname{cog}$ nitive decline in benign aging is stronger in men than in women.

Correspondence: Laura Segalà Castro, Psicologia Clínica i de la Salut, Universitat Autònoma de Barcelona, Edifici B. Campus UAB, Bellaterra (Cerdanyola del Vallès) 08193, Spain. E-mail: laurasegala@hotmail.com 


\section{SATURDAY AFTERNOON, JULY 7, 2007}

\section{Symposium 7 \\ 12:00-1:30 p.m.}

\section{Neurocognition and Psychopathology in Schizophrenia: and Integrated Vision Inside Neuroscience.}

\section{Discussant: Miguel Bernardo}

\begin{abstract}
M. BERNARDO. Neurocognition and psychopathology in schizophrenia: and integrated vision inside Neuroscience.

The cognitive deficit in schizophrenia is a core feature of the illness and not the result of symptoms and pharmacological treatment. Programa Esquizofrenia Clinic, PEC, in its line of research and training presents this symposium with part of our studies in course about cognition and psychopathology related to schizophrenia.

We test the hypothesis of endogenous Auditory Verbal Hallucinations (AVH) involving activation of auditory/linguistic association cortices that are usually activated by externally presented speech. One of the meaning of social cognition is the human ability to perceive other peoples emotions and we are studying the recognition of face emotional feelings with PET-18F-FDG technique. We revised the cerebral structural alterations related to diagnosis and their cognitive correlations using Voxel-Based-Morphometry. And to conclude, we exposed the analysis of the relationship between cognitive rehabilitation and its outcomes in daily living in patients with schizophrenia

Correspondence: Dr. Miquel Bernardo, Schizophrenia Clinic Program Hospital Clínic *Universitat de Barcelona *IDIBAPS, C/Villaroel, 170 *08036 Barcelona*Spain, email: bernardo@clinic.ub.es
\end{abstract}

E. PARELLADA. Auditory Hallucinations in First Episode Schizophrenic Patients.

Auditory verbal hallucinations (AVHs) likely result from disorders, as yet unspecified, of the neural mechanisms of language. Here we examine the functional neuroanatomy of single-word reading in patients with and without a history of AVH. METHOD: Eighteen medicated schizophrenia patients ( 8 with AVH and 10 without $\mathrm{AVH}$ ) and 12 healthy control subjects were scanned with PET (15)O-water technique under 2 conditions: reading aloud English nouns and passively looking at English nouns without reading them. RESULTS: The contrast between the 2 conditions shows higher activation in Wernicke's area during the reading condition in the patient group and a reversed laterality index for the supplementary motor area in the AVH group. CONCLUSIONS: These findings provide indications about the possible mechanisms of AVH. We suggest that the abnormal laterality of the supplementary motor area activity accounts for the failure to attribute speech generated by one's own brain to one's self and that the activation of Wernicke's area accounts for the perceptual nature (hearing) of the patient's experience. Correspondence: Dr. Miquel Bernardo, Schizophrenia Clinic Program Hospital Clínic *Universitat de Barcelona *IDIBAPS, C/Villaroel, 170 *08036 Barcelona *Spain, email: bernardo@clinic.ub.es

\section{E. FERNÁNDEZ-EGEA. Emotion assessment with PET-18F-FDG.}

The investigation into neural back-ground of emotions in normal and brain pathology using functional brain imaging techniques (fMRI, PET, SPECT) remains one of the most interesting scientific challenges in the area of psychiatry. The aim of this presentation is: 1) to present the recent neuroimaging findings concerning functioning of "emotional brain" in normal and brain pathology with particular emphasis on psychiatric disorders (schizophrenia), 2) to consolidate knowledge of neuroimaging techniques and experimental designs used in the assessment of human affect
Correspondence: Dr. Miquel Bernardo, Schizophrenia Clinic Program Hospital Clínic * Universitat de Barcelona *IDIBAPS, C/Villaroel, 170 *08036 Barcelona*Spain, email: bernardo@clinic.ub.es

N. SEGARRA. Cognition, Cerebral Structure and Outcome in Schizophrenia.

Schizophrenia patients have reduced frontal volumes (as measured by MRI) in comparison with normal controls, but among patients with schizophrenia, reduced volumes of the posterior (temporal, parietal and occipital) cortex have been associated with poor outcome. Poor outcome in our sample (across a duration of 10 years of illness) was associated with gray matter deficits in posterior cingulate while compensatory white matter increases in dorsal posterior regions may be related to better outcome. Poor-outcome patients displayed significantly more clinical symptoms, and were more severely impaired in cognitive and social functioning than good-outcome patients. Possible consequences of this may include thought disorder, disturbance of consciousness, treatment resistance, and cognitive decline indicative of a dementing process as a superimposed or inherent part of this schizophrenia subtype.

Correspondence: Dr. Miquel Bernardo, Schizophrenia Clinic Program Hospital Clínic *Universitat de Barcelona *IDIBAPS, C/Villaroel, 170 *08036 Barcelona*Spain, email: bernardo@clinic.ub.es

R. PENADÉS. Cognitive Rehabilitation in Schizophrenia and Functional Recovery.

Cognitive Remediation Therapy (CRT) will be described as a designed tool to improve neurocognitive abilities in schizophrenia. The author will present data to evaluate the effect of CRT on neurocognition, and secondarily on symptomatology and psychosocial functioning in the pathology compared to CBT, designed to improve emotional problems and positive symptoms, focusing on modification of maladaptive beliefs and schemas, but not neurocognition. Data on 40 chronic patients were randomly assigned for 4 months to one of two treatment groups: CRT or CBT. Repeated assessments were conducted before and after the treatments and at the end of a follow-up period of 6 months. Results showed that CRT produced an overall improvement on neurocognition, particularly in verbal and nonverbal memory, and executive function. CBT showed the expected treatment effect on anxiety and depression but produced only a slight non-specific improvement in working memory. Patients receiving CRT showed improvement in social functioning which remained after 6 month follow-up

Correspondence: Dr. Miquel Bernardo, Schizophrenia Clinic Program Hospital Clínic *Universitat de Barcelona *IDIBAPS, C/Villaroel, 170 *08036 Barcelona*Spain, email: bernardo@clinic.ub.es

\section{Invited Symposium 12:00-1:30 p.m.}

\section{Impact of Psychiatric and Other Co- Morbidities on Neuropsychological Functioning in Alcoholism}

\section{Chair: Omar Alhassoon}

\section{O. ALHASSOON. Impact of psychiatric and other co-morbidities} on neuropsychological functioning in alcoholism.

For several decades, researchers have been reporting a wide variety of neuropsychological deficits associated with alcohol dependence. These deficits fall into such diverse domains as novel problem solving, abstract reasoning, learning and memory, and visuospatial skills. 
However, the pattern of neuropsychological impairment does not appear to be the same for all alcoholics. In addition, up to half of the patients examined do not show any cognitive impairment. This symposium poses the question whether the variability in neuropsychological impairment found among alcoholics might be related to common co-morbidities associated with the disease. For example, 70-90\% of patients seeking treatment for alcohol dependence smoke tobacco. Dr. Durazzo will present recent data on differences in neuropsychological profiles and associated metabolic and structural neuroimaging data of smoking versus non-smoking alcoholics. Similarly, there is a high prevalence of comorbid personality disorders among substance-abusing patients in general, and alcoholics in particular. Dr. Dom's research explores the question of whether the decision making deficits associated with alcoholism are specific to the disorder itself, reflect a neurobehavioral abnormality of the underlying comorbid personality disorder, or are a characteristic vulnerability of both disorders. Dr. Uekermann's research examines the role of depression in the cognitive functioning of alcoholic patients. Depression is the most frequently diagnosed Axis-I disorder in alcoholic patient. Among alcoholic patients, 27-69\% have elevated depression scores and 15-28\% suffer from major depressive disorder. Although depression on its own has been associated with memory and executive deficits, its role in influencing the neuropsychological profile of alcoholic patients has not been well established. Finally, the explanatory role of alcoholic typologies in understanding the patterns of neuropsychological impairments in patients has not been well studied. Some alcoholic typologies use specific co-morbid disorders, such as antisocial personality disorder, to classify patients. Therefore, differences between types of alcoholics may be directly related to the distribution of co-morbid disorders in the general patient population. Dr. Alhassoon will present a direct comparison of the neuropsychological profiles and associated regional cerebral blood flow findings in two subtypes of alcoholics (anxious/depressed versus antisocial) classified based on latent class analysis of their MMPI profiles.

Correspondence: Omar M. Alhassoon, Ph.D., University of California, San Diego, School of Medicine, Department of Psychiatry, La Jolla, CA 92093,Email oalhassoon@ucsd.edu

T. DURAZZO \& D. MEYERHOFF. Comorbid chronic cigarette smoking in alcohol use disorders: The neurobiological and neurocognitive consequences.

A vast body of research indicates chronic smoking has adverse effects on human cardiac, pulmonary, and vascular function and increases risk for various forms of cancer. However, comparatively little is known about how chronic smoking affects human brain neurobiology and function. Although smoking rates continue to decrease in the general population in the developed world, they remain high in developing countries and in individuals with alcohol use disorders (AUD). Despite both the high prevalence of concurrent chronic smoking in AUD and the intense interest in biomedical consequences of AUD, surprisingly few studies have addressed the potential neurobiological or neurocognitive consequences of chronic smoking in AUD. In this presentation, we briefly review the emerging literature on the neurobiologic and neurocognitive consequences of chronic cigarette smoking. We will subsequently summarize our neuroimaging and neurocognitive studies describing the neurobiological and neurocognitive consequences of comorbid cigarette smoking and AUD, in both treatment-seeking and treatment-naïve U.S. populations. Our findings suggest comorbid chronic cigarette smoking in AUD compounds magnetic resonance-detectable abnormalities in brain morphology, metabolites, and perfusion, modulates neurocognition, and that chronic smoking adversely affects neurobiological and neurocognitive recovery in AUD. Consideration of the potential separate and interactive effects of chronic smoking and AUD may foster a better understanding of the mechanisms associated with brain injury in AUD and the factors that influence recovery from chronic and excessive al- cohol consumption. More generally, further research on comorbid smoking in AUD will generate information that is relevant to neuroimaging and neurocognitive research involving other substance use disorders and neuropsychiatric conditions in which chronic cigarette smoking is prevalent (e.g., psychostimulant abuse, mood disorders, and schizophrenia-spectrum disorders).

Correspondence: Omar M. Alhassoon, Ph.D., University of California, San Diego, School of Medicine, Department of Psychiatry, La Jolla, CA 92093,Email oalhassoon@ucsd.edu

\section{G. DOM. Decision-making deficits in alcohol-dependent patients} with and without comorbid personality disorder.

Impairments in decision making are a consistent finding in substance use disorder (SUD) populations. However, decision-making deficits are not specific for SUDs and are also reported in the context of other psychiatric disorders such as antisocial and borderline personality disorders (PDs). Given the frequent comorbidity between SUD and cluster $\mathrm{B} P D$, it might be questioned whether the decision-making impairments typically reported in SUD populations reflect the addictive disorder, the cluster B PD, or a combination of the two. Within the context of this symposium, we report on a study exploring the relation between decision making, alcohol dependence and cluster B comorbidity. We compare the decision-making performance of non-substance-abusing controls on the Iowa Gambling Task (IGT) with the decision-making performance of three abstinent alcohol-dependent samples, i.e., alcoholic patients without any $\mathrm{PD}$, alcoholic patients with a cluster $\mathrm{A}$ or $\mathrm{C}$ PD, and alcoholic patients with a cluster B PD.

The results show that all three alcohol-dependent subsamples performed inferior compared with controls. Between alcoholic subsamples, the alcoholic patients with a cluster A or C PD had the highest IGT score, followed by the alcoholic patients without a PD, while the cluster B alcoholic patients were the most impaired. These findings suggest that impairments in decision making underlie both alcohol dependence and cluster B PD, and alcoholic patients with a comorbid cluster B PD are particularly impaired in their decision making. These deficits may underlie the severe problems that characterize cluster B alcoholic patients specifically in inappropriate behaviors (e.g., poly substance abuse, legal, and professional dysfunction). Clinical implications are discussed. Correspondence: Omar M. Alhassoon, Ph.D., University of California, San Diego, School of Medicine, Department of Psychiatry, La Jolla, CA 92093,Email oalhassoon@ucsd.edu

\section{J. UEKERMANN. Depression and cognitive functioning in alcoholism.}

Alcoholism is associated with a range of cognitive deficits including memory and executive impairments. The deficits have been interpreted in the context of the frontal lobe hypothesis, which asserts a specific vulnerability of the prefrontal cortex to the neurotoxic effects of alcohol. The aim of the present study was the investigation of the influence of depression on cognitive functions in alcoholism. Patients suffering from alcoholism (Alc), patients with depression, but without alcoholism (Dep) and healthy controls (HC) completed a comprehensive neuropsychological test battery. Group comparisons revealed impairments of alcoholic patient with respect to response inhibition, reasoning and free recall. No differences between depressed and non-depressed alcoholics were observed. The deficits are consistent with the frontal lobe hypothesis of alcoholism. However, the results of the investigation suggest that the deficits are not generally exacerbated by comorbid depressive symptoms.

Correspondence: Omar M. Alhassoon, Ph.D., University of California, San Diego, School of Medicine, Department of Psychiatry, La Jolla, CA 92093,Email oalhassoon@ucsd.edu 


\section{Symposium 8 \\ 12:00-1:30 p.m.}

\section{Childhood Head Injury: Incidence, Timing Effects, and Functional Outcomes.}

\section{Chair: Megan Spencer-Smith}

\begin{abstract}
J. PONSFORD, F. VARGHA-KHADEM, L. CROWE, E. HESSEN, M.M. SPENCER-SMITH \& C.F. BENJAMIN. Childhood Head Injury: Incidence, Timing Effects, and Functional Outcomes.

Symposium Description: In spite of the significant impairment that can follow childhood head injury, there are gaps in our knowledge about this condition. The aim of this symposium is to highlight key issues that merit consideration for researchers and clinicians working with head injured populations. This symposium will begin with the presentation of results of a recent retrospective audit of childhood TBI cases that presented to a children's hospital emergency department over a one-year period. In addition to enhancing our knowledge about incidence rates, the audit also collected data on the causes of childhood TBI and the medical management provided. This symposium will also include findings from a prospective study examining neuropsychological outcome in individuals 25 years after head injury, contrasting outcome after child, and adult, head injury. The results from this study demonstrate that childhood is a time of relative vulnerability for long-term effects of head injury. The third paper in this session will also examine the issue of timing of brain insult, but focuses on the development of executive function in children with brain insults sustained at different times in development. Findings from this study suggest a non-linear association between timing of brain insult and executive function development. Finally, a longitudinal prospective study investigating the relationship between cognitive and behavioral aspects of executive function after childhood head injury will be presented.

Correspondence: Megan M. Spencer-Smith, BPsySc., Psychology, Royal Children's Hospital, Gatehouse Street, Parkville, VIC 3052, Australia. E-mail:meganmss@unimelb.edu.au
\end{abstract}

L. CROWE, V. ANDERSON, F. BABL \& C. CATROPPA. Incidence of Traumatic Brain Injury in a Pediatric Population.

Objective: Traumatic brain injury (TBI) is one of the most common childhood injuries and can lead to long-term cognitive and emotional problems. Many families seek treatment for their children following TBI in hospital emergency departments (ED). While previous studies have investigated the ED attendance of children with TBIs, few give a complete picture, for example several studies only include cases that require admission to hospital or adolescents and adults are evaluated together. The purpose of this study is to provide researchers and clinicians with information on the incidence rates, causes and medical treatment of all cases of TBI presenting to a pediatric hospital ED in one calendar year.

Participants and Methods: This retrospective study analyzed information from 1000 children aged 0 to 18 years who attended an Australian pediatric hospital ED following a TBI in 2004.

Results: From the information collected a clear representation of the nature of TBI in the pediatric population is presented.

Conclusions: The presentation will highlight differences across age groups in injury presentation, mechanisms of injury and medical treatment received and will conclude with possible prevention areas.

Correspondence: Megan M. Spencer-Smith, BPsySc., Psychology, Royal Children's Hospital, Gatehouse Street, Parkville, VIC 3052, Australia. E-mail:meganmss@unimelb.edu.au
E. HESSEN, V. ANDERSON \& K. NESTVOLD. Neuropsychological Function 25 years after Mild Head Injury. A Comparison of Outcome after Pediatric and Adult Head Injuries.

Objective: Previous studies suggest that mild pediatric and adult traumatic brain injury (TBI) may have adverse long-term neuropsychological outcomes. Studies have shown that early pediatric TBI can have a more profound impact on development than injuries later in childhood. To our knowledge no study comparing very long-term neuropsychological outcome after mild pediatric and adult TBI has previously been published.

Participants and Methods: In this study 119 subjects were assessed with a standardized neuropsychological test battery 25 years after sustaining mainly mild head injuries. Of these 45 were pediatric head injuries and 74 were adult head injuries.

Results: Both the pediatric and adult groups obtained scores in the normal range. In the pediatric group a significant relationship was found between head injury severity and current neuropsychological function. The most important predictors of poor outcome were length of PTA at injury and a combination of PTA and EEG pathology within 24 hours of injury. In the adult group no significant relationships were found between head injury variables and current neuropsychological function. No significant influence of pre- and postinjury risk factors on current neuropsychological function was evident.

Conclusions: The findings support the view that children sustaining mild or complicated mild TBI may be more vulnerable to subtle longterm neuropsychological outcome than adults sustaining similar head injuries.

Correspondence: Megan M. Spencer-Smith, BPsySc., Psychology, Royal Children's Hospital, Gatehouse Street, Parkville, VIC 3052, Australia. E-mail:meganmss@unimelb.edu.au

M. SPENCER-SMITH, V. ANDERSON, P. ANDERSON \& R. JACOBS. Timing of Brain Insult Effects on Children's Executive Functions. Objective: It is clear from previous research that executive function (EF) after brain insult (BI) is modulated by a range of factors, which serve to explain the observed variance in outcome. While timing of BI has been identified as a key predictor in focal lesion studies, there are some gaps in our knowledge about age at insult and EF. EFs are thought to be particularly vulnerable to early BI due to their extended developmental trajectory, and the protracted development of brain regions underpinning these functions. We investigate the timing of BI effects on EF by examining performance on EF measures in a cohort of children with BI sustained at different times in development. We hope to identify those periods in development when BI has minimal consequences for EF outcome and those times when damage produces most deleterious consequences for EF.

Participants and Methods: Participants were 120 children aged 1016 years with BI sustained at least 1 year prior to assessment. Timing of BI groups was determined based on growth spurts in brain development. Tests from the Delis-Kaplan Executive Function System (D-KEFS) were administered. Lesion factors were also examined to determine their effects on EF

Results: A non-linear relationship between timing of $\mathrm{BI}$ and $\mathrm{EF}$ was found, with timing of BI impacting on the outcome of skills in different ways. Time periods associated with minimal or deleterious EF after BI are discussed with reference to developmental processes.

Conclusions: Timing of $\mathrm{BI}$ is relevant to our understanding of the observed variance in children's $\mathrm{EF}$.

Correspondence: Megan M. Spencer-Smith, BPsySc., Psychology, Royal Children's Hospital, Gatehouse Street, Parkville, VIC 3052, Australia. E-mail:meganmss@unimelb.edu.au 
C. BENJAMIN, D. DWYER, J. KENARDY, V. ANDERSON, R. LE BROCQUE \& R. DOB. Childhood Head Injury: Impact on Cognitive and Behavioural Measures of Executive Function.

Objective: Research has indicated a varying range of cognitive symptoms following childhood traumatic brain injury, including attentional and executive deficits. While changes following severe head injury can be marked, less research has focussed upon how outcomes relate to injury severity and how cognitive deficits manifest outside a clinical setting. In this paper we clarify the nature and prevalence of changes in complex cognitive skills following childhood head injury in a large sample of Australian children. Critically, we evaluate the relationship between performance on formal neuropsychological testing and parental ratings of behavioural functioning in everyday life.
Participants and Methods: Over two hundred 6-14 year olds admitted to a major suburban hospital following a head injury were recruited as part of an ongoing longitudinal study in Melbourne and Brisbane, Australia. Children were assessed 12 weeks post-injury with a battery of cognitive measures including the Contingency Naming Task (CNT) and parental measures of behaviour and executive function including the Behavior Rating Inventory of Executive Function (BRIEF).

Results: Findings and their implications for clinical neuropsychological practice will be discussed.

Correspondence: Megan M. Spencer-Smith, BPsySc., Psychology, Royal Children's Hospital, Gatehouse Street, Parkville, VIC 3052, Australia. E-mail:meganmss@unimelb.edu.au 